\title{
Next-Generation Perturbed Angular Correlation Spectroscopy
}

Matthias A. M. Nagl

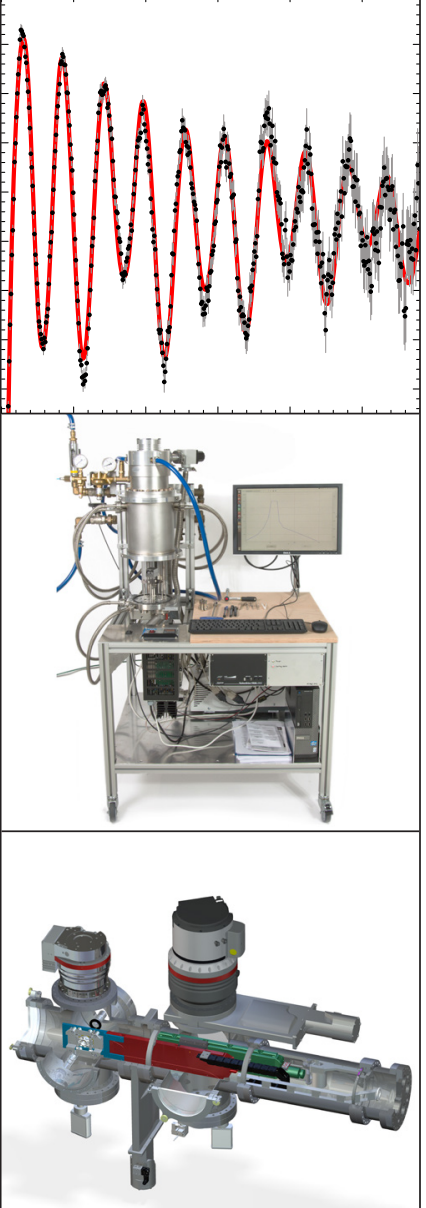





\title{
Next-Generation Perturbed Angular Correlation Spectroscopy
}

\section{DisSERTATION}

zur Erlangung des mathematisch-naturwissenschaftlichen Doktorgrades

\author{
„Doctor rerum naturalium“ \\ der Georg-August-Universität Göttingen \\ im Promotionsprogramm PROPHYS \\ der Georg-August University School of Science (GAUSS)
}

vorgelegt von

\section{Matthias A. M. Nagl}

aus Frankfurt am Main

Göttingen, 20I 4 


\section{Betreunngsausschuss}

Prof. Dr. Hans C. Hofsäss ${ }^{a}$

Dr. Ulrich Vetter ${ }^{a}$

\section{Mitglieder der Prüfungskommission}

Referent: $\quad$ Prof. Dr. Hans C. Hofsäss ${ }^{a}$

Korreferent: $\quad$ Prof. Dr. Hans-Ulrich Krebs ${ }^{b}$

2. Korreferent: Prof. Dr. Reinhard Krause-Rehberg ${ }^{c}$

\section{Weitere Mitglieder der Prüfungskommission}

Prof. Dr. Reiner Kirchheim ${ }^{b}$

Prof. Dr. Wolfram Kollatschny ${ }^{d}$

PD Dr. Kevin Kröninger ${ }^{a}$

Prof. Dr. Tim Salditt ${ }^{e}$

Tag der mündlichen Prüfung

I3. Mai 2014

\footnotetext{
${ }^{a}$ II. Physikalisches Institut, Georg-August-Universität Göttingen ${ }^{b}$ Institut für Materialphysik, Georg-August-Universität Göttingen ${ }^{c}$ Institut für Physik, Martin-Luther-Universität Halle-Wittenberg ${ }^{d}$ Institut für Astrophysik, Georg-August-Universität Göttingen ${ }^{e}$ Institut für Röntgenphysik, Georg-August-Universität Göttingen
} 


\section{Contents}

1 Introduction 1

2 Fundamentals 7

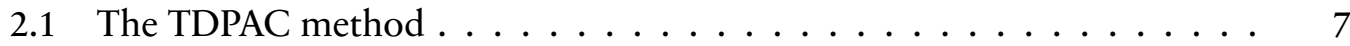

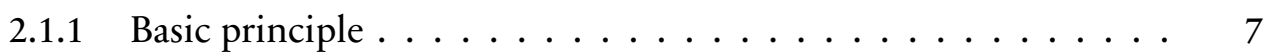

2.1.2 Angular correlation coefficients ............. . 9

2.1.3 Extranuclear perturbations . . . . . . . . . . . . . 10

2.1 .4 Perturbation factors . . . . . . . . . . . . . . . . 12

2.1.5 Practical considerations . . . . . . . . . . . . . . . . 14

2.1.6 Limitations concerning solid state applications of TDPAC . . . 15

2.2 Operating principle of analog TDPAC setups . . . . . . . . . . 17

2.2.1 Spectroscopy amplifiers . . . . . . . . . . . . . . . . . . . 19

2.2.2 Constant fraction discriminators . . . . . . . . . . 20

2.3 Scintillation detectors . . . . . . . . . . . . . . . 20

2.3.1 Operating principle of scintillation detectors . . . . . . . . . 22

2.3 .2 Scintillators ..................... 22

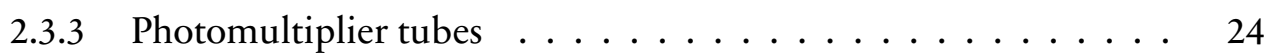

2.4 Nuclide production facilities . . . . . . . . . . . . . 25

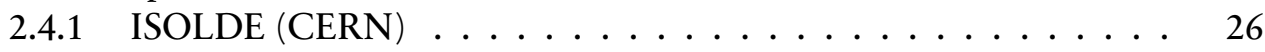

2.4 .2 LOHENGRIN $($ ILL) $\ldots \ldots \ldots \ldots \ldots \ldots \ldots$

3 Motivation and objectives 33

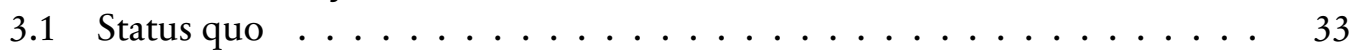

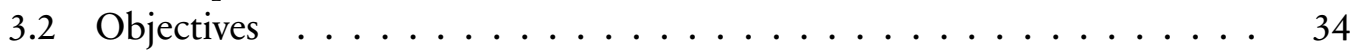

3.2.1 Implementation of a digital six-detector TDPAC setup . . . . . 34

3.2.2 Exploitation of improvements arising from the digital approach . $\quad 35$

3.2.3 Optimizations for measurements of exotic decay cascades . . . . 36

3.2.4 Investigation of improvements for detector design and signal analysis 37

3.2.5 Investigation of new candidates for measurements at ISOLDE . . 37

3.2.6 Development of new facilities for ISOLDE . . . . . . . . . 37

4 Spectrometer setup $\quad 39$

4.1 Hardware setup . . . . . . . . . . . . . . . . . . 40

4.1 .1 Scintillation detectors . . . . . . . . . . . . 41 
4.1.2 Computer equipment . . . . . . . . . . . . . . 43

4.1 .3 Auxiliary electronics . . . . . . . . . . . . . . . . 44

4.2 Software suite . . . . . . . . . . . . . . . . . . . 49

4.2.1 Software design and measurement workflow . . . . . . . . . 49

4.2.2 Data acquisition and preprocessing software . . . . . . . . 51

4.2.3 Data collection and selective recording software . . . . . . . . 53

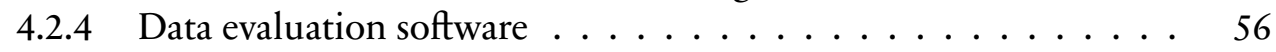

4.3 Conclusions ...................... 63

5 Signal analysis algorithms 67

5.1 Energy determination . . . . . . . . . . . . . . . 68

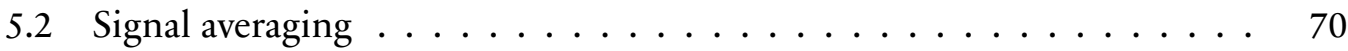

5.3 Constant trigger threshold timing $\ldots \ldots \ldots \ldots \ldots \ldots \ldots$

5.4 Emulated constant fraction discriminator . . . . . . . . . . 73

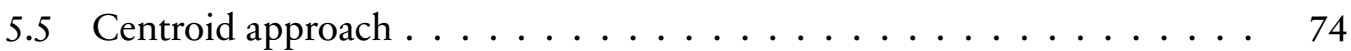

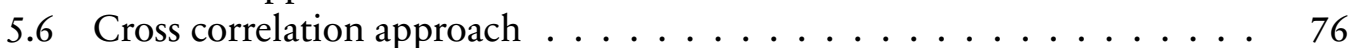

5.7 Deviation of shifted normalized waveform approach . . . . . . . . 77

5.8 Pulse shaping approach . . . . . . . . . . . . . 78

5.9 Digital constant fraction of integral values approach $\ldots \ldots \ldots 79$

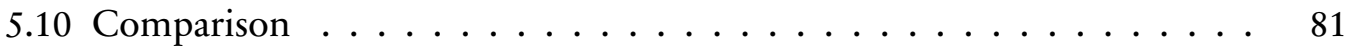

6 Gain drift compensation 85

6.1 A novel drift-compensation method . . . . . . . . . . . . . . 86

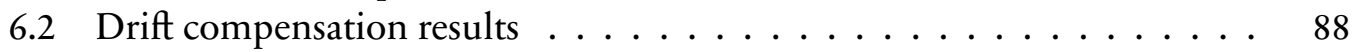

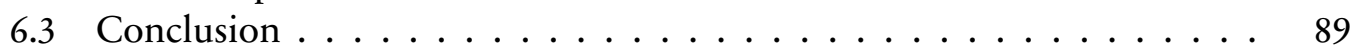

7 Ab initio scintillation detector simulations 93

7.1 Light creation and propagation in scintillation crystals . . . . . . . . . 95

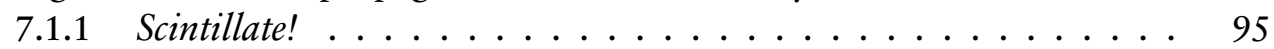

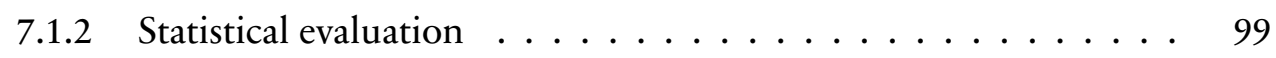

7.1 .3 Result graphs . . . . . . . . . . . . . . . . . 105

7.1 .4 Result Tables . . . . . . . . . . . . . . . . . . . . . . . 110

7.1 .5 Discussion . . . . . . . . . . . . . . . 111

7.2 Light-to-signal-conversion in photomultiplier tubes . . . . . . . . . 113

7.2.1 PMT simulation using Multiply! . . . . . . . . . . . . . . . . 113

7.2.2 Monte-Carlo simulation . . . . . . . . . . . . . . . . 113

7.3 Limitations . . . . . . . . . . . . . . . . . . . 120

7.4 Simulation results . . . . . . . . . . . . . . . . . . . . 123

7.4.1 $\mathrm{LaBr}_{3}: \mathrm{Ce} /$ Photonis XP2020 with Agilent Acqiris U1071A readout 123

7.4.2 $\mathrm{BaF2}$ / Hamamatsu R3377 with Agilent Acqiris U1064A readout . 126

7.5 Simulation based solid angle corrections . . . . . . . . . . . . . 129 
7.6 Conclusions . . . . . . . . . . . . . . . . . . . . 134

8 Test measurements 135

8.1 Fast modulations . . . . . . . . . . . . . . . . . . 135

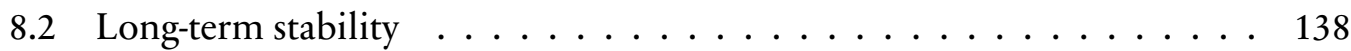

8.3 Multi-cascade evaluation . . . . . . . . . . . . . . . . . . 140

8.4 Simultaneous measurement of two probe nuclides . . . . . . . . . . 142

8.5 Conclusions . . . . . . . . . . . . . . . . . . . . 142

9 Identification of new decay cascades for the TDPAC method 145

9.1 Software . . . . . . . . . . . . . . . . . . 145

9.2 Candidate nuclides . . . . . . . . . . . . . . . . . . . . . . . . 149

9.2 .1 Categories . . . . . . . . . . . . . . . . . . . . . . . 149

9.2 .2 Parameters . . . . . . . . . . . . . . . . . 150

9.2 .3 Constraints . . . . . . . . . . . . . . . . 151

9.2 .4 Limitations . . . . . . . . . . . . . . . . . . 152

10 Facilities for probe implantation, annealing, and magnetization 173

10.1 Implantation and TDPAC facilities for ISOLDE . . . . . . . . . . 173

10.1 .1 Overview . . . . . . . . . . . . . . . . 174

10.1.2 Implantation chamber . . . . . . . . . . . . . . 178

10.1.3 Deceleration and post-acceleration chamber . . . . . . . . . 187

10.1.4 Online TDPAC chamber . . . . . . . . . . . . . . . . 190

10.1.5 Conclusions . . . . . . . . . . . . . . . . . . 194

10.2 Furnace for high temperature annealing . . . . . . . . . . . . 195

10.3 Sample magnetization . . . . . . . . . . . . . . . . . . . . 199

10.3.1 Unsuitability of flux measurements . . . . . . . . . . . . 200

10.3.2 Improved magnetization concepts . . . . . . . . . . . 202

11 Conclusions and outlook 209

11.1 Summary of achievements . . . . . . . . . . . . . . . . . . . . 209

11.2 Future of TDPAC spectroscopy . . . . . . . . . . . . . . . . . . 212

11.2.1 Fitting of $\mathrm{R}(\mathrm{t}) \operatorname{spectra} \ldots \ldots \ldots \ldots \ldots \ldots . \ldots \ldots$

11.2.2 Complementing ab initio simulations . . . . . . . . . . . 214

11.2.3 Availability of probe nuclides . . . . . . . . . . . . . 214

11.2.4 Future spectrometer designs . . . . . . . . . . . . . . . . 215

11.2.5 Synchrotron-radiation-based perturbed angular correlation . . . . 216

11.3 Transferability of results . . . . . . . . . . . . . . . 216 
$\begin{array}{ll}\text { Appendix } & 217\end{array}$

$\begin{array}{lr}\text { A Nuclides and decays } & 219\end{array}$

A.1 TDPAC probe nuclides . . . . . . . . . . . . . . . . . . . . . . 219

A.1.1 In-111 / Cd-111 . . . . . . . . . . . . . . . . . . . 219

A.1.2 Hf-181/ Ta-181 . . . . . . . . . . . . . . . . . . 221

A.1.3 Y-98 $\mu$ s-isomers populated by fission . . . . . . . . . . . . . . 221

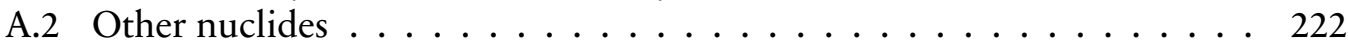

A.2.1 Na-22 . . . . . . . . . . . . . . . . . . . . . 222

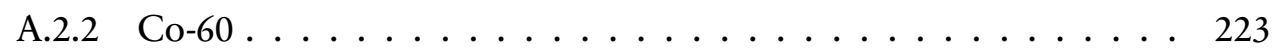

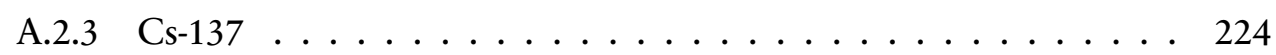

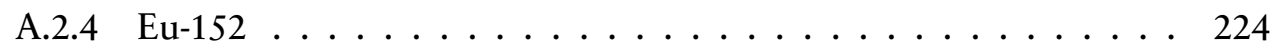

$\begin{array}{lr}\text { Bibliography } & 229\end{array}$

$\begin{array}{ll}\text { List of figures } & 243\end{array}$

$\begin{array}{lr}\text { List of tables } & 247\end{array}$

$\begin{array}{lr}\text { List of publications } & 249\end{array}$

$\begin{array}{lr}\text { Acknowledgements } & 251\end{array}$

viii 


\section{Chapter 1}

\section{Introduction}

In recent years the technological development of experimental methods in nuclear physics got new momentum due to the availability of analog-to-digital converters with sampling rates advancing to the extremely short time-scales in the nano- and subnanosecond range occurring in these experiments in combination with the availability of sufficient amounts of processing power for the real-time application of digital signal processing (DSP) techniques to converted signals. Since thereby the prerequisites are met, nuclear spectroscopy is ready for the same group of transitions that revolutionized many other areas of science and technology before: The transition from analog to digital signal processing, the transition from customized to universal hardware, and the transition from hardware defined functionality to software defined functionality. While the advantages of these transitions are not obvious at first glance, they are all the more pervasive in the long view: Digital universal hardware initially introduces additional complexity. Increased complexity however is not associated with increased cost and probability of failure if due to its versatility this kind of hardware can be used in much higher quantities in different problem areas. This finally results in higher reliability at much lower cost. Additionally, software defined functionality provides a wealth of new opportunities by reducing development effort and simultaneously making it possible to evaluate a larger amount of alternative approaches and parameters for finding the optimum choice. While areas like information and communication technology, industrial control, or radio technology are pioneering in passing the mentioned transitions, the same process is just at the beginning for nuclear spectroscopy methods. As stated above, this is mostly due to the even today challenging demands of these experimental techniques, which first had to be fulfilled by available universal hardware.

Nuclear condensed matter physics subsumes a group of tools used in condensed matter physics that originate in a number of nuclear physics discoveries made throughout the $20^{\text {th }}$ century. For instance the discovery of the positron by Carl D. Anderson ${ }^{1}$ in 1932 was followed by the development of positron annihilation spectroscopy between the 1940s and $1960 \mathrm{~s}^{2}$, which has now applications in different areas of materials science ${ }^{3}$,

\footnotetext{
${ }^{1}$ Anderson, 1932.

${ }^{2}$ Beringer and Montgomery, 1942; MacKenzie et al., 1967.

${ }^{3}$ Krause-Rehberg and Leipner, 1999; Pethrick, 1997.
} 
and positron emission tomography ${ }^{1}$ between the 1960s and 1980s, which is today a wellestablished method in medical imaging. Also Mößbauer spectroscopy as well as nuclear magnetic resonance (NMR) spectroscopy and muon spin spectroscopy ( $\mu$ SR for muon spin rotation) belong to this group of experimental methods.

Other tools developed in the context of nuclear physics like ion accelerators, synchrotrons, and nuclear reactors provide a wide range of opportunities to study condensed matter by means of Rutherford backscattering spectrometry, evaluation of channeling effects, synchrotron radiation, and neutron scattering among others.

One of the less commonly known nuclear physics methods applicable to condensed matter physics is time differential perturbed $\gamma-\gamma$ angular correlation (TDPAC) spectroscopy. Originally developed for the determination of level schemes and gyromagnetic ratios of excited nuclei, it allows the determination of these ratios in case of a precise knowledge of the magnetic fields at the probe nuclei's locations. Once the gyromagnetic factor is known, the same excited state can however be used as a probe for unknown magnetic fields. This makes it an interesting approach for characterizations in the domain of condensed matter physics as pointed out for the first time by Heer and Novey (1959).

Additionally to its sensitivity to magnetic fields, TDPAC spectroscopy can also be used to characterize electric field gradients at probe atoms' sites if the used exited state provides an electric quadrupole moment. Again, nuclear quadrupole moments are routinely determined in the context of nuclear physics experiments as property of excited nuclear states and therefore data for many nuclides and excitation levels is readily available today. The sensitivity to electric field gradients is particularly interesting for the investigation of solid state properties because the electric field gradient at the nuclei of probe atoms depends on the charge distribution around these probes and can therefore provide detailed information concerning the probes' environments.

Today TDPAC spectroscopy covers a wide range of applications in condensed matter physics. Some notable examples are:

- Surface and interface magnetism (Bertschat, 2005) since TDPAC allows studying of buried interfaces on the atomic scale

- Semiconductors studies-for instance concerning defect complexes (e.g. Abiona, Kemp, and Timmers, 2014), the diffusion of hydrogen (e. g. Deicher, 2007), or the existence and geometry of certain defect centers under different annealing conditions (e.g. Unterricker, Butz, and Saibene, 1992)

- Characterization of ceramic materials concerning phase transitions (e.g. Lopes et al., 2008)—especially under high pressure conditions (e. g. Halevy et al., 2007)—as well as ligand configuration and probe positions in the unit cell (Jürgens, 2013)

\footnotetext{
${ }^{1}$ Phelps et al., 1975.
} 
- In biochemistry, there are a large number of applications, many related to interactions between metal ions and proteins. A comprehensive overview including many references to successful studies was assembled by Hemmingsen, Sas, and Danielsen (2004). Other reviews were assembled by Chain, Ceolin, and Pasquevich (2008) and Hemmingsen, Stachura, et al. (2010).

- Electronic structure of point defects in metals; an overview was published by Zacate and Jaeger (2011).

More comprehensive overviews were assembled by Hemmingsen and Butz (2006) and also Karlsson (1995).

An important advantage of TDPAC spectroscopy in comparison to other microscopic methods is that the $\gamma$ radiation, which carries information from the sample to the detectors, is able to pass even through thin layers of metal thus allowing the measuring of samples under extreme conditions (for instance high pressure, high temperature, low temperature ultrahigh vacuum among others). Additionally, external magnetic fields are not required which predestinates the method for cases where external magnetic fields could disturb the sample's properties of interest.

Despite this convenient starting position, TDPAC spectroscopy is a challenging method. This is not due to missing knowledge about probe nuclei but due to additional requirements of the method, which will be discussed in the next chapter. Although the method is known since 1960s and the technology used in spectrometers was among the most modern available at that time, development almost stalled in the decades thereafter. One of the reasons for this situation probably was that due to the early achievements it was difficult to realize substantial progress.

The objective of this thesis is the exploitation of chances emerging due to the mentioned transitions from analog to digital signal processing, from customized to universal hardware, and from hardware defined functionality to software defined functionality in the context of TDPAC spectroscopy. Furthermore, a set of tools helping to overcome obstacles traditionally related to this experimental method thereby extending the scope of its applicability was developed. Due to similar challenges occurring throughout the whole family of nuclear condensed matter physics methods as well as nuclear spectroscopy in general, many of the achievements presented in the following chapters are also applicable to a much wider range of problems and use cases.

As shown in fig. 1.1, a successful TDPAC measurement employs a number of different challenges, which concern the preparation of samples and probes, the actual spectrometer and data taking, as well as finally the analysis and interpretation of its results. This work covers almost all of the essential parts where excellent solutions were missing or substantial improvements could be achieved due to said technological progress.

While chapter 2 summarizes basic principles of the TDPAC method and its applications, chapter 3 presents the status quo at the beginning of this work and defines its motivation and objectives. Each of the following chapters focuses on certain aspects, which 


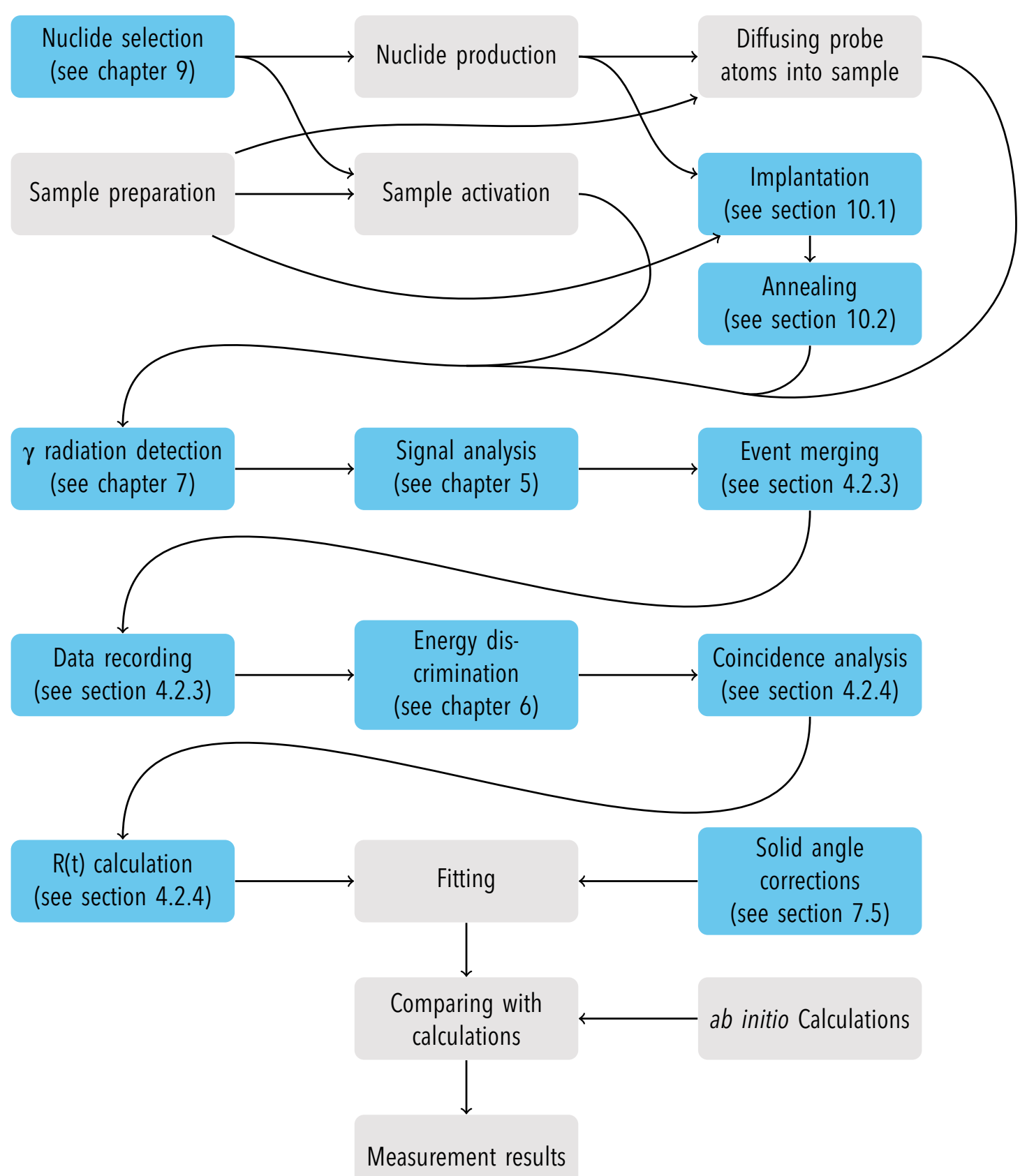

Figure 1.1: Steps of a TDPAC measurement. Highlighted steps are subject of this work. 
were investigated and improved. In chapter 11, all achievements are summarized and an outlook concerning remaining challenges is supplied. 



\section{Chapter 2}

\section{Fundamentals}

In this chapter's first sections, the principle of time differential perturbed $\gamma-\gamma$ angular correlation spectroscopy is introduced. Subsequently, several other introductory sections concerning relevant concepts and equipment follow.

\subsection{The TDPAC method}

The TDPAC method allows the measurement of magnetic fields and/or electric field gradients at the location of a population of probe atoms. From the field properties at the probe atoms' sites it is possible to draw conclusions concerning for instance the surrounding crystal structure and defects such as vacancies and impurities. Even today not many experimental techniques answering the same questions as TDPAC spectroscopy are available. Most direct methods for the measurement of magnetic fields and electric field gradients are not appropriate for microscopic measurements inside bulk material rather than at the surface. Nuclear condensed matter physics methods like nuclear magnetic resonance spectroscopy and Mößbauer spectroscopy, which rely on similar principles as TDPAC spectroscopy and address the same problem domain, are complementary rather than alternatives because of their different strengths and limitations.

A number of comprehensive articles on perturbed angular correlation exists among which Frauenfelder and Steffen (1965) as well as Hamilton (1975) are especially noteworthy. Applications in solid state physics were first emphasized by Heer and Novey (1959) and comprehensive overviews and introductions to this aspect of perturbed angular correlation experiments were published by Karlsson (1995) and Hemmingsen and Butz (2006). The following sections therefore focus on a concise overview pointing out aspects that are of special relevance for this thesis.

\subsubsection{Basic principle}

The basic requirement for a TDPAC measurement is a population of appropriately excited probe nuclei at the positions of interest for the measurement. Usually, these excited nuclei are produced by radioactive decay of a mother nuclide that was introduced in the 
examined substrate before. Alternatively, it is also possible to create excited nuclei by other means like for instance inelastic scattering of a particle beam (Nielsen et al., 1983).

For being appropriate, the excitation of the nuclei have to decay through a cascade with an intermediate level, a populating transition emitting a $\gamma$ photon, and a depopulating transition emitting another $\gamma$ photon (variants where $\alpha$ or $\beta$ particles are detected as signal of the populating process exists). An example of such an appropriate cascade is shown highlighted later in section 2.1.5.

Provided that $\gamma$ photons are not emitted isotropically with respect to the change of the $m$ quantum number during a transition, the mere detection of a populating $\gamma$ photon allows for a prediction of the amplitude of the intermediate nucleus' $m$ substates (i. e. its alignment) assuming that the used detector covers only a limited fraction of the sample's emission solid angle. This means that after detection of the first $\gamma$ photon by a given detector, the amplitudes of the emitting intermediate nucleus' $m$ substates are known. Moreover, at least some of the $m$ substates are coherently superimposed. Another view of the same situation is that by the detection of $\gamma$ photons emitted during population of the intermediate state within the limited solid angle covered by the detector a sub-population of all decaying nuclei exhibiting a certain distribution of alignments is selected.

Due to this knowledge of the nucleus' $m$ substate amplitudes and given that the direction of emission of the second $\gamma$ photon occurring due to the depopulation of the intermediate level again depends on the change of the nucleus' $m$ quantum number, the resulting direction of emission can be anisotropic, i. e. determined by the direction of the first emission.

The anisotropy of $\gamma$ emissions with respect to the change of the $m$ quantum number of the nucleus during both decays is thus a prerequisite for the anisotropy of the direction of the second emission with respect to the first. Furthermore, the amount of anisotropy of the second emission with respect to the first corresponds to both, the degree of the nucleus' alignment given the first emission's direction as well as the amount of the second emission's anisotropy with respect to this alignment.

Without extranuclear perturbations of the decay cascade, the probability to detect the populating and depopulating $\gamma$ radiation from a single nucleus at an angular difference of $\theta$ is

$$
W(\theta)=\sum_{k} A_{k k} P_{k}(\cos \theta)
$$

where $P_{k}(x)$ are Legendre polynomials. Valid values for $k$ are

$$
k=0,2, \ldots, \min \left(2 I, L_{1}+L_{1}^{\prime}, L_{2}+L_{2}^{\prime}\right),
$$

as discussed by Frauenfelder and Steffen (1965, compare pages 1023 and 1024). For all practical purposes, $k$ values bigger than 4 are discarded. Only even $k$ need to be considered since odd values yield zero due to applicable selection rules. $I$ is the intermediate level's spin whereas the $L_{1}$ and $L_{2}$ variables denote the multipole order of populating and 
depopulating $\gamma$ radiations, respectively. In case of pure multipole transitions $L_{1}^{\prime}=L_{1}$ and $L_{2}^{\prime}=L_{2}$. For mixed multipole transitions the appropriate multipole orders have to be filled in.

The $A_{k k}$ elements are called angular correlation coefficients and quantify the total anisotropy occurring due to said degree of the nucleus' alignment and the depopulating radiation's anisotropy. In case of unperturbed angular correlations, the values of both indices of these coefficients, as defined in the next section, are set to identical values. This is the reason for the duplicate index in $A_{k k}$.

\subsubsection{Angular correlation coefficients ${ }^{1}$}

Total emission anisotropies of populating and depopulating $\gamma$ photons are usually quantified by an angular correlation coefficient $A_{k_{1} k_{2}}$.

The calculation of angular correlation coefficients $A_{k_{1} k_{2}}$ is based on the orientation coefficients

$$
B_{\Lambda}\left(\gamma_{1}\right)=\frac{F_{\Lambda}\left(1,1, I_{i}, I\right)-2 \delta\left(\gamma_{1}\right) F_{\Lambda}\left(1,2, I_{i}, I\right)+\delta^{2}\left(\gamma_{1}\right) F_{\Lambda}\left(2,2, I_{i}, I\right)}{1+\delta^{2}\left(\gamma_{1}\right)},
$$

which quantify the degree of nuclear orientation after detection of the $\gamma$ photon emitted during population of the intermediate level, and the directional distribution coefficients

$$
A_{\Lambda}\left(\gamma_{2}\right)=\frac{F_{\Lambda}\left(1,1, I_{f}, I\right)+2 \delta\left(\gamma_{2}\right) F_{\Lambda}\left(1,2, I_{f}, I\right)+\delta^{2}\left(\gamma_{2}\right) F_{\Lambda}\left(2,2, I_{f}, I\right)}{1+\delta^{2}\left(\gamma_{2}\right)},
$$

which quantify the succeeding emission anisotropy of the $\gamma$ photon emitted during depopulation of the intermediate level as defined by Krane and Steffen (1970, compare eqs. 10 and 11 ).

In these equations $I$ is the intermediate level's spin while $I_{i}$ and $I_{f}$ are the initial and final levels' spins. $\delta\left(\gamma_{1}\right)$ is the multipole mixing ratio of the first emitted $\gamma$ photon originating from the transition $I_{i} \longrightarrow I$ whereas $\delta\left(\gamma_{2}\right)$ is the mixing ratio of the second $\gamma$ photon from the transition $I \longrightarrow I_{f}$.

The F-coefficients are defined by Frauenfelder and Steffen (1965, compare eq. 96) as

$$
\begin{gathered}
F\left(L, L^{\prime}, I^{\prime}, I\right)=\left[(2 L+1)\left(2 L^{\prime}+1\right)(2 I+1)(2 k+1)\right]^{\frac{1}{2}} \\
\cdot(-1)^{I \prime+I-1}\left(\begin{array}{ccc}
L & L^{\prime} & k \\
1 & -1 & 0
\end{array}\right)\left\{\begin{array}{ccc}
L & L^{\prime} & k \\
I & I & I^{\prime}
\end{array}\right\}
\end{gathered}
$$

including the Wigner 3-j and 6-j symbols.

Using eqs. (2.3) and (2.4), the angular correlation coefficients $A_{k_{1} k_{2}}$ can be calculated as

\footnotetext{
${ }^{1}$ This section contains revised parts from Nagl, Barbosa, et al. (2013)
} 


$$
A_{k_{1} k_{2}}=B_{k_{1}}\left(\gamma_{1}\right) \cdot A_{k_{2}}\left(\gamma_{2}\right)
$$

Unfortunately, $A_{k_{1} k_{2}}$ is usually defined with $k_{1}=k_{2}$ in literature (compare eq. 98 in Frauenfelder and Steffen, 1965; eq. 14.31 in Hamilton, 1975). This simplification is based on the disappearance of the interference terms in unperturbed cases. Since this precondition is usually not fulfilled under the influence of quadrupole interactions, the mixed terms are however relevant for solid state physics applications of TDPAC and are in fact often used in literature although not explicitly defined (e. g. Frauenfelder and Steffen, 1965, p. 1127).

\subsubsection{Extranuclear perturbations}

Given a population of excited nuclei with a decay cascade and anisotropically emitted $\gamma$ photons as described above, a fixed relation between the angular difference between detector positions and the probability to detect both photons belonging to a cascade occurs. Angular correlation measurements determining this relation usually use a setup involving two detectors, one of which is movable, and can for instance be applied to obtain information concerning the spins of involved nuclear levels.

In case of isomeric intermediate levels, where a certain amount of time passes between the populating and depopulating transitions, extranuclear fields can perturb the alignment of the nucleus. Prerequisite for such a perturbation is an intermediate state showing appropriate magnetic dipole or electric quadrupole moments, which provide a working point for extranuclear magnet fields or electric field gradients, respectively. Due to the hyperfine interaction said external fields remove the $m$ substates' degeneracy and cause interferences between these substates' phase factors, which exhibit energy dependentand thus now different-oscillation frequencies. The influence of the extranuclear field on the alignment of the nucleus is a function of the amount of time passing between the definition of the nucleus' alignment (i.e. the emission of the populating radiation) and the determination of the second radiation's emission direction. Since the intermediate state's life-time for a particular nucleus can be considered an exponentially distributed random number, a range of different time differences occurs for an ensemble of decaying nuclei. Therefore, for a set of fixed detectors, angular correlation probabilities can be determined depending on the time difference between both decays rather than depending on detector angles. Such a measurement may provide periodic modulations in a histogram where the abundance depending on the time difference-i.e. the individual life-time of single probe atoms' excitations-is plotted because the nucleus' alignment and thereby the emission probability towards a given detector changes periodically due to the extranuclear field. A general description of this periodically changing probability $\overline{W\left(\theta_{1}, \varphi_{1}, \theta_{2}, \varphi_{2}, T\right)}$ of detecting a coincidence using two detectors with given angles $\theta_{1}, \varphi_{1}, \theta_{2}, \varphi_{2}$ in a time interval of length $\tau_{0}$ centered at $T$ is provided by a combination of eqs. 208, 209, and 213 from Frauenfelder and Steffen (1965): 


$$
\overline{W\left(\theta_{1}, \varphi_{1}, \theta_{2}, \varphi_{2}, T\right)}=\frac{\int_{T-\tau_{0} / 2}^{T+\tau_{0} / 2} \mathrm{e}^{-\frac{t}{\tau}} W\left(\theta_{1}, \varphi_{1}, \theta_{2}, \varphi_{2}, t\right) \mathrm{d} t}{\int_{T-\tau_{0} / 2}^{T+\tau_{0} / 2} \mathrm{e}^{-\frac{t}{\tau}} \mathrm{d} t}
$$

In this notation the intermediate level's decay cancels out. $\tau$ is the life-time of the intermediate level and $W\left(\theta_{1}, \varphi_{1}, \theta_{2}, \varphi_{2}, t\right)$ is defined as

$$
\begin{aligned}
W\left(\theta_{1}, \varphi_{1}, \theta_{2}, \varphi_{2}, t\right)=\sum_{k_{1}, k_{2}, N_{1}, N_{2}} A_{k_{1} k_{2}} & {\left[\left(2 k_{1}+1\right)\left(2 k_{2}+1\right)\right]^{-\frac{1}{2}} } \\
& \times G_{k_{1} k_{2}}^{N_{1} N_{2}}(t)\left(Y_{k_{1}}^{N_{1}}\left(\theta_{1}, \varphi_{1}\right)\right)^{*} Y_{k_{2}}^{N_{2}}\left(\theta_{2}, \varphi_{2}\right) .
\end{aligned}
$$

$Y_{k}^{N}(\theta, \varphi)$ are the well-known spherical harmonics while the perturbation factor $G_{k_{1} k_{2}}^{N_{1} N_{2}}(t)$ is the only unknown component. It is defined as

$$
\begin{aligned}
G_{k_{1} k_{2}}^{N_{1} N_{2}}(t) & =\sum_{m_{a}, m_{b}}(-1)^{2 I+m_{a}+m_{b}} \sqrt{\left(2 k_{1}+1\right)\left(2 k_{2}+1\right)} \\
& \times\left(\begin{array}{ccc}
I & I & k_{1} \\
m^{\prime}{ }_{a} & -m_{a} & N_{1}
\end{array}\right)\left(\begin{array}{ccc}
I & I & k_{2} \\
m^{\prime}{ }_{b} & -m_{b} & N_{2}
\end{array}\right)\left\langle m_{b}|\Lambda(t)| m_{a}\right\rangle\left\langle m_{b}^{\prime}|\Lambda(t)| m^{\prime}{ }_{a}\right\rangle^{*}
\end{aligned}
$$

This perturbation factor completely describes the influences of extranuclear perturbations on the time dependence of the detection probability. However, the matrix elements of the contained time evolution operator $\Lambda(t)$ are not obvious.

Examples for the perturbation due to different types of external fields as well as differences occurring for different probe nuclides can be deduced from Wegner (1985) as well as Butz (1989) and the erratum in Butz (1992) where results for the aforementioned time evolution matrix and thus the perturbation factor are contained for several cases.

The equations shown above do not yet consider the finite size of used detectors. According to Lawson and Frauenfelder (1953), the angular correlation coefficients $A_{k_{1} k_{2}}$ are usually replaced by corrected values $A_{k_{1} k_{2}}^{\text {eff }}$ defined as follows:

$$
A_{k_{1} k_{2}}^{\mathrm{eff}}=Q_{k_{1} k_{2}} \cdot A_{k_{1} k_{2}}
$$

The correction factors $Q_{k_{1} k_{2}}$ can be determined using several alternative methods as described by Frauenfelder and Steffen (1965) on page 1192. 


\subsubsection{Perturbation factors}

While the generic form of the perturbation factor introduced in eq. (2.9) is applicable for all kinds of perturbations, the contained matrix elements of the time evolution operator $\Lambda(t)$ have to be determined before actual calculations are possible.

To provide an idea of perturbation factors commonly encountered in TDPAC experiments, two exemplary cases are described:

Static magnetic dipole interactions The perturbation factor in case of static magnetic dipole interactions can be written as (Frauenfelder and Steffen, 1965, page 1113)

$$
G_{k_{1} k_{2}}^{N_{1} N_{2}}(t)=\exp \left(-\mathrm{i} N \omega_{L} t\right) \delta_{k_{1} k_{2}} \delta_{N_{1} N_{2}}
$$

where $\delta_{i j}$ are Kronecker deltas and $\omega_{L}$ is the Larmor frequency, which can e.g. be calculated from tabulated magnetic moments (cf. section 9.2, here the tabulated values are denoted by $g$ for better distinguishability from the nuclear magneton $\mu_{\mathrm{N}}$ ), according to

$$
\omega_{L}=\frac{\left|g \mu_{\mathrm{N}} B\right|}{I \cdot \hbar} .
$$

For powder samples consisting of randomly oriented crystallites, averaging according to (Frauenfelder and Steffen, 1965, page 1111) yields

$$
G_{22}(t)=\frac{1+2 \cos \left(\omega_{L} t\right)+2 \cos \left(2 \omega_{L} t\right)}{5} .
$$

With eq. (2.19) from the next section now a direct relation between measured spectra and the magnetic field at the probe nuclei's locations exists. The anticipated spectra consist of two vertically shifted and superimposed cosines oscillating with the Larmor frequency and two times the Larmor frequency, respectively. An example where this relation is used for the determination of a magnetic field at the locations of probe nuclei is shown in section 8.1.

Static electric quadrupole interactions The electric field gradient can be described by a second rank Cartesian tensor $\mathscr{V}$. This tensor's components are related to the actual electric field by

$$
V_{i k}=\frac{\partial^{2} V}{\partial x_{i} \partial x_{k}}
$$

where $V$ is the electrostatic potential.

To obtain an expression for the perturbation factor, $\mathscr{V}$ is converted to the principal axes system where it assumes diagonal shape (i.e. all off-diagonal elements vanish) and 
the additional condition $\left|V_{z z}\right| \leq\left|V_{y y}\right| \leq\left|V_{x x}\right|$ is met. The deviation of the electric field gradient from axial symmetry around this coordinate system's $\mathrm{z}$ axis is quantified by

$$
\eta=\frac{\left|V_{x x}-V_{y y}\right|}{\left|V_{z z}\right|}
$$

according to Karlsson (1995, in other sources the necessary absolute values are missing) and takes values between 0 and 1. Laplace's equation holds for the electrostatic potential which means that $V_{z z}+V_{y y}+V_{x x}=0$. Due to this additional constraint, the two parameters $V_{z z}$ and $\eta$ completely describe the electric field gradient.

Another relevant convention is the definition of the quadrupole frequency

$$
\omega_{Q}=\frac{e Q V_{z z}}{4 I(2 I-1) \hbar}=\frac{2 \pi v_{Q}}{4 I(2 I-1)}
$$

where $e Q$ is the intermediate level's electric quadrupole moment (which is not related to the solid angle correction factors $Q_{k_{1} k_{2}}$ from eq. (2.10)) and $I$ its spin. $v_{Q}$ is the quadrupole interaction frequency, which is sometimes specified in literature but is of little additional use.

Unfortunately, there is no simple expression for the perturbation factor in case of electric quadrupole interactions. Usually, a series expansion with numerical determination of parameters is used. An example for this approach is the publication from Wegner (1985). Analytical approaches were published by Kuriplach, Novotný, and Procházka (1994) and Butz (1989) (with corrections in Butz, 1992).

In case of an axially symmetric electric field gradient $(\eta=0)$ the smallest frequency occurring in a TDPAC measurement is $\omega_{0}=3 \cdot \omega_{Q}$ for integer intermediate level spins and $\omega_{0}=6 \cdot \omega_{Q}$ for integer intermediate level spins, respectively. The maximal number of frequencies observable from a single probe fraction is $n(n-1) / 2$ where $n$ is the number of eigenvalues of the relevant Hamiltonian, which can be calculated from the intermediate level's spin $I$ as $n=2 I+1$. Fortunately, in many common cases eigenvalues are degenerate. For spin $5 / 2$ for instance, only three frequencies occur. Results for other spin values were summarized by Butz (1989).

Combined static magnetic and electric interactions This type of interaction can also be evaluated. An introduction can be found in Frauenfelder and Steffen (1965) beginning on page 1123.

Fractions In most real TDPAC measurements, probe atoms exist in different surroundings. For instance a number of atoms can be situated on regular lattice sites while others are located near defects or on the sample's surface. Still others could form clusters. Such fractioning of the probe atom population yields a superposition of different perturbation factors, which can be expressed by a weighted sum. The weight factors $f_{i}$ as well as each fraction's parameters can usually be determined with the help of appropriate fitting tools. 
Damping Electric field gradient values at probe atoms' sites are sensitive to variations in the probe location as well as variations in the crystal field, e. g. due to defects. In fact, even the probe's decay itself can induce displacement due to recoil and modify the probe's environment because of its changed charge. Such fluctuations among the probes belonging to a fraction of a TDPAC spectrum have a damping effect because the oscillation frequencies - which depend on the EFG strength, cf. eq. (2.16) — get slightly out of phase. This damping is usually expressed by a parameter $\delta$, which is often defined as

$$
\delta=\frac{\sigma}{\omega_{0}}
$$

with $\omega_{0}$ being the base frequency of the perturbation factor and $\sigma$ being the width of the interaction frequency's distribution (Karlsson, 1995, page 59; Frauenfelder and Steffen, 1965, page 1121). Typical examples used as estimate are Gaussian as well as Lorentzian distributions.

\subsubsection{Practical considerations}

Spectrometers used for the recording of time differential perturbed $\gamma-\gamma$ angular correlations usually consist of four or six detectors aligned at $90^{\circ}$ angles versus each other. In case of four detectors their axes share a common plane while for six detectors the axes are aligned perpendicular to the faces of a cube. Since each detector can detect populating as well as depopulating radiations, a total of eight $90^{\circ}$ detection pairs and four $180^{\circ}$ detection pairs exist in case of four detectors. For six detector setups, the number of $90^{\circ}$ pairs increases to 24 and the number of $180^{\circ}$ pairs increases to six.

From each of these detector pairs a coincidence spectrum is recorded. Before these spectra are evaluated, the zero points of their time axes are aligned in order to compensate for different delays due to deviations in cable lengths of the used detectors. Additionally, the background level arising from random coincidences is determined and subtracted. The results are then grouped according to the angular difference and for each of the two difference angles a geometric mean spectrum is calculated. This has the advantage that different detector efficiencies cancel to first order (Butz, 1989). Of course, only equivalent spectra can be averaged. Spectra belonging to a group of identical angular difference are automatically equivalent in case of a powder sample with random orientation of a sufficiently high number of crystallites. In case of single crystal samples it is usually not possible to average all spectra belonging to a group in case of six detector setups. However, the advantage of six detectors in these cases is that if digital setups with the possibility of post-measurement evaluation as introduced in chapter 4 are used, it becomes possible to study several orientations of crystal axes relative to different groups of detectors at once. The differences between such configurations and specific advantages and drawbacks were described by Wegner (1985). 
Following the averaging of equivalent spectra, the resulting two spectra are combined to a single spectrum by calculating the ratio

$$
R(t)=\frac{2}{3}(1+n) \frac{\bar{W}\left(180^{\circ}, t\right)-\bar{W}\left(90^{\circ}, t\right)}{\bar{W}\left(180^{\circ}, t\right)+n \bar{W}\left(90^{\circ}, t\right)}
$$

according to Arends et al. (1980). Usually, $n$ is set to 2. In this ratio, the exponential decay factors describing the intermediate state's life-time cancel out. Figure 2.1 shows an example for such an $R(t)$ spectrum. Arends et al. (1980) also showed that in case of powder samples consisting of randomly oriented crystallites and neglectable $A_{44}$

$$
R(t)=A_{22} G_{22}(t) .
$$

Together with eqs. (2.11) and (2.12), this allows for direct determination of the magnetic field at the probe atoms' locations. In more complicated cases, theoretical $G_{k_{1} k_{2}}^{N_{1} N_{2}}(t)$ functions can be fitted to experimental data by inserting the theoretical $G_{k_{1} k_{2}}^{N_{1} N_{2}}(t)$ into eqs. (2.8) and (2.18) and calculating the theoretical $R(t)$ spectrum before determining deviations between theoretical and measured spectra.

Although the $90^{\circ}$ arrangement of detectors is beneficial with respect to collected statistics due to the large number of identical spectra (at least for powder samples with randomly orientated perturbing fields), there are cases where different angles are preferable. For instance this can be the case if single crystal samples are analyzed and/or the electric field gradient's orientation is of interest. An example is shown by Ohtomo et al. (2002).

\subsubsection{Limitations concerning solid state applications of TDPAC}

Besides the obvious limitation that a measurement is only possible using an appropriate probe nuclide, which has to be producible and introducible into the material of interest, some additional constraints limit the TDPAC method's applicability. Most important is the limited range of field strengths that can be measured. Depending on the strength of the magnetic field or electric field gradient on one hand and the gyromagnetic factor or nuclear quadrupole moment of the intermediate state on the other hand, a number of periodicities of the interaction is expected. These periods' lengths have to fit into the maximum time difference, which is defined by the decreasing signal to noise ratio of the TDPAC signal. For long running measurements typically five times the half-life of the intermediate state is a reasonable upper limit. Additionally, the period length has to be long enough to be resolvable with the used spectrometer. Typical time resolutions are in the range between $200 \mathrm{ps}$ and 2500 ps depending on the spectrometer's performance and the energy of the detected radiations. For the spectrometer described in chapter 4 used with ${ }^{111} \mathrm{In}$, the measurement of interaction half-periods roughly between $1.5 \mathrm{~ns}$ to $400 \mathrm{~ns}$ is realistic. For measurements involving a very high number of recorded coincidences these limits can be slightly extended. 

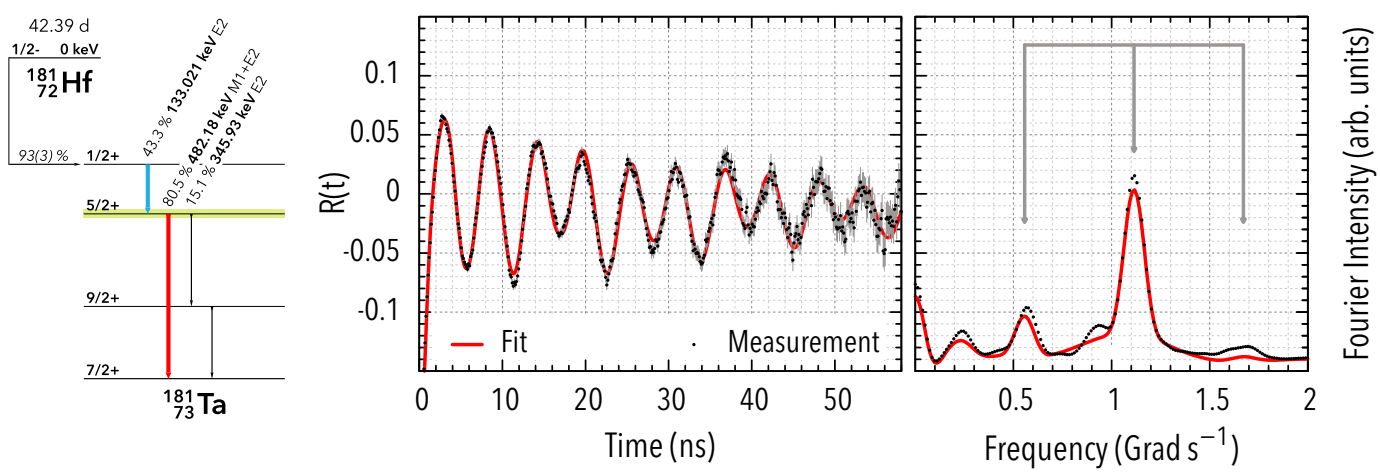

Figure 2.1: Exemplary $R(t)$ spectrum and the associated Fourier spectrum. It shows data from ${ }^{181} \mathrm{Hf}$ probe atoms implanted into an AIN sample. In the level scheme on the left the decay cascade used for the measurement is highlighted. The intermediate state's spin is $5 / 2$ and its half-life $10.8 \mathrm{~ns}$. More details concerning ${ }^{181} \mathrm{Hf}$ as TDPAC probe including a complete level scheme can be found in appendix A.1.2. Calculation of $R(t)$ from the coincidence spectra of the measurement according to eq. (2.18) removed the decay exponentials and created a spectrum, which shows distinct oscillations. Due to the decrease of signal-to-noise ratio in coincidence spectra towards larger time differences - which occurs because the signal is a modulation of the decreasing decay exponential while background noise is constant-the shown error bars are small close to $t=0$ and grow towards higher time values. Damping of the oscillations is not related to the intermediate level's decay but to small differences in the field strength sensed by different probe atoms, which makes their interaction frequencies fluctuate around a central value (see the paragraph about damping on page 14). Due to the intermediate level's spin of $5 / 2$ and the electrical quadrupole interaction observed in this measurement, three frequencies occur for each measured fraction. In the Fourier spectrum on the right the three frequencies belonging to the primary visible oscillation are indicated. For single crystal samples with uniform orientation of the electric field gradients (like the one shown here) the amplitudes of the frequency components are related to the EFG's orientation relative to the detectors. The visible frequency relation of $1: 2: 3$ is in case of spin $5 / 2$ equivalent to $\eta=0$ which means that the probes of the oscillating fraction are located on places in the crystal with axial symmetry. Angular frequencies $\omega$ in the Fourier spectrum are labelled in units of $\mathrm{rad} \mathrm{s}^{-1}$ rather than as ordinary frequency $v$ in $\mathrm{Hz}$ as usual in the TDPAC context. Both are related by $\omega=2 \pi v$. It is possible to conclude from $\eta=0$ that the probe atoms causing the oscillation were located on places in the AlN lattice with axially symmetric electric field gradients. This is true for places on parallels to the c-axis passing the $\mathrm{Al}$ and $\mathrm{N}$ atoms. The base frequency $\omega_{0}=557 \mathrm{Mrad}$ derived from a fit using Nightmare (Nédélec, 2014) can be inserted in eq. (2.16) which yields the electric field gradients strength $V_{z z}=6.2 \times 10^{22} \mathrm{~V} \mathrm{~m}^{-2}$. This value can be used for a determination of the lattice site occupied by the probe atoms using simulations as discussed in section 11.2.2. The shown spectrum is further discussed in section 8.3. 
In case of magnetic interactions, typical frequencies are the Larmor frequency $\omega_{L}$ and/or $2 \cdot \omega_{L}$ (see eq. (2.12)).

For electrical field gradients, dependent on the field gradient's symmetry parameter $\eta$ more complicated sets of frequencies occur. The lowest occurring frequency for $\eta=0$ is defined by $\omega_{0}=3 \cdot \omega_{Q}$ for integer intermediate level spins and $\omega_{0}=6 \cdot \omega_{Q}$ for half integer intermediate level spins, respectively (cf. eq. (2.16) for the definition of $\omega_{Q}$ ). An overview of the number of occurring frequencies as well as their spin and $\eta$ dependency can be deduced from Butz (1989).

A more subtle problem can emerge due to recoil occurring in $\beta$ decays. If the recoil energies exceed the displacement energy of probe atoms in the host material, it is no longer possible to extract unambiguous information concerning the probe atoms' locations from measurement data. For probes decaying with high $Q$ values (i. e. high amounts of energy released by the decay-the $\mathrm{Q}$ value is neither related to the solid angle correction factors $Q_{k_{1} k_{2}}$ from eq. (2.10) nor to the nuclear quadrupole moment $e Q$ from eq. (2.16); there somehow exist many different $\mathrm{Q}$ in the context of TDPAC), it is therefore advisable to consider recoil energies before deciding to use a certain probe.

Availability of probe nuclei can be an issue since many candidates are not commercially available. Alternative sources for exotic probe nuclei are presented in section 2.4.

Introducing of the probe nuclei into the material of interest can be realized by diffusion or implantation. Alternatively, it is sometimes possible to use neutron irradiation to activate constituents of the sample in place. This is especially an option in case of the ${ }^{181} \mathrm{Hf}$ probe due to the huge cross-section of ${ }^{180} \mathrm{Hf}$ for thermal neutrons (cf. appendix A.1.2).

Since every probe atom's decay only yields a single count in one of the coincidence histograms, it is furthermore necessary to prepare a sufficient number of probe atoms in very similar surroundings. Otherwise, the individual perturbation factors are out of phase and resulting signals are too strongly damped to extract information concerning field properties.

\subsection{Operating principle of analog TDPAC setups}

For TDPAC measurements with fixed detector setups, it is necessary to record time differences of coincident $\gamma$ events detected by different detector pairs. These events must be gated according to their energy to select only coincidences with start- and stop-signals belonging to appropriate transitions of the probe nuclei. Thus, simultaneous and precise time and energy determination for each detected $\gamma$ photon are necessary. A principal problem of analog spectrometers is, that good energy discrimination can only be obtained by extending the integration time (or shaping time for spectroscopy amplifiers (cf. section 2.2.1) because the detectors' output charge-which equals their integrated output current-is proportional to the $\gamma$ photons' energies. Signal amplitude maxima in turn are proportional to the output charge; however, they are much noisier and less 


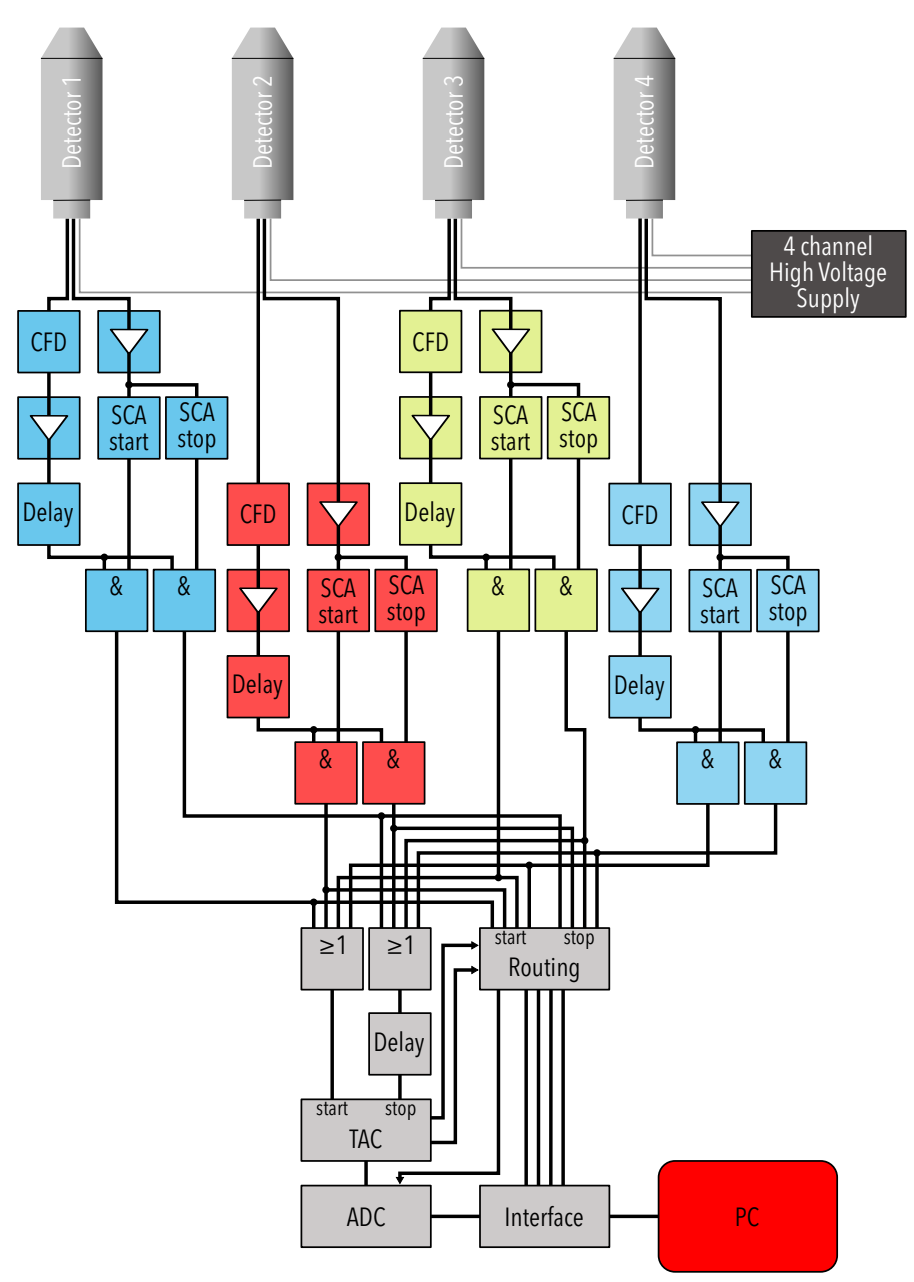

Figure 2.2: Schematic of an analog slow-fast TDPAC setup.

reliable. Integration however delays the availability of energy information necessary for the gating of quickly available time signals and therefore requires an artificial delay of time information - usually output signals of constant fraction discriminators (CFD, see section 2.2.2) - until the energy information is available. This delay is often realized using long delay cables, which make spectrometers big and heavy.

Spectrometers implementing this principle of delaying CFD signals using long cables to allow for long shaping times on the energy path are called slow-fast setups. An example schematic of such a setup is shown in fig. 2.2. In this setup, the two evaluation paths for each detector are split in the photomultiplier tubes (PMT, cf. section 2.3.3). CFDs are connected via a coupling capacitor to a dynode, which is located before the anode, while the anode supplies the energy part. The time branches consist of said CFD, an amplifier, 
and a delay line per detector. Energy branches consist of a spectroscopy amplifier with adjustable shaping time and two single channel analyzers (SCA) for the detection of start and stop signals depending on the shaped signals' amplitudes per detector. Two logical AND gates per detector output signals if the related SCA detected an appropriate amplitude while the time path sent a signal onset pulse. These AND gates thus output energy gated time signals. Start and stop time signals from all detectors are aggregated by two logical OR gates and fed into start and stop inputs of a time-to-amplitude converter (TAC). A start signal triggers a ramp generation process in the TAC, which is halted by the stop signal. The output amplitude available after this halt is proportional to the time difference between start and stop signal. An analog-to-digital converter (ADC) and additional routing circuitry are used to convert the determined time difference and flag it according to the detector combination that triggered the TAC. The resulting event, consisting of an analog value, which is proportional to the detected time difference, and information which detector sent the start and which the stop signal, is recorded by a personal computer (PC). An additional delay line between the OR-gate for stop signals and the TAC is used to compensate for differences in cable lengths of different detectors by shifting all spectra towards longer time differences. This shift is later removed from the coincidence spectra by detection of the spectra's beginning and subtraction of the corresponding time value from all channel times.

A simplified variant of the slow-fast principle is called fast-fast setup. The principal difference is that very short shaping times are used, which makes long delay lines unnecessary. In fact, energy discrimination is thus based on the signals' amplitudes around their peak instead of an integral approximation. For the spectrometer described by Bartos et al. (1993) the signal processing electronic was integrated in a single device yielding a compact spectrometer with limited energy resolution sufficient for simple nuclides like ${ }^{111}$ In (cf. appendix A.1.1).

\subsubsection{Spectroscopy amplifiers}

Spectroscopy amplifiers usually simultaneously amplify and shape incoming signals. Usually, they are used in analog spectrometers to adapt signal properties to the characteristics of downstream evaluation circuitry like single channel analyzers.

A number of different designs and extensions exist for spectroscopy amplifiers as described in depth by Leo (1994). Besides the amplifier, usual components are CR differentiators and RC integrators, which are combined to provide band-pass filtering of signals, thereby increasing the signal-to-noise ratio. Time constants of these filters are in most designs adjustable.

Considering the effect of a spectroscopy amplifier used in combination with a single channel analyzer for energy gating, it is important to note that spectroscopy amplifiers work as integrators if long shaping times are chosen. This makes it possible to evaluate signal integrals rather than amplitudes using amplitude sensitive SCAs thus reaching good 
energy discrimination properties in analog setups although there is no direct way of signal integration.

\subsubsection{Constant fraction discriminators}

Constant fraction discriminators (CFD), first described by Gedcke and McDonald (1967), are the preferred timing devices in analog nuclear spectrometers since the late 1960s. While their analog implementation is straightforward, they show outstanding performance for rise-time invariant signals. This condition is well satisfied by scintillation detectors because for them the rise-time depends primarily on the statistics of the electrons' time-of-flight in the dynode system. The statistical properties of the electrons' flight-times are uniform due to the large number of electrons produced for even single photons detected by the photo cathode (cf. section 7.2).

Figure 2.3 shows the working principle of CFD devices. The input signal is split and processed on two separate paths. One copy of the signal is delayed (usually by a delay line consisting of a piece of cable) while the other copy is inverted and multiplied by a constant factor, i. e. attenuated. The results of both paths are then added forming a bipolar signal with amplitude insensitive zero crossing.

In real setups an additional DC-offset has to be added to avoid permanent triggering on zero-crossings generated by electronic noise. This offset value is usually called walk adjust. It defines a minimum threshold, which the signal's amplitude must exceed to trigger the CFD. A variant of CFD with small delay values is called amplitude and rise-time compensated triggering (ARC, cf. Leo, 1994, p. 327).

\subsection{Scintillation detectors}

Scintillation detectors are the most common solution for $\gamma$ radiation and $\mathrm{x}$-ray detection. Although their invention by Samuel Curran (Curran and Craggs, 1949) in 1944 dates back almost seven decades, there are still not many alternatives and newer developments like detectors based on Silicon photomultipliers (SiPM) are advancements rather than substitutes.

While semiconductor detectors provide much better energy resolution, the achievable time resolution is not sufficient for most TDPAC measurement cases because it severely limits the range of measurable interaction frequencies. Ionization chamber based detectors on the other hand do not provide sufficient detection efficiency for $\gamma$ radiation. Scintillation based detectors are still the only available solution where high detection efficiency, reasonable energy and good time resolution can be combined. 


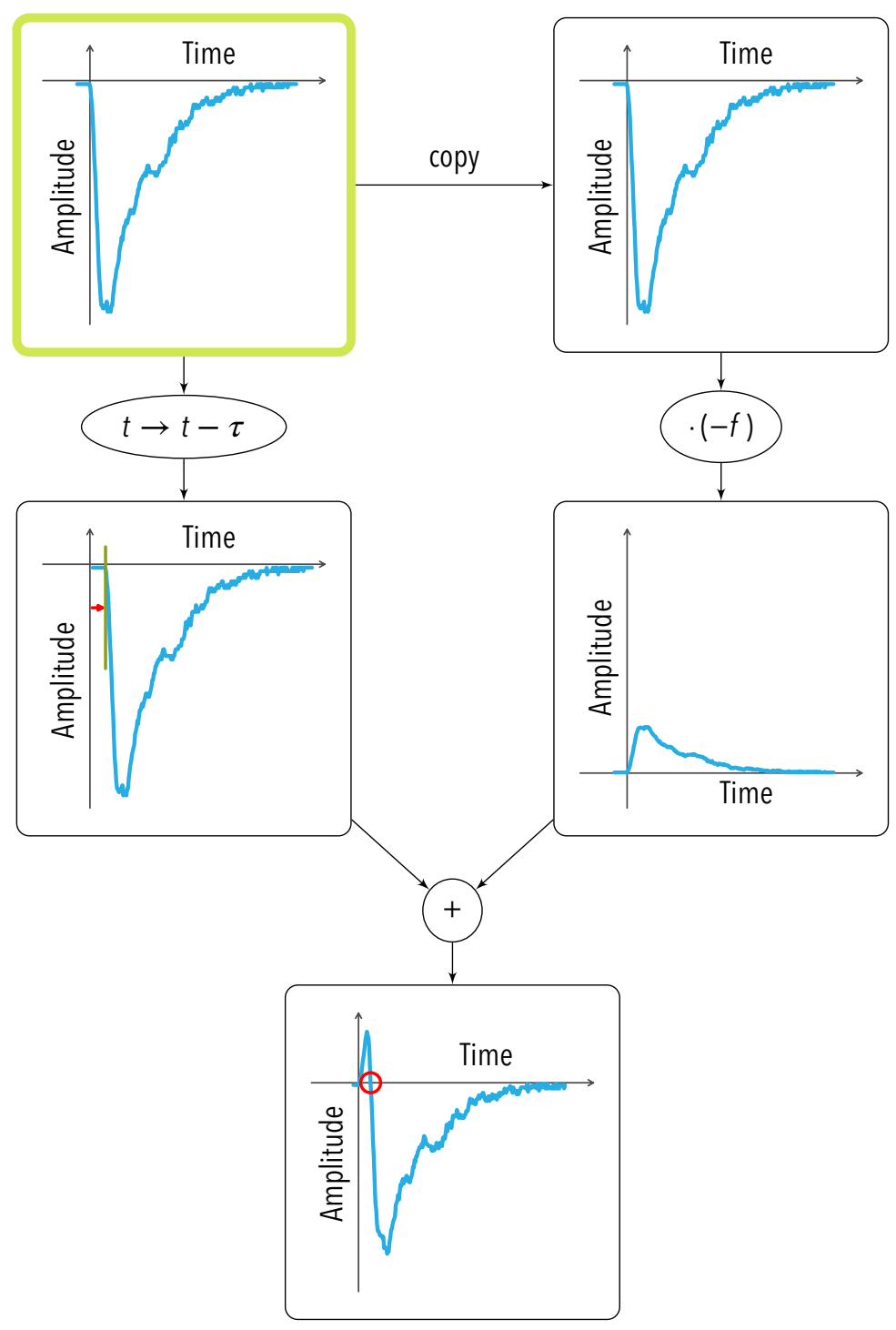

Figure 2.3: Working principle of the constant fraction discrimination (CFD) method for signal time determination. For the CFD method the input signal (highlighted by a green frame) is first copied. One of the copies is delayed (parameter $\tau$ ) while the other is inverted and attenuated by multiplication with a constant fraction value (parameter $f$ ). After this step both signals are added (mixed). The time of the resulting signal's zero crossing is used as signal time (red circle). 


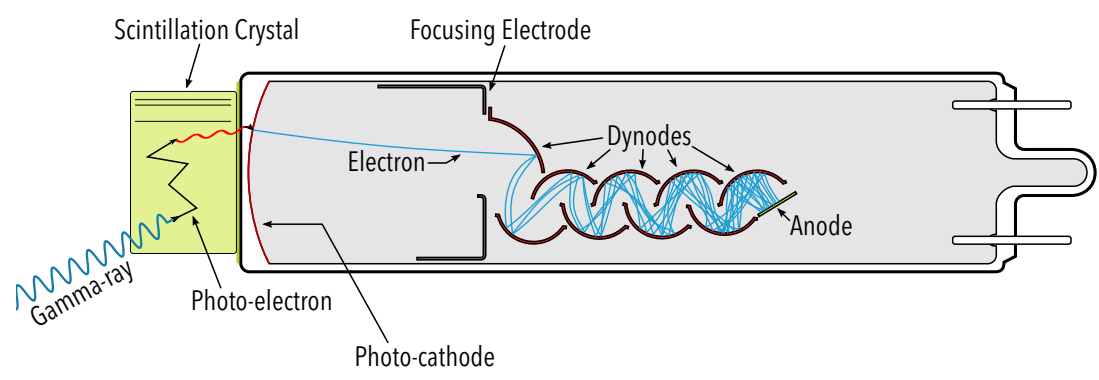

Figure 2.4: Schematic drawing of a scintillation detector (not to scale). An incident $\gamma$ photon hits the scintillation crystal and ionizes one of its atoms due to e. g. the photoelectric effect. This electron excites luminescence-centers in the scintillator, which emit visible photons as the excitation decays. These photons leave the scintillation crystal and enter the photomultiplier tube where they trigger the release of photo-electrons on the PMT's cathode. These electrons are then accelerated along an electric field between cathode and the first dynode. When they hit the dynode, several secondary electrons are emitted for each of them and are again accelerated towards the second dynode. This process is repeated until the secondary electrons created on the last dynode hit the anode.

\subsubsection{Operating principle of scintillation detectors}

Scintillation detectors typically consist of a scintillator (inorganic crystal or organic scintillation material dissolved in a subsequently polymerized solvent) and a photomultiplier tube. This scintillator is mounted on the PMT's cathode window and optically coupled by silicone oil in order to minimize reflective loss at the interface between both (cf. fig. 2.4).

A comprehensive introduction to scintillation detectors is contained in Leo (1994). The following sections will focus on aspects of special relevance for TDPAC spectroscopy.

\subsubsection{Scintillators}

The primary interaction mechanisms of $\gamma$ radiation with a scintillation crystal are:

1. Photo-effect

2. Compton scattering

3. Pair production.

The probability for each of these mechanisms to happen at the incidence of a certain $\gamma$ photon depends on the photon's energy and the scintillation material. In short, the photo-effect dominates the overall absorption of radiation at low energies whereas Compton scattering takes over for higher energy photons and finally above the threshold of 
$1.022 \mathrm{MeV}$ (which equals the cumulative mass of an electron and a positron) pair-production sets in. Since the energy deposited in the scintillator is converted to light in a series of statistical processes anyways, the most important issues concerning these interaction mechanisms are the probability that a $\gamma$ photon passes the crystal without interaction and the probability that an interaction takes place but part of the energy is lost due to scattered radiation (or annihilation radiation) leaving the crystal. While photo-effect produces fast electrons inside the crystal, which are unlikely to leave it, Compton scattering often results in incomplete energy deposition due to scattered $\gamma$ photons leaving the crystal without further interactions. Electrons and positrons produced in a pair-production process are unlikely to leave the scintillator; the annihilation radiation of the positron however is again more likely to leave without further interaction.

Atomic mass of the scintillator's constituents has a major influence on the partitioning of interaction processes among the three mechanisms as well as the probability for photons to pass the crystal undetected. For the photo-effect, the cross-section is discontinuous for energies below the K-electrons' binding energy. However, for higher energies it is proportional to $Z^{4}$ up to $Z^{5}$. For Compton interactions the cross-section is approximately related to $Z$ and for pair production (in case of $\gamma$ photons above the energy threshold) it is proportional to $Z^{2}$. This means that scintillation materials with higher atomic mass not only provide higher absorption probabilities but also a lower probability of partial energy loss because their photo-effect vs. Compton scattering ratio as well as the probability to absorb Compton scattered photons before they leave the crystal are both higher.

Due to these facts, organic scintillators are not particularly suitable for TDPAC spectrometers. They mainly consist of carbon and hydrogen-i. e. very light constituents. Therefore, even compared to NaI:Tl—which is one of the lighter inorganic scintillation materials-a plastic scintillation material like NE102A exhibits a more than two orders of magnitude smaller probability for photo-effect induced absorption of $\gamma$ rays (Leo, 1994, p. 175). Detected radiation events where not the whole energy is deposited in the scintillator usually have to be discarded in case of TDPAC measurements because they cannot be assigned to a certain energy window. Thus, almost vanishing photo-peaks as seen in case of plastic scintillators make TDPAC measurements almost impossible.

$\gamma$ photons interacting with the scintillator in one of the described ways finally ionize atoms in the crystal producing numerous electron-hole pairs. These pairs propagate to and recombine at luminescence-centers usually created by donating the crystal (Rodnyi, 1997). During recombination, visible photons that are not absorbed by the bulk material since their energy is smaller than the crystal's band gap are emitted. Ideally their energy is in the range of visible light thus being easily detectable with e. g. photomultiplier tubes.

Properties of several inorganic scintillation materials are discussed in section 7.1.1. 


\subsubsection{Photomultiplier tubes}

On light incidence, photo-electrons are emitted by the PMT's photo cathode due to the photoelectric effect. As soon as they leave the cathode material, these electrons are accelerated by means of an applied electric field towards the first dynode. Hitting the dynode material, they trigger the release of several secondary electrons, which are again accelerated towards the second dynode. The dynode system consists of several multiplication stages of this type. Electrons emitted by the last dynode are accelerated towards the anode where they are collected. The anode is discharged across a coaxial cable connection and a terminating $50 \Omega$ load resistor. Arising voltage across this resistor is measured and analyzed.

To allow electrons passing from one dynode to another, the PMT is evacuated. For scintillation materials emitting ultraviolet light like e. $\mathrm{g} . \mathrm{BaF}_{2}$, special PMT models with fused silica window exist.

Since the invention of the PMT in the 1930s (Iams and Salzberg, 1935), hundreds of models with different advantages and disadvantages have been developed. Although Photonis-which bought Burle Industries (the successor of pioneering RCA Corporation) in 2005-ceased production of PMTs in 2009, still a large number of PMT designs are being produced and developed by vendors like Hamamatsu and ET Enterprises.

The PMT parameters most relevant for TDPAC spectrometers are quantum efficiency, time properties, and amplification. Additionally, the size of the PMT window should at least match the scintillator's diameter.

Quantum efficiency $\eta(\lambda)$ describes the probability that an incoming visible photon triggers the emission of a photo-electron in the PMT's cathode, which successfully starts an amplification cascade in the dynode system. Typical values for $\eta(\lambda)$ in the visible range are between $15 \%$ and $30 \%$.

The time it takes between the emerging of a photo-electron on the cathode and the arrival of electrons on the anode is usually described by two parameters: The transit time and the transit time spread (TTS). The transit time describes the average time passing between the arrival of a light pulse on the cathode and the appearance of a pulse on the anode. It is of little interest in the TDPAC context since it is usually constant as long as the potential differences between dynodes remain unchanged and either cancels out due to the use of identical PMTs or introduces a constant offset, which is subtracted during evaluation of measurements.

TTS is usually provided as full width at half maximum (FWHM) value and quantifies the statistical fluctuations of the transit time. It is relevant for timing measurements involving few primary photo-electrons because in such cases it limits the achievable time resolution. However, in cases where dozens or even hundreds of photo-electrons are created almost simultaneously on the photo-cathode, the anode signal reveals the whole TTS distribution and therefore does not significantly influence timing performance. For $\gamma$ 
photon energies occurring in TDPAC use cases with usual inorganic scintillation materials, the number of simultaneously arriving visible photons is usually sufficiently large.

Amplification of a PMT depends on the average number of secondary electrons produced on each dynode per incoming primary electron and the number of dynodes. The secondary electron yield depends on the energy of impinging primary electrons, their angle of incidence, and the dynode material. Incidence angles are a statistical quantity, which depends on the geometry of the dynode system. Primary electrons' incidence energies are a sum consisting of the potential difference of subsequent dynodes and the emission energy during creation of the primary electron on the dynode it originates from. Usually, the latter is neglectable since the energy gained by crossing the potential difference is much higher.

For the sake of simplicity, the potential differences between subsequent dynodes are usually generated by a voltage divider connected to a single high-voltage power supply rather than a dedicated power supply per dynode. This makes supply circuitry simpleespecially for PMTs with a high number of dynodes ( 8 to 12 are typical in scintillation detector applications). The drawback of this method is that most of the current drawn from the supply flows through the voltage divider without being usable for the PMT. A common rule-of-thumb is that the current flow through the voltage divider must be at least ten times higher than the current drawn by any dynode to avoid destabilization of the voltage ratios in the divider. Improved designs are based on transistor or Zener diode stabilized voltage dividers (Hamamatsu, 2007, p. 94).

\subsection{Nuclide production facilities}

While it is possible to produce nuclides like ${ }^{111} \mathrm{In}$ and ${ }^{181} \mathrm{Hf}$ using cyclotrons or nuclear reactors (cf. appendices A.1.1 and A.1.2) and isolate as well as introduce them into samples after production, this method is not available for all possible TDPAC probes. In some cases no nuclear reaction with appropriate cross section exists for the production. Additionally, it is often difficult to isolate and process produced radioactive material from the carriers used during production. For short lived nuclei it is not possible to consecutively produce and implant the probe atoms.

For the sake of radio protection it is also desirable to combine the steps of nuclide production and implantation into samples thus making intermediate processing unnecessary.

In this section, two available alternatives to nuclide production in cyclotrons or by neutron irradiation in reactors are shown, which can be used in conjunction with TDPAC measurements. 


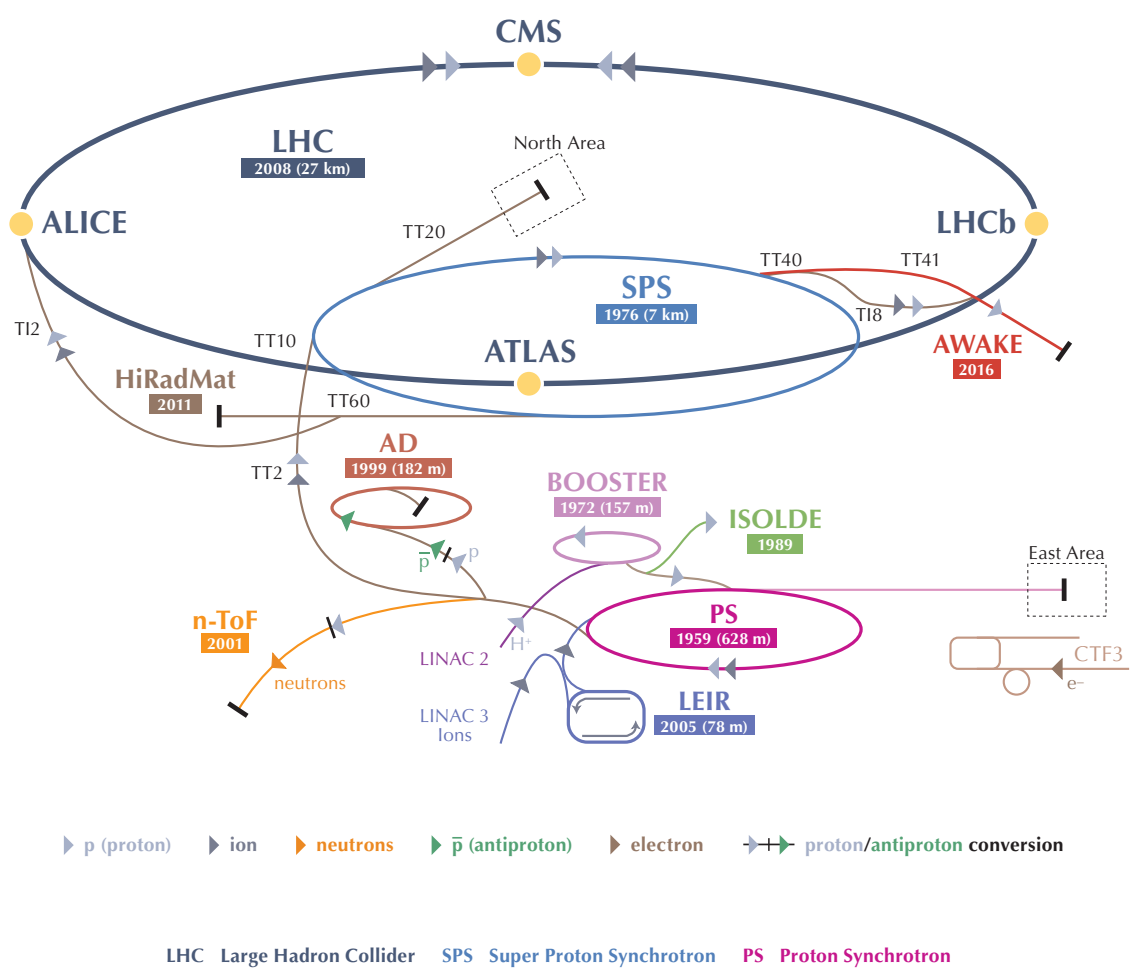

AD Antiproton Decelerator CTF3 Clic Test Facility AWAKE Advanced WAKefield Experiment ISOLDE Isotope Separator OnLine DEvice LEIR Low Energy lon Ring LINAC LINear ACcelerator n-ToF Neutrons Time Of Flight HiRadMat High-Radiation to Materials

Figure 2.5: CERN's Accelerator Complex including ISOLDE. By courtesy of Fabienne Marcastel, CERN.

\subsubsection{ISOLDE (CERN)}

The ISOLDE facility (described by Kugler et al., 1992; Kugler, 2000; Herlert, 2010) located at the Meyrin site of CERN and operated by the ISOLDE collaboration can be considered the prototype of an Isotope Separator On-Line (ISOL) facility. Since 1992, protons are delivered from CERN's PS Booster (cf. fig. 2.5) to the ISOLDE targets for nuclide production. Currently these protons' incidence energy is $1.4 \mathrm{GeV}$. The maximum average current provided is $2 \mu \mathrm{A}$.

There is a number of ISOL-facilities operational nowadays in different countries like SPIRAL (France), ISAC-I \& II (Canada), RIBF (Japan), and IGISOL (Finland) among others. A nice overview was published by Blumenfeld, Nilsson, and Duppen (2013). Due to limited yield and/or a limited choice of probe nuclides, most of them are however less appropriate for TDPAC sample preparation than ISOLDE. 


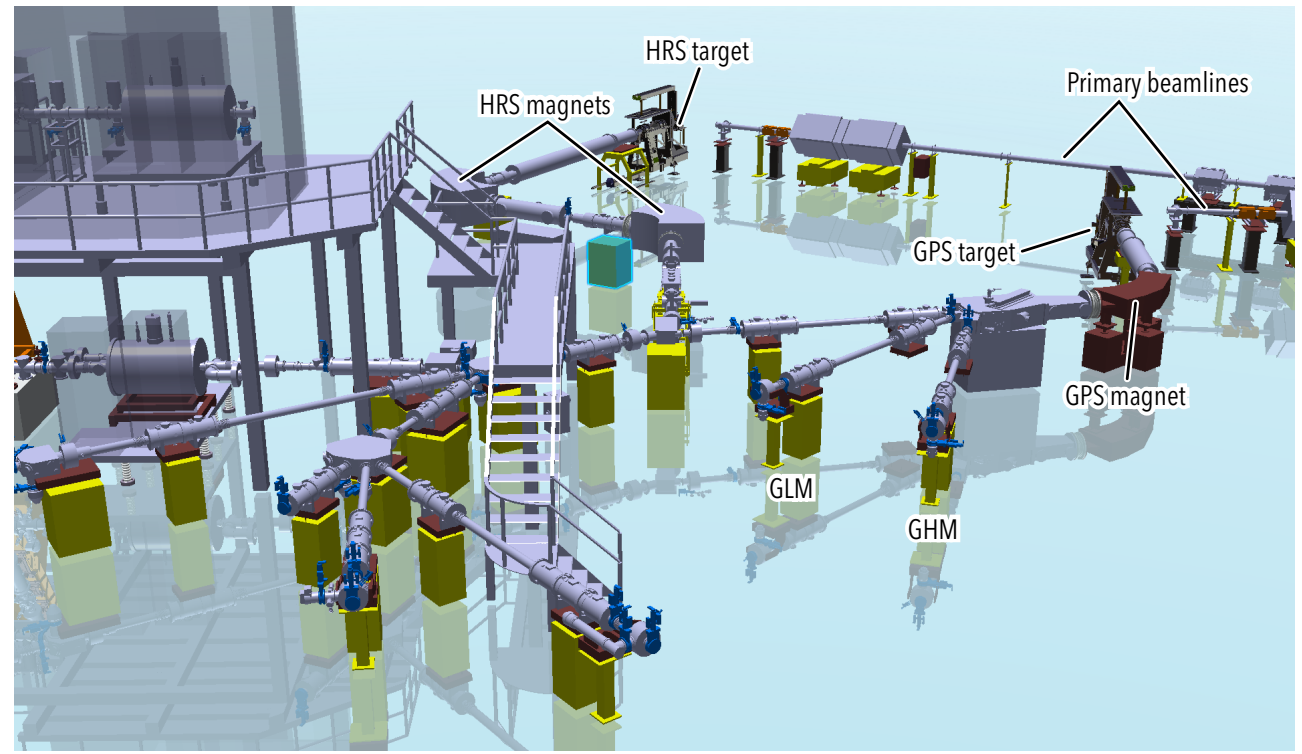

Figure 2.6: ISOLDE targets and beamlines. By courtesy of CERN.

Nuclide production at ISOLDE takes place in two targets. Due to the high energy of the primary beam, it is possible to extract this beam to air through simple blank flanges. It then passes about $1 \mathrm{~m}$ of air before entering the target chambers. Due to their high energy, parts of the protons exit the target chambers and pass another meter of air before hitting beam dumps. This design has several advantages: The primary beamline is not connected to the ISOLDE target chambers which reduces the risk of contamination and vacuum breakdown and allows for easier replacement of the target chambers, which are only connected to the ISOLDE beamline via a single valve. Additionally, the target chambers are electrically isolated from the PS Booster which makes it possible to operate them at high voltage potential. This allows acceleration of the radioactive ion beam while the actual beamline is connected to ground potential.

The two targets can be operated simultaneously since the primary beam from the PS Booster is bunched and the bending magnet, which allows to select the active target, can be switched between bunches. Figure 2.6 shows the primary beamlines entering from the right side of the image. The gaps between these beamlines and the targets are clearly visible.

ISOLDE's targets basically consist of a tantalum tube filled with a target material (usually in the shape of thin foils, liquids, or small pellets-more than 25 materials are available). These tubes usually have a length of $10 \mathrm{~cm}$ to $20 \mathrm{~cm}$ and $10 \mathrm{~mm}$ to $20 \mathrm{~mm}$ diameter (Catherall et al., 2003; Blumenfeld, Nilsson, and Duppen, 2013) and are passed by the primary proton beam along their axis. In the target material the incoming protons initiate fission, spallation and fragmentation reactions. Diffusion of reaction products to the 
surface of the target material and effusion to the transfer line is accelerated by Joule heating of the target tube to temperatures around $2000^{\circ} \mathrm{C}$. While both ends of the tube are closed, a-optionally heated - transfer line (usually a thin tube) is connected to a hole in the tube's center. From there, reaction products effuse along the transfer line to the ion source.

Several types of ion sources are available. Besides the longer established surface ion sources and plasma ion sources, the resonant ionization laser ion source (RILIS) is getting more and more popular since it provides very pure beams due to its element-specific selectivity, which complements the isobar-specific selectivity of the separation magnet anddepending on the selected nuclide-can suppress considerable amounts of contaminants.

Due to the high levels of radioactivity occurring in the target chambers, these chambers are moved, changed, and stored by mobile industrial robots. Used targets are stored for some time in shelves along the robot galleries to allow for the decay of short-lived reaction products.

After ionization and acceleration in the target font-ends, the produced radioactive ion beams pass the separators. The two target stations use different separation setups called General Purpose Separator (GPS) and High Resolution Separator (HRS). Both consist of bending magnets. The HRS, consisting of two magnets, achieves a better resolution while the GPS allows for simultaneous delivering of beams of different species to three different beamlines using a special deflector arrangement. Two of these GPS beamlines-GHM and GLM - are exclusively used for solid-state physics, biological applications, and medical applications while the third enters a merging switchyard, which allows to feed the GPS beam into the beamline otherwise distributing the HRS beam to all other experiments.

Figure 2.7 shows a picture of the ISOLDE hall. In contrast to fig. 2.6, the target area is not visible since it is hidden behind concrete shielding and the outside hill. All solid-state physics related setups are labeled. For this work, the most relevant of them is the GLM beamline since it was used for several test measurements with the new spectrometer and it is the place where the newly developed chambers shown in section 10.1 will be installed.

\subsubsection{LOHENGRIN (ILL)}

The Institut Laue-Langevin operates a high-flux reactor as a neutron source. This reactor is operational continuously for 50 days per cycle followed by a shutdown for re-fueling. Usually, four reactor cycles are provided per year. The reactor produces $57 \mathrm{MW}$ of thermal power during normal operation and provides a neutron flux of up to $1.5 \times 10^{15} \frac{1}{\mathrm{scm}^{2}}$.

Among the many instruments available at ILL, the fission product spectrometer PN1 (LOHENGRIN) is a special one since it does not provide neutrons to the user but uses them to produce exotic nuclei. A comprehensive overview of this instrument can be found in Armbruster et al. (1976) and Köster et al. (2010).

Figure 2.8 shows an overview of the LOHENGRIN setup. A thin actinide target is 


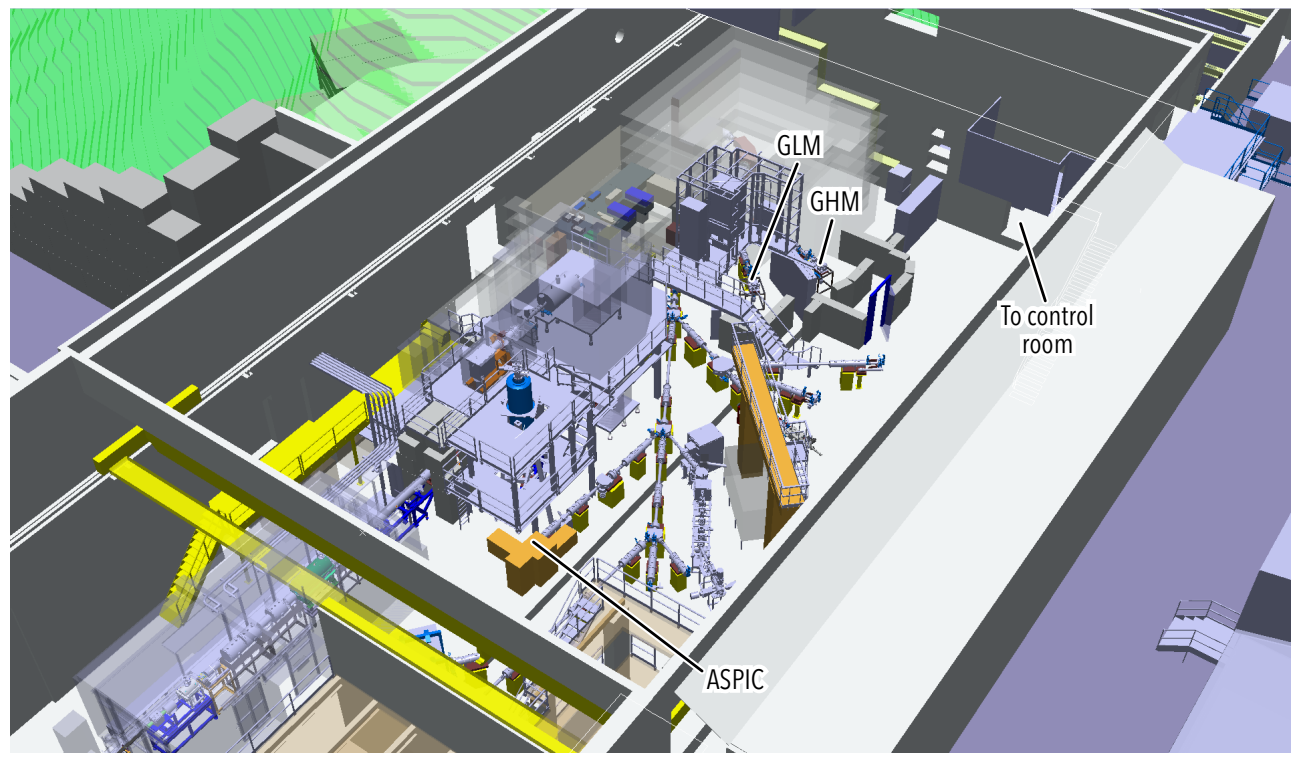

Figure 2.7: The ISOLDE Hall. By courtesy of CERN.

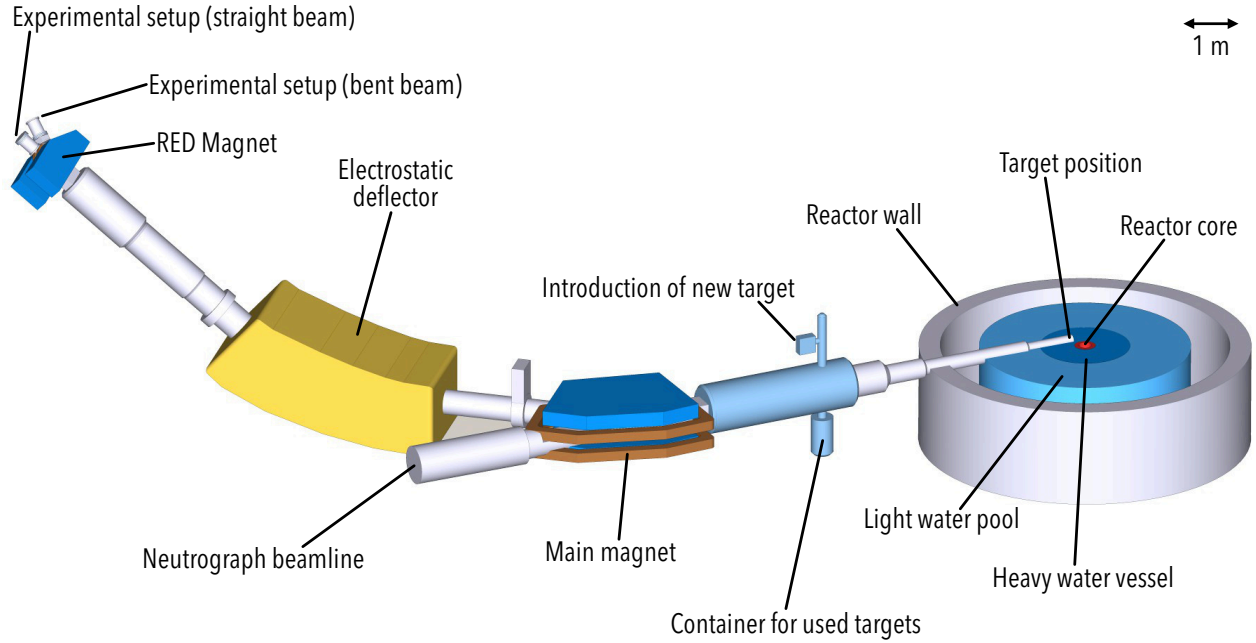

Figure 2.8: LOHENGRIN beamline. By courtesy of ILL. 
placed in the beginning of an evacuated beamline, which is located in the heavy water vessel's interior close to the reactor's core. There it is exposed to a high flux of thermal neutrons. Ionized fission fragments are emitted from the actinide layer due to recoil and guided towards a mass spectrometer consisting of a dipole magnet and an electrostatic deflector, both mounted with perpendicular deflection planes (Thomson parabola spectrometer). The ions are separated by this setup according to their mass per ionic charge and energy per ionic charge ratios.

Although originally designed for the analysis of fission fragments (Armbruster et al., 1976), LOHENGRIN is increasingly used as an radioactive ion beam facility (Köster et al., 2010). For this application, short transport times between fragment production in the actinide layer and the experiments of only $1 \mu$ s to $2 \mu$ s are an advantage concerning the yield of very short lived nuclides over facilities like ISOLDE where produced nuclides have to pass comparably slow processes like diffusion and effusion towards the ion source, ionization, and acceleration. Therefore, LOHENGRIN makes measurements based on such very short lived nuclides possible and is an interesting source for many of the TDPAC candidates shown in section 9.2.

Usability of LOHENGRIN as radioactive ion source was improved by the introduction of the reverse energy dispersion (RED) magnet, which is able to focus $40 \mathrm{~cm}$ of a certain nuclide's mass parabola to a beam-spot of only a few $\mathrm{cm}^{2}$ (Fioni et al., 1993), thereby obtaining higher count-rates, which permit TDPAC measurements.

Online TDPAC measurements at ILL The LOHENGRIN fission fragment separator is an interesting facility for online TDPAC measurements-i. e. measurements where implantation and measurements happen simultaneously_-because it can provide very short lived nuclides. Due to the high recoil energies and its rather short beamline, even nuclides with half-lives in the sub-microsecond range reach the target before decaying.

Concerning the feasibility of TDPAC measurements there are a few limitations related to LOHENGRIN's working principle. These are particularly the comparably big beam spot and low beam currents. The beam spot's size behind the RED magnet, which provides improved focusing, is about $1 \mathrm{~cm}$ by $3 \mathrm{~cm}$. Considering the small size of the scintillation crystals described in section 4.1.1 and the small distances necessary to obtain sufficient solid angle coverage using these detectors (not more than several $\mathrm{cm}$ ), the averaging effect caused by this big beam spot significantly decreases the signal to background ratio and therefore increases the necessary measurement time.

The existing target chamber available at LOHENGRIN is suitable for an adequate placement of detectors around a mounted sample. It consists of a square shaped aluminium tube with a rectangular flange at its end. This flange can be opened to change samples.

Online measurements can be performed without hardware modifications at the LOHENGRIN separator. However, if magnetization of samples is required, a changed chamber design is necessary because due to the large beam spot, it is not possible to obtain a 
homogeneous magnetic field throughout the used sample surface using a yoke construction built around the existing target chamber. Possible designs providing a satisfactory sample magnetization are be discussed in section 10.3. 



\section{Chapter 3}

\section{Motivation and objectives}

Despite analog TDPAC spectroscopy is an established method for several decades, a wealth of new approaches for improvement, optimization, and even new use-cases arose with the feasibility of all-digital setups. While the implementation and optimization of a nextgeneration spectrometer was the primary goal of this work, a number of complementing examinations were added. This chapter provides an overview of the objectives that led to the results discussed throughout the remaining chapters.

\subsection{Status quo}

When work for this thesis began, different groups were aiming to build the first digital TDPAC spectrometer able to compete with — and surpass existing — analog models. A first working proof-of-concept spectrometer had been presented by Herden, Alves, et al. (2004) at the Technische Universität Braunschweig. It uses $1 \mathrm{GS} \mathrm{s}^{-1}$ digitizers, which write data to $8 \mathrm{MiB}$ of internal memory. This memory's content is read by the host computers after it was filled. After these buffers of all four digitizers were read, a new measurement cycle is started, which involves resynchronization of the digitizers. Signal times are determined using the trigger times provided by the digitizers. Signal analysis is limited to integration of recorded waveforms for energy determination.

While this early digital spectrometer is far from being competitive with analog setups concerning its performance, it encouraged further pursuit of the approach of a software defined spectrometer. At that time it was not yet clear how well the purely software driven approach depending on general-purpose high-speed digitizers and off-the-shelf computer hardware would compete with an alternative approach using signal processing based on field-programmable gate arrays (FPGA), which was taken by a group at the University of Leipzig.

Both digital approaches were accompanied by the hope to improve spectrometer performance due to several advantages being associated with digitalization. The most important advantages from a performance point of view are the extended possibilities for signal analysis compared to analog circuitry due to the possibility to use almost arbitrarily complex algorithms and the availability of pre-trigger data. The latter is commonly 
known from storage oscilloscopes providing vastly improved signal analysis possibilities compared to simple analog oscilloscopes, which only show post-trigger parts of a signal.

Research results concerning signal processing algorithms applicable to TDPAC spectroscopy were scarce at that time since fully digital processing of detector signals was not a common method. Pioneering work from Bardelli et al. (2004) showed that good results were possible even based on moderate sampling rates. However, their results were not directly transferable since they did not detect $\gamma$ radiation but particles and used semiconductor detectors. Other groups like Saito and Hyodo (2003) also presented singular results but a comprehensive evaluation of different methods was missing.

\subsection{Objectives}

\subsubsection{Implementation of a digital six-detector TDPAC setup}

As previously stated, the implementation of a second generation all-digital TDPAC setup was a primary objective of this work. This included several tasks.

Achieved performance should be at least comparable to analog setups. To yield a setup capable of replacing its analog predecessor and gaining acceptance in the user community it was necessary to attain at least comparable performance. Relevant benchmarks are the achievable time and energy resolution as well as the count rate limit and dead time (i.e. the time that has to pass after detection of a $\gamma$ event before the same detector is ready to detect the next $\gamma$ event). Another important attribute is the achievable stability during long-term measurements since analog setups often exhibit undesirable drift effects concerning energy tuning due to a count rate dependence of the photomultipliers' amplification.

The Design should allow for easy extension with additional detectors Analog TDPAC setups exist with four, six and eight detectors (Rinneberg, Schwartz, and Shirley, 1977) and recently even with up to sixteen (Sato et al., 2008). A larger number of detectors are beneficial in many cases: For single crystal measurements due to simultaneous measurement of additional orientations and for powder samples due to improved signal-to-noise ratio (see section 2.1.5). Therefore, the hardware and software of the digital spectrometer had to be designed to support a flexible number of detectors. This allows exploiting of the better scaling of complexity in case of additional detectors compared to analog setups and of the improved evaluation possibilities mentioned in section 3.2.2.

Development of a high-performance software suite for real-time data recording and immediate evaluation The basic idea of digital TDPAC setups is to replace analog electronics by digital signal processing. In fact, this means that complexity is moved from 
hardware to software. Therefore, an appropriate software suite had to be created providing the necessary measurement functionality, which is provided by a number of electronics modules in analog setups. This software suite also plays a crucial role in fulfilling the performance goals.

Robust design allowing for long-term measurements The setup was designed for operation at ISOLDE. As maintenance proves to be difficult in case of a remote setup and since it is not possible to provide a concise introduction concerning the machine's usage to every future user, an easy to use and insensitive design had high priority.

\subsubsection{Identification and exploitation of improvements arising from the digital approach}

While the first generation of digital setups proved the feasibility of the digital approach they hardly started exploiting the new possibilities arising due to this method. In order to approach this challenge, a number of fields for improvements were identified.

Change of the measurement workflow to postpone configuration tasks and thereby accelerate data taking Analog setups require the user to adjust energy windows before measurements can start. This becomes impractical for complex energy spectra and even more for nuclides with multiple decay cascades since the number of parameters is higher in these cases and the determination of optimal values is more difficult. By recording timestamps and energy values for all occurring $\gamma$ events this task can be postponed and recording can start immediately.

Possibility of offline evaluation Between timestamp and energy values of single $\gamma$ events and the final $R(t)$ spectrum, evaluation results are influenced by many configuration parameters like energy window settings, coincidence search parameters, or fit parameters for the determination of time offsets and background levels. It is very beneficial if these parameters can be optimized offline while or even after the actual measurement has taken place.

Postponed multi-cascade evaluation Especially for nuclides with multiple decay cascades the possibility to evaluate additional cascades offline is beneficial since it allows for focusing on the most important cascades during measurement while it still remains possible to obtain information from remaining cascades afterwards.

Bias free coincidence determination Digital evaluation makes it possible to adequately treat ambiguous coincidences with multiple start- or stop-signals thus achieving 
false coincidence background levels, which are constant throughout a coincidence spectrum and not biased due to asymmetric treatment of ambiguous start- and stop-signals. This improves the precision of $R(t)$ determination by making it possible to subtract the constant background levels without disturbing the coincidence spectra.

Inclusion of an energy calibration process for improving the handling of measurements involving unusual isotopes Configuration of energy windows is traditionally done according to the energy spectra's shapes without mapping of channels (i.e. histogram bins) to actual energy values. Implementation of an energy calibration step makes it possible to determine precise and uniform window positions for all detectors at once and especially for weak or overlapping peaks, which cannot be located according to their shape. This simplifies measurements-especially those involving isotopes with a high number of $\gamma$ transitions.

Improved signal analysis Digital signal processing provides new opportunities but also challenges concerning the time and energy analysis of detector signals since wellknown and established analog methods like constant fraction discrimination cannot be easily adopted because of the limited sampling rates available. Therefore, it was an important goal to meet the challenge of finding signal processing solutions providing optimal performance.

Drift compensation Minimization of the drift of energy spectra is a prerequisite for stable measurements since it is important to prevent required parts of the energy spectra from leaving the recorded energy range as well as to prevent photo-peaks from leaving energy windows and it therefore was an objective of the spectrometer's hardware design. Moreover, the digitalization introduces additional possibilities to compensate for remaining drift thereby improving the effective energy resolution and allowing for the evaluation of nuclides with more difficult decay schemes.

\subsubsection{Optimizations for measurements involving exotic decay cascades at ISOLDE}

Measurements with unusual TDPAC nuclides, as available at ISOLDE, cause special usability and performance requirements. Since half-life is short for many candidate nuclides, measurements have to be started quickly after collection, which makes short-lived contaminants an issue that cannot be neglected. Therefore, the spectrometer has to be able to process high rates of $\gamma$ events without significant dead-time. Energy resolution has to be sufficient to separate relevant $\gamma$ events from background. Furthermore, quick online evaluations (i. e. evaluations while data is still recorded where evaluation results are continuously updated as new data arrives) are important to get feedback on collection quality while the possibility for offline evaluations is important since it provides the 
opportunity to find optimal evaluation parameters and study secondary cascades without the pressure of a beam time.

\subsubsection{Investigation of improvement approaches for detector design and signal analysis}

While digital signal processing techniques are on the rise in most areas of technology for decades, analog-to-digital converters fast enough for the implementation of DSP techniques in nuclear spectroscopy are rather new. Therefore, comprehensive studies about optimal methods for the processing of scintillation detectors' anode signals as well as detector design optimizations for digital read-out were missing. The available studies did not compare more than a few of the possible approaches and were limited to special cases.

To overcome this lack of systematic analyses, a universal evaluation approach is required. Such a method is applicable for the evaluation of timing methods and detector designs described in literature as well as for the development of improved new approaches.

\subsubsection{Investigation of new candidates for online and offline measurements at ISOLDE}

Due to the constraints of legacy spectrometers, the TDPAC method was limited to only a few probe nuclides. With the improvements described in the previous sections new candidates are to be expected and a thorough search based on nuclear databases becomes a worthwhile goal. Availability of short-lived nuclides at facilities like ISOLDE or LOHENGRIN makes it possible to accomplish experiments with nuclides where the halflife is too short for the usual sequence of nuclide production, transport, implantation, sample transport, annealing, and measurement (offline-approach) but immediate online measurements during implantation are necessary. A new compilation of short- as well as longer-lived probe nuclide candidates for the offline- and online-approaches is therefore desirable.

\subsubsection{Development of new facilities for ISOLDE}

Another objective of this work was the development of new implantation and online TDPAC chambers for ISOLDE. A total of three chambers were planned: One for efficient implantations of longer lived nuclides, one for decelerated or post-accelerated implantations, and one for online TDPAC experiments. 



\section{Chapter 4}

\section{Spectrometer setup}

When the digital TDPAC spectrometer's development started, no working setup with realtime data evaluation was available. Although the prototype setup from Herden, Röder, et al. (2008) demonstrated the feasibility of digital recording of detector signals, their synchronization method employing analog multiplexers and the necessity of resynchronizations after one of the recording hosts detected a certain number of events introduced new complications and additional dead time, which caused doubts concerning the meaningfulness of the digitizer and software based approach.

In order to resolve these doubts, initial work in the context of this thesis focused on the investigation of design improvements and methods that allowed for maximizing the spectrometer's performance by reducing dead-time and increasing throughput. Additionally, real-time merging of measurement data was an important goal because it is a prerequisite for a real-time evaluation of measured data.

Several measures were taken to achieve these goals:

1. An appropriate high-performance digitizer model supporting continuous data recording was chosen.

2. Recording computers with eight CPU cores were selected. A new software suite was developed, which - for the first time-introduced multi-threaded data evaluation to TDPAC spectroscopy and is therefore able to take advantage of multiple CPU cores.

3. A new synchronization paradigm was developed, which obviates the need for multiplexers and therefore significantly simplifies the hardware design while simultaneously avoiding distortions of the detector signals due to potentially mismatched multiplexing hardware.

4. The software suite was designed to allow for network transmission and real-time merging of recorded data.

The forthcoming sections describe the hardware as well as software design of the spectrometer in detail. Section 4.3 summarizes the results. 


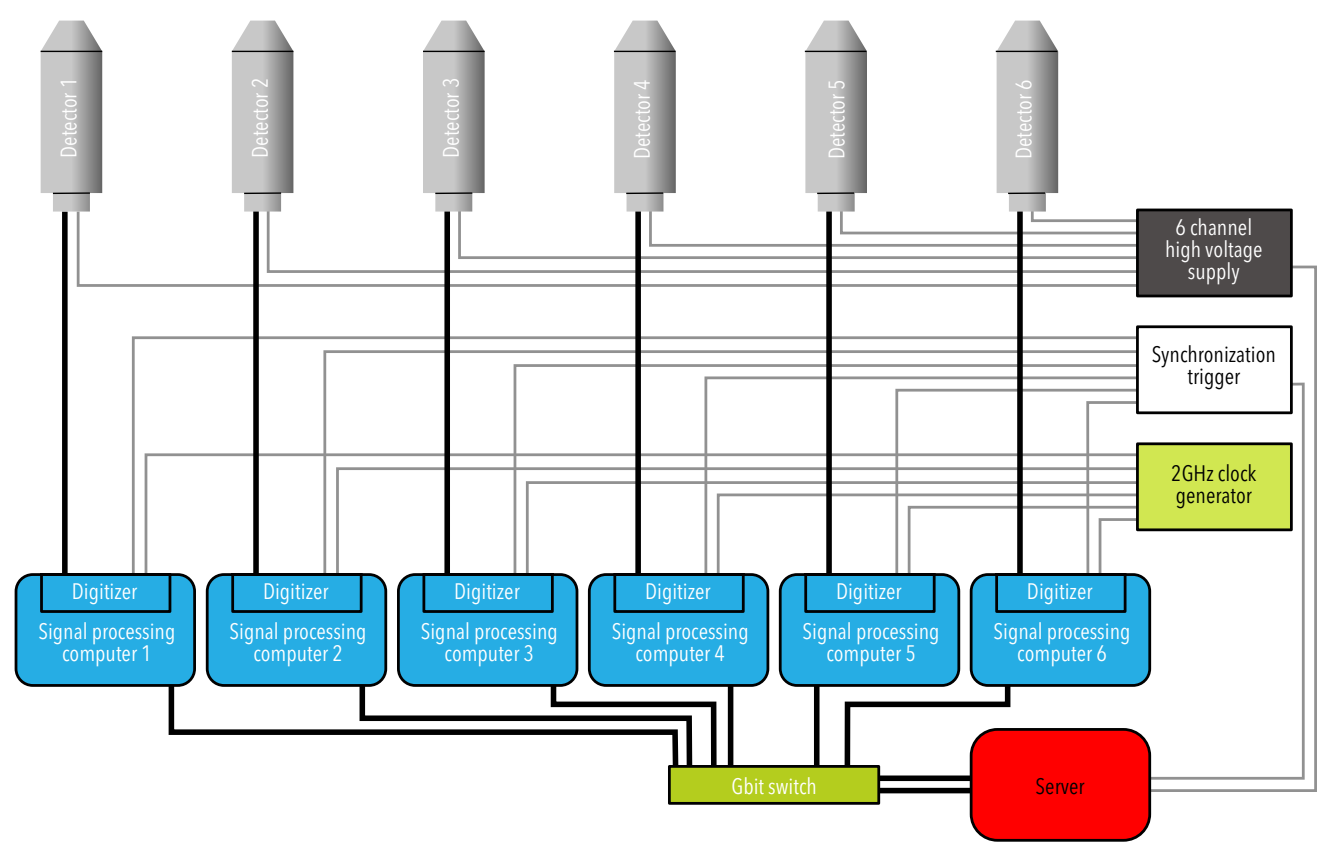

Figure 4.1: Scheme of the digital TDPAC spectrometer. The detectors' analog outputs are directly connected to high-speed digitizers. Each of these digitizers is connected to a dedicated diskless computer, which is responsible for signal processing. The preprocessed results are forwarded to a server, which selects and saves relevant $\gamma$ events. The server is also used to control the setup via a GUI based software called PacMaster. Therefore, the auxiliary electronics for power supply and digitizer synchronization is also connected to the server.

\subsection{Hardware setup}

Due to the move of spectrometer functionality from hardware to software, the remaining hardware of the spectrometer is less specialized than in case of analog setups. Of course, the careful selection of the remaining components is all the more important concerning the spectrometer's overall performance.

Figure 4.1 shows a schematic overview of the digital spectrometer's hardware setup. The current revised six detector setup is shown in fig. 4.2. Computer equipment and electronic components are contained in a road case for easy transportability. A cubical structure built from aluminium extrusion profiles visible left of the road case aligns the detectors. Each detector is held by a slidable clamp. All detectors' tips are additionally aligned by pits in a hollow PMMA cube, which contains the sample. 


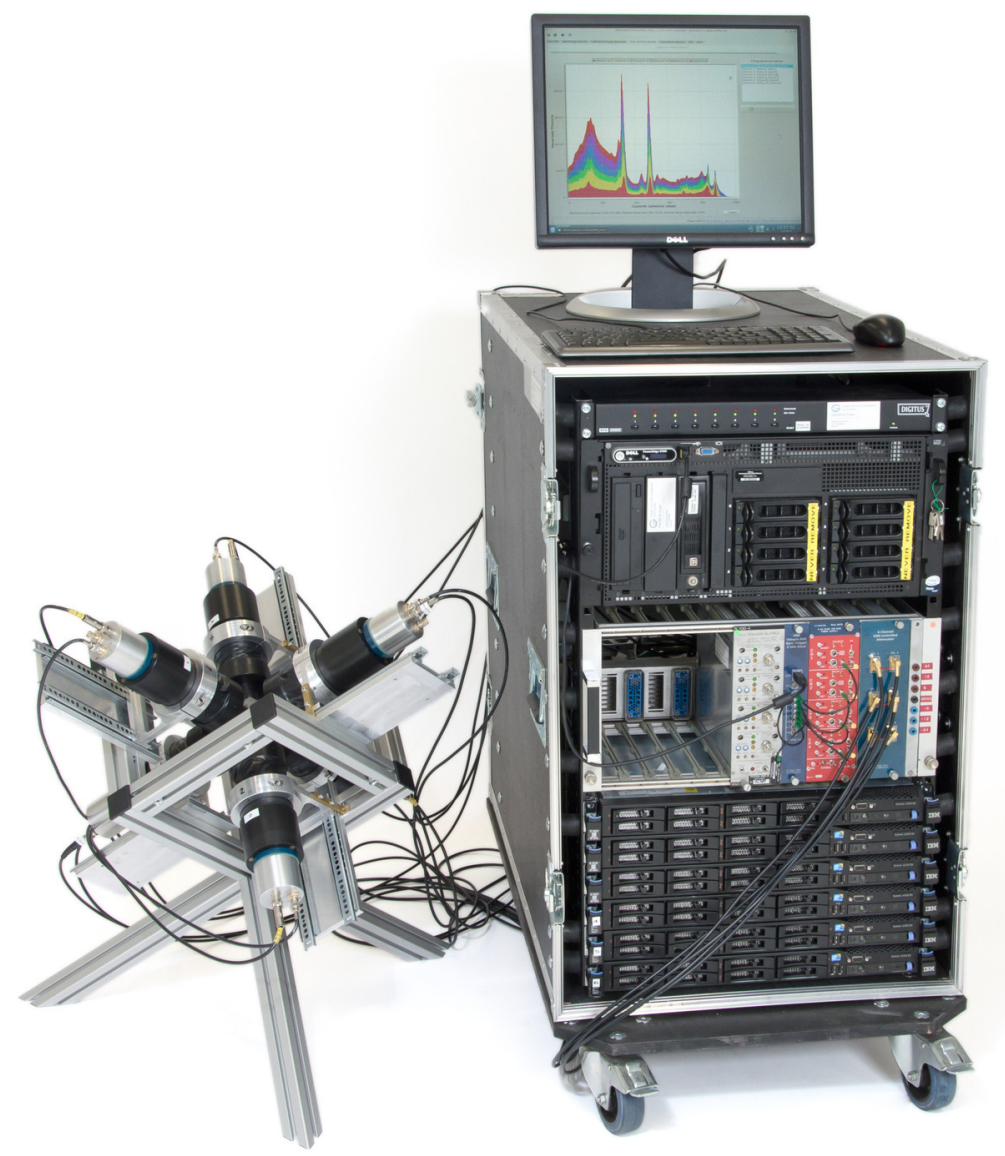

Figure 4.2: Photo of the TDPAC spectrometer with the detector holder for six detector measurements.

\subsubsection{Scintillation detectors}

For the first generation of the spectrometer, four cylindrical $\mathrm{Lu}_{1.8} \mathrm{Y}_{0.2} \mathrm{SiO}_{5}: \mathrm{Ce}$ (LYSO) crystals from Crystal Photonics with a diameter of $30 \mathrm{~mm}$ and a height of $20 \mathrm{~mm}$ where used. Since it turned out that $\mathrm{LaBr}_{3}:$ Ce scintillators are more appropriate concerning the achievable time and energy resolutions while offering much lower inherent activity which allows for measurements with weaker samples, the LYSO crystals were replaced by six $\mathrm{LaBr}_{3}$ :Ce scintillators from Saint-Gobain Crystals (Saint-Gobain, 2004a) with a diameter of $25.4 \mathrm{~mm}$ and a height of $38.1 \mathrm{~mm}$ in conjunction with the extension of the spectrometer to six detectors. The LYSO crystals are not encapsulated and have polished faces and a ground cylinder barrel, which was wrapped in white PTFE thread seal tape in order to improve light yield on the PMT's photo cathode. The $\mathrm{LaBr}_{3}$ :Ce crystals were delivered encapsulated due to their hygroscopic properties. Their housing consists of an 
aluminium shell with a thickness of $0.5 \mathrm{~mm}$ and a $5 \mathrm{~mm}$ thick light guide on the PMT facing surface. The gap between crystal and aluminium is sealed with white potting compound.

Scintillation light is detected using XP2020 photomultipliers from Photonis. This PMT model combines fast rise time with a high level of amplification due to its twelve dynode design. Therefore, the output signal on the anode reaches sufficiently high amplitudes to directly feed it into the digitizers without additional pre-amplification. Due to the high light yield of $\mathrm{LaBr}_{3}$ :Ce scintillators, a PMT model with lower amplification would however be sufficient for the current generation of the spectrometer. In order to be able to adjust the PMTs' output levels to the digitizers' maximum input amplitude, configurable attenuators were added (compare section 4.1.3).

In order to decrease light loss due to reflections at the border between scintillation crystal and the PMT window, a thin layer of silicon grease (Siliconöl M 500000 from Carl Roth $\mathrm{GmbH}+\mathrm{Co} . \mathrm{KG}$ ) was used in between. A special detector case was constructed to prevent environmental light from entering the detector while pressing the scintillation crystal on the PMT's window by applying a defined force. This is important since good mechanical contact is needed to preserve optical contact and avoid light loss at the interface between scintillator and PMT. In order to avoid damage due to the PMT's glass envelope's thermal expansion, a spring mechanism was constructed, which applies force on the PMT's socket thereby pressing the tube on the scintillation crystal, which is held by a cap adapted to the crystal's shape. This cap can be removed for simple mounting of the crystal and is connected to the cylindrical tube surrounding the PMT using a bayonet joint. $\gamma$ absorption as well as Compton scattering in the detector case was minimized by using plastics for all parts except the springs, which are located on the sample's far side.

Traditionally, voltage dividers are used in order to supply the dynodes of photomultiplier tubes with an appropriate voltage cascade from a single high voltage power supply. Passive voltage dividers consisting of a number of resistors however only provide defined voltages as long as no current is drawn off between the resistors. For high count rates the approximation of zero dynode currents (i. e. a stable voltage divider) is not justified. This introduces a count rate dependent drift in the PMTs' amplification. Because most TDPAC nuclides decay significantly during the time of a single measurement, substantially decreasing count rates are the normal case rather than an exception. Changing PMT amplification values during a single measurement however causes drifting energy spectra, which either reduce overall energy resolution or make it even necessary to continually adjust energy calibration and thus the positions of the energy windows.

In order to eliminate these problems, state of the art voltage dividers use components with variable resistance for the stages of the voltage divider connected to the last dynodes where the largest currents are drawn. Usually, either transistors controlled by a parallel resistor based divider or Zener diodes are used.

For the TDPAC spectrometer, Photonis VD124K/T dividers are being used. These volt- 
age dividers employ transistors in the last three divider stages in order to provide high PMT amplification stability up to a dynode current of $200 \mu \mathrm{A}$ (Photonis, 2009).

\subsubsection{Computer equipment ${ }^{1}$}

According to Bardelli et al. (2004), the sampling frequency, as well as the effective sampling resolution, plays a major role concerning the improvement of the achievable time resolution. To obtain a good compromise it was decided to use one Agilent Acqiris U1071A digitizer card per detector. This digitizer features a maximum sampling rate of 2 billion samples per second ( $2 \mathrm{GS} / \mathrm{s}$ ), a resolution of 8 bits (effective resolution: 6.5 bits) and a configurable input full-scale amplitude of $50 \mathrm{mV}$ to $5 \mathrm{~V}$, which can be shifted to negative voltages. However, the most important features for correlation measurements are the availability of a continuously running clock counter with ps-resolution and the possibility to use an external clock signal. Another important improvement in comparison to older digitizers used in former setups is the U1071A's ability to work in a continuous recording mode that allows for the simultaneous recording and read out of waveforms (in Agilent's documents it is called "SAR-Mode"). In this mode the digitizer divides its internal memory into several parts and uses one of these parts to record new events while another part can be read out by the host computer. After the read out has completed and the recording part is full, the parts are rotated to enable seamless recording. This ability decreases the total dead time down to the trigger rearming time of $350 \mathrm{~ns}$ per event compared to $1500 \mathrm{~ns}$ for trigger rearming and additional periodic resynchronizations that could raise the total dead time well above $10 \%$ in older digital setups (Agilent, 2009; Herden, Alves, et al., 2004; Herden, Röder, et al., 2008).

In order to provide sufficient computing power even for the real-time evaluation of very high count rates, the TDPAC spectrometer consists of one computer per digitizer (signal processing computers). Pre-processed data from these signal processing computers is collected, post-processed and saved on an additional server (master computer) providing a large amount of hard disk space.

As signal processing computers, six IBM xSeries servers x3550 M3 are used. Each of these servers uses 1 rack unit of space and is equipped with two Intel Xeon E5620 central processing units (four cores, $2.4 \mathrm{GHz}, 12 \mathrm{MiB}$ cache). Therefore, a total of eight processor cores are available complemented by $24 \mathrm{GiB}$ of RAM. Since the signal processing computers are used for real-time data evaluation only, no local hard disk space is required and they were configured to boot operating system images supplied by the master computer thus avoiding the need for local hard disks and providing simplified maintenance because only one operating system image is used by all signal processing computers. In spite of the massive computing power provided by these signal processing computers, they were much less expensive than any available FPGA option available for digitizers.

\footnotetext{
${ }^{1}$ This section contains revised parts from Nagl, Vetter, et al. (2010)
} 
The master computer is a Dell PowerEdge 2900 III. It contains two Intel Xeon 5450 CPUs (four cores, $3.0 \mathrm{GHz}, 12 \mathrm{MiB}$ cache), $16 \mathrm{GiB}$ RAM and eight hard disks (Hitachi Ultrastar 7K1000) with $1 \mathrm{~TB}$ each. The hard disks are combined to a level 6 redundant array of independent disks (RAID) using the servers internal PERC 6/i hardware RAID controller thus providing $5798 \mathrm{~GB}$ of usable space.

Master and signal processing computers are connected using a configurable Netgear Gigabit Ethernet switch (GS716T-200). The signal processing computers' ports are configured to belong to a port based virtual local area network (VLAN). The master computer uses two Gbit connections to the switch in order to optimize throughput by means of port trunking. A dedicated port of the switch is configured for an uplink connection. It belongs to the external VLAN (port based). The master's connection uses tagged VLAN support to provide access to the internal VLAN of the signal processing computers as well as the external uplink for local area network (LAN) and internet connectivity.

During normal operation, the signal processing computers run headless (without monitor or keyboard) and are controlled via the master computer. For maintenance a KVM switch (keyboard-video-mouse) is available, which allows connecting the available display and keyboard to each of the signal processing computers.

The Intelligent Platform Management Interface (IPMI) available in all signal processing computers is used to remotely boot up and shut down these computers at appropriate times. Since the signal processing computers boot off the network they cannot boot before the master computer has booted and should be shut down before the master computer shuts down. This is done automatically by the initialization sequences of the master computer. Thus, it is sufficient to boot up or shut down the master computer in order to turn the whole setup on or off.

\subsubsection{Auxiliary electronics}

Clock Determining the time difference between the incidences of $\gamma$ photons detected by two detectors involves comparisons of timestamps determined on different signal processing computers since every detector is connected to a separate signal processing computer. Therefore, the synchronization of all signal processing computers is crucial for precise coincidence time measurements. In fact, the achievable time resolution is-among other parameters-limited by the quality of synchronization.

The used Agilent Acqiris U1071A digitizers implement an integrated counter, which keeps track of the times of trigger occurrences. The counter is incremented by the sampling clock (with up to $2 \mathrm{GHz}$ ) and exact trigger times are determined by linear interpolation between the pre- and post-trigger samples.

Synchronization of digitizers in different computers requires a common external clock source to synchronize the time counters and ADCs. While it is possible to use a $10 \mathrm{MHz}$ reference clock, which is multiplied by phase locked loop (PLL) circuits integrated in the digitizers, a clock providing the sampling frequency of $2 \mathrm{GHz}$ was preferred in or- 


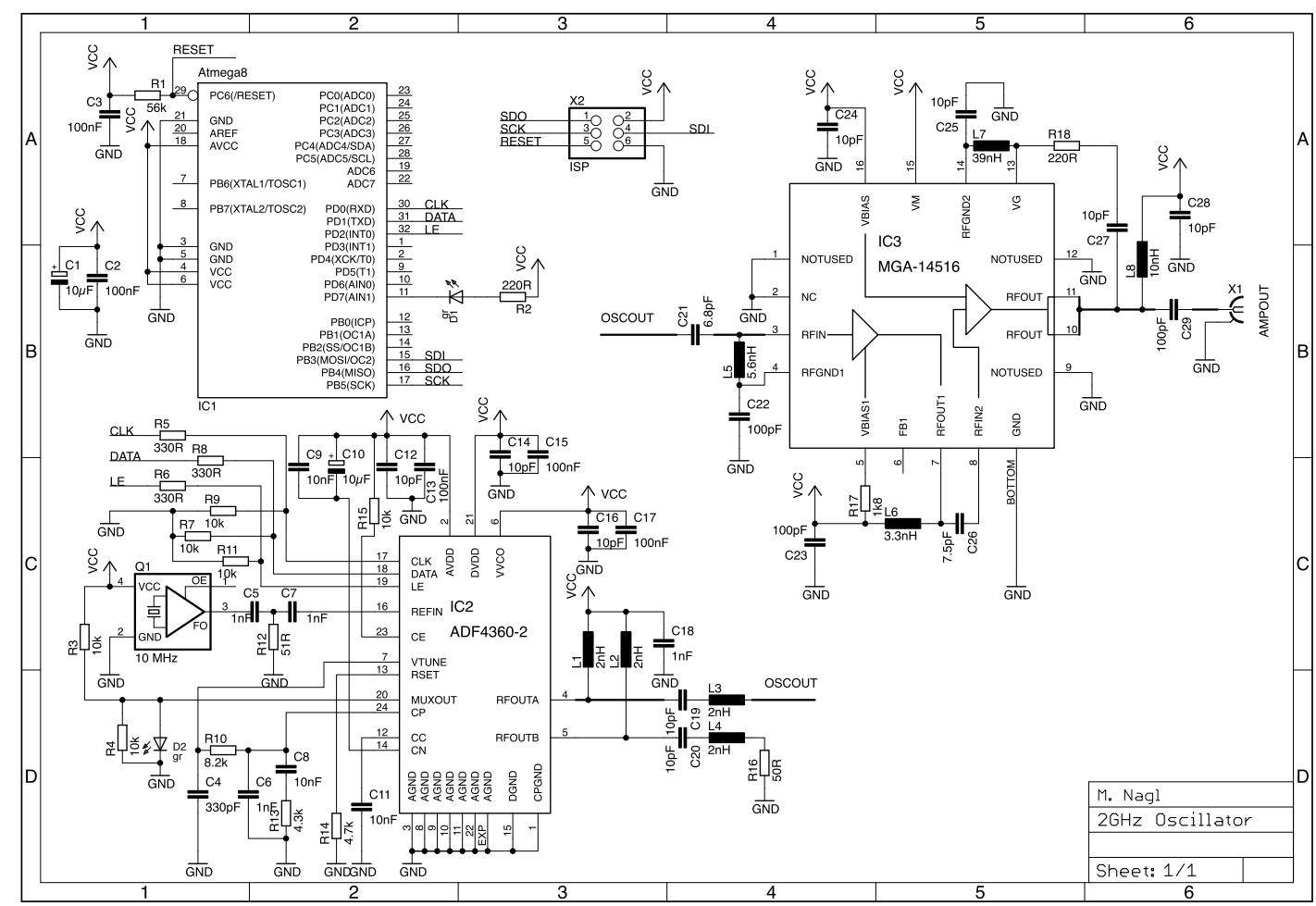

Figure 4.3: Schematic of the $2 \mathrm{GHz}$ oscillator and amplifier developed as external clock for the digitizers. The Atmega8 (IC1) is used to initialize the ADF4360-2 (IC2), which synthesizes the clock signal OSCOUT based on a reference oscillator (Q1). This signal is then amplified by a MGA-14516 (IC3). The amplified output signal is available at X1 and then fed into the power divider, which supplies it to the digitizers' clock inputs.

der to reduce jitter due to the frequency multiplication. The output signal of the clock is distributed using a passive six-way power divider (Microlab D6-69FF, frequency range $698 \mathrm{MHz}$ to $2700 \mathrm{MHz}$, amplitude balance $<0.8 \mathrm{~dB}$, compare Microlab, 2013), which provides lower jitter and temperature dependence than active solutions.

A $2.0 \mathrm{GHz}$ oscillator was designed based on the ADF4360-2 integrated synthesizer and VCO chip (Analog Devices, 2012b) because suitable clock sources were not available. This chip is able to synthesize digitally configurable frequencies from a input frequency of a $10 \mathrm{MHz}$ quartz oscillator. The clock configuration is written to the ADF4360-2's configuration registers by an additional Atmel Atmega 8 micro-controller at power up.

Because of the external clock inputs' impedance of $50 \Omega$ and the digitizers' requirement of a clock signal with peak-to-peak amplitude of $>500 \mathrm{mV}$ (compare Agilent, 2012), a minimum power of $3.98 \mathrm{dBm}$ is required per digitizer. Considering split loss of $-7.8 \mathrm{~dB}$ and insertion loss of $-1.0 \mathrm{~dB}$ at the power divider, a minimum power level of $12.78 \mathrm{dBm}$ 
is required at the dividers input. Due to insertion loss at the digitizers' clock inputs, cable losses, and limited amplitude balance of the divider, several $\mathrm{dB}$ must be added to ensure a peak-to-peak amplitude difference of at least $500 \mathrm{mV}$ for all digitizers.

To close the gap between the ADF4360-2's maximum output power of $-6 \mathrm{dBm}$ and the needed divider input power of more than $13 \mathrm{dBm}$, a suitable amplifier was needed. Since it was again very difficult to find suitable modules, an amplifier based on the MGA-14516 from Avago Technologies (Avago Technologies, 2009) was developed. This amplifier's combined gain of $31.7 \mathrm{~dB}$ is sufficient to reach the minimum amplitude for all digitizers without difficulty even in case of high losses at the cables and connectors. Figure 4.3 shows a schematic of the used clock and amplifier circuits.

Synchronization The digitizers' internal clock counters are started once at the beginning of a measurement. Since the command to start the measurement is being sent from the master computer to the signal processing computers via Ethernet, it is affected by variable latencies originating from the network hardware as well as the operating system's networking stack. Additional variable time offsets occur during the calibration process of the digitizers, which precedes the start of measurements. These latencies are in the order of milliseconds up to seconds. In order to determine matching timestamp offsets for all detector pairs making it thereby possible to search for coincident events in a limited range of time differences, a synchronization process is needed.

Synchronization was implemented by sending a steep pulse signal simultaneously to all digitizers. Each signal processing computer determines this pulse's timestamp according to its own clock counter. This timestamp is then subtracted from all subsequent timestamps from this digitizer. Since the clock counters of all digitizers are incremented simultaneously due to the common external clock, the resulting timestamps than differ by not more than several nano seconds. Remaining offsets are due to differences in cable length and unequal amplitude balance and can be eliminated after coincidence search (compare section 4.2.4).

The one-time synchronization pulse is triggered by a custom USB interface electronics shown in fig. 4.4. The TTL-signal generated by this device is fed into the trigger input of a pulse generator (currently a TGP110 from TTi). This pulse generator creates a pulse with a rise-time of less than $10 \mathrm{~ns}$ and sufficiently high positive amplitude to achieve more than $1 \mathrm{~V}$ on each digitizer's external trigger input. It is possible to use these inputs instead of the signal inputs because the trigger source of the Agilent Acqiris U1071A digitizers can be switched from the external trigger input to trigger on signal mode without stopping the internal clock counter thus preserving the offset determined by triggering on the synchronization signal after switching to normal operation. The external trigger input's impedance is again $50 \Omega$ (compare Agilent, 2012) making a sufficiently strong pulse generator necessary. The signal is distributed from the pulse generator to the digitizers by means of a six channel power divider (INSTOCK Wireless PD1160, frequency range $700 \mathrm{MHz}$ to 


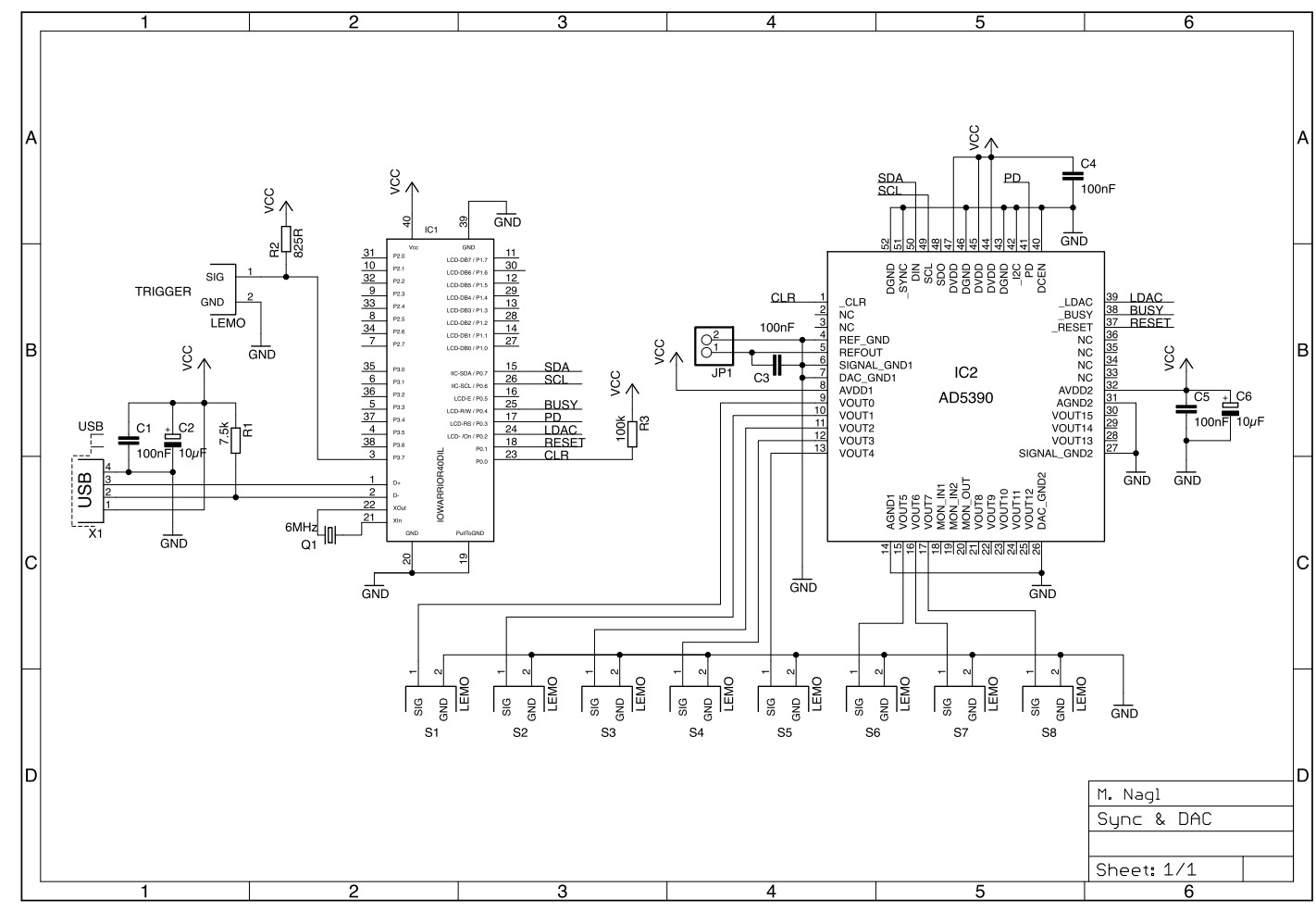

Figure 4.4: Schematic of the synchronization and digital to analog converter (DAC) interface. An IO-Warrior (IC1) provides USB to $\mathrm{I}^{2} \mathrm{C}$ and IO interfaces. One of its outputs is used as trigger for the synchronization of digitizers while a DAC (IC2) is connected to the $\mathrm{I}^{2} \mathrm{C}$ bus and outputs up to sixteen (eight connected) configurable voltages between $0 \mathrm{~V}$ and $2.5 \mathrm{~V}$ with 14 bit resolution, which are used to control the high voltage power supply channels for the PMTs.

$2700 \mathrm{MHz}$, phase balance $<5^{\circ}$, amplitude balance $<0.3 \mathrm{~dB}$, compare INSTOCK wireless, 2007).

Attenuation Due to the high light yield of $\mathrm{LaBr}_{3}$ :Ce scintillators, the output amplitude of the XP2020 photomultiplier tubes exceeds $10 \mathrm{~V}$ even for moderate $\gamma$ energies in the range of a few hundred $\mathrm{keV}$. Since these amplitudes are beyond the maximum fullscale voltage of the digitizers $(5 \mathrm{~V})$, they must be reduced in order to make measurements possible. There are two options for reducing the PMT output amplitude:

1. Reduction of the PMT voltage, which leads to less electron multiplication per dynode stage thus reducing output signal amplitudes.

2. Attenuation of the output signals to adequate maximum amplitudes. 


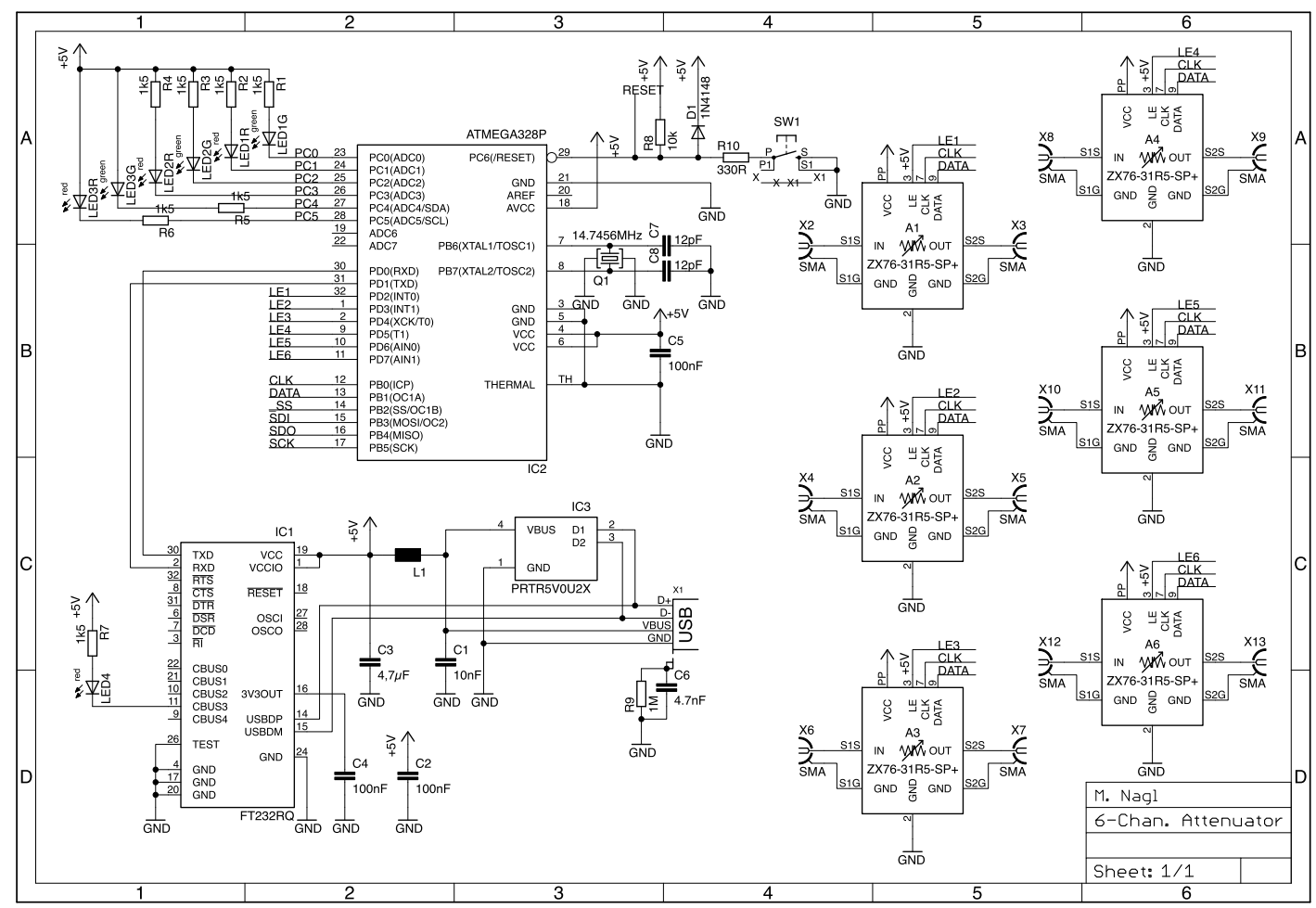

Figure 4.5: Schematic of the six channel attenuator. Six ZX76-31R5-SP+ digital step attenuators provide attenuation for the anode outputs of six detectors. They are configured by an USB interface consisting of a FT232RQ interface chip (IC1) and an Atmega328P microcontroller (IC2).

The first approach, while being very simple, reduces detector performance. Because of the reduced potential difference between subsequent dynodes, electron acceleration is lowered. This results in larger transit times and larger transit time spreads as well as lower secondary electron yield (Hamamatsu, 2007). The lower secondary electron yield on the first dynodes reduces energy resolution (less statistics means larger relative errors). Furthermore, counting statistics—which influences the achievable time resolution-is impaired.

The second approach promises better results since the detectors' energy and time resolutions are not affected by attenuation of their output signals and detector noise is attenuated as well thus preserving the signal to noise ratio. Drawbacks are merely additional thermal noise added by the attenuation device and signal distortions in case of impedance mismatching of the attenuators. Thermal noise is not relevant for the detector signals due to their high amplitude. To avoid distortions due to bad matching, high frequency attenuators with sufficient bandwidth and defined impedance must be used. 
These requirements are fulfilled by the selected digital step attenuators ZX76-31R5SP+ from Mini-Circuits (cf. Mini-Circuits, 2012). They provide a bandwidth of up to $2.4 \mathrm{GHz}$, low insertion loss (important if attenuation is disabled), high power levels with low signal distortion and a serial configuration interface that allows control of attenuation by a micro controller circuit in the range between $0 \mathrm{~dB}$ and $-31.5 \mathrm{~dB}$ with a resolution of $0.5 \mathrm{~dB}$. Configuration is done via a custom USB interface as shown in fig. 4.5. The USB interface is controlled by PacMaster, which provides GUI elements for the attenuation settings (cf. section 4.2.3). Use of the attenuators is optional.

Power supply Currently, a CAEN N472 four channel high voltage power supply is used for four of the detectors and a Wenzel N 1130-4 power supply for the remaining two detectors (both in NIM housings). A planned replacement of the power supplies by six Ultravolt 4A24-N20-F-M-25PPM modules will further decrease the amount of space needed by the spectrometer. All power supplies provide analog inputs for configuring their output voltage. The transformation ratio is $1 \mathrm{mV} \mathrm{V}^{-1}$ for the two NIM power supplies and $0.8 \mathrm{mV} \mathrm{V}^{-1}$ for the Ultravolt modules.

In order to be able to control the PMT voltage via PacMaster, the power supplies are connected to a 14 bit digital to analog converter (AD5390, compare Analog Devices, 2012a). The DAC is part of the sync device shown in fig. 4.4.

\subsection{Software suite}

Since digital signal processing implicates the move of processing complexity from hardware to software, this software plays an important role for the spectrometer's overall performance. Therefore, great care has been taken to optimize the software in a way that maximizes the advantage taken from the new approach. This section describes the software stack developed to meet this challenge.

\subsubsection{Software design and measurement workflow}

A digital implementation of a TDPAC setup allows postponing of important parts of the configuration and thus saves valuable measurement time in the beginning when the samples still provide high levels of activity. The software stack described in the following sections taps this potential while other, even newer, implementations like the one of Jäger, Iwig, and Butz (2011) are basically emulations of analog setups.

The steps for conducting a measurement are:

1. Basic setup (attenuation, PMT voltages)

2. Start of measurement

3. Eventually configuration and start of online evaluation(s) 
4. Stop of measurement

5. Offline evaluation of recorded data / Finishing of online evaluation(s)

Evaluations of the recorded data can be started anytime-online while the measurement is still running or offine after the measurement was finished. Online evaluations are updated automatically whenever new data arrives and can therefore be used as a tool to monitor measurement progress. The evaluation process consists of the following steps:

1. Selection of relevant charge ranges

2. Energy calibration / spectrum matching (charge to energy mappings)

3. Definition of energy windows

4. Setup of coincidence search settings

5. Fitting of coincidence spectra and $R(t)$ calculation

In order to make spectrometer operation as simple as possible and reduce the probability of operating errors, user interaction was limited to two software programs: One for setting up the machine parameters and controlling the measurement called PacMaster and a second for the online and/or offline evaluation of recorded data called SpectraPAC. Both programs provide a graphical user interface. The separation of measurement and evaluation into two programs is important concerning flexibility: While PacMaster runs on the spectrometer's server (cf. fig. 4.1), SpectraPAC can be used on any computer, which can access measurement data. It is possible to run several instances of SpectraPAC simultaneously on the same data file-even in online evaluation mode-allowing for evaluating of several decay cascades at once.

Data acquisition and signal processing on the signal processing computers is done using a third program called PacMan, which is remotely controlled via network by PacMaster and therefore does not provide a user interface. The signal processing computers and PacMan are designed to be operated headless and diskless. Operating system images are provided by the server via network and processed data is immediately forwarded to the server during recording. These design principles simplify software updates and ensure consistency since only a single system image exists, which is booted by all signal processing computers. At the same time cost per signal processing computer is kept low since no disks or disk controllers are necessary.

PacMan, PacMaster, SpectraPAC, and several helper tools are bundled in the Pac-Suite and are available for download (Nagl, 2008-2014). An overview of the task allocation among the main applications of Pac-Suite is shown in fig. 4.6. 


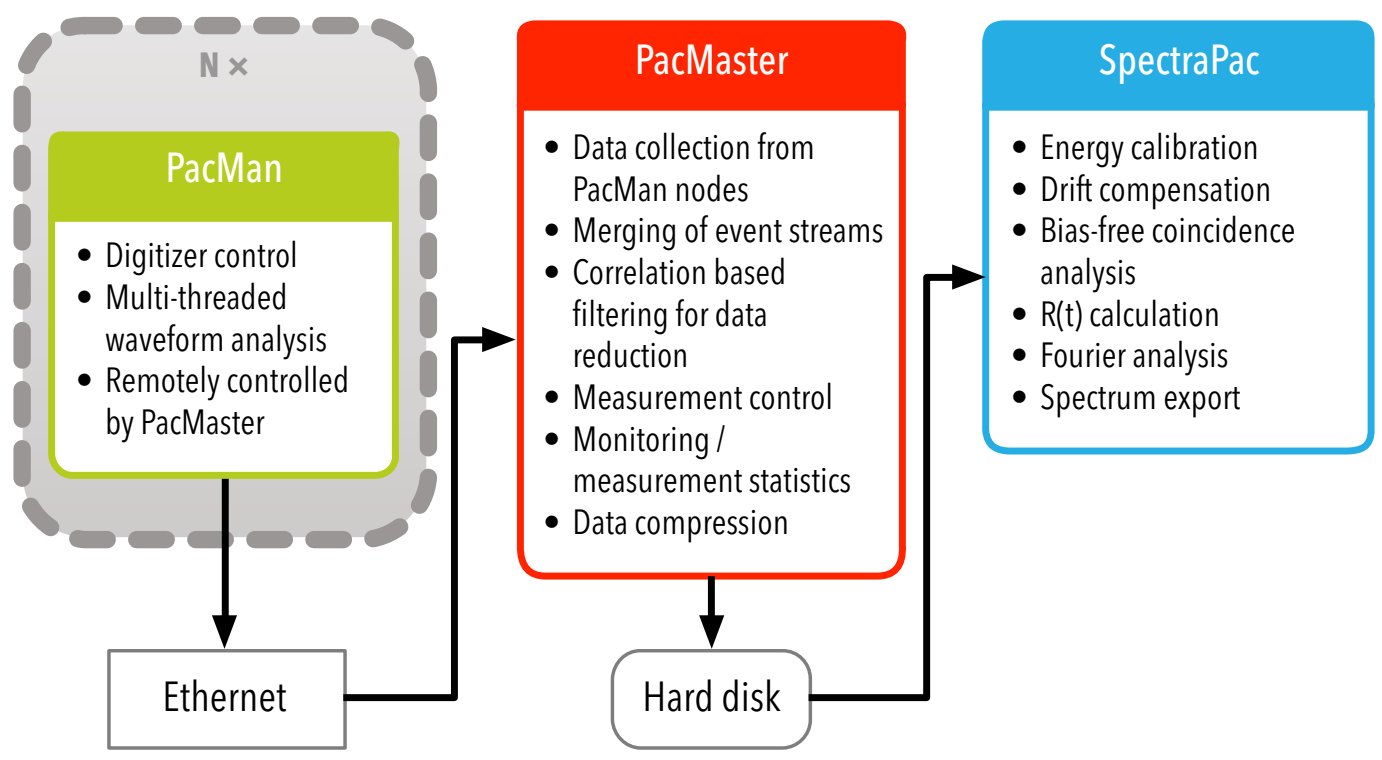

Figure 4.6: Task allocation among the PAC-Suite applications. PacMan runs on all recording computers, i. e. once per detector. It sends its results immediately via an Ethernet connection to the PacMaster instance running on the server where results from all recording computers are collected, processed, compressed, and saved to disk. From there they can be evaluated online as well as offline using SpectraPAC.

\subsubsection{Data acquisition and preprocessing software}

PacMan, the software running on the headless signal processing computers, is designed for maximum data throughput. Since each of the used signal processing computers provides eight CPU cores, a special focus was laid on parallel processing of data. This is possible because subsequent detector signals are usually independent from each other. Thus, it is possible to process the waveforms isolated on different CPU cores.

Figure 4.7 shows a schematic overview of the data flow through PacMan. Data read-out from the digitizer and dispatch via network cannot efficiently be parallelized. Therefore, there are single objects for these tasks. However, as soon as waveforms were read from the digitizer they are combined into "waveform chunks". These chunks are then distributed across the analysis threads. Thereby each thread receives the same amount of data. After an analysis thread finishes signal processing, it forwards its results to the network communication thread, which sorts the results received from all analysis threads and sends them via network to the PacMaster instance on the server.

For the actual data analysis, each analysis thread loads an analyzer object from an userselected plug-in. This means a signal processing approach can be chosen by selecting the 


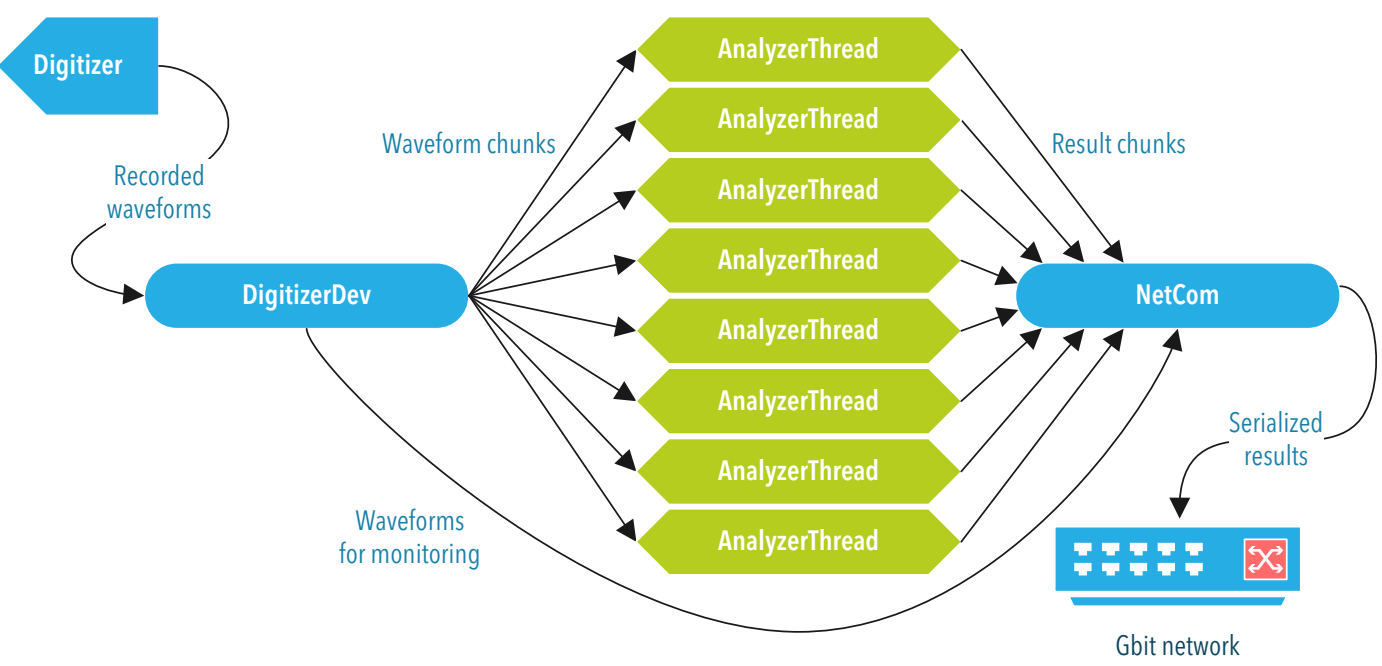

Figure 4.7: Data processing on the signal processing computers running PacMan. Data is read from the digitizer and partitioned into chunks. These chunks are then distributed across a number of analysis threads. Each of these independent threads analyzes waveforms and determines energy and time information. The results are then collected, sorted, and dispatched via an Ethernet connection by a single network communication object since sorting and sending cannot be efficiently parallelized in this case.

appropriate plug-in. A minimalistic plug-in interface simplifies the implementation of new processing approaches.

Currently two evaluation plugins are available. Both determine the event's energy by integration as described in section 5.1. The implemented timing methods are constant trigger threshold timing (cf. section 5.3) and the digital constant fraction of integral values approach (cf. section 5.9).

The number of simultaneous analysis threads run by PacMan can be remotely configured using PacMaster on the server. Selecting a number similar to the number of available CPU cores provides a good distribution of load across the cores and low peak load values. The length of input and output queues used for inter-thread communication can also be set using PacMaster but usually it is not necessary to change the default values.

Since buffering occurs only in the queues for inter-thread communication, PacMan typically uses only a few $\mathrm{MiB}$ of memory on the signal processing computers.

The controlling PacMaster instance can put PacMan into synchronization mode for the synchronization of the digitizer clocks in several signal processing computers. In synchronization mode the digitizer is configured to trigger on its external trigger input instead of the detector signal. The first detected signal on the external trigger input ends synchronization mode. This signal's timestamp is then used as offset and subtracted from all 


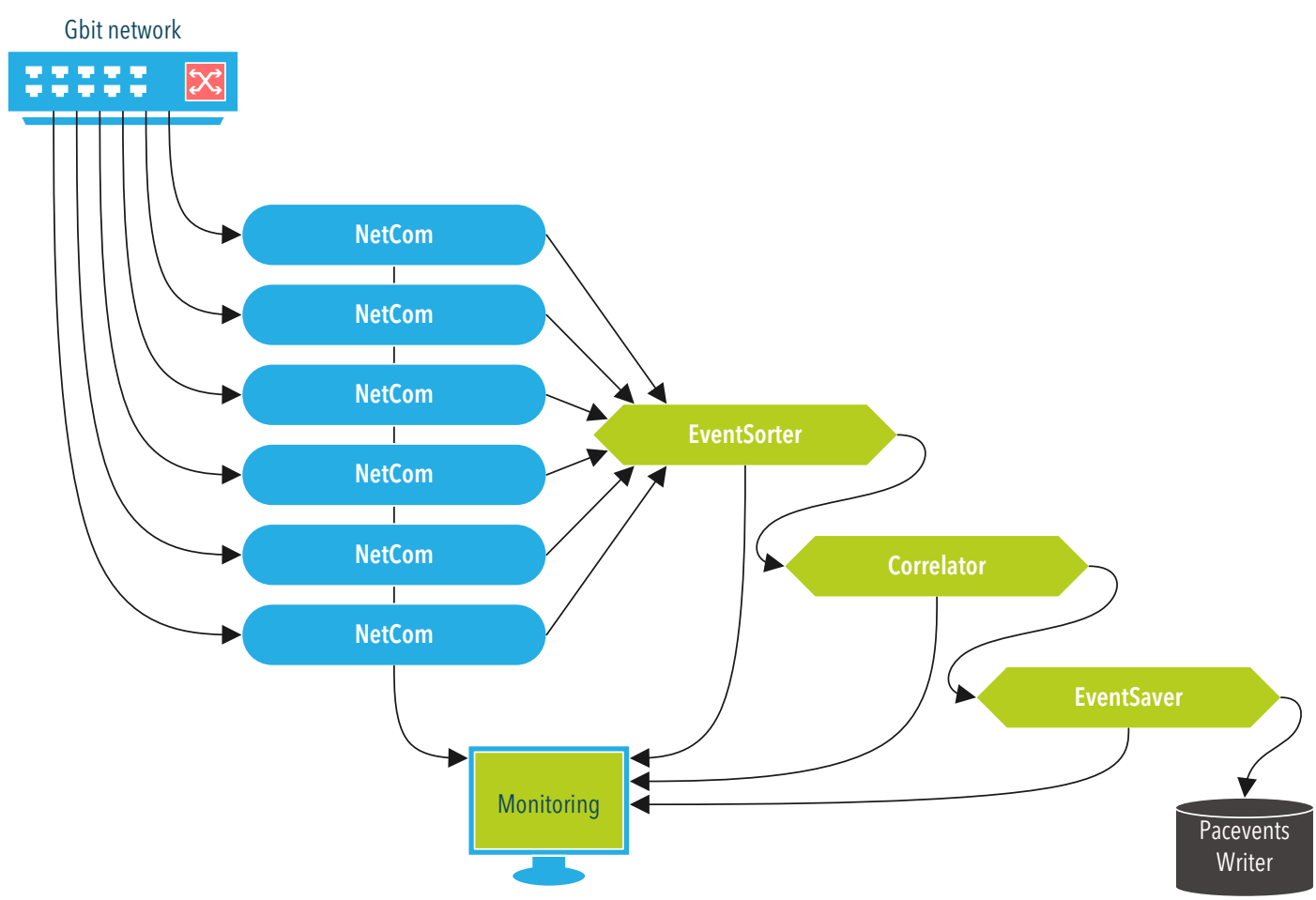

Figure 4.8: Data collection and processing on the server by PacMaster. Data is read by a signal processing computer specific network communication object (here shown for six computers). Each communication object belongs to a separate thread. Received data is then passed to a sorter thread, which unpacks the chunks of $\gamma$ events and sorts them according to their timestamps. This sorted stream of $\gamma$ events is then fed into the correlator, which implements a windowless coincidence search and allows the event saver to discard uncorrelated events.

subsequent timestamps sent to PacMaster. The synchronization sequence is controlled by PacMaster (see state machine on page 54).

\subsubsection{Data collection and selective recording software}

PacMaster is the linchpin of all measurements conducted with the digital TDPAC spectrometer. It configures and controls the signal processing computers, receives their results, sorts, filters, and compresses them, and finally saves them to the server's hard disk. At the same time it provides monitoring and diagnostic functionality allowing for quick setup and supervision of measurements. Since it does not only provide a graphical user interface but is also involved in CPU intensive data processing, it is designed multi-threaded. This allows for efficient usage of all CPU cores of the server. 
Figure 4.8 shows a schematic overview of the data flow through PacMaster. Incoming data, which arrives via network from the signal processing computers running PacMan, is received by dedicated threads and buffered in input queues. A sorter thread unpacks the event chunks and merges events from all detectors into a single queue ordered according to their timestamps. The subsequent correlator thread tags correlated events for later correlation based filtering. After correlation analysis, events are compressed using zlib (Gailly and Adler, 2013) and saved to disk. At this stage, it is possible to enable windowless filtering, which prevents isolated events that are neither preceded nor followed by other events in a certain timeframe from being saved at all.

The sorter thread, which merges events from different detectors, is particularly interesting in comparison with analog setups. In analog TDPAC setups, information from different detectors is collected at the TAC and the routing logic. Both parts are critical for the setup's timing performance and scale bad since especially the routing logic rapidly grows very complex if the number of detectors is increased. In contrast, the sorter thread processes events, which are already labeled with global timestamps. Therefore, it can use input buffers and as long as these buffers are not too small, no events are lost and it cannot impair timing performance. Adding more detectors merely means adding more input buffers which does not significantly decrease sorting performance. This design difference therefore constitutes an important advantage of the digital spectrometer over its analog predecessors concerning flexibility and extensibility.

Besides the data processing functionality, PacMaster also shows monitoring information. It provides an overview on the amount of events processed for each detector and other throughput information. Additionally, it shows energy spectra-both short-term and spanning the whole recording session-which are important for configuration because they allow to select appropriate PMT voltages, digitizer full-scale values, and attenuation values for the desired energy range. In order to monitor the signal analysis settings, PacMaster displays sample waveforms and analysis parameters. The display functionality is provided by the same plugins, which are loaded by PacMan for the processing of recorded waveforms and therefore comprises method specific information.

A special monitoring option available in PacMaster is the possibility to output raw signal data into an auxiliary file. This is helpful to collect real-life data for the development and optimization of signal processing algorithms.

State machine Because the initialization of a measurement consists of a number of steps, which are all subject to possible errors (like defective hardware, parts of the spectrometer not being switched on, ready, or initialized etc.), a state machine was implemented to make control of this system both simple and robust. Figure 4.9 shows an UML state-chart of this state machine. Its implementation made it possible to operate the spectrometer both very stable and robust while significantly simplifying the source code. Control commands for the PacMan instances on the signal processing computers 


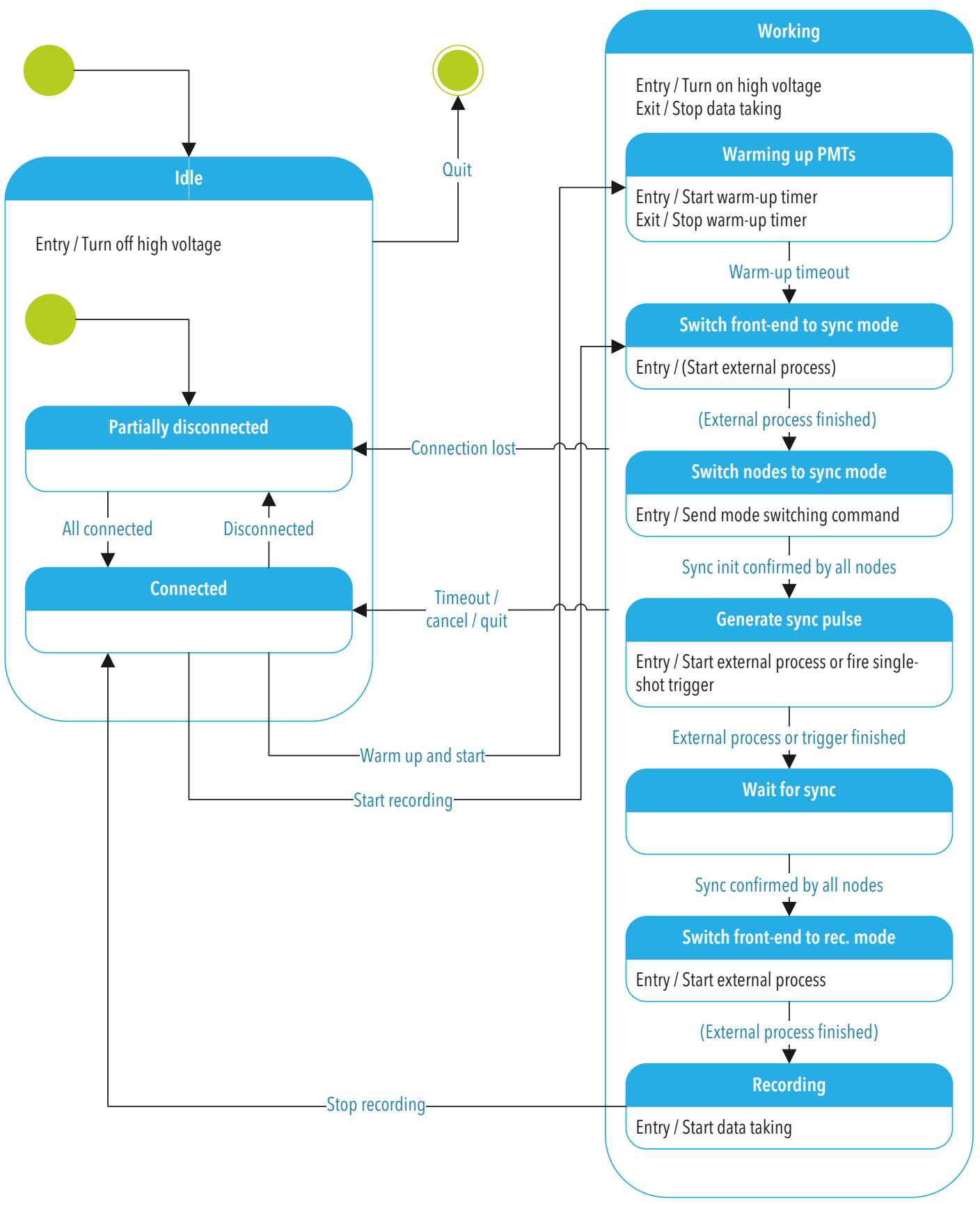

Figure 4.9: Simplified UML state-chart of the state machine implemented in PacMaster. This state machine ensures that the graphical user interface (GUI) stays in accordance with the spectrometers current state and avoids operating errors by disabling parts of the GUI not suitable for a specific state as well as it avoids data corruption on unexpected errors by defining safe shutdown procedures for these cases. 
are dispatched at state changes. Accordingly, some messages from the signal processing computers can trigger state changes.

The amplification characteristics of photomultiplier tubes and their voltage dividers show significant temperature dependence. Therefore, amplification changes after high voltage is applied until the detector temperature reaches a stable state. If measuring during this period shall be avoided, it is possible to switch on the high voltage supply before the sample is mounted or to automatically start the measurement after a user-defined warm-up time has passed. This feature is based on the "warming up PMTs" state and its transitions.

Several intermediate states are available, which make adaptation of the Pac-Suite to several spectrometer implementations feasible because specific hardware interfaces to change external parts' properties do not have to be included into PacMaster but can be realized as external commands that are then called while the states "switch front-end to sync mode", "switch nodes to sync mode", and "generate sync pulse" are entered.

For every recorded event, PacMaster writes detector, collected charge, and timestamp to disk. Its design and the design of PacMan is optimized to fulfill this task very efficiently thus allowing for high throughput - i. e. the processing of high count rates. The only analysis done before saving events is a windowless correlation analysis. This provides the possibility to filter and discard uncorrelated events thus saving disk space in case of high levels of uncorrelated background radiation.

\subsubsection{Data evaluation software}

The evaluation of data files written by PacMaster can be done using and additional application called SpectraPAC. It only reads data files and does not require lock mechanisms. Therefore, it allows for online and concurrent evaluations: If SpectraPAC hits the end of a running measurement's data file, it stops coincidence search and allows finishing the evaluation. As soon as a chunk (the actual chunk size is defined in the configuration options of PacMaster) of new data is written by PacMaster, SpectraPAC detects the availability of this data and runs an incremental evaluation. Therefore, it is possible to use SpectraPAC to monitor measurement progress. An arbitrary number of SpectraPAC instances can be started using the same data file, thus making simultaneous (online) evaluation of several decay cascades possible.

SpectraPAC's graphical user interface guides the user step-by-step from the data file to a result spectrum. These steps are:

1. Selection of signal charge ranges (optionally per detector)

2. Identification of two energies per detector for energy calibration

3. Selection of energy windows for start and stop events and additional options for the coincidence search 


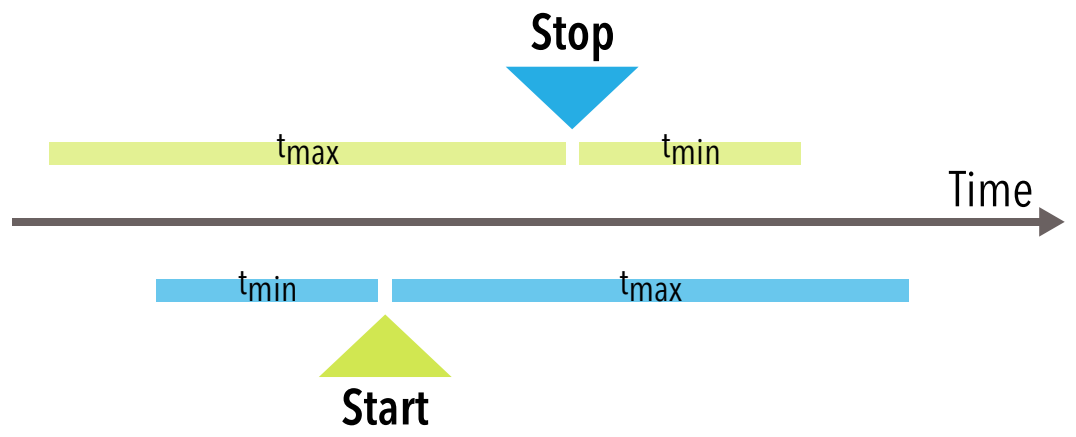

Figure 4.10: Rejection of ambiguous coincidences as implemented by SpectraPAC. The green and blue arrows symbolize the occurrence of a start and stop event, respectively. Surrounding each event there are periods of time where additional signals would be ambiguous. These periods are shown in green for additional start events and in blue for additional stop signals. Their length is determined by the min. time difference $\left(t_{\min }\right)$ and max. time difference $\left(t_{\max }\right)$ settings of the coincidence search. If more than one event from the respective category occurs in one of the highlighted periods, all coincidences involving one of the affected events are discarded.

4. Fitting of spectrum onset and background levels

\section{Export of result spectrum}

The first two steps are necessary to create a combined energy spectrum from the recorded charge values. In the first step, charge spectra are created. In the second step the user is able to select features of these spectra (i. e. characteristic peaks) and assign energy values. Based on two assignments per spectrum, a linear mapping between measured charge values and $\gamma$ energies is determined and used to convert all charge values to $\gamma$ energies. Based on these values, a combined energy spectrum for all detectors is created. This is then used to select energy windows for start and stop events.

Coincidence analysis The third step prepares the actual coincidence search. Besides the energy windows, the maximal and minimal time differences and the number of channels (i. e. histogram bins) are important parameters for the coincidence search algorithm.

There exist a number of factors introducing random offsets between the timestamps of each pair of two detectors. These are differences in the PMTs' transit times, different signal cable lengths, differences in the length of the cables connecting the synchronization signal splitter with each digitizer, different attenuations in the synchronization signal paths of the digitizers, among others. By precise matching of all offset generating factors, the overall offsets can be kept below 5 ns. Nevertheless, the remaining offsets necessitate the implementation of a reverse coincidence search (a search for stop signal preceding start signals), which is equivalent to delay lines introduced into the processing of stop signals in 
analog setups. The maximum time span of the reverse coincidence search can be selected by the min. time difference, which is therefore always negative.

By default all ambiguous cases are ignored and do not contribute to the coincidence spectra (see fig. 4.10). Counting them would introduce lag (if the first or last event were preferred as in case of some analog spectrometers) or would impair the signal to noise ratio (if all combinations were accepted). The unbiased filtering of ambiguous cases makes corrections as described by Coates (1973) superfluous. Due to this filtering, the length of the time window influences the amount of events that have to be rejected. This means that very large window settings are unfavorable-especially for high count rate measurements-because in these cases a considerable amount of events has to be discarded.

For measurements with special nuclides where start or stop signals are coincident with additional $\gamma$ photons falling into the same energy windows, selective lifting of the rejection rules was implemented. It is possible to allow multiple start and multiple stop signals and count coincidences with the $n^{\text {th }}$ event or each combination. It is also possible to selectively disable rejection of prompt signals, i. e. cases where real coincidences are often accompanied by coincidences with zero time difference.

Background and onset fitting As described before, offsets between the timestamps of each pair of detectors occur. While the reverse coincidence search avoids clipping of spectra, the specific offset values still have to be determined. This task is addressed by fitting an exponentially modified Gaussian model function

$$
\begin{array}{r}
S(t)=\quad \frac{A}{2} \cdot \exp \left[\frac{\ln 2}{T_{\mathrm{hl}}}\left(\frac{\sigma^{2} \ln 2}{2 T_{\mathrm{hl}}}-\left(t-t_{0}\right)\right)\right] \\
\cdot \operatorname{erfc}\left[\frac{\sigma \ln 2}{T_{\mathrm{hl}} \sqrt{2}}-\frac{t-t_{0}}{\sigma \sqrt{2}}\right] \\
+\frac{B}{\sigma \sqrt{2}} \cdot \exp \left[-\frac{\left(t-t_{0}\right)^{2}}{2 \sigma^{2}}\right]+N_{\mathrm{bg}}
\end{array}
$$

to each result spectrum.

The exponentially modified Gaussian (first two terms) consists of an exponentialwhich models the intermediate state's decay—convolved with a Gaussian, modeling finite time resolution. The third term models the prompt peak, which is observable in some cases, especially if the stop energy window overlaps with energies of conversion x-rays. A constant background is added as fourth term. $t_{0}$ is the relevant parameter of this fit, which can be used as time offset value. $\sigma$ is an estimate of the achieved time resolution.

Besides the time offset, the background level is another parameter that needs to be determined before further evaluation can occur since it needs to be subtracted from each spectrum. In principle it is possible to use $N_{\text {bg }}$ from the model above as background 
estimate. However, this proved not to be sufficiently precise. To understand the deviation between $N_{\text {bg }}$ and the real background level, it is important to consider that the model function eq. (4.1) in fact does not model the desired signal-i. e. the exponential decay's modulation. Since this modulation is different for spectra belonging to detector pairs with angular differences of $90^{\circ}$ and $180^{\circ}$, respectively, a systematic bias with significant effect on $N_{\mathrm{bg}}$ is introduced. The modulation cannot be modeled because it is unknown (in fact its determination is the measurement's goal).

To overcome this problem it is necessary to avoid the parts of the signal with high amplitudes. This is done by fitting a simple exponential model function

$$
U(t)=A \cdot 2^{-\frac{t-t_{\text {trart }}}{T_{\mathrm{hl}}}}+N_{\mathrm{bg}}
$$

to the lower half of the spectrum's decaying slope. This method proved to provide sufficiently precise $N_{\text {bg }}$ estimates. The initial time of the spectrum's subset, which is used for fitting, is set to 0 (by subtracting $t_{\text {start }}$ ) in order to avoid numerical problems occurring due to overly large or small values of the exponential.

Since typical numbers of coincidence spectra are 12 for four detector setups and 30 for six detector setups, automatic fitting is desirable. This requires reliable parameter estimation in order to provide useful initial values for the fit algorithm. Automatic estimation of the parameters $A, B, N_{\text {bg }}, T_{\mathrm{hl}}, t_{0}$, and $\sigma$ for eq. (4.1) as well as $A, N_{\text {bg }}$, and $T_{\mathrm{hl}}$ for eq. (4.2) is done using the following rules:

A To avoid distortions around the peak due to modulations and limited time resolution, a virtual peak amplitude is calculated by doubling the background corrected amplitude, which occurs $T_{\mathrm{hl}}$ after the peak.

B is set to $N_{\max }-N_{\mathrm{bg}}-A$, where $N_{\max }$ is the spectrums maximal amplitude.

$\mathbf{N}_{\mathrm{bg}}$ is estimated by averaging histogram bins from the range of time before the spectrum begins, i. e. the reverse coincidence range. The averaging always begins at the leftmost histogram bin and includes bins up to either half of the range between the leftmost bin and the zero point of the time axis or half the range between the leftmost bin and the spectrum peak's (i. e. highest amplitude bin's) time value, whichever is smaller. A minimum of three bins is averaged in any case.

$\mathbf{T}_{\mathrm{hl}}$ To avoid distortions due to the modulation around the spectrum's peak, the length between the peak and the spectrum's end is determined and only the range from a fourth of this length after the peak to the end is considered for $T_{\mathrm{hl}}$ determination. $N_{\text {bg }}$ is subtracted from the amplitudes in this range and the point, where half of the subset's maximum amplitude is reached, is searched from the subset's end towards its beginning. The time difference between the subset's beginning and this point is then used as estimate for $T_{\mathrm{hl}}$. 

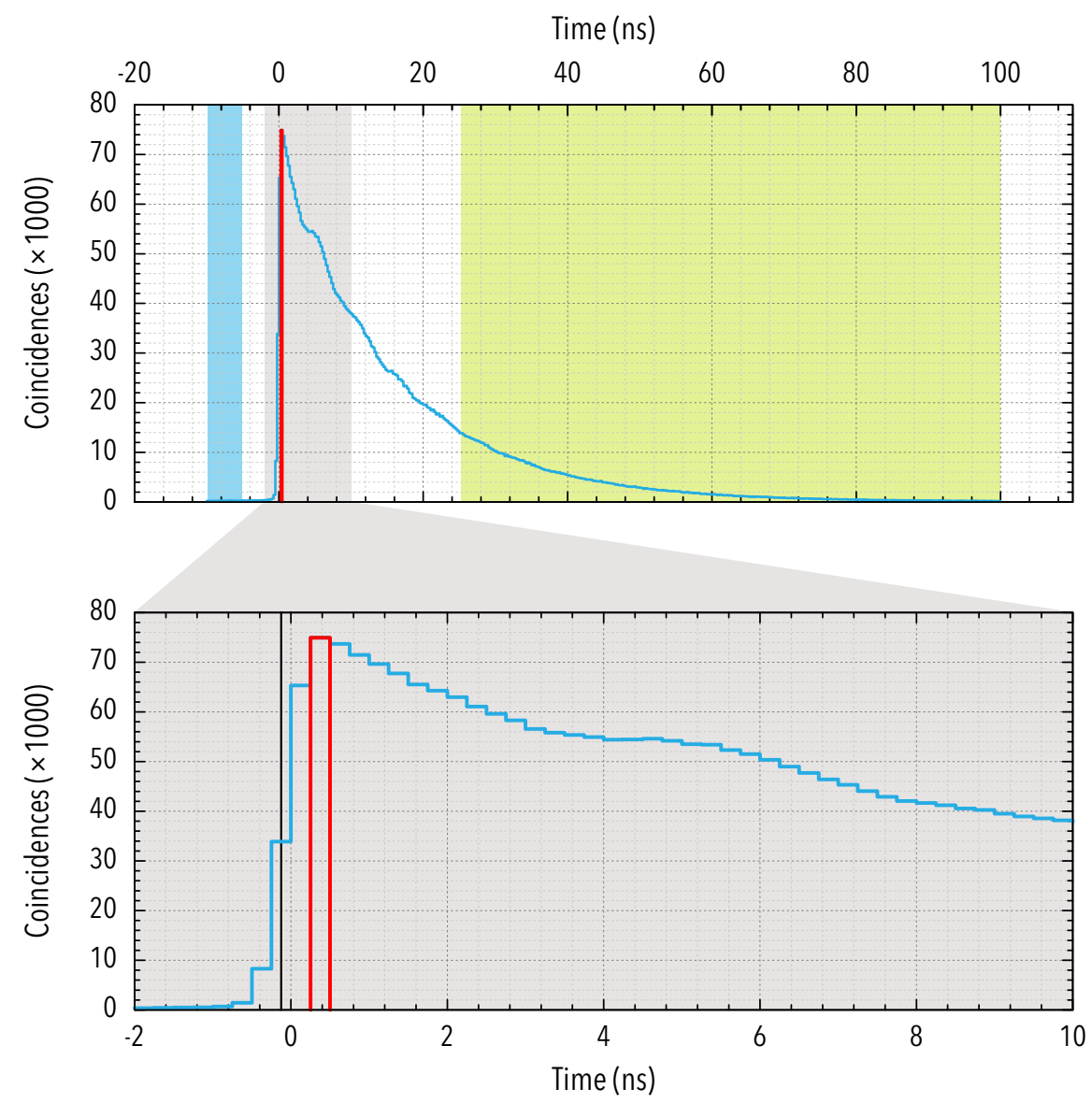

Figure 4.11: Estimation of parameters for fitting. The background level is estimated by averaging the signal range highlighted blue. The red bin denotes the spectrum's maximum, which is used for several parameter estimations. The green range is used for $T_{\mathrm{hl}}$ determination in order to avoid the range with high amplitude modulations, which is visible in the enlarged cutout. In this cutout, the $t_{0}$ estimation is marked by a vertical line.

$\mathbf{t}_{0}$ The last histogram bin with a background corrected value smaller than $20 \%$ and the first bin bigger than $80 \%$ of the background corrected peak amplitude $N_{\max }-N_{\text {bg }}$ are determined. The center between these bins is used as starting value for $t_{0}$.

$\sigma$ always starts with a value of 1 ns since this value is not exceeded by the spectrometer's time resolution regardless of the detected radiations' energies and the fit proved to be less prone to converging to local minima if the initial value of $\sigma$ is overestimated. 


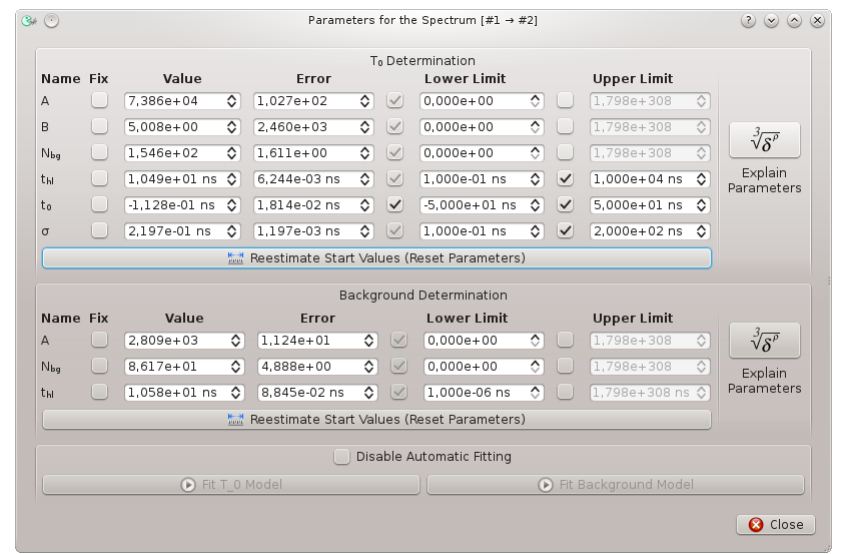

Figure 4.12: Screenshot of the dialog for manual fit parameter manipulation. This dialog can be used to optimize fitting in unusual cases where automatic parameter estimation does not yield converging fits.

An example is shown in fig. 4.11. Figure 4.12 shows the dialog for manual manipulation of fit parameters used in cases where the automatic estimators fail.

$\mathbf{R}(\mathbf{t})$ calculation Based on the $t_{0}$ and $N_{\text {bg }}$ values determined by fitting the coincidence spectra, it is possible to calculate the $R(t)$ result spectrum as well as its standard error. Coincidence spectra for identical detector-detector angles are combined using the geometric mean after aligning their time axes based on their $t_{0}$ positions. In order to align finer than the sampling interval's size, linear interpolation is used.

The background corrected number of detected $\gamma$ coincidences with a time difference from an interval around $t$ in the $i^{\text {th }} \varphi$-spectrum $\left(\varphi \in\left\{90^{\circ}, 180^{\circ}\right\}\right)$ is written as

$$
\hat{N}(i, \varphi, t)=N(i, \varphi, t)-N_{\mathrm{bg}}(i, \varphi)
$$

where $N(i, \varphi, t)$ is the uncorrected bin content and $N_{\mathrm{bg}}(i, \varphi)$ the background determined according to the previous section. For a six detector TDPAC setup, $i=1, \ldots, 24$ for $\varphi=90^{\circ}$ and $i=1, \ldots, 6$ for $\varphi=180^{\circ}$ since each of the six detectors can provide the start signal and there are four possible $90^{\circ}$ and one $180^{\circ}$ stop detectors available for every start detector.

The geometric mean of the bin at time $t$ is calculated as

$$
\bar{N}(\varphi, t)=\sqrt[n]{\prod_{i=1}^{n} \hat{N}(i, \varphi, t)},
$$

where $\hat{N}(i, \varphi, t)$ is linearly interpolated from the spectrum using the closest bin values. 
It is necessary to compute the partial derivative of the geometric mean with respect to the original spectra for error propagation:

$$
\frac{\partial \bar{N}(\varphi, t)}{\partial \hat{N}(k, \varphi, t)}=\frac{1}{n \cdot \hat{N}(k, \varphi, t)} \cdot \sqrt[n]{\prod_{i=1}^{n} \hat{N}(i, \varphi, t)}
$$

The standard error of the single spectrum bins' values is defined as

$$
\sigma_{\hat{N}(k, \varphi, t)}=\sqrt{N(k, \varphi, t)}=\sqrt{\hat{N}(k, \varphi, t)+N_{\mathrm{bg}}(k, \varphi)} .
$$

It is important to note that the background is not subtracted in this case!

Based on eqs. (4.5) and (4.6), it is now possible to calculate the standard error of the geometric mean spectrum:

$$
\begin{gathered}
\sigma_{\bar{N}(\varphi, t)}^{2}=\sum_{k=1}^{n}\left(\frac{\partial \bar{N}(\varphi, t)}{\partial \hat{N}(k, \varphi, t)} \cdot \sigma_{\hat{N}(k, \varphi, t)}\right)^{2} \\
\sigma_{\bar{N}(\varphi, t)}=\frac{\bar{N}(\varphi, t)}{n} \cdot \sqrt{\sum_{k=1}^{n} \frac{\hat{N}(k, \varphi, t)+N_{\mathrm{bg}}(k, \varphi)}{\hat{N}^{2}(k, \varphi, t)}}
\end{gathered}
$$

Since $R(t)$ calculation is based only on the mean spectra, the following abbreviations are introduced:

$$
\bar{N}_{1}=\bar{N}\left(180^{\circ}, t\right) \quad \bar{N}_{2}=\bar{N}\left(90^{\circ}, t\right)
$$

$R(t)$ is—according to eq. (2.18)—defined as

$$
R(t)=2 \cdot \frac{\bar{N}_{1}-\bar{N}_{2}}{\bar{N}_{1}+2 \cdot \bar{N}_{2}} .
$$

This definition was introduced by Arends et al. (1980) for cases with up to four coincidence spectra. The variant based on geometric means usable for larger numbers of spectra is mentioned for instance by Butz (1989).

The partial derivatives are

$$
\begin{aligned}
& \frac{\partial R(t)}{\partial \bar{N}_{1}}=\frac{6 \cdot \bar{N}_{2}}{\left(\bar{N}_{1}+2 \cdot \bar{N}_{2}\right)^{2}} \\
& \frac{\partial R(t)}{\partial \bar{N}_{2}}=\frac{-6 \cdot \bar{N}_{1}}{\left(\bar{N}_{1}+2 \cdot \bar{N}_{2}\right)^{2}}
\end{aligned}
$$


Therefore, the $\mathrm{R}(t)$ function's standard error is

$$
\begin{array}{r}
\sigma_{R(t)}^{2}=\left(\frac{\partial R(t)}{\partial \bar{N}_{1}} \cdot \sigma_{\bar{N}_{1}}\right)^{2}+\left(\frac{\partial R(t)}{\partial \bar{N}_{2}} \cdot \sigma_{\bar{N}_{2}}\right)^{2} \\
\sigma_{R(t)}=\frac{6 \cdot \sqrt{\bar{N}_{2}^{2} \cdot \sigma_{\bar{N}_{1}}^{2}+\bar{N}_{1}^{2} \cdot \sigma_{\bar{N}_{2}}^{2}}}{\left(\bar{N}_{1}+2 \cdot \bar{N}_{2}\right)^{2}},
\end{array}
$$

where $\sigma_{\bar{N}_{k}}$ can be calculated according to eq. (4.8).

The $R(t)$ spectrum determined by SpectraPAC is automatically updated as soon as the coincidence spectra change (e.g. due to new data during an online evaluation) or new values for $t_{0}$ or $N_{\mathrm{bg}}$ are provided for any of the coincidence spectra.

The $R(t)$ 's Fourier spectrum is determined using the FFTW library (Frigo and Johnson, 2005) after mirroring $R(t)$ at the y axis and multiplication with a selectable window function. Implemented window functions are square, Hann, Hamming, Kaiser-Bessel, and two different Blackman-Harris windows. Additionally, zero padding can be enabled. Compare Butz (2009) concerning zero padding and window functions.

Data flow Figure 4.13 shows the data flow through SpectraPAC, which is closely related to the evaluation steps seen by the user. In contrast to PacMan and PacMaster, SpectraPAC relies on user interaction in each of the evaluation steps. Therefore, intermediate results are forwarded forth and back between the GUI and worker threads for the different evaluation steps. These worker threads obtain measurement data via a DataReader object. For best performance, the threads are designed to access data from the saved file only linearly in each evaluation step.

\subsection{Conclusions}

Pac-Suite meets the ambitious aims defined in chapter 3 and in the current chapter's beginning. It provides the most modern and convenient user interface available while simultaneously achieving the best performance values among TDPAC spectrometers. As a benchmark for the latter, two standard measurements for the determination of time as well as energy resolution were conducted. Time resolution was tested by recording a coincidence spectrum of the ${ }^{60} \mathrm{Co}$ decay cascade (see appendix A.2.2 for more details) with transitions at $1173.2 \mathrm{keV}$ and $1332.5 \mathrm{keV}$ and an intermediate half-life of $900 \mathrm{fs}$. For the energy resolution, an energy spectrum of a decaying ${ }^{137} \mathrm{Cs}$ sample (cf. appendix A.2.3) was recorded using less than half of the digitizers' available full-scale range to prevent gain compression from distorting results towards better values. Both spectra are visible in fig. 4.14. In both cases, the measurements ran several days to provide a realistic scenario accounting for possible impairments due to drift effects in hardware components. Peaks' 


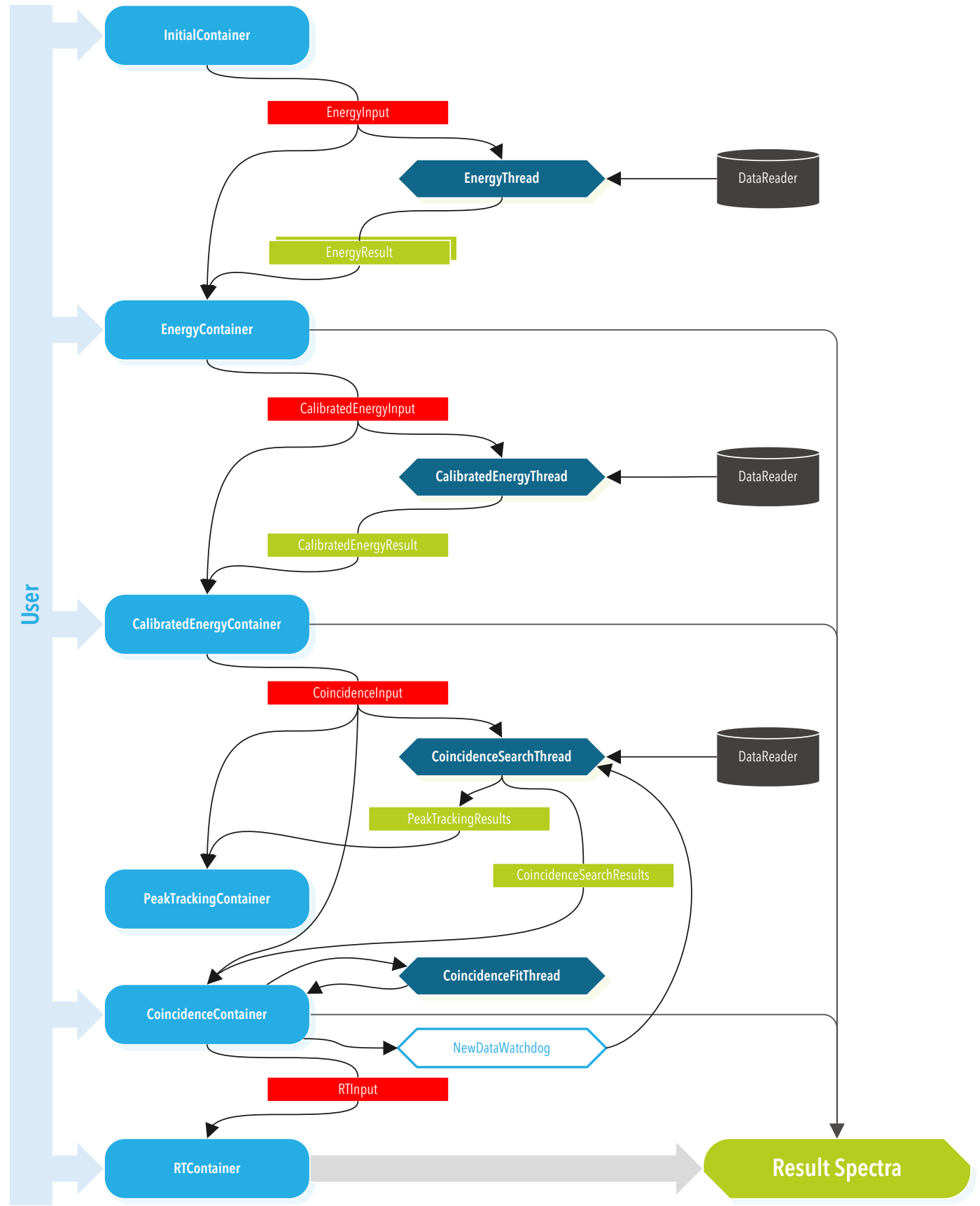

Figure 4.13: Data processing by SpectraPAC. The block names comply with the class names without the Pac prefix. Light blue container objects are persistent and contain results from the data processing threads while also managing user input and GUI functionality. On user request, these objects create input message objects (red) and invoke worker threads (dark turquoise). These worker threads read and process recorded measurement data and pass their results (green) to the subsequent containers. 


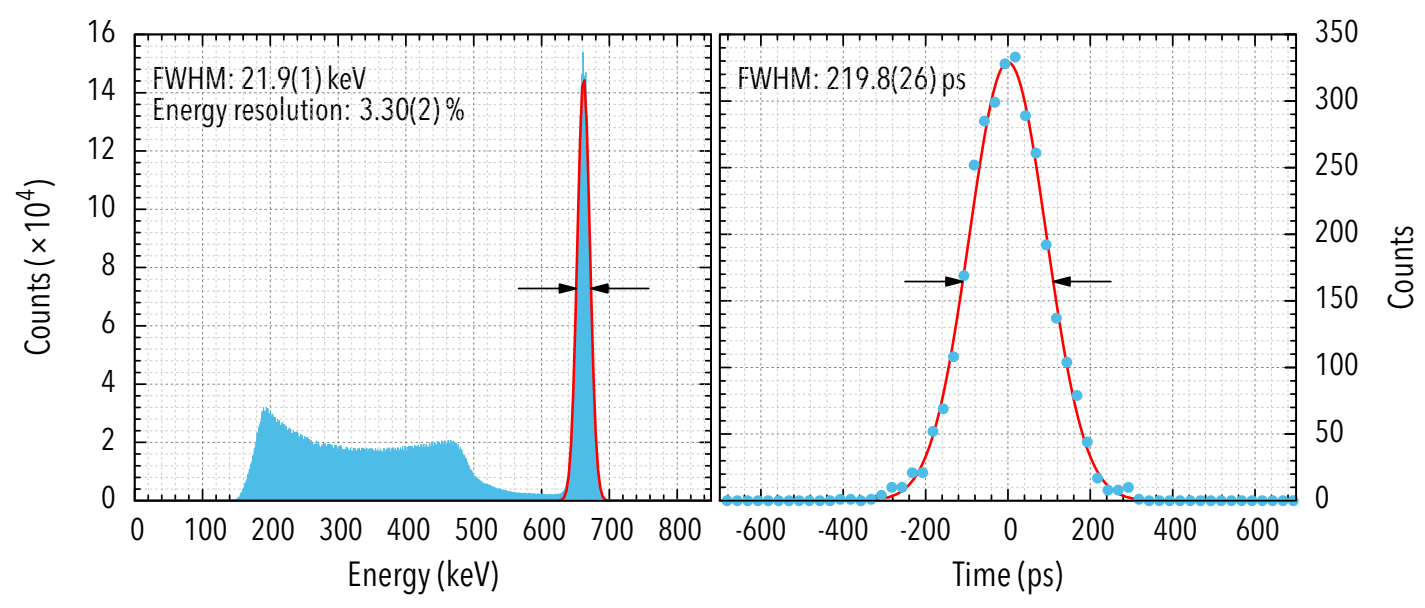

Figure 4.14: Energy resolution at the $661.7 \mathrm{keV}$ photo-peak of ${ }^{137} \mathrm{Cs}$ (left) and time resolution as determined using the $1173.2 \mathrm{keV}$ and $1332.5 \mathrm{keV}$ transitions of ${ }^{60} \mathrm{Co}$ (right). See appendices A.2.2 and A.2.3 for details concerning the used nuclides. Both FWHM values were determined by least-squares fitting of a Gaussian to the weighted data. To prevent gain compression from creating energy resolution results distorted towards apparently better values, less than half of the digitizer's full-scale range was used and the Compton edge was considered for energy calibration. Both recordings ran several days in order to demonstrate the spectrometer's long-term stability.

full width at half maximum (FWHM) were determined by least-squares fitting of a Gaussian to the weighted data. The thereby obtained time resolution is 219.8(26) ps. Energy resolution was determined based on a sum spectrum consisting of the calibrated spectra of the two randomly selected detectors also used for the time resolution benchmark by dividing the ${ }^{137}$ Cs photo-peak's FWHM by its energy. The results are a value of 3.57(2) \% in case of disabled drift compensation and 3.30(2)\% with enabled drift compensation as described in chapter 6 . This result shows that the novel drift compensation method significantly improves energy resolution even for ordinary measurements and not only in case of difficult scenarios with applied magnetic fields or similar complications.

Time as well as energy resolution are record values among TDPAC spectrometers. Table 4.1 shows a performance comparison of all published digital TDPAC setups currently available.

Despite the excellent performance values, there is still potential for improvements concerning the spectrometer's hardware. It could for instance be beneficial to replace the XP2020 PMTs by models with better timing properties and less amplification. This would render the attenuation devices redundant and eventually further improve time resolution. Moreover, in the meantime new digitizer models with up to 10 bit resolution at a sampling rate of $8 \mathrm{GS} \mathrm{s}^{-1}$ have become available. It is still to be determined how much such technical improvements would affect the spectrometer's timing performance. 
Table 4.1: Comparison of digital TDPAC spectrometers. This table lists all digital TDPAC spectrometers for which performance data is available. Due to the improved signal analysis methods employed in the spectrometer described in this work, superior performance was obtained. The second best time resolution values from Jäger, Iwig, and Butz (2011) are only reached if using $\mathrm{BaF}_{2}$ scintillators. This comes at the cost of bad energy resolution due to the low light yield of this material as shown by Webb (2012).

\begin{tabular}{lccc}
\hline Reference & Scintillator & $\begin{array}{c}\text { Time resolution } \\
{ }^{60} \mathrm{Co} / \text { FWHM in ps }\end{array}$ & $\begin{array}{c}\text { Energy resolution } \\
\% \text { at 661.7 keV }\end{array}$ \\
\hline This work & $\mathrm{LaBr}_{3}: \mathrm{Ce}$ & $\mathbf{2 1 9 . 8 ( 2 6 )}$ & $\mathbf{3 . 3 0}(\mathbf{2})$ \\
\hline Jäger, Iwig, and Butz (2011) & $\mathrm{BaF}_{2}$ & 254 & $\mathrm{n} / \mathrm{a}$ \\
Jäger, Iwig, and Butz (2011) & $\mathrm{LaBr}_{3}: \mathrm{Ce}$ & 265 & $4^{a}$ \\
Webb (2012) & $\mathrm{BaF}_{2}$ & $346.6(9)$ & 12.7 \\
Herden, Alves, et al. (2004) & $\mathrm{BaF}_{2}$ & $<400^{c}$ & $\mathrm{n} / \mathrm{a}$ \\
\hline
\end{tabular}

${ }^{a}$ value from Jäger, Iwig, and Butz (2010) according to reference in Jäger, Iwig, and Butz (2011)

${ }^{b}$ also described in Webb et al. (2013)

${ }^{c} 400 \mathrm{ps}$ are quoted for ${ }^{22} \mathrm{Na}$ while no information concerning ${ }^{60} \mathrm{Co}$ was published 


\section{Chapter 5}

\section{Signal analysis algorithms}

The reconstruction of radiation properties from detector signals is the most important challenge concerning the transition from analog to digital spectrometers. Due to the short duration of PMT signals, sampling and quantization of these signals cannot be done with far higher accuracy than necessary, i. e. it is done almost without reserve, since the performance of available digitizers is limited (cf. fig. 5.1 which shows sampling points of recorded detector signals). Therefore, specific disadvantages of the digital readout compared to analog processing occur. Thus, the art of digital processing of recorded detector signals consists in avoiding these disadvantages to achieve a better overall-performance than possible with analog setups and their specific disadvantages.

Because the limitations of analog and digital signal processing techniques are very different, digital emulation of well-established analog methods does not translate into outstanding results. Nevertheless, a comprehensive investigation of digital methods was not published up to now.

In the context of this thesis, modular evaluation software called Evaluate! was created, which allows to process raw signal data from the TDPAC spectrometer described in chapter 4 as well as simulation results created by the software described in chapter 7 . This program allows analysis of input data using all methods described below. It was also used for other evaluation tasks like spectrum generation in case of the results presented in section 7.4.

For benchmarking the algorithms presented below, two detectors from the digital TDPAC spectrometer were mounted opposite to each other with a ${ }^{22} \mathrm{Na}$ source in between. This source nuclide's basic properties are described in appendix A.2.1. It is suitable for timing tests because the two $\gamma$ photons emitted due to annihilation of positrons produced by the decay of ${ }^{22} \mathrm{Na}$ nuclei leave the sample simultaneously in opposite directions and have the same energy $(511 \mathrm{keV})$ thus showing identical behavior. Since most TDPAC decay cascades consist of $\gamma$ transitions with two different energies (cf. section 9.2), an additional recording was done using an ${ }^{152} \mathrm{Eu}$ sample. ${ }^{152} \mathrm{Eu}$ decays via two different decay channels to ${ }^{152} \mathrm{Gd}$ and ${ }^{152} \mathrm{Sm}$, respectively. The transition schemes include more transitions than in case of ${ }^{22} \mathrm{Na}$. In the decay ${ }^{152} \mathrm{Eu} \longrightarrow{ }^{152} \mathrm{Gd}$, a short lived intermediate state (half-life: $32.4 \mathrm{ps}$ ) is available. It is populated by a $778.9 \mathrm{keV}$ transition and depopulated by a $344.28 \mathrm{keV}$ transition with sufficient intensity. 


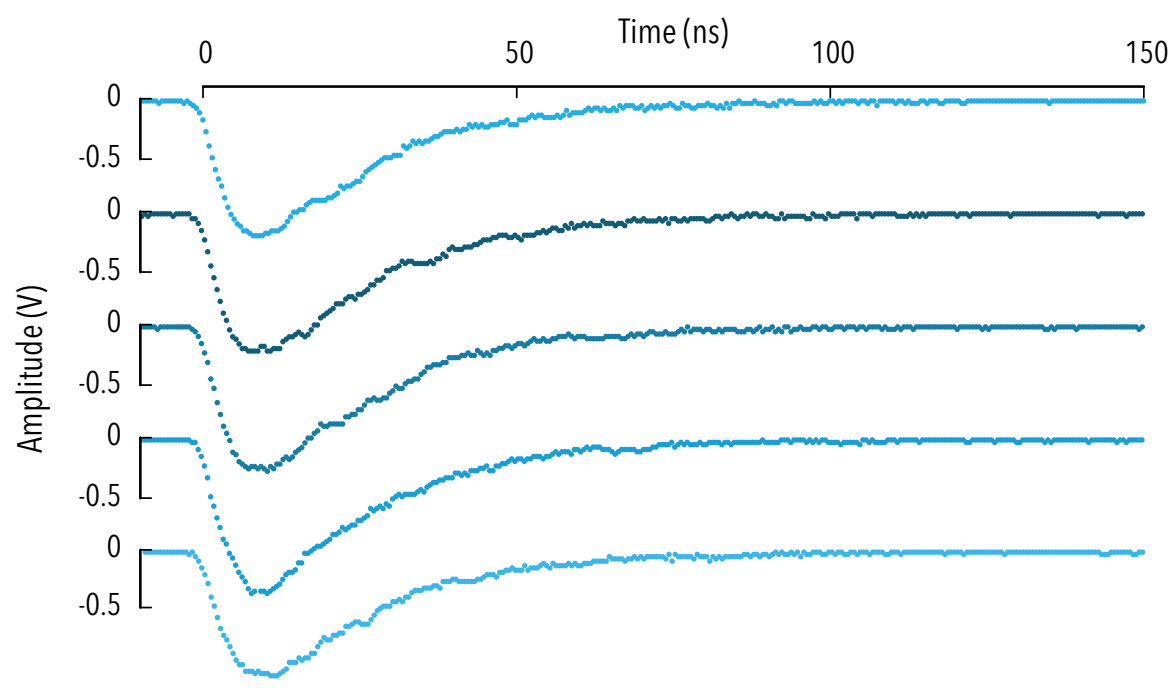

Figure 5.1: Five anode signals belonging to a $511 \mathrm{keV}$ photo-peak recorded from a detector consisting of $\mathrm{LaBr}_{3}$ :Ce crystal and a Photonis XP2020 PMT.

Since the resulting time difference distributions are not necessarily normal distributions, the time resolution values were determined directly as FWHM values instead of via Gaussian fitting or computation of the standard deviation. This was done by first fitting a parabola to four histogram bins around the peak maximum. Then the amplitude of this parabola's peak was determined. The time coordinates of the histogram crossings with half of this peak's amplitude were determined using cubic polynomial interpolation based on two bins before and two after the crossing. The difference of the left and right crossing points' time coordinates was used as FWHM value.

The detectors' high voltage supplies were configured to match the amplification of both detectors' PMTs and ensure output currents staying in the linear regime of the voltage dividers.

\subsection{Energy determination}

The amount of light created in scintillation crystals is in good approximation proportional to the absorbed radiation's energy. For usual count rates of TDPAC experiments the PMTs are amplifying sufficiently linearly. Therefore, it is legitimate to assume the charge collected on the PMT's anode to be proportional to the absorbed $\gamma$ energy.

The charge collected on the PMT's anode flows through the digitizer's $50 \Omega$ termination resistor towards ground. According to Ohm's law, the voltage measured across this resistor is proportional to the current-i. e. the charge per time. Therefore, the integral of the measured voltage over time is proportional to the absorbed $\gamma$ energy as long as the signal 


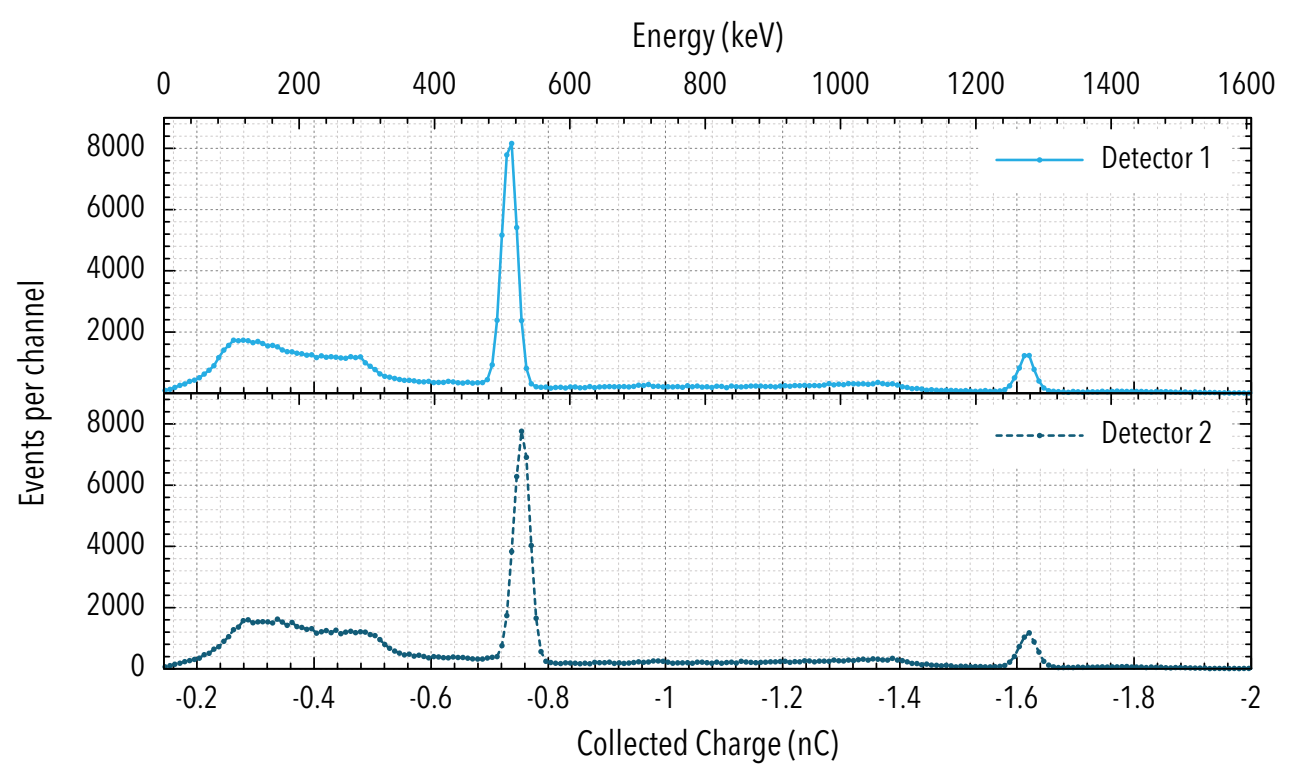

Figure 5.2: Charge spectra of a ${ }^{22} \mathrm{Na}$ sample as measured with two different detectors consisting of a $\mathrm{LaBr}_{3}$ :Ce crystal and a Photonis XP2020 PMT. The upper abscissae show the alternative energy scaling from the conducted linear energy calibration. The big peaks left of the spectra's center are the photo-peaks of the $511 \mathrm{keV}$ radiation emitted due to positron annihilation while the smaller peaks on the right are the photo-peaks belonging to the $1274.54 \mathrm{keV}$ transition visible in the decay scheme in appendix A.2.1.

is integrated sufficiently long to make the integral capture the full charge collected by the anode. The required integration time depends primarily on the decay time of the scintillator's excitations. This decay time should be sufficiently short to avoid pile-upi. e. the detection of several $\gamma$ radiations in a single integration interval—since this renders the results invalid for both events.

Integration of anode signals is one of the usual methods used for energy determination. Using digital signal analysis instead of analog circuitry simplifies the use of long integration intervals due to the redundancy of hardware delay lines for resynchronization of energy and time information and therefore usually improves the accuracy of energy analysis.

Special care needs to be taken if the digitizers' recording range is unipolar since noise from the pre- and post-signal times is then rectified and contributes to the sum. Therefore, an offset depending on the integration window's length will occur.

Figures 5.2 and 5.3 show the resulting charge spectra of ${ }^{22} \mathrm{Na}$ and ${ }^{152} \mathrm{Eu}$ samples recorded by two different detectors. Like in fig. 5.1, the integrated signals consist of 400 samples each and were recorded using the Agilent U1071A's 8 bit resolution and a sampling 


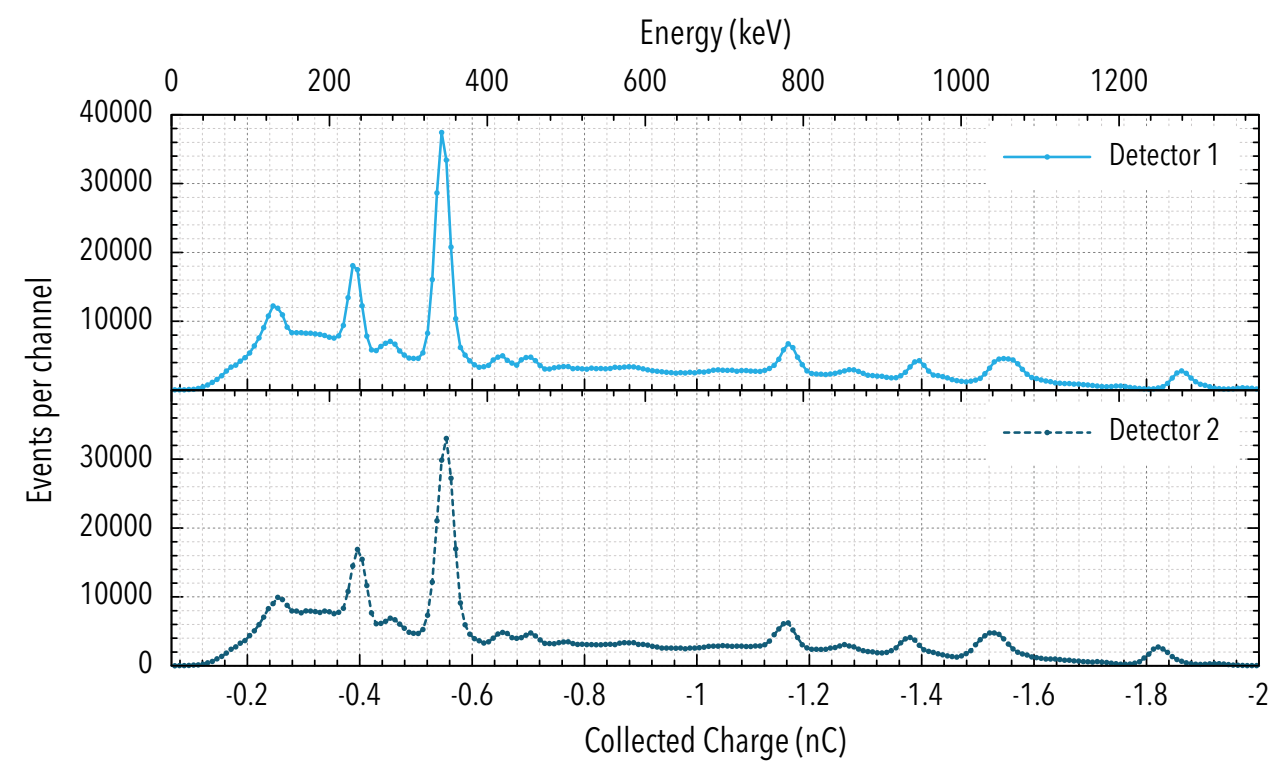

Figure 5.3: Charge spectra of a ${ }^{152} \mathrm{Eu}$ sample as measured with two different detectors consisting of a $\mathrm{LaBr}_{3}: \mathrm{Ce}$ crystal and a Photonis XP2020 PMT. Again, the upper abscissae show the alternative energy scaling from the conducted linear energy calibration. Appropriate level schemes explaining the peaks are shown in appendix A.2.4.

rate of $2 \mathrm{GS} \mathrm{s}^{-1}$. The integrals were background corrected by subtracting the average amplitude of the first ten samples (from before the signals' onset) from all sample amplitudes before integrating.

In both figures, a linear energy calibration was conducted. The resulting alternative scaling is shown at the upper abscissa of all charge spectra. Mismatching of the position of peaks and Compton edges and their values according to the decay schemes in appendices A.2.1 and A.2.4 occur due to slightly nonlinear amplification of the PMTs.

\subsection{Signal averaging}

Several of the evaluated time determination algorithms need to be initialized with an average signal shape. The challenge in obtaining such an average waveform is that the input signals have to be aligned as precisely as possible in order to avoid blurring of the average.

To solve this problem, an averaging module was created, which processes recorded events with pre-determined time origin. Algorithms without the need of averaged signals can be used for this pre-determination of signal onset times. The input signals are 


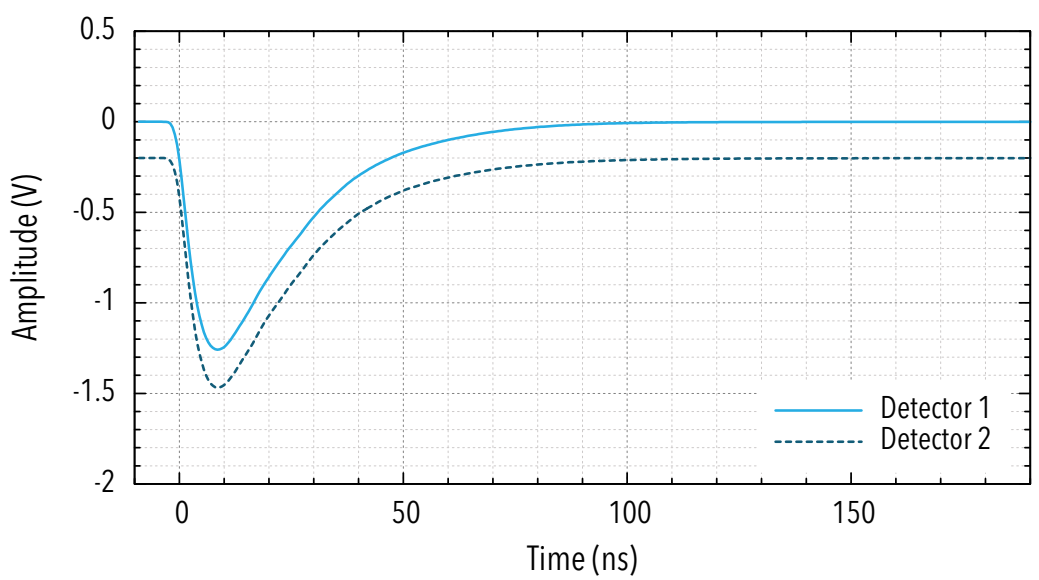

Figure 5.4: Background corrected average of aligned anode signals originating from the $511 \mathrm{keV}$ photo-peak recorded using two different detectors consisting of a $\mathrm{LaBr}_{3}$ :Ce crystal and a Photonis XP2020 PMT. The signal from detector 2 was vertically shifted by $0.2 \mathrm{~V}$ for better distinguishability.

precisely aligned according to their time origin and amplitudes at the sampling times of the averaged output signal are interpolated using cubic spline interpolation (cf. fig. 5.4).

\subsection{Constant trigger threshold timing}

The use of the time of the moment where the signal crossed a constant trigger level is the most basic and obvious approach for time determination. It is also called leading edge timing (LE) or-in case of very small threshold values-first photoelectron timing (Knoll, 1999). Since the used Agilent U1071A digitizers, like most models available today, are not capable of continuous recording due to limited memory bandwidth, a trigger has to be set up anyways. In order to be able to record signals with small maximum amplitudes, the trigger threshold is typically adjusted to be located slightly above the signal's noise level. The global timestamp of the trigger events is a value the digitizer provides readily without further signal evaluation. In order to provide timestamp values more precisely than the sampling interval's length, the used digitizers implement a linear interpolation of pre- and post-trigger amplitudes yielding an approximation of the time when the signal crossed the trigger threshold. It is possible to implement more sophisticated interpolation methods in software.

An optimized reference implementation of a software trigger algorithm was written as part of Evaluate!. It first identifies the sample $i$ immediately before the signal's crossing of the trigger threshold. The four samples around the threshold crossing, namely $\{i-1, i, i+1, i+2\}$, are then used for the determination of parameters of a cubic polyno- 


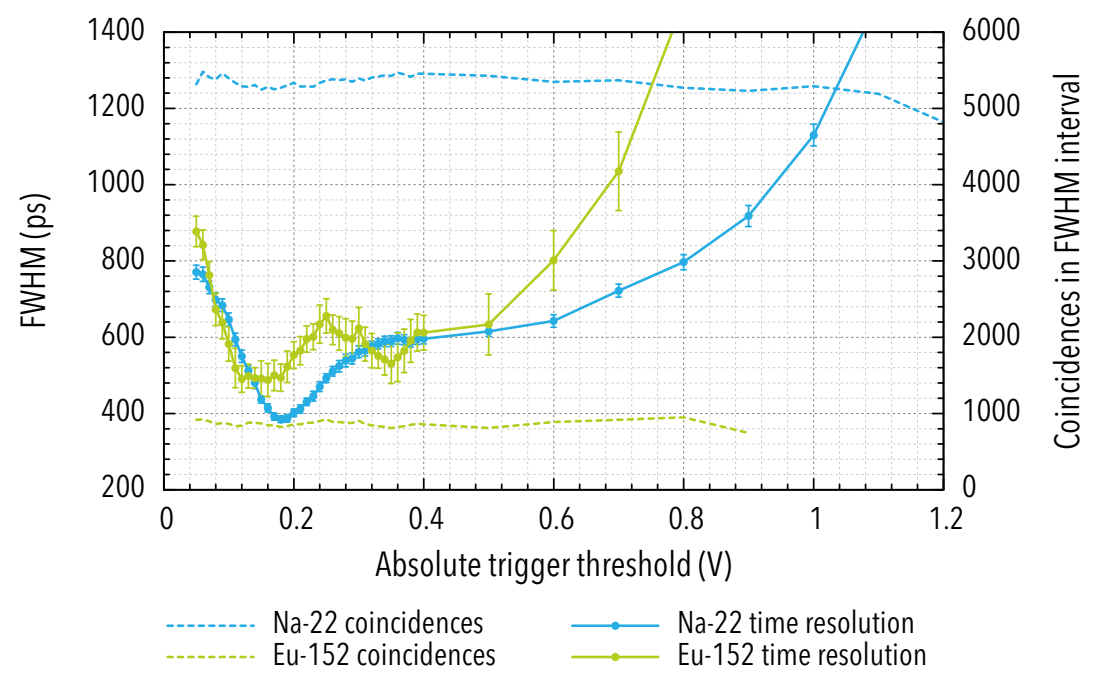

Figure 5.5: Dependence of the coincidence time resolution from the threshold value in case of the constant trigger threshold method. Both curves show minima at different trigger settings. The dashed lines indicate the number of valid coincidences found in the given datasets inside the FWHM interval. For thresholds below $0.8 \mathrm{~V}$ this is almost constant. For larger thresholds it drops to smaller values since an increasing fraction of coincidences is lost because at least one of their events does not cross the trigger threshold at all.

mial interpolation (motivated by Bardelli et al., 2004) of the signal shape in the interval ]$t_{i} ; t_{i+1}[$.

Solving

$$
\left(\begin{array}{cccc}
1 & t_{i-1} & t_{i-1}^{2} & t_{i-1}^{3} \\
1 & t_{i} & t_{i}^{2} & t_{i}^{3} \\
1 & t_{i+1} & t_{i+1}^{2} & t_{i+1}^{3} \\
1 & t_{i+2} & t_{i+2}^{2} & t_{i+2}^{3}
\end{array}\right)\left(\begin{array}{l}
a_{i} \\
b_{i} \\
c_{i} \\
d_{i}
\end{array}\right)=\left(\begin{array}{c}
y_{i-1}-y_{\mathrm{t}} \\
y_{i}-y_{\mathrm{t}} \\
y_{i+1}-y_{\mathrm{t}} \\
y_{i+2}-y_{\mathrm{t}}
\end{array}\right)
$$

via gsl_linalg_HH_svx() from the Gnu Scientific Library (Galassi et al., 2009), where $y_{i}$ is the amplitude value of the $i^{\text {th }}$ sample and $y_{\mathrm{t}}$ the trigger threshold, reveals the parameters of the cubic interpolation. It is known that the cubic polynomial defined by these parameters has a zero crossing between $t_{i}$ and $t_{i+1}$. This zero crossing's time is determined using three iterations of Newton's method. Three iterations proved to be sufficient since the solver is initialized with the linear approximation of the zero crossing.

Figure 5.5 shows the coincidence time resolution as a function of the chosen trigger threshold for the ${ }^{22} \mathrm{Na}$ and ${ }^{152} \mathrm{Eu}$ measurements described above. The evaluation is based on the described cubic polynomial interpolation of the signal around the trigger crossing.

For the ${ }^{22} \mathrm{Na}$ measurement, a very good time resolution of $385(8)$ ps is reached. The 
best resolution obtained with ${ }^{152} \mathrm{Eu}$ is significantly worse (490(35) ps). Obviously, at a certain point of the rising edge of detector signals, random fluctuations that limit time resolution have a minimum. Setting the trigger threshold to this value yields good time resolution results. Since the constant trigger threshold method, however, uses the same threshold for all signals, this minimum is not met if coincidence times between signals with different amplitudes are determined. Consequently, the resolution curve of ${ }^{152} \mathrm{Eu}$ exhibits two separated minima, which are both considerably worse than the minimum of the ${ }^{22} \mathrm{Na}$ measurement.

In order to improve the achievable time resolution, it is necessary to find a method, which automatically compensates for different signal amplitudes. This is especially important if detectors with less energy resolution are used because in these cases the energy/charge windows set to select events belonging to a certain transition have to span a wider range of signal amplitudes. For such detectors the resolution achievable using the constant threshold method is degraded even for mono-energetic coincidences like in the ${ }^{22} \mathrm{Na}$ example due to amplitude fluctuations.

\subsection{Emulated constant fraction discriminator}

The working principle of analog constant fraction discriminators described above in section 2.2.2 and Gedcke and McDonald (1967) can be emulated in digital setups. Due to the limited sampling rate, interpolation is necessary to obtain adequate resolution results. There are two possible implementations of this interpolation: Either the digitized signal's sampling granularity can be improved by piecewise interpolation before the CFD emulation is done or the CFD result signal can be interpolated. In the Evaluate! implementation of the constant fraction discriminator emulation the latter method is used because it is considerably faster than the signal interpolation approach. The delay value is thereby limited to full sampling intervals.

Analog CFD implementations' walk adjust is emulated by an adjustable offset value, which is added to the CFD signal. This prevents premature triggering on noise induced zero crossings. In the test evaluations below, the walk adjust value is set to $50 \mathrm{mV}$, which is well above the noise level of the analyzed signals (cf. fig. 5.1).

The time of the computed CFD signal's zero crossing is determined using the same cubic polynomial interpolation method, which is described in section 5.3.

A major disadvantage of the CFD method is its dependence on two parameters, fraction and delay, which makes optimization more difficult than for methods with a smaller number of parameters. To determine the relation between achievable resolution and these parameters, a series of evaluations were conducted with ${ }^{22} \mathrm{Na}$ and ${ }^{152} \mathrm{Eu}$ datasets.

Results of these evaluations are shown in fig. 5.6. For optimal parameter selection (fraction: 0.15 , delay: $3.5 \mathrm{~ns}$ ), the FWHM result is $366(9)$ ps and thus slightly better than the best results achievable with the constant threshold method for ${ }^{22} \mathrm{Na}$. In case of ${ }^{152} \mathrm{Eu}$, the 


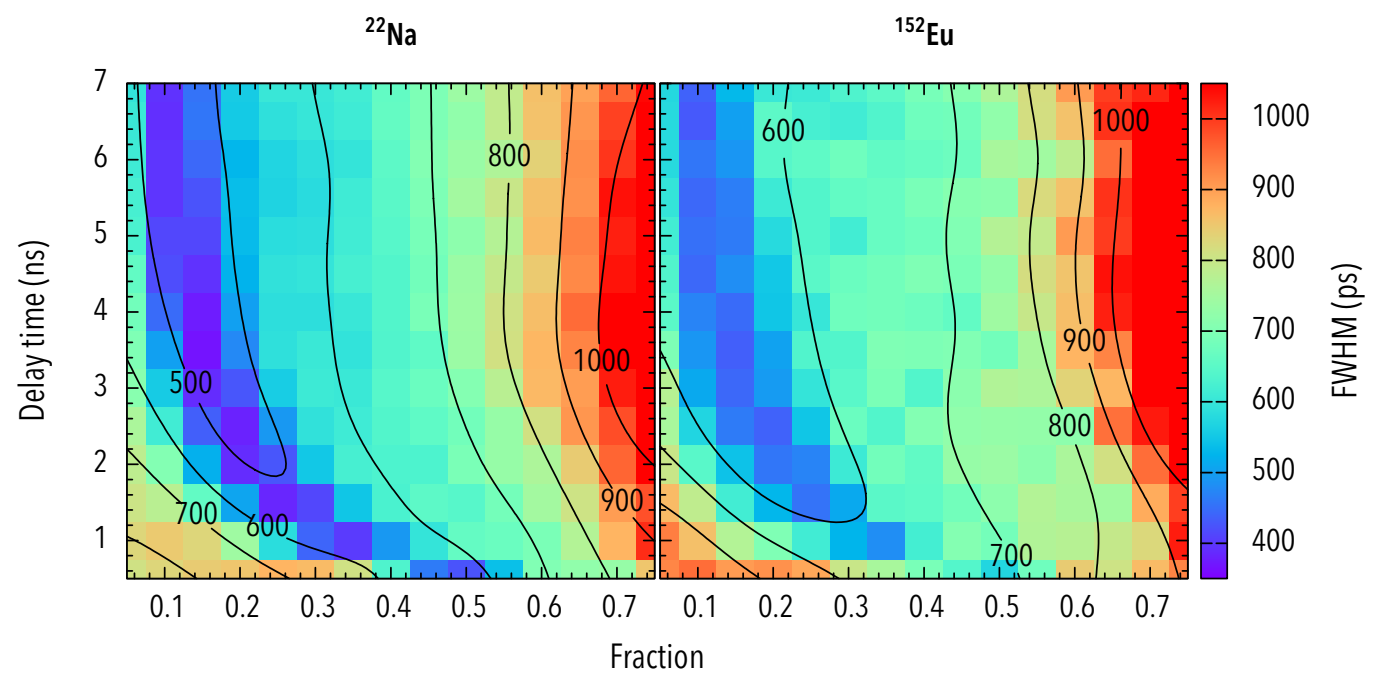

Figure 5.6: Dependence of the coincidence time resolution on the fraction and delay parameters of the CFD method.

CFD method shows its advantage: The optimal results occur at the same parameters than for ${ }^{22} \mathrm{Na}$ and the time resolution is significantly better with $432(22) \mathrm{ps}$. The parameter space map in fig. 5.6 shows combinations in blue where good resolutions are obtained. While the blue area of optimal parameter combinations is at similar positions for both decay examples, it is rather narrow and steep. This means that both parameters cannot be optimized independently and small deviations from the ideal settings result in significant degrading of performance.

Because the CFD method can be easily implemented with analog electronics and provides good performance, it is the optimal choice for analog setups. In digital setups, however, implementation effort is not different from alternative methods and therefore it is advisable to replace it with more stable approaches.

\subsection{Centroid approach}

Centroid based time evaluation methods were already considered in the 1950s (Gatti and Svelto, 1959) and a digital implementation, providing only very limited performance, was described by Jäger, Iwig, and Butz (2011).

The shape considered for computing the centroid is the area between the signal $w(t)$ and the time axis. The parameter of interest is this centroid's time coordinate. It is defined as 


$$
t_{\mathrm{c}}=\frac{\int_{t_{\min }}^{t_{\max }} t w(t) \mathrm{d} t}{\int_{t_{\min }}^{t_{\max }} w(t) \mathrm{d} t} .
$$

Jäger, Iwig, and Butz (2011) used a step approximation of the integral and calculated

$$
t_{\mathrm{c}, \mathrm{step}}=\frac{\sum_{i} t_{i} y_{i}}{\sum_{i} y_{i}}
$$

where $t_{1} \ldots t_{n}$ denote the sampling times and $f\left(t_{i}\right)$ is the measured value at time $t$. This approximation is only valid for signal shapes with low curvature.

It is possible to use a piecewise interpolation based on second order polynomials in order to obtain a better approximation of the integral. The interpolation function's parameters around sample $i$ can be efficiently computed by solving the following set of linear equations using e.g. the function gsl_linalg_HH_svx() from the Gnu Scientific Library (Galassi et al., 2009):

$$
\left(\begin{array}{ccc}
1 & t_{i-1} & t_{i-1}^{2} \\
1 & t_{i} & t_{i}^{2} \\
1 & t_{i+1} & t_{i+1}^{2}
\end{array}\right)\left(\begin{array}{l}
a_{i} \\
b_{i} \\
c_{i}
\end{array}\right)=\left(\begin{array}{c}
y_{i-1} \\
y_{i} \\
y_{i+1}
\end{array}\right)
$$

The thereby obtained set of parameters $a_{i}, b_{i}, c_{i}$ for a given interval $\left[t_{i}-\frac{\Delta t}{2}, t_{i}+\frac{\Delta t}{2}[\right.$, where $\Delta t$ denotes the sampling interval length, can be used to determine an approximation of the integrals from eq. (5.3) on this interval by integrating the interpolation polynomial. Summation of all intervals then approximates the whole integral:

$$
t_{\mathrm{c}, \mathrm{poly}}=\frac{\sum_{i}\left(\frac{1}{2} a_{i} t^{2}+\frac{1}{3} b_{i} t^{3}+\left.\frac{1}{4} c_{i} t^{4}\right|_{t_{i}-\frac{\Delta t}{2}} ^{t_{i}+\frac{\Delta t}{2}}\right)}{\sum_{i}\left(a_{i} t+\frac{1}{2} b_{i} t^{2}+\left.\frac{1}{3} c_{i} t^{3}\right|_{t_{i}-\frac{\Delta t}{2}} ^{t_{i}+\frac{\Delta t}{2}}\right)}
$$

An advantage of the centroid method is that it works without parameters and thus does not need any tuning. The FWHM time resolution results for the test cases are 1.70(5) ns for ${ }^{22} \mathrm{Na}$ and $2.7(2) \mathrm{ns}$ for ${ }^{152} \mathrm{Eu}$, which is worse than even the simple trigger threshold method with almost randomly selected threshold value. 


\subsection{Cross correlation approach}

Since the time constants defining the shape of the analyzed signals are independent from the detected $\gamma$ photon's energy and only time differences between different signals are relevant, it is possible to average signals and compare new signal with this average waveform in order to determine the new signals' time origin (i. e. the time shift compared to the average). Averaging is described in section 5.2.

A possible approach for the comparison of input signals and the average waveform is cross correlation. The correlation function exhibits a peak at the value, the time axis of the measured signal has to be shifted by in order to obtain the best match with the average signal.

Similar to convolutions, the cross correlation function's computation can be simplified by employing Fourier transformations. The Fourier transform of a cross correlation function equals the product of the complex conjugate of the Fourier transform of the first function (in this case the signal $w(t)$ ) and the Fourier transform of the second function (here the average signal $a(t)$ ). Thus

$$
(w \star a)(t)=\mathscr{F}^{-1}\left\{(\mathscr{F}\{w(t)\})^{*} \cdot \mathscr{F}\{a(t)\}\right\}
$$

where $w \star a$ is the cross correlation of the functions $w$ and $a, \mathscr{F}\{\cdot\}$ denotes the Fourier transformation and $x^{*}$ is the complex conjugate of $x$.

Since the Fourier transform of the average stays the same for all evaluated signals, it is sufficient to calculate $\mathscr{F}\{a\}$ once. Therefore, only one forward and one inverse Fourier transformation are necessary for each evaluated signal. These transformations can be computed very efficiently using the fast Fourier transform (FFT) algorithm. If the number of samples is not a power of two, it is possible to employ zero padding (Butz, 2009) to extend it to the next number that is a power of two. This is useful because the FFT algorithm works most efficiently with datasets whose size is a power of two.

Afterwards, the maximum of the cross correlation function $w \star a$ needs to be determined. This can be done by interpolating the curve in the range around the maximum sample using a parabola:

$$
h(t)=a+b t+c t^{2}
$$

The parameters $a, b, c$ can be determined like shown in eq. (5.4). The searched maximum, $t_{c c}$, is then located at

$$
t_{c c}=-\frac{b}{2 c} \text {. }
$$

Alternatively, the cross correlation can be computed on a piecewise interpolated dataset with sufficiently low granularity.

Both alternatives were tested. For the interpolated data approach, the test recordings of ${ }^{22} \mathrm{Na}$ and ${ }^{152} \mathrm{Eu}$ are extended to $512 \times 64$ samples using piecewise cubic spline interpolation. After computing the cross correlation using the Fourier method described above, 
Table 5.1: Results of the deviation of shifted normalized waveform approach for different $p$-norms.

\begin{tabular}{ccc}
\hline$p$ & $\begin{array}{c}{ }^{22} \mathrm{Na} \\
\text { FWHM (ns) }\end{array}$ & $\begin{array}{c}{ }^{152} \mathrm{Eu} \\
\text { FWHM (ns) }\end{array}$ \\
\hline 1 & $1.19(3)$ & $1.46(7)$ \\
2 & $1.22(3)$ & $1.38(5)$ \\
3 & $1.23(3)$ & $1.33(7)$ \\
4 & $1.26(3)$ & $1.33(6)$ \\
\hline
\end{tabular}

the maximum sample from the extended result data-set is picked and its position on the time axis is used as coincidence time. Additional interpolation of the maximum is not required due to the fine granularity of 6.1 ps of the extended data-set. The obtained resolution results for the test cases are $2.00(6) \mathrm{ns}$ for ${ }^{22} \mathrm{Na}$ and $2.27(10) \mathrm{ns}$ for ${ }^{152} \mathrm{Eu}$, which is in average slightly better than the results of the centroid approach but still much worse than the resolution obtainable by simpler trigger approaches.

The second approach involving the maximum interpolation using a parabola yielded a resolution of $1.21(3) \mathrm{ns}$ for ${ }^{22} \mathrm{Na}$ and $1.37(6) \mathrm{ns}$ for ${ }^{152} \mathrm{Eu}$. While being significantly better than the interpolation of input signals, it is still much worse than the methods from the trigger based group.

\subsection{Deviation of shifted normalized waveform approach}

For this approach, the normalized amplitudes of a reference waveform (i. e. an average as described in section 5.2) and the measured waveform are compared using a norm:

$$
d(\Delta t)=\sum_{i}\left\|\frac{a\left(t_{i}\right)}{\sum_{i} a\left(t_{i}\right)}-\frac{w\left(t_{i}-\Delta t\right)}{\sum_{i} w\left(t_{i}\right)}\right\|
$$

$\Delta t$ has to be varied in steps of the sampling length. Over- and underflow sample numbers occurring due to the shift in $w\left(t_{i}-\Delta t\right)$ can be wrapped around because the signal's amplitude is close to zero at both ends.

The norm can be chosen freely. Similar to the cross correlation function in the previous section, the position of the maximum of the result $d(\Delta t)$ equals the relative position of the signal's time origin. It can again be determined by interpolating as described in section 5.6.

The arbitrary selection of a norm allows modifying of the weighting of big deviations in single points. Such deviations likely occur at steep edges of the signal. Therefore, it allows assigning of higher weight to deviations at the rising edge of the detector signal. 
For testing, a $p$-norm approach was used. The evaluation was done for $p$-values of $\{1 ; 2 ; 3 ; 4\}$. Results are shown in table 5.1 . The performance is similar to the cross correlation method. The $p$ value's influence is neither strong nor consistent.

\subsection{Pulse shaping approach}

Pulse shaping is a well-known technique for the separation of slow decaying signals that overlap at high count rates (Smith, 2002). It works by convolving the signal with a special filter kernel. This kernel is chosen to transform the typically occurring signal shapes into differently shaped signals. Usually, short Gaussian pulses are chosen as target shape because delta pulses, which would be the ideal selection concerning the separability of signals, would cause alignment issues due to the finite sampling rate.

Since the target shape can be chosen arbitrarily, the technique used for pulse shaping allows transforming of real detector signals into symmetric waveforms. The center of symmetric waveforms, however, can be determined precisely and is independent from their amplitude. Therefore, it is possible to apply the pulse shaping method to the signal time determination problem.

For the evaluation of this approach, which was not described in literature before, the average signal shape according to section 5.2 was Fourier transformed. As target signal, a Gaussian was used. Its standard deviation $\sigma$ is a free parameter of the method, which can be varied for performance optimization. The Gaussian was also Fourier transformed and the filter kernel $k(\omega)$ was computed as the quotient of the transformed target signal $g(t)$ and the transformed average signal $a(t)$ :

$$
k(\omega)=\frac{\mathscr{F}\{g(t)\}}{\mathscr{F}\{a(t)\}}
$$

The steps up to here can be prepared before signal analysis starts. During the measurement, each waveform $w(t)$ has to be Fourier transformed and multiplied with the kernel function $k(\omega)$. The result then needs to be inversely transformed to yield the shaped signal

$$
s(t)=\mathscr{F}^{-1}\{\mathscr{F}\{w(t)\} \cdot k(\omega)\} .
$$

If the shape of the analyzed signals $w(t)$ is consistent with the average shape $a(t)$, the results $s(t)$ are Gaussians with the same standard deviation as selected for the target signal $g(t)$. Small values below $10 \%$ of the maximum value were discarded to reduce the influence of noise on the subsequent fitting step. This is justified, since only the position of the peak's center is relevant for time determination and the center's position can be determined based on the rising and falling edges rather than the low amplitude parts of the signal.

The isolated peaks were then fitted using the Levenberg-Marquardt nonlinear least squares algorithm as implemented by Lourakis (Jul. 2004). A Gaussian (i.e. the target 


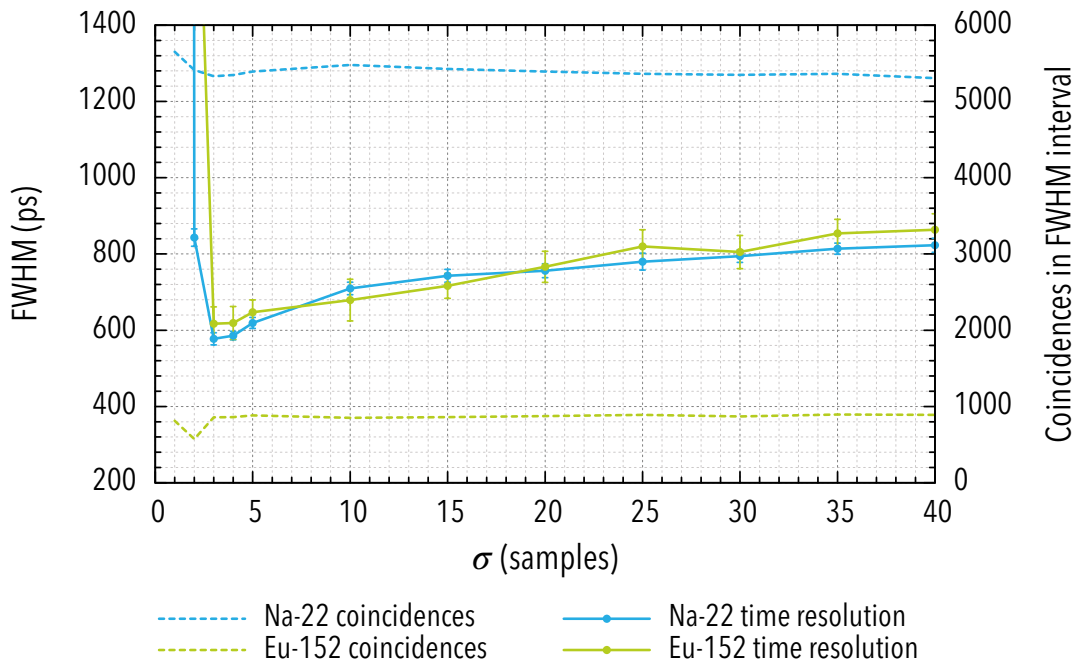

Figure 5.7: Dependence of the coincidence time resolution on the $\sigma$ parameter in case of the pulse shaping approach.

signal's shape) with fixed standard deviation $\sigma$ and free scaling and offset parameters $p_{1}$ and $p_{2}$ was used as model function

$$
\hat{g}(t)=p_{1}+p_{2} \mathrm{e}^{\frac{(t-\mu)^{2}}{2 \sigma^{2}}}
$$

The determined mean value $\mu$ was then used as the signal's timestamp value.

Figure 5.7 shows the time resolution results for different $\sigma$ values (all numbers are in units of the sampling interval). $\sigma=3$ is optimal in both cases while higher values have only a small impact on the time resolution. For $\sigma>2$, the number of coincidences is almost constant. This means that no coincidences are lost due to algorithmic instabilities. The FWHM time resolution results for the optimal $\sigma$ value of 3 sampling intervals are $578(16)$ ps for ${ }^{22} \mathrm{Na}$ and $617(44)$ ps for ${ }^{152} \mathrm{Eu}$.

\subsection{Digital constant fraction of integral values approach}

The analog CFD method and its digital emulation (cf. section 5.4) compare the detector signal with a delayed and modified copy of itself. Then they trigger on zero crossings of the resulting signal. In fact, this means that the method compares two measurements of the signal's amplitude at different times and therefore suffers from $\sqrt{2}$ times the uncertainty of a single measurement assuming the uncertainties are not correlated. In case of detector signals, the uncertainty is not only caused by the hardware but also by statistical 


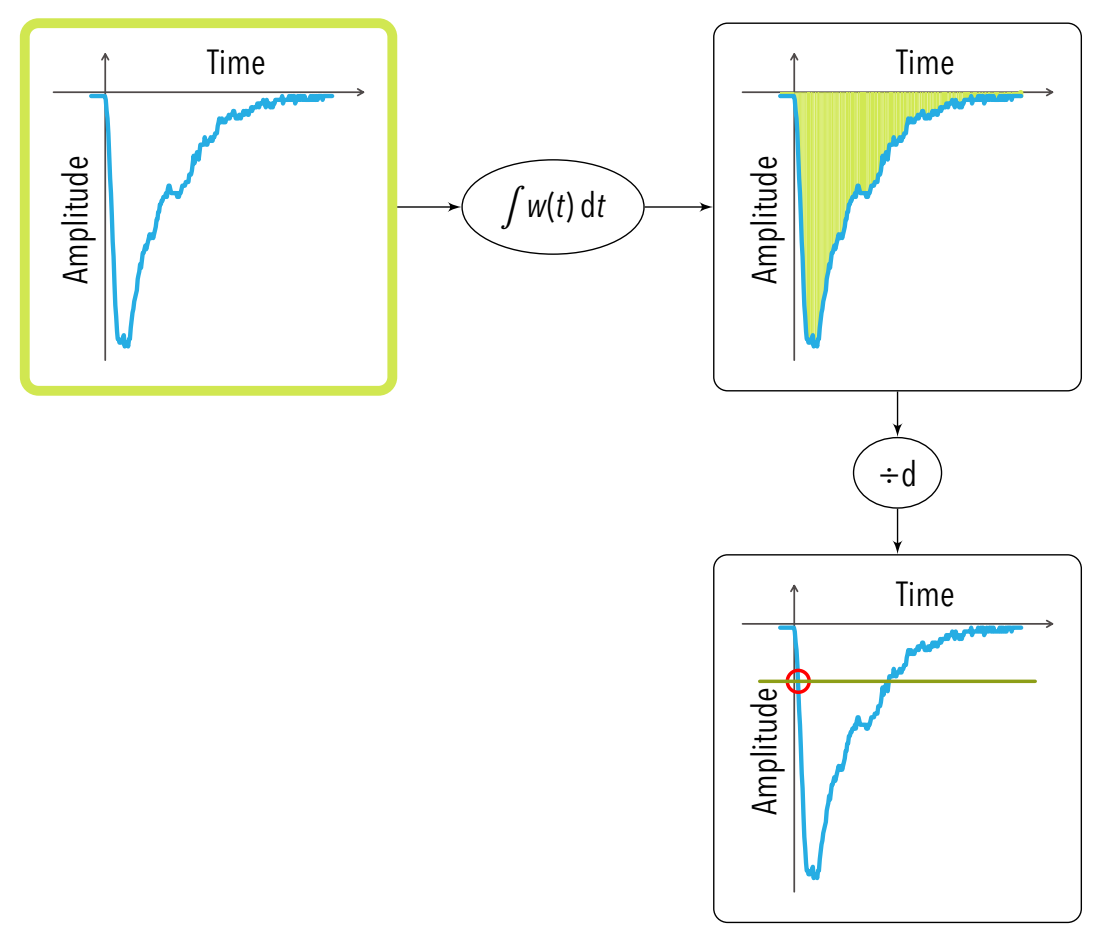

Figure 5.8: Working principles of the digital constant fraction of integral values (DCFI) method for signal time determination. For the DCFI method, the input signal (the one with the green frame) is integrated in a first step. The result is divided by a fixed divisor (parameter $d$ ) and the result is used as a variable trigger level. The crossing of the original signal and this trigger level is forwarded as the signal's timestamp.

fluctuations due to the limited number of scintillation photons contributing to the signal. This contribution also depends on the energy of the detected $\gamma$ photon.

Considering this substantial influence of noise on the results, it is an important goal to find ways to reduce the result's dependence on single measurements. A very efficient method was first described by Nagl, Vetter, et al. (2010).

Instead of comparing the signal with a delayed and scaled copy of itself, the digital constant fraction of integral values approach (DCFI, see also fig. 5.8) derives a signal specific trigger threshold from the signal's integral (i. e. the sum of all samples). This sum is hardly affected by signal noise because most noise contributions cancel out to a large extent during summation. Since the sum of all samples has to be calculated for charge/energy determination anyways, it introduces no additional computations compared to constant level triggering if it is implemented in digital setups.

The time of the signal's trigger threshold crossing can again be determined by interpolation using a cubic polynomial and root finding as described in section 5.3. An example for the interpolation is also shown in fig. 5.9. 


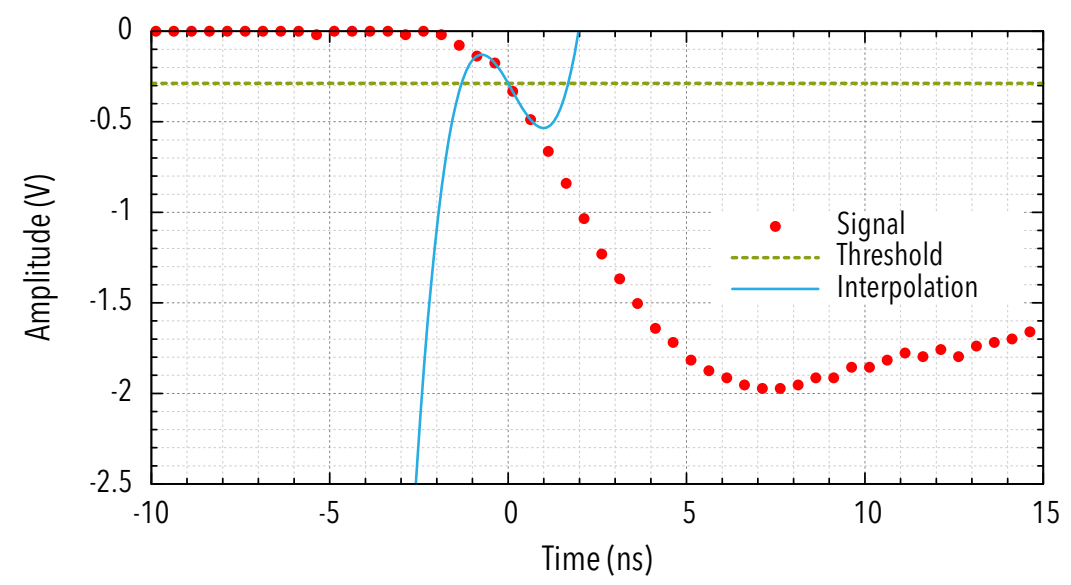

Figure 5.9: After a signal's trigger threshold (green) was determined by dividing its integral value by a constant divisor $\left(4 \times 10^{-9} \mathrm{C} \mathrm{V}^{-1}\right.$ in this example), two samples before and two samples after the point where the signal crosses the trigger level are found and the parameters of a cubic polynomial through these points (blue) are determined. The crossing of this polynomial and the trigger level between the two central samples is then found using Newton's method.

The divisor $d$ used to convert the signal's integral to a trigger level is a free parameter. Its upper limit is defined by the noise level since a typical signal divided by $d$ must still yield a trigger level well above the noise level to provide non-random results. Its lower level is defined respectively by the ratio of maximal amplitude and integral value of a typical signal. While at least two parameters, i. e. divisor and delay, have to be optimized for analog constant fraction discrimination, the DCFI approach only depends on a single parameter. This simplifies tuning.

The coincidence time resolution's dependence on the divisor $d$ is shown in fig. 5.10. The ideal setting for both test cases is reached around $d=4 \times 10^{-9} \mathrm{C} \mathrm{V}^{-1}$. The slope around the ideal value is small which accounts for stable tuning properties.

In case of optimal parameter selection $\left(4.1 \times 10^{-9} \mathrm{CV}^{-1}\right)$, the FWHM result is $375(13) \mathrm{ps}$ for ${ }^{22} \mathrm{Na}$ and $417(32)$ ps in case of ${ }^{152} \mathrm{Eu}$. In average, this is slightly better than the results of the emulated constant fraction discriminator while needing less complicated tuning due to only one free parameter and while being easier to implement in digital setups.

\subsection{Comparison}

Recently, digital evaluation of detector signals made a wide range of new analysis methods feasible. In this chapter, a number of different approaches-some well-known like 


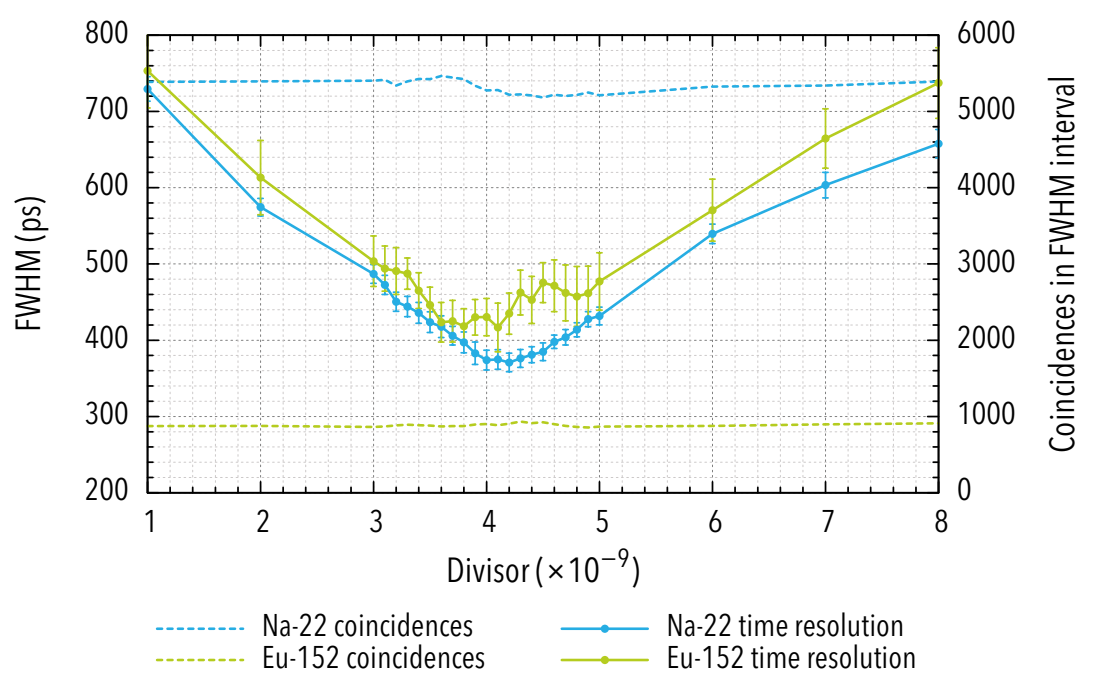

Figure 5.10: Dependence of the coincidence time resolution from the divisor value for the digital constant fraction of integral values approach.

constant trigger threshold timing and CFD, others not described in literature before like e. g. the pulse shaping approach-have been presented and evaluated.

Figure 5.11 shows a comparison of the time difference histograms of all methods for the ${ }^{152} \mathrm{Eu}$ test case. Each method was parameterized with the values yielding the best results according to the previous sections. Narrower peaks correspond to better time resolutions since the resolution is defined as these peaks' FWHM. The FWHM values are also summarized in table 5.2.

It is particularly noticeable that the group of methods that analyzes the whole signal performs badly while the trigger related methods perform significantly better. The only exception is the pulse shaping approach, which has a performance closer to the trigger group than to the whole-signal group.

Performance-wise, the digital implementation of the well-established constant fraction method-if optimized as described above-is still one of the best methods. Only the DCFI method (Nagl, Vetter, et al., 2010) performs slightly better. Concerning the simplicity of its usage and implementability, the DCFI method-which was first introduced with the first generation of the TDPAC spectrometer described in chapter 4-is certainly the better choice because it only requires a single free parameter and this parameter's optimal value is stable. 


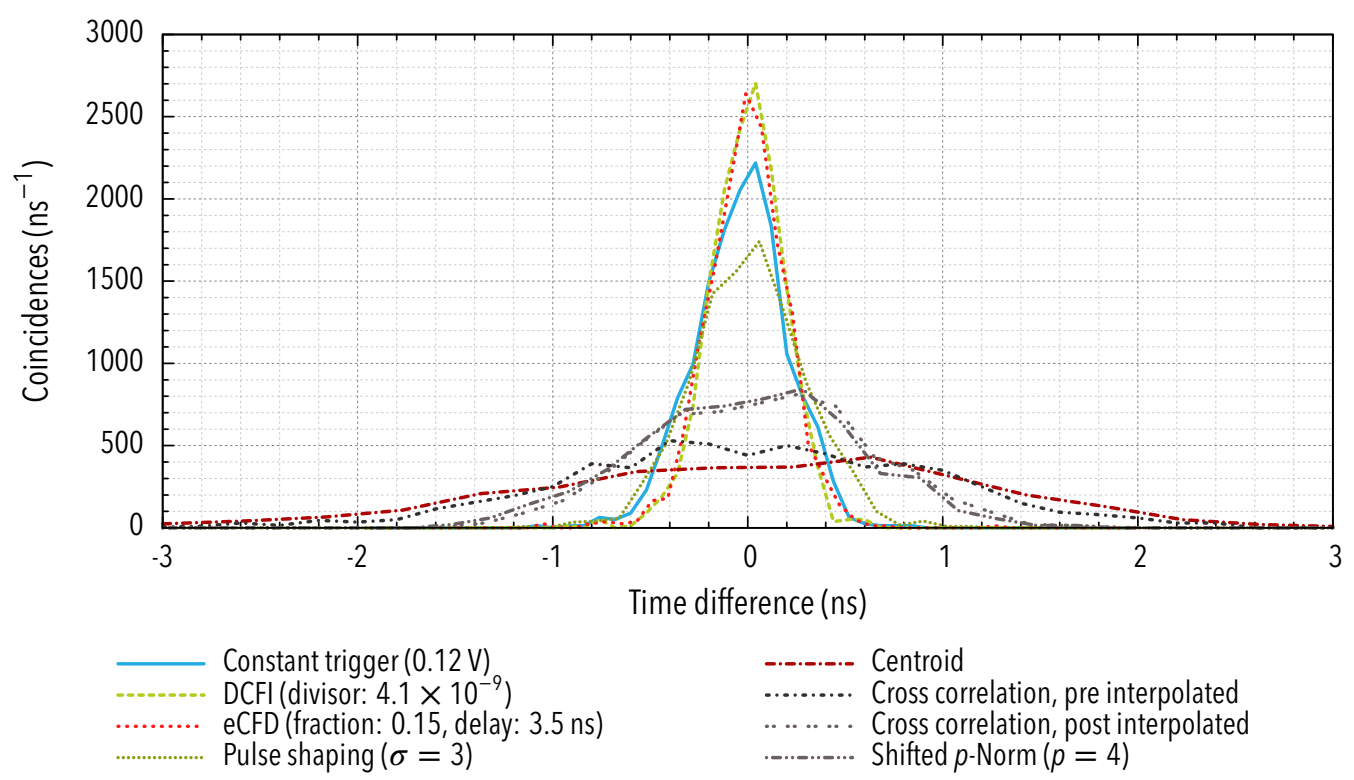

Figure 5.11: Comparison of time determination methods. All histograms were aligned to zero. Broad distributions correspond to bad time resolutions while sharper and higher peaks indicate better performing approaches. The amplitudes were normalized to show coincidences per ns. The area beneath the curves is comparable since all methods provided similar efficiencies (i. e. very low numbers of missed signals).

Table 5.2: Results of the evaluation of timing algorithms for ${ }^{22} \mathrm{Na}$ and ${ }^{152} \mathrm{Eu}$ test cases. Shown times are FWHM values. The upper group contains methods analyzing the whole signal while the lower three methods focus on a few samples around the rising edge.

\begin{tabular}{lll}
\hline Method & ${ }^{22} \mathrm{Na}(\mathrm{ns})$ & ${ }^{152} \mathrm{Eu}(\mathrm{ns})$ \\
\hline Centroid approach & $1.70(5)$ & $2.7(2)$ \\
Cross correlation approach (pre-interpolated) & $2.00(6)$ & $2.27(10)$ \\
Cross correlation approach (post-interpolated) & $1.21(3)$ & $1.37(6)$ \\
Deviation of shifted normalized waveform approach $(p=4)$ & $1.26(3)$ & $1.33(6)$ \\
Pulse shaping approach & $0.578(16)$ & $0.62(4)$ \\
\hline Constant trigger threshold timing & $0.385(8)$ & $0.49(3)$ \\
Emulated constant fraction discriminator & $0.366(9)$ & $0.43(2)$ \\
Digital constant fraction of integral values approach & $0.37(1)$ & $0.42(3)$ \\
\hline
\end{tabular}





\section{Chapter 6}

\section{Gain drift compensation}

One of the main applications of photomultiplier tubes (PMT) and successors like Silicon photomultipliers (SiPM) is radiation detection in combination with scintillators. Properties of the detected radiation, such as incidence time or energy, can be reconstructed by an analysis of the PMT's output signal. While time information is provided by the rising edges of the output signals, information concerning the energy of the detected radiation can be inferred from the collected charge, i. e. the integral of the output current across time, as long as single radiation events are separable. This is possible because the amount of scintillation light produced in scintillators is approximately proportional to the energy of the absorbed radiation, the light loss between scintillator and PMT is a statistical process, which hardly depends on the initial radiation's energy, and the PMT's gain depends only weakly on the amount of simultaneously detected light in case of the operational parameters typically occurring in radiation detectors.

Since the charge output of the PMT is approximately proportional to the detected radiation's energy, a linear conversion from measured charge values to energy values is sufficient for many applications. A calibration defining this linear conversion is usually conducted in the beginning of a measurement. The parameters for the linear conversion can be derived from the charge values of two peaks in the charge spectrum if the expected radiation energies related to these peaks are known. Higher order mapping curves can be used if necessary including information from additional peaks.

A common problem with this calibration approach is gain drift of the detectors. There are many reasons causing such drift. The leading contribution to detector drift is usually the gain-dependence on temperature and count rate.

Temperature dependence plays an important role if the calibration is done immediately after switching on the PMT's power supply because it takes a while before PMTs reach a stable operating temperature (cf. Hamamatsu, 2007, page 238). PMTs are usually supplied by a single high voltage supply, which powers the dynode stages through a voltage divider. This design can introduce a severe count rate dependence of the detector's gain because for high count rates rather high currents are drawn by the last dynode stages, which disequilibrates the voltage divider. This problem can be suppressed by high longitudinal currents, which introduce thermal problems. Newer designs use improved voltage dividers employing stabilizing mechanisms at the last stages like circuits based on Zener diodes 
or transistors (cf. Hamamatsu, 2007, page 94). However, even for such improved designs remaining effects entailed by local charge fluctuations and limited charge replenishing on the dynodes due to their own and their supply wires' impedance, remain. Another possible source of gain drift is the high sensitivity of PMTs towards external magnetic fields (cf. Hamamatsu, 2007, page 240). This effect is especially noticeable if a background field exists that makes the PMT operate in the most sensitive domain of the gain dependence on the magnetic field, i. e. the steepest part of this relation.

Since gain drift compromises long-term energy resolution of radiation measurements and especially window settings for peak discrimination, some efforts for compensation have been made before. A comprehensive overview is contained in Vickers (1997). However, all previous approaches focus on an on-the-fly adjustment of an amplifier that processes the PMT signals because they were designed for analog or mixed signal processing. Additionally, they use methods for the determination of peak positions like Gaussian fits (Conti, Eriksson, and Hayden, 2011) or coarse binning (Vickers, 1997), which are not optimal. This approach makes later corrections impossible. With the emergence of software defined detector signal evaluation, which uses digital signal processing techniques to analyze detector signals digitized using high-speed analog-to-digital converters (ADCs) immediately at the PMT's anode output (cf. Nagl, Vetter, et al., 2010), new opportunities concerning the gain drift compensation of radiation detectors are now available. In order to optimize the energy resolution of the time differential $\gamma-\gamma$ angular correlation (TDPAC) spectrometer described in chapter 4 for difficult measurement cases, such a gain drift compensation method was implemented.

\subsection{A novel drift-compensation method}

The implemented gain drift compensation method splits the signal stream from the detectors into blocks with a predefined length (default value: 5 million radiation events). For each block, charge spectra for all detectors are created by histogramming using a predefined set of bins.

Stable peak identification proved to be the most important challenge to face while implementing a peak tracking method that makes drift compensation possible. Because peaks in charge spectra of radiation detectors are usually not isolated but coalescing, the determination of peak positions can only be based on the peaks' tips rather than their edges. However, due to the small gradients around the tip, it is not possible to directly identify it in a histogram by simply searching for local maxima since there is typically more than one local maximum at the top of every single peak. Vickers (1997) overcomes this problem by increasing the histogram's bin width to almost the detector's energy resolution. This reduces the risk of manifold peaks but also significantly deteriorates tracking performance because the determination of peak positions is limited to the coarse bin width thereby providing imprecise values for the calibration. 
A better solution is to combine fine grained histograms and density estimation for peak analysis. For this purpose, a kernel density estimation (KDE) based on an Epanechnikov kernel (Wand and Jones, 1995)

$$
k(x)= \begin{cases}\frac{3}{4}\left(1-\left(\frac{x}{w}\right)^{2}\right) & ,\left|\frac{x}{w}\right| \leq 1 \\ 0 & ,\left|\frac{x}{w}\right|>1\end{cases}
$$

was implemented.

This algorithm is parameterized with a width $w$, which is chosen similar to the detector's expected energy resolution. This yields unambiguous peaks, which can be precisely located by simple channel comparisons (a KDE sample value marks a peak if it is surrounded by two smaller density values). The peaks of the density estimation are then tracked between subsequent spectra by determination of the peak location in the second spectrum that is closest to the peak in the first spectrum. The determined peak position in the second spectrum is used as start value for the peak search in the third block of data and so on. Peak positions in a block are used for calibration of all events belonging to the respective block. It is also possible to separate the number of events in each histogram and the granularity of the peak tracking into two independent parameters by the use of overlapping peak tracking histograms.

For the computation of the kernel density estimate, it is usually necessary to evaluate the kernel for each recorded event at all sampling points where it assumes finite values. Due to the random shift between the sampling points of the KDE and the single events' charge values, it is not possible to pre-compute kernel weights.

In order to simplify this process and reduce the number of computations, a method for the kernel approximation based on accumulated spectra was implemented. This is possible, assuming all events from a single charge bin have a charge value of exactly the bin's center value. Since the bin width is usually much smaller than the kernel width, the discrepancy to the usual KDE result introduced by this assumption is negligible. Because the distance between the shifted events and the KDE's sampling grid are now equal for all events, it is possible to pre-compute weights for the kernel and to multiply these weights with the count values in each bin to obtain the KDE curve.

If the weights are computed by applying the kernel function $k(x)$ to the distances between the bin center and the KDE sampling point, which are both on the same sampling grid (i. e. the distances are multiples of the bin width $\Delta x$ ), alignment artifacts occur because due to the Epanechnikov kernel's nonlinear shape, $k(i \cdot \Delta x)$ is not equal to the kernel's average value on the respective bin. This problem can be solved by calculating and using the kernel's average value

$$
w_{i}=\frac{1}{\Delta x} \int_{(i-0.5) \cdot \Delta x}^{(i+0.5) \cdot \Delta x} k(x) \mathrm{d} x
$$




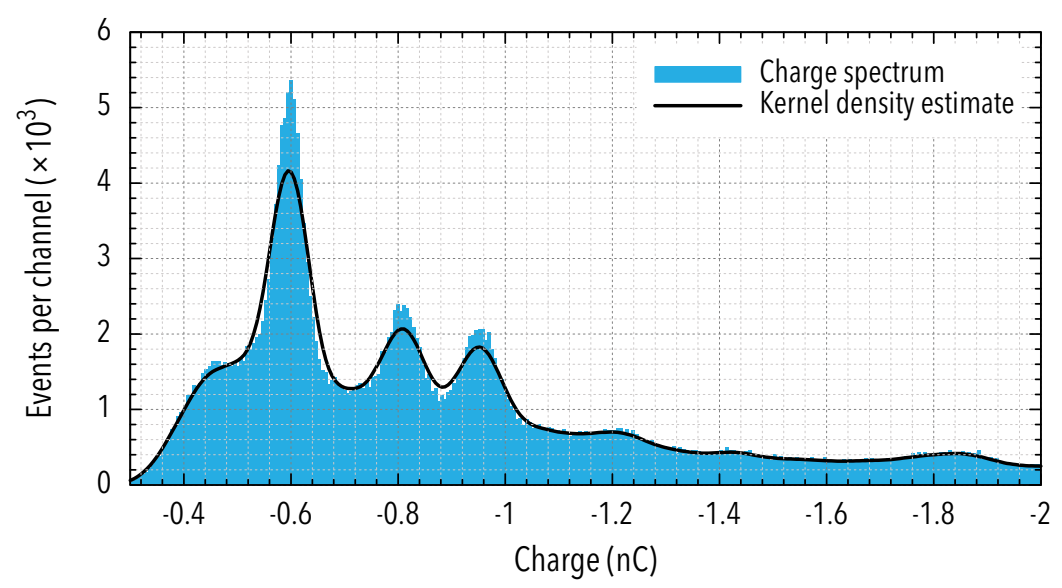

Figure 6.1: First block's charge spectrum of the decay of the ${ }^{98 \mathrm{~m}} \mathrm{Y}$ isomer from one of the four used detectors (cf. appendix A.1.3 for the decay scheme-energy assignments to peaks will be shown in fig. 6.3). The original spectrum is shown as blue histogram steps. The kernel density estimate is shown as black curve. With the kernel width set to $0.05 \mathrm{nC}$, noise on the peak tips disappears while relevant properties of the spectrum stay intact.

on the whole bin instead of its value at the bin center.

The (positive) values of $i$ where the kernel is finite are:

$$
i=0,1, \ldots,\left\lceil\frac{w}{\Delta x}+\frac{1}{2}\right\rceil
$$

Because of the kernel's symmetry, it is only necessary to calculate weight values for positive values of $i$. These values can also be used for the negative half of the kernel.

Once the weight vector is available, the KDE curve can be calculated by multiplication of each channel value $r\left(x_{k}\right)$ with the weight vector and accumulation of the bin-centered results, i. e. the KDE curve is defined as

$$
f\left(x_{k}\right)=\sum_{i=-i_{\max }}^{i_{\max }} w_{|i|} \cdot r\left(x_{k}+i \cdot \Delta x\right) .
$$

\subsection{Drift compensation results}

The drift compensation method described in the previous section was tested with data from a TDPAC measurement collected by the spectrometer described in chapter 4 .

Anode outputs of four detectors consisting of $\mathrm{LaBr}_{3}: \mathrm{Ce}$ scintillators from Saint-Gobain Crystals with a diameter of $25.4 \mathrm{~mm}$ and a height of $38.1 \mathrm{~mm}$ mounted on Photonis 
XP2020 PMTs were connected to Agilent Acqiris U1071A digitizer cards running with a sampling rate of 2 billion samples per second $(2 \mathrm{GS} / \mathrm{s})$ and a nominal resolution of 8 bit. The fifth digitizer was connected to an ionization chamber for the recording of timestamps of incoming ions.

During the measurement, a ${ }^{98 \mathrm{~m}} \mathrm{Y}$ beam (cf. appendix A.1.3 or Brant, Lhersonneau, and Sistemich, 2004 for decay information) from the fission product spectrometer LOHENGRIN at the Institut Laue-Langevin hit a nickel foil, which was mounted on an iron yoke of a neodymium magnet. The yoke was designed to create a magnetic flux through the foil with a direction parallel to the ${ }^{98 \mathrm{~m}} \mathrm{Y}$ beam and perpendicular to the foil surface. Although the yoke was built to minimize magnetic flux at the detector heads, which were mounted perpendicular to the beam direction around the nickel foil, a significant gain reduction was observable. As described above, such a magnetic field tends to increase gain drift because it makes the PMTs operate in a regime with steep slope of the gain vs. magnetic field relation. This means small fluctuations of the magnetic field have considerable influence on the gain. The size of a calibration block was set to 5 million events. However, since the ionization chamber's count rate was much higher than the detectors' count rate, the shown spectra from one of the detectors contain about 400000 events each. The scaling factor for the Epanechnikov kernel was set to $0.05 \mathrm{nC}$ because for the energy resolution of the used detectors this value leads to disappearance of noise on the peak tips while not excessively smoothing the spectra.

Figure 6.1 shows a binned charge spectrum (blue) of the ${ }^{98 \mathrm{~m}} \mathrm{Y}$ decay as recorded by one of the detectors as well as its kernel density estimate (black curve). The spectrum contains 326569 events from the first calibration block. The KDE was computed using the high performance approach described in section 6.1. At the second and third peaks' tips, the existence of several local maxima in the histogram is visible. This problem for peak localization is obviously solved by the used density estimation approach.

Figure 6.2 shows several block spectra's density estimates from different times during the measurement. The gain drift is visible by the different locations of the same peaks. Due to this drift, a classic one-time-calibration approach yields a result spectrum with very diffuse peaks, which are not even centered on the energies initially used for calibration. Obviously, peak tracking - while introducing only minimal computational effort if implemented as described-can vastly improve energy resolution as shown in fig. 6.3.

\subsection{Conclusion}

While it is advisable to exploit all available methods to reduce gain drift in radiation detectors' hardware-like stabilized voltage dividers and careful magnetic shieldingadditional drift compensation can be very helpful-especially for long running measurements-since it is often not possible to remove all of the many sources of gain drift.

The presented method provides outstanding performance at very low computational 


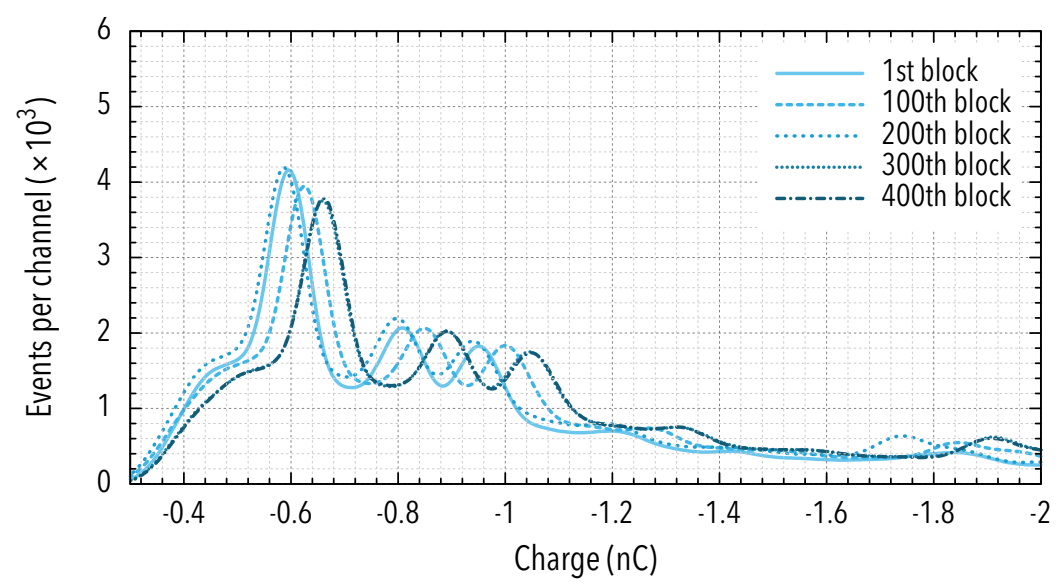

Figure 6.2: Kernel density estimates of five spectra distributed across the measurement. All spectra were recorded from the same detector.

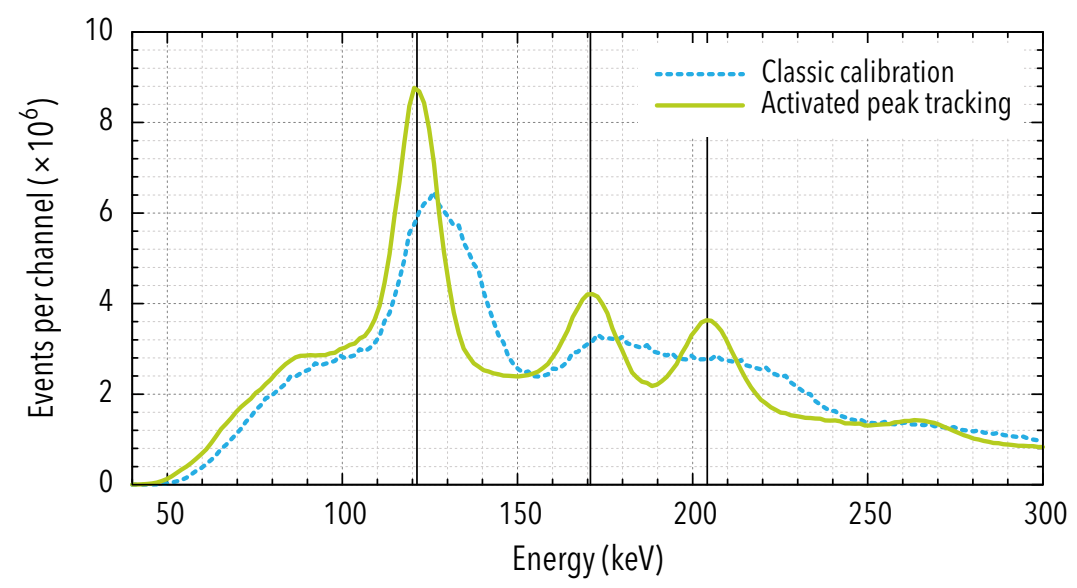

Figure 6.3: Energy spectrum of the decay of the ${ }^{98 \mathrm{~m}} \mathrm{Y}$ isomer (cf. appendix A.1.3 for the decay scheme). Both calibrations are based on the $121.3 \mathrm{keV}$ and $204.2 \mathrm{keV}$ peaks (outer black markers). Additionally, a marker for the $170.8 \mathrm{keV}$ energy was added to the plot. The green spectrum with activated peak tracking shows a better energy resolution and is not impaired by drift while the peaks of the classic spectrum exhibit significant drift towards higher energies. A total of 694515977 recorded $\gamma$ events were considered in both cases. 
cost and can easily be integrated in digital processing chains for radiation detector signals. As long as no sudden and large changes of the detectors' gain occur (i. e. in most usual drift cases caused by temperature or count rate changes), it works very stable and reliable. As an important advantage compared to older methods like those described by Vickers (1997) and related patents, it can be used online during a running measurement as well as offline while processing recorded charge values and it provides much better gain correction at lower complexity. 



\section{Chapter 7}

\section{$A b$ initio scintillation detector simulations}

Obviously, the radiation detectors are one of the key components in TDPAC spectrometers. Since the used detector design directly affects limitations of the achievable performance considering time and energy resolution, detection efficiency, and solid angle coverage, it is important to consider possible improvements of the detection hardware in order to improve overall performance of the spectrometer.

Detector designs are generally compromises because the detector applications impose numerous constraints and contrary design goals on the detector construction since parameters like detection efficiency, size, linearity, time resolution, and energy resolution need to be optimized simultaneously to different extents.

While the principle of photomultiplier tubes (PMTs) is known since the 1930s (Iams and Salzberg, 1935) and scintillation detectors based on the combination of a scintillating crystal and a PMT are among the oldest methods for x-ray and $\gamma$ detection, it is still a challenge to determine the relevant parameters for optimizing such detectors as well as the best methods (i.e. algorithms and parameters) for the processing of their output signals with regard to specific applications.

The only feasible way to a profound understanding of the relation between design decisions and performance parameters and to develop ideas for algorithms exploiting the possibilities of new digital spectrometer designs is the implementation and study of simulations of the detector itself that overcome the omission of statistical fluctuations inherent to simple Gaussian convolved exponential approaches. Such simulations can provide a well-founded idea of the limiting factors in a certain design and the parameters where tuning has the most favorable effect. Additionally, a working simulation provides a method for the evaluation and improvement of signal processing methods and a valuable tool to support the decision for a certain detector design before buying any components.

A first attempt of a Monte-Carlo simulation of whole scintillation detectors was made in cooperation with Arnold Krille from the Martin-Luther-Universität Halle-Wittenberg and is described in Krille, Nagl, et al. (2011). It implements a statistical approach for photon production similar to the one later on independently described by Seifert, Dam, and Schaart (2012) but uses it as starting point for a Monte-Carlo simulation of the amplification process in a photomultiplier tube (PMT). Statistical analysis of the simulated output signals revealed that it is not justified to neglect secondary photon transport in the 


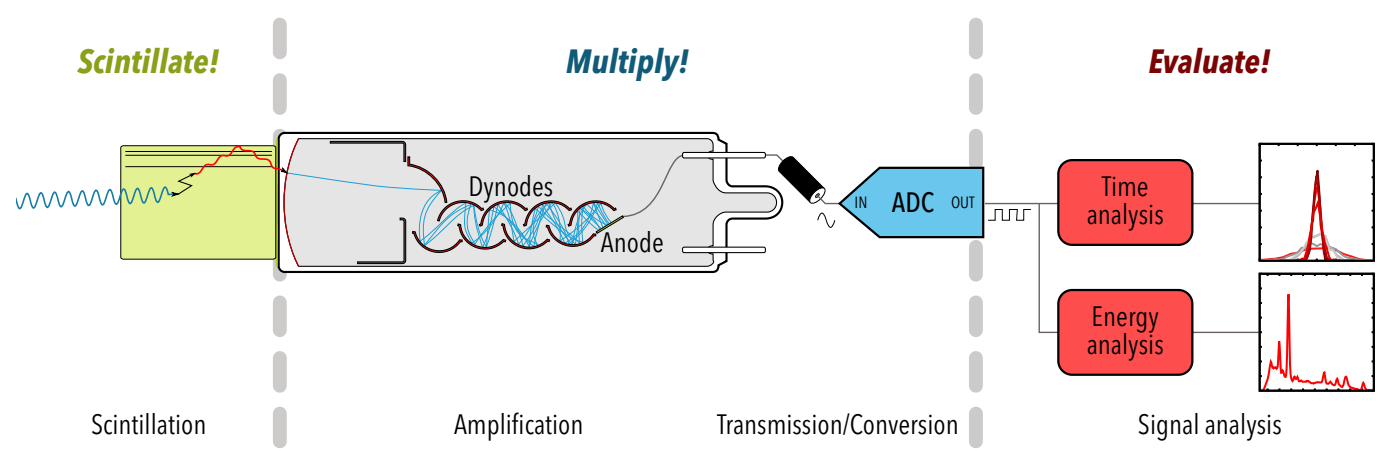

Figure 7.1: Task allocation among the Scintillate-Suite applications. This toolchain, consisting of the programs Scintillate!, Multiply!, and Evaluate!, which was developed in the framework of this thesis, allows for simulating complete radiation detection setups including the downstream signal chain and analog-to-digital conversion. Reference data from real setups can be evaluated using the same tools which allows for evaluating the quality of simulation results.

scintillation crystal for crystals with a size of more than about $1 \mathrm{~cm}^{3}$. This is consistent with a conclusion drawn by Seifert, Dam, and Schaart (2012).

Because TDPAC (and most other detector applications) rely on scintillation crystals big enough to obtain significant detection efficiencies, it is thus not sufficient to neglect photon propagation in the scintillation crystal. In order to parameterize statistical analyses, get input data for Monte-Carlo simulations, and obtain clues on how to build optimized detectors-i. e. find the application dependent best compromise- a comprehensive study of these propagation effects is necessary. Similar efforts were published by Choong (2009); however, this work is unfortunately limited to LSO scintillators and a small number of analog signal evaluation techniques and cannot be generalized since the simulation code is not available. Moreover, it uses an oversimplified PMT model.

A Geant4 (Allison et al., 2006) based simulation of the light creation and propagation process was implemented based on the lessons learned during the previously published work (Krille, Nagl, et al., 2011). Since a sufficiently realistic statistical description of light creation processes, called photon counting statistics (Seifert, Dam, and Schaart, 2012), exists and parameterization of this approach is a matter of measurements rather than simulations with satisfactory data available from literature, a focus was set on the light propagation between the point of conversion of the primary $\gamma$ photon into secondary visible photons and the photomultiplier tube's photo cathode. In order to achieve meaningful results, Geant4's implementation of realistic interaction ranges in matter was important because the precise location of $\gamma$-to-light-conversion proved to have substantial influence on the time characteristics of a certain detector setup.

Based on this approach, it is now possible to realistically simulate the whole conversion process from the $\gamma$ ray source all the way to the photo cathode even for big scintillation 
crystals including optical material properties, surface properties, painting and even optical coupling between crystal and PMT window. This allows avoiding of invalid generalization of a few special cases with unreliable results for detector optimization and provides reliable estimates of the theoretical performance limit of future detector designs.

The PMT simulation approach first implemented for Krille et al. (2011) was reimplemented as an improved and more specialized standalone software and is described in section 7.2. Task allocation among the developed detector simulation tools is shown in fig. 7.1. The toolchain called Scintillate-Suite allows for simulating of the whole signal chain thus making comparisons with real detectors easy.

\subsection{Light creation and propagation in scintillation crystals}

For the simulation of the scintillation process as well as the light propagation in the detector, a Geant4 based software called Scintillate! was created in the course of this work. This software is available for download (Nagl, 2009-2014). It outputs incidence time and position as well as photon energy for each secondary photon reaching the photo cathode.

The results of all simulation runs were post-processed using a number of scripts published alongside Scintillate!. Histograms of the incidence times of all photons hitting the simulated photo cathode as well as basic statistical evaluations were made using Python scripts. For further statistical evaluations and kernel density estimation, scripts written in $\mathrm{R}$ ( $\mathrm{R}$ Core Team, 2012) were used. As $\mathrm{R}$ proved to be too slow for the massive amount of kernel density estimates needed, an additional $\mathrm{C}++$ based command line tool was developed for this task, increasing evaluation speed by two orders of magnitude.

\subsubsection{Scintillate!}

Basically, Geant4 (Allison et al., 2006) is a framework for the simulation of particles (including photons) passing through matter. It is not a standalone application but meant to be controlled in a defined way by user supplied code that enables or disable physical interaction classes and provides geometry data.

Scintillate! was designed as a stand-alone application using Geant4 to simulate scintillation detectors consisting of a rotationally symmetric scintillator and a photomultiplier tube. It defines the geometry of this setup, allows for user modification of a wide range of typical parameters like scintillator shape, diameter, cover properties, among others and outputs simulation results in a configurable way as text files. Thus, Scintillate! provides a simplified method to use Geant4 for a special but very common simulation case.

Figure 7.2 shows an example for the implemented detector model. As Scintillate! outputs incidents on the PMT's photo cathode, only the relevant parts of the PMT are simulated. These are the PMT window, the photo cathode itself, and the glass envelope, which guides parts of the light away from the cathode (thereby reducing the overall efficiency). The photo cathode is modeled as a layer on the inner surface of the PMT window covering 


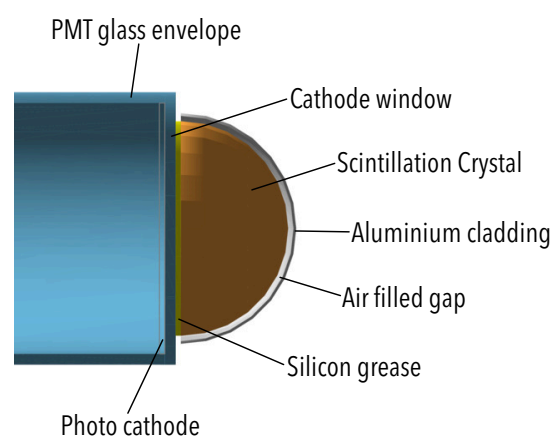

Figure 7.2: Detector model as implemented in Scintillate!. This figure shows the ellipsoidal scintillator shape. All properties of this model, including the scintillator shape, can be configured via macro files and thus without source code modifications. The thickness of the silicon grease layer between crystal and cathode window as well as the air gap between crystal and cladding are shown much larger than configured for typical simulations.

the full inner diameter. The cathode's quantum efficiency is not modeled by Scintillate!. Instead, it is part of downstream PMT simulations, which have been implemented separately.

In the current version of Scintillate!, the simulation of axially symmetric scintillation crystals of ellipsoidal, conical, and cylindrical shape is implemented. The scintillation crystal is coupled to the PMT window using a thin layer of silicon grease. The crystal is surrounded by an aluminium shell of the same shape. Between shell and crystal is an air filled gap. The default outer PMT diameter is $50.8 \mathrm{~mm}$ ( 2 inches).

The source is usually simulated as an isotropic point source on the symmetry axis in a distance of $3 \mathrm{~cm}$ from the detector's outer shell surface. Due to this parameter, a divergence realistic for many typical solid-state physics use-cases of detectors of this kind is obtained. For different applications, the distance can be changed by setting different values in the detector definition macro files read by Scintillate!. In order to save computing time, the source's emission angle was limited to values intersecting the detector model for all performed simulations.

Table 7.1 summarizes optical properties used in the detector model. To avoid infinite light paths, all absorption values were chosen finite. Because there are no consistent literature values available for the attenuation length of many materials but a strong dependence on several parameters (e.g. the purity of used materials) has to be expected, the selected values are not universal. However, as the influence of the optical attenuation on the result of the simulations is not strong as long as the attenuation length is significantly larger than the scintillator dimensions, this can typically be neglected. 
Table 7.1: Parts of the detector model and their respective optical properties. $n_{r}$ and $\lambda$ refer to the refractive indices and the optical attenuation lengths. The column $d$ contains the default setting for the respective parts' thickness.

\begin{tabular}{llcrc}
\hline Part & Material & $n_{r}$ & $\lambda(\mathrm{cm})$ & $d(\mathrm{~mm})$ \\
\hline PMT Envelope & Fused silica & $1.45-1.56^{a}$ & 200 & 2.0 \\
PMT Window & Fused silica & $1.45-1.56^{a}$ & 200 & 2.0 \\
Grease & Polydimethylsiloxane & $1.4^{b}$ & 10 & 0.1 \\
Air filled Gap & Air & 1.0 & 10000 & 0.2 \\
Shell & Aluminium & - & 0 & 0.5 \\
\hline
\end{tabular}

${ }^{a}$ Schott AG (2009)

${ }^{b}$ Mark (2009)

Table 7.2: Composition of materials used in the detector model. For materials not listed, the default values from Geant4's materials data base were used. Geant 4 allows defining of materials' compositions by number of atoms of each elemental component or by each component's fraction of mass. In Scintillate!, both methods were used. Thus, there are two types of notation used in this table.

\begin{tabular}{llc}
\hline Material & Composition & Density $\left(\mathrm{g} / \mathrm{cm}^{3}\right)$ \\
\hline Fused silica $\left(\mathrm{SiO}_{2}\right)$ & $1 \times \mathrm{Si}, 2 \times \mathrm{O}$ & $2.201^{a}$ \\
Polydimethylsiloxane & $1 \times \mathrm{Si}, 1 \times \mathrm{O}, 2 \times \mathrm{C}, 6 \times \mathrm{H}$ & $0.97^{c}$ \\
$\left(\mathrm{CH}_{3}\left[\mathrm{Si}\left(\mathrm{CH}_{3}\right)_{2} \mathrm{O}\right]_{\mathrm{n}} \mathrm{Si}_{\left.\left(\mathrm{CH}_{3}\right)_{3}\right)}\right.$ & \\
$\mathrm{LaBr}_{3}: \mathrm{Ce}^{d}$ & $36.62 \% \mathrm{La}, 63.19 \% \mathrm{Br}, 0.19 \% \mathrm{Ce}$ & $5.08^{e}$ \\
$\mathrm{NaI}_{\mathrm{T} f}$ & $15.32 \% \mathrm{Na}, 84.59 \% \mathrm{I}, 0.08 \% \mathrm{Tl}$ & $3.67^{g}$ \\
$\mathrm{Lu}_{1.8} \mathrm{Y}_{0.2} \mathrm{SiO}_{5}: \mathrm{Ce}^{b}$ & $71.43 \% \mathrm{Lu}, 4.03 \% \mathrm{Y}, 6.37 \% \mathrm{Si}$, & $7.1^{i}$ \\
& $18.14 \% \mathrm{O}, 0.02 \% \mathrm{Ce}$ & \\
\hline
\end{tabular}

${ }^{a}$ Schott AG (2009)

${ }^{b}$ The number of monomers $n$ is considered large and therefore only the monomer is considered in the determination of elemental fractions.

${ }^{c}$ Mark (2009)

${ }^{d}$ Loef et al. (2001)

${ }^{e}$ Saint-Gobain (2004a)

${ }^{f}$ Trefilova et al. (2002)

${ }^{g}$ Saint-Gobain (2005)

${ }^{b}$ Cooke et al. (2000)

${ }^{i}$ Saint-Gobain (2004b) 
Table 7.3: Scintillation material definitions used for Scintillate!. Values without citation are estimates used due to missing data. The luminosity describes the number of produced optical photons per $\mathrm{keV}$ of the incident $\gamma$ photon. $E$ is the typical energy of the produced optical photons. The next column contains the contribution of each simulated emission component to the emitted scintillation light. $\tau_{r}$ is the rise-time, which depends on transport mechanisms during the conversion from $\gamma$ to optical photons (Weber, Derenzo, and Moses, 2000). $\tau_{d}$ means the decay time of optical photon producing excited states in the scintillation material. $n_{r}$ and $\lambda$ contain the refractive index and the optical attenuation length in the scintillation material for the produced light.

\begin{tabular}{|c|c|c|c|c|c|c|c|}
\hline Material & $\begin{array}{c}\text { Luminosity } \\
\mathrm{keV}^{-1}\end{array}$ & $\begin{array}{c}E \\
\mathrm{eV}\end{array}$ & $\begin{array}{c}\text { Fraction } \\
\%\end{array}$ & $\begin{array}{l}\tau_{r} \\
\mathrm{~ns}\end{array}$ & $\begin{array}{l}\tau_{d} \\
\mathrm{~ns}\end{array}$ & $n_{r}$ & $\begin{array}{c}\lambda \\
\mathrm{cm}\end{array}$ \\
\hline \multirow{2}{*}{$\mathrm{BaF}_{2}$} & \multirow[t]{2}{*}{$11.8^{a}$} & $5.9^{a}$ & $15^{a}$ & 0 & $0.7^{a}$ & $1.54^{a}$ & $34^{b c}$ \\
\hline & & $4.0^{a}$ & $85^{a}$ & 0 & $630^{a}$ & $1.50^{a}$ & $34^{b, c}$ \\
\hline $\mathrm{LaBr}_{3}: \mathrm{Ce}$ & $75^{d}$ & $3.26^{e}$ & 100 & 0 & $16^{e}$ & $1.9^{e}$ & 100 \\
\hline \multirow[t]{2}{*}{ NaI:Tl } & \multirow[t]{2}{*}{$38^{f}$} & $3.0^{f}$ & 50 & $0^{g}$ & $250^{f}$ & $1.85^{f}$ & 100 \\
\hline & & $3.0^{f}$ & 50 & $27^{g}$ & $250^{f}$ & $1.85^{f}$ & 100 \\
\hline $\mathrm{Lu}_{1.8} \mathrm{Y}_{0.2} \mathrm{SiO}_{5}: \mathrm{Ce}$ & $32^{h}$ & $2.95^{b}$ & 100 & 0 & $41^{h}$ & $1.81^{b}$ & $42^{i}$ \\
\hline
\end{tabular}

\footnotetext{
${ }^{a}$ Saint-Gobain (2012)

${ }^{b}$ Ma and Zhu (1993)

${ }^{c}$ Worst case value for $\mathrm{BaF}_{2}$ irradiated with $10 \mathrm{kGy}{ }^{60} \mathrm{Co} \gamma$-rays.

${ }^{d}$ de Haas and Dorenbos (2008)

${ }^{e}$ Saint-Gobain (2004a)

${ }^{{ }^{S}}$ Saint-Gobain (2005)

${ }^{g}$ Weber, Derenzo, and Moses (2000)

${ }^{b}$ Saint-Gobain (2004b)

${ }^{i}$ Vilardi et al. (2006)
}

Table 7.2 shows the elemental compositions and densities as used in the material definitions. These values are used by Geant 4 for the simulation of interactions inside the respective materials.

Scintillation materials' definitions require additional properties. The used values are summarized in table 7.3. Like for the structural materials in table 7.1, values for the attenuation length could not be obtained for all materials. As measured rise-times of scintillation detectors are influenced not only by the dynamics inside the scintillation crystal but also by PMT properties, it is difficult to determine values for materials with steeply rising signals. Therefore, the rise-time values were approximated as $0 \mathrm{~ns}$ for all materials except for the slow rising component of NaI:Tl.

It is a commonly used method to manipulate light propagation in scintillation crystals by painting the crystal surfaces that are not facing the PMT window. Additionally, it is possible to polish crystal surfaces before painting or use ground surfaces. In order to evaluate the effect of such paintings and surface treatments, five different variants of each shape / material combination were simulated: 
- ground surface / unpainted

- ground surface / white painted

- polished surface / white painted

- ground surface / black painted

- polished surface / black painted

For the simulation of light propagation between crystal and paint or air, Geant4's implementation of the UNIFIED model (Levin and Moisan, 1996) is used by Scintillate!. Ground as well as polished surfaces are simulated using the "ground" finish setting. Scintillate! uses $1.3^{\circ}$ for polished and $12^{\circ}$ for ground surfaces as sigma-alpha value and an estimation of the paint's refractive index of $n_{r}=1.61$, both according to Janecek and Moses (2010). The reflectivity is set to $95.5 \%$ for white paint according to Janecek and Moses (2010) and $4.3 \%$ for black paint according to Dury et al. (2007). Unpainted crystals are simulated using a ground crystal to air surface and on the other side of the gap, between crystal and shell, an air to ground aluminium surface. Since the reflectivity of aluminium is largely dependent on the way it was machined and can vary throughout a wide range (Brandt et al., 1984), a moderate value of $70 \%$ was chosen as Scintillate! preset.

Figure 7.3 shows a cut view of a single $\gamma$ incidence as simulated by Scintillate!. A large number of secondary visible photons (green lines) were generated. Many of them reached the PMT's photo cathode after several reflections. The incidence points on the photocathode are indicated by red dots.

For the use of its result as input for photomultiplier simulations or statistical evaluations, Scintillate! outputs data files in tab-separated value format containing time, energy, and position of each visible photon's arrival on the photo cathode. For automated simulations, Scintillate! can be configured via Geant4's scripting engine.

\subsubsection{Statistical evaluation}

While being a valuable data source for downstream simulations of the PMT behavior, allowing for much more realistic results of such simulations (thereby making the simulation of a complete detector feasible), the results of Scintillate! can also be evaluated on their own. In order to draw conclusions concerning the advantages and disadvantages of certain detector designs, a statistical analysis of the simulation results was accomplished.

The following sections describe the details and meaning of examined parameters. Results follow in sections 7.1.3 and 7.1.4.

Collection efficiency The collection efficiency $\eta_{\text {col }}$ represents the fraction of produced visible photons $\left(N_{\mathrm{c}}\right)$ that are detected on the PMT's photo cathode $\left(N_{\mathrm{d}}\right)$. It is defined for 

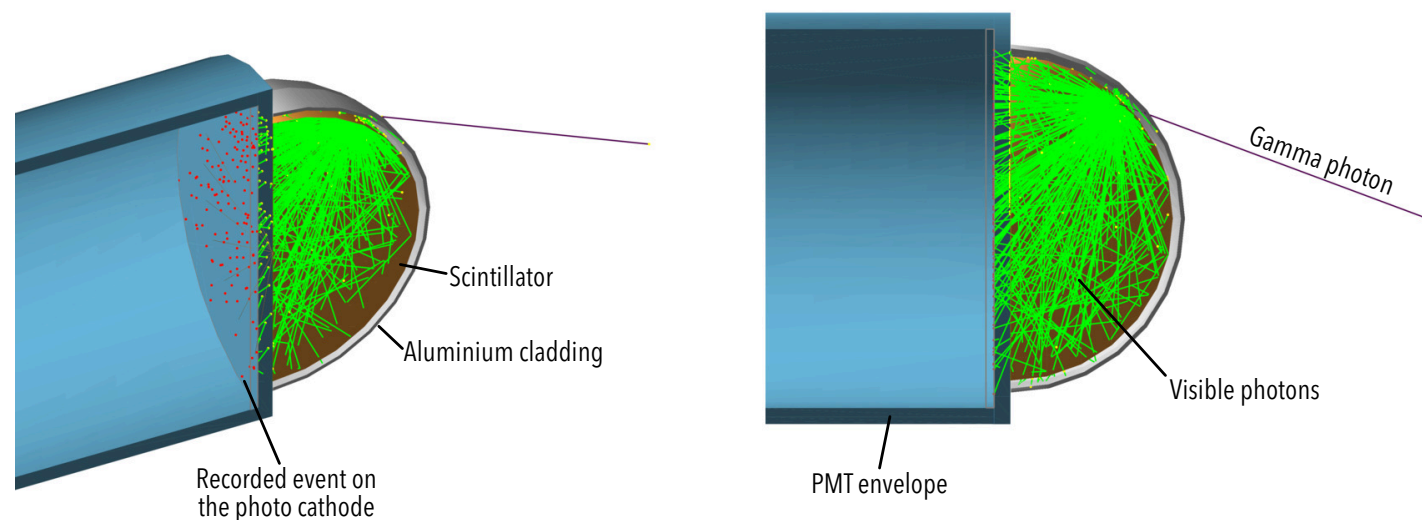

Figure 7.3: A single $\gamma$ incidence $(E=30 \mathrm{keV})$ as simulated by Scintillate!. The green lines are trajectories of visible photons while the purple line shows the original $\gamma$ photon's trajectory. Red dots highlight the points where visible photons hit the PMT's photo cathode.

all $\gamma$ events that created photons in the scintillation crystal as

$$
\eta_{\mathrm{col}}=\frac{N_{\mathrm{d}}}{N_{\mathrm{c}}}
$$

It was determined for every simulated $\gamma$ incidence that created at least a single secondary photon. The plots in section 7.1.3 show a percentile display of this surviving fraction. All events were binned according to their surviving fraction percentage and the fraction of the total number of events contained in each bin was then plotted. Peaks in the lower $\mathrm{x}$-axis range therefore mean big secondary photon loss while peaks in the higher $\mathrm{x}$-range represent efficient light transport towards the photo cathode. For this study, the secondary photon luminosity was set to $100 \mathrm{keV}^{-1}$ for all materials. This has no influence on the determined ratio but makes the results comparable for all materials and avoids sample size induced artifacts.

Arrival time of the first visible photon This parameter contains the geometry dependence of the arrival time of each event's first visible photon on the photo cathode. The zero point of the used time axis is set to the start time of the $\gamma$ photon in the source, $3 \mathrm{~cm}$ away from the simulated detector. The distribution represents the scintillator geometry dependent component of the time distribution as it would be obtained by perfect 
first-photoelectron triggering with an ideal PMT. Other contributions to the real firstphotoelectron time distribution are the scintillation material dependent time constants defining the process of photon creation in the scintillation material-which were set to zero here in order to isolate the geometry dependent component-and the limited time resolution of the used photon detection method, e. g. the photomultiplier tube. The used bin width is $10 \mathrm{ps.}$

First quartile of the photon arrival times These plots show the geometry induced time value of the first quartile. For each event, the time of the first quartile-i. e. the time at which $25 \%$ of the arriving photons already arrived at the photo cathode while $75 \%$ are still propagating through the crystal (photons that did not arrive at all were discarded) was determined and these results were binned using a bin width of $10 \mathrm{ps}$ and plotted as a histogram. These distributions help to assess the simultaneity of arrival times given a certain geometry. Since many well-established event time determination methods-like e. g. constant fraction discrimination-exhibit a better performance for signals with a short rise-time, a high degree of geometric simultaneity is desirable for high performance detectors.

According to the recommendation from Hyndman and Fan (1996), the quartile values $t_{\mathrm{fq}}$ were estimated from the photon incident times $t_{i}$ on the photo cathode as

$$
t_{\mathrm{fq}}=t_{\lfloor h\rfloor}+(h-\lfloor h\rfloor)\left(t_{\lfloor h\rfloor+1}-t_{\lfloor h\rfloor}\right)
$$

where

$$
h=\frac{1}{4}\left(N+\frac{1}{3}\right)+1
$$

$N$ is the total number of detected photons for a particular $\gamma$ event and $t_{i}$ is the incidence time of the $i^{\text {th }}$ earliest photon in the ordered set of detected photons. $\lfloor h\rfloor$ denotes the floor function, returning the largest integer smaller than $h$.

As described in section 7.1.2, only the fast component of $\mathrm{BaF}_{2}$ scintillation light was considered for quartile determination.

Kernel density estimation based simulated trigger time In many usage scenarios of scintillation detectors, first photon triggering is not possible due to dark current and noise. Furthermore, it is usually preferable to require more than one photon for triggering since it is well known that triggering on the first secondary photon hitting the detector (i. e. the first photo-electron produced in a PMT based detector) does not yield the best time resolution achievable for real detector designs (Seifert, Dam, Vinke, et al., 2012; Fishburn and Charbon, 2010).

As the light-to-signal conversion in photomultiplier tubes can be roughly approximated using a kernel density estimate (the photon arrival events on the photo-cathode can be 


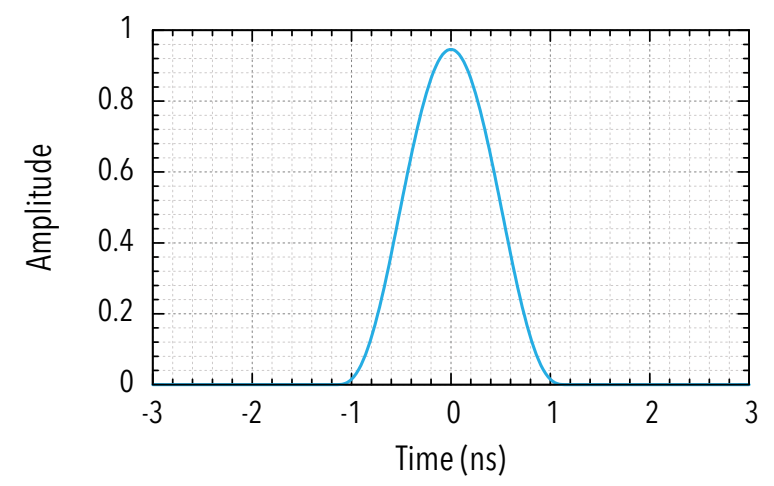

Figure 7.4: Shape of the triweight kernel with bandwidth $b=0.5 \mathrm{~ns}$ as used for the kernel density estimations.

considered unit impulse functions and the anode signal an approximation of their density), such an estimate was computed to obtain statistical results that can be compared to output signal properties of real detectors.

A canonical triweight kernel according to Wand and Jones (1995) was chosen, as it combines high efficiency (as defined there) with modest computing time requirements. Additionally, in contrast to a Gaussian kernel it is defined on a finite support, which makes it causal, and it has a shape more similar to single-photon output signals from photomultipliers than an Epanechnikov kernel. The chosen bandwidth is $0.5 \mathrm{~ns}$ which yields a kernel shape similar to single photon signals of real photomultiplier tubes (see fig. 7.4 and Hamamatsu, 2007).

The triweight kernel is defined as

$$
K(u)=\frac{35}{32}\left(1-u^{2}\right)^{3} \mathbf{1}_{\{|u| \leq 1\}}
$$

with $\mathbf{1}_{\{|u| \leq 1\}}$ being the indicator function and the input values being rescaled according to

$$
\begin{gathered}
u=\frac{t}{h} \\
h=b \cdot \sqrt[5]{\frac{9450}{143}},
\end{gathered}
$$

where $b$ denotes the selected kernel bandwidth.

In order to obtain a parameter comparable to real measurements, a trigger level was defined at the kernel density estimation amplitude of ten simultaneously arriving photons. In contrast to the parameters discussed above, the simulation of photon creation time characteristics was enabled for the kernel density based analysis in order to obtain results comparable with real detectors. 


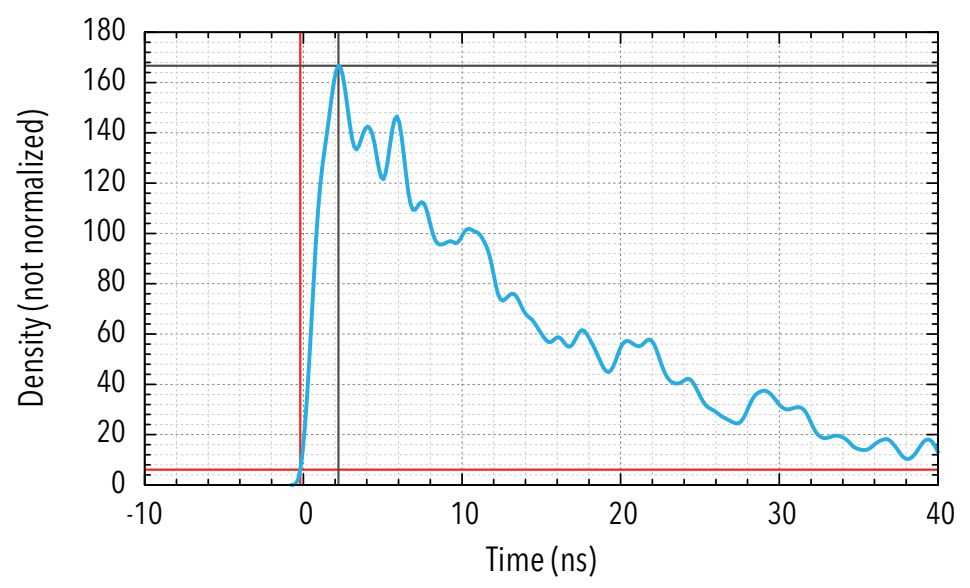

Figure 7.5: Kernel density estimate of detected photons produced by a $511 \mathrm{keV} \gamma$ photon hitting a cylindrical $\mathrm{LaBr}_{3}:$ Ce crystal $(\varnothing=40 \mathrm{~mm}, d=40 \mathrm{~mm}$, ground and white painted surface). The two parameters extracted for statistical evaluation and the related amplitudes are marked red (simulated trigger time and amplitude) and grey (maximum's time and amplitude).

In order to make the trigger level constant and independent from the number of produced secondary photons, the usual normalization of the density estimate (division by the size of the set whose density is estimated) was omitted.

An example for the kernel density estimations' result curves is shown in fig. 7.5.

The time of the crossing of kernel density and trigger level was determined for all simulated events and plotted as histogram using a bin width of 20 ps. Due to the extent of the used kernel's support to negative values and the omission of an additional delay as it occurs in real photomultipliers, some of the histograms contain events at times smaller than zero. This is not non-causal because a simple shift using a constant time offset is sufficient to guarantee all occurring times being positive.

Time of the kernel density estimation's maximum In addition to the simulated trigger times, the time of each kernel density estimation's maximum was determined. The same kernel settings as in the previous section were used. Histograms containing the distribution of these maxima are shown alongside the simulated trigger times. The used bin width is 20 ps.

Figures of merit In order to achieve quantitative comparability of the results, average and the unbiased sample standard deviation (with applied Bessel's correction) were determined for each of the statistical parameters described in the preceding sections.

Since the performed Geant 4 based simulations comprise Compton scattering and multi- 


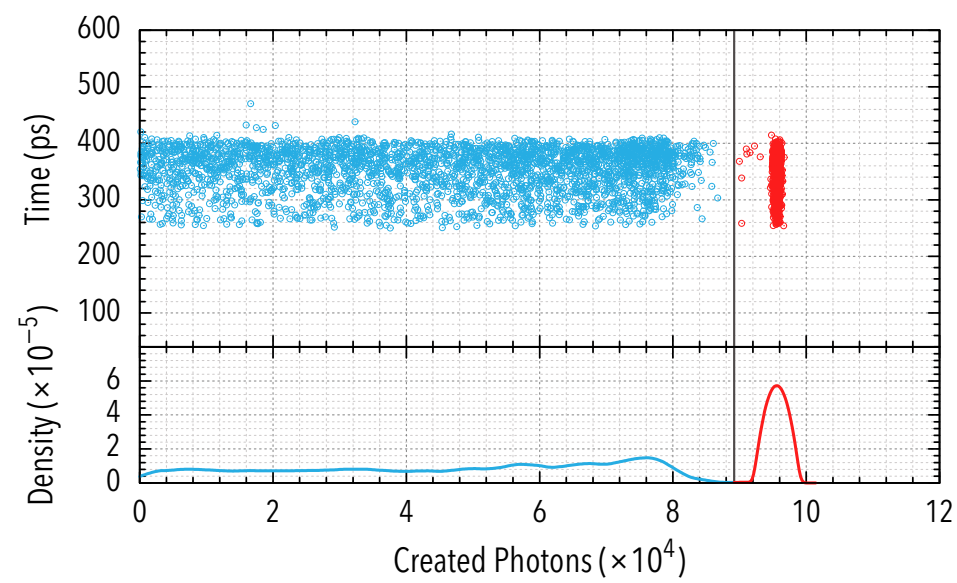

Figure 7.6: Example for the employed cluster separation of photo peak and Compton events. The scatter plot shows the time of the first quartile versus the number of secondary photons created for each $\gamma$ event of a simulation run for a cylindrical $\mathrm{LaBr}_{3}:$ Ce crystal $(\varnothing=20 \mathrm{~mm}, d=40 \mathrm{~mm}$, ground and white painted surface) irradiated by $1274.53 \mathrm{keV}$ primary $\gamma$ photons. In the region of low photon numbers, several outliers are visible which makes it necessary to separate photo peak and Compton events before determining the photo peak events' figures of merit. The separation problem was solved by means of a density estimate shown as curve in the lower diagram. The density estimate's minimum proved to provide a stable criterion for the automatic separation of photo peak and Compton events.

interaction events, a number of outliers occurred among the considered parameters' results. Figure 7.6 shows a scatter plot of the first quartile times as described on page 101 versus the number of created secondary photons. Obviously, the average of the quartile times is at about $350 \mathrm{ps}$. Several outliers are visible in the range of low numbers of created photons. This happens due to unusual multi-interaction events similar to the one shown in fig. 7.7. Since most usage scenarios of scintillation detectors discard the Compton events altogether and focus on detected events belonging to the photo peak, the figures of merit are limited to events belonging to the photo peak. This avoids asymmetric outliers, which would otherwise severely bias the results.

Automated separation of photo peak and Compton events requires a stable algorithm that is not obstructed by single events in the gap separating both groups. Considering photo peak and Compton range as clusters, several clustering algorithms were evaluated. Because of the big difference in cluster size, k-means and distribution based approaches (Everitt et al., 2011) are not suitable. A density based approach showed to provide the best results. It was implemented using an Epanechnikov Kernel (Wand and Jones, 1995) and the R implementation of Silverman's rule of thumb (Silverman, 1986; R Core Team, 


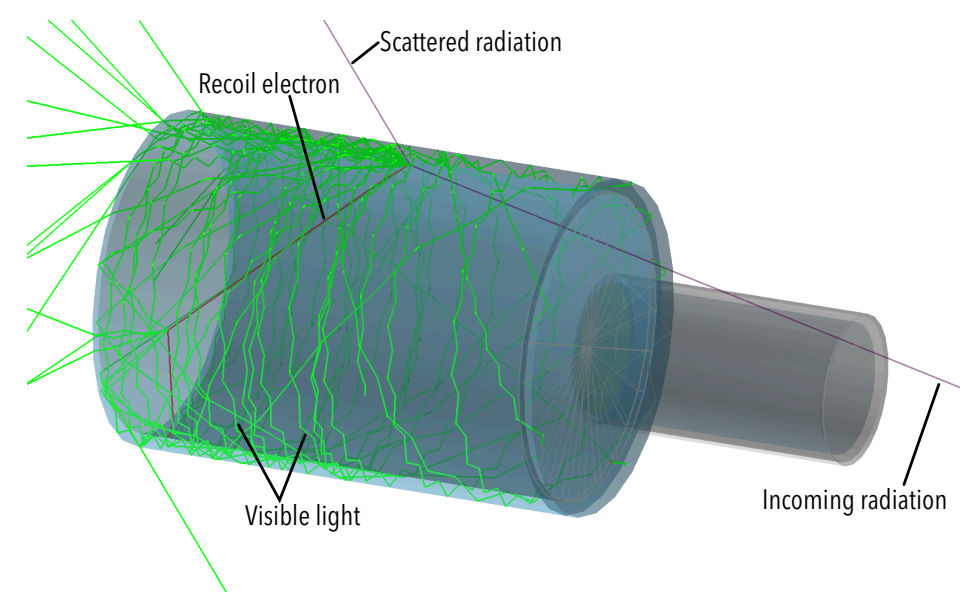

Figure 7.7: Example for an irregular event where the $\gamma$ photon passes the scintillation crystal without interaction followed by photon production due to subsequent Compton interaction inside the glass envelope of the photomultiplier. Again, the green lines are trajectories of visible photons while the purple line shows the original $\gamma$ photon's trajectory.

2012). A scaling factor of 0.3 proved to provide reliable and stable clustering results for all analyzed data-sets. Again, see fig. 7.6 for an example.

Since the theoretical distributions of the studied parameters cannot be determined analytically, the standard errors of the figures of merit were determined using the bootstrap method (Efron and Tibshirani, 1994) with a bootstrap sample size of 2000.

\subsubsection{Result graphs}

Figure 7.8 shows the statistical evaluation results for $\mathrm{LaBr}_{3}$ :Ce scintillators. Likewise, fig. 7.9 contains results of the density estimation based evaluations of the same material. Each analysis is based on a set of 10000 simulated $\gamma$ events. The version of Geant 4 used for all simulations was 4.9.6 Patch-02.

Each result plot contains graphs of ten different combinations of scintillator surface and $\gamma$ energy. Dark gray lines show results for ground and black painted surfaces while black lines indicate polished black painted surfaces. Likewise light red lines show results for ground and white painted surfaces while dark red lines represent polished white surfaces. Additionally, blue lines indicate unpainted surfaces with aluminium cover. A detailed description of the simulated surface properties can be found in section 7.1.1.

In order to provide an idea of the results' energy dependence, two $\gamma$ energies were simulated. Dashed lines show results for a $\gamma$ energy of $511 \mathrm{keV}$ while solid lines depict results for a $\gamma$ energy of $1274.53 \mathrm{keV}$. 


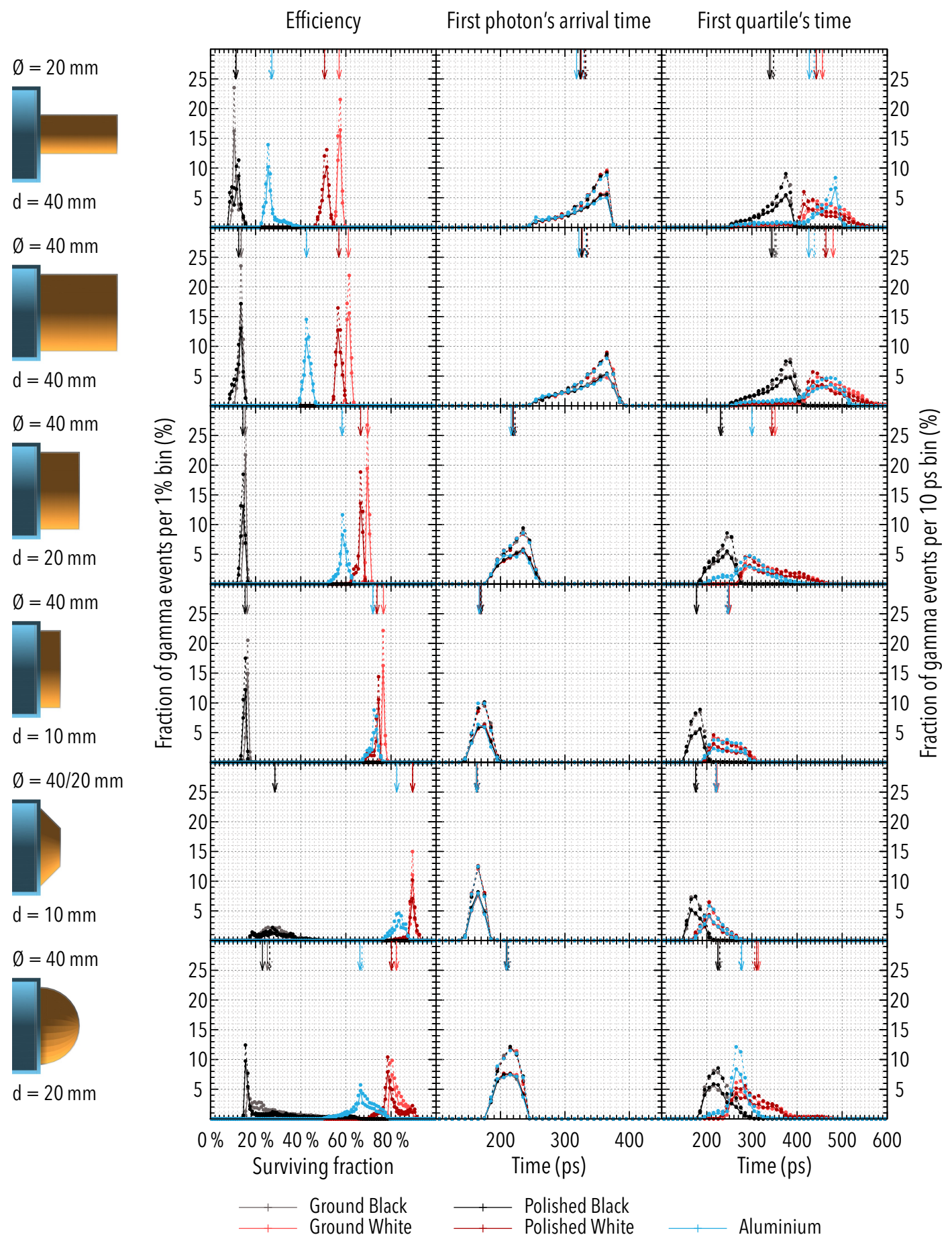

Figure 7.8: Simulation results for $\mathrm{LaBr}_{3}$ :Ce crystals. 


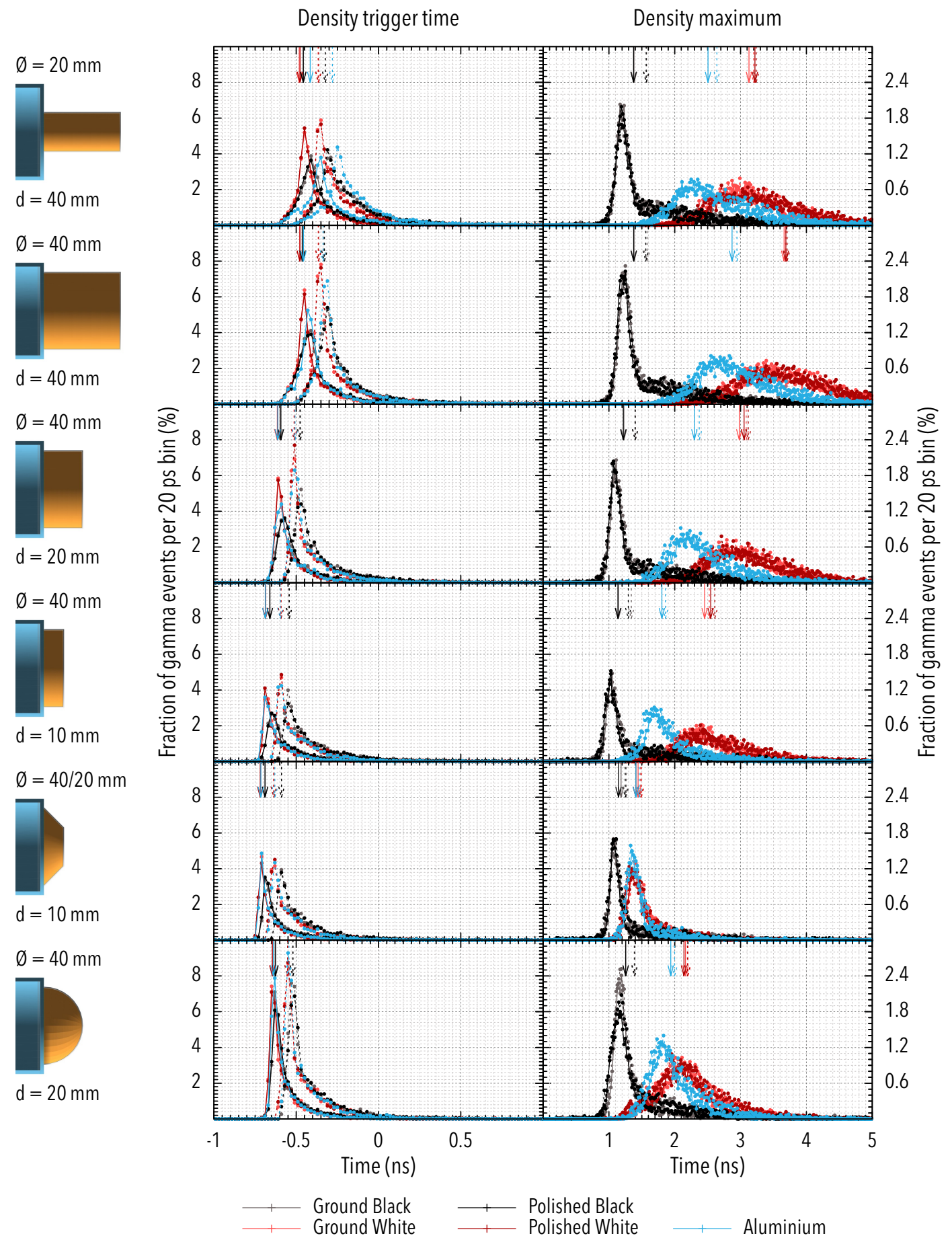

Figure 7.9: Density estimation based results for $\mathrm{LaBr}_{3}$ :Ce crystals. 
Table 7.4: Figures of merit for $\mathrm{LaBr}_{3}$ :Ce scintillators

\begin{tabular}{|c|c|c|c|c|c|c|c|c|c|c|c|c|c|}
\hline \multicolumn{3}{|c|}{ Scintillation Crystal Properties } & \multirow{2}{*}{$\frac{\text { Efficiency }}{\eta_{\text {int }}, \%}$} & \multicolumn{2}{|c|}{ Collection Efficiency } & \multicolumn{2}{|c|}{ First Photon's Arrival } & \multicolumn{2}{|c|}{ First Quartile } & \multicolumn{2}{|c|}{ Density Trigger Time } & \multicolumn{2}{|c|}{ Density Maximum } \\
\hline Geometry & Energy & Surface & & $\bar{\eta}_{\mathrm{col}}, \%$ & $s\left(\eta_{\mathrm{col}}\right), \mathrm{pp}$ & $\bar{t}_{\mathrm{fp}}, \mathrm{ps}$ & $s\left(t_{\mathrm{fp}}\right), \mathrm{ps}$ & $\bar{t}_{\mathrm{fq}}, \mathrm{ps}$ & $s\left(t_{\mathrm{fq}}\right), \mathrm{ps}$ & $\bar{t}_{\mathrm{tr}}, \mathrm{ps}$ & $s\left(t_{\mathrm{tr}}\right), \mathrm{ps}$ & $\bar{t}_{\mathrm{m}}, \mathrm{ns}$ & $s\left(t_{\mathrm{m}}\right), \mathrm{ns}$ \\
\hline \multirow{6}{*}{$\varnothing=20 \mathrm{~mm}$} & \multirow{10}{*}{$1274.53 \mathrm{keV}$} & Ground White & $46.6(2)$ & $57.11(2)$ & $0.7(1)$ & $332.3(7)$ & $29(5)$ & $457(1)$ & $52(1)$ & $-367.1(8)$ & $34(7)$ & $3.20(1)$ & $0.5(1)$ \\
\hline & & Ground Black & & $11.19(3)$ & $1.3(3)$ & $332.1(7)$ & $28(5)$ & $348.3(7)$ & $32(6)$ & $-324(1)$ & $53(1)$ & $1.57(1)$ & $0.6(1)$ \\
\hline & & Polished White & & $50.66(3)$ & $1.5(3)$ & $330.6(7)$ & $29(5)$ & $442(1)$ & $60(1)$ & $-363.9(8)$ & $35(8)$ & $3.24(1)$ & $0.6(1)$ \\
\hline & & Polished Black & & $11.00(4)$ & $1.6(3)$ & $332.1(6)$ & $29(5)$ & $347.7(8)$ & $32(6)$ & $-323(1)$ & $52(1)$ & $1.57(1)$ & $0.6(1)$ \\
\hline & & Aluminium & & $26.72(8)$ & $3.1(7)$ & $328.8(7)$ & $31(5)$ & $438(2)$ & $63(1)$ & $-280(2)$ & $69(1)$ & $2.64(1)$ & $0.5(1)$ \\
\hline & & Ground White & $34.1(2)$ & $57.00(3)$ & $0.6(2)$ & $325(1)$ & $30(7)$ & $456(2)$ & $54(1)$ & $-482(2)$ & $38(1)$ & $3.13(2)$ & $0.38(9)$ \\
\hline \multirow{4}{*}{$\mathrm{d}=40 \mathrm{~mm}$} & & Ground Black & & $11.41(6)$ & $1.4(3)$ & $325(1)$ & $30(7)$ & $341(1)$ & $34(8)$ & $-458(2)$ & $48(1)$ & $1.38(2)$ & $0.4(1)$ \\
\hline & & Polished White & & $50.54(6)$ & $1.4(3)$ & $325(1)$ & & $443(3)$ & & $-477(2)$ & $39(1)$ & & $0.4(1)$ \\
\hline & & Polished Black & & $11.24(7)$ & $1.6(4)$ & $324(1)$ & $30(7)$ & $339(1)$ & $33(8)$ & $-457(2)$ & $47(1)$ & $1.37(1)$ & $0.3(1)$ \\
\hline & & Aluminium & & $27.2(1)$ & $4(1)$ & $318(1)$ & $33(7)$ & $427(3)$ & $70(2)$ & $-415(3)$ & $66(2)$ & $2.51(2)$ & $0.4(1)$ \\
\hline \multirow{8}{*}{$\varnothing=40 \mathrm{~mm}$} & \multirow[t]{10}{*}{$511 \mathrm{keV}$} & Ground White & $51.2(2)$ & 61.19(1) & $0.7(1)$ & $335.0(6)$ & $30(5)$ & $480.3(9)$ & $47(9)$ & $-366.4(6)$ & $33(7)$ & $3.69(1)$ & $0.6(1)$ \\
\hline & & Ground Black & & 13.4 & & & & & & & $48(8)$ & & $0.5(1)$ \\
\hline & & Polished White & & $56.93(2)$ & $1.0(2)$ & $334.1(6)$ & $30(5)$ & $462(1)$ & $55(1)$ & $-364.0(7)$ & $34(7)$ & $3.71(1)$ & $0.6(1)$ \\
\hline & & Polished Black & & $12.38(3)$ & $1.6(3)$ & $333.7(6)$ & $30(5)$ & $351.4(7)$ & $34(5)$ & $-328(1)$ & $51(9)$ & $1.56(1)$ & $0.5(1)$ \\
\hline & & Aluminium & & $42.62(3)$ & $1.3(2)$ & $332.5(6)$ & $31(5)$ & $438(1)$ & $59(1)$ & $-336.6(9)$ & $45(8)$ & $2.94(1)$ & $0.52(9)$ \\
\hline & & Ground White & $37.3(2)$ & 61.11(2) & $0.6(1)$ & $327(1)$ & $31(6)$ & $480(2)$ & $49(1)$ & $-479(1)$ & $36(8)$ & $3.66(2)$ & $0.5(1)$ \\
\hline & & Ground Black & & $13.55(3)$ & $0.9(2)$ & $326.4(9)$ & $30(6)$ & $346(1)$ & $34(7)$ & $-464(1)$ & $45(9)$ & $1.38(1)$ & $0.29(9)$ \\
\hline & & Polished White & & $56.90(3)$ & $0.9(2)$ & $325(1)$ & $31(6)$ & $464(2)$ & $57(1)$ & $-477(1)$ & $37(9)$ & $3.69(2)$ & $0.5(1)$ \\
\hline \multirow[t]{2}{*}{$\mathrm{d}=40 \mathrm{~mm}$} & & Polished Black & & $12.48(5)$ & $1.6(3)$ & $326(1)$ & $32(6)$ & $343(1)$ & $36(7)$ & $-461(1)$ & $48(1)$ & $1.38(1)$ & $0.30(9)$ \\
\hline & & Aluminium & & $42.53(4)$ & $1.3(3)$ & $322(1)$ & $33(6)$ & $426(2)$ & $65(1)$ & $-456(2)$ & $47(1)$ & $2.87(1)$ & $0.38(8)$ \\
\hline \multirow{8}{*}{$\varnothing=40 \mathrm{~mm}$} & \multirow[t]{5}{*}{$511 \mathrm{keV}$} & Grol & $42.8(2)$ & 69.697( & 0.40 & $222.9(4)$ & 17 & 3 & 44 & -51 & 20 & & $0.50(9)$ \\
\hline & & Ground Blac & & $15.335(8)$ & $0.35(6)$ & $222.0(4)$ & 17( & $233.9(5)$ & $21(3)$ & $-478.7(7)$ & $30(5)$ & $1.40(1)$ & $0.5(1)$ \\
\hline & & Polished White & & $66.46(2)$ & $1.0(2)$ & $221.9(4)$ & $17(3)$ & $345(1)$ & $44(7)$ & $-510.9(4)$ & 19(4) & $3.12(1)$ & $0.5(1)$ \\
\hline & & Polished Black & & $14.27(2)$ & $0.7(1)$ & $222.6(4)$ & $17(3)$ & $233.5(5)$ & $20(3)$ & $-474.7(7)$ & $32(6)$ & $1.40(1)$ & $0.5(1)$ \\
\hline & & Aluminium & & $58.58(4)$ & $1.8(4)$ & $221.4(4)$ & $17(3)$ & $300.6(9)$ & $40(7)$ & $-507.1(6)$ & $25(4)$ & $2.372(9)$ & $0.41(8)$ \\
\hline & \multirow[t]{5}{*}{$1274.53 \mathrm{keV}$} & Ground White & $29.5(2)$ & $69.73(1)$ & $0.35(7)$ & $218.9(7)$ & $17(4)$ & $351(2)$ & $45(1)$ & $-615.1(7)$ & $17(4)$ & $2.98(1)$ & $0.35(8)$ \\
\hline & & Ground Black & & $15.35(1)$ & $0.32(7)$ & $219.3(7)$ & $17(4)$ & $231.2(8)$ & $20(4)$ & $-598(1)$ & $29(6)$ & $1.23(1)$ & $0.3(1)$ \\
\hline & & Polished Wh & & $66.54(4)$ & $0.9(3)$ & $219.9(7)$ & $17(4)$ & $344(2)$ & $43(9)$ & $-612.3(7)$ & $18(4)$ & $3.05(1)$ & $0.38(9)$ \\
\hline \multirow{2}{*}{$\mathrm{d}=20 \mathrm{~mm}$} & & Polished Black & & $14.35(3)$ & $0.7(1)$ & $219.1(7)$ & $16(3)$ & $229.9(8)$ & $19(4)$ & $-593(1)$ & $29(6)$ & $1.22(1)$ & $0.25(9)$ \\
\hline & & Aluminium & & $58.31(8)$ & $1.8(5)$ & $216.4(7)$ & $16(3)$ & $300(2)$ & $37(9)$ & $-612(1)$ & $23(5)$ & $2.30(1)$ & $0.27(7)$ \\
\hline
\end{tabular}


Table 7.4: Figures of merit for $\mathrm{LaBr}_{3}$ :Ce scintillators (continued)

\begin{tabular}{|c|c|c|c|c|c|c|c|c|c|c|c|c|c|}
\hline \multicolumn{3}{|c|}{ Scintillation Crystal Properties } & \multirow{2}{*}{$\frac{\text { Efficiency }}{\eta_{\text {int }}, \%}$} & \multicolumn{2}{|c|}{ Collection Efficiency } & \multicolumn{2}{|c|}{ First Photon’s Arrival } & \multicolumn{2}{|c|}{ First Quartile } & \multicolumn{2}{|c|}{ Density Trigger Time } & \multicolumn{2}{|c|}{ Density Maximum } \\
\hline Geometry & Energy & Surface & & $\bar{\eta}_{\mathrm{col}}, \%$ & $s\left(\eta_{\mathrm{col}}\right), \mathrm{pp}$ & $\bar{t}_{\mathrm{fp}}, \mathrm{ps}$ & $s\left(t_{\mathrm{fp}}\right), \mathrm{ps}$ & $\bar{t}_{\mathrm{fq}}, \mathrm{ps}$ & $s\left(t_{\mathrm{fq}}\right), \mathrm{ps}$ & $\bar{t}_{\mathrm{tr}}, \mathrm{ps}$ & $s\left(t_{\mathrm{tr}}\right), \mathrm{ps}$ & $\bar{t}_{\mathrm{m}}, \mathrm{ns}$ & $s\left(t_{\mathrm{m}}\right), \mathrm{ns}$ \\
\hline \multirow{9}{*}{$\varnothing=40 \mathrm{~mm}$} & \multirow{10}{*}{$1274.53 \mathrm{keV}$} & Ground White & $31.4(2)$ & $76.61(1)$ & $0.31(6)$ & $170.2(3)$ & $10(2)$ & $249.2(8)$ & $27(5)$ & $-593.0(5)$ & $17(3)$ & $2.52(1)$ & $0.42(9)$ \\
\hline & & Ground Black & & $16.27(1)$ & $0.37(8)$ & $170.4(3)$ & $10(2)$ & $177.9(3)$ & $12(2)$ & $-545.3(7)$ & $24(5)$ & $1.34(1)$ & $0.5(1)$ \\
\hline & & Polished White & & $73.64(4)$ & $1.3(3)$ & $170.7(3)$ & $10(2)$ & $245.6(8)$ & $26(5)$ & $-592.2(5)$ & $17(3)$ & $2.61(1)$ & $0.5(1)$ \\
\hline & & Polished Black & & $15.21(2)$ & $0.5(1)$ & 170.1(3) & $10(2)$ & $177.1(3)$ & $12(2)$ & $-541.6(8)$ & $26(5)$ & $1.29(1)$ & $0.5(1)$ \\
\hline & & Aluminium & & $72.19(5)$ & $1.7(4)$ & $169.3(3)$ & $9(2)$ & $244.8(7)$ & $25(4)$ & $-596.6(6)$ & $20(4)$ & $1.859(9)$ & $0.31(8)$ \\
\hline & & Ground White & $19.9(2)$ & $76.67(1)$ & $0.26(7)$ & $168.2(6)$ & $10(2)$ & $250(1)$ & $26(6)$ & $-686.3(8)$ & $13(4)$ & $2.45(2)$ & $0.3(1)$ \\
\hline & & Ground Black & & $16.28(2)$ & $0.31(9)$ & $169.7(6)$ & $10(3)$ & $177.8(7)$ & $12(3)$ & $-661(1)$ & $20(6)$ & $1.14(2)$ & $0.2(1)$ \\
\hline & & Polished White & & $73.81(7)$ & $1.3(5)$ & $167.9(6)$ & $9(2)$ & $249(2)$ & $26(7)$ & $-686.6(7)$ & $11(3)$ & $2.55(2)$ & $0.3(1)$ \\
\hline & & Polished Black & & $15.32(3)$ & $0.5(1)$ & $169.4(6)$ & $10(3)$ & $176.6(7)$ & $11(3)$ & $-660(1)$ & $22(6)$ & $1.14(1)$ & $0.3(1)$ \\
\hline $\mathrm{d}=10 \mathrm{~mm}$ & & Aluminium & & $72.0(1)$ & $1.8(5)$ & $166.8(5)$ & $9(2)$ & $247(1)$ & $25(6)$ & $-687.9(9)$ & $15(4)$ & $1.81(1)$ & $0.19(7)$ \\
\hline \multirow{8}{*}{$\varnothing=40 / 20 \mathrm{~mm}$} & $511 \mathrm{keV}$ & Ground White & $29.3(2)$ & $89.62(2)$ & $0.6(1)$ & $164.6(2)$ & $7(1)$ & $219.4(7)$ & $22(4)$ & $-636.2(5)$ & $16(3)$ & $1.495(7)$ & $0.22(6)$ \\
\hline & & Ground Black & & $28.8(2)$ & $5(1)$ & $164.4(2)$ & $7(1)$ & $177.4(4)$ & $13(2)$ & $-589.7(6)$ & $19(4)$ & $1.26(1)$ & $0.3(1)$ \\
\hline & & Polished White & & $89.53(5)$ & $1.4(4)$ & $164.4(2)$ & $7(1)$ & $220.7(7)$ & $22(4)$ & $-636.0(5)$ & $16(3)$ & $1.484(7)$ & $0.22(7)$ \\
\hline & & Polished Black & & $28.5(2)$ & $7(1)$ & $164.5(2)$ & $7(1)$ & $177.5(4)$ & $13(2)$ & $-589.3(7)$ & $21(4)$ & $1.25(1)$ & $0.3(1)$ \\
\hline & & Aluminium & & $82.64(8)$ & $2.3(5)$ & $164.6(2)$ & $7(1)$ & $219.1(7)$ & $20(4)$ & $-630.1(6)$ & $17(3)$ & $1.461(7)$ & $0.20(6)$ \\
\hline & $1274.53 \mathrm{keV}$ & Ground White & $19.0(2)$ & $89.66(3)$ & $0.5(2)$ & $163.0(5)$ & $8(2)$ & $223(1)$ & $19(6)$ & $-716.7(7)$ & $10(3)$ & $1.444(7)$ & $0.11(4)$ \\
\hline & & Ground Black & & $28.5(3)$ & $5(1)$ & $163.2(5)$ & $8(2)$ & $175.8(8)$ & $12(3)$ & $-689.5(9)$ & $13(4)$ & $1.18(1)$ & $0.2(1)$ \\
\hline & & Polished White & & $89.59(9)$ & $1.4(6)$ & $163.4(5)$ & $7(2)$ & $221(1)$ & $21(6)$ & $-718.6(7)$ & $11(3)$ & $1.410(8)$ & $0.11(5)$ \\
\hline \multirow{2}{*}{$\mathrm{d}=10 \mathrm{~mm}$} & & Polished Black & & $28.5(4)$ & $6(2)$ & $162.7(4)$ & $7(2)$ & $175.0(8)$ & $12(3)$ & $-689.5(9)$ & $13(4)$ & $1.15(1)$ & $0.2(1)$ \\
\hline & & Aluminium & & $82.6(2)$ & $2.3(7)$ & $162.7(5)$ & $7(2)$ & $221(1)$ & $19(6)$ & $-714.7(8)$ & $11(4)$ & $1.409(6)$ & $0.10(5)$ \\
\hline \multirow{8}{*}{$\varnothing=40 \mathrm{~mm}$} & $511 \mathrm{keV}$ & Ground White & $52.0(2)$ & $82.60(6)$ & $2.7(5)$ & $211.9(3)$ & $13(2)$ & $311(1)$ & $44(8)$ & $-552.1(5)$ & $22(4)$ & $2.203(9)$ & $0.40(7)$ \\
\hline & & Ground Black & & $26.3(2)$ & $9(2)$ & $211.6(3)$ & $13(2)$ & $228.2(5)$ & $23(4)$ & $-523.0(5)$ & $23(4)$ & $1.384(9)$ & $0.39(9)$ \\
\hline & & Polished White & & $80.48(9)$ & $4.0(7)$ & 211.9(3) & $13(2)$ & $306.2(8)$ & $38(7)$ & $-552.7(5)$ & $23(4)$ & $2.193(9)$ & $0.43(8)$ \\
\hline & & Polished Black & & $26.2(3)$ & $13(2)$ & $212.5(3)$ & $14(2)$ & $228.9(5)$ & $24(4)$ & $-518.9(6)$ & $28(5)$ & $1.394(9)$ & $0.4(1)$ \\
\hline & & Aluminium & & $67.2(1)$ & $6(1)$ & $212.2(3)$ & $14(2)$ & $275.3(5)$ & $23(5)$ & $-544.2(4)$ & $18(3)$ & $1.999(8)$ & $0.34(7)$ \\
\hline & $1274.53 \mathrm{keV}$ & Ground White & $35.8(2)$ & $82.35(9)$ & $2.5(6)$ & $209.6(5)$ & $14(3)$ & $314(2)$ & $43(1)$ & $-645.0(6)$ & $14(3)$ & $2.16(1)$ & $0.29(7)$ \\
\hline & & Ground Black & & $25.2(3)$ & $9(2)$ & $210.2(5)$ & $13(3)$ & $226.2(8)$ & $21(5)$ & $-628.3(6)$ & $16(4)$ & $1.254(8)$ & $0.20(8)$ \\
\hline & & Polished White & & $80.2(1)$ & $3.3(9)$ & $208.7(5)$ & $13(3)$ & $310(2)$ & $38(9)$ & $-644.5(5)$ & $13(3)$ & $2.14(1)$ & $0.29(8)$ \\
\hline \multirow[t]{2}{*}{$\mathrm{d}=20 \mathrm{~mm}$} & & Polished Black & & $23.0(4)$ & $10(3)$ & $209.5(5)$ & $13(3)$ & $224.0(8)$ & $21(5)$ & $-625.6(7)$ & $19(4)$ & $1.26(1)$ & $0.26(9)$ \\
\hline & & Aluminium & & $66.3(2)$ & $5(1)$ & $208.8(5)$ & $13(3)$ & $277.2(9)$ & $22(6)$ & $-639.1(5)$ & $14(3)$ & $1.939(9)$ & $0.23(6)$ \\
\hline
\end{tabular}


Each distribution's average value computed according to section 7.1.2 is depicted by an arrow in the same color and style as the particular graph.

The y-axis is in all cases scaled according to the total percentage of $\gamma$ events (base: 10 000) yielding an $\mathrm{x}$ value in the corresponding bin. The bin width is $10 \mathrm{ps}$ for the statistical evaluations and 20 ps for the kernel density estimation based results.

The x-axis of the collection efficiency diagrams shows the percentage of created photons reaching the photo cathode. All other x-axes represent the time after the primary $\gamma$ photon's emission in the source, which is a point source in $3 \mathrm{~cm}$ distance from the scintillator's aluminium cover.

In most cases, the contents of all bins of one graph do not add up to $100 \%$. This is due to $\gamma$ photons passing the scintillator without creating secondary photons. Thus, the integral value of each curve equals to the detection efficiency for the given $\gamma$ energy minus the (small) fraction of events that generated very few secondary photons which were lost on their way to the photo-cathode altogether. The determined detection efficiency of all simulated detector types, i. e. the fraction of $\gamma$ events where at least one visible photon was created, is shown in the column titled Efficiency in table 7.4.

\subsubsection{Result Tables}

While the diagrams in section 7.1.3 provide a concise overview on trends in the results, a more in-depth analysis requires information concerning the exact position of average values, data precision and - in this case most important-the results' variances. The analyzed parameters' variances are important because of their direct contribution to the achievable time and energy resolution while $\gamma$ energy dependent shifts in the average values of timing properties are influencing the performance of coincidence measurements including $\gamma$ events with different energies.

The result table (table 7.4) contains one row for every combination of scintillator geometry, $\gamma$ energy, and surface finish. The first data column contains the detection efficiency $\eta_{\text {int }}$-i. e. the probability for each $\gamma$ photon to create at least one secondary photon while passing the scintillation crystal. Since this detection efficiency does not depend on the crystal's surface properties, values of all surface variants were averaged. After the detection efficiency, five groups of columns follow, each of which contains average $(\bar{x})$ and unbiased sample standard deviation $(s(x))$ of one of the parameters described in section 7.1.2. The standard deviation instead of the variance is specified in the table in order to provide units comparable to the average values. All values are rounded according to and followed by their standard error (in units of the least significant figure) determined according to section 7.1.2. As also described in this section they were determined by only considering scintillation events belonging to the photo peak. Of course, the detection efficiency includes Compton events. 


\subsubsection{Discussion}

Unsurprisingly, the collection efficiency of white painted scintillators is much better than that of black painted and also of unpainted with aluminium enclosure. Interestingly, the collection efficiency of all black painted cylindrical scintillators is smaller than $20 \%$ regardless of their proportions. It seems like the solid angle of the PMT window's part that is open to photons seen from the scintillator's interior is surprisingly small andif all photons that are not emitted into this solid angle are lost due to black paint- the reduction of efficiency is dramatic. The longer crystal variants show a larger variance of efficiency because the occurring solid angles vary stronger depending on the depth of the interaction point (i.e. the point where the incoming $\gamma$ is converted to visible light). The conical and spherical black painted crystals' efficiency shows a much larger variance and higher average values. This can presumably be attributed to a higher probability for photons to enter the PMT after a single reflection. Single reflections are still possible in case of black painted crystals due to the remaining reflectivity of $4.3 \%$ (cf. section 7.1.1) and the probability for reflections at the inner surface of the scintillation material (before the light actually leaves the crystal and enters the paint). While in cylindrical crystals most photons that are not initially directed towards the PMT window need at least two reflections before hitting it - which is very unlikely in case of black painted surfaces-the conical and spherical shapes guide a larger amount of light towards the PMT with only one reflection. The large variance values for these shapes, however, have a negative impact on energy resolution since the number of photons not only varies due to statistics of the creation process but additionally due to transport. Comparably large variances not only occur for black painted variants of the conical and spherical crystals, but also for the white painted as well as unpainted. Therefore, these shapes must be considered inappropriate for applications with high demands concerning energy resolution.

In all cases, ground and white painted surfaces exhibit the best collection efficiencies closely followed by polished and white painted variants. For the cylindrical shapes, efficiency grows with smaller thickness and decreases for smaller diameters-both due to a higher average number of reflections necessary for light to enter the PMT. In all cases, the unpainted crystals show a medium efficiency and high variance which happens due to the mixture of reflections at the crystal to air interface and reflections at the aluminium surface.

First photon arrival time statistics, as shown in the second column, provides an idea of the time resolution's crystal shape dependence since the first few photons showed to contribute most information concerning the $\gamma$ incidence time (cf. section 5.10). While the average of the arrival times is of little interest because it only contributes a constant delay, which usually cancels out in case of coincidence measurements, the variance is all the more relevant. Considering the results, an obvious correlation between crystal thickness and variance exists. The thinner crystals are, the smaller the variance. Other shape parameters, including the diameter, are negligible. Significant differences between the 
different surface properties are not visible because since reflected light always has to pass longer trajectories, it does not contribute to the first photon's arrival time statistics. Additional effects, occurring due to the time characteristics of light creation in the scintillation material, were intentionally suppressed in this statistic by setting the time constants to zero in order to isolate the contribution of light propagation in the crystals. In case of the thick crystals, skewness with noticeably smaller slope towards small time values occurs. In fact, this happens due to the distribution of the interaction points across the crystals longitudinal axis with exponentially decreasing interaction probabilities. Although the total time in case of a straight photon path does not depend on the interaction point's position because the speed of the incident $\gamma$ photon is similar to the speed of visible light (considering the refractive index), the average time after which an actually created secondary photon reaches the PMT rises with the distance between PMT and interaction point because secondary photons are emitted isotropically and in case of distant interaction points the average time lost by secondary photons with trajectories not perpendicular to the PMT window is larger. In all cases, the amplitudes for $511 \mathrm{keV} \gamma$ photons are larger than for $\gamma$ energies of $1274.53 \mathrm{keV}$ because of the higher fraction of $\gamma$ photons passing the scintillator without interaction in the latter case.

First quartile times of black painted crystals are almost identical to the related first photon arrival times. This is reasonable, because most of the small number of secondary photons reaching the PMT in case of black painted crystals propagate rather parallel and since only the first quarter is relevant for the quartile's time, a further selection of the shortest trajectories occurs. White painted and unpainted scintillators exhibit a shift towards later times and rather skewed distributions. Obviously, the complex interdependence between interaction points and trajectories leads to a considerable blurring of the quartile times. This effect can be considered an explanation of the surprising discovery in section 5.10 that the late part of a detector signal cannot be used to improve time resolution.

The density based evaluations are, in contrast to the previously discussed evaluations, based on simulations with realistic scintillation process timing characteristics. For the trigger simulations, a big difference between black and white painted crystals is noticeable (see table 7.4). Obviously, the reduced number of photons overcompensates the advantage of smaller divergence of trajectories in case of black painted scintillators. Considering this result, it is better to detect reflected but early emitted photons than to restrict detection to a small number of photons with defined trajectories. The visible dependence of the position of average times on the incident $\gamma$ energy is due to different signal amplitudes, which cause a walk effect as discussed in section 5.3. Negative values on the abscissa occur due to the symmetric kernel used for the density estimation and are not acausal, because in real detectors, additional shift due to the PMT's transit time occurs, which was not artificially added here.

The density maxima, as shown in the last column of graphs, exhibit a very broad distribution. Obviously, they are not usable for precise time determination. Although the variance seems to be smaller in case of black painted scintillators regarding the distri- 
bution plots, this is not always the case due to pronounced tails of the distributions. A considerable shift of the average values from the distributions' maxima towards later times is, however, consistently visible which illustrates the blurring effect of light propagation considering scintillator timing properties and emphasizes the importance of a comprehensive simulation to understand timing relevant mechanisms in scintillation detectors.

As expected, the interaction efficiencies are correlated with the crystal thickness and are higher for $511 \mathrm{keV}$ than for $1274.53 \mathrm{keV} \gamma$ photons due to higher absorption of radiation with lower energies. To understand the interaction efficiency values in table 7.4 , it is important to remember that an isotropic point source with $3 \mathrm{~cm}$ distance to the detector's tip was simulated. The efficiency is the fraction of all $\gamma$ photons entering the detector's tip thereby triggering at least one secondary photon inside the scintillator. It is therefore related to the angle dependent effective thickness of the crystals.

\subsection{Light-to-signal-conversion in photomultiplier tubes}

As described in this chapter's introduction, the simulation of light propagation in scintillation crystals introduced in the previous section was initially implemented as a refinement of a detector simulation approach focusing on signal formation in photomultiplier tubes. The first proof-of-concept implementation was based on the EPOS software suite (Krille, 2011).

\subsubsection{PMT simulation using Multiply!}

For improved simulations, a specialized Monte-Carlo simulation of physical processes in PMTs called Multiply! was developed. It reads photon incidence times from Scintillate! result files and simulates multiplication processes in the PMT triggered by these photons. Results of the simulated multiplication subsequently pass a simulated analog-to-digital conversion as realized by digitizers in real setups. The resulting data stream is finally written to a file, which is equivalent to signal dump files provided by PacMaster as described in section 4.2.3. Therefore, immediate comparisons of simulation results and measured data from real detectors become possible.

\subsubsection{Monte-Carlo simulation}

Multiply! covers the second part of the conversion of $\gamma$ radiation into electrical pulses in real scintillation detectors as described in section 2.3.1. The first part from $\gamma$ emission in a source up to the incidence of visible photons on the PMT's photo cathode is modeled by Scintillate! as described in section 7.1.1.

The second part, covered by Multiply!, consists of the generation of photo electrons at the photo cathode, the multiplication process including time characteristics and statistical 


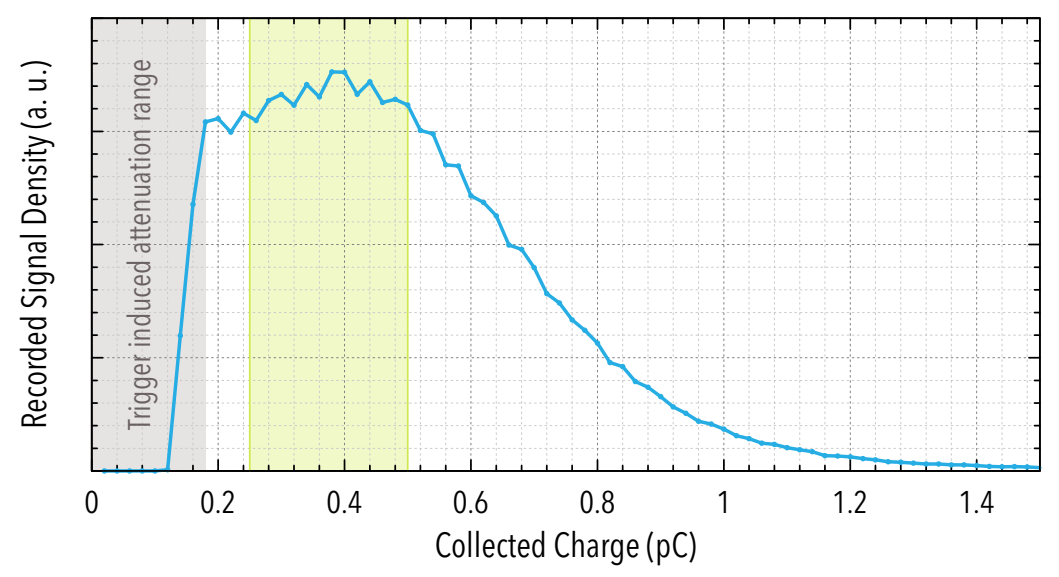

Figure 7.10: Anode output charge spectrum (integral values) of Cerenkov light, which emerged in the PMT's window while irradiated with radiation from a ${ }^{22} \mathrm{Na}$ sample. The interval highlighted green is the range where signals for the subsequent averaging (see fig. 7.11) were taken from. This spectrum's channel width is $20 \mathrm{fC}$. The anode signals forming the spectrum were recorded with a sampling rate of $4 \mathrm{GS} \mathrm{s}^{-1}$, an input bandwidth of $1 \mathrm{GHz}$, a resolution of 8 bit and a full-scale setting of $200 \mathrm{mV}$. The clipping visible at the left edge of the spectrum originates from the trigger threshold of the digitizer, which had to be set to a value above the background noise and thereby prevented small signals from being recorded.

fluctuations, the accumulation of charge on the anode, the transmission of signals to a digitizer and the analog-to-digital conversion.

Time characteristics The goal of this simulation approach was to obtain the best possible ab-initio simulation relying solely on parameters published by the PMT manufacturers. Due to this constraint, it was not possible to simulate electric field profiles and electron trajectories in the PMT's dynode system like done by tools like CST Particle Studio (Computer Simulation Technology AG, 2013) since the 3D data necessary for such an in-depth modeling is not available. The different electron trajectories, however, have an influence on the timing characteristics of output signals. Therefore, an appropriate replacement for the trajectory simulation had to be found.

An analysis of anode signal shapes fortunately revealed sufficient similarities between different PMT models after a normalization based on the rise-time value provided in most PMT data sheets. This can be seen by comparing e. g. fig. 7.11 and the signal shape shown in fig. 20 of Photonis (2007a). This observation was utilized by modeling the time characteristics of one example PMT (a Hamamatsu R3377 in a H3378-50 assembly) and using the result as a template for the simulation of other PMT models.

To obtain an average output signal as created by single photons hitting the photo cath- 


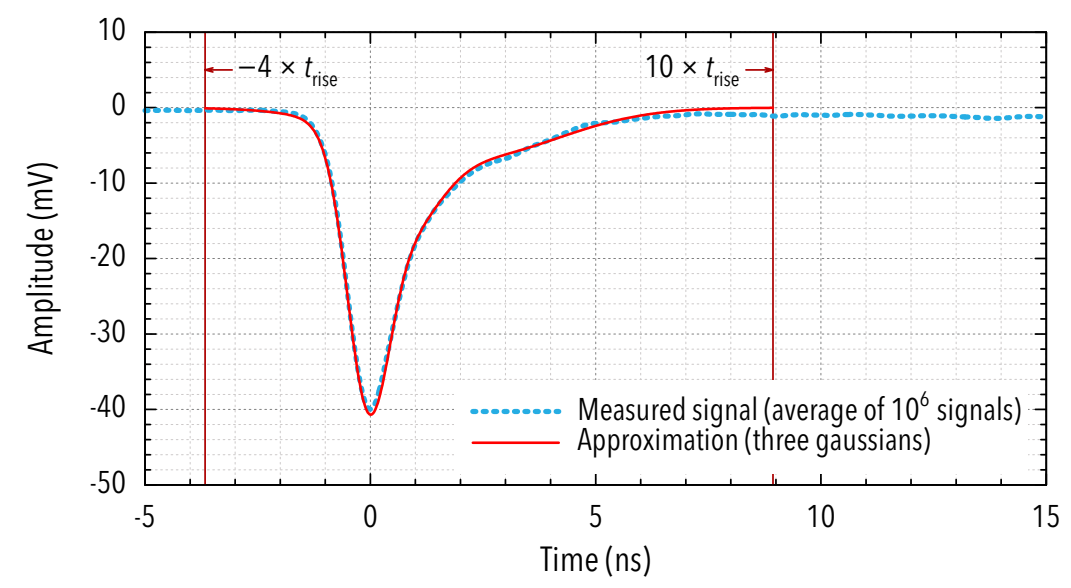

Figure 7.11: The blue points are the result of averaging $10^{6}$ Cerenkov signals from the charge window highlighted in fig. 7.10. Before averaging, the recorded signals were interpolated using cubic splines. The interpolation results were used to find each signal's minimum and align all signals according to their minima using a resolution ten times finer than the sampling interval of $250 \mathrm{ps}$. The averaged signal was modeled using a superposition of three Gaussians (red curve). This model was then used to simulate the anode output produced by single cathode electrons starting an amplification cascade. For this purpose, the signals were clipped according to the vertical red markers.

ode, Cerenkov light created in the PMT window due to radiation from a ${ }^{22} \mathrm{Na}$ sample was recorded. It is safe to assume that either single photons are produced by this process or, if multiple photons originate from the same particle passing the window, these photons at least arrive almost simultaneously. To avoid noise and other spurious signals from affecting the average, only signals from a defined charge window as shown in fig. 7.10 were selected.

Figure 7.11 shows the resulting signal, which consists of $10^{6}$ single signals that were aligned according to their interpolated minimum. This signal can be rescaled in units of the PMT's $10 \% / 90 \%$ rise-time and approximated by a superposition of three Gaussians (red curve). In order to decrease computing time in simulation runs, the Gaussians were clipped according to the red markers four times the rise-time before the signal's minimum as well as ten times the rise-time after the minimum. The parameters of the Gaussians approximating the signal are summarized in table 7.5 .

The uniform shape of single photo-electron signals emerges due to the high number of secondary electrons created in the dynode system combined with a dynode design providing a limited range of possible transit times between two dynodes as found in modern PMTs. Statistical fluctuations between different events mainly concern the trajectory between photo-cathode and first dynode since there is only a single electron available. 
Table 7.5: Parameters of three Gaussians determined by fitting averaged single photo-electron output signals as shown in fig. 7.11. The abscissa was scaled according to the $10 \% / 90 \%$ rise-time before fitting.

\begin{tabular}{lccc}
\hline & scaling & $\mu$ in units of $t_{r}$ & $\sigma$ in units of $t_{r}$ \\
\hline Gaussian 1 & 0.387528 & 0 & 0.511585 \\
Gaussian 2 & 0.243813 & 0.916979 & 0.866997 \\
Gaussian 3 & 0.368659 & 2.57607 & 2.20351 \\
\hline
\end{tabular}

However, this effect only causes fluctuations in the total amount of time needed for the signal to pass through the PMT but not concerning the signal shape. These fluctuations are quantified in PMT data sheets by the transit time spread value (TTS) and are thus a known parameter for the simulations.

Consequently, the time characteristics of PMT signals are simulated as combination of a fixed signal shape scaled by the PMT's rise-time value and a random time shift, which simulates the transit time properties. The shift values are drawn from a Gaussian distribution with the TTS value as $\sigma$. The transit time is not simulated because it is constant for all signals and therefore not relevant for coincidence measurements.

The sampling of the simulated signals is adjusted according to the user-supplied settings for sampling rate and samples per signal.

Amplification The second aspect of the signal generation that had to be simulated is the amplification. Regarding fig. 7.10 it is obvious that for single photo-electron signals the range of possible amounts of charge collected on the anode is broad. This effect results from statistical fluctuations of the small number of electrons available at the beginning of the multiplication process. Since an electron hitting a dynode surface triggers a large number of collisions of which only few lead to the emission of secondary electrons, the number of secondary electrons created on each dynode by a single incident electron can be approximated using a Poisson distribution. The Poisson distribution's parameter $\lambda$ corresponds to the average number of secondary electrons produced by a single primary electron hitting a dynode.

Due to the infinite divisibility of the Poisson distribution, it is possible to simplify the computation of the number of secondary electrons emitted from a dynode that is hit by $m$ primary electrons. For Poisson distributed random numbers,

$$
\sum_{i} \operatorname{Pois}\left(\lambda_{i}\right) \sim \operatorname{Pois}\left(\sum_{i} \lambda_{i}\right)
$$

holds. This means that the sum of $m$ Poisson distributed random numbers with a parameter $\lambda_{k, i}$ is equivalent to a single Poisson distributed random number with a parameter $\lambda_{k}$, 
which is the sum of all $\lambda_{k, i}$. Consequently, it is sufficient to compute only one random number per dynode stage with $\lambda_{k}=m \cdot \lambda_{k, 0}$ where $m$ is the number of incident electrons on the dynode (i.e. either the result from the previous dynode or the number of electrons emitted from the photo-cathode in case of the first dynode) and $\lambda_{k, 0}$ is the average number of secondary electrons produced by the $k$ th dynode per incident electron. It is assumed that $\lambda_{k, i}=\lambda_{k, 0} \forall i$ because in general all incident electrons cross the same potential difference between two dynodes and thus carry the same energy which results in comparable secondary electron production probabilities.

To put it briefly, a $\lambda_{k}$ value for each dynode step is needed to simulate the amplification process in a PMT. To derive this set of values from data sheet values, it is possible to split the overall gain value across the dynodes. Usually, this value $G$ and the number of amplification steps (i.e. dynodes) $n$ is known. To obtain even better results, it is possible to choose an uneven splitting to model the unequal distribution of potential differences implemented by many voltage divider designs. Because of the almost linear relation between electron energy and secondary electron yield in the range of typical potential differences occurring in PMTs (Sommer, 1972), it is possible to approximate the splitting of gain across dynodes by the distribution of potential differences. Usually, the ratios of voltage values can be derived from the PMT data-sheet (cf. Photonis, 2009). These relative gain values are denoted as $a_{1}, \ldots, a_{n}$ hereafter. If the voltage ratios are not known, it is usually a good approximation to set $a_{i}=1 \forall i$.

The average overall gain of the PMT (from the data-sheet) can be split into a product of average dynode gain values

$$
\bar{G}=\prod_{i=1}^{n} \bar{g}_{i}
$$

Due to the known gain ratios, it is possible to split dynode gains into a base gain value $\bar{g}$ and a per-dynode weight factor $a_{i}$ :

$$
\bar{G}=\bar{g}^{n} \cdot \prod_{i=1}^{n} a_{i}
$$

It is possible to calculate the base gain value if the $a_{i}$ are normalized to fulfill the condition $\left(a_{i}^{\prime}\right.$ being the normalized $\left.a_{i}\right)$

$$
\prod_{i=1}^{n} a^{\prime}{ }_{i}^{!}=1
$$

This condition is met using the following normalization:

$$
a_{i}^{\prime}=\frac{a_{i}}{\sqrt[n]{\prod_{i=1}^{n} a_{i}}}
$$


It results in a modified form of eq. (7.9):

$$
\bar{G}=\left(\bar{g}^{\prime}\right)^{n} \cdot \underbrace{\prod_{i=1}^{n} a^{\prime}}_{=1}
$$

Now the base gain value $\bar{g}^{\prime}$ can be calculated as

$$
\bar{g}^{\prime}=\sqrt[n]{\bar{G}}
$$

After deriving $\bar{g}^{\prime}$ and each dynode's $a^{\prime}{ }_{i}$ according to this method, it is now possible to implement an iterative simulation of the full amplification process:

$$
G_{i}=\text { Pois }\left(G_{i-1} \cdot a_{i}^{\prime} \cdot \bar{g}^{\prime}\right),
$$

where $G_{i}$ denotes the simulated total gain (i. e. the number of secondary electrons created for a single initial photo-electron) after the $i^{\text {th }}$ dynode. In this step, it is assumed that the number of secondary electrons follows a Poisson distribution. Due to the random function Pois $(\lambda)$, the result of this iterative calculation is different for each run, even for identical input parameters. The Poisson distribution's parameter $\lambda$ consists of the total gain of the previous step $G_{i-1}$, the dynode's normalized relative gain value $a_{i}^{\prime}$, and the modified dynode base gain value $\bar{g}^{\prime}$. The average total gain result of the iterative simulation equals the data sheet value $\bar{G}$ because the Poisson distribution's expected value $E(\operatorname{Pois}(\lambda))$ equals $\lambda$.

Time discretization Since signals are finally sampled by an analog to digital converter, it makes sense to define the sampling grid of this converter before calculations with real signals in the time domain are done. Using the final sampling grid as early as possible avoids alignment artifacts as well as unnecessary oversampling.

The grid of sampling points is determined by the sampling rate of the simulated converter as well as the number of samples recorded for a single signal. Pre-trigger-recording, which is an important feature of high-speed digitizers that allows the processing of complete signals including the part of their rising edge that occurred before the trigger level's crossing, can be simulated by defining a positive horizontal offset value.

In order to improve the quality of timing simulations, it is important to randomize the signal onset position relative to the sampling grid. Such a randomization can be enabled in Multiply! using the jitter option.

Accumulation The determination of time and amplification for each photo-electron created on the photo cathode as described above results in a number of scaled and shifted single-electron signals. This separation of photo-electrons into independent amplification 
cascades is legitimate as long as the charge replenishment at the dynodes works sufficiently well to keep the appropriate voltage differences (i.e. as long as the used voltage divider operates in its stable regime).

For providing of a simulated anode signal, however, it is necessary to merge all singleelectron cascades into a common signal. In real PMTs this takes place on the anode where all secondary electrons, produced by cascades on the last dynode, accumulate. Multiply! models this accumulation of charge by calculating the sum of all single-electron result signals.

Signal processing In order to recover information concerning the detected radiation, anode signals of scintillation detectors need to be processed using suitable hardware and, in case of digital setups, software. Even digital setups require a minimal processing chain consisting of a connection between PMT anode and downstream processing hardware (usually a cable and several connectors) and an analog to digital converter including necessary filters (at least a bandwidth limiting filter) and eventually amplifiers.

Thermal noise in the analog signal chain is simulated in Multiply! by adding additive white Gaussian noise (AWGN) to the signal amplitudes. The parameter $\sigma$ of the AWGN is a parameter of the simulation that can be selected according to the properties of the used (or planned) setup.

Multiply! also simulates the total attenuation of the signal chain, which can be set as negative value $G$ in $\mathrm{dB}$. The signal amplitudes $V_{0}(t)$ are then transformed to attenuated values $V_{a}(t)$ according to

$$
V_{a}(t)=V_{0}(t) \cdot 10^{\left(G_{20}\right)} \text {. }
$$

Attenuation is simulated after the addition of noise. If noise sources are expected after attenuation in real setups, the noise level set in the simulation can be appropriately adjusted.

Digitizers used for analog-to-digital conversion in real digital spectrometers convert the analog input signal into a stream of integer values. Due to the limited resolution $M$ (in bit) of the integers representing single sample points, quantization noise occurs. This step is simulated by mapping signal amplitudes $V_{a}(t)$ to a defined integer range representing a selectable amplitude range $\left[V_{\min }, V_{\max }\right]$. After this step, which includes rounding of the original continuous amplitudes, the integers are converted back to quantized amplitude values $V_{q}(t)$. A single quantization step $\Delta V$ is defined as

$$
\Delta V=\frac{V_{\max }-V_{\min }}{2^{M}-1} .
$$

The number of possible states is reduced by one to obtain a mapping of the minimum and maximum integers onto $V_{\min }$ and $V_{\max }$.

The complete quantization is implemented as

$$
V_{q}(t)=\left\lfloor\frac{V_{a}(t)-V_{\min }}{\Delta V}+0.5\right\rfloor \cdot \Delta V+V_{\min } .
$$


Data output The result signals are written to disk to allow for further processing. The files are in a simple character-separated value text format, which is identical to the format used by PacMaster for saving raw signal data as described in section 4.2.3.

A schematic illustration of the PMT simulation implemented in Multiply! is shown in fig. 7.12.

\subsection{Limitations}

Simulations of systems as complex as scintillation detectors rely on simplifications of the occurring processes. Simplifications that reduce the computational effort or simplify calculations have to be chosen carefully to avoid an unnecessary decrease of result quality while simplifications that are necessary due to missing knowledge of parameters usually cannot be negated. Simplifications of the second type are related to the following issues:

Electron trajectories in the dynode system determine the time properties of output signals as well as the efficiency of secondary electron production. Unfortunately, it is not easily possible to provide a simulation of trajectories since shape details of the dynode system are business secrets of the PMT vendors and not accessible without actually destroying a PMT. Moreover, a simulation of a full set of trajectories would require a lot of computing power because of the large numbers of secondary electrons created in the last steps of the amplification process (in the order of $10^{6}$ to $10^{8}$ ) and the high number of photo-electrons created for each primary $\gamma$ photon in case of high luminosity scintillation materials like $\mathrm{LaBr}_{3}$ : $\mathrm{Ce}$ (in the order of $10^{3}$ to $10^{4}$ ).

Secondary electron production in the dynode system is, in fact, more complicated than a single Poisson distribution based approach implies. Examples for more realistic approaches were described by Ganachaud and Cailler (1979) and Furman and Pivi (2002) where Ganachaud and Cailler simulated interactions between electrons and matter inside the material while Furman and Pivi confine themselves to a probabilistic model of secondary electron production. In both cases, knowledge concerning the dynode geometry and trajectory properties, like the incidence angles on dynodes, are required. As stated above, such information cannot be obtained from data sheets.

Afterpulses occur at random times after photon detection by PMTs due to residual gas ionization or light emitted by dynodes due to electron incidence (Photonis, 2007a). Due to the statistical distribution of the times of their occurrence, they are visible in fig. 7.11 as a slight deviation of the falling edge's asymptote from the zero level. This deviation is neglected by the implemented model described above. Therefore, after normalization the model signals' center of gravity is shifted towards earlier times. Since afterpulses result in a higher amount of average charge collected at the anode per detected photon, this neglect 

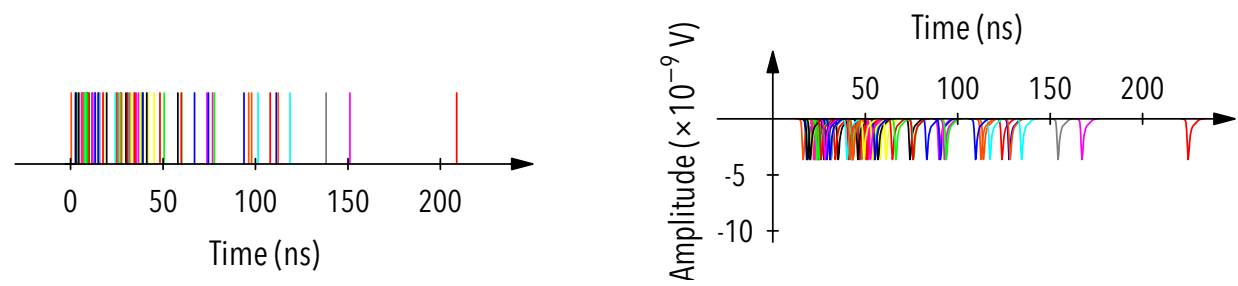

C) Simulate amplification

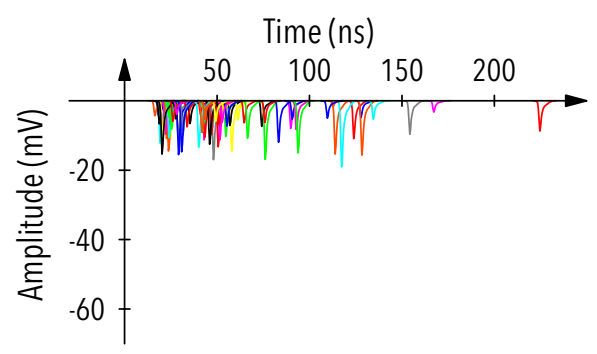

d) Accumulate signals

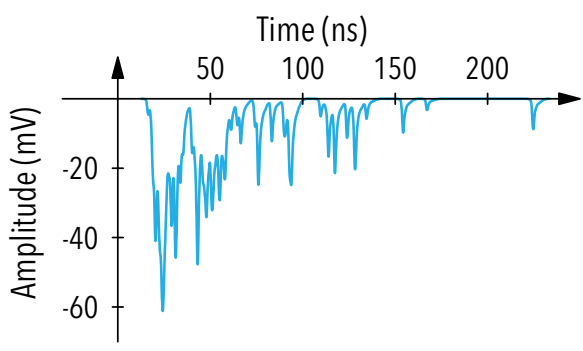

e Add additive white Gaussian noise

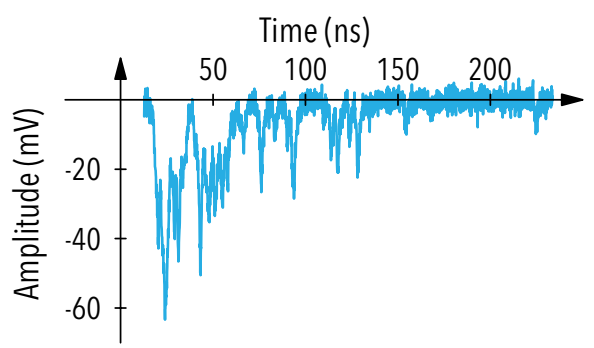

\section{f Quantize}

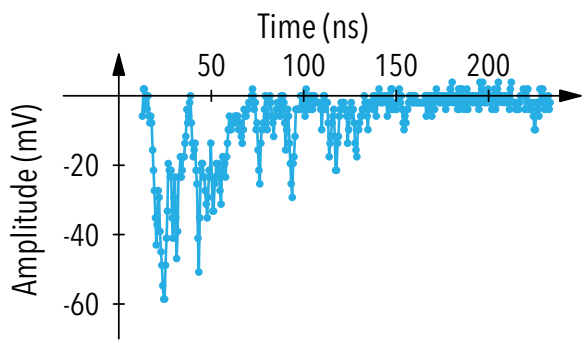

Figure 7.12: Simulated signals are generated in a step-by-step process. (a) Photon incidence times are read from the result file of a prior Scintillate! simulation run. (b) The set of delta peaks from $(a)$ is convolved with the typical anode signal shape of a signal created by a single cathode electron. Each signal's zero time is shifted by the sum of the PMT's transit time and a Gaussian distributed random number representing the transit time spread. (c) The amplitude of each single cathode electron's signal is modified by a gain factor determined by subsequently drawing Poisson distributed random numbers for each dynode stage. (d) The signals created in stages (a) to (c) are accumulated simulating the charge collection on the anode. (e) Additive Gaussian white noise is added as a simulation of electrical noise. (f) The digitizer's quantization (i.e. its limited resolution) is simulated. 


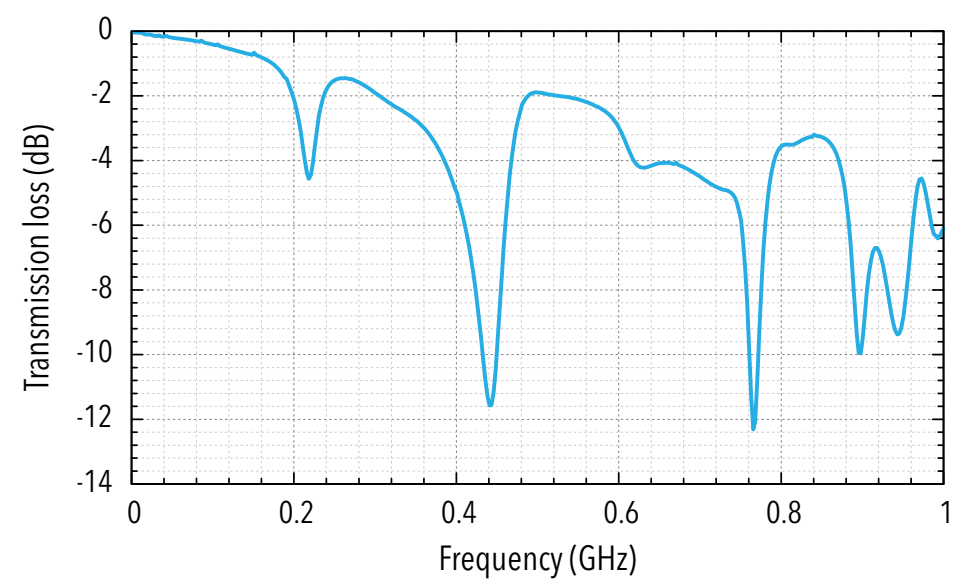

Figure 7.13: Attenuation of signals passing the voltage divider, i. e. the path between the anode port of the PMT socket and the BNC connector for the transmission cable on the used VD124K/T voltage dividers from Photonis. Obviously, the socket and divider's attenuation varies heavily across the used frequency range. This is due to missing impedance matching in the assembly and high peak frequencies occurring in the signals.

can also have influence on the energy spectra of simulated signals. Taking into account afterpulses is not possible obeying the approach of relying solely on data sheet values since, unfortunately, PMT suppliers do not publish statistical information concerning afterpulses.

Variable attenuation occurs in the signal path of scintillation detectors because due to steep rise-times, high frequency components up to about $1 \mathrm{GHz}$ exist. Usually, the anode signals are transmitted across the voltage divider circuit board using traces without impedance matching towards badly matched connectors (cf. fig. 7.13). Additionally, almost all parts of the signal chain (including the digitizers) exhibit bandwidth limits reaching into the frequency spectrum of the recorded signals. Therefore, real attenuation is not a constant but has a frequency dependency. This is intentionally not simulated by current versions of Multiply! because characteristics of this attenuation are hard to determine and not published.

The effective resolution of digitizers is smaller than their nominal resolution. For the Agilent Acqiris U1071A model, it is defined as 6.5 bit to 7.1 bit-depending on the signal's frequency spectrum (cf. Agilent, 2012). The behavior of the least significant bits is therefore not very well defined (they are not just random). There is no possibility to properly simulate this hardware limitation. 


\subsection{Simulation results}

As a test for the simulation stack described in the current chapter, several real setups were simulated and the results were evaluated using Evaluate! and compared to identical evaluations of measured data.

\subsection{1 $\mathrm{LaBr}_{3}: \mathrm{Ce}$ / Photonis XP2020 with Agilent Acqiris U1071A readout}

For the first comparison, the detectors of the spectrometer described in chapter 4 and one of this spectrometer's digitizers were simulated.

As radiation source, $\mathrm{a}^{22} \mathrm{Na}$ sample was used in the real spectrometer and simulated in the simulation. The properties of ${ }^{22} \mathrm{Na}$ sources are described in appendix A.2.1.

Measurement data was recorded using two detectors of the spectrometer described in chapter 4. For the energy evaluation, data of one of these detectors was evaluated. In order to cover the necessary energy range of the ${ }^{22} \mathrm{Na}$ decay, the PMT was operated at a high voltage setting of $1785 \mathrm{~V}$. This results in slight deviations of several parameters from data sheet values since these are typically determined at the maximum voltage of $2500 \mathrm{~V}$ but the decrease in voltage was necessary to avoid an unacceptable level of nonlinear behavior of the PMT and to avoid anode signals with amplitudes exceeding the digitizer's $5 \mathrm{~V}$ fullscale maximum.

For the simulation data, two runs of Scintillate! were accomplished: One for the detection of annihilation radiation at $511 \mathrm{keV}$ and a second one for the radiation produced by the de-excitation of ${ }^{22} \mathrm{Ne}$ nuclei with $1274.54 \mathrm{keV}$.

The parameters for Scintillate! were adjusted according to the properties of the scintillation crystal used for the measurement. Set values are listed in table 7.6.

For the PMT simulation with Multiply!, parameters were defined according to data sheets as shown in table 7.7. The transit time spread and the quantum efficiency are only specified for a supply voltage of $2500 \mathrm{~V}$ in the data sheets. Therefore, it is expected that the used values deviate slightly from real values in the measurement.

Charge spectrum The results of both, a charge spectrum of the real measurement and the simulation of this measurement, are shown in fig. 7.14.

Considering that the simulated curve was created by an ab initio simulation with the limited information available from the data sheets, a very good agreement of the measured and simulated energy spectra was obtained. The differences are similar to those usually occurring between identical detectors due to production-related tolerances of the PMTs. The higher radiation level left of the main peak (i. e. the $511 \mathrm{keV}$ peak) towards lower energies in the measurement occurs due to Bremsstrahlung induced by the produced positrons (cf. appendix A.2.1). The measured source consists of ${ }^{22} \mathrm{Na}$ in a Teflon matrix while in the simulation a point source of gamma photons was assumed. Therefore, Bremsstrahlung is missing from the simulation. While it is generally possible to 


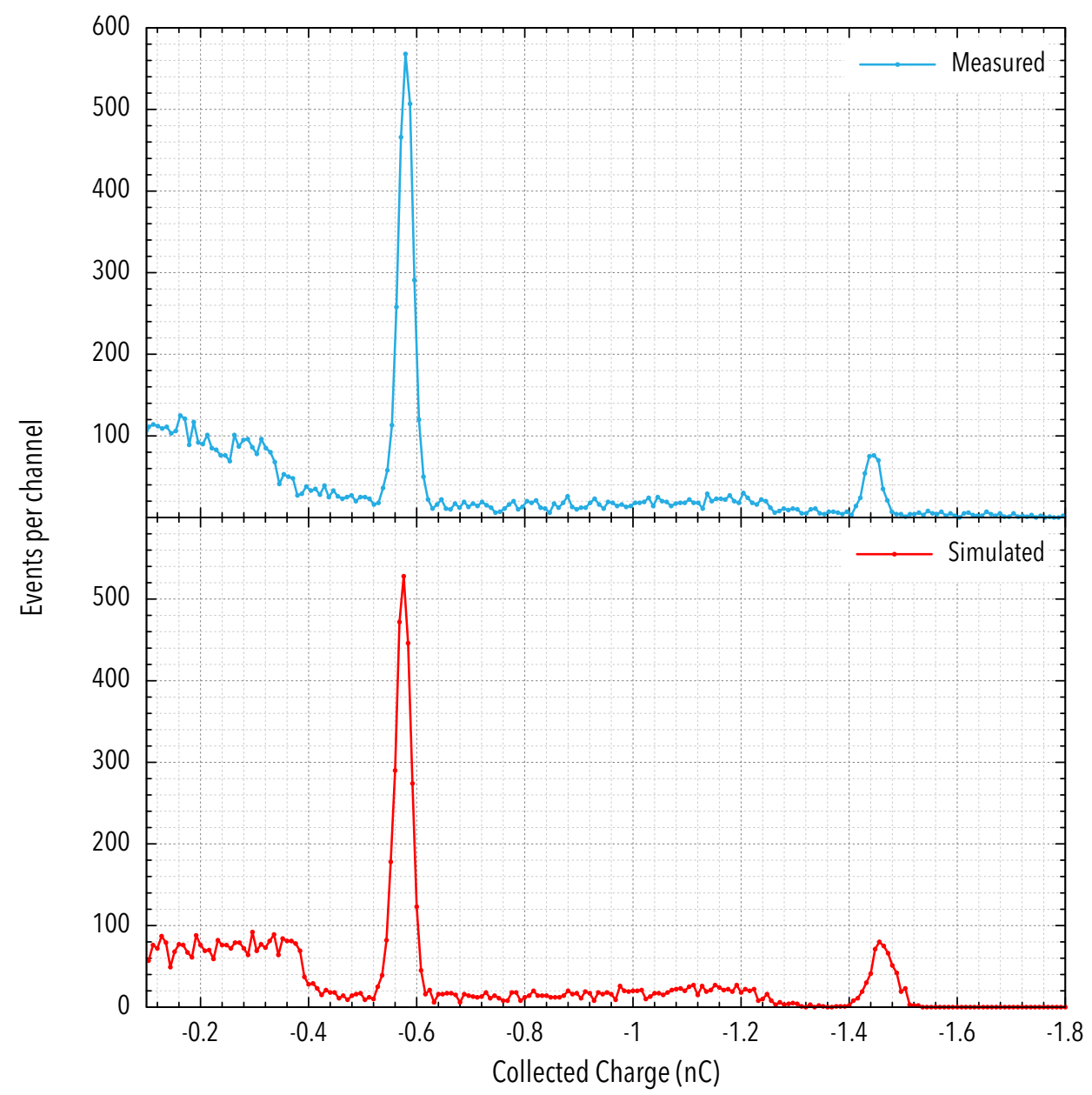

Figure 7.14: Measured (top) and simulated (bottom) charge spectra of the radiation of a ${ }^{22} \mathrm{Na}$ sample. Both spectra are very similar. Since the PMT anode collects negatively charged electrons, the abscissa was reversed to show small radiation energies on the left and bigger energies on the right. The higher amount of $\gamma$ events recorded in the measurement in the range left of the main peak $(511 \mathrm{keV})$ occurs due to Bremsstrahlung generated in the used sample by the emitted positrons before their annihilation. Measurement data was zero-corrected by a constant shift of $175 \mathrm{pC}$ corresponding to $2.2 \mathrm{LSB}$ per sample. The necessity of this correction is ascribed to a slightly asymmetric behavior of the used analog to digital converters. It is still well below the cumulative systematic errors of the digitizer occurring due to its limited effective number of bits (cf. section 7.3) and accuracy. For the used parameters, a value equivalent to 5.6 LSB — and therefore even worse than the observed deviation-is specified for the U1071A's DC accuracy. 
Table 7.6: Scintillate! settings for the simulation of a measurement conducted with the TDPAC spectrometer described in chapter 4.

\begin{tabular}{lll}
\hline Parameter & Value & Unit \\
\hline Crystal shape & Cylindrical & \\
Crystal material & $\mathrm{LaBr}_{3}: \mathrm{Ce}$ & \\
Crystal diameter & 25.4 & $\mathrm{~mm}$ \\
Crystal height & 38.1 & $\mathrm{~mm}$ \\
Crystal surface & $\mathrm{Ground}$, white painted \\
Thickness of the crystal's aluminium shell & 0.5 & $\mathrm{~mm}$ \\
Distance to the radiation source & 5 & $\mathrm{~mm}$ \\
PMT diameter & 50.8 & $\mathrm{~mm}$ \\
Grease layer thickness & $0.1^{a}$ & $\mathrm{~mm}$ \\
Thickness of the PMT's glass envelope & $2^{a}$ & $\mathrm{~mm}$ \\
Thickness of the PMT's window & $2^{a}$ & $\mathrm{~mm}$ \\
Gap between crystal and the aluminium enclosure & $0.5^{a}$ & $\mathrm{~mm}$ \\
\hline
\end{tabular}

${ }^{a}$ this value was estimated

even simulate the production of Bremsstrahlung using Geant4, this was intentionally not implemented in Scintillate! to avoid undue complexity. A very good agreement of peak widths (i. e. energy resolution) was obtained. A slightly smaller level of Compton events in the simulated spectrum occurs due to neglecting of the sample holder and other solid parts close to the source that contribute to the scattering of $\gamma$ radiation.

Signal shape The comparison of single signals in fig. 7.15 shows a redistribution of amplitude from the peak to the falling edge of the signal. Since the time difference between the parts with lower and the parts with higher amplitude is significantly larger than the single-electron signal shown in fig. 7.11 and the beginning of the rising edge is identical for both signals, the difference is not strongly related to a deviating PMT rise-time value. Instead, it occurs due to the irregular frequency response of the voltage divider, which was described in section 7.3, as well as the simplification made by simulating the $\operatorname{LaBr}_{3}: \mathrm{Ce}$ scintillator using a single pair of time constants for the rising and falling edge. According to Glodo et al. (2005) a fractionation of rise- and decay-time properties is necessary. However, the parameters depend on the Ce concentration and are therefore unknown due to missing information in the scintillator's data sheet. 
Table 7.7: Multiply! settings for the simulation of a measurement conducted with the TDPAC spectrometer described in chapter 4.

\begin{tabular}{lcc}
\hline Parameter & Value & Unit \\
\hline Quantum efficiency & $23^{a}$ & $\%$ \\
PMT gain & $1.5 \times 10^{6 b}$ & \\
Relative gain per dynode & $4,1.2,1.8,1,1,1,1,1,1$, \\
& $1.5,1.5$ and $3^{c}$ & \\
$10 \% / 90 \%$ rise time & $1.6^{b}$ & $\mathrm{~ns}$ \\
Transit time spread & $0.25^{d}$ & $\mathrm{~ns}$ \\
Attenuation & $-6.5^{e}$ & $\mathrm{~dB}$ \\
Noise $\sigma$ & $40^{f}$ & $\mathrm{mV}$ \\
ADC resolution & $8^{g}$ & $\mathrm{bit}$ \\
Sampling rate & $2^{g}$ & $\mathrm{GS} \mathrm{s}{ }^{-1}$ \\
Conversion range & -5 to 0 & $\mathrm{~V}$ \\
Time before signal onset & $10^{b}$ & $\mathrm{~ns}$ \\
Onset jitter & $\mathrm{enabled}^{b}$ & \\
Samples per signal & $400^{b}$ & \\
\hline
\end{tabular}

${ }^{a}$ Photonis (2007a), p. 9 and Photonis (2007b)

${ }^{b}$ Photonis $(2007 \mathrm{~b})$

cPhotonis (2009)

${ }^{d}$ Photonis (2007a), p. 19

${ }^{e}$ estimate according to section 7.3

$f_{\text {this value was estimated }}$

${ }^{g}$ cf. section 4.1 .2

${ }^{b} \mathrm{cf}$. section 7.2.2

\subsection{2 $\mathrm{BaF}_{2}$ / Hamamatsu R3377 with Agilent Acqiris U1064A readout}

A second test was accomplished by comparing data from a different detector system with a corresponding simulation. Again, a ${ }^{22} \mathrm{Na}$ sample was used as radiation source (see appendix A.2.1 for its properties). The measurement data was recorded by Arnold Krille using a positron annibilation lifetime spectroscopy (PALS) setup at Martin-Luther-University Halle. The detector consisted of $\mathrm{a} \mathrm{BaF}_{2}$ crystal mounted on a Hamamatsu H3378-50 PMT assembly consisting of a R3377 PMT and a suitable voltage divider. Anode signals were recorded using an Agilent Acqiris U1064A digitizer operated at $4 \mathrm{GS} \mathrm{s}^{-1}$.

The parameters used for the crystal's simulation with Scintillate! were chosen according to table 7.8 .

For the PMT simulation with Multiply!, data-sheet parameters as shown in table 7.9 were used. 


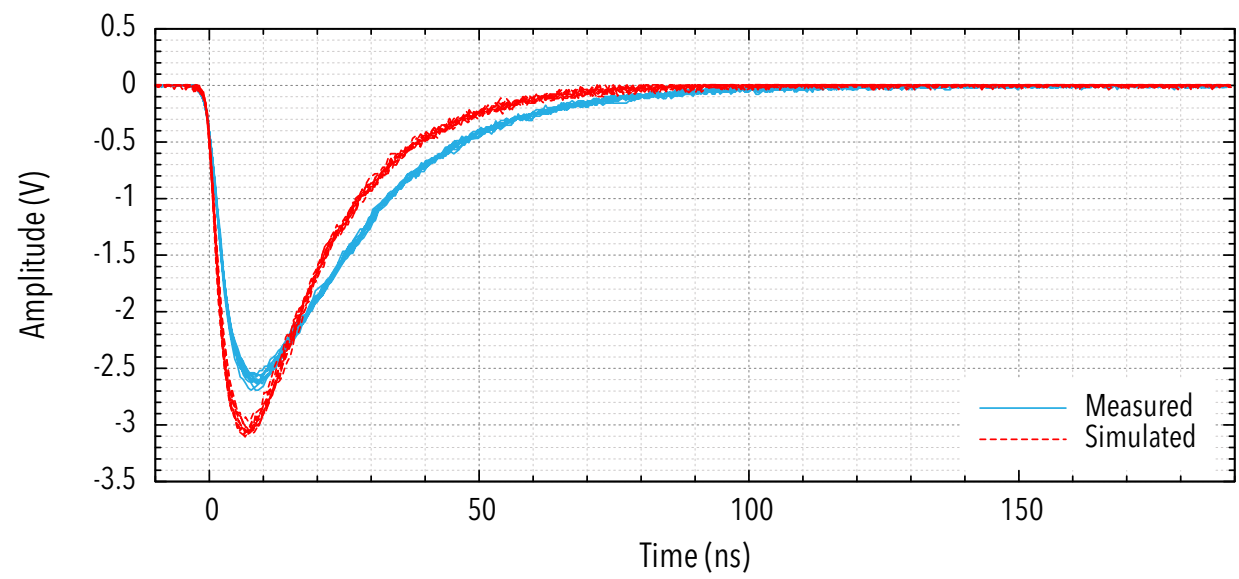

Figure 7.15: Ten measured and ten simulated anode signals from the $1274.54 \mathrm{keV}$ photo-peak of a ${ }^{22} \mathrm{Na}$ sample's radiation. While the rising edge is identical in both cases, the measured curve shows a redistribution of amplitude from the peak towards the falling edge. This difference is attributed to the irregular frequency response of the voltage divider (cf. section 7.3) and the simplified simulation of decay properties using a single decay component while according to Glodo et al. (2005) several are necessary. The simplification was necessary since the Ce content of the used scintillators is not specified by the vendor.

Table 7.8: Scintillate! settings for the simulation of a measurement conducted with a $\mathrm{BaF}_{2}$ crystal mounted on a Hamamatsu H3378-50 PMT assembly.

\begin{tabular}{lll}
\hline Parameter & Value & Unit \\
\hline Crystal shape & \multicolumn{2}{l}{ Cylindrical } \\
Crystal material & $\mathrm{BaF}_{2}$ & \\
Crystal diameter & 40 & $\mathrm{~mm}$ \\
Crystal height & 10 & $\mathrm{~mm}$ \\
Crystal surface & Ground, not painted \\
Thickness of the crystal's aluminium shell & 0.5 & $\mathrm{~mm}$ \\
Distance to the radiation source & 5 & $\mathrm{~mm}$ \\
PMT diameter & 51 & $\mathrm{~mm}$ \\
Grease layer thickness & $0.1^{a}$ & $\mathrm{~mm}$ \\
Thickness of the PMT's glass envelope & $2^{a}$ & $\mathrm{~mm}$ \\
Thickness of the PMT's window & $2^{a}$ & $\mathrm{~mm}$ \\
Gap between crystal and the aluminium enclosure & $0.2^{a}$ & $\mathrm{~mm}$ \\
\hline
\end{tabular}

${ }^{a}$ this value was estimated 
Table 7.9: Multiply! settings for the simulation of measurements conducted with a Hamamatsu H3378-50 PMT assembly.

\begin{tabular}{lcc}
\hline Parameter & Value & Unit \\
\hline Quantum efficiency & $21^{a}$ & $\%$ \\
PMT gain & $1000000^{b}$ & \\
Relative gain per dynode & $6.1,1.2,1.8,1,1,1$, \\
& 1.5 and $3^{c}$ & \\
$10 \%$ / 90\% rise time & $0.7^{c}$ & $\mathrm{~ns}$ \\
Transit time spread & $0.37^{c}$ & $\mathrm{~ns}$ \\
Attenuation & $-6^{d}$ & $\mathrm{~dB}$ \\
Noise $\sigma$ & $10^{c}$ & $\mathrm{mV}$ \\
ADC resolution & $7^{f}$ & $\mathrm{bit}$ \\
Sampling rate & $4^{g}$ & $\mathrm{GS} \mathrm{s}$ \\
Conversion range & -0.55 to $0.45^{-1}$ & $\mathrm{~V}$ \\
Time before signal onset & $50^{b}$ & $\mathrm{~ns}$ \\
Onset jitter & enabled $^{b}$ & \\
Samples per signal & $2000^{b}$ & \\
\hline
\end{tabular}

\footnotetext{
${ }^{a}$ average value for the relevant wavelengths according to Hamamatsu (2013)

${ }^{b}$ value for an operating voltage of $2500 \mathrm{~V}$ according to Hamamatsu (2013)

${ }^{c}$ Hamamatsu (2013)

${ }^{d}$ estimate according to section 7.3

${ }^{e}$ this value was estimated

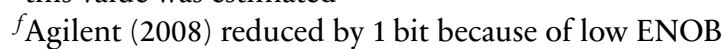

${ }^{g}$ Agilent (2008)

${ }^{b}$ cf. section 7.2.2
}

Due to the low amount of light produced by $\mathrm{BaF}_{2}$ scintillators (cf. table 7.3), energy resolution of detectors based on this material is rather limited. Since most of the light is emitted as part of the slow fraction, long integration times are necessary to be able to distinguish even the well separated peaks of ${ }^{22} \mathrm{Na}$ samples. However, during these long integration times, the probability for signal pile-up becomes high even in case of weak samples.

Nevertheless, $\mathrm{BaF}_{2}$ scintillators are very common and frequently used although in the meantime superior $\mathrm{LaBr}_{3}$ :Ce based scintillators have become available. This is due to $\mathrm{BaF}_{2}$ 's lower cost and slightly better timing resolution, which emerges due to the very steep rise and decay times of the fast component. This component, although providing very low luminosity, yields distinct peaks because its photons arrive almost simultaneously. 
Charge spectrum Both charge spectra are shown in fig. 7.16.

The obvious difference in the range of $-25 \mathrm{pC}$ to $0 \mathrm{pC}$ is not a real difference but a triggering artifact. In contrast to the simulated signal, the measured signal was not triggered by actual gamma emission but by the signal's amplitude. However, due the background noise level this trigger level cannot be set arbitrarily low. Therefore, the smaller a signal is, the higher is its probability to not trigger a recording.

The $511 \mathrm{keV}$ peak in both spectra is almost identical. A tail of the $1274.54 \mathrm{keV}$ peak towards higher energies in the measured spectrum occurs due to pile-up, which was not simulated. Since the slow component's amplitude is in the range of only a few LSB, the effective number of bits provided by the digitizer, namely 5.0 bit to $6.5 \mathrm{bit}$, had noticeable influence on the measurement results. This effect is hard to simulate because the behavior of the non-significant bits is neither random nor defined. However, an approximation was obtained by reducing the resolution of the simulated digitizer to 7 bit.

Signal shape The shapes of signals originating from measurement and simulation, as visible in fig. 7.17, are almost identical. Slight differences in the peak amplitude can be attributed to attenuation in the signal path, which exhibits a frequency dependency in real measurements. Two of the ten measured signals from the $1274.54 \mathrm{keV}$ photo-peak region contain additional pulses, which occurred due to pile-up of signals as already described above.

\subsection{Simulation based solid angle corrections}

Many TDPAC experiments require knowledge about the solid angle correction factors $Q_{k_{1} k_{2}}$ introduced in eq. (2.10). Obviously, these factors are necessary as soon as precise quantitative evaluations of measured $R(t)$ amplitudes (rather than only frequencies) are desired. While the electric field gradients' and magnetic fields' strengths can be determined by the frequencies of the coincidence rate's (and thus $R(t)$ 's) modulation and the asymmetry-parameter $\eta$, which quantifies the electric field gradient's asymmetry, depends on the observed frequency ratios, amplitudes are relevant for the determination of the field gradient's orientation.

In simple experiments involving powder samples, the perturbation factor $G_{k_{1} k_{2}}^{N_{1} N_{2}}(t)$ introduced in eq. (2.9) vanishes for $k_{1} \neq k_{2}$ (cf. Frauenfelder and Steffen, 1965, page 1110). Since the contribution of the term with $k_{1}=k_{2}=4$ can be neglected in many cases due to small $A_{44}$, only a single angular correlation coefficient, namely $A_{22}$, remains and therefore only a single solid angle correction factor, $Q_{22}$ is needed. This single factor usually does not impair evaluations and can be compensated for by introducing a free parameter in the fit of $R(t)$.

If, however, mixed or higher order terms cannot be neglected-as in single crystal measurements - the different values of $Q_{k_{1} k_{2}}$ modify the impact of these terms on the 


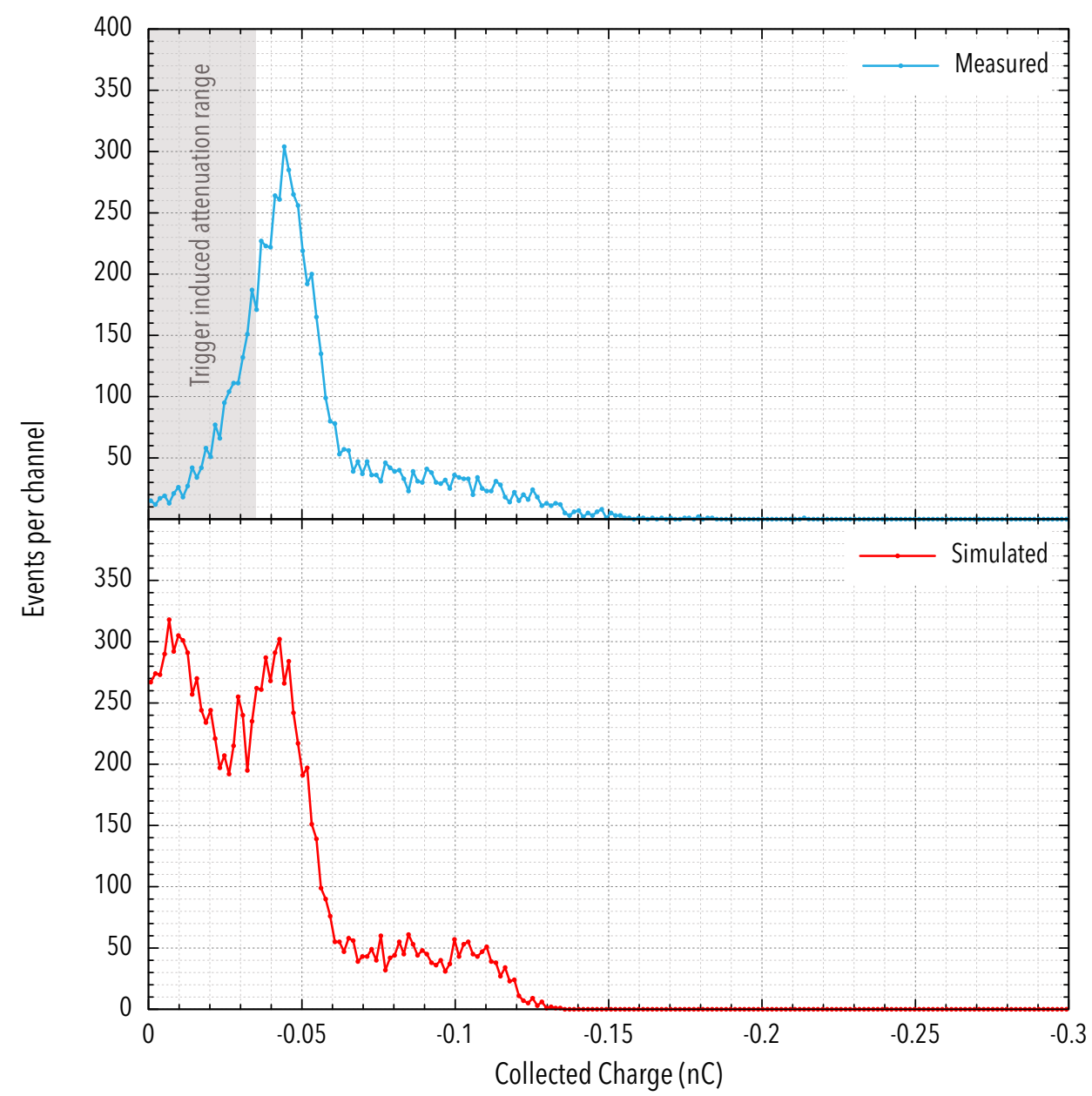

Figure 7.16: Measured (top) and simulated (bottom) charge spectra of the radiation of a ${ }^{22} \mathrm{Na}$ sample. The used detector consists of $\mathrm{a} \mathrm{BaF}_{2}$ crystal and a Hamamatsu R3377 PMT. The difference visible at very small charge values occurs due to triggering in case of the measurement. Since the trigger has to be set to values higher than the signal's noise level for the real measurement to avoid random triggering, the probability for signals to exceed the trigger threshold decreases at small $\gamma$ energies (the affected range is highlighted gray). Thereby, increasing attenuation occurs towards decreasing energies. This effect, however, does not occur in the simulation because there the simulated recordings are triggered by the simulated $\gamma$ emission. The other parts of the spectrum again show a very good agreement. Due to the low signal amplitudes and the very long decay constant of the slow part of the $\mathrm{BaF}_{2}$ signals, the limitation of the effective number of bits (ENOB) has considerable effect on the energy resolution of this detector setup. Additional blurring occurred especially at the upper limit of the measured charge spectrum due to signal pile-up also visible in fig. 7.17. 


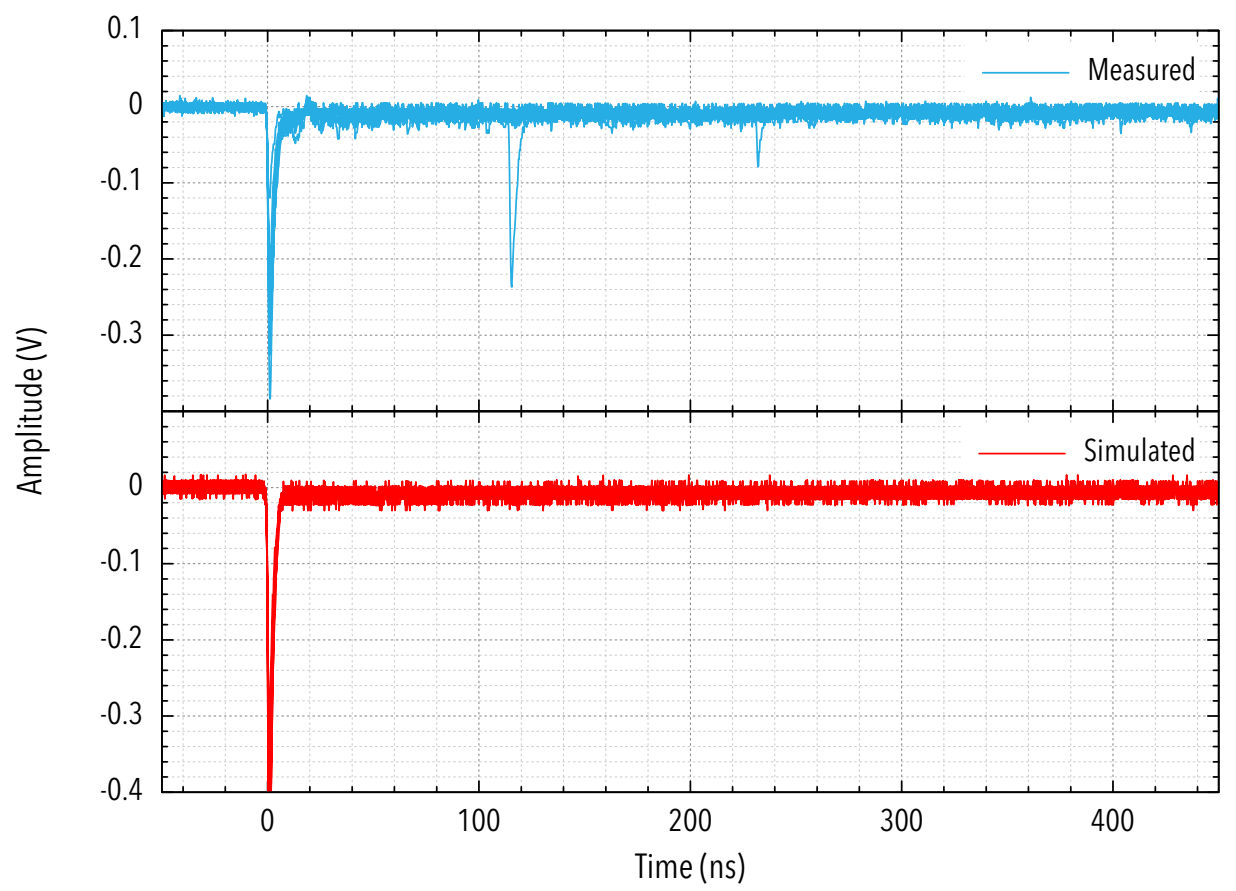

Figure 7.17: Ten measured (top) and ten simulated (bottom) anode signals of the $1274.54 \mathrm{keV}$ photo-peak of a ${ }^{22} \mathrm{Na}$ sample's radiation. Signal shapes are almost identical. The measured signals' bump $20 \mathrm{~ns}$ after the main peak is attributed to signal reflection in the used cabling. The main peak of the simulated signals is slightly less damped than that of the measured signals which is due to the frequency independent approximation of attenuation. Among the measured signals, two show additional peaks. This occurred due to signal pile-up. This explains the shallower ending of the measured spectrum in fig. 7.16 towards high absolute charge values.

correlation function $W\left(\theta_{1}, \varphi_{1}, \theta_{2}, \varphi_{2}, t\right)$ and it is not possible to handle them adequately by free fit parameters. In case of the usually used axially symmetric detectors, it is according to Frauenfelder and Steffen (1965) possible to express $Q_{k_{1} k_{2}}$ as

$$
Q_{k_{1} k_{2}}=Q_{k_{1}}\left(E_{\text {start }}\right) \cdot Q_{k_{2}}\left(E_{\text {stop }}\right),
$$

where

$$
Q_{k}(E)=\frac{J_{k}(E)}{J_{0}(E)}
$$

and $J_{k}(E)$ is defined as

$$
J_{k}(E)=\int_{0}^{\frac{\pi}{2}} \varepsilon(E, \theta) P_{k}(\cos \theta) \sin (\theta) \mathrm{d} \theta .
$$


$\varepsilon(E, \theta)$ is the angular efficiency of the used detectors for $\gamma$ radiation with energy $E$. Of course, $\varepsilon(E, \theta)$ also depends on the distance between sample and detectors, which is not mentioned because it is considered constant. $\theta$ denotes the emission angle of $\gamma$ radiation emitted from the point source, i. e. the angle between the detector axis and the $\gamma$ photon's direction of propagation.

Frauenfelder and Steffen (1965) suggest several methods for the $\varepsilon(E, \theta)$ determination:

1. Angle-independent approximation

2. Analytical calculation based on the scintillation material's absorption coefficients for $\gamma$ radiation

3. Experimental determination using collimated $\gamma$ beams

The first method obviously provides only a vague guess since it totally neglects the angle dependent length of $\gamma$ trajectories in conically shaped scintillators. The second method provides better results. However, it still neglects the energy and angle dependent escape probability of Compton scattered photons. Moreover, it depends on the availability of energy dependent absorption coefficients. Collimated $\gamma$ beams at defined energies, as necessary for the third method, are not easy to obtain. Methods like the one described by Lawson and Frauenfelder (1953) require extensive shielding setups to provide sufficient collimation.

Monte-Carlo simulations of the scintillation process and light propagation as realized by Scintillate! establish a new method: The simulation of perfectly collimated $\gamma$ radiation. Since the simulation covers Compton effect as well as trajectory simulation of secondary particles and radiations, it can provide much better results than analytical approximations based on absorption coefficients. Moreover, employing the clustering technique introduced above (see page 104), it is possible to consider only events contributing to the photo-peak thereby filtering events with escaped Compton scattered $\gamma$ photons and obtaining more precise results.

Figure 7.18 shows results of the $\varepsilon(E, \theta)$ function determined using said method for all energy values occurring in chapter 8 . Due to the simulation of $10000 \gamma$ photons per angle, a precision better than $1 \%$ was obtained for all simulated angles and energies. Obviously, the efficiency depends heavily on $\gamma$ energy and begins to decrease-especially at higher energies-at angles much smaller than the angular limit where the $\gamma$ photons do not cross the scintillator anymore (marked gray in the plot).

The simulation results were subsequently used as a piecewise constant approximation of $\varepsilon(E, \theta)$. The piecewise constant approach made it possible to evaluate the integral from eq. (7.20) independently of $\varepsilon(E, \theta)$ on the intervals defined by the simulated angles. Integration and multiplication followed by division according to eq. (7.19) yielded the results summarized in table 7.10.

These $Q_{k}(E)$ values were then used together with eqs. (2.10) and (7.18) to calculate values for $A_{k_{1} k_{2}}^{\text {eff }}$, which are summarized in table 7.11. 


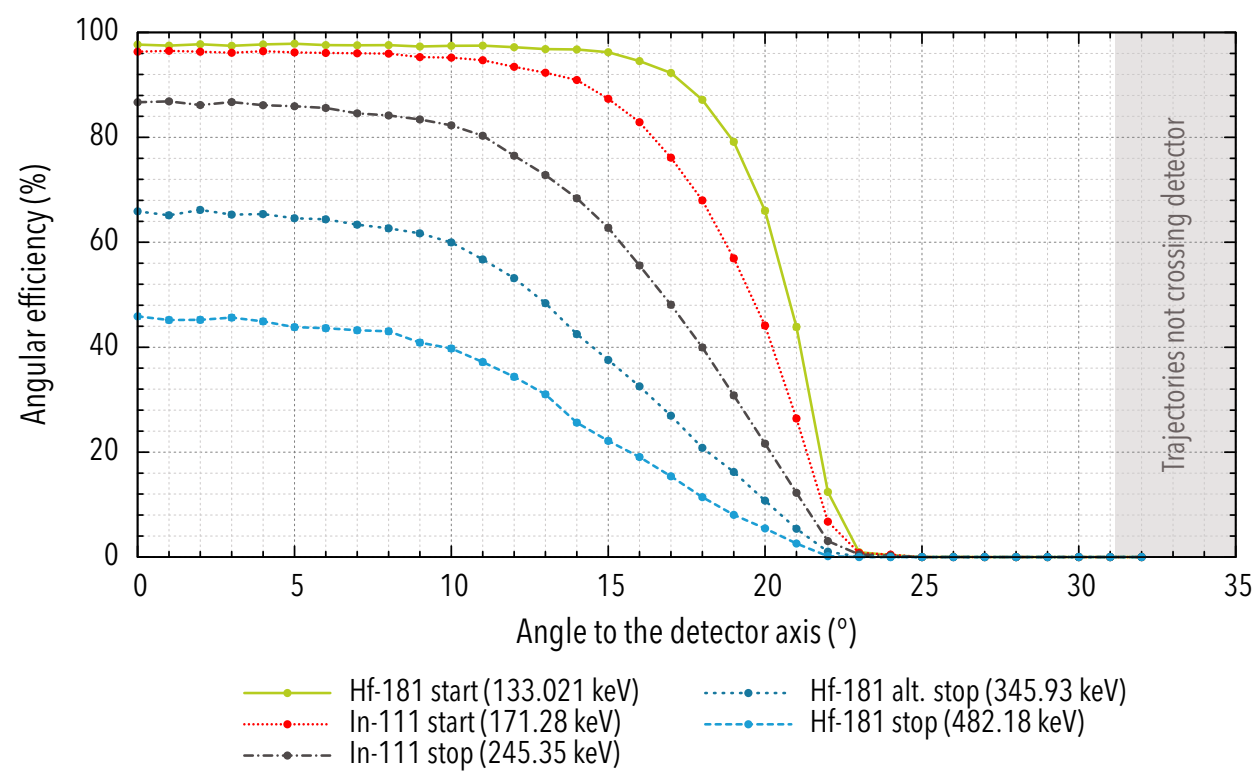

Figure 7.18: Angular efficiencies for different $\gamma$ energies. Error bars are plotted but not visible since they are too small in all cases. A point source on the detector's axis at a distance of $20 \mathrm{~mm}$ from the detector cladding's tip was simulated. This cladding consists of $0.5 \mathrm{~mm}$ thick aluminium, which surrounds the scintillator with an additional $0.5 \mathrm{~mm}$ gap in between. The abscissa shows the angle between the $\gamma$ trajectory and the detector axis. For angles larger than $\theta_{\max }=\arctan \left(\frac{25.4 \mathrm{~mm}}{2 \cdot(20 \mathrm{~mm}+1 \mathrm{~mm})}\right) \sim 31.2^{\circ}$, the emitted $\gamma$ passes the scintillator without actually entering the crystal.

Table 7.10: Solid angle correction factors required for evaluations in chapter 8 . The last column contains the effective solid angle coverage, i.e. the fraction of the full solid angle a perfect detector, which captures all photons and does not suffer from Compton scattered photons escaping the scintillator, would have to cover to obtain the same detection rate.

\begin{tabular}{llcccc}
\hline Probe & Role & Energy $(\mathrm{keV})$ & $Q_{2}$ & $Q_{4}$ & Eff. coverage (\%) \\
\hline${ }^{181} \mathrm{Hf}$ & start & 133.021 & 0.905 & 0.707 & 3.07 \\
& stop & 482.18 & 0.934 & 0.793 & 0.81 \\
& alt. stop & 345.93 & 0.930 & 0.780 & 1.29 \\
\hline${ }^{111}$ In & start & 171.28 & 0.912 & 0.729 & 2.68 \\
& stop & 245.35 & 0.922 & 0.758 & 1.98 \\
\hline
\end{tabular}


Table 7.11: $A_{k_{1} k_{2}}^{\text {eff }}$ for ${ }^{181} \mathrm{Hf}$ and ${ }^{111}$ In. $A_{k_{1} k_{2}}$ values calculated by Nuclei (cf. chapter 9) were multiplied with $Q_{k_{1} k_{2}}$ values calculated according to eq. (7.18) using $Q_{k}(E)$ values from table 7.10.

\begin{tabular}{lccrrrr}
\hline Probe & Start energy $(\mathrm{keV})$ & Stop energy $(\mathrm{keV})$ & $A_{22}^{\text {eff }}$ & \multicolumn{1}{c}{$A_{24}^{\text {eff }}$} & \multicolumn{1}{c}{ eff } & $A_{44}^{\text {eff }}$ \\
\hline${ }^{181} \mathrm{Hf}$ & 133.021 & 482.18 & -0.270 & -0.043 & -0.243 & -0.039 \\
& & 345.93 & 0.086 & 0.006 & 0.078 & 0.005 \\
\hline${ }^{111} \mathrm{In}$ & 171.28 & 245.35 & -0.150 & -0.142 & -0.001 & -0.001 \\
\hline
\end{tabular}

\subsection{Conclusions}

As shown exemplarily by the comparisons above, the implemented simulation toolchain provides outstanding results while only relying on data sheet values of the used detector components. For the first time, it provides an easy to use toolset for the estimation of the performance of small scintillation detectors. The simulated detector variants cover almost all designs usually used in spectroscopy applications like TDPAC and positron annihilation lifetime spectroscopy. While it was usual practice to buy detectors based on assumptions before being able to investigate their performance in these areas of nuclear spectroscopy, it is now easily possible to evaluate design decisions before buying parts by using the simulation system developed in the context of this thesis. In combination with the results from chapter 5 , it is even possible to pre-evaluate time determination algorithms and their optimal parameterization before even building the detectors. While being relatively common in the design of large scale detectors like those at the LHC, this approach was not used routinely for spectrometers before due to the lack of simple to use tools like those presented above.

Moreover, the implemented scintillator simulation software allows for a straightforward determination of solid angle correction factors, which was a difficult task before. This makes it possible to improve the quality of data evaluation by considering mixed and higher order terms which is especially useful in case of single crystal measurements. 


\section{Chapter 8}

\section{Test measurements}

During the development of the new TDPAC spectrometer described in detail in chapter 4, several test measurements were performed in order to evaluate its working principle and performance and optimize the evaluation toolchain. A selection of these measurements demonstrating certain properties and advantages of the spectrometer design is shown in the following sections.

SpectraPAC, the evaluation software described in section 4.2.4, outputs $R(t)$ spectra and their Fourier transformations. Fitting of the measured modulations and thereby extracting parameters relevant for the physical interpretation requires additional software. A very convenient tool for this task was written by Heinrich (2001). It is called winfit and allows the evaluation of electrical as well as magnetic hyperfine interactions in powder samples. To simplify the use of winfit for the evaluation of results of SpectraPAC, the latter provides a special export functionality, which outputs its $R(t)$ result in a format that can be imported into winfit. In the following section, winfit version 3.0.4 was used for fitting.

\subsection{Fast modulations $\left({ }^{111} \mathrm{In} \text { in iron foil }\right)^{1}$}

An early test made with the first version of the spectrometer based on four LYSO detectors involved ${ }^{111} \mathrm{In}$ (cf. appendix A.1.1) implanted into an iron foil sample with a size of $10 \mathrm{~mm} \times 10 \mathrm{~mm} \times 0.1 \mathrm{~mm}$ using an energy of $400 \mathrm{keV}$. The surface of the foil was positioned parallel to the detector plane.

Due to the high internal magnetic field strength (Lindgren and Vijay, 1981), this kind of sample is appropriate for testing the behavior of a spectrometer in case of interactions with high frequencies. Provided these interaction frequencies approached the time resolution of the spectrometer, the signal would be damped and details would vanish.

The measurement took $86 \mathrm{~h}$ and contains a total number of $4.82 \times 10^{10} \gamma$ events. This number does not include $\gamma$ events that were temporally isolated-i. e. events that were not preceded or followed by another $\gamma$ photon in a period of up to $1000 \mathrm{~ns}$ - and thus filtered. The PacMaster output file containing information about every single recorded $\gamma$

\footnotetext{
${ }^{1}$ This section contains revised parts from Nagl, Vetter, et al. (2010)
} 


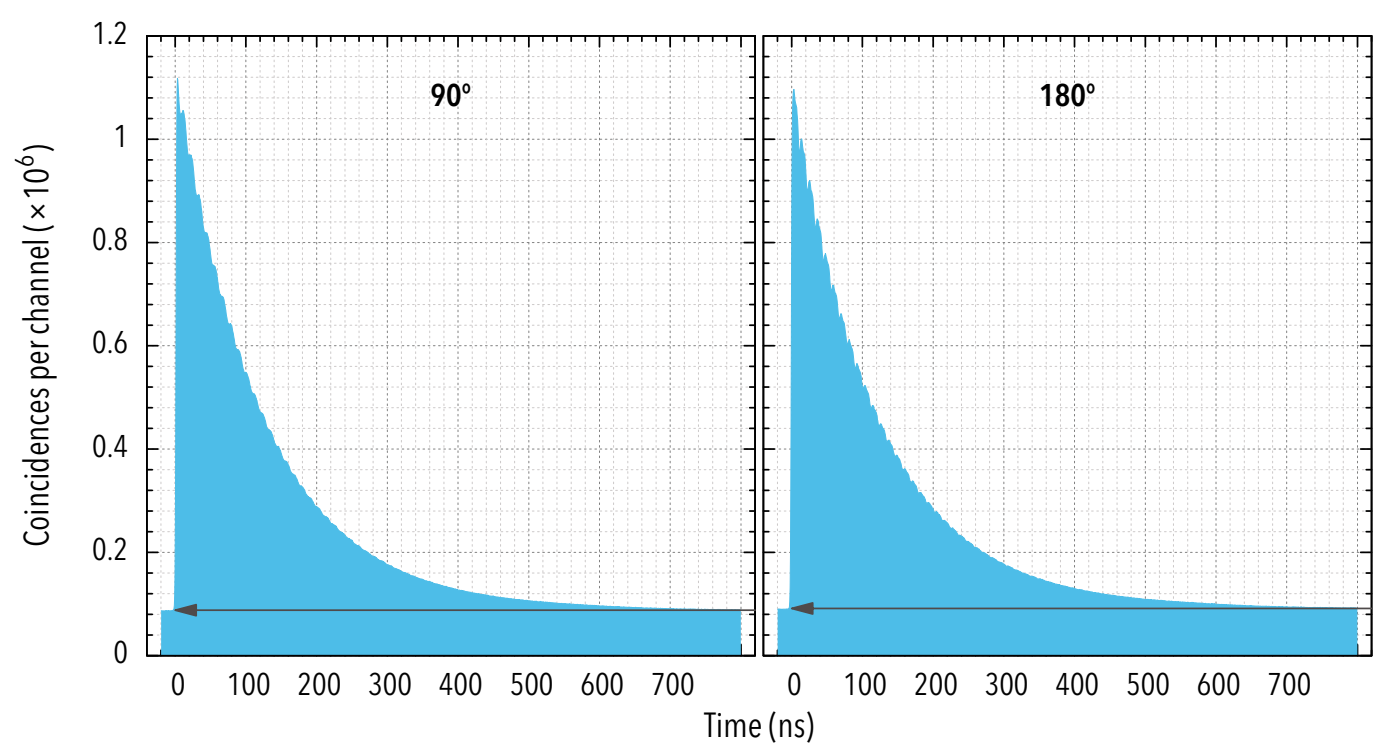

Figure 8.1: Two of the twelve recorded coincidence spectra of a sample, where ${ }^{111}$ In was implanted into a $100 \mu \mathrm{m}$ thick Fe foil using an ion energy of $400 \mathrm{keV}$. The surface normal of the foil was aligned perpendicular to the detector plane. Background estimation using an exponential fit of the decay yielded the background levels denoted by grey arrows. Obviously, these results are in perfect agreement with the background level before the spectra's onsets which demonstrates the success of the bias free coincidence determination described in section 4.2.4.

event usable for evaluation with current and future versions of SpectraPAC has a size of $554 \mathrm{GiB}$.

Two exemplary coincidence spectra of the measurement are shown in fig. 8.1. Despite the high count rate of more than 150000 correlated counts per second (additional background was filtered by PacMaster, cf. section 4.2.3), the background level determined by exponential fitting as described on page 58 and indicated by the grey arrows in the plots is in perfect agreement with the background level left of the spectrum towards negative time differences. This illustrates the benefit of the bias free coincidence analysis implemented in SpectraPAC as described on page 57.

Figure 8.2 shows SpectraPAC-generated evaluation results of this measurement. Due to the large amount of recorded gamma events, a good signal to noise ratio and a reasonably small error was accomplished up to about six times the half-life of the used intermediate state $(84.5 \mathrm{~ns}$, cf. fig. A.1 on page 220).

The Fourier transformation of the $R(t)$ spectrum shows the Larmor frequency $\omega_{L}$ and its doubled value $2 \omega_{L}$. Using eq. (2.12), the measured Larmor frequency (558.71(3) $\mathrm{Mrad} \mathrm{s}^{-1}$ ) can be translated into the magnetic field at the probe atoms' sites, which amounts to 


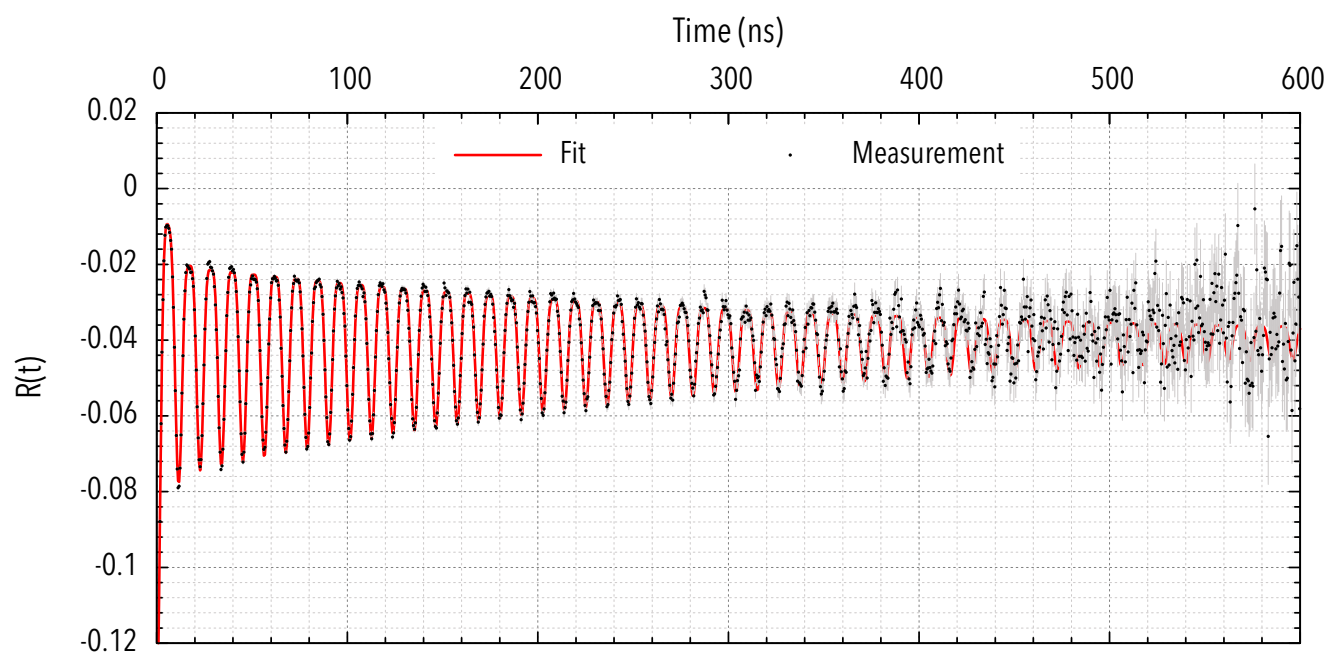

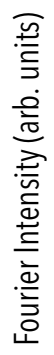

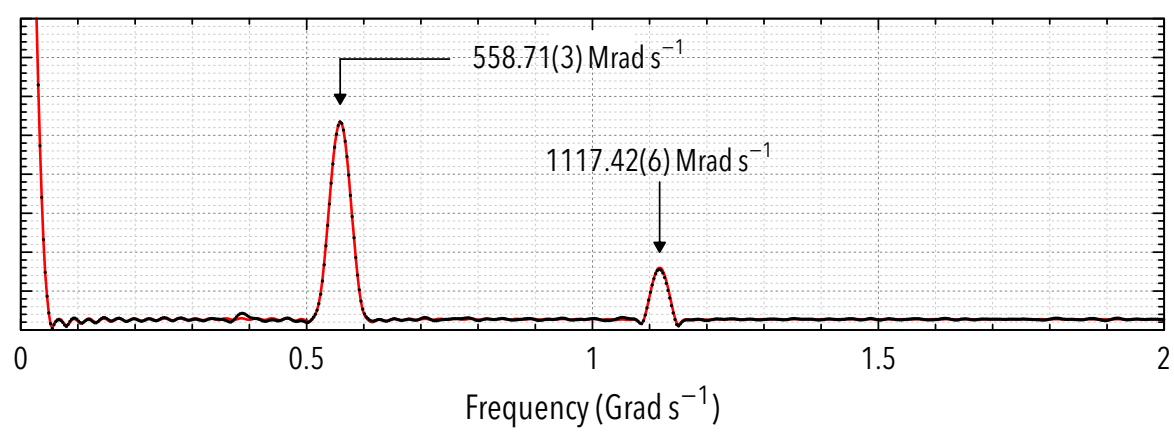

Figure 8.2: $R(t)$ spectrum and the corresponding Fourier spectrum (power spectrum) of a sample where ${ }^{111}$ In was implanted into a $100 \mu \mathrm{m}$ thick Fe foil using an ion energy of $400 \mathrm{keV}$. The surface normal of the foil was aligned perpendicular to the detector plane. Dots in the $R(t)$ spectrum correspond to the measurement results at the chosen channels' centers (channel width: $500 \mathrm{ps}$ ). Vertical grey bars indicate the absolute error of each channel $( \pm \sigma)$. Continuous lines represent the fit result of winfit. No temper treatment was conducted to anneal radiation damage, which is considered the reason for the slight damping of the $R(t)$ functions as well as an additional frequency background visible in the Fourier spectrum (two fractions were fitted - the primary one modeling the oscillations and a second one representing unordered surroundings with large damping). The magnetic hyperfine interaction is observable by the Larmor frequency $\omega_{L}$ and $2 \cdot \omega_{L}$. The Larmor frequency can be used to calculate the magnetic field at the probe atoms' sites. This calculation results in a magnetic flux density of 38.1(1) T. 
$|B|=38.1(1) \mathrm{T}$ and is in good agreement with the value reported in literature (Lindgren and Vijay, 1981).

\subsection{Long-term stability ( ${ }^{181} \mathrm{Hf}$ in AIN annealing sequence)}

Excellent time resolution properties of a spectrometer are even more important in case of ${ }^{181} \mathrm{Hf}$ probes compared to ${ }^{111}$ In due to the short half-life of its intermediate level of only $10.8 \mathrm{~ns}$ (for decay properties of ${ }^{181} \mathrm{Hf}$, see appendix A.1.2). As a test case, ${ }^{181} \mathrm{Hf}$ was implanted into a 0001-oriented AlN layer on top of $5 \mathrm{~mm} \times 5 \mathrm{~mm}$ sapphire substrate pieces. After implantation of ${ }^{181} \mathrm{Hf}$ using an ion energy of $160 \mathrm{keV}$ and a tilt angle of $7^{\circ}$ for avoiding of channeling effects at the Bonn Isotope Separator, the sample was repeatedly annealed at increasing temperatures and analyzed using the digital TDPAC spectrometer with the four used detectors aligned parallel to the sample's surface plane. For annealing, an early prototype of the furnace described in section 10.2 was used. The nominal temperature of each annealing step was maintained for $2 \mathrm{~min}$. Due to bad pressure conditions before the upgrade of the vacuum system described in section 10.2, the sample was damaged during the annealing at $1200^{\circ} \mathrm{C}$. Spectra of annealing steps with $1200^{\circ} \mathrm{C}$ and $1300^{\circ} \mathrm{C}$ were therefore recorded again using a spare sample into which ${ }^{181} \mathrm{Hf}$ had been identically implanted as into the initial one.

Figure 8.3 shows spectra of the complete annealing sequence. Since the AlN layers on the used samples have a defined orientation of crystal axes and can therefore be considered single crystals, winfit was not an appropriate fitting tool because it only supports fitting spectra from samples with unordered crystallites-i. e. powder samples. Instead, Nightmare (Nédélec, 2014) was used. According to Geruschke, Lorenz, and Vianden (2009), several probe atom environments were assumed: A primary fraction of probe atoms on places with an axially symmetric electric field gradient $(\eta=0)$ and a $V_{z z}$ orientation parallel to the 0001-axis as well as two fractions on sites close to defects and in highly disordered environments, respectively. The fit parameters determined for the spectrum recorded after annealing at $1000^{\circ} \mathrm{C}$ were copied for the other spectra's evaluation and only the fraction ratios and the primary fraction's $\delta$ value-describing the fluctuations of EFG strengths sensed by probes within a fraction-were adjusted for each spectrum. Other parameters remained fixed, to provide comparable results. Percentages of the undisturbed fraction as well as its $\delta$ value are shown in fig. 8.3 alongside the spectra. The excellent matching of fit and measurements confirms the assumed EFG orientation and symmetry. It is possible to conclude from $\eta=0$ that the probe atoms causing the primary oscillation were located on places in the AIN lattice with axially symmetric electric field gradients. This is true for places on parallels to the c-axis passing the $\mathrm{Al}$ and $\mathrm{N}$ atoms.

After implantation, the visible modulations exhibited strong damping noticeable by the comparably high $\delta$ values. This occurred due to deviations in the surroundings of the probe atoms because of implantation induced defects. Annealing at raised tempera- 


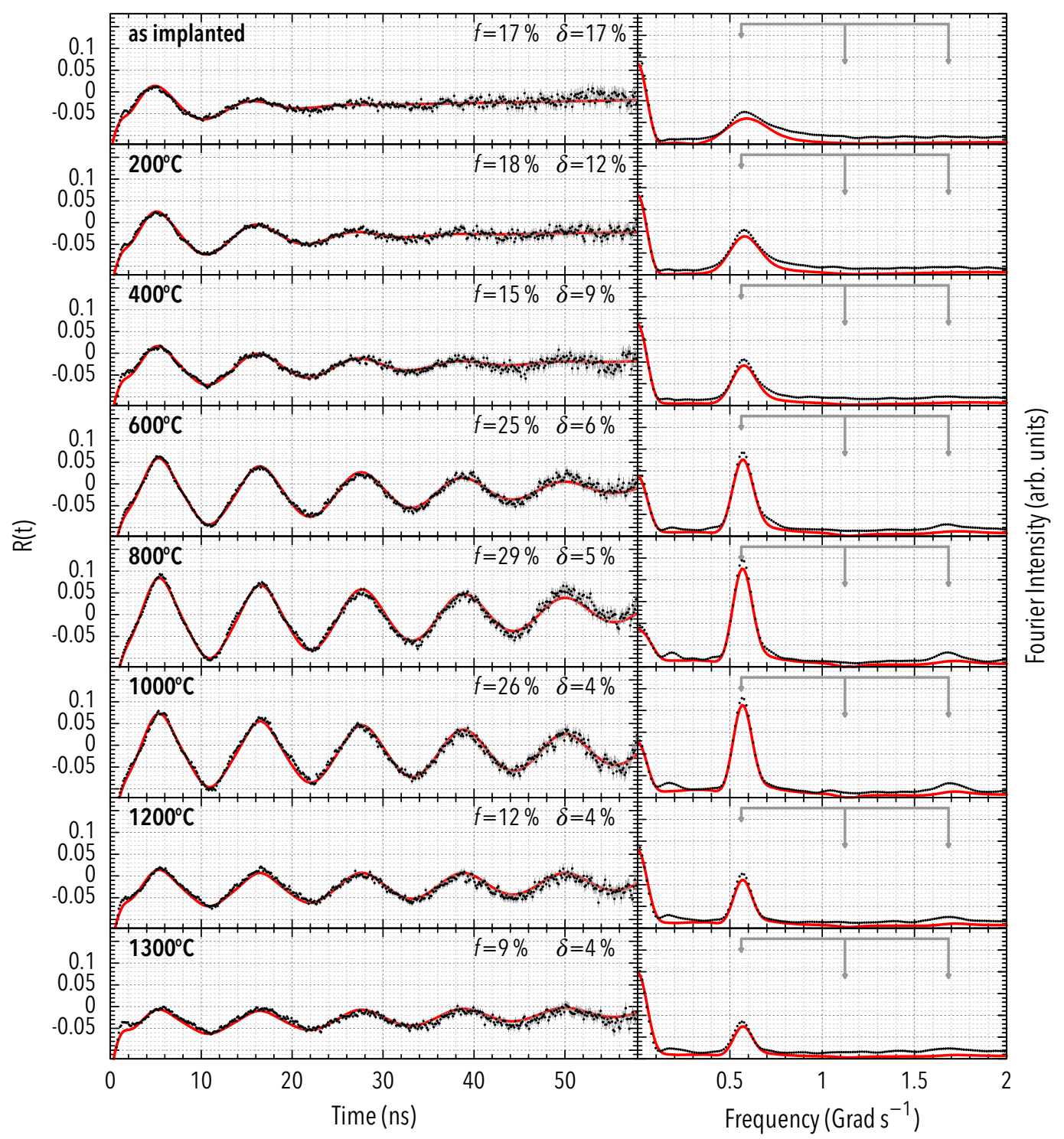

Figure 8.3: Annealing sequence of ${ }^{181} \mathrm{Hf}$ implanted AlN measured using four detectors located parallel to the sample surface. Defect induced damping (quantified by $\delta$ ) of the axially symmetric fraction decreased with increasing annealing temperatures. At temperatures above $800^{\circ} \mathrm{C}$, the axially symmetric fraction decreased probably due to nitrogen loss. The primary fraction's base frequency $\omega_{0}$ was determined as $562 \mathrm{Mrad}$, which corresponds to $V_{z z}=6.3 \times 10^{22} \mathrm{~V} \mathrm{~m}^{-2}$. 
tures reduced the amount of defects and thus signal damping and increased the amount of probes belonging to the undisturbed fraction as visible by growing $f$ values towards higher temperatures. Above $800^{\circ} \mathrm{C}$, however, $f$ and thereby the visible signal amplitude started to decrease again which probably happened due to evaporation of nitrogen from the AlN layer. A second set of measurements where the sample's surface normal was aligned in the detector plane and spanned a $45^{\circ}$ angle with the detector axes yielded spectra similar to those shown by Geruschke, Lorenz, and Vianden (2009).

Among the measurements shown above, the lowest sample activity was reached for the measurement after annealing at $1300^{\circ} \mathrm{C}$. Due to the low residual activity, the measurement ran for almost 44 days. In spite of the long measurement times, none of the measurements was interrupted prematurely or suffered from drift although the spectrometer refrains from resynchronizations during measurements as discussed in chapter 4 . This can be considered a proof for the excellent stability of the synchronization concept discussed there.

\subsection{Multi-cascade evaluation}

One of the advantages of the new TDPAC spectrometer is the possibility of simultaneous evaluation of several decay cascades of a population of probe nuclei. This allows achieving of more information from the same amount of probe atoms thus improving statistics or reducing measurement time.

There are many probe nuclides, which offer more than one cascade (see tables 9.1 to 9.3 in chapter 9). In fact, even the standard probe ${ }^{181} \mathrm{Hf} \longrightarrow{ }^{181} \mathrm{Ta}$ has two alternative depopulating transitions of the intermediate level suitable for TDPAC measurements.

In fig. 8.4, two spectra from an exemplary measurement involving ${ }^{181} \mathrm{Hf}$ probe atoms in an AlN sample prepared as described in section 8.2 and annealed at $1000^{\circ} \mathrm{C}$ are shown. In each case, a simplified version of the level scheme of the ${ }^{181} \mathrm{Hf}$ decay as shown in fig. A.2 with highlighted decay cascade was added left of the spectrum. Due to the unequal distribution of decay properties, measurement statistics of the alternative cascade are much worse in case of ${ }^{181} \mathrm{Hf}$ probes. However, the concept of multi-cascade measurements obviously works and provides additional information. The spectra exhibit different properties since the final states have different spins and the depopulating $\gamma$ photons have different multipolarities, which results in different anisotropy values. Again, Nightmare was used for fitting and the fractions and parameters found by fitting the upper spectrum were copied to the fit of the alternative one. Setting the appropriate $A_{k_{1} k_{2}}^{\text {eff }}$ (cf. section 7.5) yielded a result, which describes the measured signal quite well. Differences in the alternative cascade's Fourier spectrum occurred due to its bad signal-to-noise ratio, which let the background of the measured Fourier spectrum significantly exceed the Fourier transformation of the fitted spectrum.

Unfortunately, up to now there is no fitting software available allowing for simultane- 

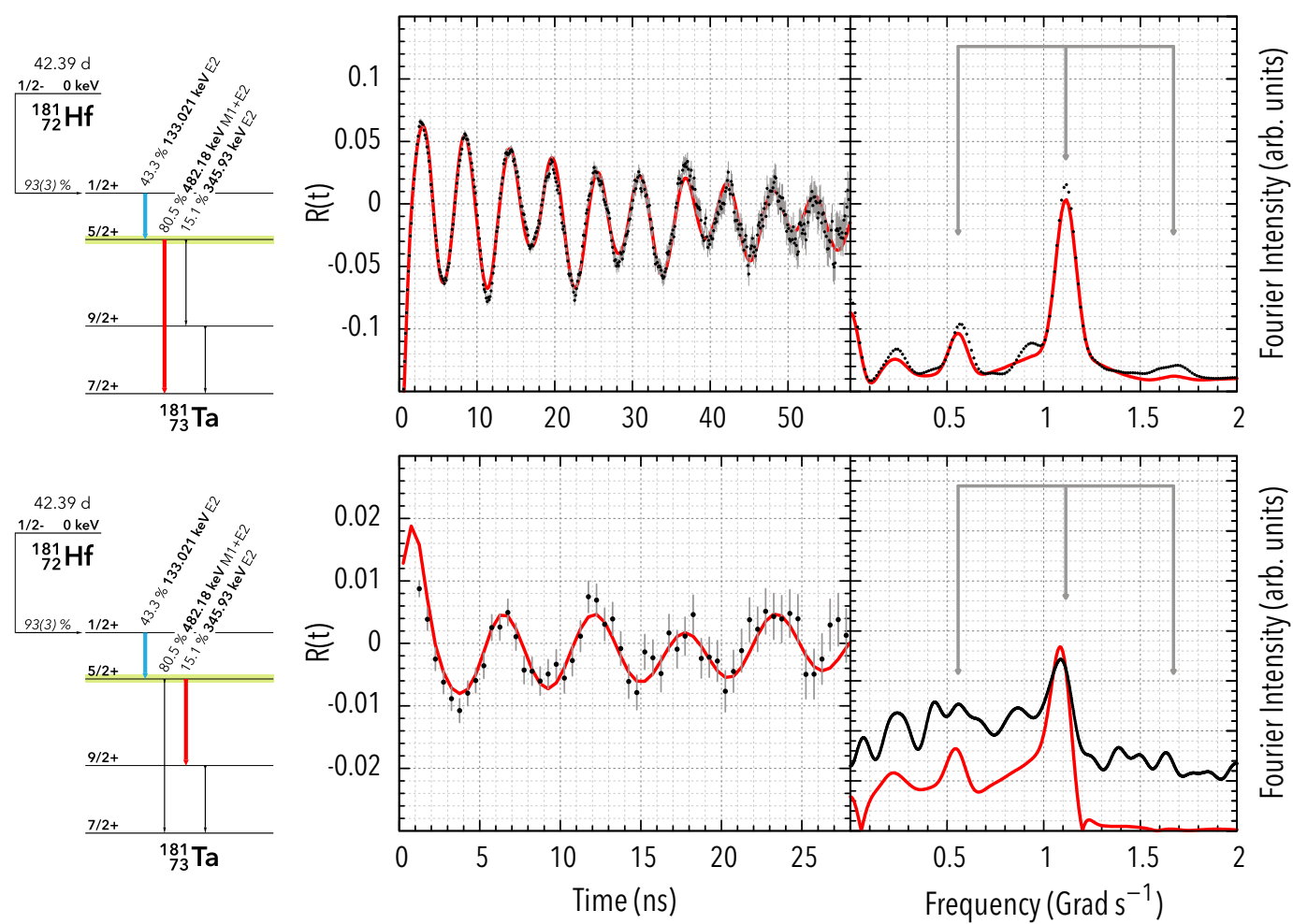

Figure 8.4: Evaluation of two concurrent decay channels of ${ }^{181} \mathrm{Hf}$, which was implanted into an AlN sample and measured with the sample's surface normal lying in the detector plane and spanning an angle of $45^{\circ}$ with all four detectors. On the left of the spectra, the decay cascade as well as the intermediate level is shown highlighted in simplified level schemes. Statistics for the lower evaluation is much worse due to the reduced probability of its depopulating transition. Differences in the amplitudes occur due to different anisotropies while the frequency of the modulation stays constant since it only depends on properties of the intermediate state rather than the depopulating transition. The primary fraction's base frequency $\omega_{0}$ was determined as $557 \mathrm{Mrad}$ for both spectra, which corresponds to $V_{z z}=6.2 \times 10^{22} \mathrm{~V} \mathrm{~m}^{-2}$. This is very close to the value determined for the measurement shown in section 8.2 , which originates from the same sample but another detector geometry.

ous fitting of spectra with a common set of parameters (cf. section 11.2.1) and manual optimization is a tedious task. Hopefully, future TDPAC fit software will exploit the possibilities emerging due to multi-cascade evaluations by allowing coupled fitting of several spectra. 


\subsection{Simultaneous measurement of two probe nuclides}

As a demonstration of the spectrometer's ability of measuring several decay channels at once and allowing to evaluate them separately online as well as afterwards, another ${ }^{181} \mathrm{Hf}$ in AlN sample prepared as described in section 8.2 was co-implanted with ${ }^{111} \mathrm{In}$ as described in section 8.1. The result was then measured once as implanted and once after a 2 min annealing step at $1000^{\circ} \mathrm{C}$. For both measurements, a geometry with the sample's surface normal lying in the detector plane and spanning an angle of $45^{\circ}$ with all four detectors was used. In both cases, the primary oscillation was successfully fitted assuming $\eta=0$ and a $V_{z z}$ orientation parallel to the 0001-axis. The determined base frequencies of the annealed samples' spectra were $557 \mathrm{Mrad}$ for the ${ }^{181} \mathrm{Hf}$ probes $\left(V_{z z}=6.2 \times 10^{22} \mathrm{~V} \mathrm{~m}^{-2}\right)$ and $31.8 \mathrm{Mrad}$ for the ${ }^{111} \mathrm{In}$ probes $\left(V_{z z}=1.1 \times 10^{22} \mathrm{~V} \mathrm{~m}^{-2}\right)$. The difference emerges due to the different electron configuration of both probe types.

Figure 8.5 shows an energy spectrum of the measurements and the results of the ${ }^{181} \mathrm{Hf}$ and ${ }^{111}$ In evaluations. Directly after the implantations, both, the ${ }^{181} \mathrm{Hf}$ as well as the ${ }^{111} \mathrm{In}$ spectrum showed strong damping and a broad frequency distribution, which is an indicator for a defect rich environment of both probe species. After annealing at $1000^{\circ} \mathrm{C}$, both probe species exhibited weakly damped oscillations. Strongly damped fast oscillations visible in the beginning of the ${ }^{111}$ In signal are in good agreement with Schmitz et al. (2009).

\subsection{Conclusions}

The shown example measurements demonstrate that the goals defined in sections 3.2.1 and 3.2.2 were achieved by the chosen spectrometer design. It is the first spectrometer allowing for online multi-cascade and multi-probe evaluations. The concept of postponing as many configuration tasks as possible already proved its value since it allowed applying advanced evaluation techniques like the drift correction described in chapter 6 even to older data-sets, thereby improving results without the necessity to repeat measurements.

Recently, the digital spectrometer was also successfully used for measurements investigating properties of MAX phases using ${ }^{181} \mathrm{Hf}$ probes. These measurements were presented by Jürgens (2013). 

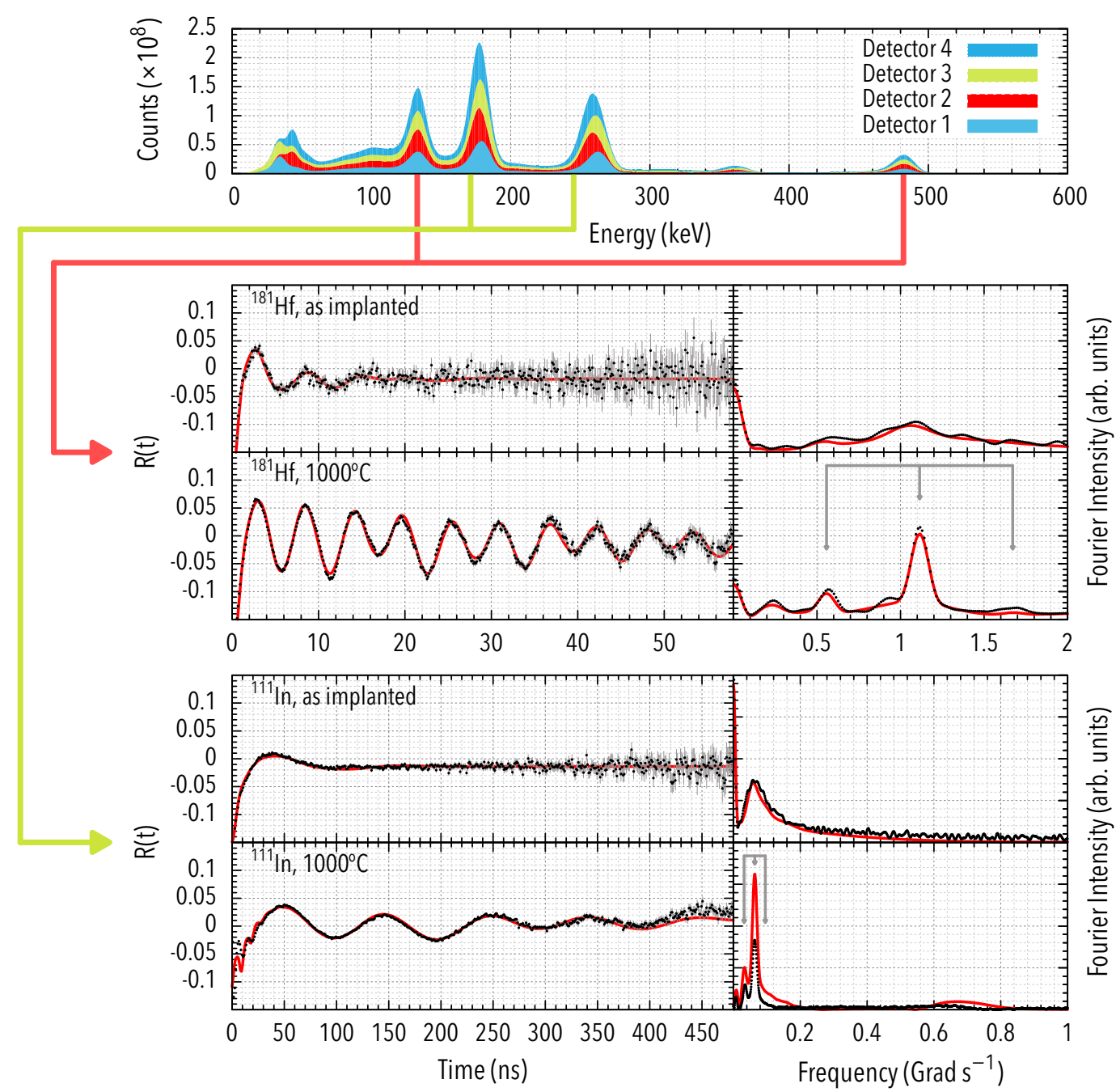

Figure 8.5: Simultaneous evaluation of ${ }^{181} \mathrm{Hf}$ and ${ }^{111} \mathrm{In}$ probe populations, which were implanted into an AlN sample. Both spectra originate from a single measurement. The upper energy spectrum shows the photo peaks that were selected for each of the probe nuclides' evaluation. The slight misalignment in case of the ${ }^{111}$ In related photo peaks is due to deviations from the linear energy calibration. Both probes indicate a defect rich environment after implantation which recovered after annealing. The quick oscillation in the beginning of the annealed ${ }^{111}$ In is explained as point defect trapped by the indium probes by Schmitz et al. (2009). 



\section{Chapter 9}

\section{Identification of new decay cascades for the TDPAC method ${ }^{1}$}

The flexible configuration and quick setup of digital TDPAC spectrometers, like the one presented in this work, raises hopes for the feasibility of measurements based on new candidate nuclides, opening a wider scope concerning e.g. valence, bonding behavior, and atomic radius than offered by the established probe nuclides. This would substantially extend the use cases of the TDPAC method towards new materials and scientific questions.

The task of identifying useful isotopes for TDPAC experiments is solvable due to the availability of comprehensive high quality nuclear structure databases like the ENSDF (Evaluated Nuclear Structure Data File 2012) and XUNDL (Experimental Unevaluated Nuclear Data List 2012). The machine readable form, in which data is accessible by means of these databases, makes it possible to automate large parts of the search tasks thereby reducing the risk of missing promising candidates.

Existing universal tools for the search in these databases like e.g. NuDat (Sonzogni, 2013) and the Live Chart of Nuclides (IAEA Nuclear Data Section, 2013) allow for a search of nuclides according to nuclear properties like $\mathrm{Q}$ values, $\gamma$ energy, or half-life. However, up to now, no solution existed for the search of nuclear decay cascades with properties suitable for TDPAC measurements. Particularly the calculation of angular correlation coefficients according to section 2.1.2 is not implemented in any of the universal tools although sufficiently high values for these coefficients are an important prerequisite considering the suitability of decay cascades for TDPAC and manual calculations are prone to errors.

\subsection{Software}

To address this issue, a software tool named Nuclei for the search and examination of decay cascades as well as helping in setting up TDPAC spectrometers during measurements based on ENSDF data was developed in the context of this work. This tool is licensed under the GPL and freely available for download from Nagl (2012-2014) and Barbosa and Correia (2012) in versions for Linux, MacOS X, and Windows.

\footnotetext{
${ }^{1}$ This chapter contains revised parts from Nagl, Barbosa, et al. (2013)
} 


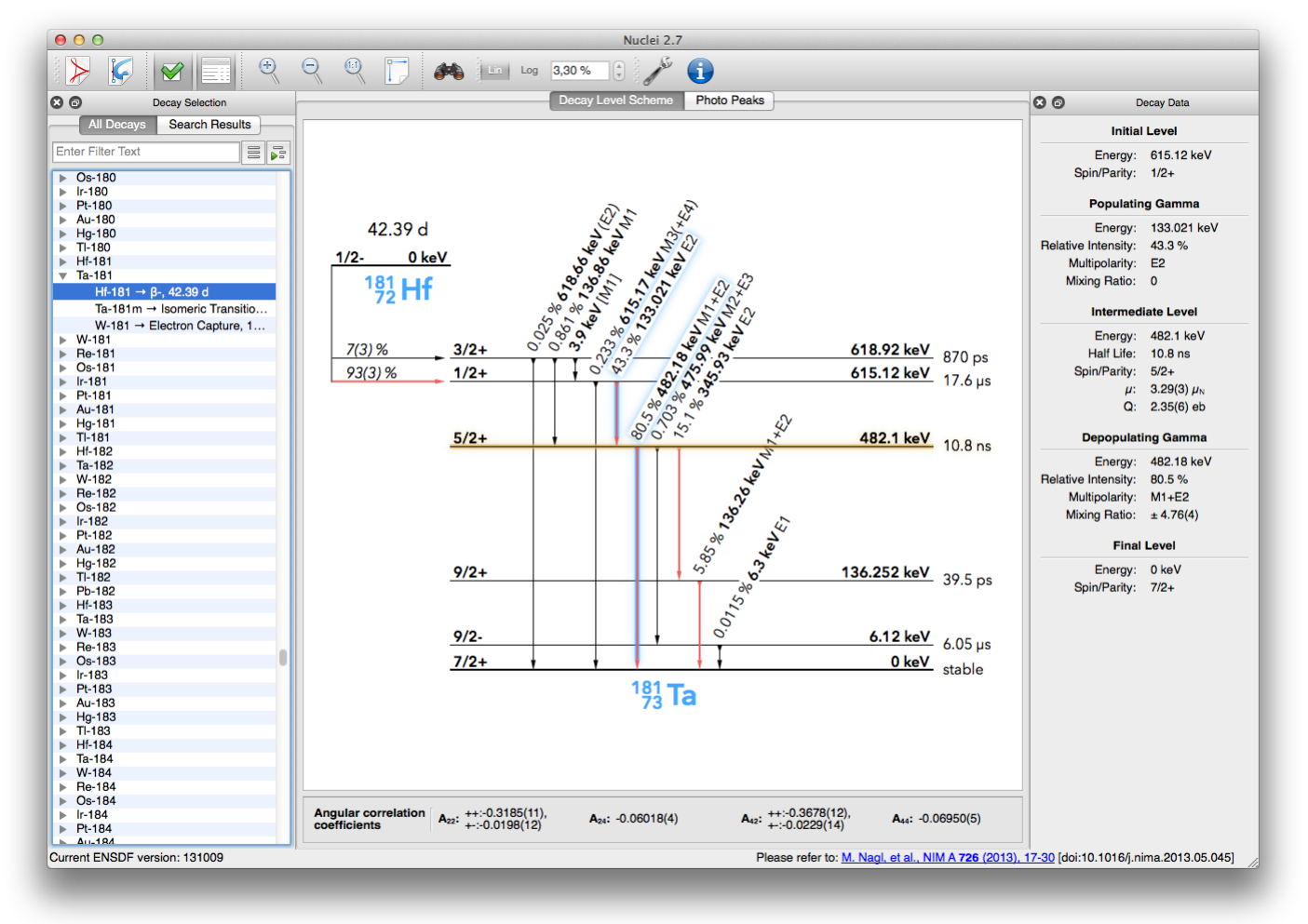

Figure 9.1: Screenshot of the decay level scheme view of Nuclei.

Edited results of three relevant search runs of this tool grouped by the parent nuclides' half-life including the most important parameters for TDPAC measurements are shown below in section 9.2.

Nucle $i$ uses mixing ratio and spin values from the ENSDF in order to calculate $B_{\Lambda}\left(\gamma_{1}\right)$ and $A_{\Lambda}\left(\gamma_{2}\right)$ as introduced in section 2.1.2 for each possible decay cascade. It then uses these results to determine $A_{22}, A_{24}, A_{42}$, and $A_{44}$ according to eq. (2.6).

It automatically downloads the most recent ENSDF database during its first startup. The downloaded files are then parsed to make relevant data accessible for automated processing.

Figure 9.1 shows the main window of the user interface of Nuclei. In the left part, a list of all daughter nuclides found in the ENSDF database is visible. After unfolding the sub-branch of a daughter nuclide, all available parent nuclides and decays become visible. If one of these decays is selected, the appropriate decay scheme is shown in the program window's central part.

In this decay scheme, two $\gamma$ transitions can be selected by mouse clicks. Detailed data for selected transitions and the intermediate level is shown in the program window's right 


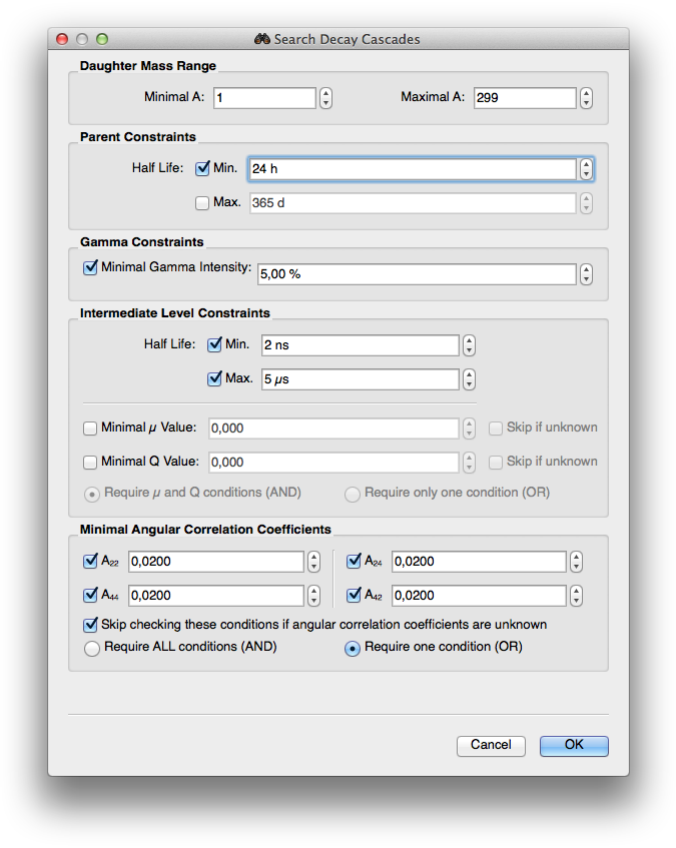

Figure 9.2: Screenshot of the search dialog of Nuclei.

part. As soon as a decay cascade (i.e. two $\gamma$ transitions with a common energy level) is selected, angular correlation coefficients are calculated according to section 2.1.2 using libAkk (Barbosa and Correia, 2012) and shown at the bottom of the central part. libAkk computes the 3-j and 6-j symbols from eq. (2.5) using the GNU Scientific Library (Galassi et al., 2009). Uncertainties from the ENSDF are propagated and shown as uncertainty in units of the least significant figure. Since possible correlations of the parameters' uncertainties are neglected, the resulting uncertainties can be considered as worst case estimates. In cases where no uncertainty value is available or the given value is "approximate" in the ENSDF, results are prefixed by a tilde $(\sim)$. If only upper or lower limits are given for mixing ratios, the values are considered unknown for the calculation of angular correlation coefficients.

Because experimental values for $A_{\lambda}$ and $B_{\lambda}$ are usually not contained in the ENSDF records, these values are calculated using $\delta$ values from the ENSDF and eqs. (2.3) and (2.4).

Nuclei's tool bar contains buttons, which allow for the export of decay schemes as PDF or SVG files including the highlighted decay path for easy utilization in publications (see e. g. figures in appendix A). Additional buttons allow opening and closing of both side panels containing decay selection as well as decay information. Four buttons are usable to adjust the zoom levels of decay schemes and photo peaks.

Figure 9.2 shows the search dialog available by clicking the tool button showing binoc- 


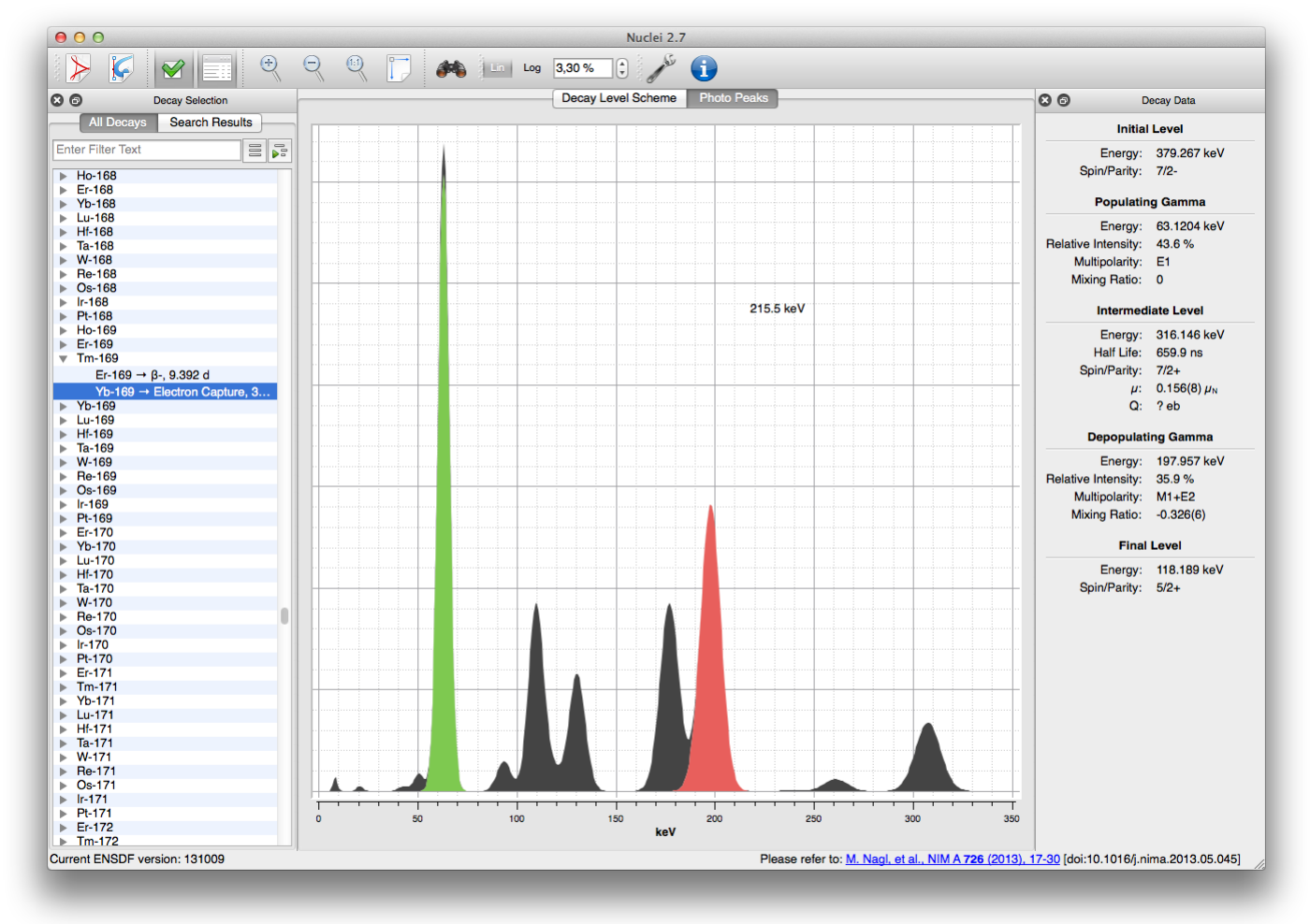

Figure 9.3: Screenshot of the photo peak view of Nuclei.

ulars in the main window. It allows defining limits for the parent nuclide's as well as intermediate level's half-life, magnetic dipole and/or electric quadrupole moments, angular correlation coefficients, $\gamma$ intensities and the mass range of the search. For nuclear moments and angular correlation coefficients, it is also selectable if checks should be skipped in case of unknown values i.e. if entries containing unknown values should be added to the search results as if the unknown value matched the criteria or if they should be ignored. For these properties, it is additionally selectable if all criteria must match or if it is sufficient if at least one matches. The results of a search run are afterwards shown instead of the nuclide list in the main window's left part.

For new TDPAC nuclides, the interpretation of energy spectra can be rather cumbersome and-much worse during a measurement-time consuming. Nuclei is able to show photo peak spectra for each selected decay in order to simplify this work. Figure 9.3 shows the spectrum for ${ }^{169} \mathrm{Yb}$ as an example. Compton scattering as well as pair production is ignored for these spectra to avoid detector specific behavior and keep it simple since the shown photo peaks are usually sufficient for the tuning of TDPAC setups.

If a $\gamma$ cascade was selected, the start and stop components are highlighted green and 
red, respectively, in the photo peak view. Other $\gamma$ contributions are plotted stacked onto the selected transitions in order to provide an idea about intensity relations.

Energy resolution as well as linear or logarithmic plot styles can be changed in the tool bar. Additionally, it is possible to change the font properties of decay schemes as well as the matching tolerance for decay data and adopted levels: Since ENSDF data consists of results from many different experiments, data sets are not always perfectly consistent. Especially information concerning nuclear moments is often only available from the adopted levels data sets and not from the decay data sets. To yield as complete output as possible, an automatic matching of these data sets was implemented. By default, deviations of up to $0.5 \%$ from the $\gamma$ energies and $4 \%$ from the level energies in the decay data set are tolerated and the closest matches are chosen. The algorithm evaluates the XREF records as described in the ENSDF manual (Tuli, 2001) in case of energy level matching. Therefore, the maximal tolerance can be set higher in case of level matching compared to $\gamma$ matching since the XREF mechanism provides additional protection against matching of wrong pairs. Unfortunately, XREF records alone are not sufficient and have to be complemented by a search for the closest level because they do not provide exact energy matching information in most cases.

\subsection{Candidate nuclides}

In this section, results of the search for TDPAC candidate nuclides using Nuclei as described in section 9.1 are presented.

\subsubsection{Categories}

The search results are grouped according to the parent nuclides' half-life periods because this property makes a big difference concerning feasibility of measurements using different setups. There are three groups:

Off-site contains parent nuclides with a half-life longer than $24 \mathrm{~h}$. These nuclides can reasonably be produced at one site (e.g. ISOLDE) and used for measurements at another site. Furthermore, it is possible to apply processing steps common to materials science or biochemistry like annealing or dissolving and introducing into biological systems after probe production and implantation.

On-site consists of parent nuclides with a half-life between $10 \mathrm{~min}$ and $24 \mathrm{~h}$. These nuclides can be transported between production and measurement and allow for limited processing as described for off-site candidates but under normal conditions it is not feasible to transport them across long distances.

Online contains parent nuclides with a half-life shorter than $10 \mathrm{~min}$. For these nuclides measurements should take place in the same chamber as implantation as there 
would hardly be enough time for a transfer between implantation chamber and measurement setup. Special combined TDPAC and implantation setups, as described in section 10.1.4, are necessary for this kind of measurements. Since implantation and measurement usually occur simultaneously, intermediate processing is not possible. It is however possible to implant into and measure heated or cooled samples.

\subsubsection{Parameters}

The candidate tables contain the following columns:

\section{Decay Parameters}

Daughter The daughter nuclide

Parent The parent nuclide

Half-Life The parent nuclide's half-life

Intermediate Level Parameters

Energy The energy of the intermediate level of each decay cascade in $\mathrm{keV}$

Half-Life The intermediate level's half-life

Spin-Parity The intermediate level's spin and parity

Q The intermediate level's electric quadrupole moment (in electron-barns)

$\boldsymbol{\mu}$ The intermediate level's magnetic dipole moment (in units of the nuclear magneton $\left.\mu_{\mathrm{N}}\right)$

\section{Cascade Parameters}

Initial Energy Energy of the cascade's initial level in keV above the ground state

Final Energy Energy of the cascade's final level in $\mathrm{keV}$ above the ground state

\section{Angular correlation coefficients}

Parameter Sign Combination In cases where the sign of one or both mixing ratios of the populating and depopulating $\gamma$ transitions was undefined, angular correlation coefficients for all possible combinations were computed. This field contains the combination of signs used to compute the values in the respective row. The upper 
sign is the one, which was used for the populating $\gamma$ 's mixing ratio while the lower sign was used for the depopulating $\gamma$ 's mixing ratio. If one of the signs was defined in the database, only the other one was varied. In cases where both signs were defined, this field remains empty

$\boldsymbol{A}_{k_{1} k_{2}}$ These four fields contain the computed angular correlation coefficients

\subsubsection{Constraints}

Search constraints had to be defined for the candidate table. The selected choice tries to achieve a good compromise between completeness and conciseness by choosing values discussed below. For each parameter, the corresponding values of the most commonly used TDPAC daughter nuclides— ${ }^{111} \mathrm{Cd}$ and ${ }^{181} \mathrm{Ta}$ - are specified as an example.

For all groups, the intermediate half-life was restricted to the range between $2 \mathrm{~ns}$ and $5 \mu \mathrm{s}$. Half-life values below the lower bound make measurements difficult because the difference between the time resolution limit of PAC setups and the intermediate state's life-time limit would allow only for a small range of frequencies to be measurable. For half-life values above the upper bound, the needed number of decays for a successful measurement grows disproportional as only a small number falls into a given interval of time. Therefore, the signal to noise ratio becomes increasingly problematic. Intermediate half-life values of ${ }^{111} \mathrm{Cd}$ and ${ }^{181} \mathrm{Ta}$ are $84.5 \mathrm{~ns}$ and $10.8 \mathrm{~ns}$.

Because nuclear moments are still missing in the ENSDF for many intermediate states, no restrictions were defined for these properties. Hopefully, the results of this search will motivate the determination of additional nuclear moments. The information about moments in the ENSDF is distributed between the decay and adopted levels records. These two sources for information can only be matched by means of the level energies. As energies originate from different sources, they are not perfectly equal in most cases. To make information from adopted levels records available, Nuclei uses a fuzzy matching, which was limited to a maximal energy difference of $4 \%$ by default (in conjunction with XREF filtering, compare section 9.1) for the candidate search. In tables 9.1 to 9.3, unknown moments are flagged by question marks. The electric quadrupole moment values of the most commonly used TDPAC levels of ${ }^{111} \mathrm{Cd}$ and ${ }^{181} \mathrm{Ta}$ are $0.77 \mathrm{eb}$ and $2.35 \mathrm{eb}$. The corresponding magnetic moments are $-0.766 \mu_{\mathrm{N}}$ and $3.29 \mu_{\mathrm{N}}$. If available from Stone (2012), Firestone and Shirley (1996), or Mertzimekis (2013), nuclear moments missing in the ENSDF data were added. These cases are highlighted in red and followed by a reference to the particular source.

Angular correlation coefficient filtering was activated and the chosen lower limit was 0.02 for the absolute value of all $A_{k_{1} k_{2}}$ as defined in eq. (2.6) and computed by Nuclei. A single absolute value above 0.02 was considered sufficient to add an entry to the search results. Off-site candidates were added to the table even in cases where $A_{k_{1} k_{2}}$ values could not be calculated due to unknown parameters. For on-site and online candidates, these 
cases were filtered. $A_{22}$ values of the most common decay cascades of ${ }^{111} \mathrm{Cd}$ and ${ }^{181} \mathrm{Ta}$ are -0.178 and -0.319 . For ${ }^{181} \mathrm{Ta}$, the table contains two $A_{k_{1} k_{2}}$ values as one of the mixing ratios' signs is missing in the ENSDF although it is well known (Wichert and Recknagel, 1986).

The $\boldsymbol{\gamma}$ intensity of all transitions involved in the decay cascades was limited to at least $3 \%$ for off-site candidates, $3 \%$ for on-site candidates, and $5 \%$ for online candidates as off-site and on-site measurements are generally less limited in terms of measurement time whereby online measurements need a better coincidence rate and thus more $\gamma$ intensity to become feasible. Using the ENSDF normalization records, intensities are calculated as the ratio of emitted $\gamma$ photons to the number of decayed parent atoms, i. e. the absolute $\gamma$ intensity.

\subsubsection{Limitations}

A search based on the ENSDF is of course limited by the integrity and quality of data available from this database. Fortunately, the ENSDF is actively maintained and probably the best source for nuclear data available today. It is, however, advisable to verify results of particular interest.

The following tables are based on an ENSDF snapshot from 2013-10-09.

A few well-known but missing nuclear moments were added manually to the result table. These values are highlighted.

Nuclides that were already used for TDPAC measurements but are missing in the candidate tables most likely did not match the search criteria. The definition of these constraints is based on practical considerations concerning today's TDPAC setups. 
Table 9.1: Off-site candidates (parent half-life: $t_{1 / 2}>24 \mathrm{~h}$, minimal $\gamma$ intensity: $3 \%$, intermediate level's half-life: $2 \mathrm{~ns}<t_{1 / 2}<5 \mu \mathrm{s}$, lower angular correlation coefficient limit: 0.02 if known). The column $\star$ contains the sign combination of mixing ratios used for the computation of results contained in the particular row (compare section 9.2.2).

\begin{tabular}{|c|c|c|c|c|c|c|c|c|c|c|c|c|c|c|}
\hline \multirow{3}{*}{ Daughter } & \multicolumn{2}{|l|}{ Decay } & \multicolumn{5}{|c|}{ Intermediate } & \multirow{3}{*}{$\begin{array}{c}\text { Initial } \\
\text { Energy } \\
\mathrm{keV}\end{array}$} & \multirow{3}{*}{$\begin{array}{c}\frac{\text { Final }}{\text { Energy }} \\
\mathrm{keV}\end{array}$} & \multicolumn{5}{|c|}{ Angular correlation coefficients } \\
\hline & Parent & Half-Life & Energy & Half-life & Spin/Parity & Q & $\mu$ & & & $\star$ & $A_{22}$ & $A_{24}$ & $A_{42}$ & $A_{44}$ \\
\hline & & & $\mathrm{keV}$ & & & $\mathrm{eb}$ & $\mu_{\mathrm{N}}$ & & & & & & & \\
\hline${ }^{44} \mathrm{Sc}$ & ${ }^{44} \mathrm{Ti}$ & $59.1 \mathrm{a}$ & 67.868 & $154.8 \mathrm{~ns}$ & $1-$ & $\pm 0.21(2)$ & $0.342(6)$ & 146.191 & 0 & & 0.05 & 0 & 0 & c \\
\hline \multirow[t]{2}{*}{${ }^{72} \mathrm{Ga}$} & ${ }^{72} \mathrm{Zn}$ & $46.5 \mathrm{~h}$ & 16.4 & $39.2 \mathrm{~ns}$ & $2-$ & $?$ & $?$ & 161.1 & 0 & & 0.05 & 0 & 0 & c \\
\hline & & & & & & & & 207.9 & 0 & & 0.05 & 0 & 0 & ( \\
\hline \multirow[t]{2}{*}{${ }^{83} \mathrm{Kr}$} & ${ }^{83} \mathrm{Rb}$ & $86.2 \mathrm{~d}$ & 9.4051 & $155.1 \mathrm{~ns}$ & $7 / 2+$ & $0.495(10)$ & $-0.943(2)$ & 561.957 & 0 & $\left(\begin{array}{l}+ \\
+\end{array}\right)$ & $0.0563(4)$ & $9.6(12) \cdot 10^{-6}$ & 0 & c \\
\hline & & & & & & & & & & $(\stackrel{ \pm}{ \pm})$ & $0.0437(4)$ & $9.6(12) \cdot 10^{-6}$ & 0 & c \\
\hline${ }^{99} \mathrm{Tc}$ & ${ }^{99} \mathrm{Mo}$ & $2.749 \mathrm{~d}$ & 181.094 & $3.44 \mathrm{~ns}$ & $5 / 2+$ & $?$ & $3.48(4)$ & 920.637 & 0 & & $0.103(4)$ & $7.6(8) \cdot 10^{-3}$ & $0.119(5)$ & $8.8(9) \cdot 10^{-3}$ \\
\hline${ }^{99} \mathrm{Ru}$ & ${ }^{99} \mathrm{Rh}$ & $16.1 \mathrm{~d}$ & 89.76 & $20.5 \mathrm{~ns}$ & $3 / 2+$ & $0.231(12)$ & $-0.284(6)$ & 618.09 & 0 & & $-0.254(4)$ & 0 & 0 & c \\
\hline \multirow[t]{2}{*}{${ }^{100} \mathrm{Rh}$} & ${ }^{100} \mathrm{Pd}$ & $3.63 \mathrm{~d}$ & 74.78 & $214 \mathrm{~ns}$ & $(2+)$ & $?$ & $4.324(8)$ & 158.8 & 32.68 & & $?$ & $?$ & $?$ & \\
\hline & & & & & & & & 158.8 & 0 & & 0.175 & 0 & 0 & c \\
\hline \multirow[t]{2}{*}{${ }^{106} \mathrm{Pd}$} & ${ }^{106} \mathrm{Ag}$ & $8.28 \mathrm{~d}$ & 2305.75 & $2 \mathrm{~ns}$ & $4-$ & $?$ & ? & 2756.85 & 2084.06 & & $0.082(6)$ & $1.2(4) \cdot 10^{-3}$ & 0 & c \\
\hline & & & & & & & & 2756.85 & 1557.71 & & 0.05 & 0 & 0 & c \\
\hline${ }^{111} \mathrm{Cd}$ & ${ }^{111}$ In & $2.8047 \mathrm{~d}$ & 245.35 & $84.5 \mathrm{~ns}$ & $5 / 2+$ & $0.77(12)$ & $-0.7656(25)$ & 416.63 & 0 & & $-0.1782(22)$ & $-0.206(3)$ & $-1.28(5) \cdot 10^{-3}$ & $-1.47(6) \cdot 10^{-3}$ \\
\hline${ }^{120} \mathrm{Sn}$ & ${ }^{120} \mathrm{Sb}$ & $5.76 \mathrm{~d}$ & 2284.9 & $5.55 \mathrm{~ns}$ & 5- & $\pm 0.033(2)$ & $-0.280(25)$ & 2482.2 & 2195.1 & & -0.07143 & 0 & -0.011 & c \\
\hline${ }^{126} \mathrm{Sb}$ & ${ }^{126} \mathrm{Sn}_{\mathrm{n}}$ & $230000 \mathrm{a}$ & 104.6 & $553 \mathrm{~ns}$ & $(3+)$ & $?$ & $?$ & 127.9 & 17.7 & & -0.07143 & -0.007034 & 0 & c \\
\hline \multirow[t]{2}{*}{${ }^{131}{ }_{I}$} & ${ }^{131} \mathrm{Te}$ & $33.25 \mathrm{~h}$ & 1797.08 & $5.9 \mathrm{~ns}$ & $9 / 2-, 11 / 2-, 13 / 2-$ & $\sim \pm 0.65$ & $-1.2(4)$ & 1899.14 & 1596.45 & & $?$ & $?$ & ? & \\
\hline & & & & & & & & 1899.14 & 1556.16 & & $?$ & $?$ & $?$ & \\
\hline${ }^{132} \mathrm{I}$ & ${ }^{132} \mathrm{Te}$ & $3.204 \mathrm{~d}$ & 49.72 & $7.14 \mathrm{~ns}$ & $3+$ & $\pm 0.20(7)$ & $2.06(18)^{1}$ & 277.86 & 0 & & -0.07143 & 0 & -0.06448 & c \\
\hline \multirow[t]{2}{*}{${ }^{133} \mathrm{Cs}$} & ${ }^{133} \mathrm{Ba}$ & $10.551 \mathrm{a}$ & 80.9979 & $6.283 \mathrm{~ns}$ & $5 / 2+$ & $-0.33(2)$ & $3.45(2)$ & 383.849 & 0 & $\left(\begin{array}{l}+ \\
+\end{array}\right)$ & $0.146(13)$ & $1.19(13) \cdot 10^{-3}$ & $1.2(22) \cdot 10^{-4}$ & $1(18) \cdot 10^{-6}$ \\
\hline & & & & & & & & & & $( \pm)$ & $-0.032(4)$ & $1.19(13) \cdot 10^{-3}$ & $-3(5) \cdot 10^{-5}$ & $1(18) \cdot 10^{-6}$ \\
\hline
\end{tabular}

Continued on next page 
Table 9.1: Off-site candidates (continued)

\begin{tabular}{|c|c|c|c|c|c|c|c|c|c|c|c|c|c|c|}
\hline \multicolumn{3}{|c|}{ Decay } & \multicolumn{5}{|c|}{ Intermediate } & \multirow{2}{*}{$\begin{array}{c}\text { Initial } \\
\text { Energy } \\
\mathrm{keV}\end{array}$} & \multirow{2}{*}{$\begin{array}{c}\frac{\text { Final }}{\text { Energy }} \\
\text { keV }\end{array}$} & \multicolumn{5}{|c|}{ Angular correlation coefficients } \\
\hline Daughter & Parent & Half-Life & $\begin{array}{c}\text { Energy } \\
\mathrm{keV}\end{array}$ & Half-life & Spin/Parity & $\begin{array}{l}\mathrm{Q} \\
\mathrm{eb}\end{array}$ & $\begin{array}{c}\mu \\
\mu_{\mathrm{N}}\end{array}$ & & & $\star$ & $A_{22}$ & $A_{24}$ & $A_{42}$ & $A_{44}$ \\
\hline & & & & & & & & 437.011 & 0 & $\begin{array}{l}(+) \\
+ \\
( \pm)\end{array}$ & $\begin{array}{r}-0.188(4) \\
0.041(3)\end{array}$ & $\begin{array}{l}-1.53(9) \cdot 10^{-3} \\
-1.53(9) \cdot 10^{-3}\end{array}$ & $\begin{array}{r}-0.217(4) \\
0.047(4)\end{array}$ & $\begin{array}{l}-1.77(11) \cdot 10^{-3} \\
-1.77(11) \cdot 10^{-3}\end{array}$ \\
\hline${ }^{140} \mathrm{Ce}$ & ${ }^{140} \mathrm{La}$ & $40.2852 \mathrm{~h}$ & 2083.26 & $3.474 \mathrm{~ns}$ & $4+$ & $\pm 0.35(7)$ & $4.35(10)$ & 2412.02 & 1596.24 & & $-0.099(5)$ & $-0.067(3)$ & $-6.5(16) \cdot 10^{-4}$ & $-4.4(11) \cdot 10^{-4}$ \\
\hline \multirow[t]{2}{*}{${ }^{143} \operatorname{Pr}$} & ${ }^{143} \mathrm{Ce}$ & $33.039 \mathrm{~h}$ & 57.356 & $4.14 \mathrm{~ns}$ & $5 / 2+$ & $?$ & $3.4(1)$ & 350.622 & 0 & & $0.203(12)$ & $1.9(8) \cdot 10^{-4}$ & $0.049(9)$ & $4.7(21) \cdot 10^{-5}$ \\
\hline & & & & & & & & 721.923 & 0 & & $?$ & $?$ & $?$ & ? \\
\hline \multirow[t]{8}{*}{${ }^{147} \mathrm{Eu}$} & ${ }^{147} \mathrm{Gd}$ & $38.06 \mathrm{~h}$ & 625.27 & $765 \mathrm{~ns}$ & $11 / 2-$ & $?$ & $7.05(3)$ & 995.17 & 229.323 & & $-0.171(22)$ & $-0.093(12)$ & $-1.1(9) \cdot 10^{-3}$ & $-6(5) \cdot 10^{-4}$ \\
\hline & & & & & & & & 995.17 & 0 & & $-0.30(4)$ & $0.044(6)$ & $-1.9(16) \cdot 10^{-3}$ & $2.9(24) \cdot 10^{-4}$ \\
\hline & & & & & & & & 1244.31 & 229.323 & & $0.070(19)$ & $0.038(10)$ & 0 & 0 \\
\hline & & & & & & & & 1244.31 & 0 & & $0.12(3)$ & $-0.018(5)$ & 0 & 0 \\
\hline & & & & & & & & 1554.29 & 229.323 & $\left(\begin{array}{l}+ \\
+\end{array}\right)$ & $-0.43(3)$ & $-0.233(17)$ & $-0.06(3)$ & $-0.034(14)$ \\
\hline & & & & & & & & & & $\left(\begin{array}{l}- \\
+\end{array}\right)$ & $0.25(6)$ & $0.14(3)$ & $-0.06(3)$ & $-0.034(14)$ \\
\hline & & & & & & & & 1554.29 & 0 & $\left(\begin{array}{l}+ \\
+\end{array}\right)$ & $-0.75(5)$ & $0.111(8)$ & $-0.11(5)$ & $0.016(7)$ \\
\hline & & & & & & & & & & $\left(\begin{array}{l}- \\
+\end{array}\right)$ & $0.44(10)$ & $-0.066(15)$ & $-0.11(5)$ & $0.016(7)$ \\
\hline${ }^{149} \mathrm{Eu}$ & ${ }^{149} \mathrm{Gd}$ & $9.28 \mathrm{~d}$ & 496.386 & $2.45 \mu \mathrm{s}$ & $11 / 2$ & $?$ & $7.0(3)$ & 795.044 & 149.732 & & $-0.181(19)$ & $-0.187(23)$ & $-4.0(11) \cdot 10^{-3}$ & $-4.1(12) \cdot 10^{-3}$ \\
\hline \multirow[t]{6}{*}{${ }^{153} \mathrm{Eu}$} & ${ }^{153} \mathrm{Sm}$ & $46.5 \mathrm{~h}$ & 103.18 & $3.8 \mathrm{~ns}$ & $3 / 2+$ & $\pm 1.254(13)$ & $2.048(6)$ & 172.853 & 83.3673 & $\left(\begin{array}{l}+ \\
+\end{array}\right)$ & $7.6(6) \cdot 10^{-3}$ & 0 & 0 & 0 \\
\hline & & & & & & & & & & $\left(\begin{array}{l}- \\
+\end{array}\right)$ & $-0.0375(7)$ & 0 & 0 & 0 \\
\hline & & & & & & & & 172.853 & 0 & $\left(\begin{array}{l}+ \\
+\end{array}\right)$ & $-0.0129(10)$ & 0 & 0 & 0 \\
\hline & & & & & & & & & & $(\stackrel{+}{-})$ & $1.88(23) \cdot 10^{-3}$ & 0 & 0 & 0 \\
\hline & & & & & & & & & & $\left(\begin{array}{l}- \\
+\end{array}\right)$ & $0.0637(15)$ & 0 & 0 & 0 \\
\hline & & & & & & & & & & $(\overline{-})$ & $-9.3(9) \cdot 10^{-3}$ & 0 & 0 & 0 \\
\hline \multirow[t]{2}{*}{${ }^{153} \mathrm{Gd}$} & ${ }^{153} \mathrm{~Tb}$ & $2.34 \mathrm{~d}$ & 41.54 & $4.08 \mathrm{~ns}$ & $5 / 2-$ & $?$ & $?$ & 212.012 & 0 & $\left(\begin{array}{l}+ \\
+\end{array}\right)$ & $-0.046(7)$ & $0.0167(12)$ & 0 & 0 \\
\hline & & & & & & & & & & $(\stackrel{+}{-})$ & $0.300(5)$ & $0.0167(12)$ & 0 & 0 \\
\hline${ }^{156} \mathrm{Gd}$ & ${ }^{156} \mathrm{Eu}$ & $15.19 \mathrm{~d}$ & 88.966 & $2.2 \mathrm{~ns}$ & $2+$ & $-1.93(4)$ & $0.774(8)$ & 1154.13 & 0 & & $0.2490(6)$ & $0.4455(11)$ & 0 & 0 \\
\hline
\end{tabular}

Continued on next page 
Table 9.1: Off-site candidates (continued)

\begin{tabular}{|c|c|c|c|c|c|c|c|c|c|c|c|c|c|c|}
\hline \multicolumn{3}{|c|}{ Decay } & \multicolumn{5}{|c|}{ Intermediate } & \multirow{2}{*}{$\begin{array}{c}\text { Initial } \\
\text { Energy } \\
\mathrm{keV}\end{array}$} & \multirow{2}{*}{$\begin{array}{c}\frac{\text { Final }}{\text { Energy }} \\
\mathrm{keV}\end{array}$} & \multicolumn{5}{|c|}{ Angular correlation coefficients } \\
\hline Daughter & Parent & Half-Life & $\begin{array}{c}\text { Energy } \\
\mathrm{keV}\end{array}$ & Half-life & Spin/Parity & $\begin{array}{l}\mathrm{Q} \\
\mathrm{eb}\end{array}$ & $\begin{array}{c}\mu \\
\mu_{\mathrm{N}}\end{array}$ & & & $\star$ & $A_{22}$ & $A_{24}$ & $A_{42}$ & $A_{44}$ \\
\hline & & & & & & & & 1168.14 & 0 & & 0.3571 & 0.6389 & 0.6389 & 1.143 \\
\hline & & & & & & & & 1242.47 & 0 & & -0.25 & -0.4472 & 0 & 0 \\
\hline & & & & & & & & 2186.74 & 0 & & $0.54(6)$ & $0.97(10)$ & $-0.23(8)$ & $-0.42(14)$ \\
\hline & ${ }^{156} \mathrm{~Tb}$ & $5.35 \mathrm{~d}$ & 88.967 & $2.21 \mathrm{~ns}$ & $2+$ & $-1.93(4)$ & $0.774(8)$ & 288.2 & 0 & & 0.102 & 0.1825 & 0.00507 & 0.00907 \\
\hline & & & & & & & & 1154.13 & 0 & & $0.2490(6)$ & $0.4455(11)$ & 0 & 0 \\
\hline & & & & & & & & 1248 & 0 & & $-0.269(4)$ & $-0.481(7)$ & $-0.04531(4)$ & $-0.08105(7)$ \\
\hline & & & & & & & & 1510.53 & 0 & & 0.102 & 0.1825 & 0.00507 & 0.00907 \\
\hline & & & & & & & & 1934.29 & 0 & & -0.07143 & -0.1278 & 0 & 0 \\
\hline \multirow[t]{2}{*}{${ }^{158} \mathrm{Gd}$} & ${ }^{158} \mathrm{~Tb}$ & $180 \mathrm{a}$ & 79.5132 & $2.52 \mathrm{~ns}$ & $2+$ & $-2.01(4)$ & $0.762(8)$ & 261.457 & 0 & & 0.102 & 0.1825 & 0.00507 & 0.00907 \\
\hline & & & & & & & & 1041.64 & 0 & & -0.07143 & -0.1278 & 0 & 0 \\
\hline \multirow[t]{3}{*}{${ }^{160} \mathrm{Dy}$} & ${ }^{160} \mathrm{~Tb}$ & $72.3 \mathrm{~d}$ & 86.7877 & $2.02 \mathrm{~ns}$ & $2+$ & $\pm 1.8(4)$ & $0.723(19)$ & 283.822 & 0 & & 0.102 & 0.1825 & 0.00507 & 0.00907 \\
\hline & & & & & & & & 966.169 & 0 & & $0.24910(5)$ & $0.44560(10)$ & 0 & 0 \\
\hline & & & & & & & & 1049.1 & 0 & & $-0.2598(12)$ & $-0.4647(21)$ & $-0.045396(10)$ & $-0.081206(18)$ \\
\hline \multirow[t]{2}{*}{${ }^{165} \mathrm{Er}$} & ${ }^{165} \mathrm{Tm}$ & $30.06 \mathrm{~h}$ & 47.16 & $4 \mathrm{~ns}$ & $5 / 2+$ & $?$ & $?$ & 507.429 & 0 & & $?$ & ? & $?$ & ? \\
\hline & & & & & & & & 853.514 & 0 & & $?$ & $?$ & $?$ & ? \\
\hline${ }^{168} \mathrm{Er}$ & ${ }^{168} \mathrm{Tm}$ & $93.1 \mathrm{~d}$ & 1094.04 & $109 \mathrm{~ns}$ & $4-$ & $?$ & $0.96(4)$ & 1541.55 & 895.794 & & $0.076(12)$ & $1.2(6) \cdot 10^{-3}$ & $2.6(6) \cdot 10^{-3}$ & $4.2(23) \cdot 10^{-5}$ \\
\hline \multirow[t]{5}{*}{${ }^{172} \mathrm{Yb}$} & ${ }^{172} \mathrm{Lu}$ & $6.7 \mathrm{~d}$ & 1172.39 & $8.33 \mathrm{~ns}$ & $3+$ & $\pm 2.9(4)$ & $0.65(4)$ & 1263.04 & 260.27 & & $-0.212(23)$ & $0.1144(22)$ & $-0.025(3)$ & $0.0137(3)$ \\
\hline & & & & & & & & 1263.04 & 78.7427 & & $0.32(3)$ & $0.571(5)$ & $0.038(4)$ & $0.0685(8)$ \\
\hline & & & & & & & & 1375.82 & 260.27 & & $0.048(5)$ & $-0.0260(5)$ & $4.8(5) \cdot 10^{-3}$ & $-2.56(5) \cdot 10^{-3}$ \\
\hline & & & & & & & & 1375.82 & 78.7427 & & $-0.072(7)$ & $-0.1300(11)$ & $-7.1(7) \cdot 10^{-3}$ & $-0.01281(11)$ \\
\hline & & & & & & & & 2073.12 & 78.7427 & & $0.017(5)$ & $0.030(8)$ & $2.4(7) \cdot 10^{-4}$ & $4.3(11) \cdot 10^{-4}$ \\
\hline \multirow[t]{2}{*}{${ }^{181} \mathrm{Ta}$} & ${ }^{181} \mathrm{Hf}$ & $42.39 \mathrm{~d}$ & 482.1 & $10.8 \mathrm{~ns}$ & $5 / 2+$ & $2.35(6)$ & $3.29(3)$ & 615.12 & 136.252 & & 0.102 & 0.007855 & 0.1178 & 0.00907 \\
\hline & & & & & & & & 615.12 & 0 & $\left(\begin{array}{l}+ \\
+\end{array}\right)$ & $-0.3185(11)$ & $-0.06018(4)$ & $-0.3678(12)$ & $-0.06950(5)$ \\
\hline
\end{tabular}


Table 9.1: Off-site candidates (continued)

\begin{tabular}{|c|c|c|c|c|c|c|c|c|c|c|c|c|c|c|}
\hline \multicolumn{3}{|c|}{ Decay } & \multicolumn{5}{|c|}{ Intermediate } & \multirow{2}{*}{$\begin{array}{c}\frac{\text { Initial }}{\text { Energy }} \\
\mathrm{keV}\end{array}$} & \multirow{2}{*}{$\begin{array}{c}\frac{\text { Final }}{\text { Energy }} \\
\mathrm{keV}\end{array}$} & \multicolumn{5}{|c|}{ Angular correlation coefficients } \\
\hline Daughter & Parent & Half-Life & $\begin{array}{c}\text { Energy } \\
\text { keV }\end{array}$ & Half-life & Spin/Parity & $\begin{array}{l}\mathrm{Q} \\
\mathrm{eb}\end{array}$ & $\begin{array}{c}\mu \\
\mu_{\mathrm{N}}\end{array}$ & & & $\star$ & $A_{22}$ & $A_{24}$ & $A_{42}$ & $A_{44}$ \\
\hline & & & & & & & & & & $( \pm)$ & $-0.0198(12)$ & $-0.06018(4)$ & $-0.0229(14)$ & $-0.06950(5)$ \\
\hline${ }^{187} \mathrm{Re}$ & ${ }^{187} \mathrm{~W}$ & $24 \mathrm{~h}$ & 206.252 & $555.3 \mathrm{~ns}$ & 9/2- & $\pm 3.04(5)$ & $5.11(9)$ & 685.797 & 134.247 & & $-0.137(9)$ & $-2(4) \cdot 10^{-5}$ & $-0.085(6)$ & $-1(3) \cdot 10^{-5}$ \\
\hline${ }^{194} \mathrm{Pt}$ & ${ }^{194} \mathrm{Ir}$ & $171 \mathrm{~d}$ & 1485.1 & $3.45 \mathrm{~ns}$ & (7-) & ? & $1.8(6)$ & 2047.5 & 1373.5 & & 0.102 & 0.04897 & 0.0189 & 0.00907 \\
\hline${ }^{198} \mathrm{Au}$ & ${ }^{198 \mathrm{~m}_{\mathrm{Au}}}$ & $2.272 \mathrm{~d}$ & 312.1 & $124 \mathrm{~ns}$ & $5+$ & $?$ & $-1.11(2)$ & 516.2 & 214.89 & & $0.096(23)$ & 0 & $7(6) \cdot 10^{-4}$ & 0 \\
\hline & & & & & & & & 645.92 & 214.89 & & -0.3 & 0 & 0.2309 & 0 \\
\hline${ }^{219} \mathrm{Rn}$ & ${ }^{223} \mathrm{Ra}$ & $11.43 \mathrm{~d}$ & 4.47 & $15.4 \mathrm{~ns}$ & $(9 / 2+)$ & $?$ & $?$ & 158.64 & 0 & & -0.131 & -0.08127 & 0 & 0 \\
\hline${ }^{237} \mathrm{U}$ & ${ }^{241} \mathrm{Pu}$ & $14.29 \mathrm{a}$ & 160 & $3.1 \mathrm{~ns}$ & $5 / 2+$ & ? & $?$ & 274 & 11.5 & $\left(\begin{array}{c}+ \\
+\end{array}\right)$ & $6.2(8) \cdot 10^{-3}$ & $2.62(9) \cdot 10^{-3}$ & 0 & 0 \\
\hline & & & & & & & & & & $( \pm)$ & $0.0896(6)$ & $2.62(9) \cdot 10^{-3}$ & 0 & 0 \\
\hline${ }^{246} \mathrm{Am}$ & ${ }^{246} \mathrm{Pu}$ & $10.84 \mathrm{~d}$ & 43.81 & $4.3 \mathrm{~ns}$ & $(1+)$ & ? & $?$ & 223.74 & 16.22 & & ? & ? & $?$ & ? \\
\hline
\end{tabular}

Table 9.2: On-site candidates (parent half-life: $10 \mathrm{~min}<t_{1 / 2}<24 \mathrm{~h}$, minimal $\gamma$ intensity: $3 \%$, intermediate level's half-life: $2 \mathrm{~ns}<t_{1 / 2}<$ $5 \mu \mathrm{s}$, lower angular correlation coefficient limit: 0.02 ). The column $\star$ contains the sign combination of mixing ratios used for the computation of results contained in the particular row (compare section 9.2.2).

\begin{tabular}{|c|c|c|c|c|c|c|c|c|c|c|c|c|c|c|}
\hline \multicolumn{3}{|c|}{ Decay } & \multicolumn{5}{|c|}{ Intermediate } & \multirow{2}{*}{$\begin{array}{c}\text { Initial } \\
\text { Energy } \\
\mathrm{keV}\end{array}$} & \multirow{2}{*}{$\begin{array}{c}\frac{\text { Final }}{\text { Energy }} \\
\mathrm{keV}\end{array}$} & \multicolumn{5}{|c|}{ Angular correlation coefficients } \\
\hline Daughter & Parent & Half-Life & $\begin{array}{c}\text { Energy } \\
\text { keV }\end{array}$ & Half-life & Spin/Parity & $\begin{array}{l}\mathrm{Q} \\
\mathrm{eb}\end{array}$ & $\begin{array}{c}\mu \\
\mu_{\mathrm{N}}\end{array}$ & & & $\star$ & $A_{22}$ & $A_{24}$ & $A_{42}$ & $A_{44}$ \\
\hline \multirow[t]{2}{*}{${ }^{28} \mathrm{Al}$} & ${ }^{28} \mathrm{Mg}$ & $20.915 \mathrm{~h}$ & 30.6383 & $2.07 \mathrm{~ns}$ & $2+$ & $?$ & $4.3(4)$ & 972.24 & 0 & & $-0.072(5)$ & $\left(-5 \cdot 10^{-8}\right)$ & $-0.129(8)$ & $\left(-8 \cdot 10^{-8}\right)$ \\
\hline & & & & & & & & 1620.1 & 0 & & $0.087(17)$ & $\left(6 \cdot 10^{-8}\right)$ & $3(3) \cdot 10^{-3}$ & $2(21) \cdot 10^{-9}$ \\
\hline \multirow[t]{2}{*}{${ }^{48} \mathrm{~V}$} & ${ }^{48} \mathrm{Cr}$ & $21.56 \mathrm{~h}$ & 308.24 & $7.09 \mathrm{~ns}$ & $2+$ & $?$ & $0.44(2)$ & 420.55 & 0 & $\left(\begin{array}{l}+ \\
+\end{array}\right)$ & $-0.077(9)$ & $-3.8(5) \cdot 10^{-3}$ & $-3(11) \cdot 10^{-5}$ & $-2(6) \cdot 10^{-6}$ \\
\hline & & & & & & & & & & $\left(\begin{array}{l}- \\
+\end{array}\right)$ & $-0.066(9)$ & $-3.3(5) \cdot 10^{-3}$ & $-3(11) \cdot 10^{-5}$ & $-2(6) \cdot 10^{-6}$ \\
\hline${ }^{62} \mathrm{Cu}$ & ${ }^{62} \mathrm{Zn}$ & $9.193 \mathrm{~h}$ & 40.8 & $4.57 \mathrm{~ns}$ & $2+$ & ? & $1.1(1)$ & 548.29 & 0 & & 0.175 & 0 & 0 & 0 \\
\hline
\end{tabular}

Continued on next page 
Table 9.2: On-site candidates (continued)

\begin{tabular}{|c|c|c|c|c|c|c|c|c|c|c|c|c|c|c|}
\hline \multicolumn{3}{|c|}{ Decay } & \multicolumn{5}{|c|}{ Intermediate } & \multirow{2}{*}{$\begin{array}{c}\text { Initial } \\
\text { Energy } \\
\text { keV }\end{array}$} & \multirow{2}{*}{$\begin{array}{c}\frac{\text { Final }}{\text { Energy }} \\
\mathrm{keV}\end{array}$} & \multicolumn{5}{|c|}{ Angular correlation coefficients } \\
\hline Daughter & Parent & Half-Life & $\begin{array}{c}\text { Energy } \\
\text { keV }\end{array}$ & Half-life & Spin/Parity & $\begin{array}{l}\mathrm{Q} \\
\mathrm{eb}\end{array}$ & $\begin{array}{c}\mu \\
\mu_{\mathrm{N}}\end{array}$ & & & $\star$ & $A_{22}$ & $A_{24}$ & $A_{42}$ & $A_{44}$ \\
\hline & & & & & & & & 637.45 & 0 & & 0.175 & 0 & 0 & 0 \\
\hline \multirow[t]{2}{*}{${ }^{66} \mathrm{Ga}$} & ${ }^{66} \mathrm{Ge}$ & $2.26 \mathrm{~h}$ & 43.81 & $18 \mathrm{~ns}$ & $1+$ & $?$ & $?$ & 234.065 & 0 & $\left(\begin{array}{l}+ \\
+\end{array}\right)$ & $-0.11(4)$ & 0 & 0 & 0 \\
\hline & & & & & & & & & & $\left(\begin{array}{l}- \\
+\end{array}\right)$ & $0.23(6)$ & 0 & 0 & 0 \\
\hline${ }^{73} \mathrm{As}$ & ${ }^{73} \mathrm{Se}$ & $7.15 \mathrm{~h}$ & 67.11 & $4.95 \mathrm{~ns}$ & $5 / 2$ & $\pm 0.356(12)$ & $1.63(10)$ & 427.66 & 0 & & $-0.057(4)$ & 0 & $-8.2(8) \cdot 10^{-3}$ & 0 \\
\hline \multirow[t]{2}{*}{${ }^{77} \mathrm{Br}$} & ${ }^{77} \mathrm{Kr}$ & $74.4 \mathrm{~m}$ & 129.63 & $9.3 \mathrm{~ns}$ & $5 / 2+$ & $\sim \pm 0.4$ & $3.30(3)$ & 276.21 & 0 & $\left(\begin{array}{c}+ \\
+\end{array}\right)$ & $0.29(3)$ & 0 & $0.016(8)$ & 0 \\
\hline & & & & & & & & & & $\left(\begin{array}{l}- \\
+\end{array}\right)$ & $-0.04(5)$ & 0 & $0.016(8)$ & 0 \\
\hline${ }^{83} \mathrm{Br}$ & ${ }^{83} \mathrm{Se}$ & $22.3 \mathrm{~m}$ & 1091.9 & $4.1 \mathrm{~ns}$ & $9 / 2+$ & $?$ & $?$ & 1810.07 & 866.71 & & $0.03(8)$ & $2(7) \cdot 10^{-4}$ & $4(6) \cdot 10^{-3}$ & $2(6) \cdot 10^{-5}$ \\
\hline${ }^{86} \mathrm{Sr}$ & ${ }^{86} \mathrm{Y}$ & $14.74 \mathrm{~h}$ & 2229.89 & $5 \mathrm{~ns}$ & $4+$ & $?$ & $?$ & 3055.9 & 1076.76 & & $-0.063(13)$ & $-0.043(9)$ & $-1(4) \cdot 10^{-5}$ & $\left(-8 \cdot 10^{-6}\right)$ \\
\hline${ }^{86} \mathrm{Y}$ & ${ }^{86} \mathrm{Zr}$ & $16.5 \mathrm{~h}$ & 242.8 & $28.5 \mathrm{~ns}$ & $2-$ & $?$ & $-1.06(6)$ & 271.9 & 0 & & -0.07143 & -0.003549 & 0 & 0 \\
\hline \multirow[t]{2}{*}{${ }^{106} \mathrm{Pd}$} & ${ }^{106} \mathrm{Rh}$ & $2.18333 \mathrm{~h}$ & 2306.78 & $2 \mathrm{~ns}$ & $4-$ & $?$ & $?$ & 2757.94 & 2085.07 & & $0.082(6)$ & $1.2(4) \cdot 10^{-3}$ & 0 & 0 \\
\hline & & & & & & & & 2757.94 & 1557.8 & & 0.05 & 0 & 0 & 0 \\
\hline${ }^{111} \mathrm{Cd}$ & ${ }^{111 \mathrm{~m}} \mathrm{Cd}$ & $48.54 \mathrm{~m}$ & 245.4 & $84.5 \mathrm{~ns}$ & $5 / 2+$ & $0.77(12)$ & $-0.7656(25)$ & 396.22 & 0 & & 0.1786 & 0.2062 & -0.003749 & -0.004329 \\
\hline \multirow[t]{6}{*}{${ }^{116} \mathrm{Sn}$} & ${ }^{116} \mathrm{Sb}$ & $60.3 \mathrm{~m}$ & 2365.94 & $350 \mathrm{~ns}$ & 5- & $\pm 0.26(1)$ & $-0.376(3)$ & 2773.3 & 2266.14 & & $-0.058(13)$ & $-0.034(8)$ & $-4(8) \cdot 10^{-5}$ & $-2(4) \cdot 10^{-5}$ \\
\hline & & & & & & & & 2773.3 & 1293.56 & & $-0.102(23)$ & $0.016(4)$ & $-7(13) \cdot 10^{-5}$ & $1.0(21) \cdot 10^{-5}$ \\
\hline & & & & & & & & 2908.81 & 2266.14 & & 0.102 & 0.05891 & 0.01571 & 0.00907 \\
\hline & & & & & & & & 2908.81 & 1293.56 & & 0.1786 & -0.02812 & 0.02749 & -0.004329 \\
\hline & & & & & & & & 3209.95 & 2266.14 & & 0.102 & 0.05891 & 0.01571 & 0.00907 \\
\hline & & & & & & & & 3209.95 & 1293.56 & & 0.1786 & -0.02812 & 0.02749 & -0.004329 \\
\hline${ }^{117}$ In & ${ }^{117} \mathrm{Cd}$ & $2.49 \mathrm{~h}$ & 659.763 & $53.6 \mathrm{~ns}$ & $3 / 2+$ & $-0.59(1)$ & $0.938(10)$ & 749.486 & 315.302 & & $-0.361(14)$ & 0 & 0 & 0 \\
\hline \multirow[t]{2}{*}{${ }^{118} \mathrm{Sn}$} & ${ }^{118} \mathrm{Sb}$ & $5 \mathrm{~h}$ & 2321.16 & $21.7 \mathrm{~ns}$ & 5- & $\pm 0.16(2)$ & $-0.300(25)$ & 2574.84 & 2280.35 & & -0.07143 & 0 & -0.011 & 0 \\
\hline & & & & & & & & 2574.84 & 1229.66 & & 0.1786 & -0.02812 & 0.02749 & -0.004329 \\
\hline \multirow[t]{2}{*}{${ }^{129} \mathrm{Cs}$} & ${ }^{129} \mathrm{Ba}$ & $2.23 \mathrm{~h}$ & 6.55 & $72 \mathrm{~ns}$ & $5 / 2+$ & $?$ & $?$ & 135.57 & 0 & $\left(\begin{array}{l}+ \\
+\end{array}\right)$ & $-0.38(4)$ & $-0.44(5)$ & $-0.015(7)$ & $-0.017(8)$ \\
\hline & & & & & & & & & & $\left(\begin{array}{l}- \\
+\end{array}\right)$ & $(0.007)$ & $(0.008)$ & $-0.015(7)$ & $-0.017(8)$ \\
\hline
\end{tabular}


Table 9.2: On-site candidates (continued)

\begin{tabular}{|c|c|c|c|c|c|c|c|c|c|c|c|c|c|c|}
\hline \multicolumn{3}{|c|}{ Decay } & \multicolumn{5}{|c|}{ Intermediate } & \multirow{2}{*}{$\begin{array}{c}\text { Initial } \\
\text { Energy } \\
\mathrm{keV}\end{array}$} & \multirow{2}{*}{$\begin{array}{c}\frac{\text { Final }}{\text { Energy }} \\
\text { keV }\end{array}$} & \multicolumn{5}{|c|}{ Angular correlation coefficients } \\
\hline Daughter & Parent & Half-Life & $\begin{array}{c}\text { Energy } \\
\text { keV }\end{array}$ & Half-life & Spin/Parity & $\begin{array}{l}\mathrm{Q} \\
\mathrm{eb}\end{array}$ & $\begin{array}{c}\mu \\
\mu_{\mathrm{N}}\end{array}$ & & & $\star$ & $A_{22}$ & $A_{24}$ & $A_{42}$ & $A_{44}$ \\
\hline & & & & & & & & 220.74 & 0 & $\left(\begin{array}{l}+ \\
+\end{array}\right)$ & $-0.55(15)$ & $-0.63(17)$ & $-0.08(12)$ & $-0.09(14)$ \\
\hline & & & & & & & & & & $\left(\begin{array}{l}- \\
+\end{array}\right)$ & $0.3(3)$ & $0.3(4)$ & $-0.08(12)$ & $-0.09(14)$ \\
\hline & ${ }^{129} \mathrm{Ba}$ & $2.16 \mathrm{~h}$ & 6.55 & $72 \mathrm{~ns}$ & $5 / 2+$ & $?$ & $?$ & 135.57 & 0 & $\left(\begin{array}{l}+ \\
+\end{array}\right)$ & $-0.38(4)$ & $-0.44(5)$ & $-0.015(7)$ & $-0.017(8)$ \\
\hline & & & & & & & & & & $\left(\begin{array}{l}- \\
+\end{array}\right)$ & $(0.007)$ & $(0.008)$ & $-0.015(7)$ & $-0.017(8)$ \\
\hline & & & & & & & & 188.93 & 0 & $\left(\begin{array}{l}+ \\
+\end{array}\right)$ & $0.097(11)$ & $0.112(13)$ & $-3.7(6) \cdot 10^{-3}$ & $-4.3(6) \cdot 10^{-3}$ \\
\hline & & & & & & & & & & $\left(\begin{array}{l}- \\
+\end{array}\right)$ & $-0.252(13)$ & $-0.291(15)$ & $-3.7(6) \cdot 10^{-3}$ & $-4.3(6) \cdot 10^{-3}$ \\
\hline & & & & & & & & 220.74 & 0 & $\left(\begin{array}{l}+ \\
+\end{array}\right)$ & $-0.55(15)$ & $-0.63(17)$ & $-0.08(12)$ & $-0.09(14)$ \\
\hline & & & & & & & & & & $\left(\begin{array}{l}- \\
+\end{array}\right)$ & $0.3(3)$ & $0.3(4)$ & $-0.08(12)$ & $-0.09(14)$ \\
\hline & & & & & & & & 426.47 & 0 & & 0.102 & 0.1178 & 0.007855 & 0.00907 \\
\hline & & & 188.93 & $2.26 \mathrm{~ns}$ & $7 / 2+$ & $?$ & $?$ & 648.42 & 6.55 & $\left(\begin{array}{l}+ \\
+\end{array}\right)$ & $0.031(8)$ & $-8.2(12) \cdot 10^{-3}$ & $3.6(9) \cdot 10^{-3}$ & $-9.5(14) \cdot 10^{-4}$ \\
\hline & & & & & & & & & & $(\stackrel{+}{-})$ & $-0.163(6)$ & $-8.2(12) \cdot 10^{-3}$ & $-0.0189(7)$ & $-9.5(14) \cdot 10^{-4}$ \\
\hline \multirow[t]{2}{*}{${ }^{130} \mathrm{Te}$} & ${ }^{130} \mathrm{Sb}$ & $39.5 \mathrm{~m}$ & 1815.24 & $9.8 \mathrm{~ns}$ & $(6+)$ & $?$ & $?$ & 2146.15 & 1632.91 & & $-0.027(4)$ & $-0.0140(19)$ & $-4.8(8) \cdot 10^{-4}$ & $-2.5(4) \cdot 10^{-4}$ \\
\hline & & & 2146.15 & $115 \mathrm{~ns}$ & $(7-)$ & $?$ & $?$ & 2404.2 & 1815.24 & & $0.091(15)$ & $1.6(4) \cdot 10^{-3}$ & $3.1(17) \cdot 10^{-3}$ & $5(3) \cdot 10^{-5}$ \\
\hline \multirow[t]{2}{*}{${ }^{130} \mathrm{Xe}$} & ${ }^{130} \mathrm{I}$ & $12.36 \mathrm{~h}$ & 1204.61 & $2 \mathrm{~ns}$ & $4+$ & $?$ & $1.7(2)^{1}$ & 1944.14 & 536.067 & & 0.102 & 0.06937 & 0.01334 & 0.00907 \\
\hline & & & 1944.14 & $2 \mathrm{~ns}$ & $6+$ & $?$ & $?$ & 2362.07 & 1204.61 & & $0.167(15)$ & $0.087(8)$ & $-0.032(4)$ & $-0.0168(20)$ \\
\hline${ }^{138} \mathrm{Ba}$ & ${ }^{138} \mathrm{Cs}$ & $33.41 \mathrm{~m}$ & 1898.71 & $2.164 \mathrm{~ns}$ & $4+$ & $?$ & $3.2(6)$ & 2445.72 & 1435.91 & & $-0.08(3)$ & $-0.055(17)$ & $-1.3(11) \cdot 10^{-3}$ & $-9(8) \cdot 10^{-4}$ \\
\hline \multirow[t]{5}{*}{${ }^{139} \mathrm{Pr}$} & ${ }^{139} \mathrm{Nd}$ & $5.5 \mathrm{~h}$ & 113.86 & $2.6 \mathrm{~ns}$ & $7 / 2+$ & $?$ & $\pm 1.19(21)$ & 822 & 0 & $\left(\begin{array}{l}+ \\
+\end{array}\right)$ & $(-0.007)$ & $-5(6) \cdot 10^{-3}$ & $\left(-7 \cdot 10^{-5}\right)$ & $-5(21) \cdot 10^{-5}$ \\
\hline & & & & & & & & & & $( \pm)$ & $-0.18(5)$ & $-5(6) \cdot 10^{-3}$ & $-2(8) \cdot 10^{-3}$ & $-5(21) \cdot 10^{-5}$ \\
\hline & & & & & & & & 851.96 & 0 & $\left(\begin{array}{l}+ \\
+\end{array}\right)$ & $(-0.009)$ & $-7(8) \cdot 10^{-3}$ & $(0.0008)$ & $6(7) \cdot 10^{-4}$ \\
\hline & & & & & & & & & & $(\stackrel{+}{-})$ & $-0.25(7)$ & $-7(8) \cdot 10^{-3}$ & $0.022(9)$ & $6(7) \cdot 10^{-4}$ \\
\hline & & & 822 & $36.8 \mathrm{~ns}$ & $11 / 2$ & ? & $6.60(5)$ & 1523.21 & 113.86 & & $-0.44(18)$ & $-0.10(7)$ & $-0.03(5)$ & $-7(11) \cdot 10^{-3}$ \\
\hline
\end{tabular}

Continued on next page

${ }^{1}$ Stone, 2012. 
Table 9.2: On-site candidates (continued)

\begin{tabular}{|c|c|c|c|c|c|c|c|c|c|c|c|c|c|c|}
\hline \multicolumn{3}{|c|}{ Decay } & \multicolumn{5}{|c|}{ Intermediate } & \multirow{2}{*}{$\begin{array}{c}\text { Initial } \\
\text { Energy } \\
\mathrm{keV}\end{array}$} & \multirow{2}{*}{$\begin{array}{c}\frac{\text { Final }}{\text { Energy }} \\
\mathrm{keV}\end{array}$} & \multicolumn{5}{|c|}{ Angular correlation coefficients } \\
\hline Daughter & Parent & Half-Life & $\begin{array}{c}\text { Energy } \\
\text { keV }\end{array}$ & Half-life & Spin/Parity & $\begin{array}{l}\mathrm{Q} \\
\mathrm{eb}\end{array}$ & $\begin{array}{c}\mu \\
\mu_{\mathrm{N}}\end{array}$ & & & $\star$ & $A_{22}$ & $A_{24}$ & $A_{42}$ & $A_{44}$ \\
\hline & & & 113.86 & $2.6 \mathrm{~ns}$ & $7 / 2+$ & $?$ & $\pm 1.19(21)$ & 2174.55 & 0 & $\left(\begin{array}{l}+ \\
+\end{array}\right)$ & $(0.003)$ & $2(3) \cdot 10^{-3}$ & 0 & 0 \\
\hline & & & & & & & & & & $( \pm)$ & $0.093(25)$ & $2(3) \cdot 10^{-3}$ & 0 & 0 \\
\hline${ }^{141} \mathrm{Pm}$ & ${ }^{141} \mathrm{Sm}$ & $22.6 \mathrm{~m}$ & 628.6 & $590 \mathrm{~ns}$ & $11 / 2$ & ? & ? & 1167.2 & 196.6 & & -0.1182 & -0.06433 & 0 & 0 \\
\hline & & & & & & & & 1313.2 & 196.6 & & $-0.407(6)$ & $-0.221(3)$ & $-0.042(4)$ & $-0.0227(24)$ \\
\hline & & & & & & & & 1414.8 & 196.6 & & $0.03(6)$ & $0.01(3)$ & $-8(6) \cdot 10^{-3}$ & $-4(3) \cdot 10^{-3}$ \\
\hline${ }^{149} \mathrm{Pm}$ & ${ }^{149} \mathrm{Nd}$ & $103.68 \mathrm{~m}$ & 114.311 & $2.53 \mathrm{~ns}$ & $5 / 2+$ & $?$ & $2.13(15)$ & 270.17 & 0 & & $0.047(4)$ & $3.9(10) \cdot 10^{-4}$ & 0 & 0 \\
\hline & & & 270.17 & $2.59 \mathrm{~ns}$ & $7 / 2$ & ? & $2.19(11)$ & 537.861 & 114.311 & & $0.24(3)$ & 0 & $0.011(6)$ & 0 \\
\hline & & & 114.311 & $2.53 \mathrm{~ns}$ & $5 / 2+$ & $?$ & $2.13(15)$ & 654.842 & 0 & & $0.047(4)$ & $3.9(10) \cdot 10^{-4}$ & 0 & 0 \\
\hline${ }^{151} \mathrm{Gd}$ & ${ }^{151} \mathrm{~Tb}$ & $17.609 \mathrm{~h}$ & 108.093 & $2.8 \mathrm{~ns}$ & $5 / 2-$ & $?$ & $-1.08(13)$ & 395.449 & 0 & & $-0.345(14)$ & $0.0361(15)$ & $-0.014(3)$ & $1.5(3) \cdot 10^{-3}$ \\
\hline & & & & & & & & 811.837 & 0 & & $0.050(17)$ & $-5.2(18) \cdot 10^{-3}$ & $-0.020(3)$ & $2.0(3) \cdot 10^{-3}$ \\
\hline & & & & & & & & 839.319 & 0 & & $0.25208(11)$ & $-0.0264(4)$ & $0.29107(13)$ & $-0.0304(4)$ \\
\hline${ }^{154} \mathrm{Eu}$ & ${ }^{154 \mathrm{~m}_{\mathrm{E}}} \mathrm{u}$ & $46 \mathrm{~m}$ & 100.88 & $50 \mathrm{~ns}$ & $4+$ & $?$ & $?$ & 136.8 & 0 & $\left(\begin{array}{l}+ \\
+\end{array}\right)$ & $8(9) \cdot 10^{-3}$ & 0 & $4.9(22) \cdot 10^{-4}$ & 0 \\
\hline & & & & & & & & & & $\left(\begin{array}{l}- \\
+\end{array}\right)$ & $0.093(9)$ & 0 & $4.9(22) \cdot 10^{-4}$ & 0 \\
\hline${ }^{156} \mathrm{Eu}$ & ${ }^{156} \mathrm{Sm}$ & $9.4 \mathrm{~h}$ & 87.58 & $12 \mathrm{~ns}$ & $1-$ & $?$ & $?$ & 125.68 & 0 & & 0.05 & 0 & 0 & 0 \\
\hline${ }^{157} \mathrm{Ho}$ & ${ }^{157} \mathrm{Er}$ & $18.65 \mathrm{~m}$ & 53.05 & $20 \mathrm{~ns}$ & $5 / 2+$ & $?$ & $?$ & 174.44 & 0 & & 0.05 & 0 & 0 & 0 \\
\hline${ }^{158} \mathrm{Gd}$ & ${ }^{158} \mathrm{Eu}$ & $45.9 \mathrm{~m}$ & 79.51 & $2.52 \mathrm{~ns}$ & $2+$ & $-2.01(4)$ & $0.762(8)$ & 1263.67 & 0 & & $-0.37(6)$ & $-0.66(11)$ & $-5(5) \cdot 10^{-3}$ & $-9(10) \cdot 10^{-3}$ \\
\hline${ }^{159} \mathrm{Dy}$ & ${ }^{159} \mathrm{Ho}$ & $33.05 \mathrm{~m}$ & 177.616 & $9.2 \mathrm{~ns}$ & $5 / 2+$ & ? & ? & 395.264 & 0 & & 0.05 & 0 & 0 & 0 \\
\hline${ }^{160} \mathrm{Dy}$ & ${ }^{160} \mathrm{Ho}$ & $25.6 \mathrm{~m}$ & 86.793 & $2.02 \mathrm{~ns}$ & $2+$ & $\pm 1.8(4)$ & $0.723(19)$ & 283.812 & 0 & & 0.102 & 0.1825 & 0.00507 & 0.00907 \\
\hline & & & & & & & & 966.172 & 0 & & $0.2485(11)$ & $0.4446(20)$ & 0 & 0 \\
\hline & & & & & & & & 1049.11 & 0 & & $-0.264(16)$ & $-0.47(3)$ & $-0.04536(15)$ & $-0.0811(3)$ \\
\hline & & & & & & & & 1155.85 & 0 & & 0.102 & 0.1825 & 0.00507 & 0.00907 \\
\hline & & & & & & & & 1285.61 & 0 & & -0.25 & -0.4472 & 0 & 0 \\
\hline & & & & & & & & 1286.72 & 0 & & $-0.0777(24)$ & $-0.139(4)$ & $-2.9(22) \cdot 10^{-6}$ & $-5(4) \cdot 10^{-6}$ \\
\hline
\end{tabular}


Table 9.2: On-site candidates (continued)

\begin{tabular}{|c|c|c|c|c|c|c|c|c|c|c|c|c|c|c|}
\hline \multicolumn{3}{|c|}{ Decay } & \multicolumn{5}{|c|}{ Intermediate } & \multirow{2}{*}{$\begin{array}{c}\text { Initial } \\
\text { Energy } \\
\mathrm{keV}\end{array}$} & \multirow{2}{*}{$\begin{array}{c}\frac{\text { Final }}{\text { Energy }} \\
\mathrm{keV}\end{array}$} & \multicolumn{5}{|c|}{ Angular correlation coefficients } \\
\hline Daughter & Parent & Half-Life & $\begin{array}{c}\text { Energy } \\
\text { keV }\end{array}$ & Half-life & Spin/Parity & $\begin{array}{l}\mathrm{Q} \\
\mathrm{eb}\end{array}$ & $\begin{array}{c}\mu \\
\mu_{\mathrm{N}}\end{array}$ & & & $\star$ & $A_{22}$ & $A_{24}$ & $A_{42}$ & $A_{44}$ \\
\hline & & & & & & & & 1398.97 & 0 & & $-0.02(4)$ & $-0.03(7)$ & $-2(3) \cdot 10^{-4}$ & $-4(6) \cdot 10^{-4}$ \\
\hline & & & & & & & & 1456.75 & 0 & & 0.3571 & 0.6389 & 0.6389 & 1.143 \\
\hline & & & & & & & & 1804.67 & 0 & & $0.53(9)$ & $0.95(16)$ & $-0.35(8)$ & $-0.62(14)$ \\
\hline & & & & & & & & 2630.71 & 0 & & $-0.28(10)$ & $-0.51(18)$ & $-4(23) \cdot 10^{-4}$ & $(-0.0007)$ \\
\hline & & & & & & & & 2701.04 & 0 & & $-0.22(15)$ & $-0.4(3)$ & $(-0.0004)$ & $(-0.0007)$ \\
\hline${ }^{162} \mathrm{Dy}$ & ${ }^{162} \mathrm{Ho}$ & $67 \mathrm{~m}$ & 80.67 & $2.25 \mathrm{~ns}$ & $2+$ & $?$ & $0.69(3)$ & 265.66 & 0 & & 0.102 & 0.1825 & 0.00507 & 0.00907 \\
\hline \multirow[t]{4}{*}{${ }^{164} \mathrm{Ho}$} & ${ }^{164 \mathrm{~m}} \mathrm{Ho}$ & $37.5 \mathrm{~m}$ & 37.34 & $2.8 \mathrm{~ns}$ & $2+$ & $?$ & $?$ & 93.98 & 0 & $\left(\begin{array}{l}+ \\
+\end{array}\right)$ & $0.017(5)$ & $8(4) \cdot 10^{-5}$ & $7(3) \cdot 10^{-5}$ & $3(2) \cdot 10^{-7}$ \\
\hline & & & & & & & & & & $(\stackrel{+}{-})$ & $0.026(7)$ & $8(4) \cdot 10^{-5}$ & $1.0(4) \cdot 10^{-4}$ & $3(2) \cdot 10^{-7}$ \\
\hline & & & & & & & & & & $\left(\begin{array}{l}- \\
+\end{array}\right)$ & $0.063(7)$ & $2.7(15) \cdot 10^{-4}$ & $7(3) \cdot 10^{-5}$ & $3(2) \cdot 10^{-7}$ \\
\hline & & & & & & & & & & $(\bar{z})$ & $0.094(8)$ & $2.7(15) \cdot 10^{-4}$ & $1.0(4) \cdot 10^{-4}$ & $3(2) \cdot 10^{-7}$ \\
\hline \multirow[t]{4}{*}{${ }^{171} \mathrm{Tm}$} & ${ }^{171} \mathrm{Er}$ & $7.516 \mathrm{~h}$ & 5.028 & $4.77 \mathrm{~ns}$ & $3 / 2+$ & $?$ & $?$ & 116.653 & 0 & $\left(\begin{array}{l}- \\
+\end{array}\right)$ & $0.1348(17)$ & 0 & 0 & 0 \\
\hline & & & & & & & & & & $(\overline{-})$ & $0.156(2)$ & 0 & 0 & 0 \\
\hline & & & & & & & & 129.044 & 0 & $\left(\begin{array}{l}+ \\
+\end{array}\right)$ & $-0.0662(3)$ & 0 & 0 & 0 \\
\hline & & & & & & & & & & $( \pm)$ & $-0.07656(24)$ & 0 & 0 & 0 \\
\hline${ }^{173} \mathrm{HF}$ & ${ }^{173} \mathrm{Ta}$ & $3.14 \mathrm{~h}$ & 107.15 & $180 \mathrm{~ns}$ & $5 / 2-$ & $?$ & $?$ & 197.4 & 0 & & -0.07143 & -0.08248 & 0 & 0 \\
\hline${ }^{177} \mathrm{Lu}$ & ${ }^{177} \mathrm{Yb}$ & $114.66 \mathrm{~m}$ & 150.25 & $130 \mathrm{~ns}$ & 9/2- & $?$ & $5.5(3)$ & 1230.73 & 0 & & 0.05 & 0 & 0 & 0 \\
\hline \multirow[t]{5}{*}{${ }^{177} \mathrm{Ta}$} & ${ }^{177} \mathrm{~W}$ & $2.2 \mathrm{~h}$ & 186.15 & $3.62 \mu \mathrm{s}$ & $5 / 2$ & ? & $2.05(13)^{1}$ & 372.57 & 0 & $\left(\begin{array}{l}+ \\
+\end{array}\right)$ & $0.1286(15)$ & 0 & $0.0138(8)$ & 0 \\
\hline & & & & & & & & & & $\left(\begin{array}{l}- \\
+\end{array}\right)$ & $-0.051(3)$ & 0 & $0.0138(8)$ & 0 \\
\hline & & & 70.47 & $70.2 \mathrm{~ns}$ & $5 / 2+$ & $?$ & $4.8(5)^{2}$ & 487.62 & 0 & $\left(\begin{array}{l}+ \\
+\end{array}\right)$ & $-0.405(11)$ & $-0.0142(12)$ & $-0.467(13)$ & $-0.0164(14)$ \\
\hline & & & & & & & & & & $( \pm)$ & $0.216(7)$ & $-0.0142(12)$ & $0.249(9)$ & $-0.0164(14)$ \\
\hline & & & & & & & & 497.41 & 0 & $\left(\begin{array}{l}+ \\
+\end{array}\right)$ & $0.283(8)$ & $9.9(9) \cdot 10^{-3}$ & 0 & 0 \\
\hline
\end{tabular}

Continued on next page

${ }^{1}$ Stone, 2012.

${ }^{2}$ Stone, 2012. 
Table 9.2: On-site candidates (continued)

\begin{tabular}{|c|c|c|c|c|c|c|c|c|c|c|c|c|c|c|}
\hline \multicolumn{3}{|c|}{ Decay } & \multicolumn{5}{|c|}{ Intermediate } & \multirow{2}{*}{$\begin{array}{c}\text { Initial } \\
\text { Energy } \\
\mathrm{keV}\end{array}$} & \multirow{2}{*}{$\begin{array}{c}\frac{\text { Final }}{\text { Energy }} \\
\text { keV }\end{array}$} & \multicolumn{5}{|c|}{ Angular correlation coefficients } \\
\hline Daughter & Parent & Half-Life & $\begin{array}{c}\text { Energy } \\
\text { keV }\end{array}$ & Half-life & Spin/Parity & $\begin{array}{l}\mathrm{Q} \\
\mathrm{eb}\end{array}$ & $\begin{array}{c}\mu \\
\mu_{\mathrm{N}}\end{array}$ & & & $\star$ & $A_{22}$ & $A_{24}$ & $A_{42}$ & $A_{44}$ \\
\hline & & & & & & & & & & $(\stackrel{+}{+})$ & $-0.151(5)$ & $9.9(9) \cdot 10^{-3}$ & 0 & 0 \\
\hline & & & & & & & & 1253.3 & 0 & $\left(\begin{array}{l}+ \\
+\end{array}\right)$ & $0.283(8)$ & $9.9(9) \cdot 10^{-3}$ & 0 & 0 \\
\hline & & & & & & & & & & $(\stackrel{+}{-})$ & $-0.151(5)$ & $9.9(9) \cdot 10^{-3}$ & 0 & 0 \\
\hline \multirow[t]{2}{*}{${ }^{181} \operatorname{Re}$} & ${ }^{181} \mathrm{Os}$ & $105 \mathrm{~m}$ & 356.75 & $96 \mathrm{~ns}$ & $5 / 2-$ & $?$ & $2.03(10)$ & 599.67 & 118.01 & $\left(\begin{array}{l}+ \\
+\end{array}\right)$ & $0.138(6)$ & 0 & $0.021(5)$ & 0 \\
\hline & & & & & & & & & & $\left(\begin{array}{l}- \\
+\end{array}\right)$ & $-0.071(14)$ & 0 & $0.021(5)$ & 0 \\
\hline \multirow[t]{2}{*}{${ }^{183} \mathrm{Re}$} & ${ }^{183} \mathrm{Os}$ & $13 \mathrm{~h}$ & 496.24 & $7.7 \mathrm{~ns}$ & $9 / 2-$ & $3.8(3)^{1}$ & $5.14(11)^{2}$ & 664.08 & 114.47 & $\left(\begin{array}{l}+ \\
+\end{array}\right)$ & $-0.01(3)$ & 0 & $1.2(12) \cdot 10^{-3}$ & 0 \\
\hline & & & & & & & & & & $\left(\begin{array}{l}- \\
+\end{array}\right)$ & $0.11(3)$ & 0 & $1.2(12) \cdot 10^{-3}$ & 0 \\
\hline \multirow[t]{4}{*}{${ }^{184}$ Ir } & ${ }^{184} \mathrm{Pt}$ & $17.3 \mathrm{~m}$ & 225.63 & $500 \mathrm{~ns}$ & $3+$ & $?$ & $?$ & 293.27 & 70.73 & $\left(\begin{array}{l}+ \\
+\end{array}\right)$ & $0.22(4)$ & $1(6) \cdot 10^{-3}$ & $0.014(4)$ & $6(4) \cdot 10^{-5}$ \\
\hline & & & & & & & & & & $( \pm)$ & $0.01(3)$ & $1(6) \cdot 10^{-3}$ & $9(22) \cdot 10^{-4}$ & $6(4) \cdot 10^{-5}$ \\
\hline & & & & & & & & & & $\left(\begin{array}{l}- \\
+\end{array}\right)$ & $-0.054(16)$ & $-2.4(17) \cdot 10^{-4}$ & $0.014(4)$ & $6(4) \cdot 10^{-5}$ \\
\hline & & & & & & & & & & $(\stackrel{-}{-})$ & $-3(8) \cdot 10^{-3}$ & $-2.4(17) \cdot 10^{-4}$ & $9(22) \cdot 10^{-4}$ & $6(4) \cdot 10^{-5}$ \\
\hline \multirow[t]{8}{*}{${ }^{185} \mathrm{Ir}$} & ${ }^{185} \mathrm{Pt}$ & $70.9 \mathrm{~m}$ & 5.8 & $5 \mathrm{~ns}$ & $9 / 2-$ & $?$ & $?$ & 158.6 & 0 & & 0.102 & 0.06333 & 0.01461 & 0.00907 \\
\hline & & & & & & & & 300.1 & 0 & $\left(\begin{array}{l}+ \\
+\end{array}\right)$ & $-0.471(10)$ & $-0.292(6)$ & $-0.10(6)$ & $-0.06(4)$ \\
\hline & & & & & & & & & & $\left(\begin{array}{l}- \\
+\end{array}\right)$ & $0.32(8)$ & $0.20(5)$ & $-0.10(6)$ & $-0.06(4)$ \\
\hline & & & 229.6 & $2.1 \mathrm{~ns}$ & $3 / 2+$ & $?$ & $?$ & 335.3 & 0 & $\left(\begin{array}{l}+ \\
+\end{array}\right)$ & $-0.0377(3)$ & 0 & 0 & 0 \\
\hline & & & & & & & & & & $\left(\begin{array}{l}- \\
+\end{array}\right)$ & $0.077(5)$ & 0 & 0 & 0 \\
\hline & & & 5.8 & $5 \mathrm{~ns}$ & $9 / 2-$ & $?$ & ? & 465.7 & 0 & $\left(\begin{array}{l}+ \\
+\end{array}\right)$ & $0.23(9)$ & $0.14(5)$ & $-0.03(4)$ & $-0.019(25)$ \\
\hline & & & & & & & & & & $\left(\begin{array}{l}- \\
+\end{array}\right)$ & $-0.40(13)$ & $-0.25(8)$ & $-0.03(4)$ & $-0.019(25)$ \\
\hline & & & & & & & & 646.6 & 0 & & -0.07143 & -0.04433 & 0 & 0 \\
\hline \multirow[t]{2}{*}{${ }^{193} \mathrm{Pt}$} & ${ }^{193} \mathrm{Au}$ & $17.65 \mathrm{~h}$ & 14.276 & $2.52 \mathrm{~ns}$ & $5 / 2-$ & $?$ & $?$ & 269.83 & 1.642 & $\left(\begin{array}{l}+ \\
+\end{array}\right)$ & $0.331(21)$ & $1.5(8) \cdot 10^{-4}$ & $0.035(10)$ & $1.6(10) \cdot 10^{-5}$ \\
\hline & & & & & & & & & & $( \pm)$ & $0.386(24)$ & $1.5(8) \cdot 10^{-4}$ & $0.041(12)$ & $1.6(10) \cdot 10^{-5}$ \\
\hline
\end{tabular}

Continued on next page

${ }^{1}$ Firestone and Shirley, 1996

${ }^{2}$ Firestone and Shirley, 1996. 
Table 9.2: On-site candidates (continued)

\begin{tabular}{|c|c|c|c|c|c|c|c|c|c|c|c|c|c|c|}
\hline \multicolumn{3}{|c|}{ Decay } & \multicolumn{5}{|c|}{ Intermediate } & \multirow{2}{*}{$\begin{array}{c}\text { Initial } \\
\text { Energy } \\
\mathrm{keV}\end{array}$} & \multirow{2}{*}{$\begin{array}{c}\frac{\text { Final }}{\text { Energy }} \\
\mathrm{keV}\end{array}$} & \multicolumn{5}{|c|}{ Angular correlation coefficients } \\
\hline Daughter & Parent & Half-Life & $\begin{array}{c}\text { Energy } \\
\mathrm{keV}\end{array}$ & Half-life & Spin/Parity & $\begin{array}{l}\mathrm{Q} \\
\mathrm{eb}\end{array}$ & $\begin{array}{c}\mu \\
\mu_{\mathrm{N}}\end{array}$ & & & $\star$ & $A_{22}$ & $A_{24}$ & $A_{42}$ & $A_{44}$ \\
\hline & & & & & & & & & & $\left(\begin{array}{l}- \\
+\end{array}\right)$ & $-0.13(4)$ & $-6(4) \cdot 10^{-5}$ & $0.035(10)$ & $1.6(10) \cdot 10^{-5}$ \\
\hline & & & & & & & & & & $(z)$ & $-0.15(4)$ & $-6(4) \cdot 10^{-5}$ & $0.041(12)$ & $1.6(10) \cdot 10^{-5}$ \\
\hline & & & 1.642 & $9.7 \mathrm{~ns}$ & $3 / 2-$ & $?$ & $?$ & 269.83 & 0 & & $-0.126(22)$ & 0 & 0 & 0 \\
\hline \multirow[t]{3}{*}{${ }^{194} \mathrm{Hg}$} & ${ }^{194} \mathrm{Tl}$ & $32.8 \mathrm{~m}$ & 1910.4 & $3.75 \mathrm{~ns}$ & 7 & $?$ & $?$ & 2138.4 & 1813.5 & $\left(\begin{array}{l}+ \\
+\end{array}\right)$ & $0.22(4)$ & $0.107(21)$ & $-0.06(3)$ & $-0.029(16)$ \\
\hline & & & & & & & & & & $\left(\begin{array}{l}- \\
+\end{array}\right)$ & $-0.39(3)$ & $-0.189(14)$ & $-0.06(3)$ & $-0.029(16)$ \\
\hline & & & & & & & & 2463.9 & 1813.5 & & -0.1068 & -0.05123 & 0 & 0 \\
\hline${ }^{196} \mathrm{Pt}$ & ${ }^{196} \mathrm{Ir}$ & $84 \mathrm{~m}$ & 1374 & $4.01 \mathrm{~ns}$ & 7 & $?$ & $-0.21(14)$ & 1821.1 & 1270.7 & & 0.102 & 0.04897 & 0.0189 & 0.00907 \\
\hline \multirow[t]{2}{*}{${ }^{198} \mathrm{~Pb}$} & ${ }^{198} \mathrm{Bi}$ & $11.6 \mathrm{~m}$ & 1823.4 & $50.4 \mathrm{~ns}$ & $(5-)$ & $?$ & $0.38(3)$ & 2141.3 & 1625.9 & & -0.07143 & 0 & -0.011 & 0 \\
\hline & & & 2141.3 & $4.19 \mu \mathrm{s}$ & $(7-)$ & $?$ & $-0.377(6)^{1}$ & 2231.3 & 1823.4 & & 0.102 & 0.04897 & 0.0189 & 0.00907 \\
\hline${ }^{199} \mathrm{Hg}$ & ${ }^{199 \mathrm{~m}_{\mathrm{Hg}}} \mathrm{Hg}$ & $42.67 \mathrm{~m}$ & 158.3 & $2.47 \mathrm{~ns}$ & $5 / 2-$ & $0.95(7)$ & $0.88(3)$ & 532.48 & 0 & & $0.251(4)$ & $0.289(5)$ & $-0.0277(17)$ & $-0.032(2)$ \\
\hline \multirow[t]{3}{*}{${ }^{204} \mathrm{~Pb}$} & ${ }^{204} \mathrm{Bi}$ & $11.22 \mathrm{~h}$ & 1273.99 & $265 \mathrm{~ns}$ & $4+$ & $\pm 0.44(2)$ & $0.224(3)$ & 2065.17 & 899.15 & & $-0.438(5)$ & $-0.298(4)$ & $-0.051(7)$ & $-0.035(5)$ \\
\hline & & & & & & & & 2185.73 & 899.15 & & 0.2473 & 0.1681 & -0.0431 & -0.0293 \\
\hline & ${ }^{204 \mathrm{~m}} \mathrm{~Pb}$ & $66.93 \mathrm{~m}$ & 1273.99 & $265 \mathrm{~ns}$ & $4+$ & $\pm 0.44(2)$ & $0.224(3)$ & 2185.88 & 899.15 & & 0.2473 & 0.1681 & -0.0431 & -0.0293 \\
\hline \multirow[t]{5}{*}{${ }^{208}$ Po } & ${ }^{208} \mathrm{At}$ & $97.8 \mathrm{~m}$ & 1524.17 & $4 \mathrm{~ns}$ & $6+$ & $?$ & $5.3(6)$ & 2041.24 & 1346.57 & & $0.022(5)$ & $0.011(3)$ & 0 & 0 \\
\hline & & & 1528.22 & $380 \mathrm{~ns}$ & $8+$ & $\pm 0.90(4)$ & $7.37(5)$ & 2160.09 & 1524.17 & & $0.025(11)$ & $0.012(5)$ & 0 & 0 \\
\hline & & & 1524.17 & $4 \mathrm{~ns}$ & $6+$ & $?$ & $5.3(6)$ & 2369.22 & 1346.57 & & -0.07143 & -0.03702 & 0 & 0 \\
\hline & & & 1528.22 & $380 \mathrm{~ns}$ & $8+$ & $\pm 0.90(4)$ & $7.37(5)$ & 2555.89 & 1524.17 & $\left(\begin{array}{l}+ \\
+\end{array}\right)$ & $-0.34(9)$ & $-0.15(4)$ & $-0.03(3)$ & $-0.013(13)$ \\
\hline & & & & & & & & & & $\left(\begin{array}{l}- \\
+\end{array}\right)$ & $0.16(12)$ & $0.07(5)$ & $-0.03(3)$ & $-0.013(13)$ \\
\hline${ }^{212} \mathrm{Rn}$ & ${ }^{212} \mathrm{Fr}$ & $20 \mathrm{~m}$ & 1502.5 & $8.8 \mathrm{~ns}$ & $4+$ & $?$ & $\pm 4.0(2)$ & 1640.8 & 1274.8 & & 0.102 & 0.06937 & 0.01334 & 0.00907 \\
\hline
\end{tabular}

${ }^{1}$ Firestone and Shirley, 1996. 
Table 9.3: Online candidates (parent half-life: $t_{1 / 2}<10 \mathrm{~min}$, minimal $\gamma$ intensity: $5 \%$, intermediate level's half-life: $2 \mathrm{~ns}<t_{1 / 2}<5 \mu \mathrm{s}$, lower angular correlation coefficient limit: 0.02 ). The column $\star$ contains the sign combination of mixing ratios used for the computation of results contained in the particular row (compare section 9.2.2).

\begin{tabular}{|c|c|c|c|c|c|c|c|c|c|c|c|c|c|c|}
\hline \multirow[b]{2}{*}{ Daughter } & \multicolumn{2}{|l|}{ Decay } & \multicolumn{5}{|c|}{ Intermediate } & \multirow{2}{*}{$\begin{array}{c}\text { Initial } \\
\text { Energy } \\
\mathrm{keV}\end{array}$} & \multirow{2}{*}{$\begin{array}{c}\frac{\text { Final }}{\text { Energy }} \\
\text { keV }\end{array}$} & \multicolumn{5}{|c|}{ Angular correlation coefficients } \\
\hline & Parent & Half-Life & $\begin{array}{c}\text { Energy } \\
\text { keV }\end{array}$ & Half-life & Spin/Parity & $\begin{array}{l}\mathrm{Q} \\
\mathrm{eb}\end{array}$ & $\begin{array}{c}\mu \\
\mu_{\mathrm{N}}\end{array}$ & & & $\star$ & $A_{22}$ & $A_{24}$ & $A_{42}$ & $A_{44}$ \\
\hline${ }^{19} \mathrm{~F}$ & ${ }^{19} \mathrm{O}$ & $26.88 \mathrm{~s}$ & 197.143 & $89.3 \mathrm{~ns}$ & $5 / 2+$ & $-0.072(4)^{1}$ & $3.607(8)^{2}$ & 1554.04 & 0 & & -0.2 & -0.2309 & 0 & 0 \\
\hline${ }^{22} \mathrm{Na}$ & ${ }^{22} \mathrm{Mg}$ & $3.8755 \mathrm{~s}$ & 583.11 & $243 \mathrm{~ns}$ & $1+$ & ? & $0.535(10)$ & 657.16 & 0 & & -0.07143 & 0 & 0 & 0 \\
\hline${ }^{30} \mathrm{Al}$ & ${ }^{30} \mathrm{Mg}$ & $335 \mathrm{~ms}$ & 244.1 & $8 \mathrm{~ns}$ & $2+$ & ? & ? & 688 & 0 & & 0.05 & 0 & 0 & 0 \\
\hline${ }^{56} \mathrm{Mn}$ & ${ }^{56} \mathrm{Cr}$ & $5.94 \mathrm{~m}$ & 26 & $8.7 \mathrm{~ns}$ & $2+$ & ? & ? & 110 & 0 & & 0.05 & 0 & 0 & 0 \\
\hline \multirow[t]{2}{*}{${ }^{57} \mathrm{Fe}$} & ${ }^{57} \mathrm{Mn}$ & $85.4 \mathrm{~s}$ & 14.4129 & $98.3 \mathrm{~ns}$ & $3 / 2$ & $0.082(8)$ & $-0.1549(2)$ & 706.399 & 0 & $\left(\begin{array}{l}- \\
+\end{array}\right)$ & $0.297(3)$ & 0 & 0 & 0 \\
\hline & & & & & & & & & & $(=)$ & $0.301(3)$ & 0 & 0 & 0 \\
\hline${ }^{68} \mathrm{Cu}$ & ${ }^{68 \mathrm{~m}} \mathrm{Cu}$ & $3.75 \mathrm{~m}$ & 84.11 & $7.84 \mathrm{~ns}$ & $2+$ & ? & ? & 721.26 & 0 & & -0.1545 & 0 & 0.007037 & 0 \\
\hline \multirow[t]{2}{*}{${ }^{75} \mathrm{Kr}$} & ${ }^{75} \mathrm{Rb}$ & $19 \mathrm{~s}$ & 178.9 & $2.08 \mathrm{~ns}$ & $(3 / 2-)$ & ? & ? & 358.11 & 0 & $\left(\begin{array}{l}+ \\
+\end{array}\right)$ & $-0.035(5)$ & 0 & 0 & 0 \\
\hline & & & & & & & & & & $\left(\begin{array}{l}- \\
+\end{array}\right)$ & $0.069(12)$ & 0 & 0 & 0 \\
\hline${ }^{77} \mathrm{Kr}$ & ${ }^{77} \mathrm{Rb}$ & $3.78 \mathrm{~m}$ & 66.5 & $118 \mathrm{~ns}$ & $3 / 2$ & ? & ? & 245.3 & 0 & & $0.021(11)$ & 0 & 0 & 0 \\
\hline${ }^{77} \mathrm{Rb}$ & ${ }^{77} \mathrm{Sr}$ & $9 \mathrm{~s}$ & 146.937 & $5.1 \mathrm{~ns}$ & $(5 / 2+)$ & ? & ? & 307.03 & 0 & & $-0.116(17)$ & 0 & $5.8(16) \cdot 10^{-3}$ & 0 \\
\hline${ }^{78} \mathrm{Br}$ & ${ }^{78 m_{B r}}$ & $119.4 \mu \mathrm{s}$ & 32.3 & $14.2 \mathrm{~ns}$ & (2-) & ? & $-1.12(4)$ & 180.9 & 0 & & -0.07143 & 0 & -0.003549 & 0 \\
\hline${ }^{79} \mathrm{Sr}$ & ${ }^{79} \mathrm{Y}$ & $14.8 \mathrm{~s}$ & 177.4 & $23 \mathrm{~ns}$ & $(5 / 2+)$ & ? & ? & 329.9 & 0 & & $0.17(5)$ & $3(18) \cdot 10^{-5}$ & $2.1(15) \cdot 10^{-3}$ & $4(23) \cdot 10^{-7}$ \\
\hline \multirow[t]{2}{*}{${ }^{87} \mathrm{Zr}$} & ${ }^{87} \mathrm{Nb}$ & $3.75 \mathrm{~m}$ & 201 & $2.44 \mathrm{~ns}$ & $(7 / 2+)$ & ? & ? & 335.8 & 0 & & $\sim 0.241$ & $\sim-0.0155$ & $\sim-0.0503$ & $\sim 0.00324$ \\
\hline & ${ }^{87 \mathrm{~m}} \mathrm{Zr}$ & $14 \mathrm{~s}$ & 201.2 & $2.44 \mathrm{~ns}$ & $(7 / 2+)$ & ? & $?$ & 336.3 & 0 & & $\sim 0.241$ & $\sim-0.0155$ & $\sim-0.0503$ & $\sim 0.00324$ \\
\hline \multirow[t]{2}{*}{${ }^{91} \mathrm{Nb}$} & ${ }^{91 \mathrm{~m}_{\mathrm{Nb}}}$ & $3.76 \mu \mathrm{s}$ & 1984.7 & $10 \mathrm{~ns}$ & $(13 / 2-)$ & ? & $8.14(13)$ & 2034.8 & 1790.6 & & 0.102 & 0.05074 & 0.01824 & 0.00907 \\
\hline & & & & & & & & 2034.8 & 0 & & $0.064(12)$ & $0.097(14)$ & $0.0114(21)$ & $0.0174(25)$ \\
\hline \multirow[t]{2}{*}{${ }^{92} \mathrm{Tc}$} & ${ }^{92} \mathrm{Ru}$ & $3.65 \mathrm{~m}$ & 270.15 & $1.03 \mu \mathrm{s}$ & $(4+)$ & ? & ? & 529.44 & 213.81 & & -0.07143 & -0.009339 & 0 & 0 \\
\hline & & & 529.44 & $100 \mathrm{~ns}$ & $(3+)$ & ? & ? & 576.9 & 270.15 & & 0.05 & 0 & 0 & 0 \\
\hline
\end{tabular}

Continued on next page

${ }^{1}$ Firestone and Shirley, 1996.

${ }^{2}$ Firestone and Shirley, 1996. 
Table 9.3: Online candidates (continued)

\begin{tabular}{|c|c|c|c|c|c|c|c|c|c|c|c|c|c|c|}
\hline \multicolumn{3}{|c|}{ Decay } & \multicolumn{5}{|c|}{ Intermediate } & \multirow{2}{*}{$\begin{array}{c}\frac{\text { Initial }}{\text { Energy }} \\
\mathrm{keV}\end{array}$} & \multirow{2}{*}{$\begin{array}{c}\frac{\text { Final }}{\text { Energy }} \\
\text { keV }\end{array}$} & \multicolumn{5}{|c|}{ Angular correlation coefficients } \\
\hline Daughter & Parent & Half-Life & $\begin{array}{c}\text { Energy } \\
\text { keV }\end{array}$ & Half-life & Spin/Parity & $\begin{array}{l}\mathrm{Q} \\
\mathrm{eb}\end{array}$ & $\begin{array}{c}\mu \\
\mu_{\mathrm{N}}\end{array}$ & & & $\star$ & $A_{22}$ & $A_{24}$ & $A_{42}$ & $A_{44}$ \\
\hline & & & 576.9 & $2 \mathrm{~ns}$ & $(2+)$ & $?$ & $?$ & 711.36 & 529.44 & & 0.05 & 0 & 0 & 0 \\
\hline${ }^{94} \mathrm{Ru}$ & ${ }^{94} \mathrm{Rh}$ & $25.8 \mathrm{~s}$ & 2498.62 & $65 \mathrm{~ns}$ & $6+$ & $?$ & $8.12(5)^{1}$ & 2644.72 & 2186.91 & & 0.102 & 0.05289 & 0.0175 & 0.00907 \\
\hline${ }^{96} \mathrm{Pd}$ & ${ }^{96} \mathrm{Ag}$ & $4.4 \mathrm{~s}$ & 2530.5 & $2.2 \mu \mathrm{s}$ & $(8+)$ & $?$ & $10.97(6)$ & 3783.5 & 2424.19 & & 0.102 & 0.04622 & 0.02003 & 0.00907 \\
\hline${ }^{98} \mathrm{Sr}$ & ${ }^{98} \mathrm{Rb}$ & $96 \mathrm{~ms}$ & 144.225 & $2.8 \mathrm{~ns}$ & $2+$ & $?$ & $\pm 0.76(14)$ & 433.52 & 0 & & 0.102 & 0.1825 & 0.00507 & 0.00907 \\
\hline${ }^{105} \mathrm{Tc}$ & ${ }^{105} \mathrm{Mo}$ & $35.6 \mathrm{~s}$ & 85.44 & $20.8 \mathrm{~ns}$ & $(5 / 2+)$ & $?$ & $?$ & 149.63 & 0 & & 0.05 & 0 & 0 & 0 \\
\hline${ }^{112}$ In & ${ }^{112 \mathrm{~m}} \mathrm{In}$ & $2.81 \mu \mathrm{s}$ & 350.5 & $690 \mathrm{~ns}$ & $7+$ & $\pm 1.03(3)$ & $4.72(4)$ & 613.2 & 162.89 & & -0.07143 & -0.03428 & 0 & 0 \\
\hline${ }^{115} \mathrm{Sn}$ & ${ }^{115 \mathrm{~m}} \mathrm{Sn}$ & $159 \mu \mathrm{s}$ & 613.5 & $3.26 \mu \mathrm{s}$ & $7 / 2+$ & $\sim \pm 0.26$ & $0.683(10)$ & 713.64 & 497.6 & & 0.102 & 0.07816 & 0.01184 & 0.00907 \\
\hline \multirow[t]{4}{*}{${ }^{115} \mathrm{Sb}$} & ${ }^{115 \mathrm{~m}} \mathrm{Sb}$ & $159 \mathrm{~ns}$ & 1300.2 & $6.2 \mathrm{~ns}$ & $11 / 2$ & $?$ & $5.53(8)$ & 2516.9 & 723.6 & & 0.102 & 0.05554 & 0.01666 & 0.00907 \\
\hline & & & & & & & & 2516.9 & 0 & & 0.1786 & -0.02651 & 0.02916 & -0.004329 \\
\hline & & & & & & & & 2638.5 & 723.6 & & 0.102 & 0.05554 & 0.01666 & 0.00907 \\
\hline & & & & & & & & 2638.5 & 0 & & 0.1786 & -0.02651 & 0.02916 & -0.004329 \\
\hline \multirow[t]{6}{*}{${ }^{117} \mathrm{Sb}$} & ${ }^{117 \mathrm{~m}_{\mathrm{Sb}}}$ & $355 \mu \mathrm{s}$ & 1322.91 & $3.8 \mathrm{~ns}$ & $11 / 2$ & $?$ & $5.35(9)$ & 2323.07 & 1160.04 & & -0.07143 & 0 & -0.01166 & 0 \\
\hline & & & & & & & & 2323.07 & 527.26 & & 0.102 & 0.05554 & 0.01666 & 0.00907 \\
\hline & & & & & & & & 2323.07 & 0 & & 0.1786 & -0.02651 & 0.02916 & -0.004329 \\
\hline & & & & & & & & 2412.76 & 1160.04 & & -0.07143 & 0 & -0.01166 & 0 \\
\hline & & & & & & & & 2412.76 & 527.26 & & 0.102 & 0.05554 & 0.01666 & 0.00907 \\
\hline & & & & & & & & 2412.76 & 0 & & 0.1786 & -0.02651 & 0.02916 & -0.004329 \\
\hline \multirow[t]{2}{*}{${ }^{120} \mathrm{Sn}$} & ${ }^{120} \mathrm{In}$ & $47.3 \mathrm{~s}$ & 2284.08 & $5.55 \mathrm{~ns}$ & 5- & $\pm 0.033(2)$ & $-0.280(25)$ & 2481.43 & 2194.25 & & -0.07143 & 0 & -0.011 & 0 \\
\hline & & & & & & & & 2749.51 & 2194.25 & & $0.036(9)$ & 0 & $6(8) \cdot 10^{-5}$ & 0 \\
\hline \multirow[t]{2}{*}{${ }^{122} \mathrm{Sn}$} & ${ }^{122}$ In & $10.8 \mathrm{~s}$ & 2245.89 & $7.9 \mathrm{~ns}$ & 5- & $?$ & $?$ & 2409.14 & 2142.14 & & -0.07143 & 0 & -0.011 & 0 \\
\hline & & & & & & & & 2653.08 & 2142.14 & & $0.036(9)$ & 0 & $6(8) \cdot 10^{-5}$ & 0 \\
\hline
\end{tabular}

Continued on next page

${ }^{1}$ Firestone and Shirley, 1996. 
Table 9.3: Online candidates (continued)

\begin{tabular}{|c|c|c|c|c|c|c|c|c|c|c|c|c|c|c|}
\hline \multicolumn{3}{|c|}{ Decay } & \multicolumn{5}{|c|}{ Intermediate } & \multirow{3}{*}{$\begin{array}{c}\frac{\text { Initial }}{\text { Energy }} \\
\mathrm{keV}\end{array}$} & \multirow{3}{*}{$\begin{array}{c}\text { Final } \\
\text { Energy } \\
\mathrm{keV}\end{array}$} & \multicolumn{5}{|c|}{ Angular correlation coefficients } \\
\hline Daughter & Parent & Half-Life & Energy & Half-life & Spin/Parity & Q & $\mu$ & & & $\star$ & $A_{22}$ & $A_{24}$ & $A_{42}$ & $A_{44}$ \\
\hline & & & $\mathrm{keV}$ & & & $\mathrm{eb}$ & $\mu_{\mathrm{N}}$ & & & & & & & \\
\hline${ }^{122} \mathrm{Sb}$ & ${ }^{122 \mathrm{~m}} \mathrm{Sb}$ & $4.191 \mathrm{~m}$ & 61.413 & $1.7 \mu \mathrm{s}$ & $3+$ & $0.41(4)$ & $2.983(12)$ & 137.472 & 0 & & -0.07143 & 0 & -0.007034 & 0 \\
\hline${ }^{123} \mathrm{Cs}$ & ${ }^{123} \mathrm{Ba}$ & $2.7 \mathrm{~m}$ & 94.57 & $9 \mathrm{~ns}$ & $(5 / 2+)$ & $?$ & $?$ & 214.57 & 0 & & -0.07143 & -0.08248 & 0 & 0 \\
\hline & & & & & & & & 231.63 & 0 & & $-0.10(7)$ & $-0.12(9)$ & $-1(5) \cdot 10^{-4}$ & $-1(6) \cdot 10^{-4}$ \\
\hline${ }^{124} \mathrm{Sn}$ & ${ }^{124} \mathrm{In}$ & $3.7 \mathrm{~s}$ & 2204.5 & $270 \mathrm{~ns}$ & $5-$ & $?$ & $?$ & 2324.87 & 2101.59 & & -0.07143 & 0 & -0.011 & 0 \\
\hline & & & 2324.87 & $3.1 \mu \mathrm{s}$ & $(7-)$ & $?$ & $?$ & 2568.01 & 2204.5 & & $-0.114(21)$ & $-0.055(10)$ & $-2(12) \cdot 10^{-5}$ & $\left(-1 \cdot 10^{-5}\right)$ \\
\hline & & & 2204.5 & $270 \mathrm{~ns}$ & 5- & $?$ & $?$ & 2568.01 & 2101.59 & & $0.045(9)$ & 0 & $\left(7 \cdot 10^{-6}\right)$ & 0 \\
\hline${ }^{124} \mathrm{Cs}$ & ${ }^{124 \mathrm{~m}} \mathrm{Cs}$ & $6.3 \mathrm{~s}$ & 301.1 & $69 \mathrm{~ns}$ & $(4-)$ & $?$ & $?$ & 397.65 & 242.87 & $\left(\begin{array}{l}+ \\
+\end{array}\right)$ & $-0.16(3)$ & 0 & $0.020(11)$ & 0 \\
\hline & & & & & & & & & & $\left(\begin{array}{l}- \\
+\end{array}\right)$ & $0.29(4)$ & 0 & $0.020(11)$ & 0 \\
\hline & & & & & & & & 397.65 & 211.62 & $\left(\begin{array}{l}+ \\
+\end{array}\right)$ & $-0.16(3)$ & 0 & $0.020(11)$ & 0 \\
\hline & & & & & & & & & & $\left(\begin{array}{l}- \\
+\end{array}\right)$ & $0.29(4)$ & 0 & $0.020(11)$ & 0 \\
\hline${ }^{126} \mathrm{~S} \mathrm{n}$ & ${ }^{126} \mathrm{In}$ & $1.64 \mathrm{~s}$ & 2161.51 & $10.8 \mathrm{~ns}$ & 5- & $?$ & $?$ & 2218.96 & 2049.71 & & -0.07143 & 0 & -0.011 & 0 \\
\hline${ }^{127} \mathrm{Cs}$ & ${ }^{127 \mathrm{~m}} \mathrm{Cs}$ & $55 \mu \mathrm{s}$ & 66 & $24.88 \mathrm{~ns}$ & $(5 / 2+)$ & $\pm 0.58(12)$ & $\pm 2.7(5)$ & 138.6 & 0 & & -0.2 & -0.2309 & 0 & 0 \\
\hline & & & & & & & & 272.2 & 0 & & -0.07143 & -0.08248 & 0 & 0 \\
\hline & & & & & & & & 451.1 & 0 & & 0.1786 & 0.2062 & -0.003749 & -0.004329 \\
\hline${ }^{127} \mathrm{Ba}$ & ${ }^{127} \mathrm{La}$ & $5.1 \mathrm{~m}$ & 81.31 & $75 \mathrm{~ns}$ & $(5 / 2+)$ & $?$ & ? & 195.6 & 56.26 & & $0.10(3)$ & 0 & $4(4) \cdot 10^{-4}$ & 0 \\
\hline${ }^{130} \mathrm{Sn}$ & ${ }^{130} \mathrm{In}$ & $290 \mathrm{~ms}$ & 2084.8 & $52 \mathrm{~ns}$ & $(5-)$ & $?$ & $?$ & 2214.6 & 1995.57 & & 0.08667 & 0 & 0 & 0 \\
\hline & & & & & & & & 2214.6 & 1946.84 & & -0.07143 & -0.011 & 0 & 0 \\
\hline & ${ }^{130} \mathrm{In}$ & $540 \mathrm{~ms}$ & 2084.89 & $52 \mathrm{~ns}$ & $(5-)$ & $?$ & $?$ & 2214.7 & 1995.66 & & 0.08667 & 0 & 0 & 0 \\
\hline & & & & & & & & 2214.7 & 1946.93 & & -0.07143 & -0.011 & 0 & 0 \\
\hline${ }^{132} \mathrm{Sn}$ & ${ }^{132} \mathrm{In}$ & $207 \mathrm{~ms}$ & 4416.29 & $3.95 \mathrm{~ns}$ & $(4+)$ & $?$ & $?$ & 4715.91 & 4041.2 & & 0.102 & 0.06937 & 0.01334 & 0.00907 \\
\hline & & & 4715.91 & $20.1 \mathrm{~ns}$ & $(6+)$ & $?$ & $?$ & 4848.52 & 4416.29 & & 0.102 & 0.05289 & 0.0175 & 0.00907 \\
\hline & & & 4416.29 & $3.95 \mathrm{~ns}$ & $(4+)$ & $?$ & $?$ & 4942.53 & 4041.2 & & -0.07143 & -0.04856 & 0 & 0 \\
\hline & ${ }^{132 \mathrm{~m}} \mathrm{Sn}$ & $2.03 \mu \mathrm{s}$ & 4415.5 & $4 \mathrm{~ns}$ & $(4+)$ & $?$ & $?$ & 4714.7 & 4041.1 & & 0.102 & 0.06937 & 0.01334 & 0.00907 \\
\hline
\end{tabular}


Table 9.3: Online candidates (continued)

\begin{tabular}{|c|c|c|c|c|c|c|c|c|c|c|c|c|c|c|}
\hline \multicolumn{3}{|c|}{ Decay } & \multicolumn{5}{|c|}{ Intermediate } & \multirow{3}{*}{$\begin{array}{c}\text { Initial } \\
\text { Energy } \\
\mathrm{keV}\end{array}$} & \multirow{3}{*}{$\begin{array}{c}\frac{\text { Final }}{\text { Energy }} \\
\mathrm{keV}\end{array}$} & \multicolumn{5}{|c|}{ Angular correlation coefficients } \\
\hline \multirow[t]{3}{*}{ Daughter } & Parent & Half-Life & Energy & Half-life & Spin/Parity & $\mathrm{Q}$ & $\mu$ & & & $\star$ & $A_{22}$ & $A_{24}$ & $A_{42}$ & $A_{44}$ \\
\hline & & & $\mathrm{keV}$ & & & $\mathrm{eb}$ & $\mu_{\mathrm{N}}$ & & & & & & & \\
\hline & & & 4714.7 & $20.2 \mathrm{~ns}$ & $(6+)$ & $?$ & $?$ & 4847 & 4415.5 & & 0.102 & 0.05289 & 0.0175 & 0.00907 \\
\hline${ }^{132} \mathrm{Sb}$ & ${ }^{132} \mathrm{Sn}$ & $39.7 \mathrm{~s}$ & 85.55 & $15.62 \mathrm{~ns}$ & $(3+)$ & $?$ & $?$ & 1078.31 & 0 & & $0.27(5)$ & $-0.047(10)$ & $-0.071(19)$ & $0.012(3)$ \\
\hline \multirow[t]{2}{*}{${ }^{132} \mathrm{Te}$} & ${ }^{132} \mathrm{Sb}$ & $2.79 \mathrm{~m}$ & 1774.77 & $145 \mathrm{~ns}$ & ${ }^{6+}$ & $?$ & $4.7(5)$ & 1925.31 & 1671.33 & & -0.07143 & -0.03702 & 0 & 0 \\
\hline & ${ }^{132} \mathrm{Sb}$ & $4.1 \mathrm{~m}$ & 1774.56 & $145 \mathrm{~ns}$ & $6+$ & $?$ & $4.7(5)$ & 1925.23 & 1671.03 & & -0.07143 & -0.03702 & 0 & 0 \\
\hline${ }^{132} \mathrm{Xe}$ & ${ }^{132 \mathrm{~m}} \mathrm{Xe}$ & $8.39 \mathrm{~ms}$ & 2214.06 & $87 \mathrm{~ns}$ & $(7-)$ & $\pm 0.010(5)$ & $-0.06(3)$ & 2752.16 & 2040.46 & & 0.1786 & 0.0857 & -0.009021 & -0.004329 \\
\hline${ }^{134} \mathrm{I}$ & $134 \mathrm{~m}_{\mathrm{I}}$ & $3.52 \mathrm{~m}$ & 44.4 & $10 \mathrm{~ns}$ & $(5+)$ & $?$ & $?$ & 316.5 & 0 & & -0.125 & 0 & 0.005249 & 0 \\
\hline \multirow[t]{2}{*}{${ }^{136} \mathrm{Ce}$} & ${ }^{136 \mathrm{~m}} \mathrm{Ce}$ & $2.2 \mu \mathrm{s}$ & 2366.8 & $5 \mathrm{~ns}$ & $6+$ & $?$ & $?$ & 2990.1 & 1314.4 & & 0.102 & 0.05289 & 0.0175 & 0.00907 \\
\hline & & & 2214.4 & $5 \mathrm{~ns}$ & $6+$ & $?$ & $?$ & 2990.1 & 1314.4 & & 0.102 & 0.05289 & 0.0175 & 0.00907 \\
\hline${ }^{138} \mathrm{Ba}$ & ${ }^{138} \mathrm{Cs}$ & $2.91 \mathrm{~m}$ & 1899 & $2.164 \mathrm{~ns}$ & $4+$ & $?$ & $3.2(6)$ & 2090.7 & 1436 & & 0.102 & 0.06937 & 0.01334 & 0.00907 \\
\hline \multirow[t]{2}{*}{${ }^{145} \mathrm{Pr}$} & ${ }^{145} \mathrm{Ce}$ & $3.01 \mathrm{~m}$ & 62.65 & $4 \mathrm{~ns}$ & $5 / 2+$ & $?$ & $?$ & 347.18 & 0 & & 0.05 & 0 & 0 & 0 \\
\hline & & & & & & & & 786.91 & 0 & & 0.05 & 0 & 0 & 0 \\
\hline \multirow[t]{4}{*}{${ }^{145} \mathrm{Gd}$} & ${ }^{145} \mathrm{~Tb}$ & $30.9 \mathrm{~s}$ & 27.3 & $11.5 \mathrm{~ns}$ & $3 / 2+$ & $?$ & $?$ & 1014.9 & 0 & $\left(\begin{array}{l}+ \\
+\end{array}\right)$ & $-0.040(17)$ & 0 & 0 & 0 \\
\hline & & & & & & & & & & $( \pm)$ & $-0.08(3)$ & 0 & 0 & 0 \\
\hline & & & & & & & & 1415.3 & 0 & $\left(\begin{array}{l}+ \\
+\end{array}\right)$ & $-0.048(5)$ & 0 & 0 & 0 \\
\hline & & & & & & & & & & $( \pm)$ & $-0.092(4)$ & 0 & 0 & 0 \\
\hline${ }^{147} \mathrm{Pr}$ & ${ }^{147} \mathrm{Ce}$ & $56.4 \mathrm{~s}$ & 93.29 & $12 \mathrm{~ns}$ & $(7 / 2+)$ & $?$ & $?$ & 362.03 & 0 & & 0.1071 & 0 & 0 & 0 \\
\hline${ }^{149} \mathrm{Dy}$ & ${ }^{149 \mathrm{~m}} \mathrm{Dy}$ & $490 \mathrm{~ms}$ & 1073.2 & $12.5 \mathrm{~ns}$ & $(13 / 2+)$ & $?$ & $?$ & 2251.8 & 0 & & 0.1786 & -0.02422 & 0.03192 & -0.004329 \\
\hline${ }^{150} \mathrm{Ce}$ & ${ }^{150} \mathrm{La}$ & $590 \mathrm{~ms}$ & 97 & $3.3 \mathrm{~ns}$ & $2+$ & $?$ & $?$ & 305.7 & 0 & & 0.102 & 0.1825 & 0.00507 & 0.00907 \\
\hline${ }^{151} \mathrm{Er}$ & ${ }^{151 \mathrm{~m}_{\mathrm{Er}}}$ & $580 \mathrm{~ms}$ & 1140.3 & $10 \mathrm{~ns}$ & $(13 / 2+)$ & $?$ & $?$ & 2239.4 & 0 & & 0.1786 & -0.02422 & 0.03192 & -0.004329 \\
\hline${ }^{151} \mathrm{Tm}$ & ${ }^{151 \mathrm{~m}} \mathrm{Tm}$ & $24 \mathrm{~ns}$ & 2655.67 & $451 \mathrm{~ns}$ & $(27 / 2-)$ & $?$ & $?$ & 3987.88 & 2515.27 & & 0.102 & 0.03912 & 0.02366 & 0.00907 \\
\hline${ }^{152} \mathrm{Nd}$ & ${ }^{152} \mathrm{Pr}$ & $3.63 \mathrm{~s}$ & 72.6 & $4.5 \mathrm{~ns}$ & $2+$ & $?$ & $?$ & 236.7 & 0 & & 0.102 & 0.1825 & 0.00507 & 0.00907 \\
\hline${ }^{152} \mathrm{~Tb}$ & ${ }^{152 \mathrm{~m}} \mathrm{~Tb}$ & $4.2 \mathrm{~m}$ & 342.2 & $960 \mathrm{~ns}$ & 5- & $?$ & $?$ & 501.74 & 283.29 & & -0.125 & 0 & 0.005249 & 0 \\
\hline${ }^{154} \mathrm{Sm}$ & ${ }^{154} \mathrm{Pm}$ & $2.68 \mathrm{~m}$ & 82.004 & $3.02 \mathrm{~ns}$ & $2+$ & $-1.87(4)$ & $0.78(4)$ & 1706.78 & 0 & & $0.256(15)$ & $0.46(3)$ & $-0.016(7)$ & $-0.029(13)$ \\
\hline
\end{tabular}

Continued on next page 
Table 9.3: Online candidates (continued)

\begin{tabular}{|c|c|c|c|c|c|c|c|c|c|c|c|c|c|c|}
\hline \multicolumn{3}{|c|}{ Decay } & \multicolumn{5}{|c|}{ Intermediate } & \multirow{2}{*}{$\begin{array}{l}\text { Initial } \\
\text { Energy } \\
\mathrm{keV}\end{array}$} & \multirow{2}{*}{$\begin{array}{c}\frac{\text { Final }}{\text { Energy }} \\
\mathrm{keV}\end{array}$} & \multicolumn{5}{|c|}{ Angular correlation coefficients } \\
\hline Daughter & Parent & Half-Life & $\begin{array}{c}\text { Energy } \\
\mathrm{keV}\end{array}$ & Half-life & Spin/Parity & $\begin{array}{l}\mathrm{Q} \\
\mathrm{eb}\end{array}$ & $\begin{array}{c}\mu \\
\mu_{\mathrm{N}}\end{array}$ & & & $\star$ & $A_{22}$ & $A_{24}$ & $A_{42}$ & $A_{44}$ \\
\hline \multirow[t]{6}{*}{${ }^{155} \mathrm{Dy}$} & ${ }^{155 \mathrm{~m}} \mathrm{Dy}$ & $6 \mu \mathrm{s}$ & 39.384 & $3.34 \mathrm{~ns}$ & $5 / 2$ & $?$ & ? & 86.767 & 0 & $\left(\begin{array}{l}+ \\
+\end{array}\right)$ & $1.2(7) \cdot 10^{-3}$ & $-7(4) \cdot 10^{-4}$ & $-8.3(18) \cdot 10^{-5}$ & $5.1(9) \cdot 10^{-5}$ \\
\hline & & & & & & & & & & $( \pm)$ & $-0.016(10)$ & $-7(4) \cdot 10^{-4}$ & $1.15(20) \cdot 10^{-3}$ & $5.1(9) \cdot 10^{-5}$ \\
\hline & & & & & & & & & & $\left(\begin{array}{l}- \\
+\end{array}\right)$ & $-0.0158(23)$ & $9.7(6) \cdot 10^{-3}$ & $-8.3(18) \cdot 10^{-5}$ & $5.1(9) \cdot 10^{-5}$ \\
\hline & & & & & & & & & & $(\overline{-})$ & $0.22(1)$ & $9.7(6) \cdot 10^{-3}$ & $1.15(20) \cdot 10^{-3}$ & $5.1(9) \cdot 10^{-5}$ \\
\hline & & & 132.195 & $51 \mathrm{~ns}$ & $9 / 2+$ & $?$ & $?$ & 234.33 & 86.767 & $\left(\begin{array}{l}+ \\
+\end{array}\right)$ & $-0.119(14)$ & 0 & $0.0107(24)$ & 0 \\
\hline & & & & & & & & & & $\left(\begin{array}{l}- \\
+\end{array}\right)$ & $0.230(17)$ & 0 & $0.0107(24)$ & 0 \\
\hline${ }^{156} \mathrm{Sm}$ & ${ }^{156} \mathrm{Pm}$ & $26.7 \mathrm{~s}$ & 75.89 & $2 \mathrm{~ns}$ & $2+$ & $?$ & ? & 249.71 & 0 & & 0.102 & 0.1825 & 0.00507 & 0.00907 \\
\hline${ }^{161} \mathrm{Er}$ & ${ }^{161 \mathrm{~m}} \mathrm{Er}$ & $7.5 \mu \mathrm{s}$ & 189 & $84 \mathrm{~ns}$ & $9 / 2+$ & $?$ & $?$ & 397 & 144 & & 0.05 & 0 & 0 & 0 \\
\hline \multirow[t]{2}{*}{${ }^{162} \mathrm{Dy}$} & ${ }^{162} \mathrm{~Tb}$ & $7.6 \mathrm{~m}$ & 80.66 & $2.19 \mathrm{~ns}$ & $2+$ & $?$ & $0.69(3)$ & 888.19 & 0 & & $\sim 0.25$ & $\sim 0.447$ & $\sim 0$ & $\sim 0$ \\
\hline & & & & & & & & 962.97 & 0 & & $-0.185(16)$ & $-0.33(3)$ & $-0.04561(4)$ & $-0.08158(8)$ \\
\hline${ }^{164} \mathrm{Dy}$ & ${ }^{164} \mathrm{~Tb}$ & $3 \mathrm{~m}$ & 73.37 & $2.39 \mathrm{~ns}$ & $2+$ & $-2.08(15)$ & $0.684(23)$ & 242.22 & 0 & & 0.102 & 0.1825 & 0.00507 & 0.00907 \\
\hline${ }^{169} \mathrm{Hf}$ & ${ }^{169} \mathrm{Ta}$ & $4.9 \mathrm{~m}$ & 28.8 & $82 \mathrm{~ns}$ & $(7 / 2+)$ & $?$ & $?$ & 177 & 0 & & 0.05 & 0 & 0 & 0 \\
\hline \multirow[t]{5}{*}{${ }^{173} \mathrm{Ta}$} & ${ }^{173} \mathrm{~W}$ & $7.5 \mathrm{~m}$ & 130.2 & $5 \mathrm{~ns}$ & $7 / 2+$ & $?$ & ? & 166 & 0 & $\left(\begin{array}{l}+ \\
+\end{array}\right)$ & $0.030(6)$ & 0 & $1(6) \cdot 10^{-4}$ & 0 \\
\hline & & & & & & & & & & $\left(\begin{array}{l}- \\
+\end{array}\right)$ & $0.070(6)$ & 0 & $1(6) \cdot 10^{-4}$ & 0 \\
\hline & & & 166 & $225 \mathrm{~ns}$ & $9 / 2-$ & $?$ & $2.66(8)^{1}$ & 623.6 & 130.2 & $\left(\begin{array}{l}+ \\
+\end{array}\right)$ & $0.068(7)$ & $3.0(17) \cdot 10^{-4}$ & 0 & 0 \\
\hline & & & & & & & & & & $(\stackrel{+}{ \pm})$ & $0.115(7)$ & $3.0(17) \cdot 10^{-4}$ & 0 & 0 \\
\hline & & & & & & & & 623.6 & 0 & & -0.131 & -0.08127 & 0 & 0 \\
\hline${ }^{177} \mathrm{Yb}$ & ${ }^{177 \mathrm{~m}} \mathrm{Yb}$ & $6.41 \mathrm{~s}$ & 104.5 & $4.48 \mathrm{~ns}$ & $(7 / 2-)$ & $?$ & ? & 331.5 & 0 & & -0.125 & 0 & 0.02611 & 0 \\
\hline${ }^{179} \mathrm{Os}$ & ${ }^{179} \mathrm{Ir}$ & $79 \mathrm{~s}$ & 145.4 & $500 \mathrm{~ns}$ & $(7 / 2-)$ & $?$ & ? & 242.9 & 100.2 & & 0.05 & 0 & 0 & 0 \\
\hline${ }^{181} \mathrm{Ta}$ & ${ }^{181 \mathrm{~m}} \mathrm{Ta}$ & $18.9 \mu \mathrm{s}$ & 482 & $10.8 \mathrm{~ns}$ & $5 / 2+$ & $2.35(6)$ & $3.29(3)$ & 615 & 135 & & 0.102 & 0.007855 & 0.1178 & 0.00907 \\
\hline & & & & & & & & 615 & 0 & $\left(\begin{array}{l}+ \\
+\end{array}\right)$ & $-0.3185(11)$ & $-0.06018(4)$ & $-0.3678(12)$ & $-0.06950(5)$ \\
\hline
\end{tabular}

Continued on next page 
Table 9.3: Online candidates (continued)

\begin{tabular}{|c|c|c|c|c|c|c|c|c|c|c|c|c|c|c|}
\hline \multicolumn{3}{|c|}{ Decay } & \multicolumn{5}{|c|}{ Intermediate } & \multirow{2}{*}{$\begin{array}{c}\text { Initial } \\
\text { Energy } \\
\mathrm{keV}\end{array}$} & \multirow{2}{*}{$\begin{array}{c}\frac{\text { Final }}{\text { Energy }} \\
\mathrm{keV}\end{array}$} & \multicolumn{5}{|c|}{ Angular correlation coefficients } \\
\hline Daughter & Parent & Half-Life & $\begin{array}{c}\text { Energy } \\
\mathrm{keV}\end{array}$ & Half-life & Spin/Parity & $\begin{array}{l}\mathrm{Q} \\
\mathrm{eb}\end{array}$ & $\mu$ & & & $\star$ & $A_{22}$ & $A_{24}$ & $A_{42}$ & $A_{44}$ \\
\hline & & & & & & & & & & $(\stackrel{ \pm}{-})$ & $-0.0198(12)$ & $-0.06018(4)$ & $-0.0229(14)$ & $-0.06950(5)$ \\
\hline \multirow[t]{2}{*}{${ }^{181} \mathrm{Pt}$} & ${ }^{181} \mathrm{Au}$ & $13.7 \mathrm{~s}$ & 116.66 & $300 \mathrm{~ns}$ & $(7 / 2-)$ & $?$ & $?$ & 166.64 & 93.93 & & 0.1071 & 0 & 0 & 0 \\
\hline & & & & & & & & 276.02 & 93.93 & & 0.05 & 0 & 0 & 0 \\
\hline \multirow[t]{5}{*}{${ }^{184} \mathrm{Os}$} & ${ }^{184 \mathrm{~m}} \mathrm{Os}$ & $23.6 \mathrm{~ns}$ & 773.9 & $2.2 \mathrm{~ns}$ & $6+$ & $?$ & ? & 1274.7 & 383.8 & & 0.102 & 0.05289 & 0.0175 & 0.00907 \\
\hline & & & & & & & & 1613.2 & 383.8 & & $0.176(3)$ & $0.0911(14)$ & 0 & 0 \\
\hline & & & & & & & & 1717.6 & 383.8 & & $-0.046(15)$ & $-0.024(8)$ & $-1.7(8) \cdot 10^{-3}$ & $-9(4) \cdot 10^{-4}$ \\
\hline & & & 1274.7 & $2.2 \mathrm{~ns}$ & $8+$ & ? & ? & 1870.9 & 773.9 & & 0.102 & 0.04622 & 0.02003 & 0.00907 \\
\hline & & & & & & & & 2366 & 773.9 & & 0.102 & 0.04622 & 0.02003 & 0.00907 \\
\hline \multirow[t]{15}{*}{${ }^{185} \mathrm{Pt}$} & ${ }^{185} \mathrm{Au}$ & $4.25 \mathrm{~m}$ & 200.89 & $728 \mathrm{~ns}$ & $5 / 2-$ & $?$ & $?$ & 424.09 & 181.09 & $\left(\begin{array}{l}+ \\
+\end{array}\right)$ & $\sim-0.124$ & $\sim 0$ & $\sim 0.0327$ & $\sim 0$ \\
\hline & & & & & & & & & & $\left(\begin{array}{l}- \\
+\end{array}\right)$ & $\sim 0.33$ & $\sim 0$ & $\sim 0.0327$ & $\sim 0$ \\
\hline & & & & & & & & 424.09 & 103.41 & $\left(\begin{array}{c}+ \\
+\end{array}\right)$ & $\sim 0.177$ & $\sim 0.205$ & $\sim-0.0467$ & $\sim-0.0539$ \\
\hline & & & & & & & & & & $\left(\begin{array}{l}- \\
+\end{array}\right)$ & $\sim-0.472$ & $\sim-0.545$ & $\sim-0.0467$ & $\sim-0.0539$ \\
\hline & & & & & & & & 451.87 & 181.09 & & -0.07143 & 0 & -0.005499 & 0 \\
\hline & & & & & & & & 451.87 & 103.41 & & 0.102 & 0.1178 & 0.007855 & 0.00907 \\
\hline & & & & & & & & 510.08 & 103.41 & & $\sim 0.0224$ & $\sim 0.0259$ & $\sim 0$ & $\sim 0$ \\
\hline & & & & & & & & 590.71 & 181.09 & $\left(\begin{array}{l}+ \\
+\end{array}\right)$ & $\sim-0.17$ & $\sim 0$ & $\sim 0.0241$ & $\sim 0$ \\
\hline & & & & & & & & & & $\left(\begin{array}{l}- \\
+\end{array}\right)$ & $\sim 0.348$ & $\sim 0$ & $\sim 0.0241$ & $\sim 0$ \\
\hline & & & & & & & & 590.71 & 103.41 & $\left(\begin{array}{c}+ \\
+\end{array}\right)$ & $\sim 0.242$ & $\sim 0.28$ & $\sim-0.0344$ & $\sim-0.0397$ \\
\hline & & & & & & & & & & $\left(\begin{array}{l}- \\
+\end{array}\right)$ & $\sim-0.497$ & $\sim-0.574$ & $\sim-0.0344$ & $\sim-0.0397$ \\
\hline & & & & & & & & 615.65 & 181.09 & $\left(\begin{array}{l}+ \\
+\end{array}\right)$ & $\sim-0.158$ & $\sim 0$ & $\sim 0.0111$ & $\sim 0$ \\
\hline & & & & & & & & & & $\left(\begin{array}{l}- \\
+\end{array}\right)$ & $\sim 0.293$ & $\sim 0$ & $\sim 0.0111$ & $\sim 0$ \\
\hline & & & & & & & & 615.65 & 103.41 & $\left(\begin{array}{l}+ \\
+\end{array}\right)$ & $\sim 0.225$ & $\sim 0.26$ & $\sim-0.0158$ & $\sim-0.0183$ \\
\hline & & & & & & & & & & $\left(\begin{array}{l}- \\
+\end{array}\right)$ & $\sim-0.419$ & $\sim-0.484$ & $\sim-0.0158$ & $\sim-0.0183$ \\
\hline
\end{tabular}

Continued on next page 
Table 9.3: Online candidates (continued)

\begin{tabular}{|c|c|c|c|c|c|c|c|c|c|c|c|c|c|c|}
\hline \multicolumn{3}{|c|}{ Decay } & \multicolumn{5}{|c|}{ Intermediate } & \multirow{3}{*}{$\begin{array}{c}\frac{\text { Initial }}{\text { Energy }} \\
\mathrm{keV}\end{array}$} & \multirow{3}{*}{$\begin{array}{c}\frac{\text { Final }}{\text { Energy }} \\
\mathrm{keV}\end{array}$} & \multicolumn{5}{|c|}{ Angular correlation coefficients } \\
\hline Daughter & Parent & Half-Life & Energy & Half-life & Spin/Parity & $\mathrm{Q}$ & $\mu$ & & & $\star$ & $A_{22}$ & $A_{24}$ & $A_{42}$ & $A_{44}$ \\
\hline & & & $\mathrm{keV}$ & & & $\mathrm{eb}$ & $\mu_{\mathrm{N}}$ & & & & & & & \\
\hline & & & & & & & & 728.01 & 181.09 & & $\sim-0.0944$ & $\sim 0$ & $\sim 0$ & $\sim 0$ \\
\hline & & & & & & & & 728.01 & 103.41 & & $\sim 0.135$ & $\sim 0.156$ & $\sim 0$ & $\sim 0$ \\
\hline & & & & & & & & 846.73 & 181.09 & & 0.05 & 0 & 0 & 0 \\
\hline & & & & & & & & 846.73 & 103.41 & & -0.07143 & -0.08248 & 0 & 0 \\
\hline \multirow[t]{15}{*}{${ }^{185} \mathrm{Au}$} & ${ }^{185} \mathrm{Hg}$ & $49.1 \mathrm{~s}$ & 8.9 & $4.8 \mathrm{~ns}$ & $(9 / 2-)$ & $?$ & $?$ & 107.5 & 0 & & -0.131 & -0.08127 & 0 & 0 \\
\hline & & & & & & & & 220.1 & 0 & & -0.07143 & -0.04433 & 0 & 0 \\
\hline & & & & & & & & 221.3 & 0 & & 0.102 & 0.06333 & 0.01461 & 0.00907 \\
\hline & & & 40.8 & $7 \mathrm{~ns}$ & $(3 / 2+)$ & $?$ & $?$ & 233.9 & 23.6 & $\left(\begin{array}{l}+ \\
+\end{array}\right)$ & $\sim-0.0951$ & $\sim 0$ & $\sim 0$ & $\sim 0$ \\
\hline & & & & & & & & & & $( \pm)$ & $\sim-0.256$ & $\sim 0$ & $\sim 0$ & $\sim 0$ \\
\hline & & & & & & & & & & $\left(\begin{array}{l}- \\
+\end{array}\right)$ & $\sim 0.215$ & $\sim 0$ & $\sim 0$ & $\sim 0$ \\
\hline & & & & & & & & & & $(\stackrel{-}{-})$ & $\sim 0.578$ & $\sim 0$ & $\sim 0$ & $\sim 0$ \\
\hline & & & & & & & & 291.1 & 23.6 & $\left(\begin{array}{l}+ \\
+\end{array}\right)$ & $0.026(17)$ & 0 & 0 & 0 \\
\hline & & & & & & & & & & $(\stackrel{ \pm}{ \pm})$ & $0.070(13)$ & 0 & 0 & 0 \\
\hline & & & 8.9 & $4.8 \mathrm{~ns}$ & $(9 / 2-)$ & $?$ & $?$ & 301.2 & 0 & $\left(\begin{array}{l}+ \\
+\end{array}\right)$ & $\sim 0.203$ & $\sim 0.126$ & $\sim-0.063$ & $\sim-0.0391$ \\
\hline & & & & & & & & & & $\left(\begin{array}{l}+ \\
+\end{array}\right)$ & $\sim-0.411$ & $\sim-0.255$ & $\sim-0.063$ & $\sim-0.0391$ \\
\hline & & & 40.8 & $7 \mathrm{~ns}$ & $(3 / 2+)$ & $?$ & ? & 439.5 & 23.6 & $\left(\begin{array}{l}+ \\
+\end{array}\right)$ & $-0.037(24)$ & 0 & 0 & 0 \\
\hline & & & & & & & & & & $( \pm)$ & $-0.101(18)$ & 0 & 0 & 0 \\
\hline & & & 220.1 & $26 \mathrm{~ns}$ & $(11 / 2-)$ & $?$ & $?$ & 490.2 & 8.9 & & -0.1182 & 0 & -0.06433 & 0 \\
\hline & & & & & & & & 682.3 & 8.9 & & -0.07143 & 0 & -0.01166 & 0 \\
\hline \multirow[t]{4}{*}{${ }^{187} \mathrm{Au}$} & ${ }^{187} \mathrm{Hg}$ & $114 \mathrm{~s}$ & 223.93 & $48 \mathrm{~ns}$ & $(11 / 2-)$ & $?$ & $?$ & 673.24 & 120.4 & & $-0.07(23)$ & 0 & $-0.01(4)$ & 0 \\
\hline & & & & & & & & 749.3 & 120.4 & $\left(\begin{array}{l}+ \\
+\end{array}\right)$ & $-0.2(5)$ & 0 & $0.03(9)$ & 0 \\
\hline & & & & & & & & & & $( \pm)$ & $-0.2(5)$ & 0 & $0.03(9)$ & 0 \\
\hline & & & & & & & & & & $\left(\begin{array}{l}+ \\
+\end{array}\right)$ & $0.3(9)$ & 0 & $0.03(9)$ & 0 \\
\hline
\end{tabular}


Table 9.3: Online candidates (continued)

\begin{tabular}{|c|c|c|c|c|c|c|c|c|c|c|c|c|c|c|}
\hline \multicolumn{3}{|c|}{ Decay } & \multicolumn{5}{|c|}{ Intermediate } & \multirow{3}{*}{$\begin{array}{c}\text { Initial } \\
\text { Energy } \\
\text { keV }\end{array}$} & \multirow{3}{*}{$\begin{array}{c}\frac{\text { Final }}{\text { Energy }} \\
\mathrm{keV}\end{array}$} & \multicolumn{5}{|c|}{ Angular correlation coefficients } \\
\hline Daughter & Parent & Half-Life & Energy & Half-life & Spin/Parity & $\mathrm{Q}$ & $\mu$ & & & $\star$ & $A_{22}$ & $A_{24}$ & $A_{42}$ & $A_{44}$ \\
\hline & & & $\mathrm{keV}$ & & & $\mathrm{eb}$ & $\mu_{\mathrm{N}}$ & & & & & & & \\
\hline & & & & & & & & & & 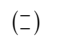 & $0.3(9)$ & 0 & $0.03(9)$ & 0 \\
\hline & ${ }^{187} \mathrm{Hg}$ & $2.4 \mathrm{~m}$ & 223.96 & $48 \mathrm{~ns}$ & $(11 / 2-)$ & ? & $?$ & 476.59 & 120.43 & & $-0.1(4)$ & 0 & $-0.06(21)$ & 0 \\
\hline${ }^{189} \mathrm{Pt}$ & ${ }^{189} \mathrm{Au}$ & $4.59 \mathrm{~m}$ & 172.7 & $464 \mathrm{~ns}$ & $9 / 2-$ & $?$ & $?$ & 493.8 & 6.3 & & $-0.160(5)$ & $-0.099(3)$ & $-0.09059(9)$ & $-0.05622(6)$ \\
\hline \multirow[t]{14}{*}{${ }^{189} \mathrm{Au}$} & ${ }^{189} \mathrm{Hg}$ & $7.6 \mathrm{~m}$ & 325.1 & $190 \mathrm{~ns}$ & 9/2- & ? & $?$ & 491.51 & 247.3 & $\left(\begin{array}{l}+ \\
+\end{array}\right)$ & $-0.34(9)$ & $-0.017(14)$ & $-0.21(5)$ & $-0.011(9)$ \\
\hline & & & & & & & & & & $( \pm)$ & $0.18(7)$ & $-0.017(14)$ & $0.11(5)$ & $-0.011(9)$ \\
\hline & & & & & & & & 770.73 & 247.3 & $\left(\begin{array}{l}+ \\
+\end{array}\right)$ & $0.84(22)$ & $0.04(3)$ & $0.14(6)$ & $7(6) \cdot 10^{-3}$ \\
\hline & & & & & & & & & & $( \pm)$ & $-0.44(18)$ & $0.04(3)$ & $-0.07(4)$ & $7(6) \cdot 10^{-3}$ \\
\hline & & & & & & & & & & $\left(\begin{array}{l}+ \\
+\end{array}\right)$ & $-0.51(16)$ & $-0.026(22)$ & $0.14(6)$ & $7(6) \cdot 10^{-3}$ \\
\hline & & & & & & & & & & $(z)$ & $0.27(12)$ & $-0.026(22)$ & $-0.07(4)$ & $7(6) \cdot 10^{-3}$ \\
\hline & & & & & & & & 911 & 247.3 & $\left(\begin{array}{l}+ \\
+\end{array}\right)$ & $0.24(6)$ & $0.012(10)$ & 0 & 0 \\
\hline & & & & & & & & & & $( \pm)$ & $-0.13(5)$ & $0.012(10)$ & 0 & 0 \\
\hline & ${ }^{189} \mathrm{Hg}$ & $8.6 \mathrm{~m}$ & 325.32 & $190 \mathrm{~ns}$ & $9 / 2-$ & $?$ & $?$ & 646.35 & 247.46 & $\left(\begin{array}{l}+ \\
+\end{array}\right)$ & $-0.19(5)$ & $-9(8) \cdot 10^{-3}$ & $-0.027(7)$ & $-1.3(11) \cdot 10^{-3}$ \\
\hline & & & & & & & & & & $( \pm)$ & $0.10(4)$ & $-9(8) \cdot 10^{-3}$ & $0.014(6)$ & $-1.3(11) \cdot 10^{-3}$ \\
\hline & & & & & & & & 712.9 & 247.46 & $\left(\begin{array}{l}+ \\
+\end{array}\right)$ & $-0.33(12)$ & $-0.017(14)$ & $0.12(4)$ & $6(5) \cdot 10^{-3}$ \\
\hline & & & & & & & & & & $( \pm)$ & $0.18(9)$ & $-0.017(14)$ & $-0.06(3)$ & $6(5) \cdot 10^{-3}$ \\
\hline & & & & & & & & & & $\left(\begin{array}{l}- \\
+\end{array}\right)$ & $0.72(20)$ & $0.04(3)$ & $0.12(4)$ & $6(5) \cdot 10^{-3}$ \\
\hline & & & & & & & & & & $(=)$ & $-0.38(16)$ & $0.04(3)$ & $-0.06(3)$ & $6(5) \cdot 10^{-3}$ \\
\hline \multirow[t]{4}{*}{${ }^{189} \mathrm{~Pb}$} & ${ }^{189 \mathrm{~m}} \mathrm{~Pb}$ & $22.2 \mu \mathrm{s}$ & 1287.25 & $2.1 \mathrm{~ns}$ & $(21 / 2+)$ & $?$ & $?$ & 1825.43 & 818.8 & & 0.102 & 0.04194 & 0.02207 & 0.00907 \\
\hline & & & & & & & & 2097.83 & 818.8 & & 0.102 & 0.04194 & 0.02207 & 0.00907 \\
\hline & & & 2097.83 & $2.1 \mathrm{~ns}$ & $(25 / 2+)$ & $?$ & $?$ & 2434.53 & 1567.45 & & 0.1786 & 0.06981 & -0.01107 & -0.004329 \\
\hline & & & & & & & & 2434.53 & 1287.25 & & 0.1786 & 0.06981 & -0.01107 & -0.004329 \\
\hline${ }^{194} \mathrm{Au}$ & ${ }^{194 \mathrm{~m}} \mathrm{Au}$ & $420 \mathrm{~ms}$ & 244.6 & $2.6 \mathrm{~ns}$ & $(7+)$ & $?$ & $?$ & 406.8 & 107.4 & & $-0.370(21)$ & $-0.178(10)$ & $-0.074(8)$ & $-0.035(4)$ \\
\hline${ }^{194} \mathrm{pb}$ & ${ }^{194} \mathrm{Bi}$ & $115 \mathrm{~s}$ & 2407.6 & $17 \mathrm{~ns}$ & $(9-)$ & ? & $-0.38(14)$ & 2581.4 & 2241.3 & & -0.07143 & -0.03092 & 0 & 0 \\
\hline
\end{tabular}

Continued on next page 
Table 9.3: Online candidates (continued)

\begin{tabular}{|c|c|c|c|c|c|c|c|c|c|c|c|c|c|c|}
\hline \multicolumn{3}{|c|}{ Decay } & \multicolumn{5}{|c|}{ Intermediate } & \multirow{2}{*}{$\begin{array}{c}\text { Initial } \\
\text { Energy } \\
\mathrm{keV}\end{array}$} & \multirow{2}{*}{$\begin{array}{c}\frac{\text { Final }}{\text { Energy }} \\
\mathrm{keV}\end{array}$} & \multicolumn{5}{|c|}{ Angular correlation coefficients } \\
\hline Daughter & Parent & Half-Life & $\begin{array}{c}\text { Energy } \\
\text { keV }\end{array}$ & Half-life & Spin/Parity & $\begin{array}{l}\mathrm{Q} \\
\mathrm{eb}\end{array}$ & $\begin{array}{c}\mu \\
\mu_{\mathrm{N}}\end{array}$ & & & $\star$ & $A_{22}$ & $A_{24}$ & $A_{42}$ & $A_{44}$ \\
\hline \multirow[t]{9}{*}{${ }^{196} \mathrm{~Pb}$} & ${ }^{196} \mathrm{Bi}$ & $5.13333 \mathrm{~m}$ & 1049.27 & $100 \mathrm{~ns}$ & $2+$ & $?$ & $?$ & 1738.59 & 0 & & 0.102 & 0.1825 & 0.00507 & 0.00907 \\
\hline & ${ }^{196} \mathrm{Bi}$ & $4 \mathrm{~m}$ & 1049.23 & $100 \mathrm{~ns}$ & $2+$ & $?$ & $?$ & 1738.62 & 0 & & 0.102 & 0.1825 & 0.00507 & 0.00907 \\
\hline & & & 1738.62 & $1 \mu \mathrm{s}$ & $4+$ & $?$ & $?$ & 1797.96 & 1049.23 & & -0.07143 & -0.04856 & 0 & 0 \\
\hline & & & 1049.23 & $100 \mathrm{~ns}$ & $2+$ & $?$ & $?$ & 1797.96 & 0 & & 0.1786 & 0.3194 & -0.00242 & -0.004329 \\
\hline & & & 1797.96 & $140 \mathrm{~ns}$ & 5- & $?$ & $\pm 0.490(15)$ & 2170.2 & 1738.62 & & -0.07143 & 0 & -0.011 & 0 \\
\hline & & & & & & & & 2170.2 & 1049.23 & & 0.1786 & -0.02812 & 0.02749 & -0.004329 \\
\hline & & & 2170.2 & $5 \mathrm{~ns}$ & 7 & $?$ & ? & 2308.6 & 1797.96 & & 0.102 & 0.04897 & 0.0189 & 0.00907 \\
\hline & & & & & & & & 2591.8 & 1797.96 & & -0.07143 & -0.03428 & 0 & 0 \\
\hline & & & 2308.6 & $52 \mathrm{~ns}$ & 9- & $?$ & $-0.33(9)$ & 2646.1 & 2170.2 & & -0.07143 & -0.03092 & 0 & 0 \\
\hline${ }^{196} \mathrm{Po}$ & ${ }^{196 \mathrm{~m}} \mathrm{Po}$ & $856 \mathrm{~ns}$ & 859.12 & $12 \mathrm{~ns}$ & $2+$ & $?$ & $?$ & 1387.75 & 0 & & 0.102 & 0.1825 & 0.00507 & 0.00907 \\
\hline \multirow[t]{2}{*}{${ }^{201} \mathrm{Hg}$} & ${ }^{201 \mathrm{~m}} \mathrm{Hg}$ & $94 \mu \mathrm{s}$ & 547.5 & $20 \mathrm{~ns}$ & $9 / 2-$ & $?$ & $?$ & 766.9 & 26.34 & $\left(\begin{array}{c}+ \\
+\end{array}\right)$ & $\sim 0.219$ & $\sim 0.136$ & $\sim-0.0325$ & $\sim-0.0202$ \\
\hline & & & & & & & & & & $\left(\begin{array}{l}- \\
+\end{array}\right)$ & $\sim-0.0519$ & $\sim-0.0322$ & $\sim 0.0646$ & $\sim 0.0401$ \\
\hline \multirow[t]{3}{*}{${ }^{203} \mathrm{~Pb}$} & ${ }^{203 \mathrm{~m}} \mathrm{~Pb}$ & $480 \mathrm{~ms}$ & 1921.98 & $42 \mathrm{~ns}$ & $21 / 2+$ & $?$ & $-0.641(21)$ & 2795.76 & 1663.61 & $\left(\begin{array}{c}+ \\
+\end{array}\right)$ & $0.19(8)$ & $0.08(3)$ & $-0.03(3)$ & $-0.012(12)$ \\
\hline & & & & & & & & & & $\left(\begin{array}{l}- \\
+\end{array}\right)$ & $-0.34(9)$ & $-0.14(4)$ & $-0.03(3)$ & $-0.012(12)$ \\
\hline & & & & & & & & 2949.11 & 1663.61 & & 0.2208 & 0.09075 & -0.04375 & -0.01798 \\
\hline \multirow[t]{4}{*}{${ }^{211} \mathrm{At}$} & ${ }^{211 \mathrm{~m}_{\mathrm{At}}}$ & $4.23 \mu \mathrm{s}$ & 1416.6 & $50 \mathrm{~ns}$ & $(21 / 2-)$ & $\pm 0.53(5)$ & $9.56(9)$ & 1927.8 & 1320.6 & & -0.07143 & -0.02936 & 0 & 0 \\
\hline & & & 2641.4 & $70 \mathrm{~ns}$ & $(29 / 2+)$ & $\pm 1.00(5)$ & $15.31(13)$ & 4177.4 & 1927.8 & & -0.125 & 0.01285 & 0 & 0 \\
\hline & & & 4177.4 & $10 \mathrm{~ns}$ & $(31 / 2+)$ & $?$ & $?$ & 4381.1 & 2641.4 & $\left(\begin{array}{c}+ \\
+\end{array}\right)$ & $-0.151(22)$ & 0 & $0.031(19)$ & 0 \\
\hline & & & & & & & & & & $\left(\begin{array}{l}- \\
+\end{array}\right)$ & $0.251(22)$ & 0 & $0.031(19)$ & 0 \\
\hline${ }^{211} \mathrm{Rn}$ & ${ }^{211} \mathrm{Fr}$ & $3.1 \mathrm{~m}$ & 540 & $4 \mathrm{~ns}$ & $5 / 2-$ & $?$ & $?$ & 1458 & 0 & & 0.102 & 0.1178 & 0.007855 & 0.00907 \\
\hline \multirow[t]{3}{*}{${ }^{212} \mathrm{At}$} & ${ }^{212 \mathrm{~m}_{\mathrm{At}}}$ & 152 us & 885.4 & $18.7 \mathrm{~ns}$ & $(11+)$ & $?$ & $\pm 5.94(11)$ & 1262.4 & 701.6 & & 0.05 & 0 & 0 & 0 \\
\hline & & & & & & & & 1262.4 & 223 & & -0.07143 & -0.02894 & 0 & 0 \\
\hline & & & 1317 & $2 \mathrm{~ns}$ & (11-) & $?$ & $?$ & 1540.6 & 223 & & 0.102 & 0.04135 & 0.02238 & 0.00907 \\
\hline
\end{tabular}


Table 9.3: Online candidates (continued)

\begin{tabular}{|c|c|c|c|c|c|c|c|c|c|c|c|c|c|c|}
\hline \multicolumn{3}{|c|}{ Decay } & \multicolumn{5}{|c|}{ Intermediate } & \multirow{2}{*}{$\begin{array}{c}\text { Initial } \\
\text { Energy } \\
\mathrm{keV}\end{array}$} & \multirow{2}{*}{$\begin{array}{c}\frac{\text { Final }}{\text { Energy }} \\
\mathrm{keV}\end{array}$} & \multicolumn{5}{|c|}{ Angular correlation coefficients } \\
\hline Daughter & Parent & Half-Life & $\begin{array}{c}\text { Energy } \\
\text { keV }\end{array}$ & Half-life & Spin/Parity & $\begin{array}{l}\mathrm{Q} \\
\mathrm{eb}\end{array}$ & $\begin{array}{c}\mu \\
\mu_{\mathrm{N}}\end{array}$ & & & $\star$ & $A_{22}$ & $A_{24}$ & $A_{42}$ & $A_{44}$ \\
\hline \multirow{12}{*}{$\begin{array}{l}{ }^{225} \mathrm{Ra} \\
{ }^{227} \mathrm{Fr}\end{array}$} & & & 1604.5 & $35.4 \mathrm{~ns}$ & $(15-)$ & $?$ & $\pm 9.46(8)$ & 1763.9 & 1540.6 & & -0.07143 & -0.02672 & 0 & 0 \\
\hline & & & & & & & & 2212.2 & 1540.6 & & -0.07143 & -0.02672 & 0 & 0 \\
\hline & & & & & & & & 2250 & 1540.6 & & 0.1786 & 0.0668 & -0.01157 & -0.004329 \\
\hline & ${ }^{225} \mathrm{Fr}$ & $4 \mathrm{~m}$ & 31.56 & $2.1 \mathrm{~ns}$ & $3 / 2-$ & $?$ & ? & 179.75 & 0 & & 0.05 & 0 & 0 & 0 \\
\hline & ${ }^{227} \mathrm{Rn}$ & $20.8 \mathrm{~s}$ & 39.88 & $2.7 \mathrm{~ns}$ & $3 / 2+$ & $?$ & ? & 144.32 & 0 & $\left(\begin{array}{l}+ \\
+\end{array}\right)$ & $\sim-0.0339$ & $\sim 0$ & $\sim 0$ & $\sim 0$ \\
\hline & & & & & & & & & & $( \pm)$ & $\sim-0.111$ & $\sim 0$ & $\sim 0$ & $\sim 0$ \\
\hline & & & & & & & & & & $(\stackrel{-}{+})$ & $\sim-0.0339$ & $\sim 0$ & $\sim 0$ & $\sim 0$ \\
\hline & & & & & & & & & & $(=)$ & $\sim-0.111$ & $\sim 0$ & $\sim 0$ & $\sim 0$ \\
\hline & & & & & & & & 306.53 & 0 & $\left(\begin{array}{l}+ \\
+\end{array}\right)$ & $\sim-0.049$ & $\sim 0$ & $\sim 0$ & $\sim 0$ \\
\hline & & & & & & & & & & $( \pm)$ & $\sim-0.16$ & $\sim 0$ & $\sim 0$ & $\sim 0$ \\
\hline & & & & & & & & & & $\left(\begin{array}{l}- \\
+\end{array}\right)$ & $\sim-0.049$ & $\sim 0$ & $\sim 0$ & $\sim 0$ \\
\hline & & & & & & & & & & $(\beth)$ & $\sim-0.16$ & $\sim 0$ & $\sim 0$ & $\sim 0$ \\
\hline
\end{tabular}




\section{Chapter 10}

\section{Facilities for probe implantation, annealing, and magnetization}

Basically, there are two different approaches for TDPAC measurements: For long lived probe nuclides, it is possible to separate nuclide production, implantation, annealing, and measurement. This approach allows using of optimized setups for each step and is usually used for the nuclides listed in tables 9.1 and 9.2. A complex implantation setup for the implantation of such probe atoms consisting of two parts has been developed within the framework of this thesis and will be presented in section 10.1. Additionally, a custom high temperature furnace design was optimized for annealing purposes (cf. section 10.2).

The second approach, which makes TDPAC measurements with short and very short lived nuclides (cf. table 9.3) possible, is to integrate several of the named steps to make the overall process sufficiently quick. Due to the short half-lives involved, it is typically necessary to do the measurements directly at the production facilities. Implantation and annealing steps usually have to be combined if annealing is necessary (hot implantation). The requirement to perform measurements during implantation introduces additional challenges because it becomes necessary to allow placing detectors, which have to be outside the beamline and implantation chambers, very close to the target, which obviously has to be inside the beam tube, while at the same time making it possible to control the target's temperature and/or magnetization. All these design goals are in conflict with the requirement to minimize $\gamma$ absorption between sample and detectors. Designs meant to meet these challenges are presented in section 10.1.4.

\subsection{New implantation and TDPAC facilities for ISOLDE}

For the purposes of this work, a major replacement of the implantation facilities available at ISOLDE was developed. The whole project consists of three new chambers with different purposes.

The first chamber, which is almost ready for commissioning by now, is a replacement of the old implantation chamber operational at the GLM branch of ISOLDE. It is described in detail in section 10.1.2. The new implantation chamber is designed to make it possible 
to shoot through it into chambers mounted downstream. Its collimator and ion optical components can thereby be used to optimize the beam properties.

The second chamber is designed to be mounted behind the implantation chamber although it can also be operated on its own. It provides the possibility for implantations using a decelerated ion beam thus implanting closer to the surface of the used samples. Alternatively, the ion beam can be post-accelerated, thereby allowing conducting of implantations using total ion energies of up to $120 \mathrm{keV}$. Its design and working principle is described in section 10.1.3. Like the implantation chamber, the deceleration chamber can also be operated in shoot-through-mode using its collimator and ion optics for beam conditioning thus allowing to use a third chamber behind it.

A third chamber is intended for online TDPAC measurements using the ISOLDE beam. It will allow for hot implantations and simultaneous measurements. Its design is based on the experiences obtained while building and optimizing the high-temperature furnace described in section 10.2. Additional design optimizations were necessary to allow for online measurements. Especially the reduction of size and $\gamma$ absorption, which is necessary to allow mounting of detectors close to the hot sample while not losing the emitted $\gamma$ radiation due to absorption, is a major design goal of this chamber. The currently preferred solution is described in section 10.1.4.

All three chambers are designed to be self-contained in order to make it possible to operate them in arbitrary combinations and replace them easily. They can be lifted via the existing crane using hooks on the top side of their supporting frames.

\subsubsection{Overview}

Rendered views of the current plans for all three chambers are shown in figs. 10.2 and 10.3. While fig. 10.2 shows an overview of the chamber ensemble, fig. 10.3 shows a horizontal cross-section revealing the interior parts. The existing ISOLDE beamline is colored blue in both images and the wall on the camera's side of the cave was suppressed. The viewing direction in both figures is from the GHM branch's side (see also fig. 2.6 for the ISOLDE hall's layout ). The rightmost chamber, connected to the ISOLDE beamline, is the new implantation chamber while the middle chamber is the deceleration and postacceleration chamber whereas the left chamber is the current design draft of the online TDPAC chamber. The space beneath the deceleration chamber is covered by a metal cage because it contains the devices that are on the chamber's interior potential of $-60 \mathrm{keV}$ to $60 \mathrm{keV}$. Beneath the implantation chamber, the chiller for the turbopumps is visible.

All three chambers are mounted on supporting frames, which allow for a precise positioning of the chambers. The brackets holding the chambers' flanges can be moved horizontally in arbitrary directions. They rest on an inner frame, which can be adjusted vertically relative to the supporting frame by four fine pitch threaded bolts. The threaded stands of the supporting frame allow for compensation of uneven floors as well as coarse vertical adjustment. 


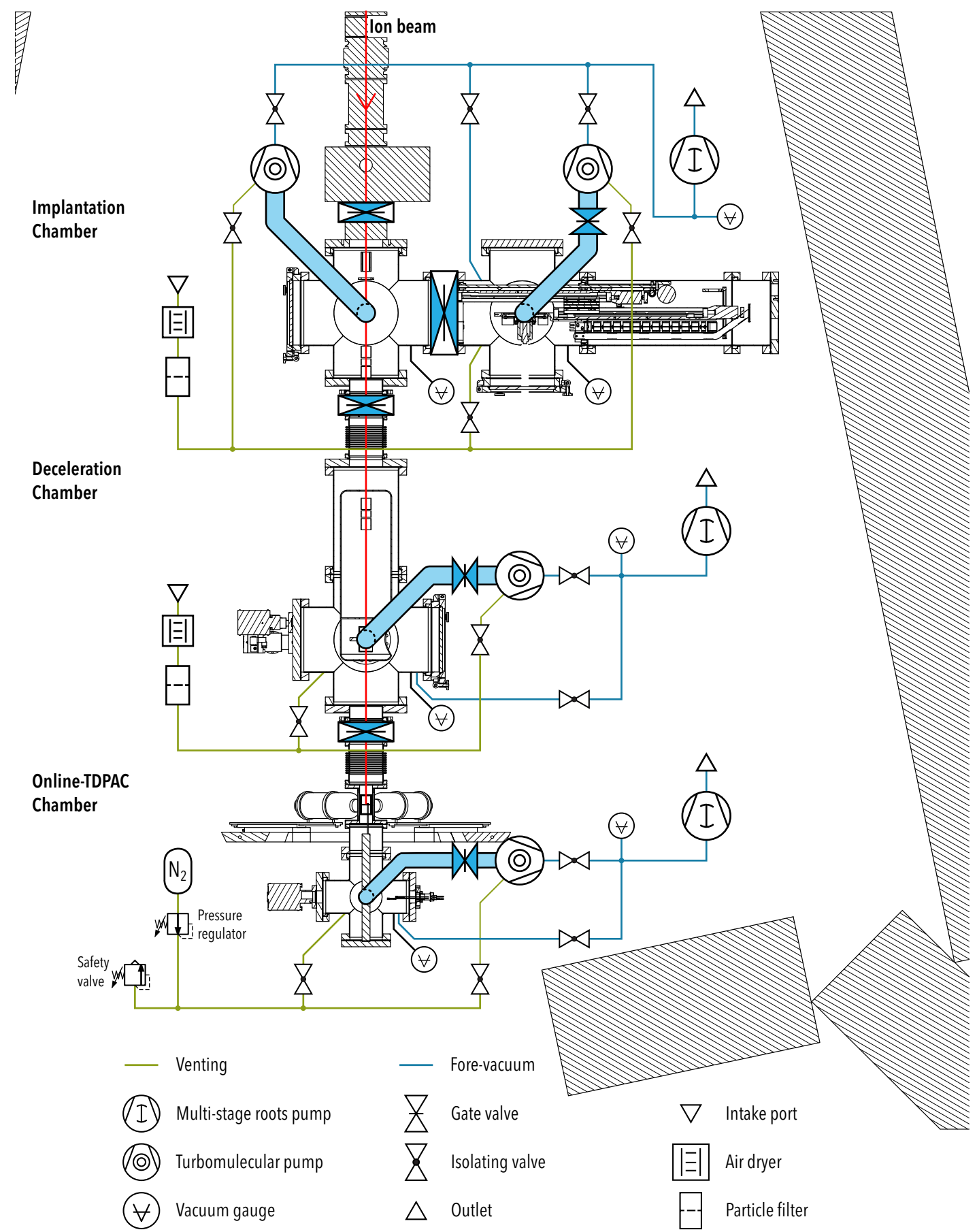

Figure 10.1: Circuit diagrams of the new ISOLDE chambers' vacuum systems. The used symbols were chosen according to DIN 28401 and DIN ISO 1219, as appropriate. 


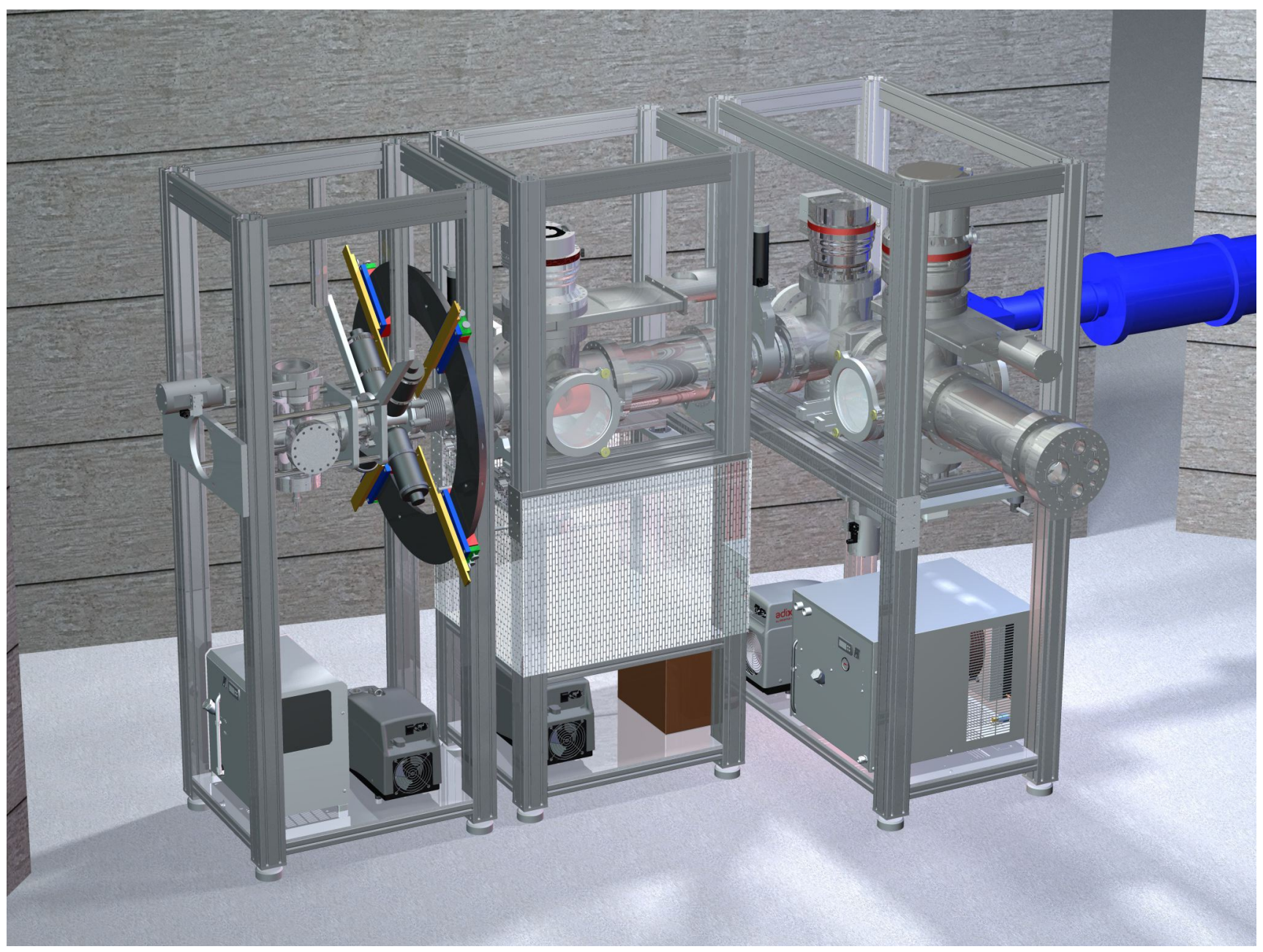

Figure 10.2: Overviews of the new implantation chambers designed for ISOLDE's GLM branch. 


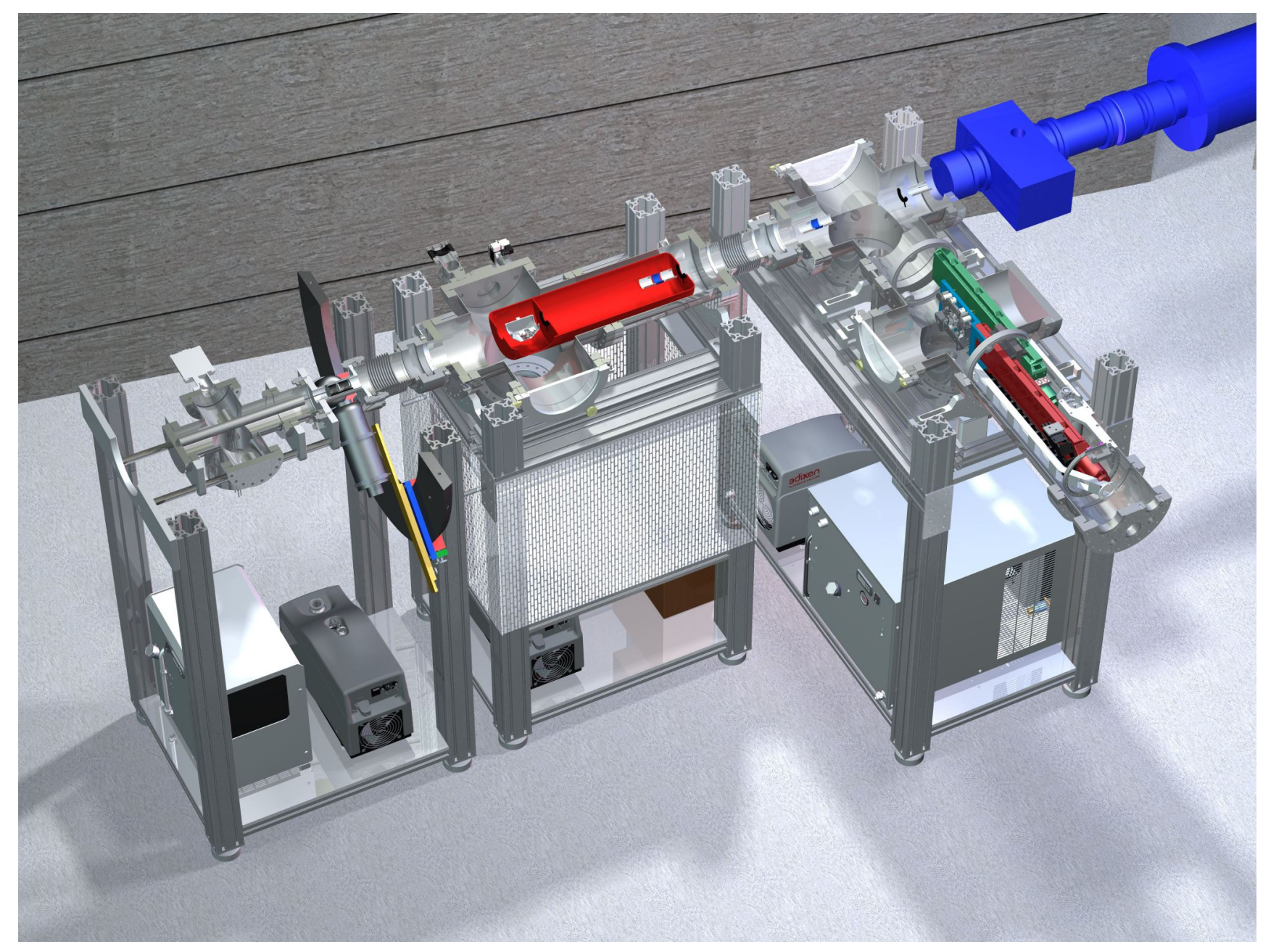

Figure 10.3: Cross section through the new implantation chambers designed for ISOLDE's GLM branch. 
The circuit diagram in fig. 10.1 contains an overview on vacuum and ventilation subsystems of all three chambers and is discussed in the chamber specific sections below.

\subsubsection{Implantation chamber}

The new implantation chamber is basically a replacement for the chamber currently used at ISOLDE. It provides the possibility to implant nuclides into arbitrary samples. Its major advantages, compared to the current solution, are the load-lock mechanism, which allows for rapid sample changing while the actual implantation chamber stays evacuated, and the possibility for tilted implantations for the avoiding of unwanted channeling effects. Figure 10.4 shows a photo of this chamber.

Chamber layout The two separable parts of the chamber, necessary for the realization of a load-lock mechanism, consist of a six-way cross with DN200 CF flanges each. These two elements are connected by a pneumatic DN200 gate valve controlled by two solenoid valves. This valve is a special design, which maintains its position in case of a power breakdown or a loss of pressure. The two solenoid valves are only activated temporarily during opening/closing of the main valve. The gate valve's position maintaining property is essential to avoid the risk of damage occurring if the valve opens while only one of the chamber parts is evacuated or if the valve closes while the sample transport system blocks its opening. Both cases are additionally prohibited by hard-wired interlocks: The closing solenoid's circuit is closed by a dedicated switch, which detects if the sample transport system is in its safe position for sample changing, while the opening solenoid's circuit is interrupted if the vacuum sensors of the two chamber parts report pressure values of different orders of magnitude. Additionally, both circuits are interrupted as soon as one of the two quick access doors is opened to avoid the risk of injuries due to a moving valve gate.

The part of the chamber used for the actual implantations is equipped with a quick access door to simplify service tasks (especially the exchange of contaminated collimators). The second chamber part provides another quick access door for sample loading and unloading.

Evacuation and ventilation Each part of the chamber is evacuated by a turbomolecular pump (see the implantation chamber part of fig. 10.1). A Pfeiffer Vacuum HiPace 700 is mounted at the continually evacuated implantation part and a HiPace 1200 is used for the sample loading part. The sample loading part's evacuation system was designed to yield a pressure below $10^{-6} \mathrm{mbar}$ in less than $10 \mathrm{~min}$ after closing the quick access door. Both turbopumps are connected to a dry multi-stage roots pump (Pfeiffer Vacuum ACP 28) acting as fore-pump. To allow for rapid evacuation of the loading/unloading chamber part, an automatized bypass system is in place. It consists of a DN200 gate valve between the chamber and the HiPace 1200 turbopump, two valves isolating the two turbopumps 


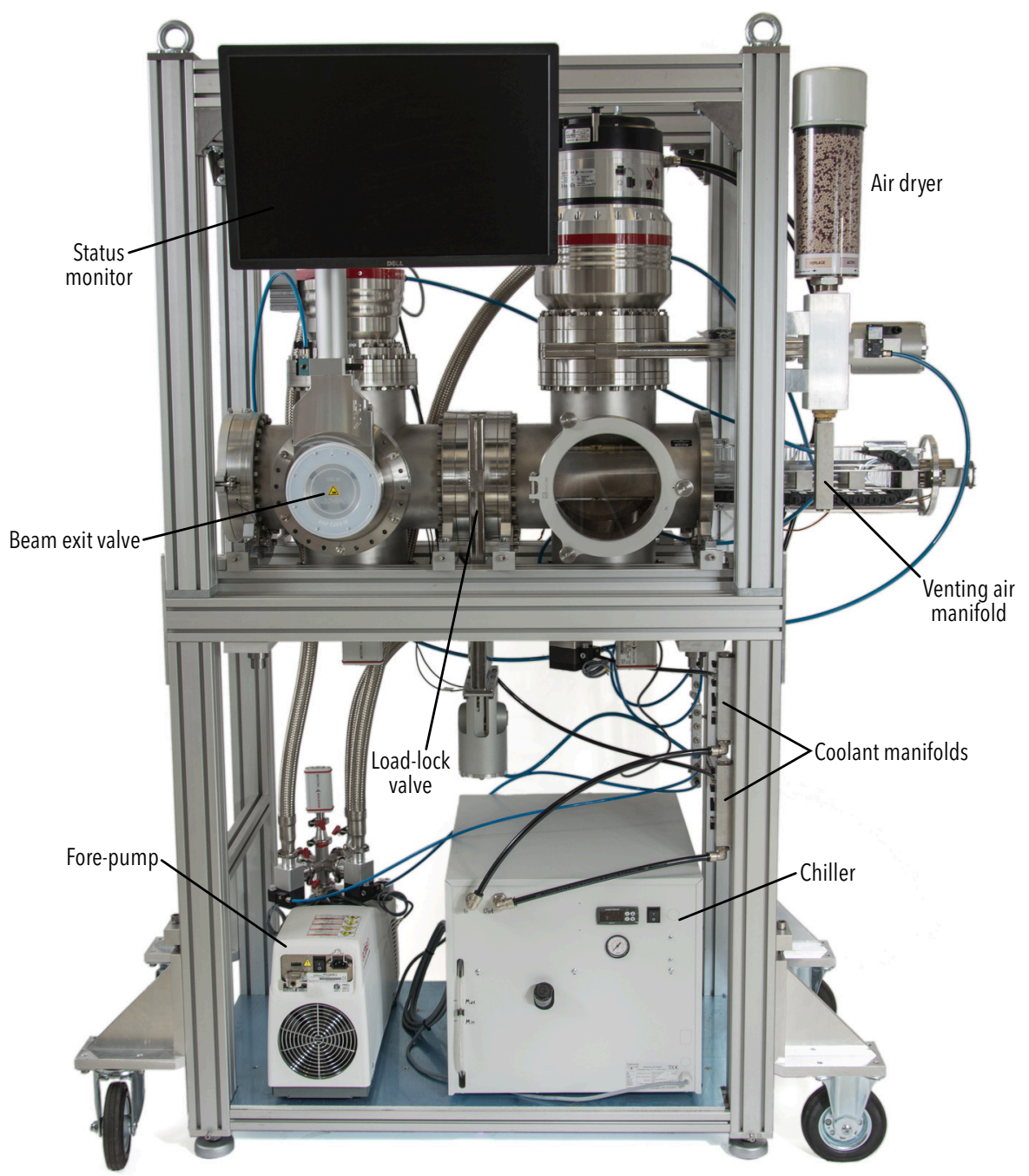

Figure 10.4: Photo of the implantation chamber with opened sample transport system.

from the fore-pump, and an additional bypass valve, which provides a direct connection between the chamber and the fore-pump. During sample change, the gate valve is closed and the HiPace 1200 only affects the volume above the valve. As soon as evacuation of the chamber starts, the two valves between fore-pump and the turbopumps are closed and the bypass valve connection fore-pump and chamber is opened. After the pressure in the fore-vacuum system reaches an appropriate level, the valves connecting turbo- and fore-pumps are reopened. As soon as these valves have opened and the pressure level in the chamber reaches a sufficiently low level, the bypass valve is closed and the gate valve is opened. All valves are equipped with pneumatic actuators, which are controlled by 
solenoid valves. The chamber control electronics takes care for proper valve operation. The actual implantation chamber is not equipped with a bypass system since it is not ventilated during normal operation.

The two turbopumps are cooled using a closed cycle cooling system based on a ProfiCool Novus PCNO 15 chiller mounted on the lower level of the supporting frame.

The chamber is ventilated using cleaned air. To reduce condensation of air moisture on the chamber's interior parts during ventilation, an air dryer reduces the humidity level of the incoming air. An additional particle filter removes dust. Three valves control ventilation of the sample loading chamber as well as ventilation of the implantation chamber's and the sample loading chamber's turbopumps. The latter two valves are triggered by the turbopump controllers. The direct ventilation valve for the sample loading chamber can only be opened if the gate valve, connecting the chamber and its turbopump, is closed. Pressure levels are measured using three vacuum gauges connected to the two chamber parts and the fore-vacuum distribution. These gauges are directly read out by the control electronics via RS485.

Sample transport The transport of samples between the loading and implantation parts of the chamber, as well as sample positioning during implantations, is based on an assembly of two PI miCos LS-110 linear stages with integrated limit switches mounted face-to-face and providing a total travel range of $610 \mathrm{~mm}$. These stages are specified to carry up to $10 \mathrm{~kg}$ and therefore provide high stiffness and thus precise positioning. The fixed stage is mounted on the tilt mechanism, which provides the possibility of tilted implantations at arbitrary angles, while the travelling stage moves the sample holder into the chamber's implantation part. Figure 10.5 shows the transport assembly inside the chamber in sample loading position. In contrast, fig. 10.6 shows the same in implanting position. In both figures, tilt is not active and the samples' surfaces are aligned perpendicular to the beam direction.

The tilt mechanism consists of two outer rings fitted into opposite flanges of the six-waycross, which constitutes the sample loading chamber. Half of these rings' width consists of an annulus gear while the other half is a flat contact surface for the inner rings' bearings. The inner rings are holding several bearings acting as wheels on the flat parts of the outer rings as well as one gear-wheel each. These gear-wheels are mounted on a common shaft, which is rotated by a stepper motor via a worm gearing. The fixed stage is mounted on the inner rings and can therefore be tilted using this mechanism. The traveling stage's slider is mounted on the slider of the fixed stage while the traveling stage's body is the base for the holding frame, which receives the removable sample holders. A cam ring, fixed on the rotating part, operates two limit switches, which are mounted on the chamber's extension tube (i.e. they do not move themselves), and are used as tilt reference switch and hard limit for the rotation avoiding damage to the cabling. To compensate the linear movement of the stages, the cable harness towards the sample holder is lead through an 


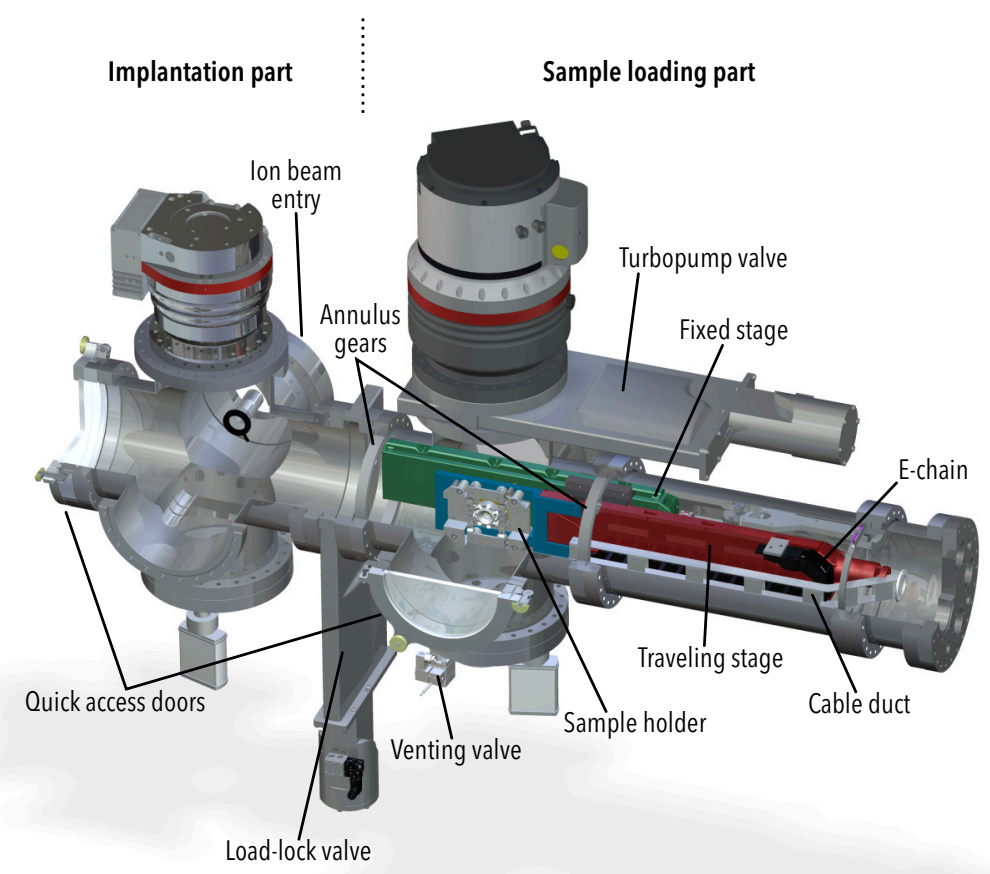

Figure 10.5: Implantation chamber with the sample transport system in sample loading position.

e-chain (igus B15). The rotation is compensated by guiding all cables connecting the rotating part with the environment through an eye on the rotational axis, which is mounted at the end of a rotating cable duct.

Figure 10.7 shows the chamber in tilted implanting position. Compared to fig. 10.6, the sample holder was rotated and is therefore tilted with respect to the ion trajectory. Figure 10.8 shows photos of the opened sample transport system in different positions.

The sample holder As a key component of the implantation chamber, the sample holder was carefully designed to fulfill the following design goals:

- Precise operation, ease of use, and robustness

- Good electrical isolation from the rest of the chamber is required for reliable precision measurements of ion currents in the pA range.

- Getting stuck of the sample holder inside the chamber has to be impossible by design. 


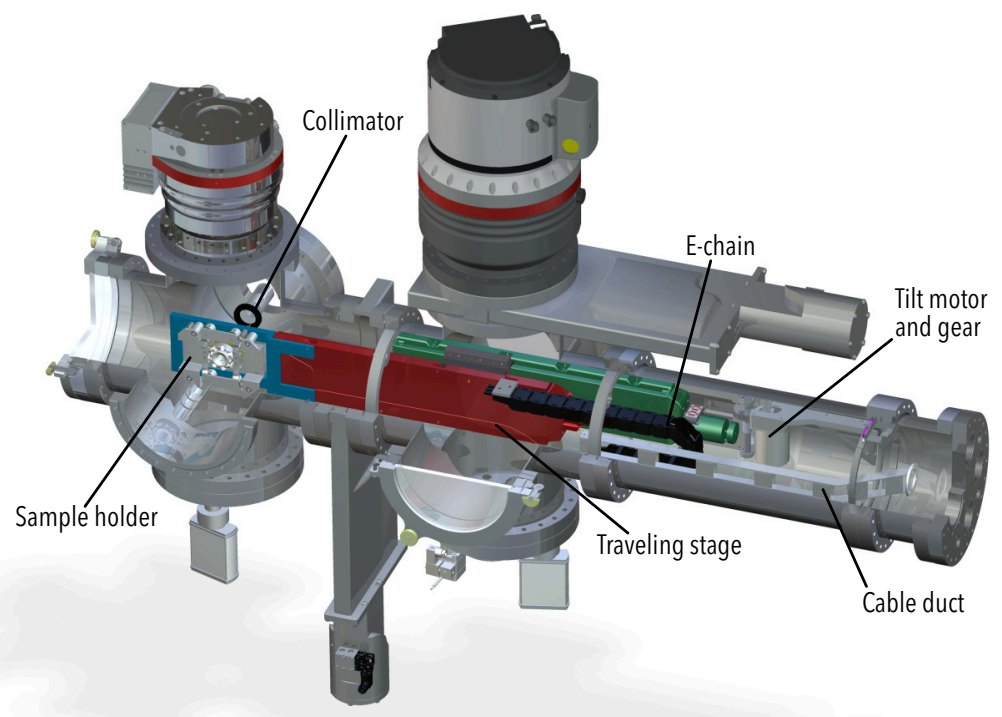

Figure 10.6: Implantation chamber with the sample transport system in implanting position.

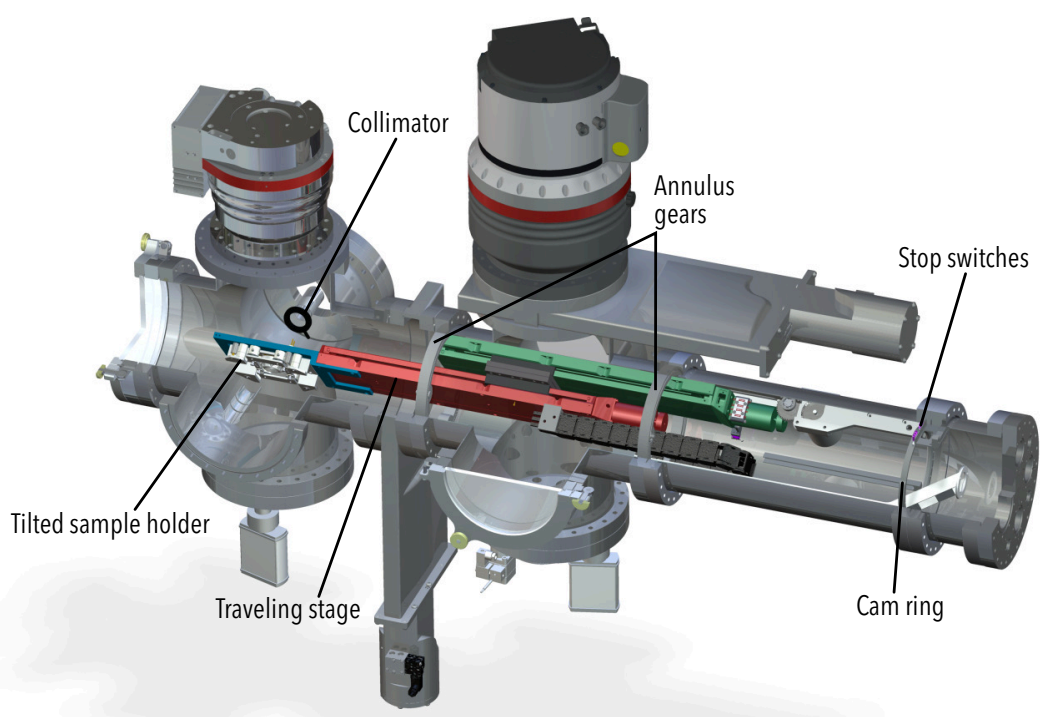

Figure 10.7: Implantation chamber with the sample transport system in tilted implanting position. 
(a) Sample transport system in sample loading position.

(b) Sample transport system in implanting position.

(c) Sample transport system in tilted implanting position.

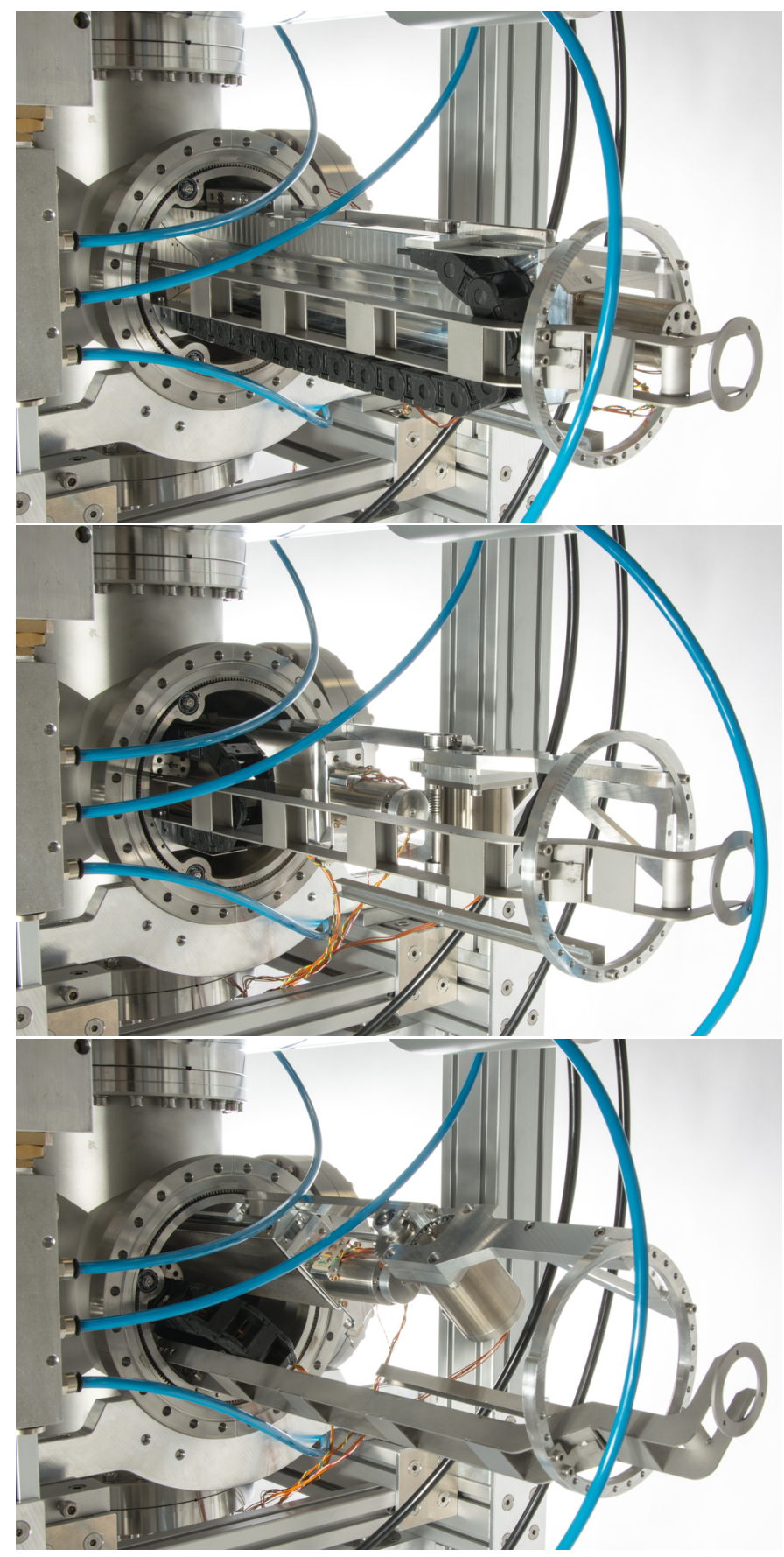

Figure 10.8: Photos of the sample transport system. The tube, which normally covers this part, was removed. 


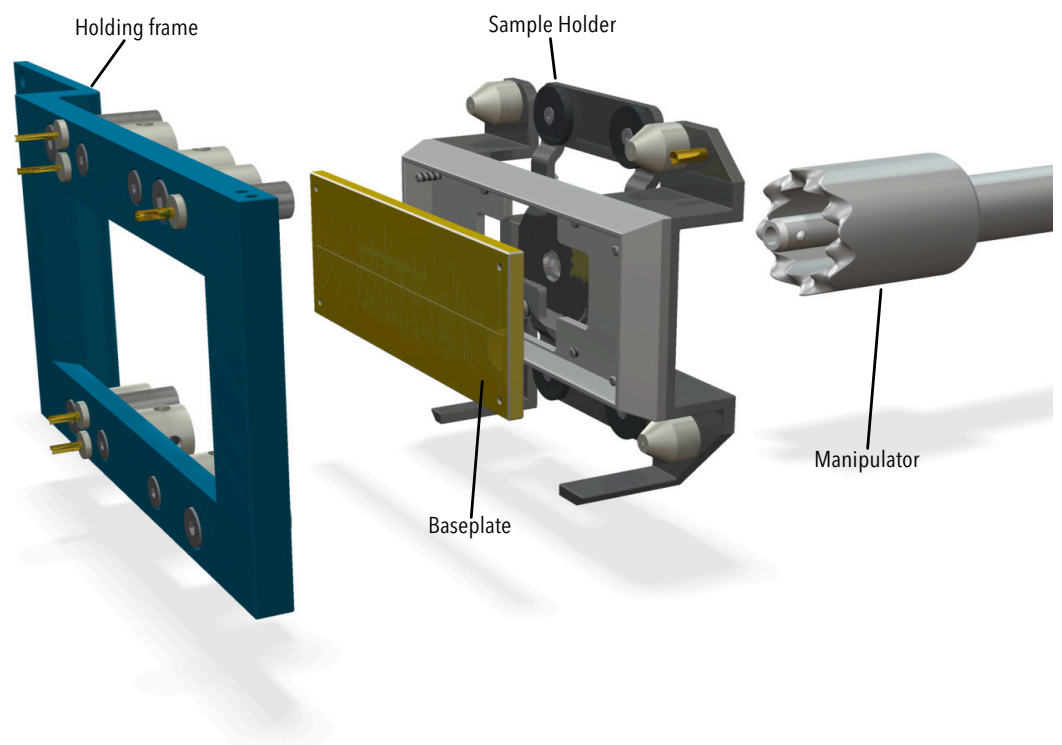

Figure 10.9: Sample holder (exploded view).

- Loading and unloading of the holder has to be possible by using a manipulator for decreased radiation exposition of the operators.

- A flat surface on the ion beam's side is desirable for decreased contamination and no artificial reduction of achievable tilt angles.

- Reliable contacting is required for ion current measurement, temperature measurement, and sample heating.

- A replaceable and modifiable sample carrying base plate with a simple shape is beneficial for easy and cheap replacement in case of contamination.

The design, developed to solve these requirements, is shown in figs. 10.9 to 10.12 . The blue holding frame shown in these images is mounted on the traveling stage of the sample transport system. A replaceable baseplate, which carries the samples and can be customized for different sample types and mounting methods, is fixed on the actual sample holder by four screws. The slot for the baseplate in the standard holder design has a depth of $10 \mathrm{~mm}$, allowing the construction of baseplates adapted to the thickness and other properties of certain samples. For special applications, it is possible to build holders with even deeper slot openings.

When inserted into the holding frame, the sample holder is properly aligned by four conically shaped positioners and their counterparts. The positioners on the side of the 


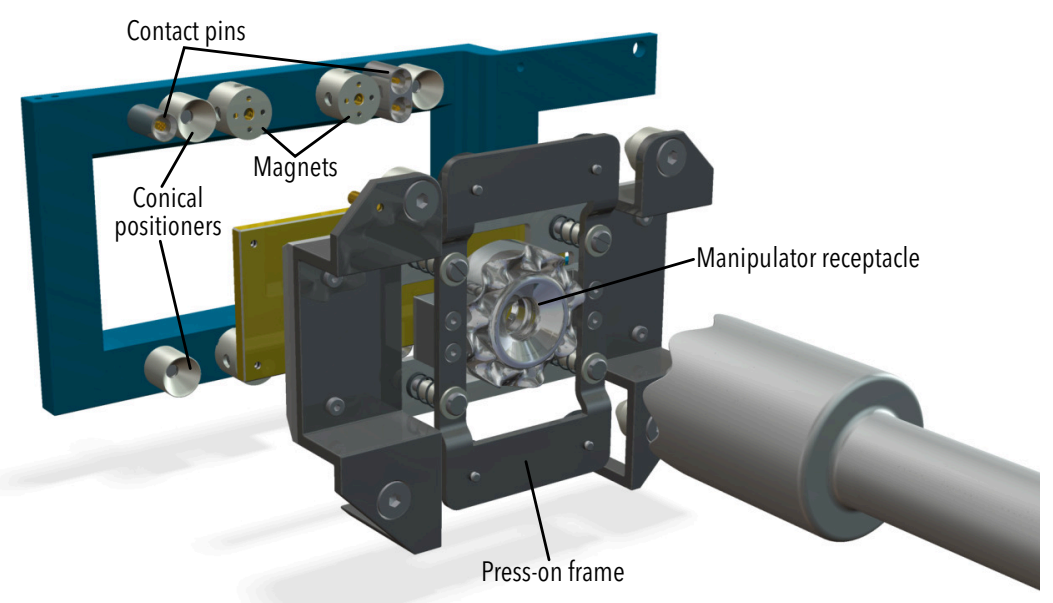

Figure 10.10: Sample holder (rear, i. e. quick access door side).

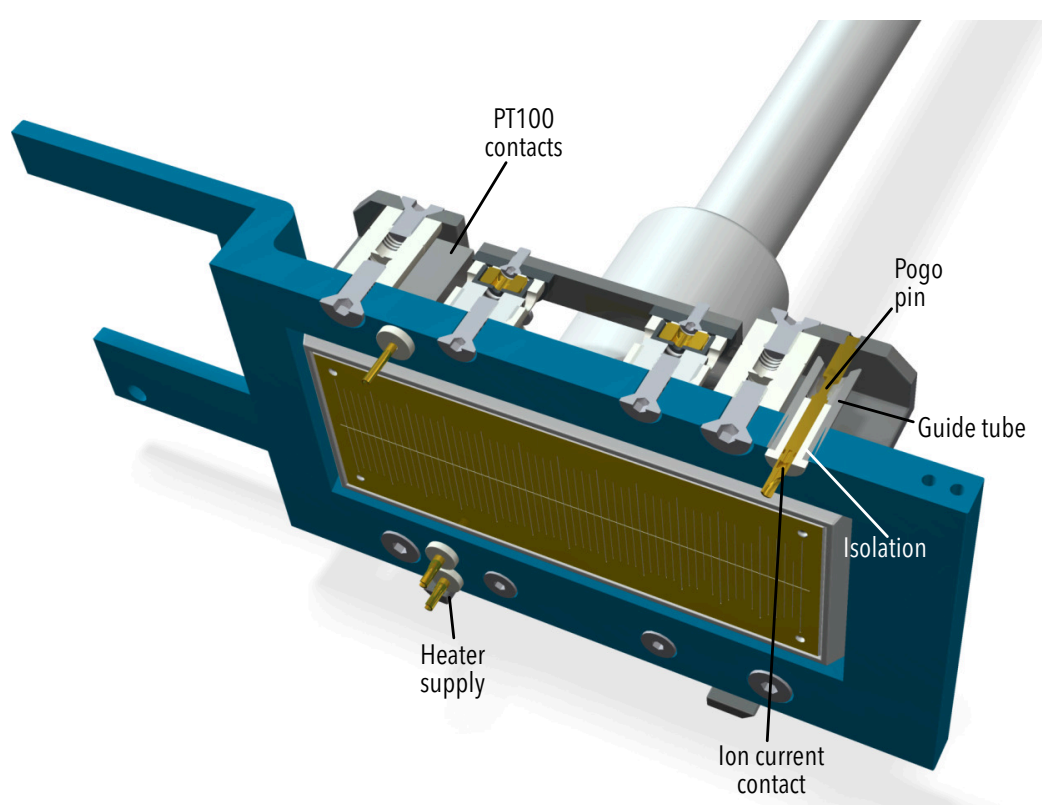

Figure 10.11: Cut through the sample holder's upper frame. 


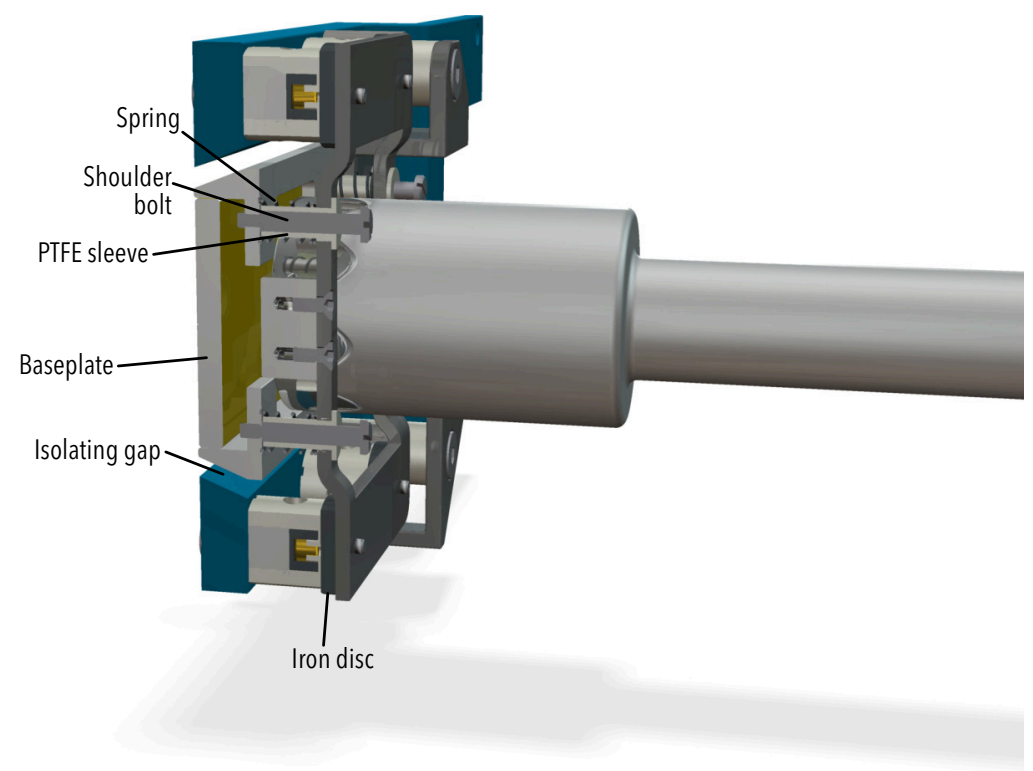

Figure 10.12: Cut through the sample holder's press-on frame.

sample holder are made of polytetrafluoroethylene (PTFE) to provide isolation of the sample holder towards the chamber. This is important to allow for ion current measurements. The sample holder is continuously pressed against the chamber sided positioners by a press-on frame, which is being held by four magnets mounted on the chamber sided holding frame. PTFE sleeves, screwed into the press-on frame, make this frame slide back and forth on shoulder bolts, which are attached to the actual holder. Springs between sample holder and press-on frame ensure proper pressing force. A cut view of this mechanism is visible in fig. 10.12. Between the holding frame and the sample holder, an isolating gap stays open once the sample holder is held by the magnets. The only contact points are the PTFE positioners and the magnet covers, which also consist of PTFE. Inside the magnet covers, the ring magnets themselves are located in iron pots, which provide shielding of the magnetic field towards the ion beam and enhanced holding force towards the sample holder. On the sample holder's press-on frame, iron discs are mounted as a counter part of the magnets.

The manipulator receptacle is attached to the press-on frame, thus allowing to apply force on this frame thereby compressing the springs until the iron discs snap onto the magnets. A safe mechanical connection between the manipulator and the receptacle is provided by a ball locking bolt (Kipp K0746.02112040), which is contained in the manipulator's tip. This bolt's locking balls snap into an indentation in the wall of the receptacle's hole. Pressing the button on the backside of the bolt, which is extended to the user's end of the manipulator, releases the locking balls and make it possible to remove 
the manipulator from the sample holder. A toothed ring around the ball locking bolt on the manipulator and this ring's counterpart on the receptacle prevent the sample holder from rotating around the locking bolt after it is removed from the holding frame.

Electrical contacts between the chamber and the sample holder are provided without the need to plug in or out connectors manually because this could expose operators to unacceptable amounts of radiation. Instead, connections for current measurement, temperature measurement and heater supply are provided by spring loaded contact probes (also called pogo-pins), which are usually used for circuit testing in the electronics industry. These pins are protected from mechanical strain by steel tubes. Between the steel tubes and the contact pins, PTFE sleeves provide isolation. For current measurement and heater supply, F73306B300G300C probes from Feinmetall are used. The temperature sensor is connected using two F77206B200G300C probes from the same vendor. Both probe types are fixed using the appropriate receptacles. This allows for easy replacement in case of defects. In fig. 10.11, a cut view of the current contact assembly is visible.

Ion optics Since focusing and sweeping of the ion beam can be done using upstream elements from the ISOLDE beamline, ion optic components between the chamber's ion entry and the sample mainly consist of a collimator and a suppressor net.

As collimator, a zero aperture stainless steel iris model from Edmund optics (57-597) with a maximum aperture of $30 \mathrm{~mm}$ is used. It is operated by a vacuum stepper motor. To allow for current measurements, it is fixed in a PTFE receptacle and contacted using two redundant probe pins. The receptacle is built in a way that allows removing the collimator quickly in order to reduce operator exposure to radiation in case of contaminations.

The suppressor net consists of an etched molybdenum screen mounted on isolators. It can be folded away to allow collimator changing as described above.

Both, the collimator and the suppressor can be moved along the beam axis using another stepper motor. This provides the possibility to move it close to the sample during perpendicular implantations and to move it to an appropriate distance from the sample in case of tilted implantations to prevent collisions between suppressor and sample holder.

To allow beam tuning while shooting through the implantation chamber in order to use the ion beam in downstream chambers like the ones described below, a XY-deflector exists between the ion entry and the collimator assembly. An additional Einzel lens at the chamber's ion exit allows focusing of the beam as needed by downstream chambers.

\subsubsection{Deceleration and post-acceleration chamber}

Control of the penetration depth of ions in matter is possible by varying the ion energy. At ISOLDE, this is, to a certain extent, possible by changing the acceleration voltage at the ion source. This voltage, however, is limited to $60 \mathrm{kV}$. Reducing it to lower values requires a readjustment of all ion optical elements along the beamline. Additionally, 
separator magnet's resolving power and yield are reduced at lower voltages. Therefore, the achievable depth range is very limited.

While spreading of implanted ions across the bulk volume of a sample is possible in certain cases by heat induced diffusion, which can take place as part of annealing, controlled implantation profiles close to the sample's surface outside the depth range reachable by acceleration voltages of about $40 \mathrm{keV}$ to $60 \mathrm{keV}$ cannot currently be obtained at ISOLDE.

To remove this limitation, an additional implantation chamber has been designed. It complements the implantation chamber described above. While the new implantation chamber with its load-lock system and rapid pumping facilities provides efficient series implantations, the deceleration chamber allows variations in the implantation energy while not providing load-lock facilities. Due to the overall design, it is possible to use both chambers alternatingly, i. e. by changing samples in one of them while simultaneously implanting in the other and vice versa. Thereby, a $100 \%$ usage of available beam time is possible.

Chamber layout The chamber shown in fig. 10.13 consists of a six-way cross with DN200 CF flanges and an extension tube. An inner tube (HV capsule) is mounted on isolators along the longitudinal axis of the main chamber. It contains the sample holder, an Einzel lens, and two collimators, as well as necessary support equipment like motors. This HV capsule, including its interior parts, can be put on an adjustable potential of $-60 \mathrm{kV}$ to $60 \mathrm{kV}$. The voltage is supplied by two Heinzinger PNC 60000 power suppliesone for positive and the other for negative voltages. Due to the reverse voltage protection of these power supplies, it is possible to connect their outputs without the need for an additional high-voltage relay.

Control electronics for the motors inside the high voltage part, which drive the two collimators and rotate the sample holder, as well as the supply for the Einzel lens and the picoamperemeters for measuring the current on the collimators and the sample, are contained in an isolated box beneath the chamber. This box $(H V b o x)$ is surrounded by three layers. An inner metal layer set to the same voltage as the HV capsule, an isolating layer of PMMA, and an outer metal layer set to ground voltage. This design provides a defined surface of the HV part, which prevents arcing and avoids damage to sensitive devices in its interior while also ensuring electrical safety during operation. At the chamber's bottom flange, a DN100 ceramic tube is mounted, which isolates the chamber from this tube's bottom flange. This isolated flange projects into the high voltage part of the isolated box and carries a custom feedthrough assembly. All connections between the electronics in the HV box and the HV capsule inside the chamber are connected through this assembly. Inside the ceramic tube, the cables are guided by an inner aluminium tube, which prevents them from getting in contact with the grounded parts of the chamber and which completes the Faraday cage made from HV box and HV capsule. The devices in the HV box are supplied with power by an isolating transformer (Tauscher TU- 


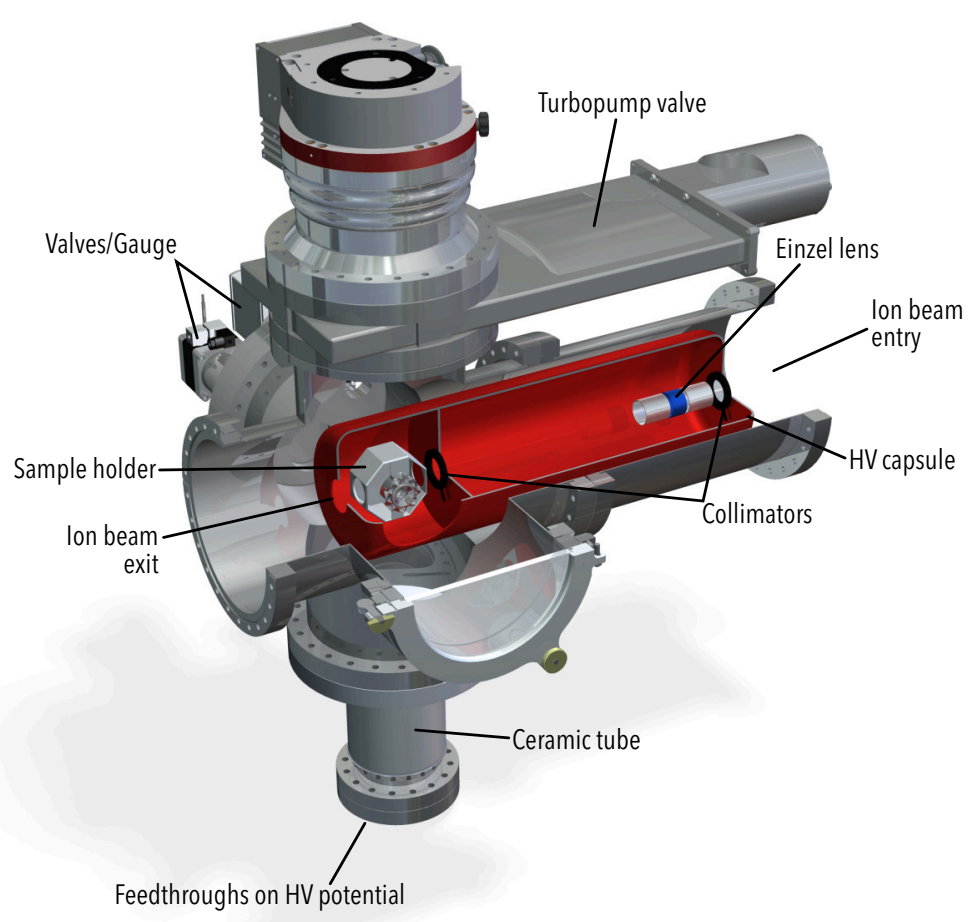

Figure 10.13: Deceleration / post-acceleration chamber.

VFCP UI168/75 500VA). An embedded ARM single board computer inside the HV box controls all other devices and is connected to the external control computer using two Ethernet media converters and a $100 \mathrm{Mbit}$ fiber connection. The Einzel lens is supplied by two Ultravolt high voltage modules (35A24-P15 and 35A24-N15) and a Gigavac high voltage relay (G61C871). The negative supply is meant to allow for defocusing if needed. The relay makes it possible to select the appropriate supply. It is necessary, since the Ultravolt modules are not equipped with reverse voltage protection.

To avoid arcing, the high voltage supply is interrupted as soon as the pressure in the chamber exceeds $10^{-5}$ mbar. Additionally, a hard interlock prevents activation of the HV supplies as long as the quick access door is not closed.

Evacuation and ventilation As visible in fig. 10.1, the vacuum system of the deceleration chamber is very similar to that of the implantation chamber. The most important difference is the missing of a separately pumped implantation part. The valves (including the bypass system) and the air cleaning system are the same as described on page 178. Due to the simpler layout of the chamber, a smaller air-cooled turbopump (Pfeiffer Vacuum 
HiPace 800) and fore-pump (Pfeiffer Vacuum ACP 15) are used. Therefore, a separate chiller is not needed. The chamber is equipped with its own fore-pump to allow for easy repositioning without the need to connect/disconnect fore-vacuum and control connections.

The sample holder A lead glass shielded quick access door is available for sample changing. The lead glass' duty is the shielding of Bremsstrahlung created due to the deceleration or post-acceleration of ions. The sample holder is accessible through a cutout in the HV capsule. It consists of an octagonal wheel with six faces for samples and two open faces allowing for shooting through the holder in one of the rotational positions to reach downstream chambers.

To allow shooting through, the space between the faces is hollow. The octagonal wheel is mounted on a plate in the back, which is held by a magnet and positioners similar to the sample holder of the implantation chamber. A receptacle, which is compatible to the implantation chamber's manipulator, is mounted on the side of the holder that faces the quick access door.

The sample holder can be arbitrarily rotated using a motor located in the HV capsule.

Ion optics As collimators, the same zero aperture stainless steel iris model from Edmund optics (57-597) as in the implantation chamber is used. The collimator at the entrance of the HV capsule allows for beam tuning before the high voltage supply is enabled. During implantations it will stay open. A second collimator is located in front of the sample. Both collimators are motorized.

Strong focusing, occurring due to the potential difference between the HV capsule and the beamline, is avoided by focusing the ion beam on the opening at the capsule's entry. The decelerated but diverging beam inside the chamber is then re-focused using the Einzel lens inside the HV capsule.

The HV capsule is divided into two compartments. The first (in the direction of the beam) contains the Einzel lens, which allows for compensation of said focusing effect. In order to provide a room free of electric field gradients around the sample, a second compartment is separated by the collimator and a surrounding wall. This compartment contains the sample holder and the suppressor net.

\subsubsection{Online TDPAC chamber}

As shown in chapter 9, the majority of candidate nuclides for TDPAC measurements has a half-life shorter than $10 \mathrm{~min}$. It is very difficult to conduct measurements with classical TDPAC spectrometers, which are designed for long running measurements with separated implantation, annealing, and measurements steps, using these nuclides.

For making successful measurements using this large selection of probe candidates possible, it is necessary to integrate said three steps. This means that it becomes necessary to 
implant into a hot sample for instant annealing and run a measurement simultaneously. Additionally, implantation has to take place at a facility that allows the production of short-lived nuclides.

To solve this task, a special online implantation chamber for use at ISOLDE was designed and will shortly be built. The combination of implantation, annealing, and measurement is challenging due to contradicting design goals. While an efficient measurement requires to move the detectors close to the samples to cover a considerable fraction of the solid angle, a high-temperature annealing setup requires space for heat shields (cf. section 10.2). Moreover, heat shields made from heat-resistant materials like tantalum exhibit very high absorption values for lower energy $\gamma$ radiation. E. g. even thin sheets of tantalum considerably reduce detected $\gamma$ radiation in the sub-100 keV range, which occurs in many decay cascades usable for TDPAC (see table 9.3). In turn, light materials with low $\gamma$ absorption are usually not sufficiently heat resistant for use as heat shield. Also for other parts of the setup, minimization of $\gamma$ absorption is a challenge. For example, heating elements without metal case are necessary in order to avoid shadowing radiation emitted towards the heating element.

The requirements for the online TDPAC chamber are:

- The sample should be heatable to at least $1000^{\circ} \mathrm{C}$.

- The ISOLDE ion beam must reach the target.

- A possibility to mount detectors around the sample in the minimal possible distance is required.

- The chamber is required to protect the detectors from the sample heater's heat.

- Efficient sample changing needs to be possible.

- It should be possible to apply a magnetic field during measurements.

Chamber layout Figures 10.14 to 10.16 show the current stage of the chamber design. The sample is mounted on a disc heater. A round $25 \mathrm{~mm}$ BORALECTRIC model, similar to the one used in section 10.2, consisting of a pyrolytic boron nitride substrate and pyrolytic graphite traces ensures low $\gamma$ absorption. The screws holding the heater are aligned diagonally with respect to the detectors.

A multi-layer heat shield around the sample and heater reduces heat radiation. It is planned as threefold tube around the sample with terminating discs at both ends. While the discs can be made of tantalum, or another appropriate metal with high $\gamma$ absorption, because they are not in between sample and detectors, the tubes are planned to consist of glassy carbon (HTW, 2014). This material shows outstanding temperature resistance while 


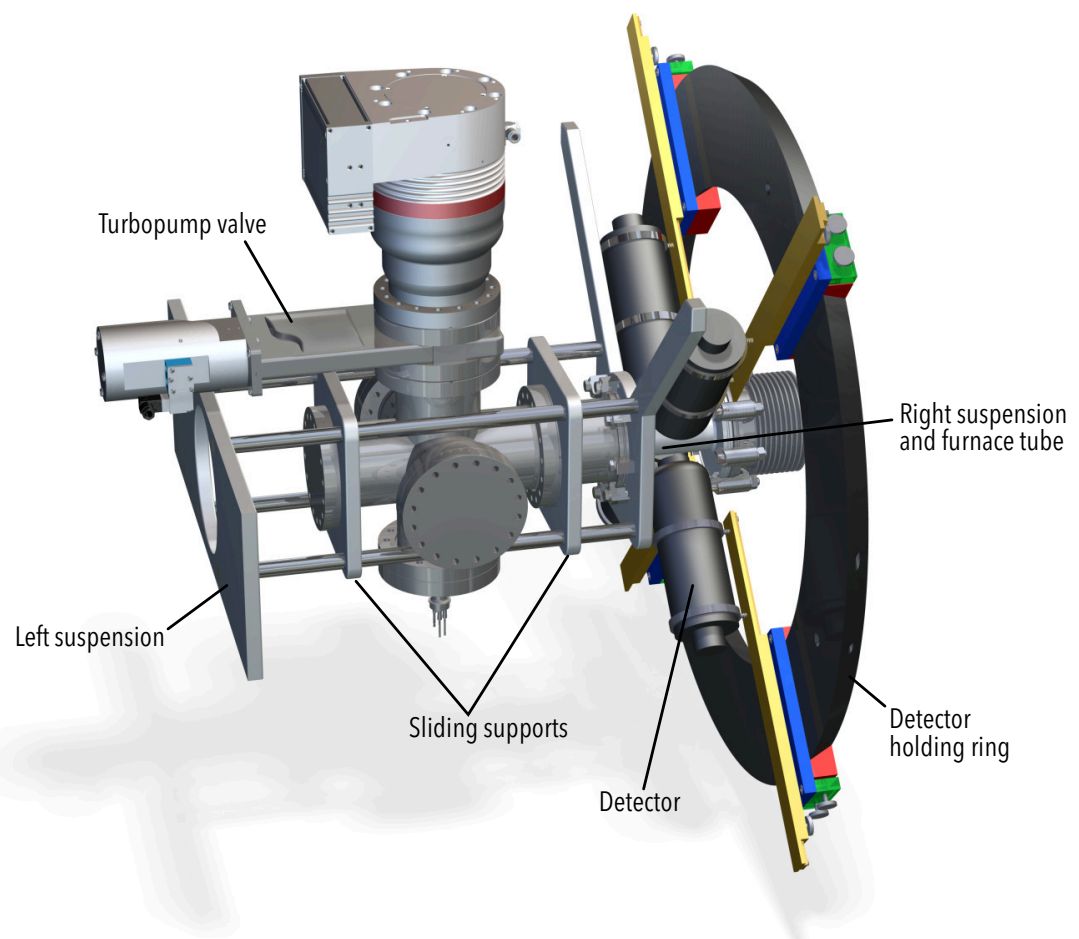

Figure 10.14: The online TDPAC chamber (closed).

consisting of pure carbon. Therefore, it exhibits very low $\gamma$ absorption. Simultaneously, it is mechanically strong, i. e. thin tubes or even foils are sufficient for shielding.

Due to the absence of ferromagnetic materials around the furnace, the online TDPAC chamber is also compatible to the Helmholtz style magnetization setup which will be introduced in section 10.3.2.

Outside the heat shield, a custom aluminium tube will act as vacuum chamber. To avoid damage to the tube and protect the detectors from the remaining heat passing the heat shield, the tube is planned double walled with cooling water flowing between the inner and outer walls. On one end of this tube, an ISO-F flange will provide the chamber opening for sample mounting. This furnace tube, as well as the heat shield, is visible in the cut view in fig. 10.16 .

Figures 10.14 and 10.15 show the opening mechanism. The main part of the chamber, consisting of a six-way cross with DN100 CF flanges, an adaptor tube to an ISO-K flange, and auxiliary parts, is held by two sliders, which slide on four steel bars using four linearmotion bearings each. The BORALECTRIC heater together with the rear heat shield is attached to this chamber and is therefore moved out of the furnace tube and the remaining parts of the shielding if the chamber is moved away from the furnace tube. After mounting a sample, the chamber is moved back and the clamps between the ISO-K and 


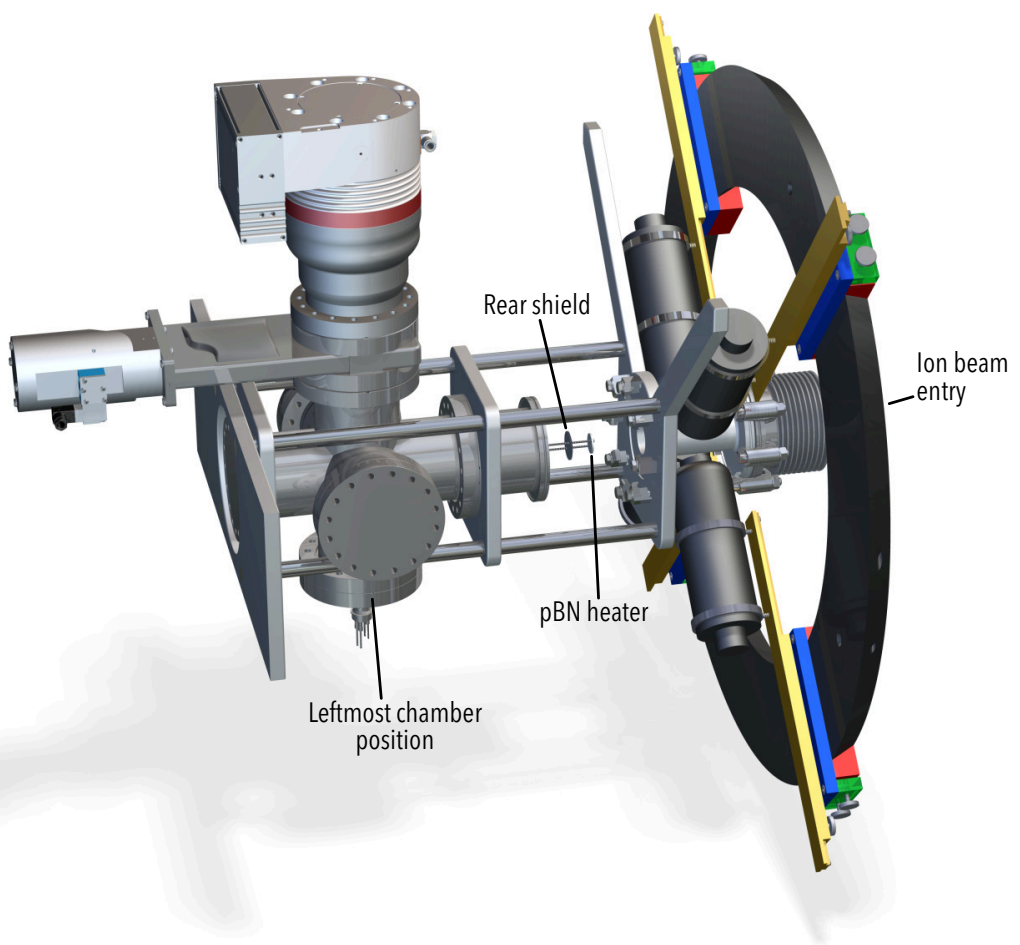

Figure 10.15: The online TDPAC chamber (open).

ISO-F flanges are closed. The steel bars are suspended on the furnace flange, which in turn is connected to the upper part of the support frame. The other side of the bars is directly mounted on the support frame.

A turbopump, valves for bypass operation, and feedthroughs for heater supply and temperature measurement are attached to the flanges of the six-way cross.

The supporting frame, which is similar to the frames of the other two chambers and will provide the possibility to move the chamber via crane, also carries a chiller and a fore-pump. The chiller will provide the cooling water for the furnace tube.

Evacuation and ventilation As shown in fig. 10.1, the vacuum system of the online TDPAC chamber is almost identical to the vacuum system of the deceleration chamber described on page 189. The only difference besides the smaller valves and turbopump is the intended use of ventilation using dry nitrogen instead of dried and cleaned air to extend the lifetime of the BORALECTRIC heater and further reduce the amount of humidity reaching the chamber while venting. 


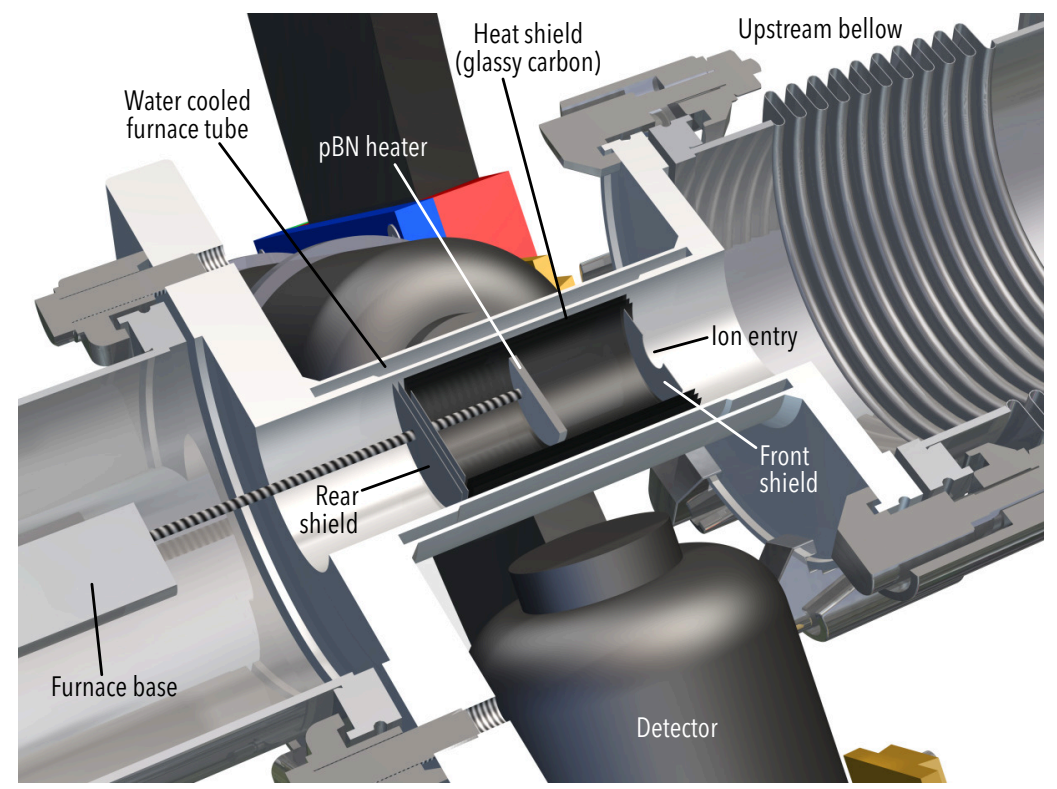

Figure 10.16: The online TDPAC chamber's furnace core.

\subsubsection{Conclusions}

The new ensemble of chambers for the GLM branch at ISOLDE will provide a plentitude of new possibilities. Normal implantations will be possible more efficiently and safely with the implantation chamber.

Decelerated and post-accelerated implantations, as permitted by the second chamber, will provide new possibilities for implantation depth control. This is especially interesting for the emission channeling method, but also extends the research scope of TDPAC at ISOLDE.

An online TDPAC chamber will provide the opportunity to conduct measurements with a large number of short lived nuclides, identified in chapter 9, at high temperatures in combination with small sample-to-detector distances and minimal $\gamma$ absorption. It can be combined with the spectrometer described in chapter 4 .

The implantation chamber as well as the deceleration chamber are designed to allow mounting downstream chambers and use the integrated ion-optical components for beam conditioning. All chambers are constructed as self-contained units, which allow for easy replacement and even temporary usage at different beamlines. 


\subsection{Furnace for high temperature annealing}

If ion implantation techniques are used to insert radioactive probe nuclei into solid samples, implantation induced defects emerge in these samples. Since these defects influence the probe nuclei's environments-usually in a non-uniform way-they impair the recovery of information concerning the probes' surroundings and often make it difficult if not impossible to extract useful information from measurements.

Therefore, implantation of TDPAC probe nuclei usually requires subsequent annealing, which recovers defects in the samples and/or allows the probe nuclei to diffuse to welldefined places in the host matrix. Subject to the host material and the type of probe nuclei, a broad spectrum of annealing temperatures and durations can be appropriate. To avoid unintentional chemical reactions of the host material and/or the probe atoms during annealing, a suitable atmosphere-or vacuum-is necessary. In particular this demand is not met by many available furnace constructions. Fusing the samples into evacuated or gas-filled silica bulbs before annealing is a possible workaround. However, this method introduces additional processing steps and does not provide control of the pressure conditions at the sample during annealing. It therefore makes the annealing a hardly defined process. Especially pressure in evacuated silica bulbs must be expected to dramatically rise during annealing due to outgassing of the sample itself as well as the silica's surface. All these issues considered, annealing can be regarded as the least defined process in many TDPAC experiments.

In order to overcome the limitations described above, a high-temperature furnace was developed and optimized in the course of this work. The initial prototype consisted of a water cooled double-walled tube, terminated by a blank flange on the top and an adapter flange on the bottom. Both flanges were implemented including machined channels for water cooling. A heater based on a pyrolytic boron nitride substrate with embedded conductive traces made from pyrolytic graphite (BORALECTRIC) inside a heat shield assembly made from tantalum sheet metal was mounted inside this cooled vacuum chamber. An external pumping unit was connected using a flexible vacuum hose. The BORALECTRIC heater was controlled using a temperature controller supplied from Tectra in standalone mode allowing manual adjustment of a target temperature. This controller also evaluates the signal of a thermocouple, which is located in a lateral hole of the BORALECTRIC element.

Tests using this existing setup showed that although it was working, there were problems concerning the pressure during operation. During the rise of temperature at the heating element, pressure usually rose from $10^{-6} \mathrm{mbar}$ to values above $10^{-4} \mathrm{mbar}$. The pressure at the sample location had to be considered even higher since the gauge was mounted at the connection of the hose towards the external pumping unit.

To overcome the bad pumping performance, a heat shield consisting of two bent steel parts was added close to the upper end of the cooled chamber walls. Both parts are fixed using solid weld joints for good heat transfer towards the chamber walls. They were bent 


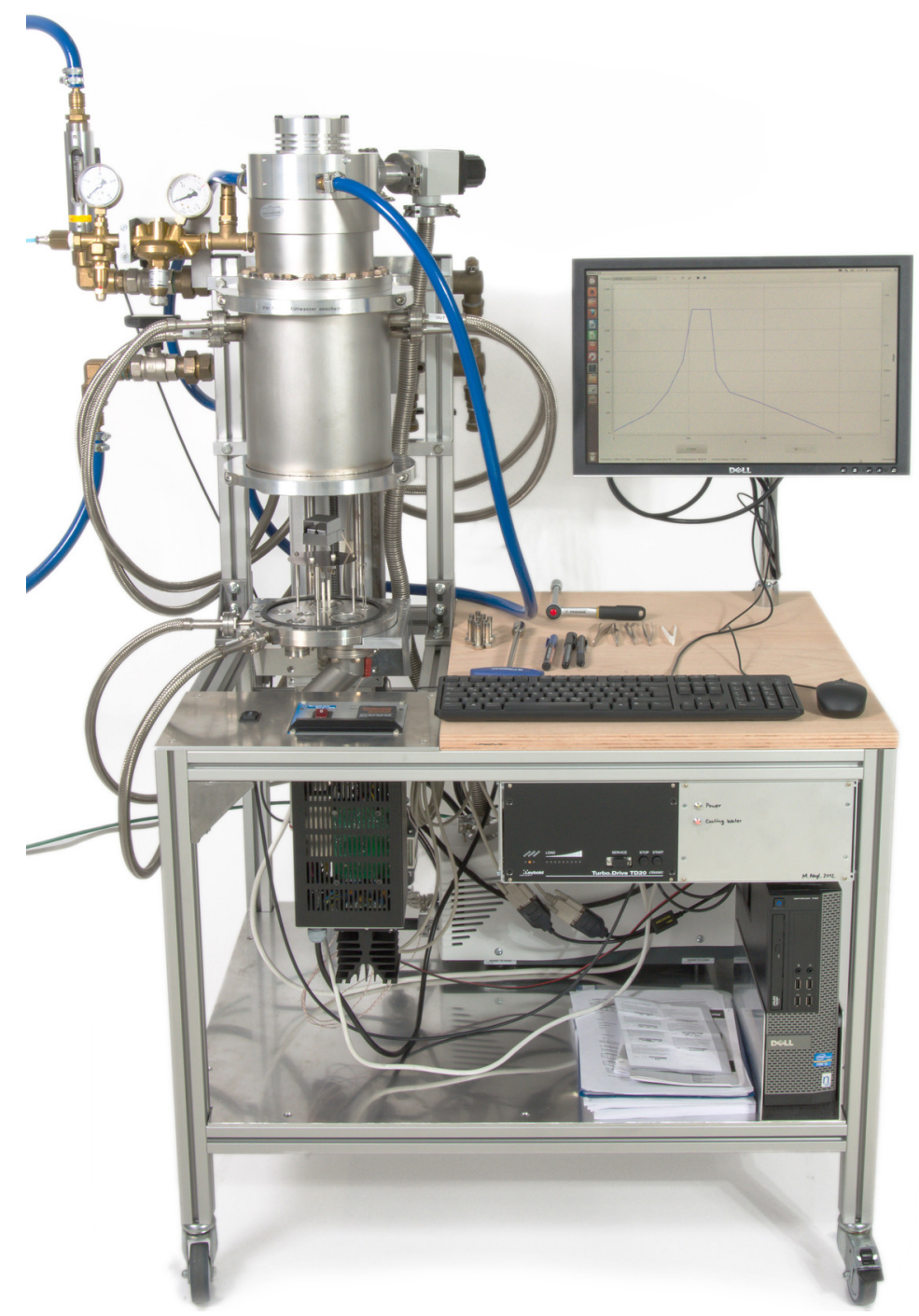

Figure 10.17: High temperature vacuum furnace. The actual furnace chamber is located in the upper part. The turbopump is located on top of the chamber. The coolant distribution is mounted on the back side of the chamber with the flowmeter visible behind the nitrogen pressure regulator left of the chamber, which is used for venting. Beneath the chamber, the opened bottom flange, which carries the furnace assembly, is visible. In the image, this bottom flange is on its lowest position for sample changing. Before closing the chamber, it is moved upwards by the motorized linear stage located behind the flange. Beneath the table, the necessary control devices (furnace controller, turbopump controller, a control computer, and the custom built controller for the linear stage and coolant monitoring) are visible. 


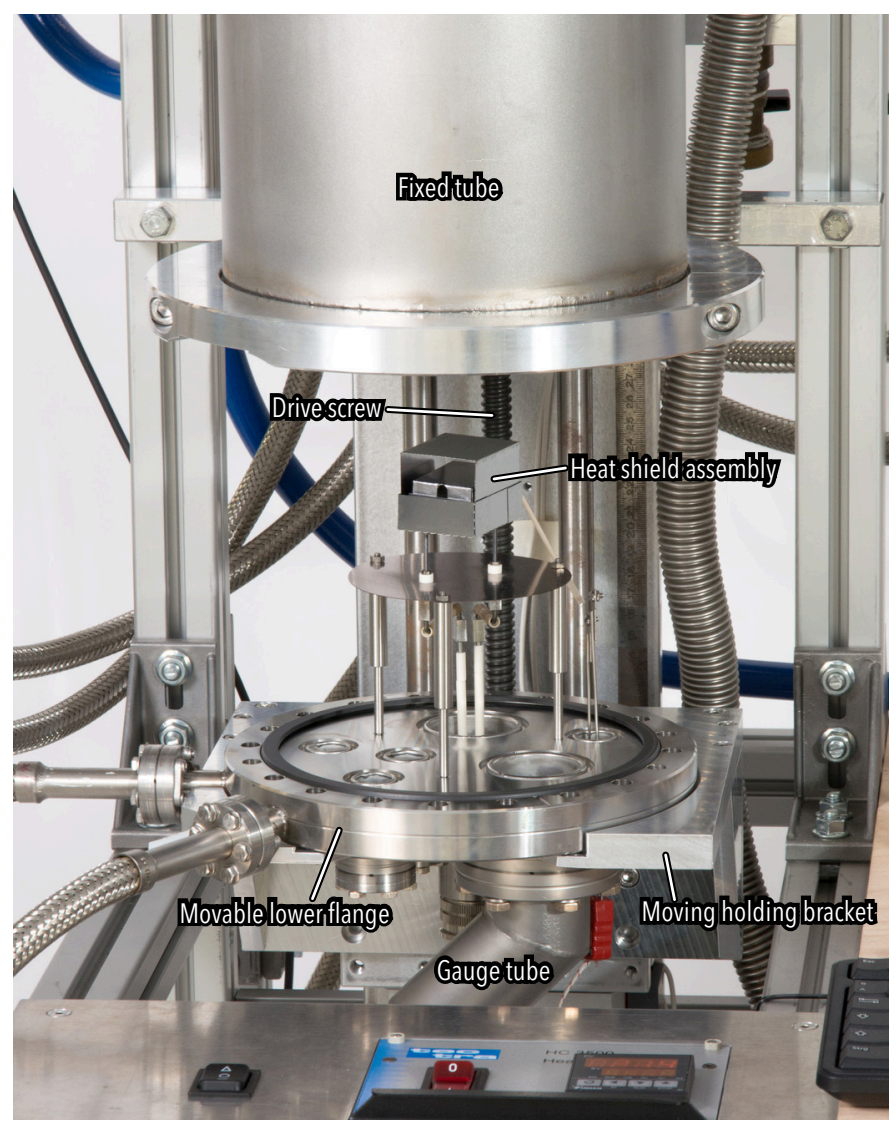

Figure 10.18: Core of the high temperature vacuum furnace. In the center of the image, the tantalum heat shield assembly around the BORALECTRIC heating element is visible. This assembly is mounted on the bottom flange, which is held by a bracket moving up and down along a motorized linear stage. Below the bottom flange, the double-angled tube holding the gauge is partly visible.

in a way to provide a maximal opening while still blocking the line-of-sight between the heater assembly and the upper flange of the chamber.

The blank flange above the heat shield was replaced by a turbopump of equal size. The gauge remained at the chamber's bottom-now providing much more reliable values since the pump is located at the opposite end of the chamber. It was mounted at the end of a double-angled tube to prevent heat radiation from hitting its sensor.

Due to the turbopump and the heat shield, sample changing through the top flange is no longer possible. Mounting of the pump at the tube's bottom end was avoided to prevent parts of the furnace or sample from being able to fall into the pump. To make sample changing through the bottom flange possible and avoid samples falling off the heating el- 


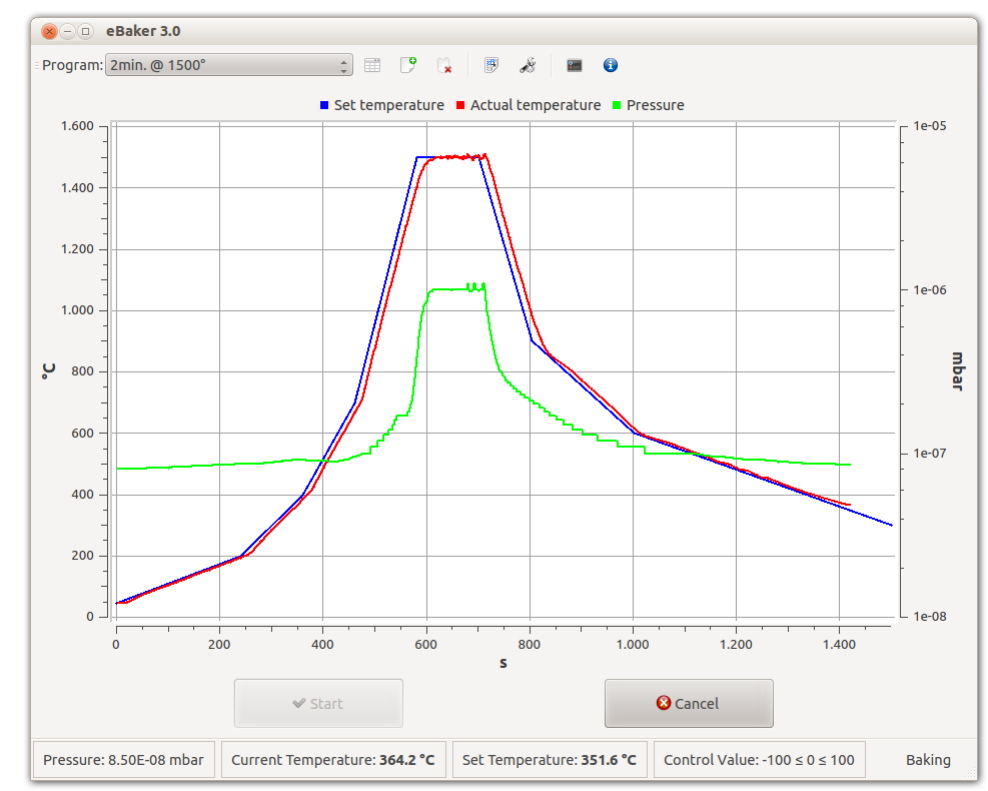

Figure 10.19: Screenshot of eBaker after annealing for $2 \mathrm{~min}$ at $1500^{\circ} \mathrm{C}$.

ement during manual mounting of the bottom flange, a motorized linear stage and a bracket holding the bottom flange were added. The whole furnace, including the newly added dry multi-stage roots pump (Pfeiffer Vacuum ACP 15) generating fore-vacuum, was mounted on a wheeled frame.

Electronics for safe limit switching of the linear stage motor was developed. A flowmeter for the monitoring of coolant flow was added and combined with custom electronics, which switches off the heater as soon as coolant flow stops.

Figure 10.17 shows the improved furnace. The heater assembly is visible above the bottom flange. This flange is held by a bracket, which is moved up or down by the motorized stage mounted behind the chamber. The Tectra controller and the switch for moving the stage are located in front of the furnace while the turbopump controller and the custom electronics are mounted beneath the table surface.

Figure 10.18 shows the furnace core in more detail. The BORALECTRIC element is located inside a double walled tantalum heat shield, which was added to reach temperatures of more than $1500^{\circ} \mathrm{C}$. This assembly is mounted on a disc, which itself is fixed on the bottom flange. On the left side of this flange, the coolant supply hoses are visible. Below, there is the bended tube, which protects the gauge from the heating element's thermal radiation.

To achieve better reproducibility, a software tool named eBaker was written, which allows to define temperature profiles. It then transfers these profiles to the Tectra controller and starts an annealing run on request. During the annealing run, the current temper- 
ature is recorded and plotted as shown in fig. 10.19. The recorded data can be exported for documentation purposes. For simultaneous monitoring of the pressure inside the furnace, an interface to the used gauge (Pfeiffer Vacuum MPT 100) was implemented. The pressure is recorded and plotted alongside the temperature and can also be exported.

While the foregoing modifications and improvements significantly improved the pressure level during heating, there was still a significant rise in pressure-especially after longer periods of venting. This occurred due to air moisture condensing on the interior parts of the chamber during venting because of the expansion and cooling of the air while entering the chamber.

As a solution for this problem, venting with dry nitrogen was implemented. A vacuum valve is connected to the turbopump's venting connection. This valve is connected to a double stage pressure regulator with a low output pressure range of 0 bar to 1.5 bar and a safety relief valve with a rated pressure of 0.6 bar. This implementation avoids the risk of massive excess pressure during ventilation and is necessary since, due to the chamber design, it is not possible to release a door as pressure limiting measure. Nitrogen pressure levels slightly above atmospheric pressure inside the furnace can be obtained to avoid leaking in of air if the furnace is not operational for longer periods. To allow for flushing the chamber with nitrogen, a second valve was added between turbopump and fore-pump. Flushing is possible by alternating opening and closing of this valve and the nitrogen venting valve.

\subsection{Sample magnetization}

Providing external magnetic fields for aligning the sample magnetization or even directly providing a defined field inside samples is very helpful in many cases. It allows, for instance, to increase the modulation amplitude of TDPAC spectra by preventing parts of the probe population from being exposed to a field parallel to one of the detector axes, because in these cases these parts' contribution to the spectrum is unperturbed-and therefore useless (Hamilton, 1975). Moreover, certain field geometries allow measuring of $\omega_{L}$ rather than $2 \cdot \omega_{L}$ which reduces the time resolution requirements, thus rendering experiments with higher Larmor frequencies possible (Raghavan and Raghavan, 1971). Due to these advantages, external alignment of sample magnetization is implemented in many TDPAC experiments aiming at the measurement of magnetic fields (e.g. Ohkubo et al., 1993; Sasanuma et al., 2004). Usually, a design based on one or several permanent magnets and a pot-shaped yoke is implemented (see for example Müller, 2009, p. 15). While being a good solution for simple offline cases employing small sample sizes, i. e. cases where implantation and measurement do not have to occur simultaneously, this method is not feasible for online measurements. Especially the large beam spot at the LOHENGRIN separator (cf. section 2.4.2) makes it challenging to combine homogeneous fields and a simultaneous beam entry perpendicular to the detector plane. 


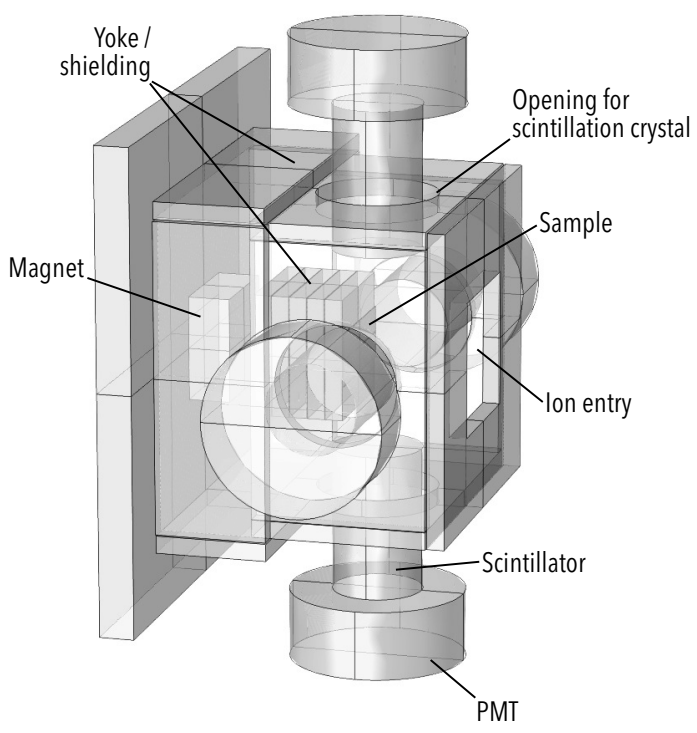

Figure 10.20: Magnetization setup tested at the LOHENGRIN target. The inner stack of soft iron plates guided the magnetic flux towards the sample. The gap between this stack and the magnet was filled by the chamber's rear cover. The ion entry was sufficiently big to embrace the chamber's wall. The outer construction was meant to guide the magnetic flux and provide shielding for the PMTs.

The design aims for magnetization setups in the context of online TDPAC are:

- Minimal $\gamma$ absorption between sample and detectors

- Free ion path perpendicular to the detector plane with a diameter appropriate for the beam spot's size

- Homogeneous perpendicular magnetic flux across the whole sample surface

- Possibility to move detectors close to the sample

- Sufficient shielding to reduce the field strength at the PMT's photo cathode to tolerable levels well below $1 \mathrm{mT}$ (Photonis, 2007b)

\subsubsection{Unsuitability of flux measurements}

A first attempt to address the preconditions described above was made in preparation of a beam time at the LOHENGRIN separator in 2013. The designed yoke was built around the existing sample chamber. 


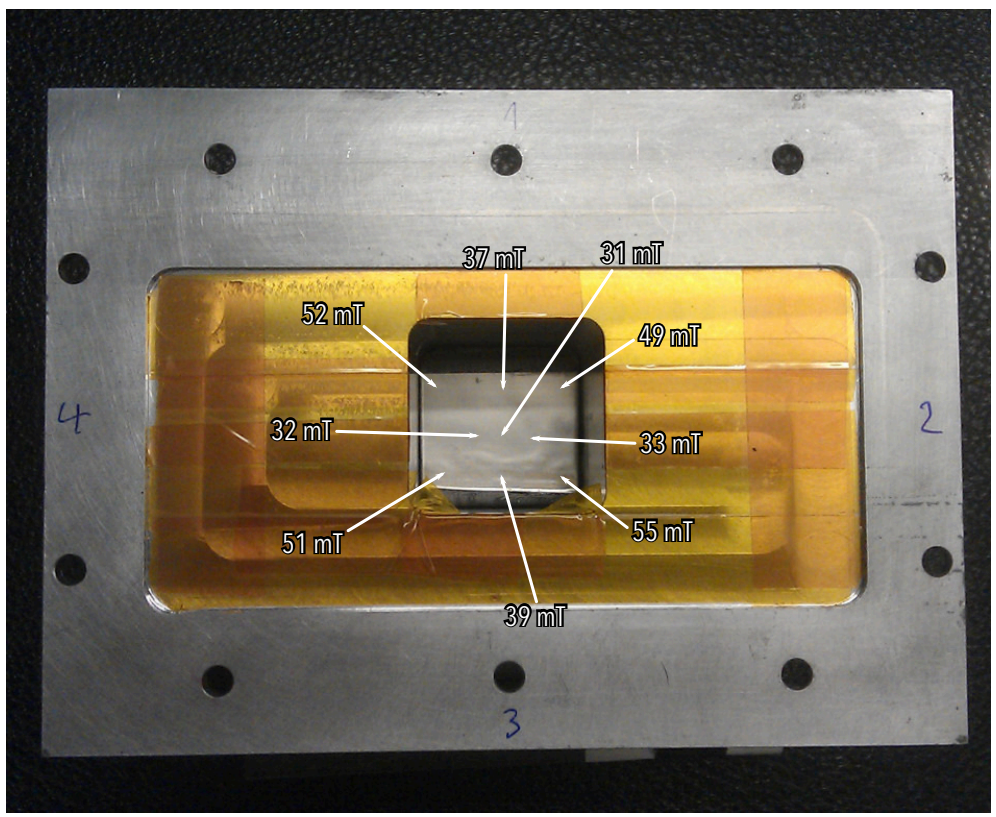

Figure 10.21: Surface-perpendicular magnetic flux values above the sample foil measured using a magnetometer. The foil is mounted inside the LOHENGRIN target chamber. The size of this chamber's opening is approximately $35 \mathrm{~mm} \times 35 \mathrm{~mm}$.

Figure 10.20 shows a transparent drawing of the yoke construction, the magnet, and the sample. A stack of soft iron plates was located behind the sample inside the chamber. The rear cover of the aluminium chamber was located between this stack and a cuboidal $\mathrm{Nd}_{2} \mathrm{Fe}_{14} \mathrm{~B}$ magnet. Around the chamber, a yoke consisting of soft iron pieces was build. The detector heads of the four scintillation detectors used for the measurement extended into the yoke through suitable holes in the iron pieces. Measurements of the perpendicular flux at the surface of the used sample (a nickel foil) with a magnetometer showed good agreement with simulation results obtained by modeling the setup using version $4.3 \mathrm{~b}$ of COMSOL Multiphysics (compare fig. 10.21 and fig. 10.22). The saturation curve of nickel was adopted from Field Precision LLC (2013).

Although these results seemed to indicate a success in realizing the design aims described above, a closer examination revealed an important insufficiency of both, the measurement as well as this simulation: Both quantify the magnetic flux above the sample's surface rather than inside the sample.

However, the flux relevant for TDPAC measurements is the flux beneath the sample's surface. A simulation of the field at that position resulted in figs. 10.23 and 10.24.

Obviously, in case of ferromagnetic samples, it is not sufficient to measure magnetic flux values close but outside the sample because the high permeability of these materials massively influences the field profile inside the sample. The only reliable method for the 


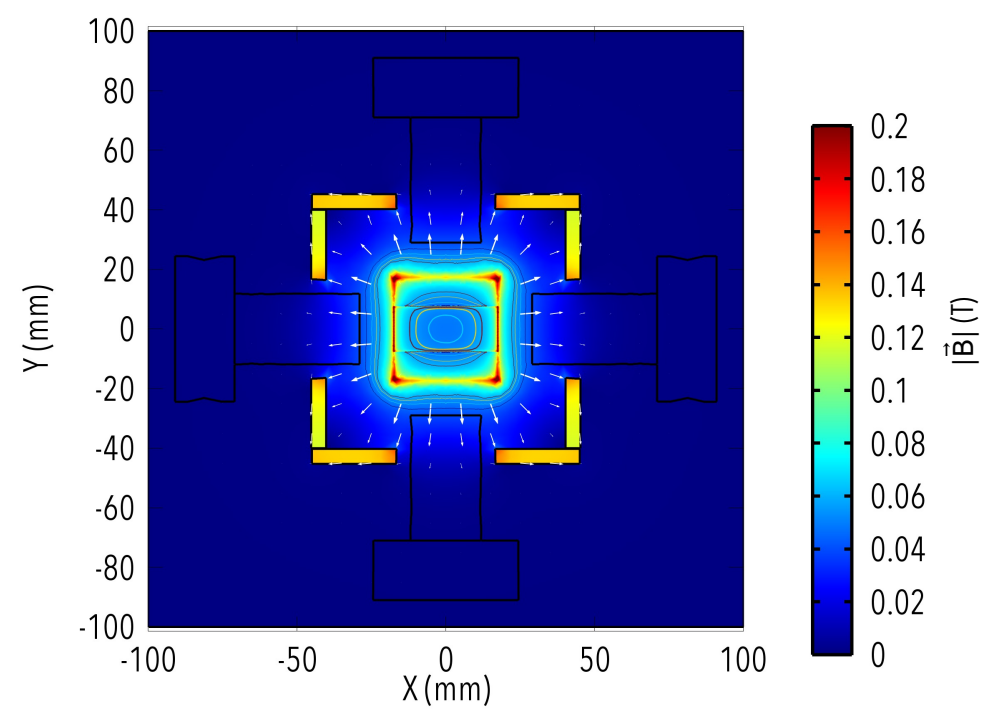

Figure 10.22: Simulated magnetic flux values $10 \mu \mathrm{m}$ above the sample foil's surface. The blue contour line corresponds to a total flux of $45 \mathrm{mT}$, the yellow one to $50 \mathrm{mT}$, and the dark one to $60 \mathrm{mT}$. In order to improve the visibility of the flux values across the sample surface, the color scale was limited to $0.2 \mathrm{~T}$. White arrows visualize the direction of magnetic flux in the figure's cut plane.

prediction of flux values inside samples is a careful simulation. To improve the magnetic flux homogeneity inside a sample according to the design aims, improved yoke designs are required.

The maximum flux at the PMT photo cathodes is $1.75 \mathrm{mT}$ according to the simulations. This is at the limit of what PMTs with additional mu-metal shielding can tolerate without a complete loss of their output signal. However, this field strength already significantly degrades gain and reduces gain stability. Compare chapter 6 for consequences of and countermeasures against this degradation.

\subsubsection{Improved magnetization concepts}

To address the inhomogeneous flux across the sample's surface described above, two new magnetization designs were developed:

Disc-shaped magnet The most basic design approach is to put the sample directly on top of a permanent magnet. Figure 10.25 shows this kind of setup including additional magnetic shielding to prevent a drop of PMT performance. A round shape of magnet 


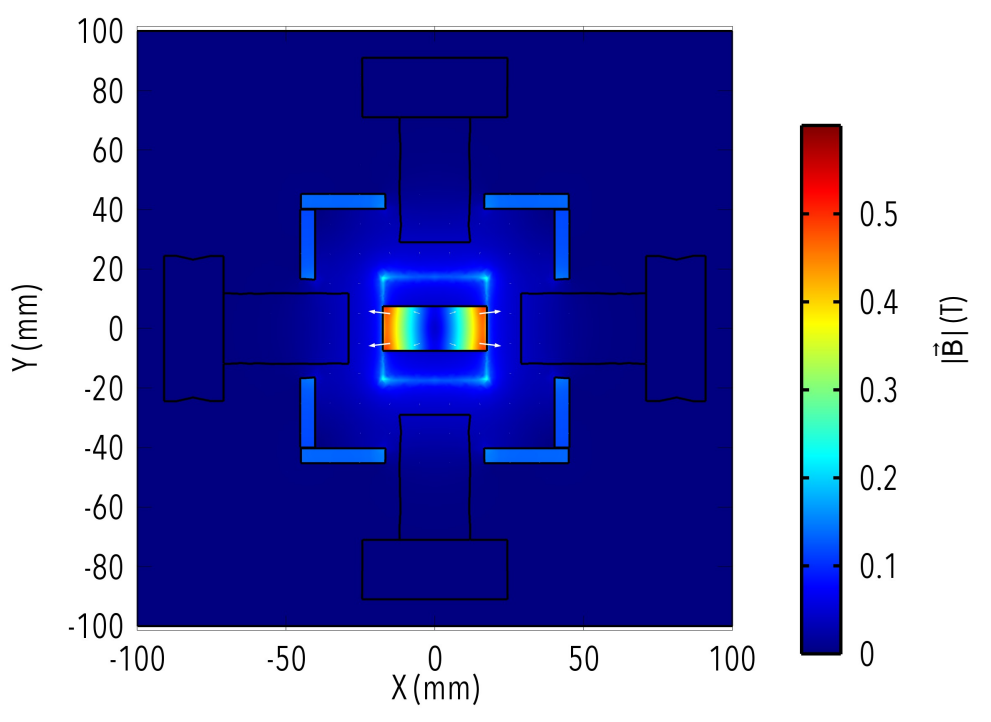

Figure 10.23: Simulated magnetic flux values $10 \mu \mathrm{m}$ beneath the sample foil's surface. Towards the sample's edge, a growing flux parallel to the sample's surface, which exceeds the perpendicular component everywhere but in a small central region of the sample, is visible. White arrows visualize the direction of magnetic flux in the figure's cut plane.

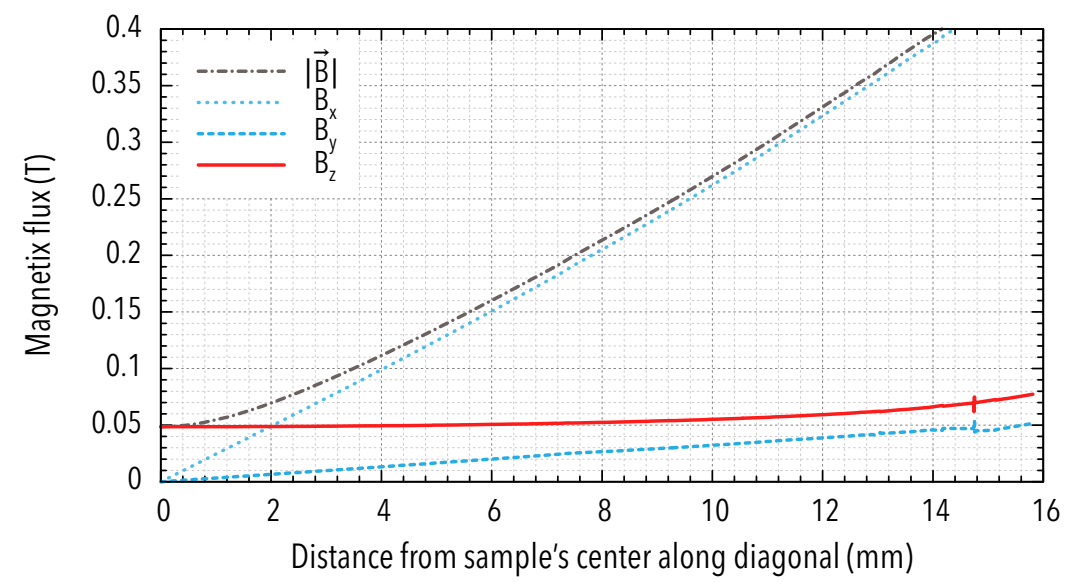

Figure 10.24: Results of the flux simulation $10 \mu \mathrm{m}$ beneath the sample foil's surface along the samples diagonal. The distance values denote the distance from the center. The component of the magnetic field perpendicular to the sample's surface $\left(\mathrm{B}_{z}\right)$ only exceeds the other components in the central region. In the lateral regions of the sample, the components parallel to the surface are much stronger. This inhomogeneity of the magnetic flux makes meaningful measurements very difficult. 


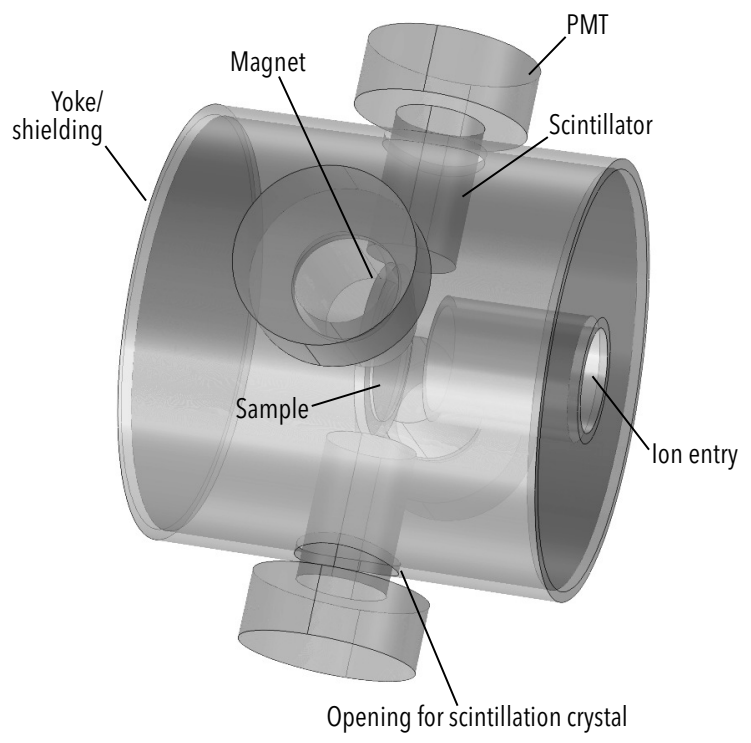

Figure 10.25: Homogeneous magnetization setup based on a single disc-shaped magnet.

and sample is preferable to improve homogeneity and avoid unnecessary distortions of the magnetic field.

The disadvantage of this method is the high $\gamma$ loss, which occurs because the constituents of permanent magnets are usually high- $Z$ materials and therefore shadow almost half of the low energy radiation emitted from the sample towards the detectors (since the magnet covers almost half of the solid angle) while increasing the Compton background. This problem cannot be solved by inserting a spacer with low $\gamma$ absorption between magnet and sample because in this arrangement an almost homogeneous flux only occurs if the sample is located directly on the magnet's surface.

Figures 10.26 and 10.27 show the flux through a plane $10 \mu \mathrm{m}$ beneath the sample's surface whereas fig. 10.28 provides an overview of the flux on a plane cutting through two detectors and the ion beam entry.

Given the simplicity of the setup, the homogeneity of the magnetization across the sample is satisfactory and the flux of $0.44 \mathrm{mT}$ at the PMT position is acceptable. However, for coincidence measurements the loss of a large amount of gamma radiation emitted in backward direction inside the magnet is a major drawback because if both $\gamma$ photons belonging to an examined decay cascade are in an energy range absorbed by the magnet, the coincidence rate drops to not more than a quarter of the original rate that could be obtained without magnets. This is a severe problem-especially in cases with low ion production rates and limited beam times like at the ILL and in many cases also at ISOLDE. 


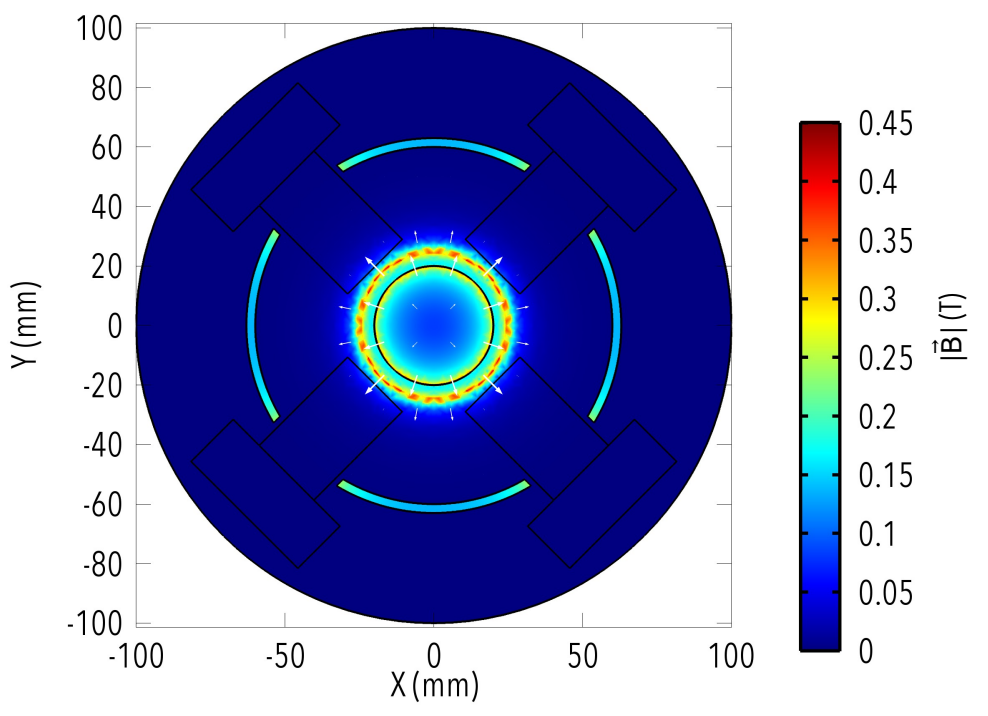

Figure 10.26: Simulated magnetic flux values $10 \mu \mathrm{m}$ beneath the sample foil's surface in the arrangement with a single disc-shaped permanent magnet. The inner part, which is hit by the $3 \mathrm{~cm}$ by $1 \mathrm{~cm}$ beam spot, shows a rather homogeneous flux with a major perpendicular and only small parallel components relative to the sample's surface. White arrows visualize the direction of magnetic flux parallel to the figure's cut plane.

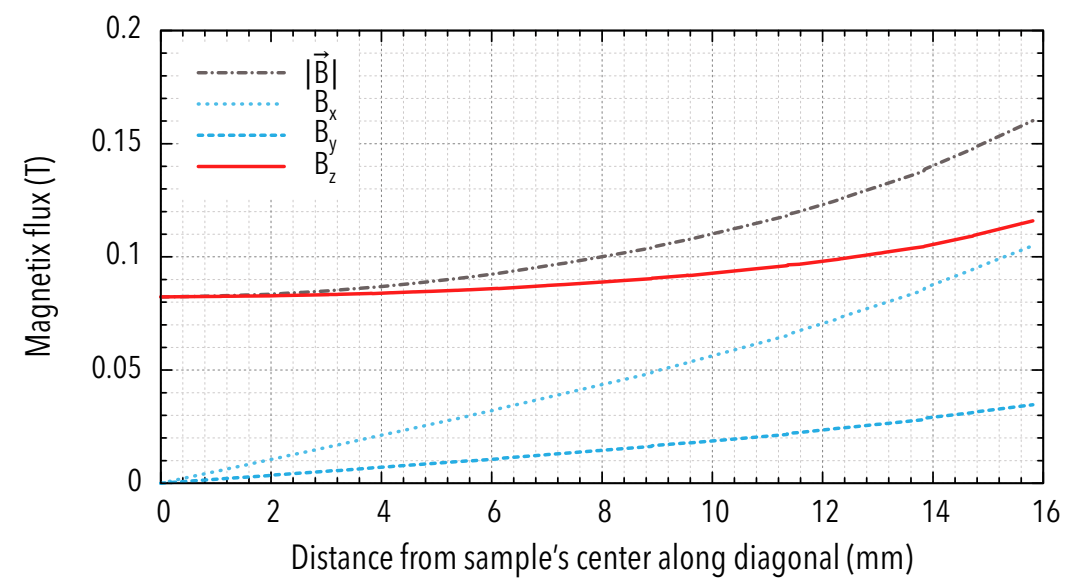

Figure 10.27: Results of the flux simulation $10 \mu \mathrm{m}$ beneath the sample foil's surface along the sample's diagonal in an arrangement with a single disc-shaped permanent magnet. The distance values denote the distance from the center. The homogeneity is much better than in the original design. The parallel components of the magnetic field stay smaller than the perpendicular component across the whole sample surface. 


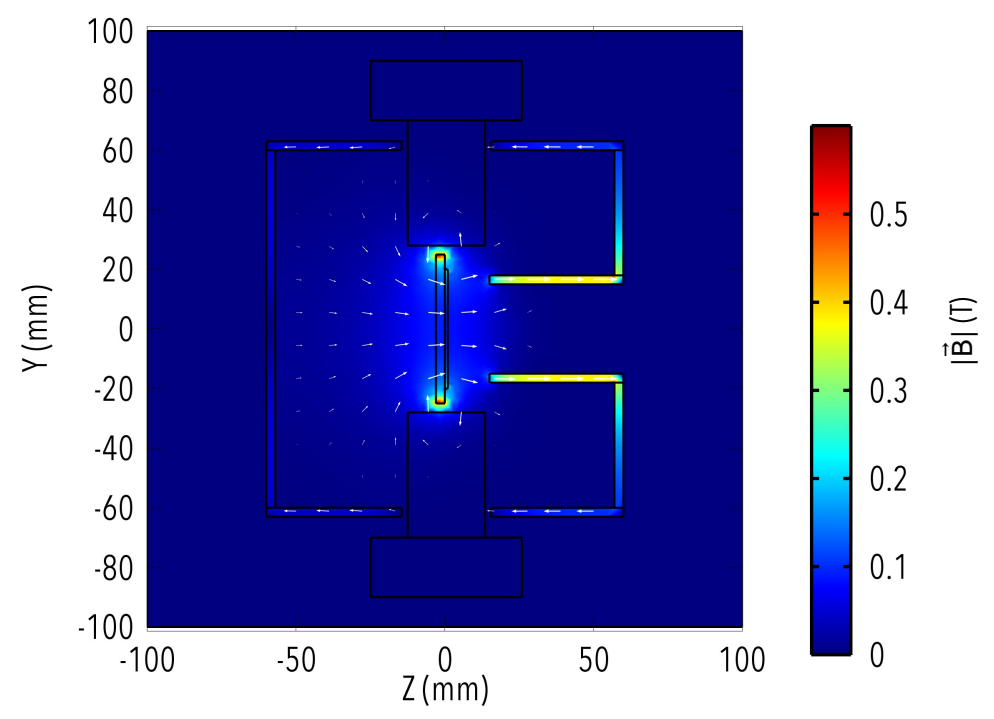

Figure 10.28: Simulated magnetic flux values on a plane cutting through two detectors and the ion beam entry in the arrangement with a single disc-shaped permanent magnet. A logarithmic scaling is used for the arrow length in order to cover a larger range of values. White arrows visualize the direction of magnetic flux parallel to the figure's cut plane.

Helmholtz configuration In order to eliminate the loss of radiation related to the magnetic-disc-setup described in the previous section, a second approach was developed. It is based on a configuration of two ring shaped permanent magnets inspired by Helmholtz coils together with several iron parts used for optimal field shaping. A drawing of the setup is shown in fig. 10.29.

Unlike the previous setups, this one allows to use an isolatedly mounted sample without magnets or yoke parts protruding into the space between sample and detectors. A good level of homogeneity throughout the relevant part of the sample is obtained (cf. figs. 10.30 and 10.31).

Figure 10.32 shows the flux on a cut plane through two of the detectors and the ion entry.

An additional shielding tube reduces the magnetic flux at the photo cathodes to values below $0.9 \mathrm{mT}$. The drawbacks of this design are the difficult handling of the big permanent magnets and the higher amount of Compton scattering due to high- $\mathrm{Z}$ material located close to the sample. However, this increase is expected to be negligible considering the improved coincidence rates. 


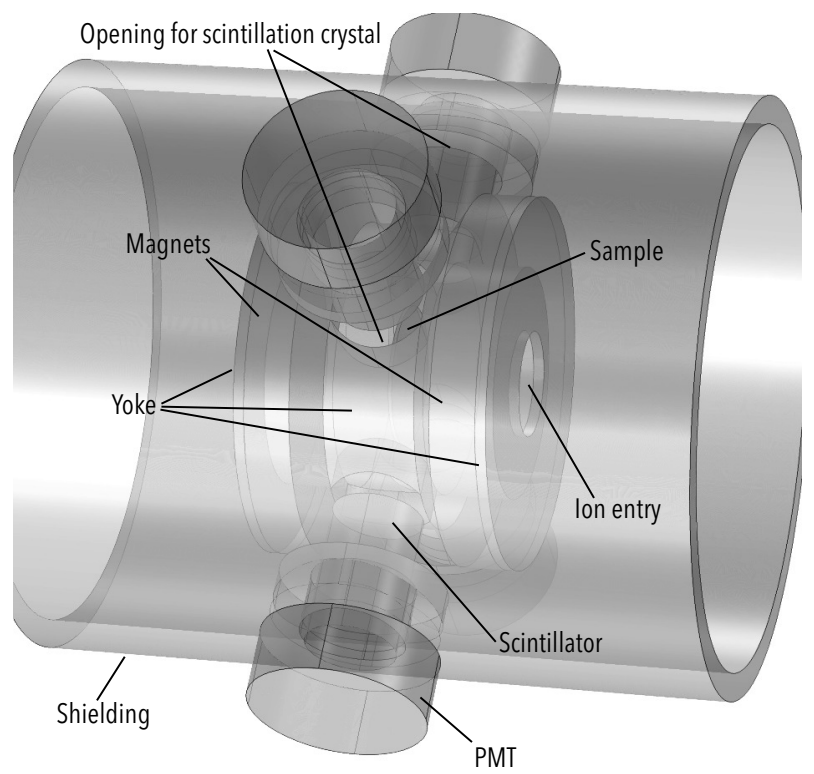

Figure 10.29: Magnetization setup with improved (i. e. lower) $\gamma$ absorption based on two ringshaped magnets.

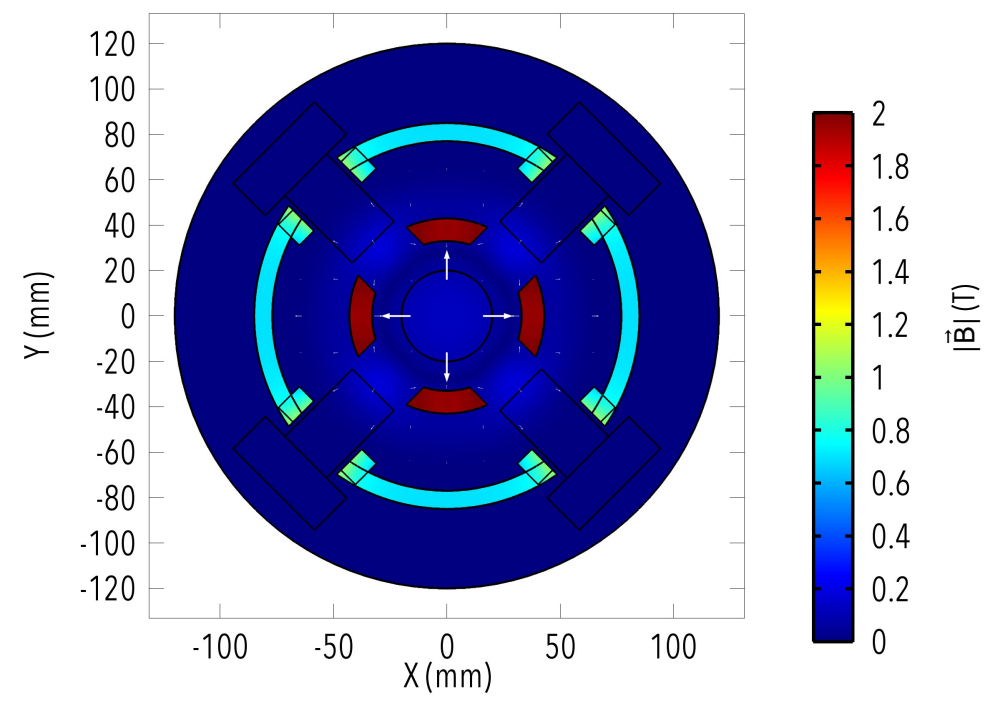

Figure 10.30: Simulated magnetic flux values $10 \mu \mathrm{m}$ beneath the sample foil's surface in the Helmholtz configuration of two ring-shaped permanent magnets. White arrows visualize the direction of magnetic flux parallel to the figure's cut plane. 


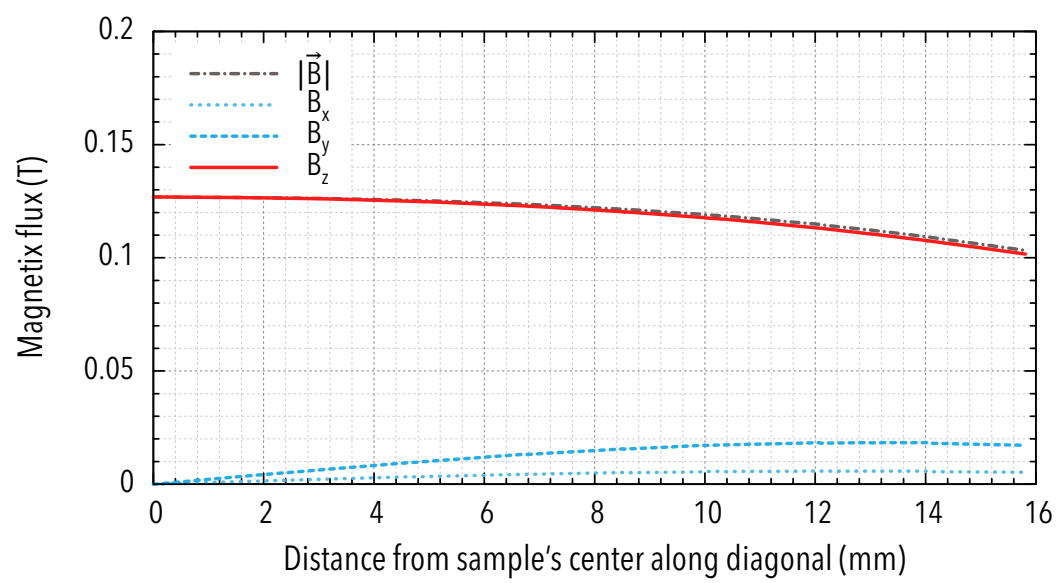

Figure 10.31: Results of the flux simulation $10 \mu \mathrm{m}$ beneath the sample foil's surface along the samples diagonal in the Helmholtz configuration of two ring-shaped permanent magnets. The distance values denote the distance from the center. The homogeneity is significantly better than even in the design with a disc-shaped magnet.

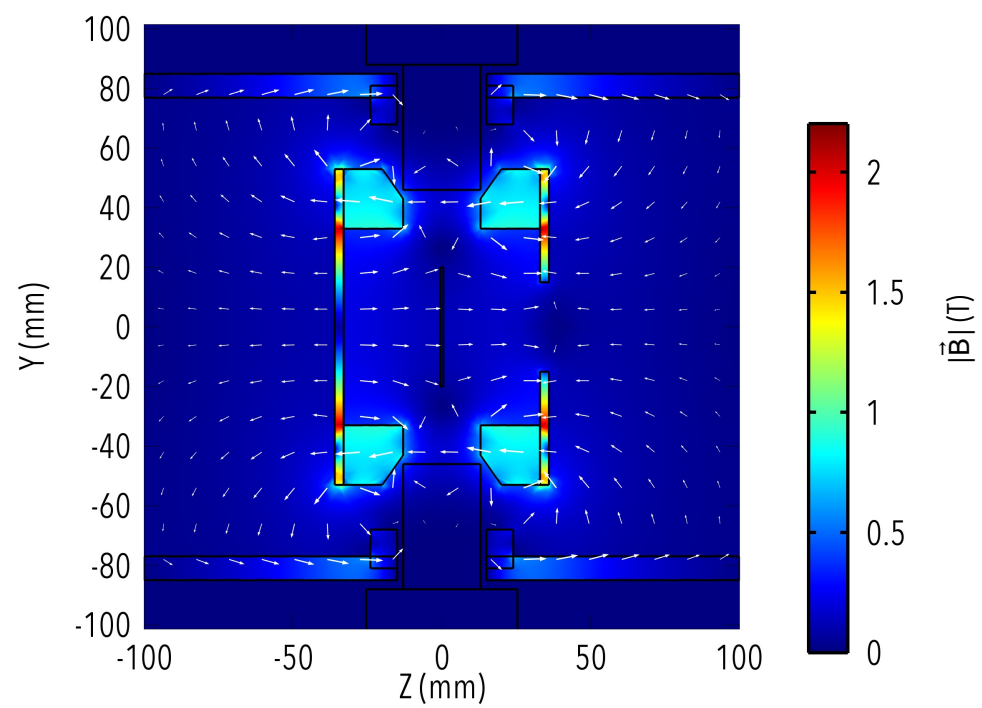

Figure 10.32: Simulated magnetic flux values on a plane cutting through two detectors and the ion beam entry in the Helmholtz configuration of two ring-shaped permanent magnets. A logarithmic scaling is used for the arrow length in order to cover a larger range of values. White arrows visualize the direction of magnetic flux parallel to the figure's cut plane. 


\section{Chapter 11}

\section{Conclusions and outlook}

Based on the conclusion of the general feasibility of a digital TDPAC spectrometer drawn as a consequence of the results published by Herden, Alves, et al. (2004), the consequent exploitation of related chances in the context of this thesis yielded the most advanced TDPAC spectrometer currently available. Simultaneously, solving of a number of related problems led to a tool-set, which closed essential gaps thereby opening up new opportunities for the TDPAC method in general.

\subsection{Summary of achievements}

The implementation of the new digital TDPAC spectrometer described in chapter 4 was a success in terms of performance as well as reliability and usability. Doubts, present in the TDPAC community, concerning the capabilities of the design approach-especially in case of high count rates-were successfully resolved. Due to the newly developed continuous measurement method exploiting the SAR-mode of the used Agilent digitizers, dead-time was successfully reduced to only $350 \mathrm{~ns}$ per event. Moreover, thanks to a custom $2 \mathrm{GHz}$ clock providing a stable and precise common timing signal, measurements with the new spectrometer can run for months, despite only a single synchronization process is required in the beginning. Additionally, the sophisticated coincidence search implemented in the evaluation software SpectraPAC-which exceeds the possibilities of previous setups-ensures bias-free results even at highest count-rates. Achieved time and energy resolutions are very close to the limits of the used scintillation materials and better than those of any other available digital TDPAC spectrometer (cf. section 4.3).

The question, if a purely software driven approach depending on general-purpose highspeed digitizers and off-the-shelf computer hardware is able to compete with an FPGA based solution, can now be answered: It is not only competitive but the superior approach since it provides better performance per development effort and cost. Although the construction of an FPGA based spectrometer in Leipzig started several years before the spectrometer described in chapter 4 (cp. Agne, Das, and Butz, 2005), a first working implementation was finished half a year later (Jäger, Iwig, and Butz, 2010). This implementation was not substantially better than the earlier software driven prototype from Herden, Alves, et al. (2004) except for better detectors, which are not an achievement of 
the spectrometer's working principle. In contrast to the design described in chapter 4 , it does neither implement improved control of the coincidence search compared to analog models nor does it allow postponing of configuration tasks. It is eventually not much more than a digital emulation of analog setups. Although in the meantime the capabilities of the FPGA based spectrometer were slightly improved (Jäger, Iwig, and Butz, 2011), development of each improvement is cumbersome in their case since signal processing has to be defined on a very low level to be FPGA compatible. The same usually involves just a small number of code lines in case of the software driven spectrometers. Signal analysis was transferred to plugins in Pac-Suite and sophisticated and highly optimized libraries like the Gnu Scientific Library (Galassi et al., 2009) or FFTW (Frigo and Johnson, 2005) are available, which not only simplify implementation but also debugging as well as testing of new algorithms. In the meantime, the first version of the software driven spectrometer described in Nagl, Vetter, et al. (2010) was replicated by a group from McGill University (Webb et al., 2013).

Another important advantage of software driven spectrometers is the usability of unused CPU time for simulations. For this purpose, the TORQUE Resource Manager (Adaptive Computing, 2014) was set up as a batch system on all computers belonging to the spectrometer and prioritization of CPU and disk access was optimized to always favor Pac-Suite, i. e. measurements, over simulations. Despite continuously running measurements, remaining CPU time was sufficient to process all simulations discussed in chapter 7 as well as the massive WIEN2k simulations described by Jürgens (2013).

Due to the use of transistor stabilized voltage dividers for the supply of the used photomultiplier tubes, low detector gain drift was achieved. Remaining drift is successfully compensated by the newly developed automatic drift compensation method described in chapter 6, which was integrated into SpectraPAC.

Extensibility is provided due to the modular concept, which allows adding of additional detectors by simply adding another recording computer. The only required connections are a free Ethernet switch port and a free connector at each of the power splitters used for clock and sync signals. Care has been taken to avoid artificially limiting the number of supported detectors in the developed software, namely PacMaster and Spectra$P A C$. While analog setups quickly grow impracticably complex by the addition of more detectors due to increasing complexity in their routing logic and usually suffer from an increased amount of discarded events due to pile-up at the central time-to-amplitude converter, the new digital spectrometer scales extraordinarily. For instance, the update from the first generation setup using four LYSO detectors to the current state with six $\mathrm{LaBr}_{3}$ detectors was a matter of days and did not require changes to the software.

Shifting complexity from hardware to software required the development of a powerful software suite. Pac-Suite, which includes the aforementioned programs PacMaster and SpectraPAC as well as the software running on the recording computers (PacMan) and a set of additional tools, provides all the functionality necessary to operate a digital spectrometer and evaluate its results. The design of PacMan, which sends data immediately after pro- 
cessing via a custom, highly efficient, networking protocol to a PacMaster instance running on a central computer, made it possible to operate headless as well as diskless recording computers. This allows for the use of very small systems. In principle the currently used 1 rack unit systems could be replaced by even smaller form-factors since only sufficient processing power alongside a PCI slot for the digitizer and a Gbit Ethernet connection are necessary. Pac-Suite's optimized binary file format makes it possible to run single or multiple online as well as offline evaluations of measurements and provides easy file handling due to the limitation to a single file per measurement. The measurement workflow implemented by Pac-Suite differs significantly from that of analog spectrometers in order to exploit the greater flexibility of the digital approach. For instance, an energy calibration step was introduced into the evaluation, which allows for the aforementioned drift compensation. Energy windowing is now postponed after this calibration step thereby making it possible to use one set of energy windows for all detectors and making it easy to tune window settings after the actual measurement was finished. This also simplifies multi-cascade evaluations as shown in chapter 8 . Configuration tasks, necessary before the start of an actual measurement, were reduced to the absolute minimum. In fact, only the digitizer's full-scale range and the high-voltage settings have to be chosen in advance. By using calibration sources, it is even possible to prepare this beforehand.

Long-term stability of the setup was proven by various test-measurements many of which were running for more than a month as well as maintenance free permanent operation for periods spanning more than a year. Transport to beam times at ISOLDE and the ILL was possible without problems and the spectrometer was immediately operational in all these cases.

In order to obtain optimal timing results, comprehensive investigations concerning the optimization of signal processing algorithms were conducted as described in chapter 5 . These investigations led to the development of a new timing method providing outstanding performance while reducing the tuning effort. Despite the limited sampling rate and resolution of available digitizers, the new timing method is at least competitive with optimized analog timing methods.

To simplify design evaluation for future spectrometers and provide a deeper understanding of the performance-limiting mechanisms in the detection process, a simulation toolchain for the involved scintillation crystal, the photomultiplier tube, and downstream processing was implemented allowing for simulation driven detector design. These efforts are described in detail in chapter 7. Besides providing said evaluation and optimization opportunities, this toolchain can also be used for the determination of solid angle correction factors $Q_{k_{1} k_{2}}$ as introduced in section 2.1.3. An example of this application is described in section 7.5.

Since the lifting of constraints of previous spectrometers is assumed to have influence on the variety of usable probe nuclides and available information concerning the anisotropy of decay cascades was often fragmentary and faulty, a comprehensive software-based search for candidate nuclides and decay cascades was implemented. In contrast to former 
manual attempts to identify promising candidates, which resulted in several candidate lists of varying quality, this search software, which uses data from the well maintained ENSDF database, allows for easy repeating of searches every time a new database version is released. Additionally, it provides a visualization of decay schemes and predicts energy spectra, which is very useful functionality for the evaluation of measurements employing unusual nuclides. In fact, the development of the energy spectrum prediction was directly motivated by experiences gained during ISOLDE beam times.

The design and implementation of auxiliary facilities like a custom high-temperature furnace and magnetization devices as well as new solid-state physics infrastructure for ISOLDE (cf. chapter 10) can be considered a perfect complement for the TDPAC related efforts described above. While annealing facilities like said furnace and magnetization facilities are directly related to typical TDPAC applications, the ISOLDE facilities are important to increase the usability of exotic nuclides identified by means of the aforementioned nuclide search software. Whereas the new implantation chamber allows for increased implantation efficiency and control due to a very precise load-lock system and the opportunity of tilted implantations, the deceleration and post-acceleration chamber provides better control of implantation profiles. Finally, the planned online TDPAC chamber will provide the possibility to exploit the newly identified short lived TDPAC candidate nuclides.

\subsection{Future of TDPAC spectroscopy}

Despite its unique advantages, TDPAC spectroscopy was only playing a minor role among condensed matter physics methods for many years. One of the reasons for this discrepancy is the need for radioactive probe atoms, which requires adequate laboratories and appropriate admission for the handling of unsealed radioactive sources. Moreover, in case of the implantation of probe atoms, suitable ion accelerators are necessary. Finally, for probes other than ${ }^{111} \mathrm{In}$, which is commercially available, a production facility must be available. However, considering the success of methods like neutron scattering or methods relying on synchrotron radiation, these drawbacks alone cannot explain why TDPAC spectroscopy is regarded such an exotic approach.

Another, less obvious, difference compared to methods of similar complexity is that TDPAC was neither commercialized nor implemented in the context of a major research institution. This means that all existing spectrometers are individual prototypes and most supporting tools were only developed for internal use in the groups owning the spectrometers. Therefore, a large number of solutions exist for the control of analog spectrometers as well as fitting of spectra but none of them has reached a level of quality, reliability, and availability which would establish it as a standard.

Hopefully, the publication of software tools created in the context of this thesis including their complete source code contributes to solving this issue. Pac-Suite was intention- 
Table 11.1: Tools for the fitting of TDPAC spectra. For the older program Depack ${ }^{a}$ no information could be obtained.

\begin{tabular}{|c|c|c|c|c|}
\hline & winfit & Nightmare ${ }^{b}$ & PolyPacFit & $\mathrm{NNFit}^{b}$ \\
\hline $\begin{array}{l}\text { Graphical user } \\
\text { interface }\end{array}$ & $\checkmark$ & $\checkmark$ & $x$ & $x$ \\
\hline $\begin{array}{l}\text { Fits single } \\
\text { crystal spectra }\end{array}$ & $x$ & $\checkmark$ & $x$ & $\checkmark$ \\
\hline $\begin{array}{l}\text { Available for } \\
\text { download }\end{array}$ & $x$ & $x$ & $\checkmark$ & $x$ \\
\hline $\begin{array}{l}\text { Source code } \\
\text { available }\end{array}$ & $\checkmark$ & $x$ & $\checkmark$ & $\checkmark$ \\
\hline $\begin{array}{l}\text { Documentation } \\
\text { available }\end{array}$ & $\checkmark$ & $x$ & $\checkmark$ & $\checkmark$ \\
\hline References & $\begin{array}{l}\text { Heinrich (2001) and } \\
\text { Heinrich (2005) }\end{array}$ & Nédélec (2014) & $\begin{array}{l}\text { Zacate, Evenson, } \\
\text { et al. }(2010) \text { and } \\
\text { Zacate }(2014)\end{array}$ & Barradas (1992) \\
\hline
\end{tabular}

${ }^{a}$ mentioned by Bertschat (2005), among others. However, the cited publication is hardly accessible

${ }^{b}$ Nightmare uses fit routines derived from NNFit

ally designed in a way that allows for easy adaptation to any digital spectrometer. It is even possible to use it for the control of FPGA based spectrometers if the communication module of PacMaster is adapted and the FPGA does the same data analysis usually handled by PacMan.

\subsubsection{Fitting of $R(t)$ spectra}

A remaining issue is the quality and availability of fit software. Fitting is necessary to extract information concerning magnetic fields and/or electric field gradients from the $R(t)$ spectra. Only few tools can be obtained including source code and most programs are limited to the fitting of spectra from powder samples (see table 11.1). Additionally, documentation quality is a frequent issue concerning available TDPAC fit software. Software with a modern GUI, improved usability, and documentation combined with single crystal fit abilities would really help in making TDPAC spectroscopy more successful than it is today. Moreover, it would be beneficial if a fitting tool supported multi-cascade evaluations since this possibility would improve evaluation results in cases where appropriate data is available from digital spectrometers. 


\subsubsection{Complementing ab initio simulations}

Successful fitting of TDPAC spectra yields information concerning the strength of a magnetic field or the magnitude, symmetry, and orientation of an electric field gradient. The last step of a successful application of the TDPAC method in condensed matter physics is usually concluding from these field related information to environmental details of the probe nuclei. While this last step was limited to simple cases in the past, it is now possible for many rather complicated cases due to the availability of ab initio simulation tools for molecular and crystal properties. For instance Jürgens et al. (2011) and Jürgens (2013) used WIEN2k (Schwarz and Blaha, 2003) to simulate electric field gradients at different lattice sites which made it possible to compare these results with TDPAC measurements and thereby conclude where the probe atoms are located. Heinrich (2005) similarly used ADF (Scientific Computing \& Modelling, 2014) for modeling electric field gradients in molecules. These simulation tools close the last gap concerning conclusions from TDPAC measurements to statements concerning the microscopic structure of investigated samples, i. e. concerning the actual interpretation of measurement results.

\subsubsection{Availability of probe nuclides}

As mentioned before, there are several methods available allowing for the production of probe nuclides. ${ }^{111} \mathrm{In}$, for instance, can be produced in cyclotrons using the nuclear reaction ${ }^{112} \mathrm{Cd}(\mathrm{p}, 2 \mathrm{n}){ }^{111}$ In whereas ${ }^{181} \mathrm{Hf}$ can be produced by irradiation of ${ }^{180} \mathrm{Hf}$ with thermal neutrons in a reactor. Production of nuclides with these methods is possible in a large number of facilities worldwide and therefore supply is not a problem.

However, more exotic nuclides, which cannot be produced using simple nuclear reactions, require the use of ISOL facilities or fission product separators. The most wellknown and established facilities of this kind are ISOLDE (CERN) and LOHENGRIN (ILL), which were introduced in chapter 2. Of course, available beam time at these facilities is limited.

However, at least for ISOL facilities the outlook is positive since a number of new installations is currently being built. While the future will show if projects like SPES ${ }^{1}$, SPIRAL2 ${ }^{2}$, ISAC-II ${ }^{3}$, or ARIEL ${ }^{4}$ are suitable concerning the requirements of TDPAC spectroscopy, there is at least the chance that due to the availability of additional facilities it will become easier rather than more difficult to apply for beam time at ISOLDE, which is a proven nuclide source for solid-state physics experiments. Simultaneously, the possibilities for solid-state physics at ISOLDE will significantly increase due to the new chambers presented in chapter 10. At ILL, a new ion tracking chamber with much better time

\footnotetext{
${ }^{1}$ Laboratori Nazionale di Fisica Nucleare, 2014.

${ }^{2}$ GANIL, 2014.

${ }^{3}$ TRIUMF, 2014b.

${ }^{4}$ TRIUMF, 2014a.
} 
resolution is currently being planned which will improve gating on ion incidences and could eventually allow for coincidence experiments with spin-polarized fission fragments. This would remove the requirement of a decay cascade and thereby massively increase the choice of nuclides.

Altogether, the availability of exotic probe nuclei tends to increase rather than decrease in future, making it possible to exploit the extended choice of possible probes presented in chapter 9.

\subsubsection{Future spectrometer designs}

One of the outstanding results of the move to a digital spectrometer design as described in this work is the possible omission of the massive increase in routing complexity associated with former designs, which prevented an increase in the number of detectors. Time and energy evaluation is now for all channels completely separated from the other channels as well as from remaining evaluation steps (i.e. it scales proportional to the number of detectors) whereas merging of data as well as the coincidence search as described in section 4.2 scale proportional to the count-rate rather than strongly overproportional with the number of detectors.

This means that a segmentation of detectors-which alone does not increase the countrate-is now possible without a significant increase in complexity. By means of segmentation, the dilemma that either the anisotropy decreases due to solid angle corrections if the detectors are moved close to the sample or the count-rate drops and therefore the signal-to-noise ratio decreases if the detectors are moved further away from the sample, can be solved. By using for example eight appropriately shaped scintillators, resulting in a segmented ring around the sample, solid angle coverage (cf. table 7.10) could be considerably improved while obtaining much better statistics due to a total number of $16 \times 45^{\circ}$, $16 \times 90^{\circ}, 16 \times 135^{\circ}$, and $8 \times 180^{\circ}$ coincidence spectra. Due to the better solid angle coverage of such a segmented ring, the coincidence rate of each of these spectra could be comparable to the coincidence rate of a current four detector setup-which only provides a total of twelve coincidence spectra compared to 56 of the segmented eight-detector setup.

Provided that advanced evaluation methods are developed, which make it possible to exploit the additional information, such an improved detector setup will allow for yielding more information from much weaker samples than even the spectrometer described in this thesis, although the new spectrometer already considerably improved the chance for successful online measurements compared to older designs in case of measurements using nuclides with low production yields.

Another interesting new trend is the development of fully digital SiPMs where no analog signal is produced but instead the number of triggered avalanche photodiodes is counted (Philips, 2014). Since the area of each individual photodiode is very small, the probability that two photons from a scintillation crystal hit the same photodiode is low and therefore counting of triggered cells provides good energy information. If this 
kind of sensor was further optimized to supply excellent energy and timing information, it could eventually replace the evaluation of analog signals in future spectrometers (i. e. it could replace the PMT as well as PacMan including the recording computers).

\subsubsection{Synchrotron-radiation-based perturbed angular correlation}

A rather new approach in the TDPAC context is the excitation of intermediate states by means of synchrotron radiation instead of radioactive decays followed by the same measurement principle as in case of normal TDPAC spectroscopy. This method is also called SRPAC. The first successful implementation was described by Baron et al. (1996). In the meantime, several successful studies were performed using this method. For instance from Dattagupta (2004) and Sergueev et al. (2006).

\subsection{Transferability of results}

In chapter 1, the progression towards the transition from analog to digital signal processing, the transition from customized to universal hardware, and the transition from hardware defined functionality to software defined functionality and its advantages were mentioned. These transitions in the context of TDPAC spectroscopy were the overarching framework of this thesis.

A side effect of said transitions is a better transferability of results to different problem domains since they imply a high degree of modularity in combination with universal building blocks. An example for this transferability is the successful collaboration with positron annihilation spectroscopists for the definition of simulation objectives, which motivated the work described in chapter 7 . In this context it became apparent that in several areas of nuclear spectroscopy people were and still are currently working to solve very similar problems. For instance, digital time determination, which was investigated systematically in chapter 5 , is a current topic not only in the context of TDPAC spectroscopy but also for time of flight experiments (Bardelli et al., 2004), positron annihilation spectroscopy (Saito and Hyodo, 2003), nuclear spectroscopy in general (Pasquali et al., 2007; Sun et al., 2012), and positron emission tomography (Xie et al., 2009). Drift compensation, as described in chapter 6, is also applicable to many areas of nuclear physics as shown by former approaches to this problem as summarized by Vickers (1997).

Similarly, the experiences gained concerning the high-speed online processing of converted signals in the context of the TDPAC spectrometer's realization are applicable to an even wider range of subjects. The spectrometer and its software suite can easily be adapted to any kind of coincidence measurement (e. g. PET, PALS, time of flight) even in completely different areas like e. g. for Lidar or Radar setups. 
Appendix 



\section{Appendix A}

\section{Nuclides and decays}

Throughout this work, a number of nuclides were used repeatedly. This section contains brief descriptions of their specific properties. Transitions which occur for $\geq 5 \%$ of decays are highlighted red in all decay schemes. If not otherwise specified, decay data was taken from the Evaluated Nuclear Structure Data File (2012).

\section{A.1 TDPAC probe nuclides}

\section{A.1.1 ${ }^{111} \mathrm{In} \longrightarrow{ }^{111} \mathrm{Cd}$}

${ }^{111}$ In is for TDPAC what ${ }^{57} \mathrm{Co}$ is for Mößbauer spectroscopy: The most popular nuclide available. As visible in fig. A.1, it decays to ${ }^{111} \mathrm{Cd}^{*}$, which de-excites almost completely through two transitions and a single intermediate level. Its intermediate level exhibits ideal properties for TDPAC measurements.

It is populated and depopulated by two transitions, which emit $\gamma$ photons with energies of $171.28 \mathrm{keV}$ and $245.35 \mathrm{keV}$. These energies are sufficiently different to be separable even with $\mathrm{BaF}_{2}$ scintillators or analog fast-fast spectrometers (cf. section 2.2). The intermediate level's half-life equals $84.5 \mathrm{~ns}$, which is comfortably more than the time resolution limit of even simple spectrometer designs. Additionally, both the magnetic moment as well as the quadrupole moment of the intermediate level are considerably high $\left(-0.766 \mu_{\mathrm{N}}\right.$ and $0.77 \mathrm{eb}^{1}$ ) providing sensitivity for magnetic fields (cf. section 8.1) as well as electric quadrupole moments (see e. g. Jürgens et al., 2011). Due to its spin of $5 / 2+$, the intermediate level is split to three energy levels in presence of an electric field gradient. The resultant three frequencies observed in TDPAC measurements can easily be detected and allow the determination of the asymmetry parameter $\eta$, which corresponds to the asymmetry of the electric field gradient tensor at the probe atoms' sites. The relation between this tensor's asymmetry and frequencies determined by TDPAC measurements was summarized by Wegner (1985) as well as Butz (1989).

${ }^{111}$ In is used for a number of medical applications like antibody or blood cell labeling (Nordion, 2011) and is therefore commercially available. Due to its convenient half-life

\footnotetext{
${ }^{1}$ The unit for electric quadrupole moments is electron-barn (eb). However, the e is often omitted in literature.
} 


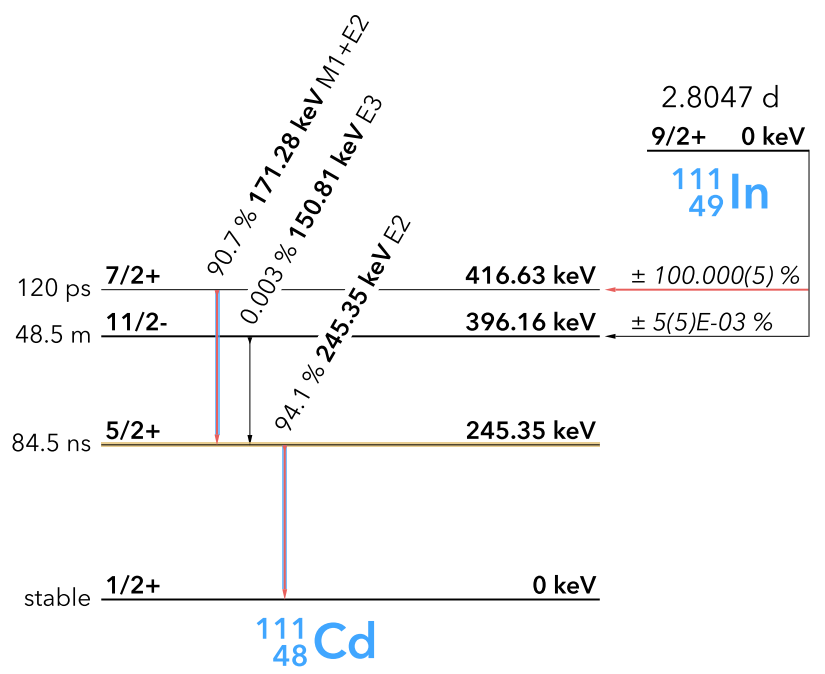

Figure A.1: Decay scheme of ${ }^{111}$ In.

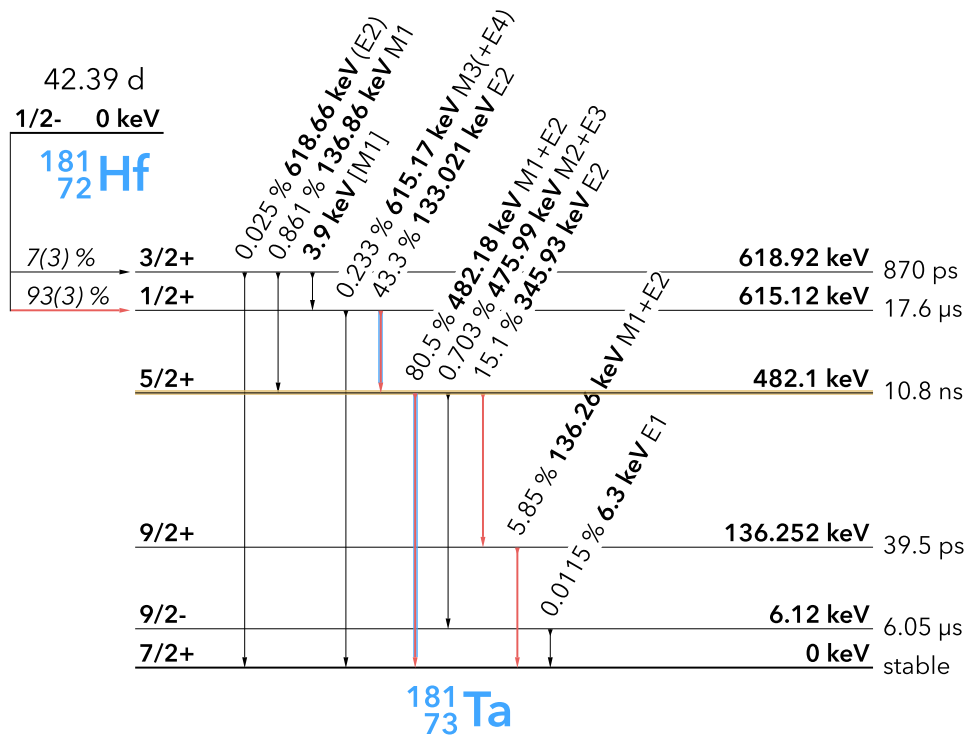

Figure A.2: Decay scheme of ${ }^{181} \mathrm{Hf}$. 
of $2.8 \mathrm{~d}$, it is transportable and comparably safe since possible contaminations occurring during preparation or implantation decay within weeks.

\section{A.1.2 ${ }^{181} \mathrm{Hf} \longrightarrow{ }^{181} \mathrm{Ta}$}

${ }^{181} \mathrm{Hf}$ is the second most popular TDPAC mother nuclide. Unlike ${ }^{111} \mathrm{In}$, it is not commercially available but can be produced very efficiently in research reactors by the nuclear reaction ${ }^{180} \mathrm{Hf}(\mathrm{n}, \gamma){ }^{181} \mathrm{Hf}$, which has a huge cross section of $13.04(7) \mathrm{b}$ for thermal neutrons (Mannhart, 1975).

According to the decay scheme visible in fig. A.2, ${ }^{181} \mathrm{Hf}$ decays mostly to a rather long lived (half-life: $17.6 \mu \mathrm{s}$ ) intermediate state of ${ }^{181} \mathrm{Ta}$. This level decays via a transition emitting $133 \mathrm{keV} \gamma$ photons to an intermediate level (spin: 5/2+), which is suitable for TDPAC measurements. Its - compared to the intermediate level of ${ }^{111} \mathrm{Cd}$ short-half-life of $10.8 \mathrm{~ns}$ is compensated by higher nuclear moments (magnetic moment: $3.29 \mu_{\mathrm{N}}$, electric quadrupole moment: $2.35 \mathrm{eb}$ ). Nevertheless, it puts higher demands on the spectrometer's time resolution since the higher frequencies caused by higher nuclear moments and therefore stronger interactions must be resolvable.

Depopulation of the intermediate level takes place via two alternative decays: A stronger one towards the ground state of ${ }^{181} \mathrm{Ta}$ emitting $482.18 \mathrm{keV} \gamma$ photons and a weaker transition emitting $345.93 \mathrm{keV} \gamma$ photons. While analog spectrometers are limited to the evaluation of one decay path, the spectrometer described in chapter 4 allows evaluating of both alternatives (see section 8.3).

\section{A.1.3 ${ }^{98} \mathrm{Y} \mu \mathrm{s}$-isomers populated by fission}

The level scheme of ${ }^{98} Y$ contains a double decay cascade usable for TDPAC measurements. The first cascade has an intermediate level with a half-life of $35.8 \mathrm{~ns}$ and an angular correlation of $A_{22}=0.2$, assumed the spin assignment of $2^{-}$from the Evaluated Nuclear Structure Data File (2012) data is valid. If the spin assignment of 4 - from Brant, Lhersonneau, and Sistemich (2004) is valid, $A_{22}$ vanishes and the first cascade is not usable for TDPAC measurements. The second cascade follows directly with an intermediate level's half-life of $620 \mathrm{~ns}$ and $A_{22}=0.1$. Unfortunately, this double-cascade is not efficiently populated in the $\beta^{-}$decay ${ }^{98} \mathrm{Sr} \longrightarrow{ }^{98} \mathrm{Y}$. It is, however, populated by deexcitation of ${ }^{98} \mathrm{Y}$ isomers produced by fission as shown in fig. A.3. Such fission fragments can be produced at LOHENGRIN (cf. section 2.4.2) and live-due to their isomeric nature-sufficiently long for transport and implantation into a sample. These properties make ${ }^{98} \mathrm{Y}$ a promising candidate for online TDPAC experiments. The nuclear moments of the two intermediate levels still have to be determined. 


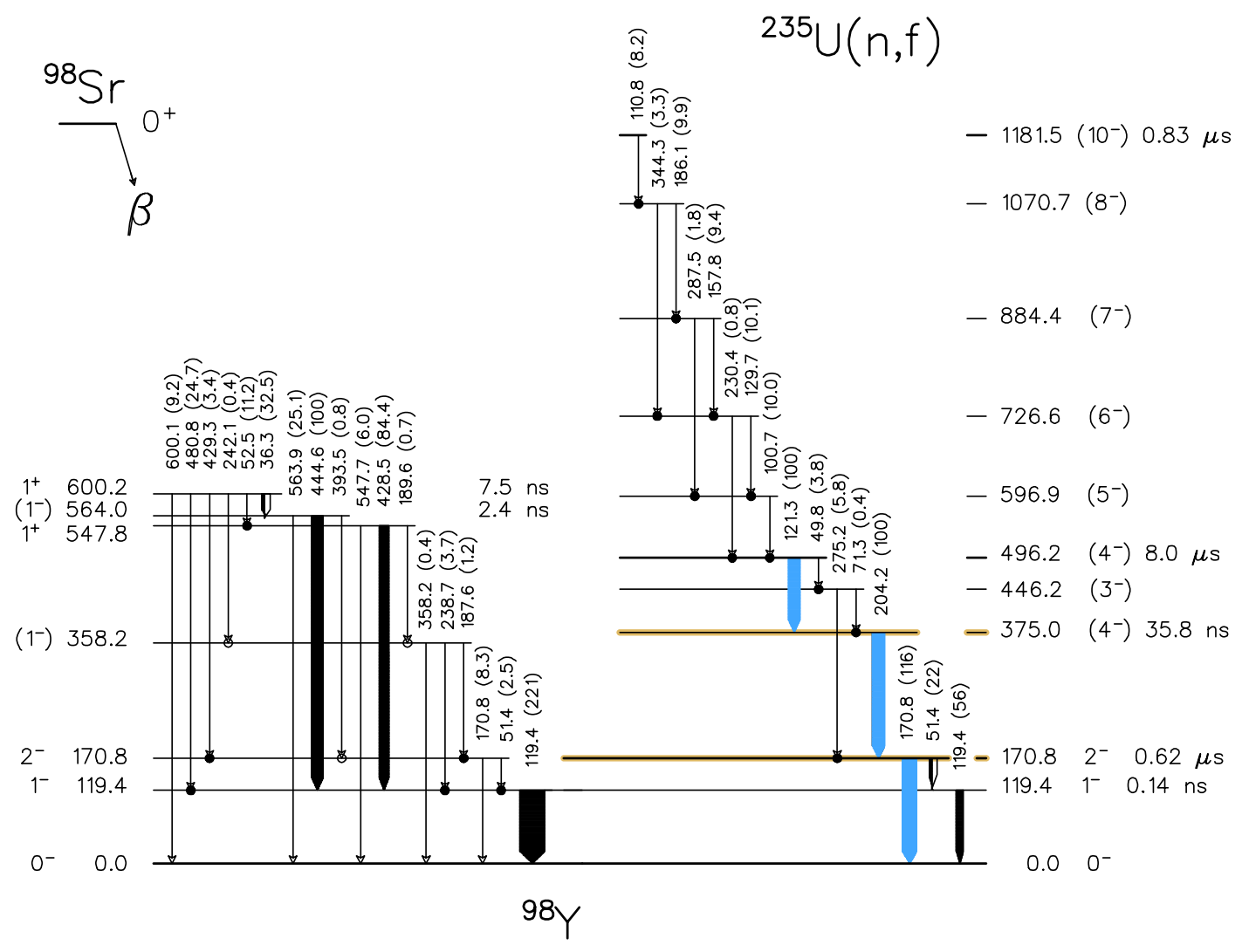

Figure A.3: Partial level scheme of ${ }^{98} \mathrm{Y}$. The left side shows levels from the ${ }^{98} \mathrm{Sr} \beta^{-}$-decay while the right side shows the complete set of levels observed in the decay of $\mu$ s-isomers populated by fission of ${ }^{235} \mathrm{U}$. The double decay cascade is highlighted. By courtesy of Gerard Lhersonneau. Adapted with permission from Brant, Lhersonneau, and Sistemich (2004). Copyrighted by the American Physical Society.

\section{A.2 Other nuclides}

\section{A.2.1 ${ }^{22} \mathrm{Na}$}

${ }^{22} \mathrm{Na}$ decays by means of a $\beta^{+}$decay to ${ }^{22} \mathrm{Ne}$. After the decay, almost $100 \%$ of the daughter nuclei are in an excited state and emit another $\gamma$ photon with an energy of $1274.54 \mathrm{keV}$ before reaching the ground state. Due to these decay properties, the energy spectrum of radiation emitted from a ${ }^{22} \mathrm{Na}$ source only contains two photo-peaks. One at $1274.54 \mathrm{keV}$ originating from the radiation emitted by the excited ${ }^{22} \mathrm{Ne}$ nuclei and a second at $511 \mathrm{keV}$ due to the annihilation of positrons produced in the $\beta^{+}$decay of ${ }^{22} \mathrm{Na}$.

Figure A.4 shows the decay scheme of ${ }^{22} \mathrm{Na}$ with its single $\gamma$ transition. 


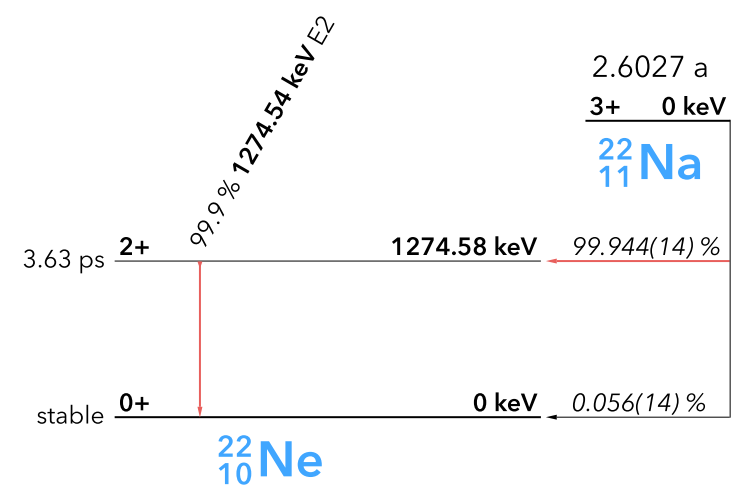

Figure A.4: Decay scheme of ${ }^{22} \mathrm{Na}$.

Due to the single transition, ${ }^{22} \mathrm{Na}$ produces rather clean energy spectra. Since the annihilation results in two $\gamma$ photons each with an energy of $511 \mathrm{keV}$ emitted in opposite directions, it is perfectly usable as calibration source for coincidence measurements because if only $511 \mathrm{keV}$ events are selected from recordings of two detectors mounted on both sides of a sample, a high amount of coincident events from simultaneously emitted photons is obtained.

According to Bé et al. (2010), $9.64 \%$ of ${ }^{22} \mathrm{Na}$ nuclei decay by an electron capture process rather than a $\beta^{+}$decay. However, their $K_{\alpha}$ energies as well as Auger electron energies are well below $1 \mathrm{keV}$ and therefore not interfering with recordings of higher energetic $\gamma$ emissions. Positrons emitted during ${ }^{22} \mathrm{Na}$ 's $\beta^{+}$decay have a maximum kinetic energy of $1821.02 \mathrm{keV}$ while the vast majority belongs to a decay channel with a maximum kinetic energy of $546.44 \mathrm{keV}$ and an average energy of $215.62 \mathrm{keV}$. Before their annihilation, these positrons can cause the emission of Bremsstrahlung visible in energy spectra at energies of several hundred $\mathrm{keV}$.

\section{A.2.2 ${ }^{60} \mathrm{Co}$}

${ }^{60} \mathrm{Co}$ decays via a $\beta^{-}$decay to ${ }^{60} \mathrm{Ni}$. More than $99 \%$ of the resulting excited ${ }^{60} \mathrm{Ni}$ nuclei deexcite following a single decay cascade with very short half-life of $900 \mathrm{fs}$ as shown in fig. A.5. Since this time is several orders of magnitude shorter than time resolutions achievable with current detectors, ${ }^{60} \mathrm{Co}$ is used as a standard source for the determination of time resolution. Transition energies in said decay cascade are comparably high with $1173.23 \mathrm{keV}$ for the first and $1332.49 \mathrm{keV}$ for the second transition. In cases where time resolution at lower energies is of interest, it is possible to select coincident Compton events generated by a ${ }^{60} \mathrm{Co}$ source from the energy range of interest. 


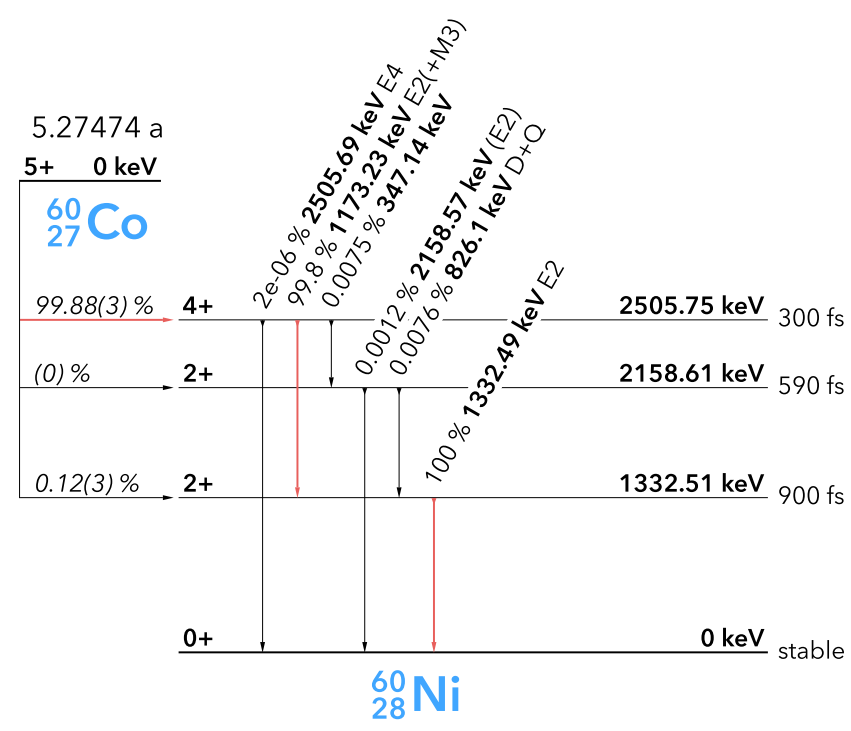

Figure A.5: Decay scheme of ${ }^{60} \mathrm{Co}$.

${ }^{60} \mathrm{Co}$ is commercially available and is used as a standard $\gamma$ source for a number of industrial, medical, and scientific applications (QSA Global, 2014).

\section{A.2.3 ${ }^{137} \mathrm{Cs}$}

${ }^{137} \mathrm{Cs}$ decays via a $\beta^{-}$decay primarily to the isomer ${ }^{137 \mathrm{~m}} \mathrm{Ba}$ with a half-life of $2.552 \mathrm{~min}$. ${ }^{137 m} \mathrm{Ba}$ deexcites via a single transition emitting $\gamma$ radiation with an energy of $661.657 \mathrm{keV}$ (cf. fig. A.6).

${ }^{137} \mathrm{Cs}$ sources are used as calibration source for radiation measurement equipment, in industrial gauging applications, and for wireline measurements in oil exploration (Raims Ltd., 2014) and are therefore commercially available. Due to the single transition in the decay scheme, the photo-peak at $661.657 \mathrm{keV}$ is isolated and free of background. This simplifies determination of its width and therefore makes ${ }^{137} \mathrm{Cs}$ a standard source for the determination of the energy resolution of $\gamma$ detection devices.

\section{A.2.4 ${ }^{152} \mathrm{Eu}$}

${ }^{152} \mathrm{Eu}$ decays via a $\beta^{-}$decay to ${ }^{152} \mathrm{Gd}$ and via an electron capture process to ${ }^{152} \mathrm{Sm}$ with probabilities of $27.92 \%$ and $72.08 \%$, respectively (Firestone and Shirley, 1996). The transition schemes include more transitions than in the case of ${ }^{22} \mathrm{Na}$. In the decay ${ }^{152} \mathrm{Eu} \longrightarrow{ }^{152} \mathrm{Gd}$, a short lived intermediate state (half-life: $32.4 \mathrm{ps}$ ) is available. It is populated by a $778.9 \mathrm{keV}$ 


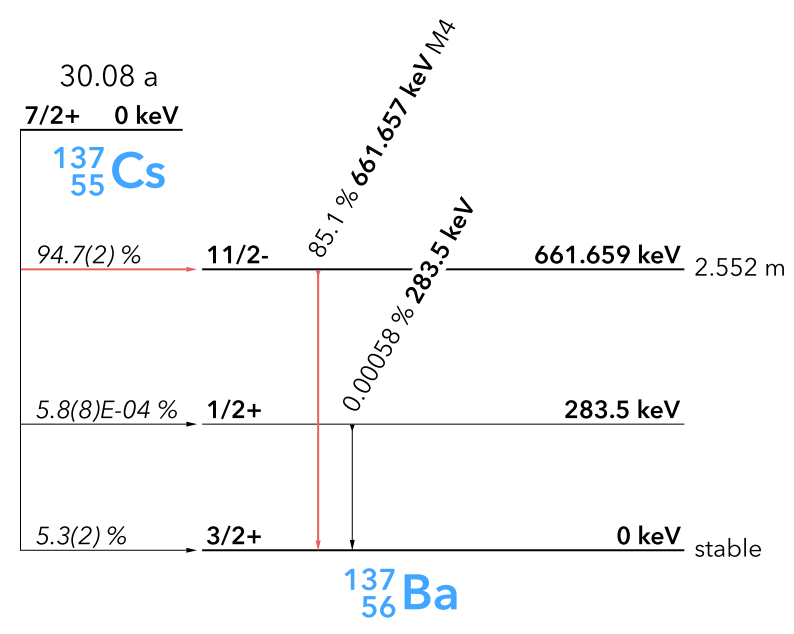

Figure A.6: Decay scheme of ${ }^{137}$ Cs.

transition and depopulated by a $344.28 \mathrm{keV}$ transition with sufficient intensity for timing experiments. Both channels' decay schemes are shown in figs. A.7 and A.8.

${ }^{152} \mathrm{Eu}$ is commercially available (e. g. Eckert $\&$ Ziegler, 2008) and usually used in calibration sources because of its comparably large number of different $\gamma$ transitions, which span a broad range of energies and allow for quick precision calibrations of semiconductor detectors, and because of its convenient half-life of 13.537 years, which allows for long usage periods without replacement of the source. 
Nૂ

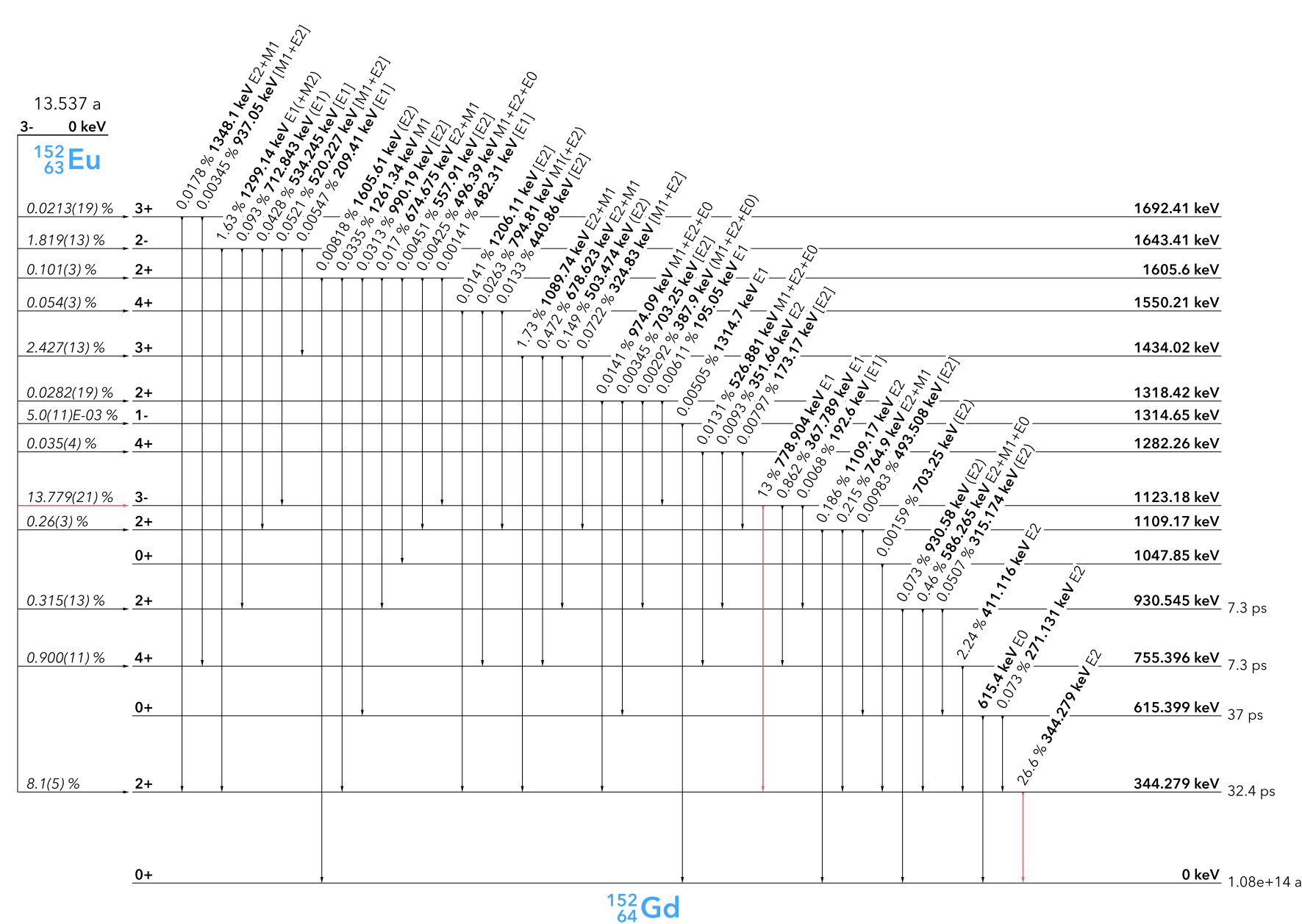

Figure A.7: Decay scheme of ${ }^{152} \mathrm{Eu} \longrightarrow{ }^{152} \mathrm{Gd}$. 


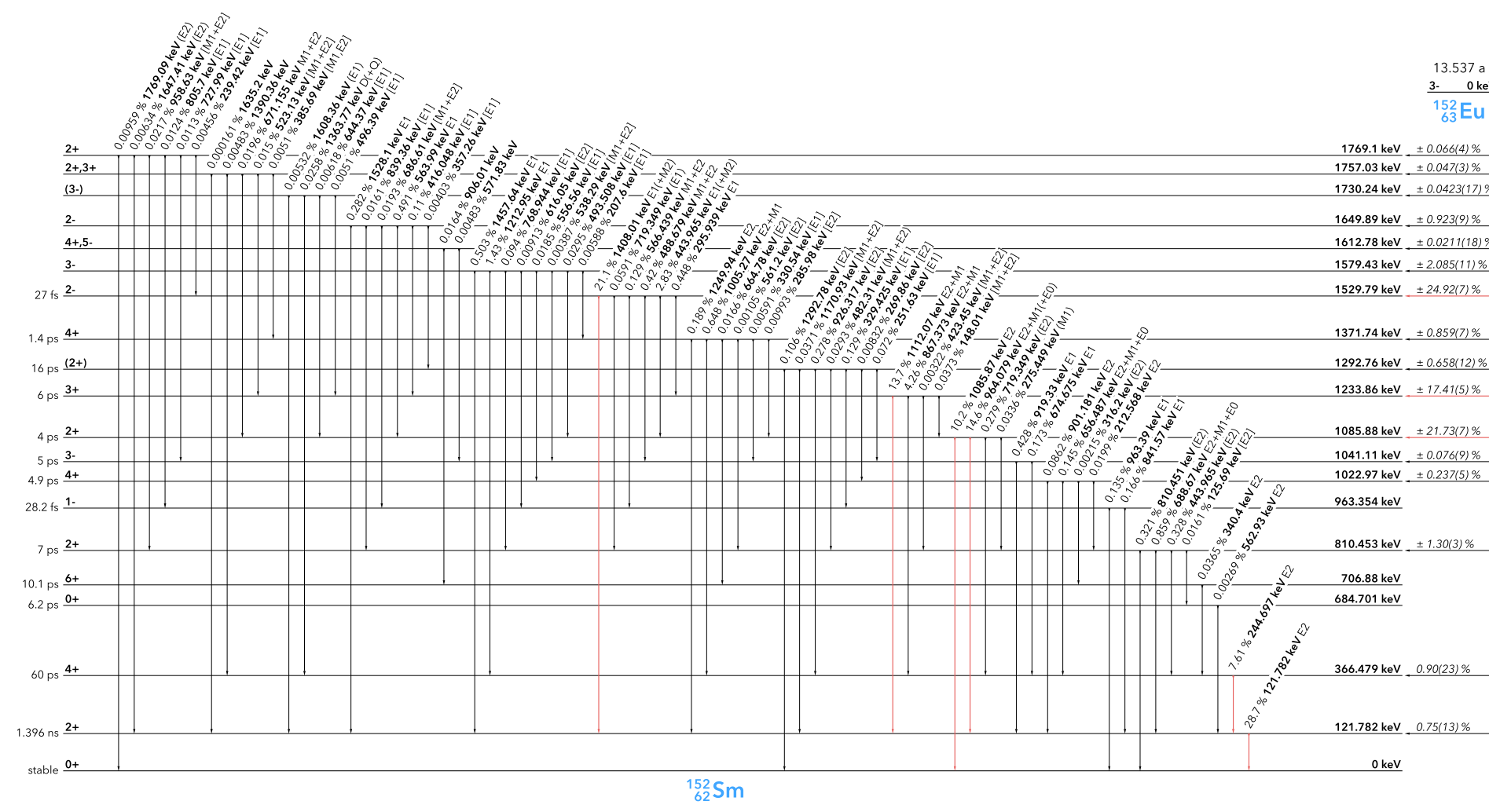

Figure A.8: Decay scheme of ${ }^{152} \mathrm{Eu} \longrightarrow{ }^{152} \mathrm{Sm}$ 



\section{Bibliography}

Abiona, A. A., W. Kemp, and H. Timmers (2014). "Pd-vacancy complex in Ge: TDPAC and ab initio study”. In: AIP Conference Proceedings 1583.1, pp. 105-108. DoI: 10.1063/1.4865614 (cit. on p. 2).

AdaPtive Computing (2014). TORQUE Resource Manager. uRL: http://ww. adaptivecomputing. com/products/open-source/torque/ (cit. on p. 210).

Agilent (2008). Agilent U1064A data sheet. Agilent Technologies. URL: http://cp.literature . agilent.com/litweb/pdf/5989-7444EN.pdf (cit. on p. 128).

- (2009). User Manual Agilent Acqiris 8-Bit Digitizers. Agilent Technologies. uRL: http:// www.agilent.com (cit. on p. 43).

- (2012). Agilent U1071A data sheet. Agilent Technologies. URL: http : / / cp . literature . agilent.com/litweb/pdf/5989-7100EN.pdf (cit. on pp. 45, 46, 122).

Agne, T., S. K. Das, and T. Butz (2005). "ISOLDE: Development of a Fully-Digital, UserFriendly PAC-Spectrometer". In: The Physics Institutes of Universität Leipzig. Ed. by M. GrundMANN. Universität Leipzig. ISBN: 3-934178-64-2. URL: http://www .uni-leipzig.de/ exph2/report_2005.pdf (cit. on p. 209).

Allison, J. et al. (2006). "Geant4 developments and applications". In: IEEE Transactions on Nuclear Science 53.1, pp. 270-278. ISSN: 0018-9499. DOI: 10.1109/TNS.2006.869826 (cit. on pp. 94, 95).

Analog Devices (2012a). AD5390 Data Sheet. Analog Devices. Norwood, USA. url: http: //www.analog.com/static/imported-files/data_sheets/AD5390_5391_5392.pdf (cit. on p. 49).

- (2012b). Integrated Synthesizer and VCO ADF4360-2. Data sheet. Analog Devices. Norwood, USA. URL: http://www.analog. com/static/imported-files/data_sheets/ADF4360-2.pdf (cit. on p. 45).

Anderson, C. D. (1932). “The apparent existence of easily deflectable positives”. In: Science 76.1967, pp. 238-239. DoI: 10.1126/science.76.1967.238 (cit. on p. 1).

Arends, A., C. Hohenemser, F. Pleiter, H. Waard, L. Chow, and R. Suter (1980). "Data reduction methodology for perturbed angular correlation experiments". In: Hyperfine Interactions 8.1-3, pp. 191-213. ISSN: 0304-3843. DOI: 10.1007/BF01026869 (cit. on pp. 15, 62).

Armbruster, P. et al. (1976). "The recoil separator Lohengrin: Performance and special features for experiments". In: Nuclear Instruments and Methods 139, pp. 213-222. ISSN: 0029-554X. DOI: 10.1016/0029-554X(76)90677-7 (cit. on pp. 28, 30).

Avago Technologies (2009). MGA-14516 High Gain, High Linearity Active Bias Low Noise Amplifier. Data sheet. Avago Technologies. URL: http://www.avagotech.com/docs/AV02-1049EN (cit. on p. 46). 
Barbosa, M. B. and J. G. Correia (2012). libAkk. urL: http://nuclei. sourceforge. net/ (cit. on pp. 145, 147).

Bardelli, L., G. Poggi, M. Bini, G. Pasquali, and N. Taccetti (2004). "Time measurements by means of digital sampling techniques: a study case of 100 ps FWHM time resolution with a $100 \mathrm{MSample/s,} 12$ bit digitizer". In: Nuclear Instruments and Methods in Physics Research Section A 521.2-3, pp. 480-492. ISSN: 0168-9002. DOI: D0I : 10.1016/j. nima.2003.10.106 (cit. on pp. 34, 43, 72, 216).

Baron, A. Q. R., A. I. Chumakov, R. Rüffer, H. Grünsteudel, H. F. Grünsteudel, and O. Leupold (1996). "Single-nucleus quantum beats excited by synchrotron radiation". In: EPL (Europhysics Letters) 34.5, p. 331. DoI: 10.1209/epl/i1996-00460-0 (cit. on p. 216).

Barradas, N. P. (1992). NNFIT the PAC MANual. Manual (cit. on p. 213).

Bartos, A., K. Schemmerling, T. Wenzel, and M. Uhrmacher (1993). "A compact \{TDPAC\} spectrometer". In: Nuclear Instruments and Methods in Physics Research Section A 330.1-2, pp. 132-139. ISSN: 0168-9002. DoI: 10.1016/0168-9002(93)91314-D (cit. on p. 19).

BÉ, M.-M. et al. (2010). Table of Radionuclides. Vol. 5. Monographie BIPM-5. Pavillon de Breteuil, F-92310 Sèvres, France: Bureau International des Poids et Mesures. ISBN: 92822-2234-8. URL: http: / / www . bipm . org/utils/common/pdf /monographieRI/Monographie_BIPM5_Tables_Vol5.pdf (cit. on p. 223).

Beringer, R. and C. G. Montgomery (1942). "The Angular Distribution of Positron Annihilation Radiation". In: Phys. Rev. 61 (5-6), pp. 222-224. DoI: 10.1103/PhysRev.61.222 (cit. on p. 1).

Bertschat, H. (2005). "Surface and Interface Magnetism on the Atomic Scale". In: Frontiers in Magnetic Materials. Ed. by A. NarliKar. Springer Berlin Heidelberg, pp. 503-530. ISBN: 978-3-540-24512-4. DOI: 10.1007/3-540-27284-4_17 (cit. on pp. 2, 213).

Blumenfeld, Y., T. Nilsson, and P. V. Duppen (2013). "Facilities and methods for radioactive ion beam production". In: Physica Scripta 2013.T152, p. 014023. Dor: 10.1088/ 0031-8949/2013/T152/014023 (cit. on pp. 26, 27).

Boенм, B. W. (1981). Software Engineering Economics. Prentice-Hall Advances in Computing Science \& Technology Series. Upper Saddle River, New Jersey: Prentice-Hall. ISBN: 9780138221225 (cit. on p. 249).

Brandt, J. L., W. B. Frank, G. P. КосH, and J. J. Mills (1984). Aluminum: Properties and Physical Metallurgy. Ed. by J. E. Натсн. American Society for Metals. IsBn: 9780871701763 (cit. on p. 99).

Brant, S., G. Lhersonneau, and K. Sistemich (2004). "Shape coexistence in the odd-odd neutron-rich nucleus ${ }^{98} \mathrm{Y}$ studied in the interacting boson model”. In: Phys. Rev. C 69 (3), p. 034327. Dor: 10.1103/PhysRevC.69.034327 (cit. on pp. 89, 221, 222).

Butz, T. (1989). "Analytic perturbation functions for static interactions in perturbed angular correlations of $\gamma$-rays". In: Hyperfine Interactions 52.3, pp. 189-228. ISSN: 0304-3843. DOI: 10.1007/BF02651311 (cit. on pp. 11, 13, 14, 17, 62, 219). 
- (1992). "Analytic perturbation functions for static interactions in perturbed angular correlations of $\gamma$-rays". In: Hyperfine Interactions 73.3-4. Erratum, pp. 387-388. ISSN: 03043843. DOI: 10.1007/BF02418614 (cit. on pp. 11,13).

Butz, T. (2009). Fouriertransformation für Fußgänger. Wiesbaden: Vieweg + Teubner. ISBN: 978-3834805386 (cit. on pp. 63, 76).

Catherall, R., J. Lettry, S. Gilardoni, and U. Köster (2003). "Radioactive ion beams produced by neutron-induced fission at ISOLDE". In: Nuclear Instruments and Methods in Physics Research Section B 204. 14th International Conference on Electromagnetic Isotope Separators and Techniques Related to their Applications, pp. 235-239. ISSN: 0168-583X. DOI: 10.1016/S0168-583X(02)01915-8 (cit. on p. 27).

Chain, C., M. Ceolin, and A. Pasquevich (2008). "PAC research in biology". In: HFI/NQI 2007. Ed. by A. Pasquevich, M. Rentería, E. Saitovitch, and H. Petrilli. Springer Berlin Heidelberg, pp. 619-626. IsBN: 978-3-540-85319-0. DoI: 10.1007/978-3-540-85320$6 \_96$ (cit. on p. 3).

Choong, W.-S. (2009). "The timing resolution of scintillation-detector systems: Monte Carlo analysis". In: Physics in Medicine and Biology 54.21, p. 6495. Dor: 10 . 1088 / 0031 9155/54/21/004 (cit. on p. 94).

CoATES, P. (1973). "Distortion in the measurement of time-interval distributions". In: $\mathrm{Nu}$ clear Instruments and Methods 113.2, pp. 311-312. IssN: 0029-554X. DOI: 10.1016/0029554X(73)90851-3 (cit. on p. 58).

Computer Simulation Technology AG (2013). CST PARTICLE STUDIO. Website. url: https://www.cst.com/Products/CSTPS (cit. on p. 114).

Conti, M., L. Eriksson, and C. Hayden (2011). "Monitoring Energy Calibration Drift Using the Scintillator Background Radiation". In: IEEE Transactions on Nuclear Science 58.3, pp. 687-694. ISSN: 0018-9499. DOI: 10.1109/TNS.2011.2144621 (cit. on p. 86).

Cooke, D. W., K. J. McClellan, B. L. Bennett, J. M. Roper, M. T. Whittaker, R. E. Muenchausen, and R. C. Sze (2000). "Crystal growth and optical characterization of cerium-doped $\mathrm{Lu}_{1.8} \mathrm{Y}_{0.2} \mathrm{SiO}_{5}$ ”. In: Journal of Applied Physics 88.12, pp. 7360-7362. Dor: 10.1063/1.1328775 (cit. on p. 97).

Curran, S. C. and J. D. Craggs (1949). Counting Tubes. 1st ed. New York: Academic Press Inc. (cit. on p. 20).

DatTagupta, S. (2004). "Synchrotron radiation-based perturbed angular correlation (SRPAC) - an application to glass transition”. In: Radiation Physics and Chemistry 70.4-5. Indo-US Workshop on Radiation Physics with Synchrotrons and Other New Sources, pp. 511-514. ISSN: 0969-806X. DOI: 10.1016/j. radphyschem.2003.12.029 (cit. on p. 216).

DE HaAs, J. T. M. and P. Dorenbos (2008). “Advances in Yield Calibration of Scintillators”. In: IEEE Transactions on Nuclear Science 55.3, pp. 1086-1092. ISSN: 0018-9499. DOI: 10. 1109/TNS.2008.922819 (cit. on p. 98).

Deicher, M. (2007). "Characterization of defects in semiconductors using radioactive isotopes”. In: Physica B: Condensed Matter 389.1, pp. 51-57. ISSN: 0921-4526. DoI: 10.1016/j. physb.2006.07.035 (cit. on p. 2). 
Dury, M. R., T. Theocharous, N. Harrison, N. Fox, and M. Hilton (2007). "Common black coatings - reflectance and ageing characteristics in the $0.32-14.3$ micro meter wavelength range". In: Optics Communications 270.2, pp. 262-272. ISSN: 0030-4018. DOI: 10.1016/j.optcom.2006.08.038 (cit. on p. 99).

ECKert \& Ziegler (2008). Industrial Radiation Sources. Catalogue. URL: http: / / www . ezag. com/fileadmin/ezag/user-uploads/isotopes/isotopes/5_industrial_sources.pdf (cit. on p. 225).

Efron, B. and R. J. Tibshirani (1994). An Introduction to the Bootstrap. Monographs on Statistics and Applied Probability 57. Boca Ratom: Chapman \& Hall. IsBn: 0-412-042312 (cit. on p. 105).

Evaluated Nuclear Structure Data File (2012). International Network of Nuclear Structure and Decay Data Evaluators. URL: http: //www. nndc.bnl.gov/ensdf/ (cit. on pp. 145, 219, 221).

Everitt, B. S., S. Landau, M. Leese, and D. Stahl (2011). Cluster Analysis. 5th ed. Wiley Series in Probability and Statistics 848. Wiley. ISBN: 978-0-470-74991-3 (cit. on p. 104).

Experimental Unevaluated Nuclear Data List (2012). uRL: http : / / ww . nndc . bnl . gov / ensdf / ensdf/xundl.jsp (cit. on p. 145).

Field Precision LLC (2013). Saturation curves for soft magnetic materials. Website. URL: http://www.fieldp.com/magneticproperties.html (cit. on p. 201).

Fioni, G., H. Faust, M. Gross, M. Hesse, P. Armbruster, F. Gönnenwein, and G. MünzenBERG (1993). "Reduction of energy dispersion on a parabola mass spectrometer". In: Nuclear Instruments and Methods in Physics Research Section A 332.1-2, pp. 175-180. ISSN: 0168-9002. DOI: 10.1016/0168-9002(93)90756-8 (cit. on p. 30).

Firestone, R. B. and V. S. Shirley (1996). Table of Isotopes. Ed. by R. B. Firestone and V. S. SHIRLEY. 8th ed. Wiley-Interscience. ISBN: 978-0471356332 (cit. on pp. 151, 161164, 224).

Fishburn, M. and E. Charbon (2010). "System Tradeoffs in Gamma-Ray Detection Utilizing SPAD Arrays and Scintillators”. In: IEEE Transactions on Nuclear Science 57.5, pp. 25492557. ISSN: 0018-9499. DOI: 10.1109/TNS.2010.2064788 (cit. on p. 101).

Frauenfelder, H. and R. M. Steffen (1965). "Angular distribution of nuclear radiation”. In: Alpha-, Beta-, and Gamma-Ray Spectroscopy. Ed. by K. Siegbahn. Vol. 2. NorthHolland Publishing Company. Chap. XIX(A), pp. 997-1198 (cit. on pp. 7-14, 129, 131, 132).

Frigo, M. and S. G. Johnson (2005). "The Design and Implementation of FFTW3". In: Proceedings of the IEEE 93.2. Special issue on "Program Generation, Optimization, and Platform Adaptation”, pp. 216-231. DOI: 10.1109/JPROC.2004.840301 (cit. on pp. 63, 210).

Furman, M. A. and M. T. F. Pivi (2002). "Probabilistic model for the simulation of secondary electron emission”. In: Phys. Rev. ST Accel. Beams 5 (12), p. 124404. DoI: 10.1103/ PhysRevSTAB.5.124404 (cit. on p. 120).

Gailly, J.-L. and M. Adler (2013). zlib. Website. UrL: http://www.zlib.net/ (cit. on p. 54). 
Galassi, M., J. Davies, J. Theiler, B. Gough, G. Jungman, P. Alken, M. Booth, and F. Rossi (2009). GNU Scientific Library Reference Manual. Ed. by B. GougH. 3rd ed. Network Theory Ltd. URL: http://www.gnu.org/software/gsl/ (cit. on pp. 72, 75, 147, 210).

Ganachaud, J. and M. Cailler (1979). "A Monte-Carlo calculation of the secondary electron emission of normal metals: I. The model”. In: Surface Science 83.2, pp. 498-518. ISSN: 0039-6028. DoI: 10.1016/0039-6028(79)90059-1 (cit. on p. 120).

GANIL (2014). SPIRAL2. Website. URL: http://pro.ganil-spiral2.eu/spiral2 (cit. on p. 214).

Gatti, E. and V. Svelto (1959). "Theory of time resolution in scintillation counters". In: Nuclear Instruments and Methods 4.4, pp. 189-201. ISSN: 0029-554X. DoI: 10.1016/0029554X(59)90001-1 (cit. on p. 74).

Gedcke, D. and W. McDonald (1967). "A constant fraction of pulse height trigger for optimum time resolution”. In: Nuclear Instruments and Methods 55, pp. 377-380. ISSN: 0029-554X. DOI: 10.1016/0029-554X(67)90145-0 (cit. on pp. 20, 73).

Geruschke, T., K. Lorenz, and R. Vianden (2009). "Alloy and lattice disorder in Hf implanted". In: Physica B: Condensed Matter 404.23-24. Proceedings of the 25th International Conference on Defects in Semiconductors, pp. 4882-4885. ISSN: 0921-4526. DoI: 10.1016/j.physb.2009.08.253 (cit. on pp. 138, 140).

Glodo, J., W. Moses, W. Higgins, E. V. D. Van Loef, P. Wong, S. Derenzo, M. Weber, and K. SHAH (2005). "Effects of Ce concentration on scintillation properties of $\mathrm{LaBr}_{3}: \mathrm{Ce}$ ". In: IEEE Transactions on Nuclear Science 52.5, pp. 1805-1808. ISSN: 0018-9499. DOI: 10. 1109/TNS.2005.856906 (cit. on pp. 125, 127).

HaLevy, I. et al. (2007). "XRD, TDPAC and LAPW study of $\mathrm{Hf}_{10} \mathrm{~B}_{2}$ under high pressure". In: Hyperfine Interactions 177.1-3, pp. 57-64. ISSN: 0304-3843. DoI: 10.1007/s10751-0089707-8 (cit. on p. 2).

Hamamatsu (2007). Photomultiplier Tubes Basics and Applications. 3rd ed. Hamamatsu. uRL: http://www. hamamatsu.com/resources/pdf/etd/PMT_handbook_v3aE.pdf (cit. on pp. 25, 48, 85, $86,102)$.

- (2013). Photomultiplier tubes R2083, R3377. Data sheet. URL: http://www. hamamatsu . com/ resources/pdf/etd/R2083_R3377_TPMH1227E04.pdf (cit. on p. 128).

Hamilton, W. D. (1975). "Gamma-ray angular distribution and correlation measurements (I) experimental methods using radioactive sources". In: The electromagnetic interaction in nuclear spectroscopy. Ed. by W. D. Hamilton. Amsterdam: North-Holland Publishing Company. Chap. 14, pp. 645-700. IsBN: 0-7204-0275-1 (cit. on pp. 7, 10, 199).

Heer, E. and T. B. Novey (1959). "The Interdependence of Solid State Physics and Angular Distribution of Nuclear Radiations". In: Solid State Physics. Ed. by F. SeItz and D. Turnbull. Vol. 9. Academic Press, pp. 199-255. DoI: 10.1016/S0081-1947(08)60566- 4 (cit. on pp. 2, 7).

HeInRICH, F. (2001). "Untersuchung der Korrosion von HILUMIN-Batterieelektroden mittels zeitdifferentieller $\gamma-\gamma$-Winkelkorrelationsspektroskopie”. Diplomarbeit. Leipzig, Germany: Universität Leipzig (cit. on pp. 135, 213). 
Heinrich, F. (2005). "Der elektrische Feldgradient in Makromolekülen - Experiment und Rechnung”. Dissertation. Leipzig, Germany: Universität Leipzig (cit. on pp. 213, 214).

Hemmingsen, L. and T. Butz (2006). "Perturbed Angular Correlations of $\gamma$-rays (PAC) Spectroscopy”. In: Encyclopedia of Inorganic Chemistry. John Wiley \& Sons, Ltd. ISBN: 9780470862100. DOI: 10.1002/0470862106.ia322 (cit. on pp. 3, 7).

Hemmingsen, L., K. N. Sas, and E. Danielsen (2004). "Biological Applications of Perturbed Angular Correlations of $\gamma$-Ray Spectroscopy". In: Chemical Reviews 104.9. PMID: 15352785, pp. 4027-4062. DOI: 10.1021/cr030030v (cit. on p. 3).

Hemmingsen, L., M. Stachura, P. Thulstrup, N. Christensen, and K. Johnston (2010). "Selected applications of perturbed angular correlation of $\gamma$-rays (PAC) spectroscopy in biochemistry". In: Hyperfine Interactions 197.1-3, pp. 255-267. ISSN: 0304-3843. DOI: 10.1007/s10751-010-0248-6 (cit. on p. 3).

Herden, C. H., M. A. Alves, K. D. Becker, and J. A. Gardner (2004). "A new generation TDPAC spectrometer". In: Hyperfine Interactions 159.1-4, pp. 379-383. DoI: 10.1007/ s10751-9128-x (cit. on pp. 33, 43, 66, 209).

Herden, C. H., J. Röder, J. A. Gardner, and K. D. Becker (2008). "Fully digital time differential perturbed angular correlation (TDPAC) spectrometer". In: Nuclear Instruments and Methods In Physics Research Section A 594.2, pp. 155-161. DOI: D0I : 10.1016/j . nima. 2008.05.001 (cit. on pp. 39, 43).

Herlert, A. (2010). “The ISOLDE Facility”. In: Nuclear Physics News 20.4, pp. 5-12. DoI: 10.1080/10619127.2010.529735 (cit. on p. 26).

HTW (2014). Glassy carbon. Website. Hochtemperatur-Werkstoffe GmbH. uRL: http://www. htw-germany. com/technology. php5? lang=en\&nav0=2 (cit. on p. 191).

Hyndman, R. J. and Y. Fan (1996). "Sample Quantiles in Statistical Packages". In: The American Statistician 50.4, pp. 361-365. ISSN: 00031305. URL: http : / / www . jstor . org / stable/2684934 (cit. on p. 101).

IAEA Nuclear Data Section (2013). Live Chart of Nuclides. Website. url: http : / / www nds.iaea.org/relnsd/vcharthtml/VChartHTML.html (cit. on p. 145).

Iams, H. and B. Salzberg (1935). "The Secondary Emission Phototube". In: Proceedings of the Institute of Radio Engineers 23.1, pp. 55-64. ISSN: 0731-5996. DOI: 10.1109/JRPROC. 1935. 227243 (cit. on pp. 24, 93).

INSTOCK wIRELESS (2007). PD1160 data sheet. INSTOCK wireless components. URL: http: //instockwireless.com/power_divider_pdf/power_divider_pd1160_35.pdf (cit. on p. 47).

JäGER, M., K. IwIG, and T. Butz (2010). “A user-friendly fully digital TDPAC-spectrometer”. In: Hyperfine Interactions 198.1-3, pp. 167-172. ISSN: 0304-3843. DoI: 10.1007/s10751-0100201-8 (cit. on pp. 66, 209).

JäGER, M., K. IwIG, and T. Butz (2011). “A compact digital time differential perturbed angular correlation-spectrometer using field programmable gate arrays and various timestamp algorithms”. In: Review of Scientific Instruments 82.6, 065105, p. 065105. DOI: 10. 1063/1.3599417 (cit. on pp. 49, 66, 74, 75, 210). 
JaneceK, M. and W. W. Moses (2010). "Simulating Scintillator Light Collection Using Measured Optical Reflectance”. In: IEEE Transactions on Nuclear Science 57.3, pp. 964970. ISSN: 0018-9499. DOI: 10.1109/TNS.2010.2042731 (cit. on p. 99).

Jürgens, D., M. Uhrmacher, H.-G. Gehrke, M. Nagl, U. Vetter, C. Brüsewitz, H. Hofsäss, J. Mestnik-Filho, and M. W. Barsoum (2011). "Electric field gradients at ${ }^{111} \mathrm{In} /$ ${ }^{111} \mathrm{Cd}$ probe atoms on A-sites in 211-MAX phases". In: Journal of Physics: Condensed Matter 23.50, p. 505501. DoI: 10.1088/0953-8984/23/50/505501 (cit. on pp. 214, 219).

Jürgens, D. (2013). "Untersuchung der elektrischen Hyperfeinwechselwirkung in $\mathrm{M}_{(n+1)}$ $\operatorname{AX}_{(n)}$-Phasen mittels der gestörten $\gamma$ - $\gamma$-Winkelkorrelation”. Dissertation. Göttingen, Germany: Georg-August-Universität (cit. on pp. 2, 142, 210, 214).

Karlsson, E. B. (1995). Solid State Phenomena As Seen by Muons, Protons, and Excited Nuclei. Oxford: Oxford University Press. IsBn: 978-0-19-853778-6 (cit. on pp. 3, 7, 13, 14).

Knoll, G. F. (1999). Radiation Detection and Measurement. 3rd ed. New York: John Wiley \& Sons. ISBN: 9780470131480 (cit. on p. 71).

Köster, U., H. Faust, T. Materna, and L. Mathieu (2010). "Experience with in-pile fission targets at LOHENGRIN". In: Nuclear Instruments and Methods in Physics Research Section A 613.3. Target and Stripper Foil Technologies for High Intensity Beams_-Proceedings of the 24th World Conference of the International Nuclear Target Development Society, pp. 363-370. ISSN: 0168-9002. DOI: 10.1016/j.nima.2009.09.078 (cit. on pp. 28, 30).

Krane, K. S. and R. M. Steffen (1970). "Determination of the $\frac{E 2}{M 1}$ Multipole Mixing Ratios of the Gamma Transitions in $\mathrm{Cd}^{110}$ ". In: Phys. Rev. C 2 (2), pp. 724-734. DoI: 10.1103/PhysRevC.2.724 (cit. on p. 9).

Krause-Rehberg, R. and H. S. Leipner (1999). Positron Annihilation in Semiconductors. Vol. 127. Springer Series in Solid-State Sciences. Berlin: Springer-Verlag. ISBN: 978-3540-64371-5 (cit. on p. 1).

Krille, A., M. Nagl, U. Vetter, H. Hofsäss, and R. Krause-Rehberg (2011). "First results of ab initio simulations of scintillation detector characteristics". In: Journal of Physics: Conference Series 262.1, p. 012033. URL: http://stacks.iop.org/1742-6596/262/i=1/ $\mathrm{a}=012033$ (cit. on pp. 93-95).

Krille, A. (2011). EPOS. Website. URL: https://sun.hzdr.de/trac/epos (cit. on p. 113).

Kugler, E., D. Fiander, B. Johnson, H. Haas, A. Przewloka, H. Ravn, D. Simon, and K. Zimmer (1992). "The new CERN-ISOLDE on-line mass-separator facility at the PSBooster". In: Nuclear Instruments and Methods in Physics Research Section B 70.1-4, pp. 4149. ISSN: 0168-583X. DOI: 10.1016/0168-583X(92)95907-9 (cit. on p. 26).

Kugler, E. (2000). “The ISOLDE facility”. In: Hyperfine Interactions 129.1-4, pp. 23-42. ISSN: 0304-3843. DOI: 10.1023/A:1012603025802 (cit. on p. 26).

Kuriplach, J., I. Novotný, and I. Procházka (1994). "Comments on time-differential perturbed angular correlation functions for static axial electric quadrupole interaction". In: Hyperfine Interactions 88.1, pp. 13-18. ISSN: 0304-3843. DOI: 10.1007/BF02068697 (cit. on p. 13). 
Laboratori Nazionale di Fisica Nucleare (2014). SPES Project. Website. url: https : //web.infn.it/spes/ (cit. on p. 214).

Lawson, J. S. and H. Frauenfelder (1953). "The Correction for Finite Angular Resolution in Directional Correlation Measurements". In: Phys. Rev. 91 (3), pp. 649-652. Dor: 10. 1103/PhysRev.91.649 (cit. on pp. 11, 132).

Leo, W. R. (1994). Techniques for Nuclear and Particle Physics Experiments. 2nd ed. Berlin: Springer -Verlag. ISBN: 3-540-57280-5 (cit. on pp. 19, 20, 22, 23).

Levin, A. and C. Moisan (1996). "A more physical approach to model the surface treatment of scintillation counters and its implementation into DETECT". In: Nuclear Science Symposium, 1996. Conference Record., 1996 IEEE. Vol. 2, pp. 702-706. DoI: 10.1109/ NSSMIC. 1996.591410 (cit. on p. 99).

Lindgren, B. and Y. K. ViJaY (1981). "Pressure and Temperature-dependence of the Cdhyperfine Field In Fe, Co, Ni and Gd”. In: Hyperfine Interactions 9.1-4, pp. 379-384. DoI: 10.1007/BF01020948 (cit. on pp. 135, 138).

Loef, E. V. D. van, P. Dorenbos, C. W. E. van EijK, K. Krämer, and H. U. Güdel (2001). "High-energy-resolution scintillator: $\mathrm{Ce}^{3+}$ activated $\mathrm{LaBr}_{3}$ ". In: Applied Physics Letters 79.10, pp. 1573-1575. DoI: 10.1063/1.1385342 (cit. on p. 97).

Lopes, A. M. L., J. P. Araújo, V. S. Amaral, J. G. Correia, Y. Tomioka, and Y. Tokura (2008). "New Phase Transition in the $\operatorname{Pr}_{1-x} \mathrm{Ca}_{x} \mathrm{MnO}_{3}$ System: Evidence for Electrical Polarization in Charge Ordered Manganites". In: Phys. Rev. Lett. 100 (15), p. 155702. DOI: 10.1103/PhysRevLett.100.155702 (cit. on p. 2).

Lourakis, M. (Jul. 2004). levmar: Levenberg-Marquardt nonlinear least squares algorithms in C/C++. Website. uRL: http://www.ics.forth.gr/ lourakis/levmar/ (cit. on p. 78).

MA, D. and R. ZHU (1993). "On optical bleaching of barium fluoride crystals". In: Nuclear Instruments and Methods in Physics Research Section A 332.1-2, pp. 113-120. IssN: 01689002. DoI: 10.1016/0168-9002(93)90747-6 (cit. on p. 98).

MacKenzie, I. K., T. L. Khoo, A. B. McDonald, and B. T. A. McKee (1967). "Temperature Dependence of Positron Mean Lives in Metals”. In: Phys. Rev. Lett. 19 (17), pp. 946-948. DOI: 10.1103/PhysRevLett.19.946 (cit. on p. 1).

Mannhart, W. (1975). "Messung von thermischen Aktivierungs-Wirkungsquerschnitten mit hoher Genauigkeit". In: Zeitschrift für Physik A Atoms and Nuclei 272.3, pp. 273-277. ISSN: 0939-7922. DOI: 10.1007/BF01438020 (cit. on p. 221).

Mark, J. E. (2009). Polymer Data Handbook. 2nd ed. Oxford University Press. ISBN: 978-019-518101-2. URL: http://www.knovel.com/web/portal/browse/display?_EXT_KNOVEL_DISPLAY_ bookid=26208VerticalID=0 (cit. on p. 97).

Mertzimekis, T. J. (2013). Magnetic and Electric Moments. Website. URL: http://magneticmoments. info/data/index.html (cit. on p. 151).

Microlab (2013). Power Splitters, Dx-69FF series. Data sheet. Microlab. Parsippany, USA. URL: http://fxr.com/ /media/Microlab/Datasheets/Dx69FF'/200113\%/20RA.ashx (cit. on p. 45).

Mini-Circuits (2012). ZX76-31R5-SP+Digital Step Attenuator. Data sheet. Mini-Circuits. New York, USA. URL: http://www.minicircuits.com/pdfs/ZX76-31R5-SP+.pdf (cit. on p. 49). 
MülleR, K. (2009). "Hyperfeinwechselwirkungen von ${ }^{100} \mathrm{Pd}$ in $\mathrm{Ru}, \mathrm{Ru}_{2}$ und $\mathrm{RuGa}_{3}$ ". Diplomarbeit. Rheinische Friedrich-Wilhelms-Universität Bonn (cit. on p. 199).

Nagl, M. A., M. B. Barbosa, U. Vetter, J. G. Correia, and H. C. Hofsäss (2013). “A new tool for the search of nuclides with properties suitable for nuclear solid state physics based on the Evaluated Nuclear Structure Data Files". In: Nuclear Instruments and Methods in Physics Research Section A 726, pp. 17-30. ISSN: 0168-9002. DOI: 10.1016/j . nima . 2013.05.045 (cit. on pp. 9, 145).

Nagl, M. A. (2008-2014).PAC-Suite. Project Homepage. URL: http://pac-suite.sourceforge. net (cit. on pp. 50, 249).

- (2009-2014). Scintillate-Suite. Project Homepage. URL: http://scintillate. sourceforge. net (cit. on pp. 95, 249).

- (2012-2014). Nuclei. Project Homepage. URL: http : / / nuclei . sourceforge . net/ (cit. on pp. 145, 249).

Nagl, M., U. Vetter, M. Uhrmacher, and H. Hofsäss (2010). "A new all-digital time differential $\gamma-\gamma$ angular correlation spectrometer”. In: Review of Scientific Instruments 81.7, 073501, p. 073501. DoI: 10.1063/1.3455186 (cit. on pp. 43, 80, 82, 86, 135, 210).

NÉDÉLEC, R. (2014). Nightmare (MDI). Website. URL: http : / / pacweb . hiskp . uni - bonn . de / mediawiki/index.php?title=Nightmare_(MDI) (cit. on pp. 16, 138, 213).

Nielsen, K. B., B. Toft, K. C. Mishra, S. K. Mishra, K. J. Duff, and T. P. Das (1983). "Nuclear quadrupole interaction of excited nuclear state $\left({ }^{19} \mathrm{~F}\right.$ " $\left.\mathrm{I}=5 / 2\right)$ of fluorine in fluorobenzenes". In: Journal of the American Chemical Society 105.7, pp. 1734-1737. Dor: 10.1021/ja00345a005 (cit. on p. 8).

Nordion (2011). Indium-111 Radiochemical Indium Chloride Solution. Data sheet. Nordion Inc. 447 March Road, Ottawa, ON, Canada. URL: http : / / www . nordion . com / documents / products/In-111_Can.pdf (cit. on p. 219).

Ohкubo, Y., Y. Кobayashi, K. Asai, T. Окada, and F. Ambe (1993). "TDPAC and emission Mössbauer studies of $\mathrm{Fe}_{3} \mathrm{O}_{4}\left({ }^{99} \mathrm{Ru}\right)$ ". In: Hyperfine Interactions 78.1-4, pp. 535-539. IsSN: 0304-3843. DOI: 10.1007/BF00568188 (cit. on p. 199).

Ohtomo, T., S. Nasu, K. Baba, S. Morimoto, M. Miyasaka, Y. Ōnuki, Y. Kawase, and T. SaIto (2002). " ${ }^{140} \mathrm{Ce}\left(\longleftarrow{ }^{140} \mathrm{La}\right)$ Time-Differential Perturbed Angular Correlation Study of $\mathrm{CeRu}_{2} \mathrm{Ge}_{2}$." In: Materials Transactions 43.7, pp. 1480-1485. URL: http://www.jim.or.jp/ journal/e/43/07/1480.html (cit. on p. 15).

Pasquali, G., R. Ciaranfi, L. Bardelli, M. Bini, A. Boiano, F. Giannelli, A. Ordine, and G. Poggi (2007). "A DSP equipped digitizer for online analysis of nuclear detector signals”. In: Nuclear Instruments and Methods in Physics Research Section A 570.1, pp. 126132. ISSN: 0168-9002. DOI: 10.1016/j.nima.2006.10.008 (cit. on p. 216).

Pethrick, R. A. (1997). "Positron annihilation-A probe for nanoscale voids and free volume?” In: Progress in Polymer Science 22.1, pp. 1-47. ISSN: 0079-6700. DoI: 10.1016/ S0079-6700(96)00023-8 (cit. on p. 1).

Phelps, M. E., E. J. Hoffman, N. A. Mullani, and M. M. Ter-Pogossian (1975). “Application of Annihilation Coincidence Detection to Transaxial Reconstruction Tomogra- 
phy”. In: Journal of Nuclear Medicine 16.3, pp. 210-224. URL: http://jnm. snmjournals.org/ content/16/3/210 (cit. on p. 2).

Philips (2014). Philips Digital Photon Counting. Website. URL: http : / / www . philips . com / digitalphotoncounting (cit. on p. 215).

Рнотоnis (2007a). Photomultiplier Tubes Catalogue (cit. on pp. 114, 120, 126).

- (2007b). Photomultiplier XP2020 Datasheet (cit. on pp. 126, 200).

- (2009). Voltage Divider VD124K/T (cit. on pp. 43, 117, 126).

QSA Global (2014). Cobalt-60. Website. URL: http://www.qsa-global.com/sources/industrialisotopes/cobalt-60.aspx (cit. on p. 224).

R Core Team (2012). R: A Language and Environment for Statistical Computing. ISBN 3900051-07-0. R Foundation for Statistical Computing. Vienna, Austria. URL: http://www. R-project.org (cit. on pp. 95, 104).

Raghavan, R. and P. Raghavan (1971). "A new method for differential perturbed angular correlation measurements”. In: Nuclear Instruments and Methods 92.3, pp. 435-437. ISSN: 0029-554X. DoI: 10.1016/0029-554X(71)90421-6 (cit. on p. 199).

RAIMS LTD. (2014). CESIUM 137 (CS-137) SOURCES. Website. URL: http: //www . raims . co. uk/products/sealed-radioactive-sources/cs_137/ (cit. on p. 224).

Rinneberg, H., G. Schwartz, and D. Shirley (1977). "Pac studies of spin deviations, pressure induced hyperfine field shifts, and temperature variation of supertransferred hyperfine fields". In: Hyperfine Interactions 3.1, pp. 97-107. ISSN: 0304-3843. DoI: 10.1007/ BF01021542 (cit. on p. 34).

Rodnyi, P. A. (1997). Physical Processes in Inorganic Scintillators. 1st ed. Vol. 14. Laser \& Optical Science \& Technology. CRC Press. ISBN: 9780849337888 (cit. on p. 23).

Saint-Gobain (2004a). BrilLanCe 380 Scintillation Material. Data sheet. Saint-Gobain Ceramics \& Plastics, Inc. URL: http : / / www . detectors . saint - gobain . com / uploadedFiles / SGdetectors / Documents / Product_Data_Sheets / BrilLanCe380 - data - sheet . pdf (cit. on pp. 41, 97, 98).

- (2004b). PreLude 420 Scintillation Material. Data sheet. Saint-Gobain Ceramics \& Plastics, Inc. URL: http://www.detectors.saint-gobain.com/uploadedFiles/SGdetectors/Documents/ Product_Data_Sheets/PreLude420-Data-Sheet.pdf (cit. on pp. 97, 98).

- (2005). NaI(Tl) and Polyscin NaI(Tl) Sodium Iodide Scintillation Material. Data sheet. Saint-Gobain Ceramics \& Plastics, Inc. URL: http : / / www . detectors . saint - gobain . com / uploadedFiles / SGdetectors / Documents / Product_Data_Sheets/NaI(Tl) - Data - Sheet. pdf (cit. on pp. 97, 98).

- (2012). BaF2 Barium Fluoride Scintillation Material. Data sheet. Saint-Gobain Ceramics \& Plastics, Inc. URL: http : / /www . crystals . saint - gobain . com / uploadedFiles / SG - Crystals / Documents/Barium'/20Fluoride'/20Data'/20Sheet. pdf (cit. on p. 98).

SaIto, H. and T. Hrodo (2003). "Improvement in the gamma-ray timing measurements using a fast digital oscilloscope". In: Radiation Physics and Chemistry 68.3-4. Proceedings of the 7th International Conference on Positron and Positronium Chemistry, pp. 431434. ISSN: 0969-806X. DOI: 10.1016/S0969-806X(03)00199-3 (cit. on pp. 34, 216). 
Sasanuma, T., A. Taniguchi, M. Tanigaki, Y. Ohkubo, and Y. Kawase (2004). "Measurement of the Magnetic Moment of the First Excited State in ${ }^{93} \mathrm{Sr}$ Using on-line TDPAC technique”. In: Hyperfine Interactions 159.1-4, pp. 251-255. ISSN: 0304-3843. DoI: 10.1007/s10751-005-9114-3 (cit. on p. 199).

SATo, W. et al. (2008). "Online time-differential perturbed angular correlation study with an ${ }^{19} \mathrm{O}$ beam - Residence sites of oxygen atoms in highly oriented pyrolytic graphite". In: Nuclear Instruments and Methods in Physics Research Section B 266.2, pp. 316-322. ISSN: 0168-583X. DOI: 10.1016/j.nimb.2007.11.001 (cit. on p. 34).

Schmitz, J., J. Niederhausen, J. Penner, K. Lorenz, E. Alves, and R. Vianden (2009). "Stable In-defect complexes in GaN and AlN". In: Physica B: Condensed Matter 404.2324. Proceedings of the 25th International Conference on Defects in Semiconductors, pp. 4866-4869. ISSN: 0921-4526. DoI: 10.1016/j.physb.2009.08.181 (cit. on pp. 142, 143).

Sснотт AG (2009). LITHOSIL Synthetic Fused Silica. Data sheet. Hattenbergstrasse 10, 55122 Mainz, Germany. URL: http://www . itos . de/dateien/fusedsilicia/lithotec_fused_ silica_jan2009.pdf (cit. on p. 97).

Schwarz, K. and P. Blaha (2003). "Solid state calculations using WIEN2k”. In: Computational Materials Science 28.2. Proceedings of the Symposium on Software Development for Process and Materials Design, pp. 259-273. ISSN: 0927-0256. DOI: 10 . 1016 / S0927 0256(03)00112-5 (cit. on p. 214).

Scientific Computing \& Modelling (2014). ADF. Website. URL: http://www.scm. com/ADF/ (cit. on p. 214).

Seifert, S., H. van Dam, R. Vinke, P. Dendooven, H. Lohner, F. Beekman, and D. SchaArt (2012). "A Comprehensive Model to Predict the Timing Resolution of SiPMBased Scintillation Detectors: Theory and Experimental Validation”. In: IEEE Transactions on Nuclear Science 59.1, pp. 190-204. ISSN: 0018-9499. DOI: 10.1109/TNS.2011.2179314 (cit. on p. 101).

Seifert, S., H. T. van Dam, and D. R. Schaart (2012). "The lower bound on the timing resolution of scintillation detectors". In: Physics in Medicine and Biology 57.7, p. 1797. DOI: 10.1088/0031-9155/57/7/1797 (cit. on pp. 93, 94).

Sergueev, I., U. van Bürck, A. I. Chumakov, T. Asthalter, G. V. Smirnov, H. Franz, R. RüFfer, and W. Petry (2006). "Synchrotron-radiation-based perturbed angular correlations used in the investigation of rotational dynamics in soft matter". In: Phys. Rev. B 73 (2), p. 024203. DOI: 10.1103/PhysRevB.73.024203 (cit. on p. 216).

Silverman, B. W. (1986). Density Estimation for Statistics and Data Analysis. Boca Ratom: Chapman \& Hall. ISBN: 978-0-412-24620-3 (cit. on p. 104).

Sмith, S. (2002). Digital Signal Processing: A Practical Guide for Engineers and Scientists. Burlington, MA01803, USA: Newnes. ISBN: 0-7506-7444-X. URL: http://www.dspguide.com/ (cit. on p. 78).

Sommer, A. H. (1972). "Bialkali ( $\left.\mathrm{K}_{2} \mathrm{CsSb}\right)$ Photocathode as a High-Gain Secondary Electron Emitter". In: Journal of Applied Physics 43.5, pp. 2479-2480. Dor: 10.1063/1.1661537 (cit. on p. 117). 
Sonzogni, A. (2013). NuDat 2.6. Website. NNDC, Brookhaven National Laboratory. URL: http://www.nndc.bnl.gov/nudat2/ (cit. on p. 145).

Stone, N. J. (2012). Table of Nuclear Magnetic Dipole and Electric Quadrupole Moments. International Atomic Energy Agency, Vienna, Austria. URL: http: / /ww - nds . iaea .org/ publications/indc/indc-nds-0594/ (cit. on pp. 151, 153, 158, 160, 167).

Sun, J., H. Liang, S. Xie, and B. Cheng (2012). "A fast digital timing and analysis system based on real-time dCFD technique for nuclear physics experiments". In: Science China Technological Sciences 55.9, pp. 2651-2655. ISSN: 1674-7321. DOI: 10.1007/s11431-012-49306 (cit. on p. 216).

Trefilova, L., A. Kudin, L. Kovaleva, B. Zaslavsky, D. Zosim, and S. Bondarenko (2002). "Concentration dependence of the light yield and energy resolution of NaI:Tl and CsI:Tl crystals excited by gamma, soft X-rays and alpha particles”. In: Nuclear Instruments and Methods in Physics Research Section A 486.1-2. Proceedings of the 6th International Conference on Inorganic Scintillators and their Use in Scientific and Industrial Applications, pp. 474-481. ISSN: 0168-9002. DOI: 10.1016/S0168-9002(02)00756-8 (cit. on p. 97).

TRIUMF (2014a). ARIEL. Website. URL: http://www.triumf.ca/ariel (cit. on p. 214).

- (2014b). ISAC Facilities for Rare-Isotope Beams. Website. URL: http : / / www . triumf . ca / research-program/research-facilities/isac-facilities (cit. on p. 214).

Tuli, J. K. (2001). Evaluated Nuclear Structure Data File. Manual. National Nuclear Data Center, Brookhaven National Laboratory. P.O. Box 5000, Upton, New York 11973-5000. URL: http://www.nndc.bnl.gov/nndcscr/documents/ensdf/ensdf-manual.pdf (cit. on p. 149).

Unterricker, S., T. Butz, and S. Saibene (1992). "Annealing of lattice defects in chalcopyrite semiconductors-TDPAC investigations". In: Nuclear Instruments and Methods in Physics Research Section B 63.1-2. Proceedings of the E-MRS 1991 Spring Meeting, Symposium F on Nuclear Methods in Semiconductor Physics Strasbourg, France, May 28-30, 1991, pp. 236-239. ISSN: 0168-583X. DoI: 10.1016/0168-583X(92) 95203-4 (cit. on p. 2).

ViCKers, D. S. (1997). "Gamma Camera with on the fly calibration for PMT drift”. Patent US 5677536 (cit. on pp. 86, 91, 216).

VILARDI, I. et al. (2006). "Optimization of the effective light attenuation length of YAP:Ce and LYSO:Ce crystals for a novel geometrical PET concept". In: Nuclear Instruments and Methods in Physics Research Section A 564.1, pp. 506-514. ISSN: 0168-9002. DoI: 10.1016/ j.nima.2006.04.079 (cit. on p. 98).

Wand, M. P. and M. C. Jones (1995). Kernel Smoothing. Monographs on Statistics and Applied Probability 60. Boca Ratom: Chapman \& Hall/CRC. ISBN: 0-412-55270-1 (cit. on pp. 87, 102, 104).

Webb, T., L. Nikkinen, J. Gallego, and D. Ryan (2013). "A simple digital TDPAC spectrometer". In: Hyperfine Interactions 222.1-3, pp. 103-108. ISSN: 0304-3843. DoI: 10.1007/ s10751-012-0663-y (cit. on pp. 66, 210). 
WeBb, T. A. (2012). "Digital TDPAC spectroscopy with application to the magnetic dynamics in iron-rich amorphous Fe-Hf compounds". Master Thesis. Montreal (Quebec), Canada: McGill University (cit. on p. 66).

Weber, M., S. Derenzo, and W. Moses (2000). "Measurements of ultrafast scintillation rise times: evidence of energy transfer mechanisms". In: Journal of Luminescence 87-89, pp. 830-832. ISSN: 0022-2313. DoI: 10.1016/S0022-2313(99)00430-5 (cit. on p. 98).

WeGNER, D. (1985). "Calculated perturbed angular correlations for ${ }^{111}$ In doped cubic single crystals”. In: Hyperfine Interactions 23.2, pp. 179-210. ISSN: 0304-3843. DoI: 10.1007/ BF02058076 (cit. on pp. 11, 13, 14, 219).

WheEler, D. A. (2004). SLOCCount. Website. URL: http://www.dwheeler.com/sloccount/ (cit. on p. 249).

Wichert, T. and E. Recknagel (1986). "Perturbed Angular Correlation”. In: Microscopic Methods in Metals. Ed. by U. Gonser. Topics in Current Physics 40. Berlin: SpringerVerlag. Chap. 11, pp. 317-364 (cit. on p. 152).

Xie, Q., C.-M. Kao, X. Wang, N. Guo, C. Zhu, H. Frisch, W. Moses, and C.-T. Chen (2009). "Potentials of Digitally Sampling Scintillation Pulses in Timing Determination in PET". In: IEEE Transactions on Nuclear Science 56.5, pp. 2607-2613. ISSN: 0018-9499. DOI: 10.1109/TNS.2009.2023656 (cit. on p. 216).

Zacate, M. O. (2014). PolyPacFit. Website. uRl: http://polypacfit.nku.edu (cit. on p. 213).

Zacate, M. O. and H. Jaeger, eds. (2011). Perturbed Angular Correlation Spectroscopy-A Tool for the Study of Defects and Diffusion at the Atomic Scale. Defect and Diffusion Forum 311. pp. 3-38. DOI: 10.4028/www.scientific.net/DDF.311.3 (cit. on p. 3).

Zacate, M., W. Evenson, R. Newhouse, and G. Collins (2010). "Fitting PAC spectra with stochastic models: PolyPacFit”. In: Hyperfine Interactions 197.1-3, pp. 223-227. ISSN: 0304-3843. DoI: 10.1007/s10751-010-0241-0 (cit. on p. 213). 



\section{List of figures}

1.1 Steps of a TDPAC measurement $\ldots \ldots \ldots \ldots \ldots \ldots$

2.1 Exemplary measurement results . . . . . . . . . . . . . . 16

2.2 Schematic of an analog slow-fast TDPAC setup . . . . . . . . . . . . 18

2.3 Working principle of Constant fraction discrimination . . . . . . . . 21

2.4 Schematic drawing of a scintillation detector . . . . . . . . . . . 22

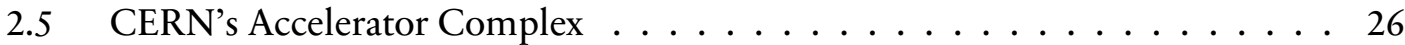

2.6 ISOLDE targets and beamlines . . . . . . . . . . . . . . . 27

2.7 The ISOLDE hall . . . . . . . . . . . . . . . . . . . . . . . . . . 29

2.8 LOHENGRIN beamline . . . . . . . . . . . . . . . . . . . . . . . . 29

4.1 Scheme of the digital TDPAC spectrometer . . . . . . . . . . . . . . . 40

4.2 Photo of the TDPAC spectrometer . . . . . . . . . . . . . . 41

4.3 Schematic of the $2 \mathrm{GHz}$ oscillator and amplifier . . . . . . . . . . . 45

4.4 Schematic of the synchronization and DAC interface . . . . . . . . . . . 47

4.5 Schematic of the six channel attenuator . . . . . . . . . . . . . 48

4.6 Task allocation among the Pac-Suite applications . . . . . . . . . . . . 51

4.7 Data processing on the signal processing computers by PacMan . . . . . . 52

4.8 Data collection and processing on the server by PacMaster . . . . . . . . . 53

4.9 State-chart of the state machine implemented in PacMaster . . . . . . . . . . 55

4.10 Rejection of ambiguous coincidences . . . . . . . . . . . . . . . . . 57

4.11 Coincidence fit parameter estimation . . . . . . . . . . . . . . . 60

4.12 Screenshot of the dialog for manual fit parameter manipulation . . . . . . . 61

4.13 Data processing by SpectraPAC . . . . . . . . . . . . . . . . 64

4.14 The TDPAC spectrometer's time and energy resolution . . . . . . . . . 65

$5.1 \quad$ Five exemplary anode signals . . . . . . . . . . . . . . . 68

5.2 Charge spectra of a ${ }^{22} \mathrm{Na}$ sample as measured with two different detectors . 69

5.3 Charge spectra of a ${ }^{152} \mathrm{Eu}$ sample as measured with two different detectors . $\quad 70$

5.4 Averaged anode signals . . . . . . . . . . . . . . . . 71

5.5 Constant threshold method: Time resolution vs. trigger threshold . . . . . . 72

5.6 CFD method: Time resolution vs. fraction and delay . . . . . . . . . 74

5.7 Pulse shaping method: Time resolution vs. $\sigma \ldots \ldots \ldots \ldots$

5.8 Working principle of the digital constant fraction of integral values method . . 80 
5.9 Cubic polynomial interpolation of the rising edge $\ldots \ldots \ldots \ldots 1$

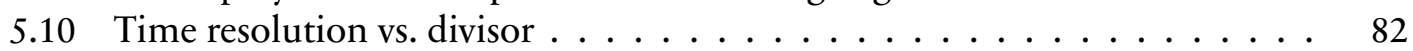

5.11 Comparison of time determination methods $\ldots \ldots \ldots 83$

6.1 Charge spectrum of the ${ }^{98 \mathrm{~m}} \mathrm{Y}$ isomer's decay . . . . . . . . . . . 88

6.2 Kernel density estimates of five spectra distributed across the measurement $\quad 90$

6.3 Energy spectrum of the ${ }^{98 \mathrm{~m}} \mathrm{Y}$ isomer's decay . . . . . . . . . . . . 90

7.1 Task allocation among the Scintillate-Suite applications . . . . . . . . . . . 94

7.2 Detector model as implemented in Scintillate! . . . . . . . . . . . . . . . . 96

7.3 A single $\gamma$ incidence as simulated by Scintillate! . . . . . . . . . . . . . . . 100

7.4 Shape of the triweight kernel with bandwidth $b=0.5 \mathrm{~ns} \ldots \ldots \ldots$

7.5 Kernel density estimate of detected photons . . . . . . . . . . . . . . . 103

7.6 Cluster separation of Compton and photo-peak components . . . . . . . . 104

7.7 Example for an irregular event . . . . . . . . . . . . . . . . . . . 105

7.8 Simulation results for $\mathrm{LaBr}_{3}$ :Ce crystals. . . . . . . . . . . . . . . . . . 106

7.9 Density estimation based results for $\mathrm{LaBr}_{3}:$ Ce crystals. . . . . . . . . . . . . 107

7.10 Charge spectrum of single photon PMT signals . . . . . . . . . . . . . . 114

7.11 Averaged signal shape of single photon PMT signals . . . . . . . . . . . 115

7.12 Overview on the PMT simulation method . . . . . . . . . . . . . 121

7.13 Attenuation of signals passing a VD124K/T voltage divider . . . . . . . . . 122

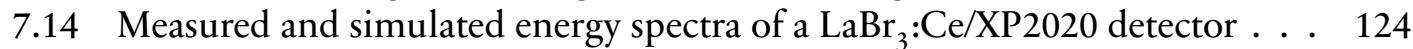

7.15 Measured and simulated signal shapes of a $\mathrm{LaBr}_{3}: \mathrm{Ce} / \mathrm{XP2020}$ detector $\ldots 127$

7.16 Measured and simulated energy spectra of $\mathrm{a} \mathrm{BaF}_{2} / \mathrm{R} 3377$ detector . . . . . . 130

7.17 Measured and simulated signal shapes of $\mathrm{a} \mathrm{BaF}_{2} / \mathrm{R} 3377$ detector . . . . . . 131

7.18 Angular efficiencies for different $\gamma$ energies . . . . . . . . . . . 133

8.1 Coincidence spectra of ${ }^{111}$ In in iron $\ldots \ldots \ldots \ldots \ldots \ldots$

$8.2 \mathrm{R}(\mathrm{t})$ and Fourier spectra of ${ }^{111} \mathrm{In}$ in iron $\ldots \ldots \ldots \ldots . \ldots . \ldots 137$

8.3 Annealing sequence of ${ }^{181} \mathrm{Hf}$ implanted into AlN . . . . . . . . . . . . . . 139

8.4 Evaluation of two cascades from a single measurement . . . . . . . . . . . 141

8.5 Simultaneous evaluation of different probe nuclides in one measurement . 143

9.1 Screenshot of the decay level scheme view of Nuclei. . . . . . . . . . . . 146

9.2 Screenshot of the search dialog of Nuclei. . . . . . . . . . . . . . . . . 147

9.3 Screenshot of the photo peak view of Nuclei. . . . . . . . . . . . . . . . 148

10.1 Circuit diagrams of the vacuum systems . . . . . . . . . . . . 175

10.2 Overview of the new implantation chambers for ISOLDE . . . . . . . 176

10.3 Cross section through the new implantation chambers for ISOLDE . . . . 177

10.4 Photo of the implantation chamber . . . . . . . . . . . . . . . . 179

10.5 Implantation chamber: Sample changing position $\ldots \ldots \ldots \ldots 181$ 
10.6 Implantation chamber: Implanting position . . . . . . . . . . . . 182

10.7 Implantation chamber: Tilted implanting position . . . . . . . . . . . 182

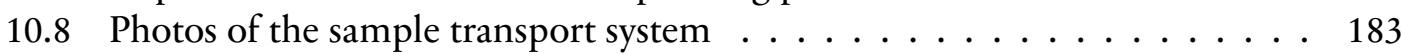

10.9 Sample holder (exploded view). . . . . . . . . . . . . . . . . . 184

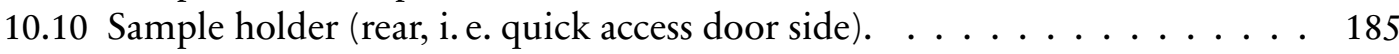

10.11 Cut through the sample holder's upper frame. . . . . . . . . . . . . . . . . . . . . . . . . . .

10.12 Cut through the sample holder's press-on frame. . . . . . . . . . . . . 186

10.13 Deceleration / post-acceleration chamber . . . . . . . . . . . . . . . . . . . . . . . . . 189

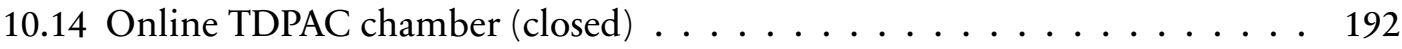

10.15 Online TDPAC chamber $($ open $) \ldots \ldots \ldots 3$

10.16 Online TDPAC chamber: furnace core . . . . . . . . . . . . . . 194

10.17 High temperature vacuum furnace . . . . . . . . . . . . . . . . . 196

10.18 Core of the high temperature vacuum furnace . . . . . . . . . . . . . 197

10.19 Screenshot of $e$ Baker after annealing for 2 min at $1500^{\circ} \mathrm{C}$. . . . . . . . . . 198

10.20 Magnetization setup tested at the LOHENGRIN target . . . . . . . . . . . 200

10.21 Measured magnetic flux above the sample's surface . . . . . . . . . . . . 201

10.22 Simulated magnetic flux above the sample's surface . . . . . . . . . . . . . 202

10.23 Simulated magnetic flux beneath the sample's surface . . . . . . . . . . . 203

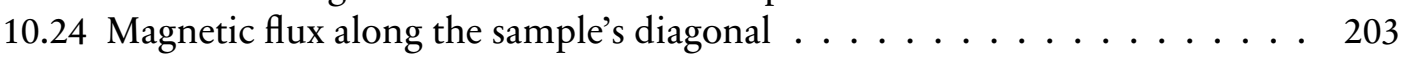

10.25 Simple magnetization setup . . . . . . . . . . . . . . . 204

10.26 Simulated magnetic flux through the sample in the disc-shaped configuration 205

10.27 Magnetic flux along the sample's diagonal in the disc-shaped configuration 205

10.28 Simulated magnetic flux through the setup in the disc-shaped configuration 206

10.29 Full-angle magnetization setup . . . . . . . . . . . . . . 207

10.30 Simulated magnetic flux through the sample in the Helmholtz configuration 207

10.31 Magnetic flux along the sample's diagonal in the Helmholtz configuration . 208

10.32 Simulated magnetic flux through the setup in the Helmholtz configuration 208

A.1 Decay scheme of ${ }^{111} \mathrm{In} \ldots \ldots \ldots 220$

A.2 Decay scheme of ${ }^{181} \mathrm{Hf} \ldots \ldots \ldots \ldots$

A.3 Decay scheme of ${ }^{98} \mathrm{Y} \ldots \ldots \ldots \ldots \ldots \ldots 222$

A.4 Decay scheme of ${ }^{22} \mathrm{Na} \ldots \ldots \ldots \ldots \ldots \ldots$

A.5 Decay scheme of ${ }^{60} \mathrm{Co} \ldots \ldots \ldots \ldots \ldots \ldots$

A.6 Decay scheme of ${ }^{137} \mathrm{Cs} \ldots \ldots \ldots \ldots \ldots \ldots$

A.7 Decay scheme of ${ }^{152} \mathrm{Eu} \longrightarrow{ }^{152} \mathrm{Gd} \ldots \ldots \ldots . \ldots \ldots$

A.8 Decay scheme of ${ }^{152} \mathrm{Eu} \longrightarrow{ }^{152} \mathrm{Sm} \ldots \ldots \ldots . \ldots . \ldots 227$ 



\section{List of tables}

4.1 Comparison of digital TDPAC spectrometers . . . . . . . . . . . . 66

5.1 Results of the deviation of shifted normalized waveform approach $\ldots \ldots \ldots 77$

5.2 Results of the evaluation of timing algorithms $\ldots \ldots \ldots . \ldots . \ldots 3$

7.1 Parts of the detector model and their respective optical properties . . . . . 97

7.2 Composition of materials used in the detector model . . . . . . . . . . 97

7.3 Scintillation material definitions used for Scintillate! . . . . . . . . . . . . 98

7.4 Figures of merit for $\mathrm{LaBr}_{3}$ :Ce scintillators . . . . . . . . . . . . . . . 108

7.5 Parameters of Gaussians approximating a single photo-electron signal . . . . 116

7.6 Scintillate! settings for the $\mathrm{XP2020} / \mathrm{LaBr}_{3}:$ Ce simulation $\ldots \ldots \ldots \ldots$

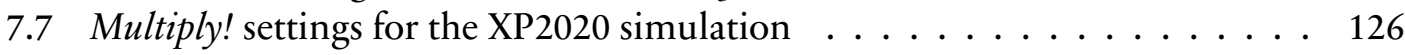

7.8 Scintillate! settings for the $\mathrm{BaF}_{2} / \mathrm{H} 3378-50$ simulation $\ldots \ldots \ldots \ldots$

7.9 Multiply! settings for the H3378-50 simulation . . . . . . . . . . . . . 128

7.10 Solid angle correction factors . . . . . . . . . . . . . . 133

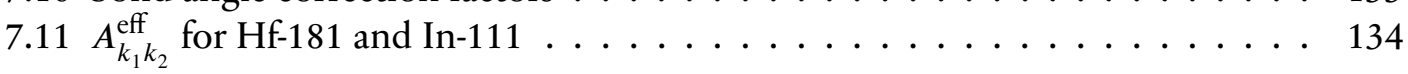

9.1 Off-site candidates . . . . . . . . . . . . . . . . . . . . . 153

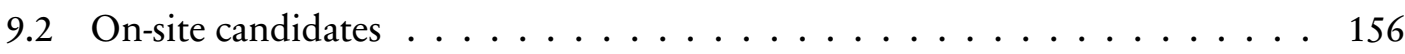

9.3 Online candidates . . . . . . . . . . . . . . . . 163

11.1 Tools for the fitting of TDPAC spectra . . . . . . . . . . . . . . . . 213 



\section{List of publications}

\section{Peer reviewed publications}

Nagl, M., U. Vetter, M. Uhrmacher, and H. Hofsäss (2010). “A new all-digital time differential $\gamma-\gamma$ angular correlation spectrometer”. In: Review of Scientific Instruments 81.7, 073501, p. 073501. DOI: $10.1063 / 1.3455186$.

Jürgens, D., M. Uhrmacher, H.-G. Gehrke, M. Nagl, U. Vetter, C. Brüsewitz, H. Hofsäss, J. MestnikFilho, and M. W. Barsoum (2011). "Electric field gradients at ${ }^{111} \mathrm{In} /{ }^{111} \mathrm{Cd}$ probe atoms on A-sites in 211-MAX phases”. In: Journal of Physics: Condensed Matter 23.50, p. 505501. DoI: 10.1088/09538984/23/50/505501.

Krille, A., M. Nagl, U. Vetter, H. Hofsäss, and R. Krause-Rehberg (2011). "First results of ab initio simulations of scintillation detector characteristics”. In:Journal of Physics: Conference Series 262.1, p. 012033.

Nagl, M. A., M. B. Barbosa, U. Vetter, J. G. Correia, and H. C. Hofsäss (2013). “A new tool for the search of nuclides with properties suitable for nuclear solid state physics based on the Evaluated Nuclear Structure Data Files". In: Nuclear Instruments and Methods in Physics Research Section A 726, pp. 17-30. ISSN: 0168-9002. DOI: 10.1016/j.nima.2013.05.045.

\section{Published software}

\begin{tabular}{|c|c|c|c|}
\hline & Name & Lines of code $e^{a}$ & $\begin{array}{c}\text { Development effort est. } \\
\text { (person-years) }\end{array}$ \\
\hline 2 & $\begin{array}{l}\text { PAC-Suite (Nagl, 2008-2014) } \\
\text { PacMan } \\
\text { PacMaster } \\
\text { SpectraPAC }\end{array}$ & 26219 & 6.17 \\
\hline & $\begin{array}{l}\text { Scintillate-Suite (Nagl, 2009-2014) } \\
\text { Scintillate! } \\
\text { Multiply! } \\
\text { Evaluate! }\end{array}$ & 5333 & 1.16 \\
\hline$\frac{n n}{n \sqrt{n d}}$ & Nuclei (Nagl, 2012-2014) & 5804 & 1.27 \\
\hline & Total: & 37356 & 8.6 \\
\hline
\end{tabular}

${ }^{a}$ generated using SLOCCount by David A. Wheeler (Wheeler, 2004)

${ }^{b}$ estimated according to the basic COCOMO model (Boehm, 1981) 



\section{Acknowledgements}

An dieser Stelle möchte ich allen danken, die einen Beitrag zum Gelingen dieser Arbeit geleistet bzw. mich in den vergangenen Jahren unterstützt haben.

Meinem Betreuer Hans Hofsäss danke ich für das entgegengebrachte Vertrauen und die wissenschaftliche Freiheit, die ich während der Promotionszeit genossen habe.

Den Korreferenten Hans-Ulrich Krebs und Reinhard Krause-Rehberg gilt mein Dank für die Begutachtung meiner Arbeit. Reinhard Krause-Rehberg möchte ich weiterhin für die Einladung zu dem von ihm organisierten Workshop „Digital Signal Processing in Nuclear Science“ danken, der einigen Einfluss auf die Richtung meiner Arbeit hatte.

Den übrigen Mitgliedern der Prüfungskommission danke ich für die konstruktive Atmosphäre während meiner Disputation.

Meinen Kollegen möchte ich für angenehme Zusammenarbeit und interessante Diskussionen danken. Besonders hervorzuheben ist hier Ulrich Vetter, der mich ursprünglich überzeugt hat nach Göttingen zu kommen und seither meine Arbeit entscheidend unterstützt hat. Weiterhin Daniel Jürgens für seine unübertreffliche Hilfsbereitschaft und Christoph Brüsewitz für all die Diskussionen zu den Untiefen der TDPAC-Methode.

Für die Umsetzung und Optimierung meiner Pläne für die neue ISOLDE-Festkörperphysik-Infrastruktur und die Fertigstellung des Hochtemperaturofens gilt mein besonderer Dank Christof Schmidt und seinen Mitarbeitern, die in diese Aufgaben hunderte Arbeitsstunden investiert haben. Für die Abwicklung einer riesigen Zahl von Bestellungen im Rahmen dieser Projekte möchte ich unserem Sekretariat danken.

Für Hilfe bei der Lösung der kleinen Probleme des Wissenschaftsalltags danke ich den Werkstätten des II. Physikalischen Instituts.

Arnold Krille gilt mein Dank für die Zusammenarbeit im Bereich der Detektorsimulation und zahlreiche interessante Diskussionen.

Ulli Köster danke ich für die Unterstützung an ISOLDE und dem ILL und die Eröffnung neuer Perspektiven bezüglich der Nutzung von LOHENGRIN als Ionenquelle für TDPAC-Studien.

I am thankful to J. G. Correia for his support during several beam times at ISOLDE as well as very insightful discussions concerning the new chambers and the TDPAC method in general.

I would also like to thank Karl Johnston for support during ISOLDE beam times and Jens Röder for helpful discussions.

I appreciate the provisioning of beam time by the ISOLDE Collaboration and by the Institut Laue-Langevin. 
Nicht zuletzt möchte ich Sajah, Amane und Mione für ihre Unterstützung und Geduld, sowie meinen Eltern für all die Kinder-Notbetreuungs-Einsätze herzlich danken!

Meinen Freunden außerhalb der Universität, beispielhaft sei hier Sebastian Thias genannt, danke ich dafür, dass sie mich davor bewahrt haben zu einem Opfer des StockholmSyndroms zu werden.

Diese Arbeit wurde finanziert vom Bundeministerium für Bildung und Forschung (Projektnummern 05KK7MG1, 05K10MG3 und 05K13MG1). 